


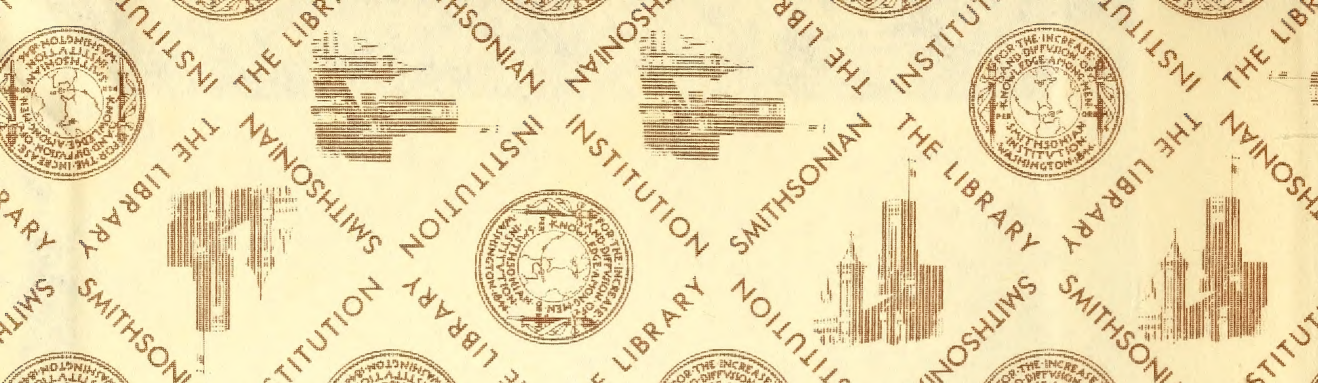

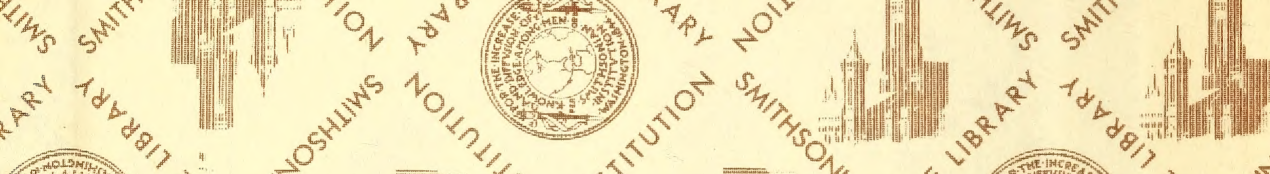

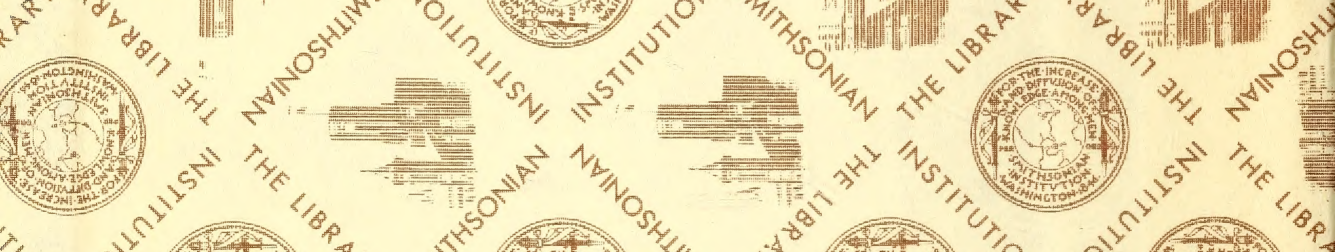

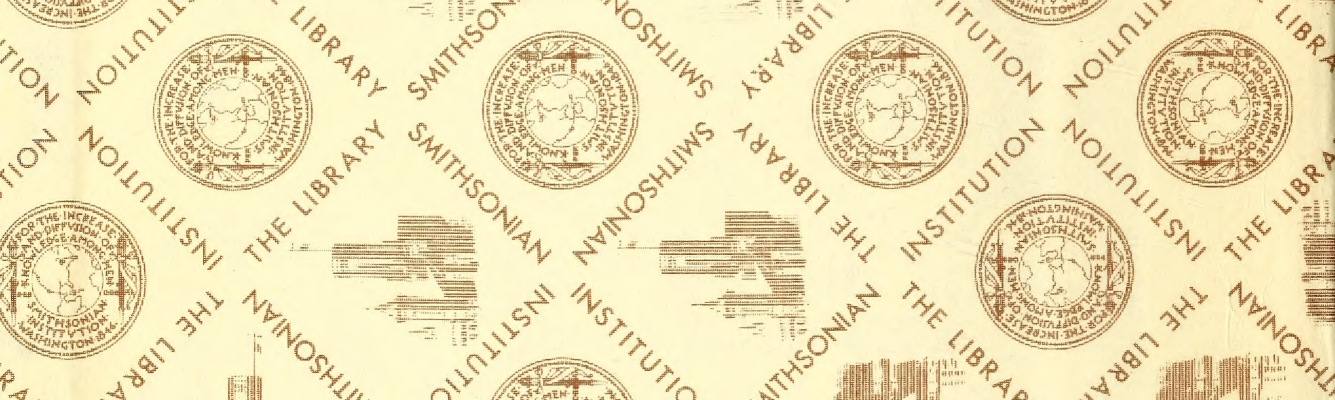

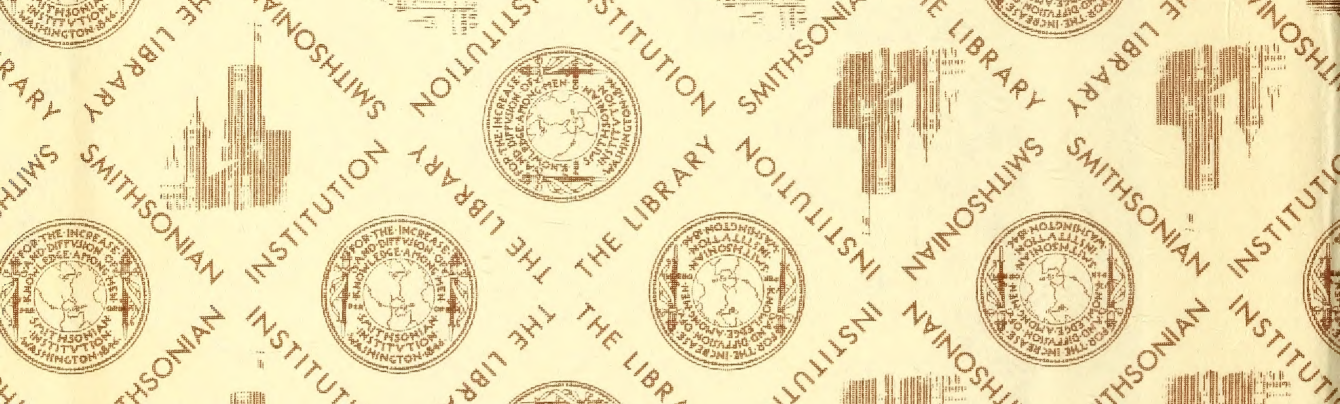

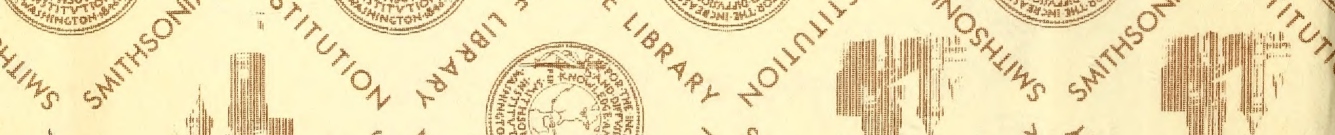

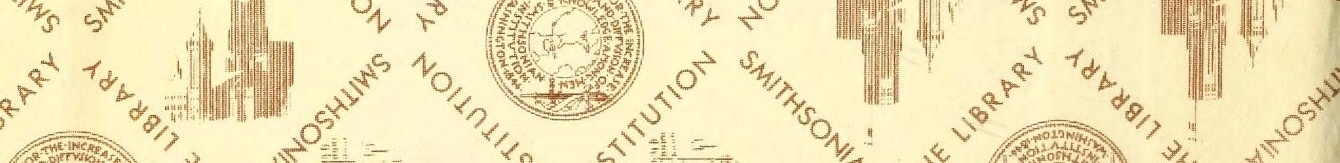

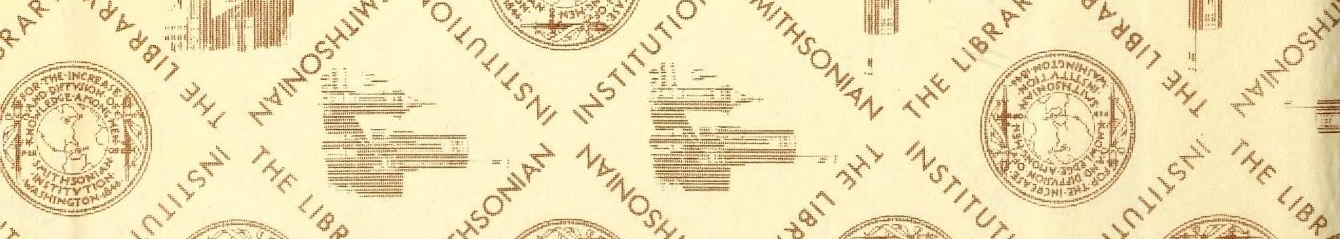

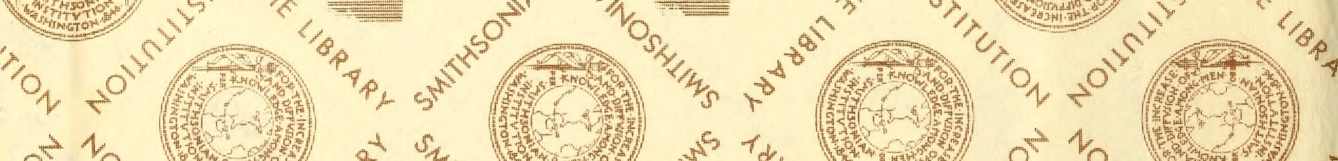

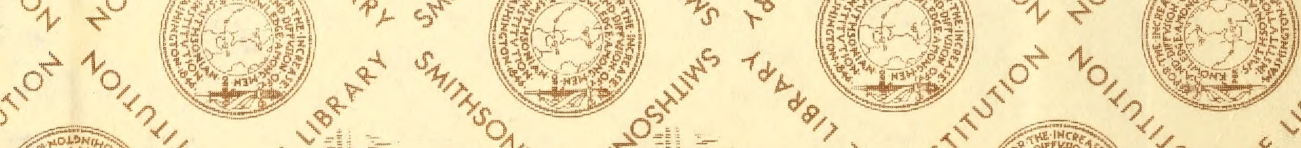

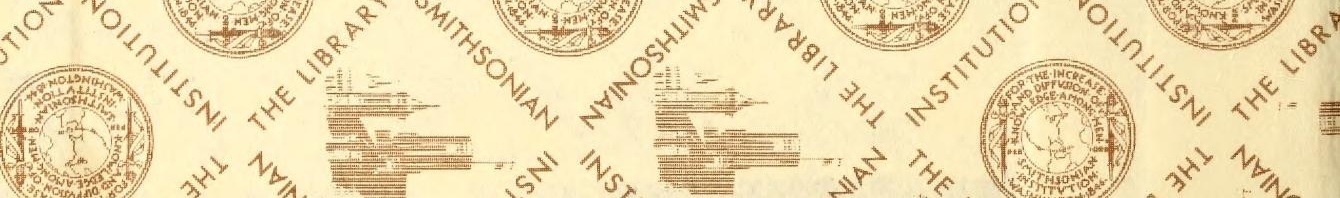

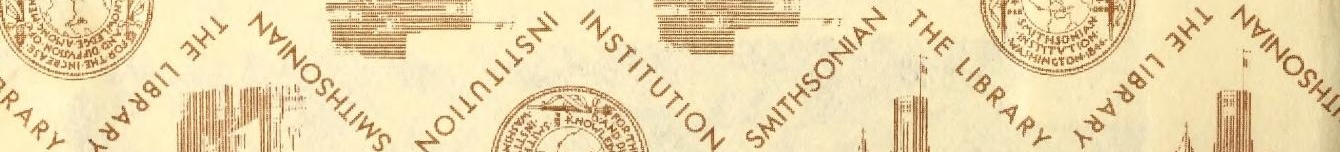




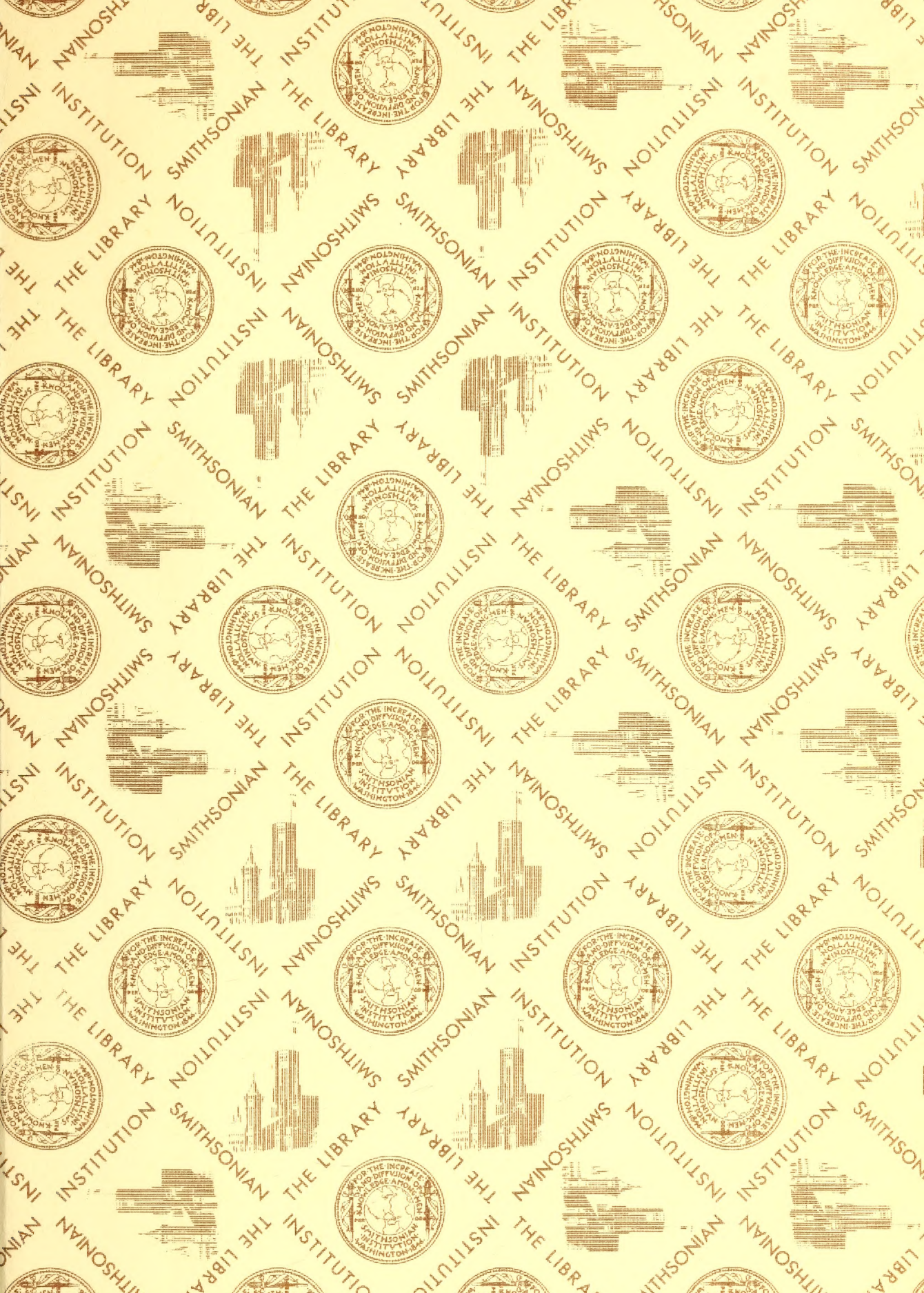





Entered according to the Act of Congress, in the year 1894, by W. L. Z U I L L,

In the Office of the Librarian of Congress at Washington.

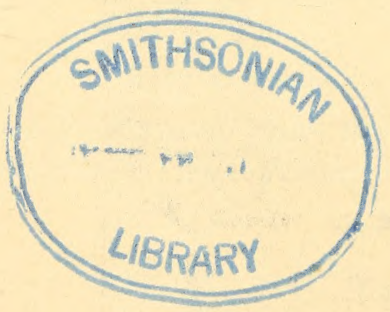

PRINTED BY WM. J. DORNAN, PHILADELPHIA. 


\section{VOLUME I.}

DISEASES OF THE DIGESTIVE ORGANS.

DISEASES OF THE URINARY ORGANS.

DISEASES OF THE GENITAL ORGANS.

DISEASES OF THE HEART AND LARGE BLOOD. VESSELS.

DISEASES OF THE SKIN. 



\section{TRANSLATOR'S NOTE.}

Durịng the nine years in which I was Professor of Veterinary Surgery and Obstetrics in the Veterinary Department of the University of Pennsylvania, the fact was daily brought to my mind with ever-increasing force that there was absolutely no satisfactory text-book on the practice of veterinary medicine for Englishspeaking students and practitioners. Those who could read German or French were particularly fortunate, as they had choice of a number of scientific and up-to-date works on this subject; the others were obliged to content themselves with what notes they could make on the lectures of their teachers.

A knowledge of this paucity in English veterinary literature, together with the conditions which were responsible for it, was the principal cause which prompted me to endeavor to fill this great void as quickly and as completely as possible. This could be done in but one way, viz., by the translation of one of the many valuable productions existing in French or German. The need for such a work was too urgent and the time too limited to permit of anything original on this subject.

It appeared to me that the very admirable production of Professors Friedberger and Fröhner would be most suited to our demands; its merits were so unquestionable and its value so thoroughly appreciated and understood, that it had even been adopted by the French as a text-book. No work has yet appeared in any language so thoroughly scientific and exhaustive as that of Friedberger and Fröhner, a translation of which I here offer to the English-speaking veterinary surgeon and student. In making this translation the latest editions both German and French have been used.

It will be seen by making a comparison with the original work that the bibliography has been excluded; this has been done for the reason that it tended to encumber the book with a mass of material of but little value to the general practitioner; those few 
readers who may have use for it are referred to the original text.

In order to make the English translation more perfect I have made use of very many of the annotations by the French translators whenever, in my opinion, they enhanced the value of the original work. In a few instances I have retained annotations of Professor Trasbot, but have carefully excluded all which appeared to me to be simply criticisms or which added nothing to the facts already contained in the text.

Throughout this work the reader will notice that I have made many additions to the text; these are indicated by being enclosed in brackets [] and signed. with the initials of the translator. This has been done only where American methods of treatment differ radically from those indicated by the authors, and it is hoped that it will increase the value of the work as a text-book.

That English-speaking veterinary students and practitioners might be provided with a work of reference compatible with the requirements of the profession was the desire actuating me in this undertaking, and is the only excuse I have to offer for the translation here presented. If I have succeeded in the accomplishment of my desire I shall feel myself amply rewarded.

I must acknowledge with thanks the very valuable services of Mr. A. F. Tilly, whose painstaking care in the reading of the proof has relieved me of much anxiety.

857 NORTH Broad STREET,

W. L. ZUILL. Philadelphia.

\section{ANNOTATIONS.}

N. D. A. . . . . Notes of the Authors.

N. D. T. - . . . Notes of French Translators.

W. L. Z. - . - . Notes of English Translator.

L. T. $\quad$ - . - Professor Trasbot. 


\section{CONTENTS OF VOL. I.}

\section{SECTION I.}

\section{DISEASES OF THE DIGESTIVE ORGANS.}

Stomatitis : Inflammation of the buccal mucous membrane.$\quad 17$

1. Stomatitis, erythematous and catarrhal . . . . . 18

2. Stomatitis, aphthous . . . . . . . . . . 20

3. Stomatitis, ulcerous . . . . . . . . . . . . . 21

Parotitis

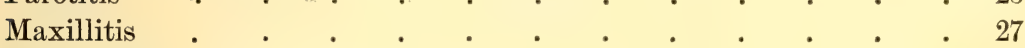

Pharyngitis . . . . . . . . . . . . . $\quad .28$

Catarrhal inflammation and purulent collection of the guttural pouches 42

Diseases of the osophagus . . . . . . . . . . 45

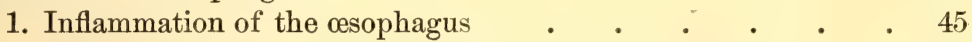

2. Dilatation of the œsophagus . . . . . . . . . . 446

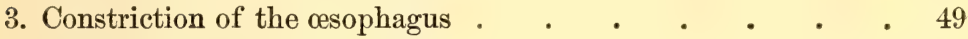

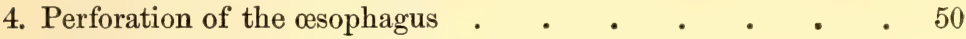

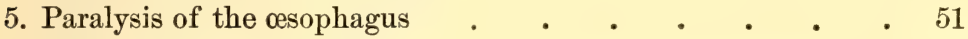

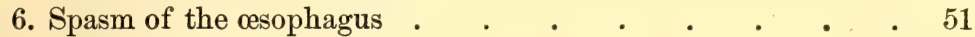

Diseases of the crop of birds . . . . . . . . 52

1. Inflammation of the mucous membrane of the crop a . 52

2. Obstruction of the crop . . . . . . . . . 53

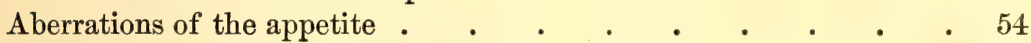

1. Pica of the ox : Licking-disease . . . . . . . 54

2. Sheep which eat wool (Mallophagia) • • • . $\quad . \quad$. 63

Acute gastro-intestinal catarrh of the horse . • • • • . $\quad$ • 66

Chronic gastro-intestinal catarrh of the horse $\quad$ • . . $\quad$. 82

Acute gastro-intestinal catarrh of the ox . . . . . $\quad$. 90

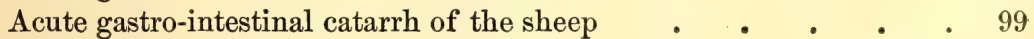

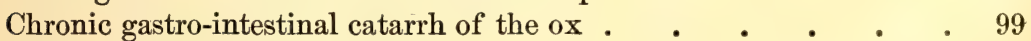

Acute tympanites of ruminants . . . . . . . $\quad . \quad 105$

Chronic tympanites of ruminants . . . . . . . . 113

Overloading of the rumen in the ox . . . . . . . . 114

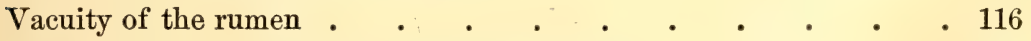

Catarrh of the stomach and duodenum of the ox $\quad$. $\quad . \quad$. 117

Gastro-intestinal catarrh of young animals . $\quad . \quad$. $\quad . \quad 118$

Gastric troubles caused by the presence of foreign bodies in the stomach

of ruminants . . . . . . . . . . . 123 
Gastro-intestinal catarrh of carnivora

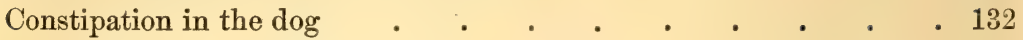

Foreign bodies swallowed by dogs . . . . . . . 135

Gastro-intestinal catarrh of pigs . . . . . . . . 138

Gastro-intestinal catarrh, constipation, and diarrhea of poultry . $\quad$. 139

Colics of the horse . . . . . . . . . . 141

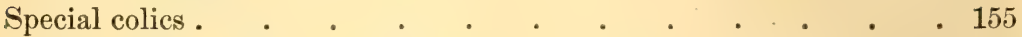

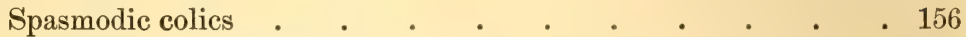

Colics from overloading . . . . . . . . . . . 157

Thrombo-embolic colic . . . . . . . . . . . . 161

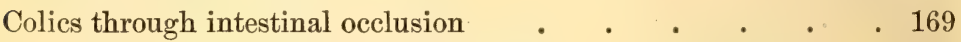

1. Coprostasis : Colics of constipation . . . . . 169

2. Colics caused by calculus or foreign bodies in the intestinal

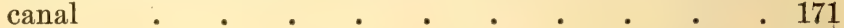

3. Colics caused by change of position of the intestine: Volvulus . . . . . . . . . . 173

4. Colics due to invagination . . . . . . . 175

5. Colics due to neoformations which diminish the intestinal calibre . . . . . . . . . . 176

6. Colics due to constriction of the intestine . . . . 177

7. Colics due to dilatation and paresis of the intestine . 178

Gaseous colics . . . . . . . . . 179

Verminous colics . . . . . . . . . . . . 180

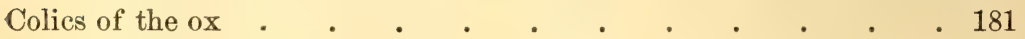

1. Colics due to invagination . $\quad . \quad$. $\quad . \quad$. $\quad . \quad$. $\quad .181$

2. Colics due to intestinal strangulation . . . . . . $\quad . \quad 183$

3. Other colics of the $\mathrm{ox} \quad$. $\quad$ • . . . . . . . 183

Colics of the $d$ log . . . . . . . . . . . . . . 184

Colics of the pig . . . . . . . . . . . 185

Ulcers of the stomach and intestine . . . . . . . . 185

Gastric and intestinal hemorrhages . . . . . . . 190

Gastro-enteritis . . . . . . . . . . . 193

Simple gastro-enteritis . . . . . . . . . . . 194

Croupous enteritis . . . . . . . . . . . . 199

Mycotic or infectious gastro-enteritis . . . . . . . 201

Mycotic gastro-enteritis of carnivora, of omnivora and poultry 204

Mycotic gastro-enteritis of herbivora . . . . . 207

Toxic gastro-enteritis . . . . . . . . . 211

I. Toxic gastro-enteritis produced by acrid poisons ․ . . . 212

Poisoning by caustic alkalies . . . . . . . 212

Poisoning by caustic acids . . . . . . . 212

Poisoning by sea salt and brine . . . . . 213

Poisoning by sulphate of soda . . . . . . . 214

Poisoning by nitre (nitrate of potash) . . . . . 215

Poisoning by tartar emetic . . . . . . . 216

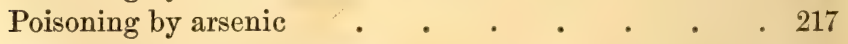

Poisoning by arsenic, acute . . . . . . . 217 
Poisoning by arsenic, chronic, in the ox . . . . 218

Poisoning by phosphorus . . . . . 。 . 219

Poisoning by mercury . . . . . . . . 221

Poisoning by lead . . . . . . . . . . 224

Poisoning by copper . . . . . . . . 225

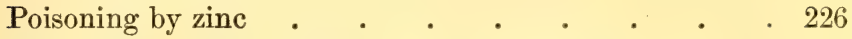

Poisoning by carbolic acid . . . . . . 227

Poisoning by acetic acid . . . . . . . 228

Poisoning by cantharides . . . . . . . 228

Poisoning by croton oil . . . . . . . . . 228

Poisoning by euphorbia . . . . . . . . 229

Poisoning by mercurialis annua et perennis . . . 229

Poisoning by daffodils . . . . . . . . 229

Poisoning by laurel . . . . . . . . . . 230

Poisoning by rape cake . . . . . . . . 230

Poisoning by pepper. . . . . . . . . 230

Poisoning by acorns . . . . . . . . 230

Poisoning by aloes . . . . . . . . . . 231

Poisoning by spurge mezereum . . . . . 231

Poisoning by wild horse-radish . . . . . 231

Poisoning by swallow-wort . . . . . . . 231

Poisoning by cotton-seed meal . . . . . . $\quad$. 231

Poisoning by turpentine plants . . . . . 232

II. Toxic gastro-enteritis caused by acrid narcotics . $\quad . \quad$. 233

Poisoning by colchicum . . . . . . . 233

Poisoning by tobacco . . . . . . . . 234

Poisoning by yew leaves . . . . . . . . 234

Poisoning by digitalis . . . . . . . . . 235

Poisoning by flax . . . . . . . . . . 236

Poisoning by box leaves . . . . . . . . 236

Poisoning by black, green, and fetid hellebore • . 236

Poisoning by veratrum . . . . . . . . 236

Poisoning by crow-foot . . . . . . . . 237

Poisoning by aconite . . . . . . . 237

Poisoning by poisonous hemlock . . . . . 237

Poisoning by American water hemlock . . . . 238

Poisoning by small hemlock . . . . . . 238

Poisoning by field poppy . . . . . . . 238

Poisoning by fennel seed . . . . . . . . 239

Various poisonings by acrid narcotic plants . . . 239

Poisoning by ergot of rye . . . . . . . . . . 239

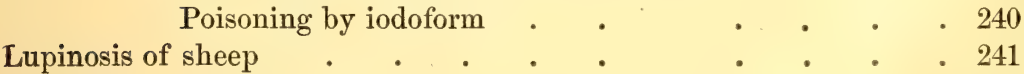

Lupinosis of the horse . . . . . . . . . 248

Trifoliosis of the horse . . . . . . . . . . . 249

Purely narcotic poisonings . . . . . . . . . . . $\quad$. 250

Poisoning by strychnine . • . . . . . 250 
Poisoning by beech-tree fruit

Poisoning by solanum

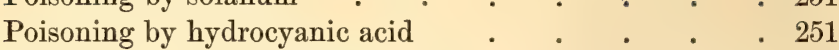

Poisoning by furrow-weed . . . . . . 252

Poisoning by alcohol . . . . . . . 252

Poisoning by horse-tail grass, scouring rush, shave-grass 252

Poisoning by oxide of carbon . . . . . . . 254

Poisoning by lathyrus cicera . . . . . . 254

Poisoning by viper bite . . . . . . . 254

Intestinal helminthiasis. . . . . . . . . . 255

I. Plathelminths . . . . . . . . . . . 256

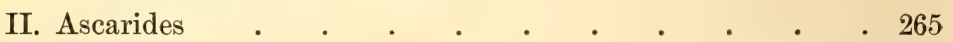

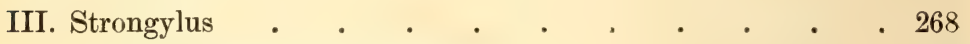

A. Sclerostoma of the horse . . . . . . . 268

B. Strongylosis of the abomasum of sheep . . . . . 269

IV. Oxyuris . . . . . . . . . . . . 274

V. Giant echinorrhynchus of the pig . . . . . . 274

VI. Various other intestinal parasites . . . . . . . 275

Diseases of the liver . . . . . . . . . . . 276

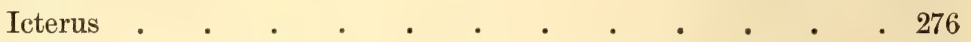

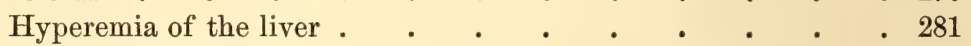

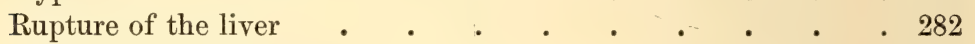

Parenchymatous hepatitis . . . . . . . . 284

Suppurative hepatitis . . . . . . . . . 285

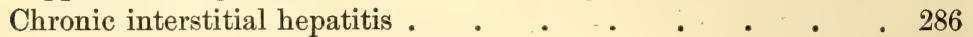

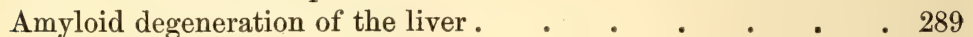

Carcinoma of the liver . . . . . . . . . . 290

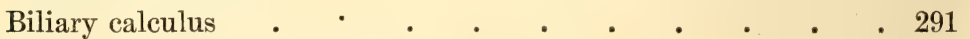

Parasitic diseases of the liver. . . . . . . . . . 292

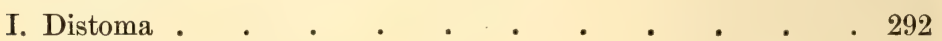

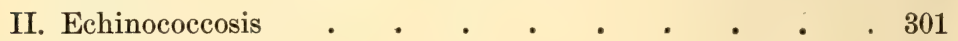

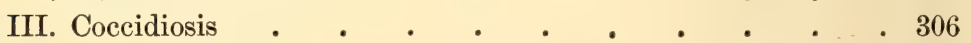

Diseases of the pancreas . . . . . . . . . 309

Pancreatitis . . . . . . . . . . . . . 309

Diseases of the spleen . . . . . . . . . . . 311

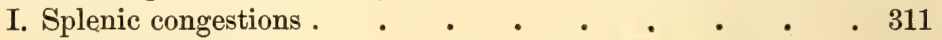

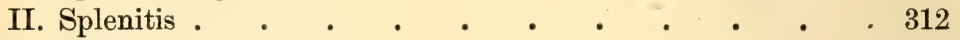

III. Rupture of the spleen . $\quad$ • . . . . . . 314

Diseases of the peritoneum . . . . . . . . . 316

Acute peritonitis . . . . . . . . . . . 316

Chronic peritonitis . . . . . . . . . . . 325

Ascites . . . . . . . . . . . . 326 


\section{SECTION II.}

\section{DISEASES OF THE URINARY ORGANS.}

Importance of urinalysis in morbid conditions, particularly in affections of the kidneys . . . . . . . . . 336

A. Chemical analysis of urine . . . . . . . . 337

B. Physical examination of urine . . . . . . . . 347

Congestion of the kidneys . . . . . . . . . . . 352

Nephritis . . . . . . . . . . . . . . . .

Acute nephritis . . . . . . . . . . . 356

Chronic nephritis . . . . . . . . . . . . 362

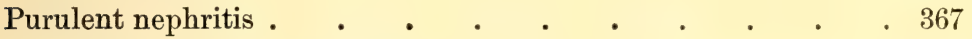

Amyloid degeneration of the kidneys . . . . . . . . . 368

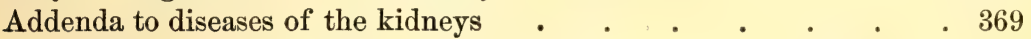

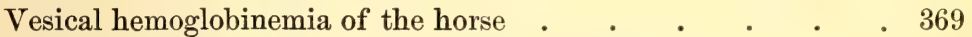

Hemoglobinemia of the ox and other ruminants . . . $\quad 380$

Pyelitis . . . . . . . . . . . . 387

Retention of the urine . . . . . . . . . . . 388

Cystitis . . . . . . . . . . . 392

Hematuria . . . . . . . . . . . 394

\section{SECTION III.}

DISEASES OF THE GENITAL ORGANS.

Parturition fever . . . . . . . . . . . . . . 396

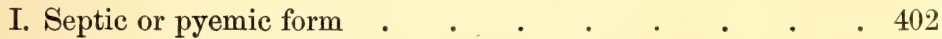

II. Toxic or paralytic form . . . . . . . . . 408

III. Puerperal septicemia complicated by paralysis . . . 414

Nymphomania: Satyriasis . . . . . . . . . . 416

Weakening of the genesic instinct . . . . . . . . . . 423

Impotency and sterility . . . . . . . . . . . . 424

Anomalies of lactation and alterations of the milk . . . . . 426

1. Agalorrhea . . . . . . . . . . 427

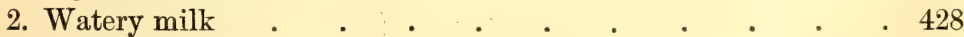

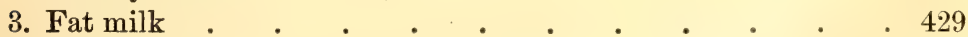

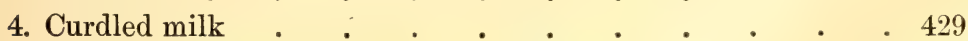

5. Milk which does not produce any butter . . . . . 430

6. Putrid milk 
7. Mucous, oily, viscous, filamentous milk . . . . . 431

8. Blue milk . . . . . . . . . . . 432

9. Foreign products in milk . . . . : . . . 435

\section{SECTION IV.}

\section{DISEASES OF THE HEART AND LARGE BLOODVESSELS.}

General anatomical and physiological considerations on diseases of the heart: Physical diagnosis . . . . . . . . 439

Hypertrophy and dilatation of the heart . . . . . . . . . 443

Pericarditis . . . . . . . . . . . . 450

I. Pericarditis of the ox and goat: Traumatic carditis and pericarditis . . . . . . . . . 450

II. Pericarditis of the horse and other domestic animals $\quad$. $\quad 456$

Palpitations: Chorea of the diaphragm $\quad$. $\quad . \quad$. $\quad . \quad 459$

Myocarditis . . . . . . . . . . . . . . . 462

Ossification of the auricles of the heart . . . . . . $\quad$. 465

Rupture of the heart . . . . . . . . . . . . . . . 466

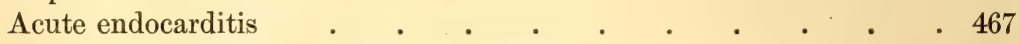

Chronic endocarditis: Valvular alterations . . . . . . . . 471

Aneurism of the aorta . . . . . . . . . . . . 481

Rupture of the large vessels of the pectoral and abdominal cavities $\quad 483$

Thrombosis of the branches of the aorta $\quad$. $\quad . \quad$. . . 484

Hematozoa . . . . . . . . . . 487

\section{SECTION $V$.}

DISEASES OF THE S SKIN.

General considerations . . . . . . . . . . . 489

I. Non-parasitic diseases of the skin . . . . . . 492

Erythematous dermatitis . . . . . . . . . 492

Eczema . . . . . . . . . . . 495

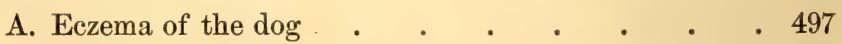

B. Eczematous diseases of the horse . . . . 504

1. Papulo-vesiculous eczema . . . . . 504

2. Squamous chronic eczema . . . . . 507 
3. Chronic impetiginous eczema of the regions which are covered by the mare . . . . . 508

4. Eczema of the flexion surfaces of the inferior articulations of the legs . . . . . . . 511

C. Eczematous diseases of the ox . . . . . 513

D. Eczematous diseases of sheep . . . . . . 521

E. Eczematous diseases of pigs . . . . . . . . 522

Urticaria . . . . . . . . . . . . 523

1. Urticaria of the horse, $\mathrm{dog}$, and ox . . . . 524

2. Urticaria of the pig . . . . . . . 525

Gangrenous dermatitis . . . . . . . 527

Bullous dermatitis . . . . . . . . . 528

Shedding of hair and wool . . . . . . : 530

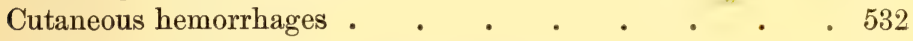

II. Skin diseases cured by vegetable parasites . . . . . 534

Depilating thrush . . . . . . . . . 535

Favus . . . . . . . . . . . . . . 541

Contagious pustulous dermatitis . . . . . . 544

III. Cutaneous diseases produced by animal parasites . . . 548

Mange of domestic mammiferous animals $\quad$. $\quad . \quad 548$

Mange of the horse $\quad . \quad$. $\quad . \quad$. $\quad . \quad$. 557

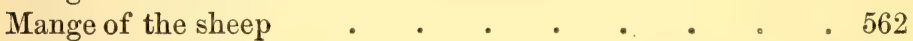

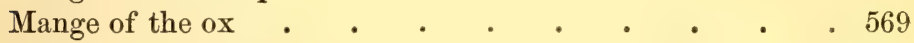

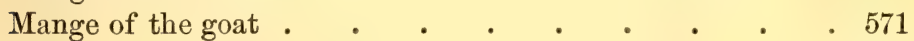

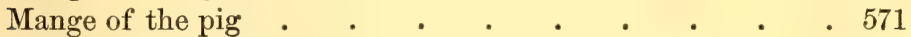

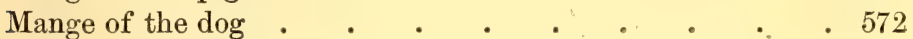

Mange of the cat . . . . . . . . . . . 576

Mange of the rabbit $\quad . \quad$. $\quad . \quad$. $\quad . \quad 577$

Mange of the ferret . . . . . . . . 578

Follicular mange . . . . . . . . • • . 578

Diseases produced by filaria . . . . . . . . 586

Skin diseases caused by insects . . . . . . . . . 587

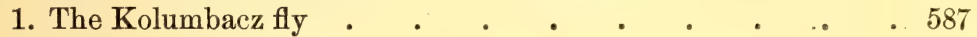

2. Hypoderma of the ox . . . . . . . . 588

3. Larvæ of the Lucilia . . . . . . . . . . 589

Lice and trichodectes . . . . . . . . . . . 590

Various other parasites of the skin . . . . . . . 591

1. Fleas . . . . . . . . . . . . 591

2. Ticks . . . . . . . . . . . . . 592

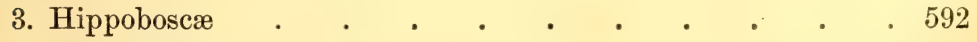

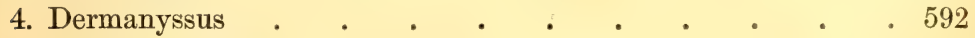

5. Autumnal lepta . . . . . . . . . . . . 593

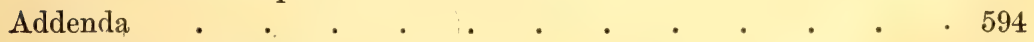

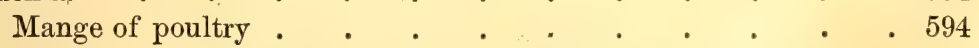

Other cutaneous diseases of poultry caused by animal parasites . 596 



\title{
PATHOLOGY AND THERAPEUTICS
}

\author{
OF THE

\section{DOMESTIC ANIMALS.}

\author{
SECTION I. \\ DISEASES OF THE DIGESTIVE APPARATUS.
}

\section{STOMATITIS: INFLAMMATION OF THE BUCCAL MUCOUS MEMBRANE.}

WE will not describe here stomatitis of a surgical order, produced by traumatic irritation (bits, foreign bodies, rough manipulations); chemicals (potash, ammonia, alum, croton oil, cantharides, emetics); hot substances (burns by drenches, by foods, by too hot drinks); and those which originate as phenomena of other affections (febrile diseases, papillomatosis of the dog and young horse, aphthous fever, petechial fever, etc.), or those which are developed by continuity of tissue, during the course of a laryngitis, or a pharyngitis, or a rhino-adenitis. Excluding these, we may recognize in inflammations of the mucous membrane of the mouth the following forms as those that are more or less characteristic :

1. Catarrhal or erythematous stomatitis;

2. Aphthous stomatitis;

3. Ulcerative stomatitis;

4. Pustulo-contagious stomatitis ;

5. Diphtheritic stomatitis;

6. Pultaceous stomatitis.

The first three must be studied as diseases of the digestive apparatus; the others belong to the list of contagious diseases. 


\section{Simple Catarrh of the Mucous Membrane of the Mouth: Erythematous (Erysipelatous) Catarrhal Stomatitis.}

Etiology. Erythematous stomatitis is observed in all our domestic animals, and particularly in the horse, whenever through the influence of any cause the prehension of food is interfered with for a sufficient length of time. It is easily produced by the different diseases of the digestive organs: the epithelium of the mucous membrane of the mouth, not being scoured off by the alimentary substance as it is exfoliated, decomposes in situ, and becomes an irritating agent. It is also frequently the result of the anorexia which accompanies the ordinary febrile diseases of long duration, in which condition both the decrease in the salivary secretion and the morbid properties of the saliva itself, especially favor the decomposition of the desquamated epithelium.

The pathogenic influence of various fungi introduced with the food is well known. Special mention is to be made of the mouldiness of oats and fodder, grain rust (Puccinia graminis), blight or black rust (Erysiphe communis), rape fungus (Polydesmus exitiosus), brown rust of grain (Tilletia caries). Zürn ascribes these rusts especially to Oidium albicans. We may also recognize the existence of a stomatitis (erysipelatous), due to eating hay that is mixed with nettles (Bombyx, or Onethocampa processionea).

Stomatitis of dentition is probably due to traumatisms caused by the exfoliation of the teeth from their alveolar cavities, and to hyperemia and inflammation of the gums.

It has also been ascribed as due to the action of cold, but stomatitis arising from this cause is extremely doubtful.

Symptoms. These vary according to the gravity of the disease. In mild cases we observe at the beginning a more or less marked dryness, and an elevation of the temperature of the mucous surfaces; later this is covered with a slimy matter, grayish, thin, bullous, and spumous, especially abundant on the upper surface of the tongue. The mucous membrane of the lips and cheeks is injected and of a more or less dark-red color (erythematous stomatitis); the anterior part of the palate may be tumefied by stasis in the venous circulation of the very vascular tissues of the submucous membranes; the mouth exhales a fetid odor.

The coated tongue is the result of an exaggerated epithelial production, due to the catarrhal condition of the mucous mem- 
brane, and from an arrest of the functional activity of this epithelial surface, because of difficulty in the prehension and mastication of food, which is taken in very small quantity; on the other hand, the lessening of the secretions leads to dryness of the mucous membrane, and consequently to opacity of the epithelial cells, which then become more visible.

When the catarrh is more intense, the mucous membrane, especially that of the lips, cheeks, and gums, becomes redder and thicker. The exfoliation of the epithelium is copious, the back of the tongue is thickly covered and streaked a dirty green or brown by the alimentary particles. In the cat the papillæ of the tongue are white or yellowish, and are quite different from the edge, which is of a bright red. In cattle and pigs we sometimes observe hypertrophy of the inflamed conical papillæ, which were wrongly looked upon as a formation of aphthæ (thrush of cattle's tongue). The odor exhaled from the buccal cavity becomes offensive. At the same time there is a hypersecretion of saliva and mucus, which run out of the corners of the mouth in the form of thick threads. When the saliva is very abundantly secreted, it sometimes flows from the mouth in an uninterrupted stream; at other times it is converted into froth through mastication. ${ }^{1}$

Certain authors devote a special chapter to abundant salivation (ptyalism), but it constitutes only a symptom common to a number of diseases, among which we find in the first place stomatitis, then affections of the stomach, intestine, uterus, ovaries, and the brain. Ptyalism is sometimes a symptom of helminthiasis, otorrhea, and parotitis. In several cases observed in the dog, we were not able to determine its cause; it is perhaps sometimes of cerebral origin. The observations made in the horse and ox, in which ptyalism has been attributed to the use of damaged fodder, where no sign of stomatitis was to be detected, seem very extraordinary (see observations of Mathieu, Paugoué, Pech, and Born).

Concerning salivation due to certain medicinal substances (mercury, pilocarpine, morphine, iodine), see treatises of materia medica and toxicology.

1 In man the normal alkaline reaction of the saliva becomes acid in different diseases, notably in pleurisy, encephalitis, rickets, dyspepsia, and diabetes (GorupBesanez). In our numerous researches upon animals, we have never recognized the acid reaction of saliva. Would this latter not be due, in man, to an acid fermentation of particles of food remaining in the cavity of the mouth, or to decayed teeth, etc.? 
We sometimes find small vesicles upon the lips, cheeks, and tongue, leaving catarrhal erosions after their rupture, tumefaction of the mucous glands, and obstruction of the excretory duct of these glands, appearing in small grayish or reddish-gray pustules, surrounded by a red zone, which may ulcerate; all this is little understood. Weber has noticed the appearance of numerous small vesicles with serous contents, in stomatitis of the horse. (Was it not a case of aphthous fever?)

We have seen ulceration of the glands in the course of glanders and in a rickety horse; Möbius has observed it after the feeding of clover. These alterations have erroneously been called follicular stomatitis by some practitioners.

The epiornitic affection known as "pip" in our poultry yards, though it is a disease especially of the respiratory organs, is often accompanied by a catarrhal inflammation of the mouth (Zürn and others). The continual half-opened condition of the bill leads to drying of the tongue, the horny covering of which becomes opaque and hard, which may be mistaken for a pathological membrane.

Treatment. The cause of the trouble must first be removed, then light solutions of carbonate of soda or of sea-salt may be used, in order to dissolve the mucus. A mouth-wash made of 1 quart of water, $\frac{1}{10}$ of a quart of vinegar, and a spoonful of sea-salt is an old and very popular remedy. Gargling with a solution of 1-3 per 100 of boric acid is very efficient. A solution of 1-4 per 100 of chloride of potassium is also very good, but deglutition of the liquid must be avoided, for in small animals this drug might provoke a dangerous bleeding.

Solutions of permanganate of potash, much used lately, are not advisable: their disinfecting action is very weak and their taste extremely disagreeable.

Any astringent sufficiently diluted may be useful, but in most cases fresh and clean spring-water alone will hasten a cure. In very serious cases a $1: 100$ solution of carbolic acid can be used.

\section{Aphthous Stomatitis (Vesicular): Aphthæ of the Mucous} Membrane of the Mouth.

The term "aphthæ" has only acquired a definite meaning since the researches of Bohn. Before these were made the term was used 
to designate any vesicular eruption on the mucous membrane of the mouth. According to Bohn, aphthæ consists of a croupous inflammation limited to the mucous membrane. It is characterized by a fibrinous exudate, pseudo-diphtheritic, while the vesicular lesion of catarrhal stomatitis consists of a sub-epithelial serous collection. The type of aphthæ is furnished by aphthous fever.

Aside from this contagious disease, it is not certain that aphthæ is developed in our domestic animals.

It is probable that the aphthoe or spongeoles of the mouth, described by Hertwig in his Treatise on the Diseases of Dogs, are false aphthæ. We have never recognized them in the horse or dog. Moreover, all authors agree that the transmission of aphthous fever to these two species is extremely rare.

Anacker has mentioned the possibility of confounding aphthous fever with the vesicular form of catarrhal stomatitis. Zeilinger ${ }^{1}$ and Kohler ${ }^{2}$ have seen aphthous fever in the horse. Haubner, Uhlich, and Adam have noticed the transmission to the dog and cat. See Aphthous Fever. (See also the article of Cauvet ${ }^{3}$ upon the aphthæ of the horse.)

\section{Ulcerative Stomatitis.}

Ulcerative stomatitis is a necrotic inflammation of the mucous membrane of the mouth, and especially of the gums. Anatomically it constitutes a higher grade of the aphthous process than the preceding form, and is recognized by the formation of ulcerations upon the mucous membrane.

Etiology. Traumatisms sometimes determine ulcerative stomatitis. It is often found in weak anemic dogs, and on those of weak and delicate constitution, and it has been observed that the smaller breeds are particularly predisposed to it. Previous diseases (such as gastro-enteritis, rickets, etc.) leaving the organism in a weak condition often help to develop it. In the cat it may be produced by the same causes. The ulcerations accompanying this form of stomatitis have been wrongly called scorbutic (Hertwig), for they may be seen to change rapidly in animals which are otherwise perfectly healthy, having never shown any hemorrhage of the mucous membrane (see Scorbutus). Their

1 Zeilinger, Adam's Wochenschr., 1864.

3 Cauvet, Journ. du Midi, 1861.

2 Kohler (communication). 
development is perhaps favored by particular individual conditions abnormally vulnerable, a lack of resistance of the mucous membrane acting in connection with an infection. In certain cases decayed teeth, or teeth covered with tartar, are the startingpoint of the disease in producing an infectious gingivitis ; nevertheless, the morbid process may start at a spot quite distant from the teeth.

We shall study ulcerative stomatitis due to mercury, lead, phosphorus, etc., in the section treating of Poisons.

We have never noticed this disease in an enzootic or contagious form, though Hackbarth has observed it in an enzootic condition upon horses pasturing in hybrid clover fields. Rivolta and Berndt have also described a very malignant ulcerative stomatitis in lambs, produced by Polydesmus exitiosus (Berndt) or bacteria (Rivolta).

Symptoms in the dog. The first signs are always localized on the gums. Their edges are of a dark-red color, and more or less tumefied near the incisors and canine teeth. After one or two days the tumefaction will have spread and the gums will show a spongy consistence; they are detached and bleed on the least friction. A little later all the diseased tissues, and especially the swollen gums, become greenish or yellowish-gray; phlegmasia progressing, the mucous membrane becomes partially necrotic. The mortified spots look spongy; they are of a glue-like, pulpy substance. If they should be removed suddenly or artificially it leaves an ulcer of one or two millimetres around the canine teeth with irregular cut edges, and forming a hard, dark-red, protruding swelling; the inside of the wound is irregular, and has a bad appearance. A thick thread of saliva, sometimes bloody or saneous, runs out of the mouth, staining the lips, nose, and cheeks. The cavity of the mouth often contains a large quantity of saliva, escaping when the jaws are distended, a movement which the dog tries to avoid. The saliva, the cavity of the mouth, and the expired air are of a fetid, cadaverous odor, which can be smelled at some distance.

The lesions upon the gums which have just been described can also be found on the lips, in the gingivo-buccal fold, on the labial commissures, more rarely on the cheeks, and exceptionally upon the lower surface of the tongue. The sores on the lips are often opposite those of the gums, which suggests an auto-infection; at 
other times their location seems to indicate the spreading of the trouble by means of the lymphatics. Some cases are met (in young dogs) where the teeth and gums are perfectly healthy, the ulcerations are on other points of the mucous membrane, and on the lips or commissures of the mouth. In the latter form we have seen the destructive ulceration spreading upon the cutaneous surface.

These accidents are always accompanied by symptoms of simple stomatitis.

Course. When the evolution of the disease is regular, and the animals young, the ulcers extend little in depth and surface. The general condition is but little influenced; fever is often absent; sometimes, however, the temperature rises to $39.5^{\circ}$; the appetite is usually retained. When once the ulcerative process is arrested and the sloughs eliminated, healing often takes place in from eight to ten days. Cases of this kind are the most numerous.

In old animals or those having suffered from other diseases, infiltration and destruction of the tissues progress rapidly; the sores become deep and sinous, the diseased teeth loosen, and their roots are covered with a grayish fetid pulp, produced by the destroyed alveolar periosteum; decay and necrosis of the maxillary may then complicate the disease. Sometimes a bucco-nasal fistula is formed with consecutive purulent rhinitis. The lips and cheeks are tumefied, hardened and deformed by an odematous infiltration surrounding the ulcerations; the lymphatic ganglions of the diseased regions are engorged. The general condition is alarming and the cure doubtful. Most frequently, persisting, intense, and increasing fever sets in, in connection with the appearance of new eruptions; the pulse becomes more frequent and weaker (in small dogs 140 to 160 pulsations per minute may be counted). Soon the appetite is entirely lost, diarrhea appears, and weakness is extreme; great stupefaction or even coma follows, and the animals die from septic infection.

Under these conditions the disease lasts from six to twelve days; it may, however, take a chronic course.

Berndt has described an ulcerous stomatitis of lambs, which is essentially a very intense stomatitis and rhinitis. He describes the symptoms thus: great weakness, staggering gait, rapid and painful breathing, short and weak cough. The mucous membrane of the mouth shows numerous erosions on the gums, on the surface 
and especially on the edges of the tongue; they are found also upon the visible parts of the pituitary mucous membrane; here they are covered with a thick dark crust. The following lesions are found at the autopsy : the nasal, laryngeal, pharyngeal, and buccal mucous membranes are inflamed and covered with erosions. The lower edge of the posterior pulmonary lobes is hepatized and of a dark-red color; the cut surface is granulous and discharges a bloody liquid. In compressing the bronchial tubes a spumous dark chocolate-colored liquid trickles out of them.

Berndt has found on microscopic examination, numerous ramified filaments and small chains of spores of the Polydesmus exitiosus, between the epithelial cells of the mucous membranes of the nose and mouth. The lambs were fed on rape cakes in which the sporules of the Ascomycetes were to be found. The progress of the disease was arrested as soon as this food was discontinued.

A contagious ulcerous stomatitis similar to the preceding case, has been described by Rivolta. It was complicated by conjunctivitis, pneumonia, and hepatitis. This author ascribes the trouble to a schizomycete (Bacterium subtile oxquorum). Gips has also described a mouth-rot in lambs.

Prognosis. The prognosis varies according to the individual. The age of the animal, extension of the lesions, and their effect upon the general condition, must be taken into consideration in order to draw our conclusions. There is much less danger for young vigorous animals than for old ones which are weak and exhausted; small lesions are much less serious than wide or confluent ulcerations. We must observe here that ulcerative stomatitis is more rarely accompanied by fatal septicemia than phagedenic accidents, with their seat in other parts of the body, for instance, in the intestine. The anatomical construction of the buccal cavity is evidently not favorable to the production of septicemia ; dentists have frequently observed this in human beings.

Treatment. Substantial food ought to be given, composed of hacked and soft substances. The animals should be put in wellventilated rooms or in the open air. The same solutions as prescribed for catarrhal stomatitis have to be used to disinfect the mouth. Carbolic acid seems to be the best therapeutic agent; it is an excellent antiseptic, it removes all bad odor and is not toxic. It may be used in a 1 to 2 per cent. solution. A solution of 1 to 2 per cent of carbolic or boric acid, corrosive sublimate in $1: 1000$, 
or a solution of tannin, etc., may be applied on the ulcerations with care. Aromatic tincture, the tincture of aloes or myrrh, and spirits of cochlearia, applied with a brush, have given good results Decayed or loose teeth must be extracted.

If septic infection already exists, the disease defies all treatment. Cauterization of the affected regions (with chloride of zinc, acetate of aluminum) cannot prevent the fatal termination.

\section{PAROTITIS: INFLAMMATION OF THE PAROTID GLAND.}

This disease, also common in man, is still designated in our animals by the vulgar name of "mumps." Its bibliography in veterinary science is very meagre; there exist but vague and incomplete descriptions of this disease, and this deficiency still remains to be remedied by statistics of well-known cases.

Etiology. The following forms of parotitis may be distinguished :

1. Traumatic parotitis, due to some mechanical lesion. It belongs to the field of surgery.

2. Idiopathic essential parotitis, produced by a miasmatic, contagious, infectious agent. It is an epizootic disease. The contagious parotitis of the goat, observed by Franze, as well as that mentioned by Hertwig on the dog, and Schüssele on the dog and cat, are all similar to mumps in man. The pathology of this form of parotitis is not yet strictly determined; the infectious agent undoubtedly reaches the gland either through the blood, or, which is more likely, through the mouth, in ascending Stenon's canal.

3. Parotitis produced by inflammatory extension. An encysted salivary calculus can become the cause of a parotitis. Toussaint has described a parotitis determined by the extension of a stomatitis along Stenton's canal through a largely dilated infundibulum. In glanders, angina, and distemper of dogs, the inflammation may spread from a neighboring tissue surrounding the parotid gland.

4. Metastatic parotitis, happening during pyemia, or septicemia.

5. Actinomycotic parotitis, due to Actinomyces bovis. It constitutes a common variety of actinomycosis, and is observed in an epizootic form. 
The disease described by Stockfleth, in his Treatise on Surgery, under the title "Chronic Inflammation of the Salivary Glands," is without doubt actinomycotic parotitis. According to his observations, animals pasturing on, or eating hay from, low swampy meadows are more exposed to contract the disease. The parotid is most frequently attacked, exceptionally the maxillary and sublingual glands are also affected. The inflammations develop slowly, the gland being hard and irregular, gradually increasing in size; they are indolent, but in most cases break down into abscess. The neighboring lymphatic ganglions almost always take part in the inflammation. As soon as the hypertrophied gland reaches a certain size it exerts pressure upon the larynx and pharynx, and becomes an obstruction to the function of respiration and deglutition. All remedial agents are absolutely useless. Extirpation of the diseased organ is the only successful treatment.

Symptoms. Three forms of parotitis are to be considered here: the idiopathic form, the form which results from an inflammation of the surrounding tissue, and the metastatic form. They present a common symptom: the swelling of the parotid gland. In the first the tumefaction is uniform, and extends over the whole organ. The other two varietics are distinguished by a limited swelling, and by consecutive abscess formation of the affected glandular lobules (see Toussaint's case). Inflammation can also spread to the surrounding tissue. In the epizootic form, observed by Franze upon the goat, the maxillary bones and glands participated in the inflammation. The patients partook of neither food nor drink.

The rational symptoms and the fever of parotitis have not yet been described.

Differential diagnosis. Parotitis has often been confounded with inflammation of the pharyngeal ganglions. The topography of these organs, and the signs furnished by the general condition of the patients, allow us to make a correct diagnosis.

Parotitis may also be simulated by purulent collection of the guttural pouches, by tumefaction produced by old jugular phthisis, by an œdematous or phlegmonous inflammation of the subcutaneous connective tissue, and by tumors of any nature developed in the parotid region.

Treatment. Parotitis showing no tendency to form abscess must be treated with moist heat and by the local application of 
ointments, of camphor, carbolic acid, or iodoform. ${ }^{1}$ When the disease shows any tendency to become chronic we may use tincture of iodine, red ointment (hyg. iod. rub.), cantharides, etc. The abscess must be opened as soon as possible. Salivary fistula, resulting from abscess formation in the gland, is to be treated in accordance with the rules of classical surgery.

\section{MAXILLITIS: INFLAMMATION OF THE MAXILLARY GLAND.}

Inflammation of the maxillary gland is usually the result of an obstruction of Wharton's canal by the introduction of foreign bodies. Most frequently it is caused by brome-grass [a kind of oats or grass belonging to the genus Bromus-W. L. z.], which when once introduced into the canal can progress as far as the gland, due to the arrangement of its barbs. The same conditions are sometimes caused by barley or oats. In exceptional cases the trouble may be produced by calculi located in the canal. Maxillitis is almost always unilateral.

The beginning of the disease is insidious. Appetite is preserved, but mastication is slow and troublesome; hard food and oats are soon left untouched. Within a few days characteristic symptoms appear. Salivation is abundant, the mouth hot, the mucous membrane injected. In examining the floor of the buccal cavity we observe a red, conical projection on the frenum of the tongue, from which a purulent fluid escapes; this is the tumefied free opening of the duct. Often food accumulates between the tongue and maxillary; on removing it we find Wharton's canal projecting and inflamed; if compressed from behind forward, a whitish purulent fluid, sometimes mixed with alimentary particles, trickles out of it. Phlegmasia of the gland is also indicated by a tumefaction in the cavity of the mouth, which is observed on the side at the base of the tongue, and is a hard or odematous, sensitive puffiness of the lingual canal.

Maxillitis ends in resolution or suppuration. If we succeed in

3 [For some years the use of moist heat has been discarded, owing to its inconvenience of application and the great difficulty of maintaining it in close apposition to the skin. In its stead we use a thick poultice of cotton-wool, which is light and clean, and in its therapeutic action far more satisfactory than moist heat.-W. L. Z.] 
expelling the foreign bodies in the salivary duct by pressure, a cure will be obtained in a few days; but if there is no intervention tumefaction of the gland increases, and leads to suppuration; sometimes the abscess opens in the mouth, sometimes the pus passes through the skin, in the inter-maxillary space. Wharton's canal becomes voluminous and its walls are often perforated, when the pus passes through it with difficulty. When there is abscessformation the saliva and pus always exhale a fetid odor. Partial gangrene of the gland is a rare complication. Salivary fistules which may persist after suppuration, or limited mortification of the gland, may become obliterated after a certain time.

The duration of the disease varies from eight to fifteen days.

Maxillitis is a benign disease. A rapid cure can always be obtained, provided it is diagnosticated at the beginning. Suppuration and gangrene give it some gravity, but fatal complications are never to be feared.

Treatment. Foreign bodies hindering the flow of the saliva must be removed from the canal ; this is the first thing to do in all cases. As a rule, a moderate pressure upon the duct, from behind forward, is sufficient; sometimes, however, it is necessary to make an incision through its walls, running parallel with its direction, and extract the irritating body by means of forceps. For several days nothing but farinaceous drinks and food easy to masticate are to be given the sick animal. The mouth has to be frequently cleansed with fresh water. As soon as the gland has formed an abscess we must make an exit for the pus, and cleanse the cavity by means of antiseptic injections. When affected by partial mortification the same agents may be used.

\section{PHARYNGITIS: PHARYNGEAL ANGINA.}

Inflammation of the mucous membrane covering the guttural cavity and its immediate neighborhood has for a long time been called angina. Exclusive localization upon the pharynx is extremely rare. Sometimes the symptoms of pharyngitis, at other times those of laryngitis, predominate in the clinical aspects of angina. The separate study of pharyngeal and laryngeal angina is necessary, in order to insure clearness and intelligence of description. 
In man and in the smaller animals we can distinguish, according to the localization of the trouble, inflammation of the velum of the palate, properly called amygdalitis, and pharyngitis; but the obstacles preventing the exploration of the throat of the horse and ox render these distinctions almost impossible during life. Thus, in veterinary pathology, pharyngeal angina designates a more or less extended inflammatory process, located upon the mucous membrane of the velum palati and on that of the tonsils and pharynx. It is usually accompanied by a less intense phlegmasia of the mucous membrane of the larynx, of the superior part of the trachea, of the buccal and nasal cavities, and sometimes also of the Eustachian tubes, as well as of the guttural pouches.

In smaller animals pharyngoscopic examination enables us to diagnosticate the following forms of the disease : Catarrhal, phlegmonous, croupous, diphtheritic, lacunary, necrotic, and parenchymatous; but in subjects of our larger species it is impossible to distinguish precisely the type of the disease. However, the intensity of the rational symptoms, the condition of the lymphatic ganglions, the duration of the trouble, etc., may point to the existence of a pharyngitis assuming such or such a character. Rejection of croupous membranes may lead to a diagnosis of croupous pharyngitis, but we could not determine with any accuracy whether they originate from the larynx or pharynx. In our study of pharyngitis we shall lay aside all former divisions.

[The croupous membranes here spoken of correspond to the false membrane of true croup, consisting as they do of a fibrinous exudate or an inflammatory transudation of coagulable lymph and fibrin, and sometimes mucin containing globules of pus. No evidence has yet been offered showing the extension of these small membranes into the trachea and bronchi as in man. In every instance coming under my own observation they were confined to the pharyngeal cavity, and principally to the Schneiderian mucous membrane, even as far forward as the external naris. These membranes differ very materially from the true diphtheritic false membrane, which consists exclusively of epithelial cells in a fibrinous network, having on its free surface exudation corpuscles or pyoid globules and granules, indicating stages of degeneration. Diphtheritic deposit also differs from the false membrane of croup, by being more thick, tough, and yellow, and less susceptible of organization. Diphtheritic exudate may be artificially 
produced in animals by the experiment of Dr. Sanderson, which consists in the local application of oil of cantharides, but the false membranes thus produced have a greater tendency to organize. -W. L. z.]

Etiology. Pharyngitis appears at times as a sporadic disease, at other times it is contagious. It is frequently found in the pig and horse, less often in the ox and dog; it is very rare in sheep. A certain individual predisposition, which has been proved to exist also in man, is one of the general causes, and is apt to favor its development; but the exact nature of this predisposition is not better known in animals than in man. At all events, weak, delicate, and young animals are more frequently affected by pharyngitis, than are old, adult, or strong horses; these, however, are not exempt from it.

The principal causes determining it are :

1. Mechanical, chemical, and thermic irritations, acting directly upon the mucous membrane. In the first division we must mention wounds and contusions produced by irritating bodies; sharp bones, fish-bones, thorns, needles, coarse food, barley balls; unskilled filling of the teeth, and also gad-fly larvæ in the mucous membrane (Garnier, Pigeaire, Mather, Christensen). Prominent tumors in the pharynx, especially actinomycomas and tuberculous neoformations in the ox, can also produce catarrh of the pharyngeal mucous membrane. As chemical irritants, we must point out non-dissolved emetic drinks, concentrated croton oil, ammonia not sufficiently diluted; phosphoric preparations, and food and drinks that are too hot.

2. Local cold, caused by breathing very cold air, especially during north or northeast winds (Schmidt); icy drinks and fodder covered with frost, under certain conditions, can produce pharyngitis. A common cold, rain, and sudden changes of temperature are enough to determine it. Too much importance has undoubtedly been ascribed to the influence of cold by the old school (diseases a frigore), but this etiological factor must not be altogether dropped in the present state of science; especially must it be taken into consideration in the pathology of pharyngitis.

3. The propagation of pharyngitis from contiguous inflammation. We have already described the influence of stomatitis, rhinitis, and laryngitis in the genesis of pharyngeal angina. It may appear during the course of any common disease. We know the pharynx 
to be the main seat of infection in strangles, and strangles and pharyngitis have often been mistaken for each other; it appears frequently as a complication of aphthous fever, hydrophobia, canine distemper, contagious pneumonia of the horse, petechial fever, anthrax, chronic and acute glanders, tuberculosis, etc.

4. Infection. Various epizootic outbreaks of angina prove the existence of an infectious agent producing pharyngitis in certain instances. Its character and course are then similar to those of a contagious disease.

We must be prudent when we observe this form of angina in the horse. A pharyngitis, with the formation of abscess of the submaxillary ganglions, existing as an epizootic, must always be considered as a symptom of strangles (streptococcus pyogenes of strangles!).

An infectious pharyngitis, often complicated by pyemia, is commonly observed in young dogs during the first week of life.

Pathological anatomy. We think it proper to first review the normal anatomical features. According to Franck, the mucous membrane of the soft palate contains a thick layer of yellowish acinous glands, secreting mucus and serum (Ellenberger's glands); in the pig alone are found numerous small lymphatic ganglions between these glands. The pharyngeal mucous membrane possesses but a very thin covering of these mixed glands. At the base of the tongue, and by the side of the amygdalæ (tonsils) are found numerous openings, which represent lymphatic follicles. Upon the superior border, in the neighborhood of the Eustachian tube and upon the dorsum of the pharynx, are also numerous lymphatic follicles. In the horse and pig the amygdalæ are situated in the posterior part of the buccal cavity, where we notice the two excavations called the foramen cæcum. In other domestic animals we find only a deep depression; it may be to this difference that we can attribute the frequency of angina in the horse and pig.

It being, as already stated, almost impossible to establish a precise diagnosis of the localizations of pharyngitis in our animals, to make an anatomo-pathological description of them would be of no practical interest.

In light cases of pharyngitis the mucous membrane, marked with a diffuse or ecchymotic redness, is thickened, covered with a mucous or muco-purulent exudate and sometimes streaked with blood; it often presents traces of erosion. In chronic pharyn- 
gitis it is pale, thickened, plicated, and covered with a thick, thread-like mucus (Bruckmüller). Inflamed glands and follicles predominate in the shape of small pimples of the size of a milletseed. In intense pharyngitis we find an odematous inflammation with very marked redness of the mucous membrane; this may even be found to have a pulpy consistence. Sometimes the exudate coating it is purulent or bloody and ichorous, sometimes it consists of a greenish, glue-like coating, under which the membrane, stripped of its epithelium, seems gnawed away. The lymphatic follicles and the mucous glands are enlarged, and may become the size of a pea; they are often found ulcerated; we observe numerous small purulent points on the mucous membrane, especially in the neighborhood of the amygdalæ, which, according to Bruckmüller, have sometimes undergone calcareous transformation.

When the lesion of the mucous membrane is communicated to the subjacent connective tissue, pharyngitis takes a phlegmonous character. We then find the cellular sub-mucous tissue infiltrated with serum and blood; it is often transformed into a purulent or gelatinous mass, which may form a more or less large abscess, often opening through the mucous membrane, or causing gangrene by excessive pressure. The purulent infiltration of the subcutaneous connective tissue may be the determining cause of abscess, fistula, or a diffuse mortification of the mucous membrane. The alimentary matters enter into the false tracts thus burrowed by the abscess formation, maintaining suppuration, and at times producing gangrene. Bruckmüller has seen these foreign bodies between the mucous and muscular walls of the œsophagus, extend from the pharynx to the stomach.

The pharyngeal ganglions always, and the sublingual glands frequently, participate in the inflammation. They are found tumefied, with hemorrhagic infiltration, or transformed into a reddish pulpy mass, or broken down into abscess. Post-pharyngeal abscesses, sometimes simple, sometimes sinuous, are more or less voluminous; they contain a thick, creamy pus (a decilitre or even more). This pus may undergo a cheesy transformation, an abundant connective-tissue hyperplasia. In serious pharyngitis the ganglions of the throat are tumefied, form abscess, and the pus which they contain finds its way to the surface through one or several fistular tracts. The mucous membrane of the larynx is 
injected, sometimes ecchymotic; in certain places it is the seat of a serous inflammation (œdema of the glottis) ; in others, but especially at the base of the epiglottis, we notice an odematous inflammation.

Generally the mucous membrane of the Eustachian tubes is thickened; the guttural pouches are sometimes filled with cheesy pus.'

As to the bronchial tubes, we often find catarrhal alterations; occasionally they are full of pus, the mucous membrane is ulcerated, and the cartilage exposed. Traumatic pneumonia is not rare; frequently there exist, associated with foci of gangrenous lobular pneumonia, cavities of the size of a pigeon's egg; at this point is formed a pleuritic exudate.

The pituitary mucous membrane is brick-red, dark-red, or violet; it is covered with purulent bloody crusts, and sometimes an exudate presenting a croupous character. According to the location, the surface of the derma is velvety, villous, or rough.

In certain cases we also find lesions of stomatitis and those of catarrhal enteritis; at the same time the rectum may be the seat of a desquamative superficial inflammation.

When the disease has developed rapidly the heart undergoes fatty degeneration; the liver is increased in size, yellowish, and fatty. These, however, are accessory lesions found in all febrile diseases.

Symptoms. The veterinarian hardly ever has an opportunity to observe the beginning of pharyngitis. Besides, at this time it would be impossible to make a correct diagnosis, for diminution of the appetite and a cough are initial symptoms in many diseases. Only after three or four days, when prehension of food is much interfered with, will the owner have his attention called to the trouble. Stiffness of the head, sensibility to pressure of the guttural region, difficulty of deglutition, and the pain provoked by this act, which is shown especially by an extending motion of the head at the time the bolus passes the pharynx, may become so intense as to cause total abstinence from food and drink: these are the signs noticed at that stage. Troublesome and painful mastication and deglutition induce accumulation of a considerable

1 We have often taken retro-pharyngeal and sub-parotid abscesses for a purulent collection in the guttural pouches; this error in diagnosis can only be recognized when the abscess is punctured.-N. D. A. 
quantity of mucus and saliva in the mouth, escaping in long transparent threads from the buccal orifice, soiling the lips and surrounding objects. When these secretions are retained in the buccal cavity they become heated and acquire an offensive odor, due to decomposition of the epithelial cells and fluids.

The animals prefer soft and liquid food to fodder; the latter, after a prolonged mastication, is usually passed in the shape of patulous balls, coated with mucus. Drinks are rejected through the nose; they contain alimentary matters and nasal mucus, which mixture is very often colored green by the chlorophyll. This rejection constitutes the pathognomonic symptom of pharyngitis. It is the result of a functional defect, through paresis or true paralysis of the muscles of deglutition. This paresis is defined by the serous infiltration, and the participation of all the muscular tissues in the inflammatory process. This condition is mainly due to the imperfect contraction of the pharyngo-staphylinus.

The knowledge of this mechanism of rejection, based upon pathological anatomy, permits us to affirm that the rejection of a quantity of matter through the nasal cavities is a symptom of serious pharyngitis, that is to say, submucous and phlegmonous.

Deglutition of liquids is painful, and generally the animals drink little, notwithstanding that they show intense thirst. They like to play with water put before them; sometimes you see them perform the act of deglutition, without any of the fluid passing into the stomach.

The mouth is hot, its mucous membrane red, and the tongue coated. The examination of the pharynx, in small animals, allows us to see the alterations mentioned above.

The pituitary membrane has a catarrhal aspect, especially in the neighborhood of the guttural orifices. A serous, spumous discharge can be seen at the very beginning of the disease, which becomes muco-purulent, whitish, gray, yellow, or green, and dries around the circumference of the nostrils.

The conjunctiva is more or less congested.

The cough is dry and irregular from the start; later it becomes heavier. Coughing is always painful, and the animals try to avoid it. When it is provoked, the mouth is emptied of its mucosalivary contents mixed with food. Its cause must be looked for in the propagation of an inflammation to the nasal mucous membrane, or in irritation exercised by the exudates upon the pharyn- 
geal mucous membrane. The least pressure of the hand upon the larynx will cause coughing; in some cases it may be noticed at every movement of the head.

During the height of the disease the breathing is accelerated, and is accompanied by a wheezing or even a rattling noise. On auscultation of the larynx and trachea we perceive the utmost variety of moist râles.

The parotido-guttural region is tumefied, especially on the surface of the inferior edge of the parotid gland. As a rule, the swelling is not the same on both sides; it is sometimes limited, sometimes diffuse, and may extend to the whole pharyngeal region. Parotidean tumefaction is mostly due to inflammation of the pharyngeal ganglions and of the sub-parotid connective tissue; it is rarely induced by an accumulation in the guttural pouches. The skin of this region is sometimes oedematous. The ganglions of the throat, the maxillary and sublingual glands are enlarged, and the thyroid body sometimes tumefied.

We may sometimes observe symptoms of intestinal catarrh; we note frequent gapings (yawnings), an arrest of peristaltic motion, and fetid, soft, and coated excrements; the urine is acid. If enteritis does not follow, the animals will still eat, and the urine retains its alkaline reaction for several weeks, notwithstanding the persistent fever; in serious pharyngitis it contains albumin; the lessening of its specific gravity seems to vary with the crisis of the disease; in three days we have seen its weight oscillate between 1001 and 1006 ; but we must add, in that case polyuria was present.

Fever is irregular. In some cases it is entirely nil; the pulsations can hardly be counted, varying from 40 to 48 per minute. In others the disease starts with a temperature of $40^{\circ}$ to $41^{\circ} \mathrm{C}$, coming suddenly and without any marked acceleration of the pulse. The temperature has been seen to keep at $40^{\circ} \mathrm{C}$. during the whole course of the disease, and the number of pulsations did not exceed 40 per minute. Generally the heart function is accelerated in proportion to the degree of temperature. ${ }^{1}$

If from the outset there is intense fever, it is the result of an infection: such is the pharyngitis of strangles. A thermic curve ascending regularly and constantly indicates phlegmonous complications; a sudden rise generally signifies the formation of an

1 Fröhner: Reportor., 1881. 
abscess of lymphatic ganglions. A sudden and considerable acceleration of the pulse is an unfavorable prognostic symptom; 80 pulsations per minute are a serious symptom in the horse, while high temperatures are perfectly compatible with a cure. We find frequently an irregular distribution of temperature on the superficial regions; the ears and other extremities are cold.

We have sometimes observed a sudden urticarial eruption, disappearing rapidly, and appearing again at some later time. This eruption, only noticed in infectious pharyngitis, is without doubt a vaso-nervous or embolic exanthema, similar to that occurring frequently in strangles.

Course. In the majority of cases, the course of pharyngitis is that of a simple acute disease; complications, however, may alter it. Chronic pharyngitis is almost always the result of slight irritations acting persistently (tumors, gad-fly larvæ, etc.). The most frequent termination in all cases of simple catarrhal angina is recovery in about eight or ten days.

When there are abscess formations in the lymphatic ganglions and submucous connective tissue, breathing becomes painful, fever increases, and a cure is often delayed from four to five weeks, whenever œdema of the glottis does not interfere to produce death by asphyxia. These abscesses very seldom open from the outside, and still more rarely from within and without at the same time, but almost always inside of the pharynx, through a limited gangrene of the mucous membrane.

Romant has, however, observed a complete pharyngeal fistula in the colt. We have seen two similar fistulæ in the horse. One of them was produced by spontaneous bursting of an abscess located at a depth of about $10 \mathrm{~cm}$. in the pharyngeal cavity, and which had been punctured with a lancet afterwards. In the other case the fistula was found after the cure; it opened into the intermaxillary space; liquids could be injected into the buccal and pharyngeal cavities through its ramified canal.

When tissues are thus dissected and perforated by suppuration, they may possibly mortify; putrid and septic poisonings are then to be feared. These complications are manifested by a very high temperature, by a gangrenous odor escaping from the nose and mouth, and often also by the presence of decomposed tissue in the discharge. Sometimes death happens after the opening of an abscess and the escape of the pus into the bronchial tubes. 
When inflammation of the ganglions results in abscess formation, without a breaking through of pus, indurations, caseations, and calcifications are produced, and a cure will always be incomplete; consecutive hypertrophies may more or less interfere with breathing or induce paralysis of the pharynx.

Pneumonia is a very serious complication, but one that is quite common in phlegmonous and necrotic pharyngitis; death often results from it. As the possibility of this complication always exists in serious cases, the veterinarian should never neglect to examine the chest with the greatest minuteness. Pulmonary inflammation is caused by foreign bodies in most cases, and it rapidly terminates in gangrene; sometimes it is due to false deglutition, at other times it is the result of unskilful administration of some drink. It may also be due to emboli starting from necrotic centres (metastatic pneumonia).

Differential diagnosis. A diagnosis of confirmed pharyngitis is easy; it is not at all the same thing, however, at the outset, or when complications exist. It may be mistaken for foreign bodies encysted in the region, or fixed upon the mucous membrane, also with tumors of the pharynx, which interfere with deglutition (actinomycomas, tuberculous productions in the ox, sarcomas in dogs), with hypertrophy of the soft palate, etc. Spasms, contractions, and paralysis of the pharynx are indicated by symptoms similar to those of pharyngitis. Parotiditis, glossitis, stomatitis, purulent collection of the guttural pouches, ptyalism, hydrophobia, and strangles, may all induce diagnostic error.

Prognosis. It varies with the intensity and extent of the inflammation and the existing complications. A great deal of care is needed in serious cases; cedema of the glottis and gangrenous pneumonia may appear at any time. Laryngitis must also be taken into serious consideration.

Treatment. Healthy animals ought to be separated from the sick as soon as pharyngitis assumes a contagious character; the latter should be placed in a well-ventilated stable, with mild temperature, and protected from draughts. We should give them green food, or very fine hay, bran or flour slop; oats and cut straw must be discontinued; fresh water should be left within the reach of the animals. The lips and nostrils must be kept perfectly clean. The mouth must be washed frequently with plain fresh water or with a slight addition of vinegar, sea-salt, chlorate of 
potassium, etc.; it is important to direct the jet of the liquid toward the cheek and not the pharynx.

Cold compress or ice applications are to be used when the region is hot, tender, and painful, or when the disease takes a phlegmonous character. Priessnitz's compresses are recommended at the time of abscess formation; it is well to dip them in carbolized water. These compresses relieve the animals, they are antiphlogistic, hasten maturation of the abscess, and reduce inflammatory tumefaction. Friction with mercurial ointment is also useful, though absorption of mercury by the skin is doubtful; this ointment has an emollient action, and its fatty substance helps to soften the integument. The compresses of Priessnitz are much to be preferred to mercurial applications.

The action of derivatives (mustard, liniments, collodion or cantharides ointment) is questionable; they are not to be recommended in all cases. If they favor the maturation of the abscesses they have no real derivative action. They may be used to rapidly dissipate a hard and circumscribed tumefaction, but they must not be used when the swelling is extensive, the local inflammation intense, and suppuration deep and diffuse; when the heart is weak, and in general, in young and lymphatic animals. Often they produce dyspnoea, enormous tumefactions, and gangrenous destruction of the connective tissue. Many horses have succumbed from their effects.

Abscesses should be opened as soon as they are found to exist; it is prudent to make an incision of the skin only, and to perforate the tissues with the finger. In this way abscesses located at a depth of ten centimetres may be opened without any risk. Their ulterior treatment is taught by surgery.

Fumigations with plain or carbolized water are frequently used, but they exert only an indifferent influence. They must not be given too hot. In the dog the local applications of astringent substances by means of an atomizer or a pharyngeal brush are very advantageous. Alum, nitrate of silver, or tannin may be used.

Dieckerhoff's intra-pharyngeal or intra-laryngeal injections (nitrate of silver solution 1:50, or Lugol's solution) are dangerous, as we have ourselves experienced. ${ }^{1}$ [The danger, however, consists more in the method of application than in the drugs used. Locally, these remedies (argenti nitras and iodine) exert a most

1 Friedberger: Münch. Jaresber., 1886-87. 
beneficial influence upon the inflamed mucous membrane, but they should be brought in contact with the parts by means of an atomizer of suitable construction. Hydrogen peroxide used in the same manner is a very valuable and efficient remedy.-W. L. z.]

In regard to internal medication we may choose from a number of medicinal agents, but most of them are objectionable. Drinks are absolutely rejected, and it is advisable to be careful in using electuaries, pills, etc. Fever and constipation require some care; injections of sea-salt and soap may be given. In certain cases hypodermatic injections of eserine are indicated (if there is no dyspnœea), or administration of tartar emetic in drinks; this latter drug is useful in combating fever and constipation. The fever often resists all antifebriles; and in order to reduce this it will be best to look to external hydrotherapy and cold injections.

Spinola lays great stress upon the good effects produced by emetics in the early stages of pharyngitis, in animals vomiting easily.

As complications, we may have to treat pneumonia ; to open the guttural pouches; to prevent asphyxia, by performing tracheotomy. The latter operation must be done at the proper time, and as the right moment may happen at any time of the day, it is a good precaution to prepare the necessary instruments in advance.

Pneumonia should be treated according to classical indications; unhappily it is very seldom brought under control, no matter what is done.

Pharyngitis of other domestic animals. The symptomatology of pharyngitis in the horse and ox, which we have just described, applies also in its principal manifestations to that of our other domestic species. It would be difficult to describe it fully in the latter, as our knowledge on this subject is yet very restricted, and the observations at hand are very few and incomplete.

1. In the pig. Pharyngitis of the pig, described by Spinola in his Treatise on the Diseases of the Pig, is quite frequent. It is hard to distinguish it from laryngitis. Idiopathic angina must be clearly separated from anthrax-angina. Among its causes Spinola mentions the ingurgitation of very cold water when the body is much overheated, which explains the frequency of this disease in the spring in pigs running in the fields, and those in localities situated along rivers coming from mountainous regions. Mechanical or chemical causes may also produce it. The symp- 
toms vary with the intensity of the disease. In the catarrhal form the fever is nearly always absent (Spinola). ${ }^{1} \quad$ We observe, principally, redness of the mucous membrane, sometimes a lacrymation, an accelerated, difficult or painful respiration, a dry cough, a hoarse voice, heat in the buccal cavity, difficulty of deglutition, retching, and even true vomiting, also a more or less pronounced red and sensitive puffiness of the throat. Appetite is lessened or entirely suppressed. The duration of the disease is from ten to fifteen days; cough becomes more and more moist, and recovery, which is the rule, becomes more and more evident; the morbid process rarely persists in an aggravated form.

According to Spinola, the symptoms of aggravated angina are : Redness and dryness of the groin and visible mucous membranes, especially those of the mouth; bright and haggard eyes; the guttural region is red, hot, tumefied, and painful; the mouth is hot, breath short and often very painful; cough abortive, short, and painful-the patients seek to avoid it; the grunt is hoarse. The appetite is lost, and deglutition is always difficult. Defecation and micturition are rare; the excrements are hard, and the urine is yellowish-brown.

Later, when the disease takes a more serious character, these symptoms increase in intensity. The mucous membranes become of a dark livid color; respiration is very painful, almost spasmodic; the patiests take a sitting position, similar to the dog's, the fore-feet spread and head stretched; they breathe through the half-opened mouth, and produce a distressed, wheezing noise; anguish is extreme; the eyes are injected and protruding; there is a stare of agony in the look; the veins of the head swell up; the groins, the lips, and the mucous membrane of the mouth become of a bluish-red shade. The animals stagger, fall, have convulsions, and die from asphyxia in from twenty-four hours to three days.

The autopsy generally reveals an intense inflammation and the existence of a croupous exudate in the pharynx, larynx, and upper

I It is difficult to recognize a feverish condition in the pig. The normal temperature, which is on an average $39.6^{\circ} \mathrm{C} ., 103.7^{\circ} \mathrm{F}$. (Siedamgrotzky) is subject to very great variation; the same is true with regard to the pulsations. We are obliged to have recourse to the contingent symptoms, which are: the fever, the dryness and lividity of the groin, the pendant ears, uncurled tail, cold extremities, the lost appetite accelerated respiration, and the tendency of the animals to remain in their litter. 
part of the trachea. ${ }^{1}$ It is the so-called membranous angina. Besides, we find signs of asphyxia (pulmonary œdema, venous hyperemia and its consequences).

When recovery is about to follow, the respiratory phenomena become less marked, and after five or ten days everything returns to normal. But, often, chronic inflammatory alterations of the peri-pharyngeal connective tissues persist, and produce a wheezing noise. Röll looks upon this as the result of the formation of small abscesses, surrounded by an infiltrated connective tissue, which are formed in the neighboring tissues of the pharynx in serious pharyngitis.

The gravity of the diagnosis depends upon the cause of the disease, on its intensity and extent, and on the means of treatment at hand to check it. It is serious in proportion to the inflammatory process in the larynx; it is usually judged by the more or less marked difficulty in the respiration and the intensity of the fever.

Treatment. The animals must be placed in a dry stable, of mild temperature, and protected from draughts. Slops made of bran and flour, green food, but especially curdled milk, should be given.

At the beginning of the disease an emetic may act as an abortive; it is often of advantage to administer a second, and sometimes a third one, after a few days (Spinola). White hellebore, in doses of 5 centigrammes to 2 grammes, is an excellent drug for this purpose; it can be replaced by ipecac, in doses of 1 to 3 grammes. The medicaments are given mixed with the food, or, if the animals do not eat, incorporated in oil. We should never have recourse to drenches, as they are always dangerous. For this reason emetics are not advisable. Clysters and hypodermatic injections are more suitable, especially subcutaneous injections of veratrine (20 to 30 milligrammes dissolved in 3 to 4 grammes of alcohol).

Counter-irritations upon the throat (ointment of cantharides, etc.) are useful in some cases, inefficient in others.

Diet, emetics, [sedatives], and derivatives may be all that is required in benign cases; in obstinate constipations the administration of oils and medicated clysters are to be recommended. For cases of any gravity there is no systematic medication. A

\footnotetext{
${ }^{1}$ Sesselmann: Preuss. Mittheil., 1853-54.
} 
local bleeding is not practicable; a general bleeding, which is difficult to obtain in any quantity (ears, tail, inferior lingual vein, great saphena), is counter-indicated, for excitement and crying must be avoided as much as possible. Nitrates, alkalines, expectorants, and ammonia are almost without effect. When deglutition is almost impossible, internal treatment must be abandoned, or there would be danger of provoking pneumonia. The veterinarian is almost always called too late, and he has often to advise killing the animals, unless the owners decide to run the chances of a cure, on account of the condition of gestation, or the value of the animals as breeding-stock.

Priessnitz's compresses are still the most useful means that may be employed, if circumstances allow it and the necessary assistance is at hand.

The convalescent animals ought to remain indoors for a certain time before following the herd.

2. In the dog. Pharyngitis of the dog is quite common, and has a remarkable tendency to return. The best trained races (shepherd and deer hounds, etc.) are most exposed to it. The disease, in most cases, starts with dysphagia and ptyalism. Soon the pharyngeal ganglions become inflamed and tumefied, but they rarely form abscess. The swelling and redness of the mucous membrane of the amygdalæ, of the soft palate, etc., are easy to recognize on examination of the pharynx. The course is ordinarily rapid; there are, however, cases where the disease lasts quite a long time (two to four weeks).

The treatment consists in the application of Priessnitz's compresses, which ought to be renewed every three hours. Applications of camphorated ointment can also be made upon the inflamed glands.

\section{CATARRHAL INFLAMMATION AND PURULENT COLLECTION OF THE GUTTURAL POUCHES.}

Etiology. If phlegmasia of the mucous membrane forming the guttural pouches, can exist as a primal affection, it is undoubtedly extremely rare. It almost always accompanies affections of the pharynx, or parotitis, and in most cases it originates from 
strangles. When once the inflammation has started in the walls of these pouches, the muco-pus secretion accumulates within, maintains the pathologic state which has given rise to it, and changes it into a chronic state. As glandered horses are now, as a rule, destroyed as soon as the disease is recognized, collections in the guttural pouches, of a glanderous nature, are altogether exceptional.

The disease is usually localized in one of the pouches; sometimes both are infected.

Symptoms. The principal symptom of purulent collection in the pouches is an intermittent discharge, appearing, as a rule, only at meal-times, during mastication and deglutition of food, particularly while swallowing liquids (Delafond). Sometimes it appears also during exercise, but is then much less abundant. It is an odorless, generous discharge, consisting of a liquid part, holding yellowish-white flakes of a variable size in suspension; it runs out of the lower nasal groove, without agglutinating the hair, and without forming crusts. When the purulent collection is double, the discharge is always bilateral; when it is single, it runs sometimes through both nostrils, sometimes only through that corresponding with the affected guttural pouch.

The subglossal ganglions are always more or less tumefied. They form an extended gland, mobile under the skin and deeper parts, less voluminous, less irregular, and much less hard than the gland of glanders.

The parotid region is often the seat of a more or less pronounced tumefaction, accompanied or not by heat, and of abnormal sensibility.

When purulent collection of the guttural pouches coexists with pharyngitis or strangles, and if the orifice of communication of the diseased pouch with the Eustachian tube is obstructed, the abscess becomes voluminous, and interferes with deglutition and respiration by a constant pressure it exercises upon pharynx and larynx. The guttural pouches, if distended to excess, may burst under the repeated efforts of deglutition and sneezing; in other cases they produce spells of suffocation and asphyxiation. Death may also occur through gangrenous pneumonia, by the introduction of food into the respiratory organs.

The disease has no tendency to resolution. Left to itself, it persists through life. The purulent matter will then thicken, 
become of a pasty consistence, and undergoes a cheesy or cretaceous change; frequently the discharge will diminish, and the functional disturbance become much reduced, sometimes even entirely disappearing.

Pathological anatomy. The alterations consist of a congestive tumefaction and hypersecretion of the mucous membrane; in the pouch is found a purulent, glairy matter, holding in suspension whitish or yellowish-white firm clots. When the disease is old the mucous membrane is pale, wrinkled, rugous, much hardened, and sclerotic, often adhering to the neighboring tissues through induration of the connective tissue which unites it to these organs; the numerous small lymphatic ganglions of the region are hard and tumefied; pus contained in the pouch is consistent and adheres to the mucous membrane, sometimes it forms veritable concretions (Barthélemy, U. Leblanc, Goubaux).

Diagnosis. When there is purulent collection of the pouches, it is indicated by a unilateral or a bilateral discharge, of a clotty character, free from odor or nearly so, and intermittent, appearing during mastication or the deglutition of solids and especially of liquids, by an engorgement of the lymphatic ganglions, and a tumefaction of the parotid region, which makes its diagnosis especially easy. It can only be mistaken for collection of the sinus. In troublesome cases, analysis of the symptoms will always allow us to distinguish these two affections. In collection of the sinus the discharge is particularly abundant during exercise; it is curdled like collection of the guttural pouches, but it exhales generally an offensive odor. Percussion of the sinus produces pain, and this, and the bulging of the bones forming the external wall of the sinus, are characteristic symptoms of the seat of the disease.

Prognosis. Collection of the guttural pouches is a serious affection. It never heals spontaneously, and the deep location of the affected organ renders it almost insusceptible to means likely to check phlegmasia of the mucous membranes. We have seen that it may cause death by asphyxia, or gangrenous pneumonia. The necessity of having recourse to an operation in order to obtain a cure is not without serious dangers, and is another circumstance aggravating the prognosis.

In fact, the only efficient treatment is the puncture of the diseased guttural pouch. It should be made through the parotid, or by practising classical hyo-vertebrotomy. Catheterization of the 
pouch, and other advised means of treatment (fumigations, revulsive applications upon the parotid region, etc.) have always been unsuccessful.

\section{DISEASES OF THE GSOPHAGUS.}

Diseases of the œsophagus are rare and hard to diagnosticate in our different domestic species. Some of these diseases, particularly obstruction by foreign bodies and oesophageal obstruction, belong to the domain of surgery. Inflammation, dilatation, contractions, ruptures, spasm, and paralysis of the œsophagus only, will be dwelt upon here.

\section{Inflammation of the Esophagus: Esophagitis.}

We distinguish a catarrhal, phlegmonous, parenchymatous, and a peri-osophagitis, according to the seat of inflammation: upon the mucous membrane, or in the submucous connective tissue, the muscular, or the peri-øesophageal connective tissue. The last three forms are ordinarily produced by foreign bodies or traumatisms.

Etiology. Catarrhal œesophagitis sometimes accompanies pharyngitis; in certain cases it is symptomatic of a general disease (bovine pest, aphthous fever, scab-rot), or of a tumor of the oesophagus; it can also be produced in the dog by parasites; by Spiroptera sanguinolenta (Bruckmüller, Johne). Caustic substances occasionally produce catarrhal cesophagitis, or even croupous, as observed in the ox after administration of ammonia for tympanites. Very hot drinks may also produce an osophagitis, by their local action upon the osophagus.

Pathological anatomy. We find hyperemia and an abundant epithelial proliferation in the catarrhal form; exudation upon other inflamed mucous membranes is wanting. Chronic catarrhs are characterized by a particular tendency to papillary hypertrophy. The special disposition of the oesophageal mucous membrane explains this exception to the rule. Its structure is very similar to that of the skin; Schütz has thoroughly studied it in the ox. In fact, it possesses an epidermis, a Malpighian network, papillæ, and a basement membrane supporting them. Therefore there is nothing surprising in the similarity of these alterations to 
the epithelial desquamations of the skin, and the papillary hyperplasias met with so frequently in chronic inflammation of the cutaneous derma. Unfortunately, most authors have not taken into account this construction of the osophageal mucous membrane, consequently our knowledge of the pathological anatomy, in the various affections of which it is the seat, is as yet very incomplete.

Symptoms. They are usually obscure and do not permit us to make a correct diagnosis, at least at the onset. Dysphagia, nausea, and abnormal sensibility to pressure exerted in the jugular groove may nevertheless afford guidance. We have observed whining, ptyalism, cough, and an absolute dysphagia in a case of croupous œsophagitis; a cast of the size of the œsophagus and about 35 centimetres long was rejected after a few days.

Treatment. A direct refrigerating action must be exerted upon the mucous membrane, by means of very cold drinks and broken ice; the patients must be sustained with cooling, non-exciting liquid nourishment; astringent solutions may be used (tannin, nitrate of silver, chloride of potassium, etc.), but not at the risk of interfering with digestion. Blistering frictions on the lower edge of the neck and shoulder, and jugular regions, produce no effect. Esophageal stricture, happening sometimes after cicatrizations of croupous ulcerations, may be prevented by introducing the osophageal sound periodically.

\section{Dilatations of the Esophagus.}

Etiology. Dilatations can be divided into the diffused, occupying the whole periphery of the oesophagus, and the circumscribed dilatations, or jabots. Ectases of the esophagus are generally accompanied by constrictions; they are produced by the same mechanism as the eccentric hypertrophies of the heart when there is valvular constriction (stenosis). In order to induce a movement of the alimentary substances through the constricted part of the oesophagus, more intense peristaltic contractions must be performed by the muscular fibres of the region lying immediately above, when the osophagus becomes enlarged, its muscular fibres atrophied, and its walls pale and thin.

Ectases without contractions are rare and seem to be caused by local inflammations. It may be that the psorospermic utricles 
(sarcosporidia) found so often in the muscular walls of the œsophagus of the horse, play a certain part in the production of simple dilatations.

Zenker divides the jabots, according to the mechanism producing them, into jabots caused by eccentric dilatation, and those produced by traction. The first are produced when the wall of the œsophagus gives way at a certain point, where resistance has been weakened under the pressure of the alimentary bolus, as happens when the organ is wounded. Others are provoked by cicatricial retraction taking place in the peri-œsophageal connective tissue, or in a neighboring organ or ganglion, or after some traumatic lesion. The mechanism producing these jabots may be likened to that of bronchiectasia consecutive to interstitial pneumonia.

Most of the jabots are of small size and of discoid form, but some of them take in almost the whole length of the osophagus. Stockfleth has observed a dilatation measuring almost one and a half metre in length, and nine centimetres in diameter at its centre; their very variable capacity may reach, and even surpass, that of a bucket. Sometimes they contract adhesions with neighboring organs, particularly with the trachea. According to Stockfleth, jabots are. almost always located on the cervical portion of the oesophagus in the horse and on the thoracic portion in the ox.

Esophageal ectasis is not very rare in animals. It is mainly found in the horse and ox, sometimes in the sheep (Ercolani), the goat (Gilis), and the dog.

Symptoms. They are almost the same in all cases, regardless of the length and diameter of the dilatation; but ectases developing slowly are shown only by very obscure signs at the start.

Before other symptoms appear, the appetite becomes capricious; at times the animals are under the influence of an insatiable craving; again, they will hardly touch their food. A loss of flesh, gradually becoming more marked, is also observed. This condition may last for years without becoming aggravated, and sometimes without seriously attracting attention. In most cases vomiting is added to the symptoms. Ingurgitated matters are often rejected through the nose, at more or less extended intervals after meals; they are always coated with mucus, and, on account of their prolonged sojourn in the osophagus, they may have undergone a slight fermentation. They are never digested, as has been stated; the nausea can only result in the rejection of a certain quantity of alimentary matter contained in the jabot. 
There is often dysphagia, and deglutition is sometimes impossible. With their hind quarters under them, the neck extended, and head low, the animals often make the most violent efforts to produce a dislodgement of the bolus (Arloing), and at the same time a very peculiar hard, gurgling bruit is heard. Some patients go through the motion of mastication and deglutition, although they have not taken food for several hours. Salivation is abundant, and we may have colics recurring periodically (Leblanc). In the bovine species affected with jabot, we may notice an irresistible tendency to lick surrounding objects. In some cases, when hay can no longer pass the dilatation, oats and cut straw may be easily swallowed.

The pathognomonic symptom of jabot is the existence of a tumor in the left jugular region, more or less elongated, and of varying dimensions, from the size of the fist to that of a man's head. The tumor appears when the animal is feeding, and may disappear under pressure of the hand. It is painful, if at the same time an osophagitis exists; at the moment of deglutition, very quick peristaltic movement may be noticed.

The sound introduced into the œsophagus comes in contact with an obstacle, arising either from post-ectasic contraction, or a culde-sac of the jabot, or from alimentary material accumulated there.

Frequently there are excessive coughing spells and dyspnoea; this may even lead to suffocation through compression of the trachea. In one case the left carotid was the seat of very energetic pulsations.

As a rule, this condition lasts for years; it terminates in death, however, sooner or later, through exhaustion and marasmus, asphyxia, or rupture of the œsophagus.

Diagnosis. Jabot may be confounded with rupture, inflammation, spasm and paralysis of the œesophagus, with pharyngitis, stomatitis, acute gastritis, with various poisonings, and even with colics. Careful examination of the patient, the use of the oesophageal sound, and also persistence of the disease, allows us to make an exact diagnosis in most cases.

Treatment. Treatment of oesophageal ectasia is rarely successful. In giving small quantities of liquid or semi-liquid food we may produce those complications which are the consequence of the disease. When food begins to accumulate in the jabot, the sound or hand massage becomes useful. Operation on the jabot 
is little known. Stockfleth, relying on the observations of Collins $^{1}$ and Moisant, ${ }^{2}$ advises excision of an elliptic flap from the wall of the dilatation, and suturing the edges of the osophageal wound. In proceeding thus, Schindelka obtained a success in the horse.

\section{Strictures of the Esophagus.}

Etiology. Constriction of the oesophagus is produced by a variable mechanism. According to Zenker, it is sometimes congenital; this is the rarest form. It may be determined by new formations comprising the canal at a certain part of its length. We may specially mention scrofulous tumors, actinomycomas, lymphomata, peri-oesophageal abscesses, tuberculosis of the bronchial and of the mediastinal ganglions, especially in the ox (lipomata, Rüffert), aneurism of the aorta, melanosis (Bruckmüller); these lesions compress the oesophagus laterally or surround it completely.

Constrictions through obturation are quite common; they are mostly due to encysted foreign bodies, to papillary fibroma in the ox (Schütz), to tumors produced by the Spiroptera sanguinolenta in the dog, and even to muscular hypertrophy (Anacker).

Constriction may, again, be provoked by cicatricial retraction consecutive to a scarification, and to a wound or a cancer of the œesophagus. This variety is called stricture. According to Stockfleth, stricture sometimes appears under the form of a thickened muff on a rugous surface, and sometimes in annular shape, the free edge of the ring advancing into the lumen of the canal and forming a kind of valve. Spasmodic constrictions will be described under Spasm of the Esophagus.

Dilatation of the osophagus always accompanies constrictions, as mentioned above; the manifestations provoked, therefore, by these two affections are almost similar in most cases. In the ox, however, constriction produces a special symptom : chronic tympanites (Luatti, Legrand, Johne, Haubner, etc.). The ordinary cause of esophageal constriction in this animal being a tuberculous ganglional hypertrophy, if tympanites is added to these vague symptoms of tuberculosis (cough, emaciation, sub-dulness, pleuritic friction), it may become of value in establishing the diagnosis of

1 Journal de Lyon, 1857.

2 Recueil Vét., 1860. 
this latter affection; in order to possess this value, however, it must not be accompanied by other digestive troubles.

Treatment. This belongs to the domain of surgery, and requires a frequent use of the esophageal sound. This method is especially applicable, in constriction by compression, in the ox. Sounds of graded sizes might also be resorted to. Dietetic indications are the same as for oesophageal ectasia.

\section{Perforation of the Esophagus.}

This accident is commonly due to foreign bodies swallowed and stopped in the osophagus, or to unskilful manipulations with the sound, sometimes resulting in laceration of the jabot. The symptoms are different, according to the location of the perforation in the cervical or thoracic portion. In the first case, the discharged alimentary matters soon form a tumor, which may become enormous, and always produces a phlegmonous inflammation. Deglutition is impossible; there is a regurgitation of food and drink through the mouth and nose. Penetration of air in the peripharyngeal connective tissue may sometimes determine a diffuse emphysematous congestion of the neck, head, and shoulders. Formation of an oesophageal tracheal fistula has been observed. The expired air then often has a gangrenous odor.

Rupture of the thoracic portion is still more serious. The patients are taken with vertigo, fall on the ground, and present all the symptoms of an intense pulmonary congestion or of severe attacks of colic. We observe trembling, nausea, anxious expression of the face, violent contraction of the muscles of the head and neck; if the animals stand, or regain the standing position, the head will be much extended upon the neck and shoulders. (Franzen.) Pneumothorax is not rare. Symptoms of suppurative pleuritis soon appear, and death follows within a short time.

There is nothing to be attempted for perforation of the jabot taking place within the thorax. But when the rupture has its seat upon the cervical portion of the œesophagus we might perform a lengthy incision in the tumor, and trim and suture the lips of the osophageal wound. (See Sonders' case, mentioned by Stockfleth. ${ }^{1}$ ) 


\section{Paralysis of the Fisophagus: Paralytic Dysphagia.}

Paresis and paralysis of the oesophagus are very rare. They seem to be under the influence of lesions of the nervous centres. Straub, who has observed two cases of this kind, ascribes them to an anterior cerebral disturbance; Hering ${ }^{1}$ remarks that paralysis is sometimes a subacute symptom of encephalitis.

The paralyzed œsophagus is filled from one end to the other with alimentary matters, and forms a large cylindrical cord in the left jugular groove. The other symptoms are those of constriction. When the trouble is symptomatic of a lesion of the encephalon, we find, besides, cerebral nervous trouble.

The treatment should be restricted to frequent use of the sound and to an application of revulsives. Administration of strychnine salts might also be tried.

We must not confound inability to drink with paralysis of the œsophagus. Animals apparently perfectly healthy and swallowing solid food without difficulty, find it impossible to perform suction. This condition is almost always congenital and results from functional defect. This defect is found in sucklings as well as in adults; it exists more in horses than in other animals. $\mathrm{Straub}^{2}$ has described such a case. We have treated an eight-yearold horse, which was brought to us with the remark: "He drinks like a dog." This horse swallowed food very well, and was eager for water; when it was presented to him he performed a kind of smacking with his lips, lapped with his tongue, and chattered with his teeth, but without being able to swallow a mouthful of water. He was given liquid food only.

\section{Spasm of the Esophagus: Esophagism.}

Primitive spasmodic constriction of the osophagus (dysphagia spastica) is very rare in comparison with symptomatic spasmodic contractions, with which they are often confounded. Spasmodic conditions similar to neurosis, defined under the name of osophagism, are often produced by an obstructing bolus in the oesophagus, a wound, or an ulcer of this canal.

Idiophatic spasm of the œsophagus has been found in two horses by Mollereau; Guilmot observed it in a horse affected by gastric

${ }^{1}$ Hering: Pathology, 1858.

${ }^{2}$ Straub: Repertor., 1880. 
trouble, and Mossé in a few mules, old and young. The spasms lasted from three-quarters of an hour to six hours. The Percheron under Mollereau's observation was taken with convulsive motions and vomiting; he was accustomed to extend his head very much upon his shoulders and gather his feet. The other horse observed by the same author used to scrape the floor while eating, seemed to have colic, could not swallow, and was taken with nausea when any pressure was exercised upon the much-extended oesophagus.

The cases described under the name of "intermittent paralysis of the œesophagus" are doubtless associated with periodic spasm of this organ.

The treatment ought to consist in the administration of antispasmodic agents-bromide of potassium, chloroform, morphine, etc. Mossé obtained a cure through œesophagotomy and the application of an osophageal tube.

The spasm of deglutition which accompanies hydrophobia, epilepsy, catalepsy, and tetanus is usually produced by contraction of the pharyngeal muscles, and of the muscular system of the superior extremity of the oesophagus. The treatment does not admit of any special medication.

\section{DISEASE OF THE BIRD'S CROP.}

The birus crop is an alimentary reservoir in which the food substances undergo a maceration which renders them more digestible. These secretions possess a neutral reaction, and, according to Foster, contain a ferment capable of transforming starch into sugar.

The pathology of the crop comprises two principal morbid conditions: 1, Catarrh or inflammation of the mucous membrane; 2, Obstruction of the organ. These affections are also commonly designated under the names of soft and hard crop.

\section{Catarrh and Inflammation of the Mucous Membrane} of the Crop.

Etiology. The catarrhal condition of the mucous membrane is ordinarily provoked by abnormal retention, desiccation and decomposition of alimentary matters, or through injection of substances having already undergone a partial decomposition. Parasites of 
the mucous membrane (Spiroptera nasuta, R., according to Robin ; Spiroptera uncinata, R., according to Zürn) can equally give rise to this affection. Intense inflammations of the mucous membrane of the crop have also at. times been observed; they result from intoxications from various corrosives: phosphorus, arsenic, and mercurial preparations.

Symptoms. The soft crop, which is quite common, shows a distention of the reservoir; this distention is soft, clammy, or tympanitic, according to its origin either from liquids or from gas. The sick bird stops eating, is under the influence of nausea, or vomiting, and fetid gases escape through its bill. They may be expelled by artificial compression of the crop. The bird becomes gradually weaker and may die from inanition.

Treatment. Zürn advises emptying the crop by methodical massage performed upon this organ and the osophagus, the patient's head being held in a dependent position ; then administering an astringent preparation, or a salicylic acid solution (in teaspoonful doses). After a twenty-four hours' diet, we may give slops, soups, and easily digested food. This regimen ought to be continued for several days.

\section{Obstruction of the Crop: Ingluvial Indigestion.}

Etiology. The obstruction of the crop is mostly due to ingestion of a too abundant quantity of indigestible matters (hard and dry grain, and all kinds of foreign bodies). Pourquier has described a case in which it was produced by the leaves of Robinia pseudo-acacia, L. In rarer cases it may be produced by pressure exerted upon the œsophagus, lungs, thoracic reservoirs, and trachea, when these organs are under the influence of an acute inflammation.

[Indigestion of the crop may also be produced by the presence in this organ of certain parasites, as the Trichosoma contortum (Crep.); this form of the disease seems to affect certain species of ducks, especially the young. Railliet and Lucet have described this form of indigestion as having existed with marked severity in the Department of the Loiret, in France, where it made many victims among Pekin ducks, and but few among the common breeds. - w. L. z.]

Symptoms. The most striking symptom is the excessive volume of the crop. In a duck observed by Zürn, this organ 
weighed 1348 grammes (membrane and contents: food, gravel, and pebbles). We have found it of enormous dimensions in a hen ; it measured $40 \mathrm{~cm}$. in circumference and $15 \mathrm{~cm}$. in diameter. It presented a hard consistence to the touch. Anorexia is usually complete. If catarrh of the crop exists at the same time, a fetid liquid runs through the bill. Sometimes the wall of the organ ruptures. If indigestion passes into a chronic state, a long, hanging crop will be formed.

Treatment. The principal indication in treatment consists in disengorgement of the crop by methodical massage. Zürn advises hydrochloric acid in doses of one to two drops, in a teaspoonful of peppermint, or calamus, which is to be administered three or four times a day. Extraction of the alimentary matters is left as a last resource. The operation is performed according to rules laid down by surgery for the performance of esophagotomy. It should be attempted only on chickens and geese, for it is usually fatal in pigeons. Zürn ascribes the gravity of the operation in the pigeon to the particular constitution of the lining membrane of the crop.

\section{ABERRATION OF THE APPETITE.}

\section{Pica of the Ox manifested by Licking: Licking-Disease} (Lecksucht).

Among the numerous aberrations of the taste, this malacia of the ox, as well as that perversion of appetite of the sheep which prompts the animals to eat the wool of their neighbors (Wollefressen) must take a special place in the treatises on internal pathology, both from a scientific point of view and from their agricultural importance. Their place belongs naturally to this section, on diseases of the digestive apparatus. Perversions of the taste, sometimes met with in other affections, such as hydrophobia, diabetes insipidus (Leblanc), etc., constitute but an accessory symptom of these morbid conditions; they do not deserve more particular mention than those taking place in our own species during the course of pregnancy, chlorosis, and hysteria. The same is true of depraved appetites (sows eating their progeny, female animals in general swallewing the placenta, birds eating their feathers, etc.); these s.rc vices, instinctive acts, or manias, rather than pathological insubles. 
Licking-disease is a special morbid condition, the principal symptom of which is an irresistible desire felt by the animals to lick, chew, and swallow if possible, the most diverse and most disgusting objects. The disease assumes a chronic course; it finally produces digestive and nutritive troubles, leading to cachexia and death. Concerning its frequency among our different domestic animals, these must be classified in the following order : ox, pig, horse, and goat.

Etiology and Pathology. The licking-disease constitutes a symptomatic morbid type rather than a pathological change, and the manifestation which generally dominates its clinical picture is common to numerous affections. Its nature has, for this reason, been very differently regarded. It has been ascribed to infection, to heredity, and to imitation; some authors have made of it a nervous disease, while others have traced it to osteomalacia; others again have ascribed it to local defects, to improper alimentation, and to the particular constitution of the soil.

1. Infection and heredity have been considered as being the cause of the disease from the earliest times, but without any sufficient reason, as a close observation of facts testifies.

2. If imitation is sometimes the cause of a condition similar to the affection which we are now considering, it is not to be admitted as being able to produce the true pica disease in the proper sense, this trouble corresponding to a profound alteration of nutrition. Indeed, Lemke's experiences are absolutely demonstrative in this respect.

3. Tscheulin considers the disease as a depravity of the sense of taste, and describes it under nervous diseases. According to Spinola and a few others, its immediate cause is probably an alteration of the sensitive nerves of the stomach, and a kind of functional trouble of the pneumogastric. Quite recently, Lemke has defined it as "a chronic disease consisting of a perversion of nutrition, produced by a lack of phosphorus, and which starts as an affection of the central organs and nerves taking a leading part in nutrition." No one will contest the participation of the nervous system and the complex symptomatic apparatus of the disease; the function of the nerves of the palate appear to be particularly affected; but it is by no means proven that the nervous symptoms are always primary, and that licking is a symptom connected with this affection in all cases. Haubner and Siedamgrotzky are 
nearer the truth in ascribing the trouble, at least in the larger number of cases, to a special and as yet undetermined nervous condition.

4. It is incontestable that the disease is sometimes under the influence of an affection of the digestive organs; this relation is especially striking when, in a healthy and well-fed flock, certain affected subjects lose flesh, and finally succumb to marasmus. At the same time, if we succeed in stopping the licking by means of anti-dyspeptics and antiphlogistics, we can safely conclude that it was produced by dyspepsia, or by catarrh of the gastro-intestinal mucous membrane. Already the old veterinary school has pointed out "acidity of the stomach" as the cause of licking; this acidity is undoubtedly connected with a gastro-intestinal affection.

5. Haubner ascribes it to defective and badly kept stables, irregular food, and lack of hygienic precautions. But Lemcke very judiciously observes that the disease is quite as frequent in the best kept stables as in those where the elementary principles of hygiene are unobserved.

6. Are there really any etiological relations existing between osteomalacia and licking-disease ? The opinions of authors vary very considerably in regard to this. According to Roloff and Röll, licking-disease would not be an essential affection, a special morbid state, but really the first symptom of osteomalacia, and in certain cases in which this last should end in a cure it would be the only manifestation of it. Other authors consider these latter cases as affording precisely the criterion establishing for the disease an independent entity.

According to Rychner, Nessler, Anacker, and Lemke, lickingdisease and osteomalacia constitute two essentially different affections, having each their particular causes. Nessler bases his opinion upon this fact: that fractures, which are very common in osteomalacia, are exceptional in licking-disease. Rychner and Anacker state that they are often associated, and easily complicate one another. According to Lemke, osteomalacia only complicates the disease of licking when the affected subjects are under the influence of a rheumatic diathesis, or if they are exposed to cold. Finally, according to Haubner, Siedamgrotzky, Spinola, and others, the licking-disease existing as a primary affection is sometimes complicated with osteomalacia, which constitutes its termination in such cases. 
We shall formulate our opinions thus :

In certain cases osteomalacia is the cause of licking (pica). But these two affections, coexisting sometimes in the same individual, are really two different morbid states. In practice we meet the disease pica without any existing osteomalacia. The close relationship of these two nutritive troubles being given, it is probable that one and the same cause may sometimes produce the one and sometimes the other.

7. The principal occasional cause of pica must be looked for in the food and in the composition of the soil. In most cases the affection is certainly the result of the absence or insufficiency of certain principles, notably of nutritive salts, in the alimentary matters. The qualifying value of these principles and these salts are not yet determined. Nessler's researches (analyses of hay and water) have especially shown the absence of soda salts. He verified these facts in the Black Forest during the years 1861, 1867, and 1868. His observations concerning the absence of phosphate of lime in food refer to osteomalacia, of which he has also studied the pathology.

The primary indications concerning the relations existing between the appearance of pica and the alimentation given to animals are of a general order and very vague. All the poor and indigestible food; coarse, washed, or muddy hay, harvested too late; too much feeding of straw ; swampy and turfy meadows, water rushes, the cyperaceæ (Cyperus, Scirpus, Carex), the different varieties of dock, etc, as well as the feeding of malt, slops, fermented nutriments, potatoes, turnips, and want or excess of seasalt, have all been charged as being the cause of the trouble. Haubner went so far as to attribute the development of the affection to the action of certain vegetable species (Meum athamanticum, Achillea millefolium, Alchemilla vulgaris, etc.). This last etiological conception, altogether hypothetic, has been absolutely negatived by Lemke's experience.

Licking-disease must be considered as the expression of several conditions of inanition, but especially of humoral anomalies, resulting mostly from the absence or insufficient quantity of certain saline matters in the food; these anomalies may also be the cause of digestive troubles, intestinal catarrh, and probably also of osteomalacia. Without regard to the cause, as soon as the anomalous condition exists the organism feels the need of remedying it; 
other morbid sensations besides those of hunger and thirst are evident; they are produced by the want of certain salts. A simple therapeutic instinct at the first start, this appetite soon degenerates ; the diseased organism can only feel abnormal sensations, and the licking-disease is a pathological craving in the clearest form it can adopt.

Frequency and distribution. The disease runs into a sporadic or enzootic condition. In some years it affects a very large number of animals. It exists constantly in the Black Forest and in many mountainous countries.

In the Black Forest they call dry farms all those upon which the disease is well established (Camerarius ${ }^{1}$ ).

Nessler states that these farms are all located upon granitic soil ; on those in regions having schistic soil the disease never prevails. Nessler explains this geological influence by the disintegrating property of the schistous rocks, which constantly furnish nutritive salts, especially soda salts, to the soil, and consequently to plants. The granitic soils, on the contrary, are composed of coarse grains, which decompose very slowly.

The disease exists endemically in certain localities, and may persist for a long time, sometimes for a period of twenty years, when the precaution of removing the animals to another locality is not taken. The disease appears in other places only in very dry years, when fodder is scarce. According to Haubner and Spinola, there are farms where it makes the raising of calves almost impossible. Cows which are imported contract it generally in two or three years.

As a general theory, pica is the appanage of poor countries and small farming; it appears mostly toward the end of the winter, when food is wanting. Permanent stabling seems to favor its development.

Cows in an advanced condition of gestation, and good milkers, are predisposed to it, while oxen are rarely attacked by it.

The loss of saline matters from the cow's organism during advanced gestation, or full lactation, is probably the cause of this difference. Calves just weaned are frequently affected by it; Lemke has observed the disease in sucklings.

Symptoms. Lemke distinguishes two periods in the disease,

1 Camerarius, Ephemerid. Nat. Cur., 1665-1721, describes them by the name of villoe tabeficioe. 
the first stage being apyretic, the second febrile. The initial stage is always apyretic.

The initial symptoms are a certain degree of inappetence, the long duration of the meal, the slowness and infrequency of the act of rumination. Soon the appetite becomes capricious, and the characteristic symptom of the disease-licking-appears.

The animals will devour part of their litter without being driven to it by hunger; in freedom and on pastures they select aqueous plants, those growing in places where manure has been deposited, also young sprouts of ligneous plants. In the stable they approach their grooms in order to lick their clothes. ${ }^{1}$

These depraved appetites observed in subjects presenting the appearances of health, exist in all species, even among men $\left(\right.$ Lasègne $\left.{ }^{2}\right)$.

These symptoms become worse from day to day; the patients at length are licking continually, day and night, the walls, woodwork and partitions, etc., and biting all objects within their reach, even the chains used to tie them (Pfeifer). They are more excitable and fretful ; their expression is anxious, sharp, and restless; hyperesthesia of the lumbo-sacral region is also often observed (Lemke).

These symptoms may persist for a shorter or longer period (two to four months) with variations in intensity; the weight is not materially reduced; milk secretion is maintained. The animals will stop licking when threatened by the voice (Lemke). The disease becomes aggravated, the appetite for matters foreign to the alimentation makes itself felt more and more; the patients swallow these substances, and it is almost impossible to prevent them from doing so, as they will refuse their food. They devour the litter soiled with excrements and urine, rotten wood, old rags, leather, excrements, etc.; they swallow earth, clay, sand, and all calcareous

1 This symptom must not be mistaken for curiosity, or the attention observed among certain bovine animals when strange people enter the stable, nor with the tendency of licking clothing, which is seen in many healthy animals kept in stables constantly. We must also point out that certain animals, otherwise perfectly healthy, seek to reach and swallow all kinds of objects. Marheincke (Magazin von Gurlt und Hertwig, 1836) describes a striking example of it. A cow, which had never been sick and was a great eater, swallowed successively a pair of cotton stockings, some bread and cheese wrapped in a piece of muslin, a cloth waistcoat with metal buttons, a child's merino dress, and a servant's cotton jacket. See Gurlt, Pathol. Anat.-N. D. A.

2 Lasègne (Etudes Médicales, 1884) reports a remarkable instance of " a well-educated young girl, who devoured nearly half of her drawing-teacher's coat!" -N. D. T. 
and argillaceous matters they can seize; rubbish, remains of mortar, pieces of tiles, glass fragments, etc.; finally they eat one another's hair (Utz). Pool water and stagnant urine are often preferred to clean water.

With lessening of the appetite, constant licking, refusal of good food, and the ingestion of the greatest variety of substances, an infrequent and short rumination is observed, and the motions of the paunch become weaker, at times being suppressed; the milk loses some of its fat (Lemke), but its quantity often remains for a long time quite as abundant (Leytze). The feces are sometimes dry, hard and coated with a thick mucus; sometimes they are soft, clammy, liquid, and have a very fetid odor; they sometimes contain clay, sand, etc., and cause tenesmus (Anacker). The urine is almost always acid, often albuminous.

Lemke has observed an increase of temperature varying from $0.5^{\circ}$ to $1.5^{\circ}$. Arterial pulsations number 70 to 80 per minute, the pulse being weak; respiration is not sensibly changed.

The animals become more and more debilitated; cachexia follows emaciation (especially after calving, the affected cows pass very quickly to a skeleton, Lemke); the mucous membranes turn pale, the hair becomes dull and dry, the skin is covered with a heavy coat of dirt, it is dry and sticks to the bones; a wrinkle cannot be made without difficulty, and disappears slowly-a symptom due to reduction of the natural elasticity of the integument, and for this reason it has been called " cooked," hard, tanned, and hide-bound.

Marasmus soon develops; the patients stand in a gathered position, the back arched; they often grind their teeth; the movements, especially those of the posterior parts, are difficult and limited, and while they are being performed, creaking of the articulations is to be heard (some authors, among others Bräuer, speak of a rheumatic affection of the posterior members). Finally, the exhausted animals fall to the ground and refuse all nourishment; the abdominal sounds cease, the pupils are dilated (Lemke), and death supervenes from inanition.

When licking-disease coexists with osteomalacia, it is manifest by certain special symptoms.

Course. Left to itself, the disease always runs a chronic course. The duration varies from a few months to a year and more; according to Lemke it lasts from six months to two years. 
A cure may be obtained within from three weeks to four months (Lemke), under the influence of a simple change of regimen. The transition from winter diet to pasture, or from pasture to winter diet, change of location, etc., may have a happy result; there is a much better chance of obtaining this result when the affection is but slightly advanced. If the causes, however, which have originated it persist, and no efficient treatment is applied, the affection ordinarily terminates in death.

Pathological anatomy. In the majority of cases the animals are killed at a stage when the disease is little advanced; then a more or less marked emaciation only can be observed, together with the catarrhal condition of the mucous membrane of the digestive tract. When the disease has terminated in death, more deeply seated alterations are found. The fat has disappeared; the adipose tissue shows a gelatinous consistence; it is often of a reddishgray color; the emaciated muscles are pale and soft; the little blood that is left is pale-red and forms half-liquid clots. We sometimes find a serous exudate in the pleura, pericardium, and peritoneum. The gastro-intestinal mucous membrane is the seat of a more or less intense catarrh. Finally, the skin becomes dry and hard; the subcutaneous connective tissue has almost entirely disappeared.

The cases that have been described as having bone lesions belong to osteomalacia, and not to licking-disease.

Prognosis. The prognosis varies with the period of the disease. It is very serious when the animals are already in an advanced stage of emaciation, and if digestive troubles exist. Change of diet and other means are then usually insufficient. In mild cases the prognosis will depend chiefly upon the causes of the affection, and the possibility of suppressing it either by changing the regimen or by removing the animals.

The gravity of the prognosis will be greatly lessened, if the happy results from apomorphine as a curative agent that have been obtained by Lemke are confirmed in practice.

Treatment. In order to enter upon a rational treatment an absolutely indispensable prerequisite is the knowledge of the cause of the disease. Unfortunately, in many cases we are reduced to mere hypothesis.

When the nutritive relation of the food does not correspond to the needs of the organism, prophylaxis must consist in a modifica- 
tion through the improvement of meadows and pastures ; this will be obtained through the use of artificial manure, Chilean nitrate, etc. Experience has demonstrated a change of alimentation to be the most natural and also the safest remedy for this trouble. The food given should come from districts where the disease does not exist; the transportation of these animals to such places should also be advised. But it is often impossible to follow these directions, on account of the expense occasioned by it, and the veterinarian is limited to having an addition of food rich in proteïn added to the regular ration. For instance, grains, leguminous food (peas), etc. saline substances, and especially seasalt.

Attempts have been made for a long time to regulate the digestion in many different ways : administration of dilute hydrochloric acid; chloride of sodium in small doses; bitters and aromatics; when the digestive juices are too acid, antacids have been given, also absorbents (lime-water, chalk, carbonate of magnesia, and bicarbonate of soda). All these means are of advantage when licking depends upon a condition of the digestive apparatus. When there are signs of osteomalacia we must resort to phosphate of lime.

Feser has advised hydrochlorate of apomorphine in hypodermatic injections. This remedy has given some good results when tried by Hackl and Reindl. Lemke, basing his statements upon an extensive experience, has recently proclaimed it to be a specific against licking. He has treated 226 cows, 21 oxen, and 141 calves. Licking disappeared within a few days in all these animals; a certain degree of inappetence, only, persisted from eight to fifteen days.

When the trouble is old, 0.1 gramme will be the dose for the cow; when recent, 0.2 gramme. For the calf the doses are the same. The injections are given once a day for three days at a time; the animals remain cured for a period of three to five months, when the causative surroundings are not changed; when the cause of the trouble is suppressed at the same time the cure is permanent.

According to Lemke, apomorphine injections, practised every three months, keep the animals in flesh for a period of three years, in regions where the disease is endemic, and where cattle must be removed yearly or every other year. 
The observations of Väth, Gassner, and Ostertag ${ }^{1}$ concerning the efficiency of apomorphine are very favorable. Hafner, however, has mentioned one unsuccessful case.

We shall say but few words on licking-disease in the pig and the horse.

1. We must not mistake it, in the pig, for osteomalacia. Sometimes both affections coexist. ${ }^{2}$

Spinola states that the disease is observed in the pig, in countries where it is common in the ox; it also resembles the latter in its course, its duration, and its ending. The sick pig shows a special craving for decayed peas and tree bark.

2. Horses, and particularly young ones, are sometimes affected. The patients lick and bite the surrounding objects constantly; they refuse part of their ration in order to eat their litter, even the manure. There are, however, but few isolated cases found among this species, and it is probably due to gastric trouble. We observe a caprice in many young horses which must not be mistaken with licking; we refer to the habit in the young, of eating the mother's tail and mane.

We have seen licking disappear rapidly in a young horse which was given lime-water; Lippold ${ }^{3}$ has seen in the adult horse a morbid condition similar to licking-disease.

\section{Wool-eating Sheep (Mallophagia) (Wollefressen).}

This affection is of the same nature as licking-disease. The harm done to wool, the loss which results from it, and the mortality it occasions among lambs, give it a certain importance from an economical point of view.

Etiology. The recorded opinions upon the etiology and nature of the affection now to be described are quite as diverse as those which have been advanced concerning the morbid condition last mentioned. There are two principal theories: in the first, the affection is looked upon as the result of imitation; in the second, it is ascribed to diseases of nutrition. At the present time it it would be arbitrary to exclude either in an absolute manner. Both incriminated causes, it would seem, take a certain part in the production of the disease.

1 Väth, Gassner, and Ostertag (unpublished communications).

2 Jansen : Preuss. Mittheil., 1873-74.

3 Lippold: Sächs. Jahresber., 1883. 
1. The theory of imitation is especially defended by Spinola. According to him, inaction and weariness are the starting-point of the defect. The principal arguments which he advances in favor of his opinion are the following: At the beginning the disease exists in one animal; later it is generally observed only among certain groups of animals of the same sheepfold; its course has often been stopped by a strict separation, through isolation of the "wool-eaters" (Spinola, Burmeister); finally, we may see it appear before the winter stalling, that is to say, before the time when ordinarily diseases of nutrition occur.

2. The disease has long been looked upon as being the result of a disturbance of nutrition, produced by food that is either insufficient or is deficient in its chemical composition. Among modern authors, Lemke especially has reinstated this theory by basing it upon experiences the results of which permit us to exclude the influence of imitation. The disease is particularly noticed in winter, at the time when the food is insufficient or is composed too exclusively of potatoes and malt residues, substances which are poor in nitrogen, or when dry fodder is wanting, etc. In spring it disappears under the influence of a change of diet. In lambs it happens, ordinarily, when the milk-giving ewes are fed too sparingly, and when there is a deficiency of milk, or if its chemical composition is poor. In one sheepfold where the lambs, not finding a sufficient quantity of mother's milk, had eaten wool. May saw the disease disappear when the young animals had been supplied with cow's milk. The trouble has also been charged to damp, turfy, and acid pastures, as well as to excess or lack of seasalt (Haubner-Siedamgrotzky).

Frequency. Mallophagia is especially found among improved races, particularly among merinos, and, as stated, at the time the animals are fed in the sheepfold-that is to say, during the winter. Often the trouble appears suddenly, eight or fifteen days after the animals are placed on winter diet, and disappears of its own accord as soon as the sheep go to pasture.

Symptoms. Young lambs from two to six weeks old begin to nibble their mothers' wool, preferably that of the limbs, abdomen, and tail. At first this act seems an amusement, a simple caprice, but the habit soon becomes a craving, and the lambs eat indiscriminately the wool of all sheep. Ordinarily they preserve a healthy appearance, but at times they swallow considerable quan- 
tities of long, coarse wool ; nutrition is then affected and digestive troubles appear; the animals lose flesh, their development is delayed, they are periodically constipated, and some will succumb with all the symptoms of gastro-enteritis. At the autopsy an inflammation of the stomach is found, produced by woollen balls about the size of a hazel-nut, and by numerous woollen locks, which may become introduced into the pyloris and obstruct it.

When the disease first appears it is found upon one subject only-mostly, it is said, on a wether or a pregnant ewe. The young animals seem to be looking for particles of food in their fleece. May and other authors state that they prefer to nibble wool that has been soiled by urine or excrements. At the outset the first wool-eater will attack several sheep, but soon he pursues one and the same subject only, and imitators will not be slow to take part in the work. As a general rule, the first victim is completely shorn, when the company leaves him to look for another. With the number of eaters, however, that of the sufferers increases also. Sometimes in a short period the trouble spreads among a large part of the flock, and occasions the loss of a considerable quantity of wool. It may persist for months without particularly altering the general condition of the animals; ordinarily not the least fever is observed, the appetite does not seem lessened, and rumination is not at all disturbed. However, in some cases constipation appears, also paleness of the mucous membrane, emaciation, dryness of the wool, etc. Nevertheless, mallophagia does not produce cachexia, marasmus, and death, like licking-disease in the ox; it leads neither to osteomalacia nor to osteoporosis.

The animals, as a rule, practise this vicious habit only during the day and in the intervals between meals; but when the trouble is of long standing, they can be seen in dark sheepfolds eating one another's wool almost without interruption, even during the night.

The sheep will very seldom attack his own fleece; this enables us to distinguish "wool-disease" from lumbar prurigo and certain pruriginous eczemas.

Prognosis. The affection referred to is much less serious than licking-disease; it may, however, produce death in lambs. All the prognostic considerations-relative to change of locality, diet, pasture, and the advantages which may be obtained in using apomorphine-which have been formulated in regard to lickingdisease are also to be applied here. 
Treatment. In the first place, alimentation must be modified. Change of alimentary substances and the feeding of more heatproducing nourishment should be advised. Keeping in the open air is to be preferred to stalling. The lambs especially ought to be abundantly fed; May advises their separation from the mothers after the third week, and letting them suck several times daily.

In order to insure the disappearance of the disease in a short. time, it will only be necessary to separate at the beginning of the trouble the first wool-eaters, as well as the first victims, from the flock. Salts, alkalines, stomachics, etc., may be tried. Spinola advises to keep the stables dark during the intervals between meals; but this measure seems to us irrational, and resort to it is unnecessary.

Quite recently Lemke obtained extraordinary results through subcutaneous administration of chlorhydrate of apomorphine upon 800 sheep. The dose should be 0.1 to 0.2 gramme (consequently the same as for the ox). The curative effect is said to last for about four months.

If the results obtained by apomorphine should be confirmed, therapeutics will have made an enormous stride forward. It is desirable that experiments be made in order to solve this interesting question.

\section{ACUTE GASTRO-INTESTINAL CATARRH IN THE HORSE.}

\section{Acute Catarrhal Gastro-enteritis: Acute Dyspepsia. ${ }^{1}$}

Introduction. We shall study in this chapter catarrhal inflammation of the stomach and of the intestine, and the morbid condi-

1 Friedberger and Fröhner devote special chapters to the study of gastro-intestinal catarrh and gastro-enteritis. Gastro-intestinal catarrh is especially characterized by a modification of the gastric and intestinal secretions, by an exudative process, and by alterations which are generally limited to the mucous membrane. In gastroenteritis, phlegmasia is more intense, the symptoms are more marked, the anatomopathological alterations more extended and decper; the process spreads often to the submucous connective tissue, to the muscular coating, to the subserous and even serous connective tissue. The authors have undoubtedly adopted this division in order to be able to introduce dyspepsias into a classification based on pathological anatomy. There is no essential difference between these affections. They differ only from one another by the degree of intensity of intestinal phlegmasia, and in practice we may meet with all the intermediary degrees between mild gastro-intestinal catarrh and very acute gastro-enteritis.-N. D. T. 
tions defined under the names of indigestion, overloading of the stomach, gastric trouble, gastric condition, gastric fever, etc. In doing this, we follow an old arrangement which obtains on account of the difficulty of recognizing in the horse the exact seat and degree of intensity of the diseases of the stomach and intestine. Acute catarrh may undoubtedly affect the stomach and intestine separately, and may even extend over a more or less restricted portion of the latter; besides, there are numerous intermediary degrees between the hyperemic condition of the mucous membrane during digestion and its intense catarrhal state. But in the horse, where vomiting is not observed as a characteristic symptom of gastritis, or where diarrhea is often absent on account of the favorable influence of the great length of the digestive tract, it is hardly possible to distinguish catarrhal inflammation of the stomach from that of the intestine. However, when it is recognized that these affections are mostly concomitant and produced by the same causes, this weak point of the veterinarian's diagnosis is not so important as it at first appears.

Diagnostic signs which could establish the degree of intensity of the affection are also wanting. Mild cases were formerly designated dyspepsia. Dyspepsia comprised all affections of the digestive apparatus not accompanied by any appreciable alteration of the mucous membrane of the stomach, and consisting essentially of physiological conditions. It is more than probable that these dyspepsias are found in our domestic animals, especially in convalescent, old, or anemic subjects ; but whilst they may be suspected, it is impossible to demonstrate their existence. It is also very difficult to tell where dyspepsia ends and catarrh begins, for dyspepsia very frequently terminates in catarrh. For these reasons we do not consider it necessary to regard dyspepsia as a pathological entity.

In human medicine we formerly distinguished several varieties of dyspepsia. At one time digestive troubles produced by the ingestion of excessive quantities of alimentary matter, or of food of an improper composition (dyspepsia ab ingestis) were in question, at another time dyspepsia was due to the quantitative or qualitative modification of the normal gastric juice. The anomalies of quantity consisted of an insufficient secretion of the gastric juice, which might be also poor in its active principles; they were found in anemia, hydremia, in "stupefaction" of the gastric nerves, in old 
subjects, or when coarse food was partaken of (dyspepsia torpida). The qualitative alterations were represented by an excess or absence of acids in the gastric juice (dyspepsia ab acido, dyspepsia acida). These essential dyspepsias were opposed to symptomatic dyspepsias, which accompany febrile diseases.

Physiological considerations on acute gastro-intestinal catarrh in the horse. No department of special pathology requires to a greater degree an exact knowledge of the physiological phenomena which take place in the gastro-intestinal apparatus. It is of importance to bear in mind the following points :

1. Mastication favors digestion by increasing the salivary secretion and the surface of the alimentary particles upon which the gastric juice may perform its action. Insufficiently masticated food produces troubles of digestion.

2. Small meals, at short intervals, are digested more rapidly and more completely than heavy meals given at long intervals. These latter frequently produce digestive troubles and gastric catarrh.

3 . In the horse, on account of the large quantity of saliva swallowed during the meal, the reaction of the stomach is alkaline or neutral as long as the ingestion of food lasts; it reaches its minimum of acidity immediately after eating $(0.8$ per 1000$)$; the proportion of acids increases then gradually as high as 2 per 1000 . When this proportion is passed there is abnormal acidity. Acidity of the gastric juice of carnivorous animals is physiologically greater than that of the horse. In febrile diseases such as influenza, intense gastric catarrh, etc., the contents of the stomach are of a neutral or even alkaline reaction, which is due to insufficient acidification of the gastric juice (Ellenberger and Hofmeister).

4. The first acid in the digestion of the horse is lactic acid': the digestive action of this agent upon starch is considerable; its maximum effect corresponds with the second hour of digestion. Hydrochloric acid and pepsin transform albuminoid matters into peptones. The maximum activity of albumin digestion takes place three or four hours after absorption of the food, in ordinary meals; in heavier meals, between the sixth and eighth hour. Therapeutic administration of hydrochloric acid during the period of lactic digestion is injurious, injection of large quantities of water during the period of hydrochloric acid digestion is equally so on account of the extreme dilution of this acid (Ellenberger and Hofmeister).

5. Digestion of albumin can only take place when acid is present. 
The function of the horse's stomach is specially favored by a solution of hydrochloric acid of $2: 1000$, which may be replaced by a lactic acid solution of $2: 100$; the same effect is obtained by a $1: 1000$ solution of hydrochloric acid, added to a $5: 1000$ solution of lactic acid. When the hydrochloric acid concentration exceeds the proportion of $5: 1000$ the pepsin action is lost, and the excess of lactic acid may produce catarrh of the stomach (Ellenberger and Hofmeister). Admitting the normal contents of the stomach to be 10 litres, the proper dose of hydrochloric acid will be 20 grammes (in a solution of $2: 1000$ ), supposing that there was no trace of this acid in the stomach.

6. The action of pepsin is favored by water and a temperature of $37^{\circ}$ to $55^{\circ} \mathrm{C}$. Cold is therefore hurtful. The inflamed mucous membrane of the stomach does not furnish any pepsin. Exaggerated quantities of pepsin interfere with digestion (Ellenberger and Hofmeister).

7. Epidermic productions are not digested by the horse, viz., epidermis, hair, feathers, wool, nails, and horn; the same is true of wax (wax pills !). Bones, elastic tissues, straw, bran, and cellulose, especially when these substances are old, can only be digested with difficulty.

8. The normal peristaltic movements of the stomach assist digestion by increasing the secretion of the gastric juice and producing a more thorough mixture of the alimentary matters. When these movements are no longer produced, on account of weakness of the stomach, or the participation of the muscular wall in the inflammation of the mucous membrane, digestion is interfered with, dilatation of the stomach takes place, etc. The peristaltic movements are also suppressed when hydrochloric acid gives out, for this agent is the principal stimulant of gastric action.

9. The mucous membrane of the stomach is protected from the digestive action of the gastric juice: by the blood which irrigates the mucous membrane abundantly, the alkaline property of which counteracts the acidity of the digestive fluids; by means of a coating spread upon the surface of the mucous membrane, and finally, by the gastric epithelium.

[That the stomach is capable of digesting all albuminoid substances and yet escapes auto-digestion is a fact that has never yet been satisfactorily explained; if the alkalinity of the blood neutralizes the gastric juice and prevents self-digestion of the 
stomach, we would expect this condition to favor the action of the pancreatic secretion-which acts best in an alkaline medium-and that the intestinal wall would be digested. The mucus may be removed from the walls of the stomach, and even the epithelium itself, without any injurious effect of the digestive fluids upon this organ being produced, as may be seen in wounds and traumatisms of the stomach wall by foreign bodies contained in it.-W. L. z.]

When for any reason there is an interference with the circulation in the mucous membrane, or passive anemia, as happens in catarrhs, emboli, etc., the action of the gastric juice will appear in so-called peptic ulcerations. But if, on the contrary, there is hypersecretion of mucus on account of gastric catarrh, the mucous membrane is covered with a heavy exudate which neutralizes part of the hydrochloric acid, and prevents the gastric juice from penetrating into the chyme. ${ }^{1}$

10. The different ferments (acetic, 'butyric, lactic, and alcoholic acids, etc.), which are always found in alimentary matters, are destroyed in the stomach through the action of the normal gastric juice. When the secretion of this latter becomes diminished, these acids are stirred up and produce abnormal fermentations; hydrates of carbon are found, the production of which irritate the mucous membrane of the stomach, and also that of the intestine.

11. The peptones formed in the stomach are absorbed in quite a considerable quantity through the gastric mucous membrane. But when the mucous membrane is the seat of a catarrh, absorption of peptones is no longer performed; they exist in excessive quantities, and act unfavorably upon the digestion of albumin (Ludwig).

12. The reaction of the intestinal contents is alkaline in the small intestine, the cæcum, and in the inferior portion of the colon; it is sometimes acid, and at other times alkaline in the upper portion of the colon. When fecal matters are arrested in colics and in catarrh, acid reaction of the intestinal contents is ordinarily found (Ellenberger and Hofmeister, Tereg). An augmentation or

1 The researches made by Prof. Hayem have shown that, in normal digestion, free hydrochloric acid exists in a very small proportion, and may even be wanting altogether. The gastric juice is said to derive its acidity from the organic combinations of this acid (Hayem: Bull. Soc. Méd. des Hôpitaux, 1889).-N. D. T.

[The presence of free hydrochloric acid in the gastric juice is abundantly proven by the experimentations of Prout, Schmidt, Bertholet, Bidder, Heidenhain, Rothschild, and others. (See Smith's "Physiology of Domestic Animals," page 349.)w. L. z.] 
a diminution in the absorption of certain salts is a consequence of this acidity. Fever diminishes the quantity of the intestinal secretions, the qualities of which are also changed; these modifications give occasion to digestive troubles.

13. Certain gases are formed in the cæcum of the horse, provoked by cellulose fermentation $\left(\mathrm{CH}_{4}, \mathrm{CO}_{2}, \mathrm{SH}_{2}, \mathrm{H}\right)$; these and also the products of albumin decomposition (phenol, indol, skatol) are often produced in excess in intestinal catarrh, colics, etc., and determine symptoms of poisoning, or they are more abundantly eliminated through the urine.

14. The normal stay of alimentary matters in the intestines of the horse is from three to four days.

Etiology. Acute gastro-intestinal catarrh is a very frequent disease in all our domestic species, and particularly in the horse; it may result from causes exerting a direct or an indirect action upon the mucous membrane. Certain individuals have a predisposition to it. These are weak, anemic, poorly fed and emaciated subjects, very young and also old horses, animals recovering from a serious disease, also those in poor condition from debilitating diet consisting of a poor quality of food, and such as have previously suffered from this disease, etc. In very fine and delicate thoroughbred horses an attack may sometimes occur through the most trivial causes.

Among the direct irritations the action of which, influencing the mucous membrane, may provoke catarrh may be mentioned : green food, food and drinks that are very hot or that are covered with frost or entirely frozen, soups, very hot malt and slops; unclean or decayed food and drinks, mouldy or slimy hay or oats, damp straw and sour bread; also alimentary matters going through any description of fermentation or decomposition, such as potatoes, turnips, fruit, grass, and germinating grains; finally, acid milk in young sucklings. All irritating alimentary substances may have the same bad influence, especially those containing poisonous plants (colchicum, tobacco, digitalis, etc.). The abuse of acrid medicines and purgatives, again, is harmful. Gastro-intestinal catarrh may also be produced through substances hard to digest, or altogether indigestible, as foreign bodies, ligneous fibres, elastic tissue; exclusive alimentation of grain, or of leguminous grains, which are of difficult digestion and frequently provoke a great swelling of the stomach; this. is also the case in poor mastication 
of food (in old horses, and in the young during the eruption of teeth). We must also mention the ingestion of food and drink in too large quantities. Indigestion through overloading the stomach, which is very often the starting-point of catarrh, is usually produced through the change from poor to abundant feeding, or when easily digested food is replaced by more or less indigestible substances. Animals are also very much exposed to catarrhal phlegmasia of the stomach or intestine when subjected to an abrupt change from dry to green food, or the reverse; or if, when unaccustomed, they partake of large quantities of watery grass fermenting too easily-for instance, green clover. It can be produced, likewise, in the horse by a prolonged abstinence, by heavy meals separated by long intervals, by irregular eating, and may occur when the horses get loose during the night and ransack the oat-box.

The indirect causes of gastro-intestinal catarrh are: any violent efforts, and work immediately after meals - the muscles attracting the blood to the periphery and the digestive organs becoming anemic; sudden changes of temperature-for instance, cold, momentarily congesting the digestive mucous membrane, and thus producing the first stage of catarrh ; great heats, very low atmosspheric pressure, etc., may also cause the trouble. Besides these causes, catarrh may also be produced through a weakened constitution, or colds resulting from badly built, unclean, and damp stables with defective ventilation. In some countries gastro-intestinal catarrh assumes an epizootic form at certain periods. Infectious catarrh is caused by the introduction of certain micro-organisms into the intestine; notwithstanding its specific cause, it is here often difficult to distinguish it from common acute catarrh or plain gastroenteritis. Finally, we observe sometimes a symptomatic, secondary, gastro-intestinal catarrh accompanying various febrile diseases-for instance, pneumonia, canine distemper, puerperal fever, petechial fever, etc.

Pathological anatomy. We rarely have occasion to make an autopsy upon animals that have died from essential gastro-intestinal catarrh ; it is, indeed, exceptional to see it produce death. When, however, it coexists with another affection which carries off the sick animal, we can observe minutely any alterations it has produced in the stomach and intestine.

1. Alterations of the stomach. The pyloric portion of the mucous membrane is tumefied; sometimes it is uniformly red, 
at other times it is marked with large ecchymoses, assuming frequently a ramified disposition. These interstitial hemorrhages are of various dimensions; they are found punctiform, lenticular, or more extended and irregular; they give the inflamed membrane a red-speckled, spotted, or streaky appearance (hemorrhagic catarrh of older authors).

The mucus, secreted in considerable quantity, forms a mucofibrinous or muco-purulent covering on the surface of the mucous membrane, which may have been colored a more or less dark red by the blood of superficial hemorrhages; in microscopic examination we detect the presence of mucous corpuscles, leucocytes, and desquamated epithelial cells undergoing dissolution.

- No histological study of alterations of the mucous membrane, in the horse, has been made. According to Ziegler's researches on man, this membrane is found to present degeneration of the cylindrical epithelium of the excretory tubes of the glands, abundance of granulations of the epithelium of the pepsin glands, cellular infiltration of the inter-glandular and submucous connective tissue, lastly swelling and desquamation of the endothelium of the lymphatics.

2. Alterations of the intestine. They are nearly similar to those of the stomach. The mucous membrane is tumefied and raised by the serous infiltration of the subjacent connective tissue; the muscular layer is also infiltrated; often the whole intestinal wall can be torn very easily. Inflammatory hyperemia is generally limited to small surfaces, sometimes it is spread over the entire intestine. In certain cases the papillæ and villi alone are inflamed, they are of a bright or dark red tint, and are more or less projecting. The inflammation may finally be localized aronud the solitary or agminated follicles. In this last variety of the disease, designatcd follicular catarrh, the follicles protrude on the surface of the mucous membrane in the form of whitish-gray nodules, varying in size from a millet- to a hemp-seed, and surrounded by a reddish zone. This condition has been found in the large intestine by Röll; it is also observed in the small intestine. When the inflammed follicles are subjected to a purulent transformation, or if they are used up by the friction from alimentary matters, there will be found crater-like cavities remaining in their place, which must not be mistaken for intestinal ulcers, and which may be properly called follicular ulcers. We may observe more or less diffuse epithelial 
desquamations in serious catarrh, which are called catarrhal erosions.

The product of the catarrhal mucous membrane consists of a fluid rich in epithelial cells; the variable proportion of the latter gives it a serous, mucous, or pus-like consistence. It is ordinarily of a grayish or reddish color; sometimes the hemorrhages color it a more or less intense red. When the epithelial destruction is particularly marked, the catarrh is said to be desquamative.

The mesenteric ganglions always participate in the inflammation.

Histological examination of the mucous membrane has not been made. Ziegler has found, in man, cellular infiltration of the connective tissue surrounding Lieberkühn's glands. This lesion is constant in cases of catarrh of medium intensity.

Symptoms. These are more or less marked, in proportion to the degree and extent of the trouble, its causes, and the degree of resistance of the animal.

1. Symptoms of acute gastric catarrh. The reduced appetite constitutes the first and often the only symptom visible to the owner. There is either entire inappetence, or the animals eat little and slowly; the appetite is irregular, capricious, and ordinarily the patients prefer straw and coarse food to good nutriments ; aberrations of taste may be observed, as "licking," or an irresistible desire to eat their litter, especially when soiled with excrement and urine. Thirst is usually diminished; some animals refuse water absolutely. The irritation of the terminal branches of the vagus nerve may cause frequent yawnings by reflex action, which may be seen to last for hours at a time.

In the horse, overloading of the stomach occasionally produces nausea and even true vomiting, but without serious danger to the animal and without the manifestation of severe colics. Numerous facts demonstrate, that these mild vomitings, caused by gastric catarrh or overloading of the stomach, are not so rare as is generally admitted. These vomiting spells are harmless; they are quite common, especially in the mountains, where horses left at liberty often eat considerable quantities of the leaves of hellebore, Veratrum album. (See Colics from Overeating.)

As a rule, the buccal mucous membrane presents very marked modifications. It may be found pale, dirty yellow, or livid red; at the beginning of the disease it is dry, later it is covered with a 
thick slimy mucus, frothy and bad-smelling; the buccal cavity contains a considerable accumulation of thick thread-like mucus, of an alkaline reaction. Lampas is often noticed, and as the animals eat but little, the tongue is coated. Finally, cases are quite common where gastro-intestinal catarrh is complicated with a true catarrhal stomatitis.

In the early stages of the disease examination of the belly gives no indication of the condition, but later on it becomes emptied and tucked up. The peristaltic movements of the intestine may continue normal ; in some cases they are suppressed ; the excrements are dry and coated, and are passed in small quantities, and often at very short intervals ; undigested alimentary matters are frequently found. There is no change in the normal constituents of the urine, and its modifications are important.

The general condition varies according to the intensity of the trouble and the degree of resistance possessed by the animal ; we observe stupefaction, laziness, and a tender condition during work; they become tired rapidly and perspire at the least effort ; breathing is painful. Fever is often absent, and when it exists is always moderate; the rectal temperature increases but a few tenths of a degree, and the pulsations hardly exceed the normal by more than ten per minute. This febrile condition persists, however, for a few days only. In rare cases we observe chills at the initial period; the temperature of the body is irregularly distributed, the ears and the extremities being cold; all the mucous membranes are more or less injected.

Sometimes febrile manifestations exist, at other times they are wanting. It is, therefore, illogical to substitute the term "acute catarrh of the stomach" by "gastric fever." If we wish to become more thoroughly acquainted with these morbid conditions formerly designated by the collective name of "gastric fever" we should remember the following facts:

1. Formerly, all apyretic diseases of the stomach were designated under the names of dyspepsia, status gastricus, difficult digestion, etc.; these were in opposition to the group of pyretic diseases of this organ, which were known as "gastric fever." But at the present time it is more rational to separate them into pyretic gastric catarrh and apyretic gastric catarrh.

2. For a long time we have noticed that there are subjects in which an apparently benign gastric affection-or, to speak more 
clearly, an acute catarrh of the stomach-develops with intense fever. These rare cases are characterized by the disproportion which exists between the gastric symptoms, properly so-called, and the intense persisting fever ( 40 to $41^{\circ} \mathrm{C}$., with 70 pulsations or more per minute), accompanied with serious disturbances of the general condition (great muscular weakness and considerable depression of the senses). It was difficult to classify them elsewhere than under gastric fever, where most of them could be properly considered. But, even here, this denomination is but significative; at all events, it is superfluous.

What is the significance of these gastric cases accompanied by intense fever? They seem to depend upon several causes:

1. In anemic animals having little resistive power, acute catarrhal fever is more intense, and it leads more easily to debility and exhaustion.

2. Catarrhs that are of an infectious nature ordinarily develop with intense fever. They often assume an epizootic form; the infecting agent is a micro-organism coming from without, or a product of decomposition formed in the stomach at the expense of the ingested nutriments.

3. The symptoms described in a number of cases indicate that they were not gastric catarrh. This has been confounded with other diseases. Thus "gastric catarrh accompanied by an intense febrile condition" is often the initial period of an infectious febrile disease, the diagnosis of which only becomes possible later on. Intoxications occurring after alimentation with tainted fodder, or which are caused by resorption of certain abnormal products of digestion, etc., have also been mistaken for gastric catarrh. It is very likely that a large number of cases of the so-called typhoid gastro-enteritis belong to these intoxications.

2. Symptoms of acute intestinal catarRh.-Contrary to what is observed in acute gastric catarrh, where the appetite is always impaired, we find that in intestinal catarrh the animals always continue to eat as usual, at least during the first period of the disease. When there is any diarrhea, thirst is sometimes excessive. The general condition is satisfactory, and the disease may proceed without fever; but ordinarily its localization upon the intestine leads to more serious general troubles than when the stomach is the part affected.

The abdominal pains are more frequent and more intense than 
in gastric catarrh. The abdomen is sometimes distended by gases, and is quite sensitive to palpation. As a rule, the peristaltic movements are very active, and noisy borborygmi are heard even at a distance; but at certain periods these movements do not any longer occur, and the intestinal noises are hardly perceptible. At the beginning the feces are large and soft; they are often coated with a thick mucus; sometimes they may be covered with whitish or yellowish (proctitis) croupous membranes. Numerous undigested grains of oats and long and coarse fragments of fodder are seen in the excrements, which smell putrid or sour. A greenish-yellow liquid, which soils the tail and the inside of the hind legs, sometimes runs from the anus immediately after defecation.' There is a constant escape of bad-smelling gases, but especially at times of defecation.

Loose diarrhea appears; this is an important symptom from a diagnostic point of view. The excrements, which were watery and soft, are changed into a thick pulp, which becomes softer; then the feces are altogether liquid and have an acid or putrid odor; weakness becomes excessive, the anus remains gaping, and there is a continual escape of matter from it (colliquative diarrhea). These matters, going through decomposition, are of an acid reaction (fat acids); but this is of little value as a diagnostic symptom, for in the normal state the excrements often have an acid reaction.

In certain cases of acute catarrh, when phlegmasia is localized upon the small intestine, diarrhea may be absent; the diarrheic fluid is entirely reabsorbed, the intestinal contents become more and more compact as they approach the rectum, and the excrements are moulded. On the other hand, the existence of an intestinal catarrhal affection is not always indicated by diarrhea. A green diet may influence diarrhea; this may even be produced through a simple acceleration of peristaltic movements of reflex origin, through a consecutive stasis of blood, as are frequently observed after a sudden taking of cold.

The urine, which is hardly altered in gastric catarrh, is always much modified in enteritis. As soon as diarrhea exists the urine is excreted in less quantity, and its specific gravity is increased; it often changes its color, ordinarily it becomes clearer, more transparent, and loses its sediment-these modifications are more or less marked. The proportion of indican increases : this fact is well established in human medicine; we have recognized it in the horse in 
cases of active fermentation and decomposition of the alimentary matters in the intestines. It is always rich in phosphates, while these salts are almost always absent in normal urine. Its reaction becomes almost always neutral or even acid.

Depending upon numerous observations, Fröhner has established the fact that in herbivora the urine is acid only in inflammatory diseases of the gastro-intestinal canal when not in a state of inanition. Febrile diseases, even those of long duration, do not impair its alkaline reaction if the animals continue to eat and it is not complicated by gastro-intestinal catarrh. We have many times had occasion to confirm the exactness of these observations. The stated opinions of Hofmeister and Siedamgrotzky, ${ }^{1}$ that the acid reaction is noticed after a long abstinence in intestinal catarrhs and in all the other cases of intense fever, is not confirmed by facts.

Various hypotheses have been advanced concerning the acidity of urine in herbivora. Fröhner, depending upon the fact that the absorbing activity of the intestinal mucous membrane is modified when affected by inflammatory trouble, and that the phosphatic salts alone continue to be absorbed by this membrane, has ascribed the acidity of the urine to its greater richness in phosphates. Tereg thinks that the acids existing in the contents of the intestine affected by catarrh act upon the earthy insoluble phosphates, rendering them soluble and consequently absorbable. Finally, Siedamgrotzky and Hofmeister admit that the expulsion of phosphates by urine is more abundant because the diseased digestive glands eliminate them in smaller quantities.

Course and duration. When the catarrh is slight and accompanied by a weak febrile reaction it generally ends in a cure. This will take place mostly within a few days; it is rarely delayed more than six or eight. The fever disappears little by little, appetite returns, alvine evacuations are less frequent, and the scybala gradually become of firmer consistence. A slight constipation usually follows diarrhea. The return of the urine to an alkaline reaction is a favorable sign, even when still rich in phosphates (basic phosphate of lime).

But when the causes of the disease persists there is either a slight gradual attenuation of the catarrh, which passes into a chronic form, or it becomes complicated. In the latter case fever increases ;

1 Hofmeister and Siedamgrotzky : Mikroskopischen u. chemischen Diagnostik, 1884. 
the persisting diarrhea becomes dysenteric and colliquative; the abdominal pains are frequent and violent, the flanks are tucked up, and the animals are greatly emaciated. The hair is dull and bristly; the extremities are cold, the muscular weakness is more and more accentuated; the animals remain quiet, with the back arched and the feet gathered together. They are tired, exhausted; they stagger, lie down, and remain in a recumbent position for whole days, and refuse all food. Convalescence is very slow even when in such serious cases a cure is obtained; but the disease leads mostly to marasmus and death, especially in old, exhausted horses.

Differential diagnosis. When acute gastro-intestinal catarrh pursues its course without fever, it cannot be mistaken for any other affection of the intestine. In certain cases it is difficult to determine whether the disease is primitive, or if it constitutes only an added symptom of a pre-existing trouble; therefore no diagnosis of gastro-intestinal catarrh must be made before having excluded the existence of all the other morbid conditions of the digestive apparatus. It is sometimes quite difficult to differentiate between the true intestinal catarrh and gastritis. The intensity of the fever, the general disturbances, and finally the recognition of the causes, permit us, however, to make a diagnosis. When the follicular organs are ulcerated we may very easily mistake the appearing colliquative diarrhea for true infectious dysentery. The course of the disease, the anamnesis, etc., also furnish a clue for diagnosis.

There are cases where we may assert the localization of the catarrh upon a definite portion of the gastro-intestinal mucous membrane.

When there are symptoms of icterus, we conclude that the catarrh is located upon the first portion of the small intestine. We may recognize the participation of the stomach in the catarrhal infection by the perception of frequent and violent borborygmi in the left hypochondriac region.

Catarrhs of the duodenum complicated by an inflammation of the mucous membrane of the biliary canal (ductus choledochus), and icterus through retention of the bile, were formerly designated under the name of "bilious fever." But it is very probable that this expression has often been used to designate certain febrile diseases of the liver. Hematogenic icterus appearing after serious febrile affections which are accompanied by a certain degree of blood-dissolution (infectious diseases) has been specially designated under the name of bilious fever, bilious condition, etc. It was 
admitted that bilious fever could assume a more serious form and acquire a putrid character (typical bilious fever of the older authors).

In catarrhal affections which are limited to the large intestine we observe, as special symptoms, colics and diarrhea. The expulsion of coated fecal matter indicates a localization of the catarrh in the posterior portion of the large intestine and the rectum (catarrhal proctitis).

Frequent expulsions of small quantities of excrements, tenesmus, violent efforts and complaints at the time of defecation, arching of the back, etc., are all so many symptoms of rectal catarrh.

The expulsion of excrements covered with mucus was formerly looked upon as a mucous fever. We follow Röll's example in considering this disease, which is particularly common in ruminants and dogs-as a variety of acute gastro-intestinal catarrh. Any desquamative or exudative process, in fact, was formerly called mucous fever ; this becomes evident in the description given by Spinola, who assigns the following as the principal symptoms of the trouble: paleness of the mucous membrane, a copious exudation upon the whole intestinal tract, excrements coated with a large quantity of mucus, tendency to diarrhea, etc. This old nomenclature must be abandoned to history.

Treatment.-The prophylaxis consists in the rational application of dietetic principles relating to the alimentation and keeping of the horse; strict observation of the animals protects them from most of the etiological influences which play the principal rôle in the development of the disease.

The curative treatment itself must be mainly hygienic. In many cases attention to the diet alone will overcome the trouble. Heavy and exciting foods must be avoided, and those selected should be dispensed in small quantities at a time. When the disease is traced to overloading of the stomach with alimentary matter it will be well to subject the animals to entire abstinence for several days. Drinking-water must be exposed to the air for some time, and must be given immediately after meals, and not a few hours after. The patients should be well covered, and the whole surface of the skin should be rubbed several times daily. It is also advisable to give them a little exercise in fresh air.

The internal medication will depend upon the various indications:

1. In a case of overloading the stomach, the alimentary matters should be expelled as quickly as possible. We frequently use for 
this purpose a purgative electuary, having a base of soda salts, and emetics : tartar emetic 6 grammes, sulphate of soda 300 grammes; rye-flour and water (q. s.), or calomel given in small doses (3 grammes). A subcutaneous injection of eserine may also be given (sulphate of eserine 0.1 gramme, distilled water 10 grammes), or of eserine and pilocarpine (of each 0.1 gramme).

2. In order to stop abnormal fermentations of the stomach and intestine, hydrochloric acid is recommended (dose, 10 to 15 grammes a few hours after meals). Table salt (20 to 30 grammes), alkaline carbonates, especially bicarbonate of soda ( 25 to 30 grammes) may also be used to counteract it. Gastro-intestinal catarrh, accompanied by much flatulence, with acidity of the contents of the intestine, yields quite rapidly to hyposulphite of soda (hyposulphite of soda 150 grammes, calamus 25 grammes, althæa powder, and water q. s. to make an electuary. Creolin in doses of 5 to 15 grammes is useful as an antizymic (creolin 15 grammes; pulv. licorice-root 15 grammes, althæa powder, water $q$. s. for three pills. Give one pill a day).

3. When weakness of the stomach predominates, we must use the so-called stomachics, at the same time giving hydrochloric acid. Pepsin must first be recommended (10 to 15 grammes), then the alkalines, table salt sulphate of soda, and ammonia (sulph. of soda 300 grammes, chloride of sodium 100 grammes, chlorhydrate of ammonia 50 grammes; to be given in a dose of a tablespoonful per meal). As vegetable stomachics, we administer white mustard (50 grammes of powder in electuary), pepper, ginger (10 grammes), gentian (25 grammes), powdered rhubarb (15 grammes); bicarbonate of soda and powdered licorice, of each 10 grammes, althæa powder, and water q. S., the electuary to be given in three doses; aloes ( 5 grammes in a pill), the whole group of bitters (absinthe, gentian, etc.), and also stimulant essences (auise, fennel, cumin, juniper cones, etc.).

4. Very small doses of calomel may be successfully used for diarrhea in the early stages; this agent is particularly useful when there is evacuation of fetid excrements. We must then attempt to stop diarrhea by a special diet (such as dry food, roasted oats and flour, and some mucilaginous preparations). When these means are not sufficient, we resort to astringents, beginning with oakbark and cinchona (25 grammes), sulphate of iron and alum (10 grammes), tannin (5 grammes). We then give them sugar of lead. 
(5 grammes), and lastly nitrate of silver ( 0.5 gramme to 1 gramme) in pills with the ammonia bolus, or in weak solution in distilled water, boiled or spring water. Opium is one of the most efficient agents (100 to 150 grammes of plain opium tincture in a mucilaginous decoction; to be given at one time). Röll has extolled tincture of nux vomica.

5. In catarrh localized on the posterior part of the large intestine and rectum, we try to act directly upon the mucous membrane by means of clysters. When diarrhea exists, it is proper to use a solution of nitrate of silver per rectum. This proceeding is of special advantage in cases where intestinal catarrh takes a slow course. Alum and tannin solutions, etc. (1-2 per 100), are frequently used to prevent catarrhal tendencies.

\section{CHRONIC GASTRO-INTESTINAL CATARRH IN THE HORSE.}

\section{Chronic Catarrhal Gastro-enteritis: Chronic Dyspepsia.}

The considerations developed in discussing the acute form of this disease might be repeated here, in order to demonstrate the propriety of understanding chronic catarrh of both the stomach and intestine as being included in the same description. As pointed out by Röll, gastro-intestinal catarrh is a frequent disease in the horse. It usually constitutes an essential morbid condition, but in some cases it is merely a phenomenon of another organic affection (alterations of stomach and intestine, disease of the lungs, heart, and kidneys). This latter variety of chronic catarrh is particularly found in old horses.

Etiology. This is a disease common to all periods of life, some animals, however, are more particularly exposed to contract it : the grave troubles of nutrition, cachectic or anemic conditions, chronic intoxications, exhaustion from excessive fatigue, etc., undoubtedly predispose to the affection.

Chronic gastro-intestinal catarrh may follow the acute form, when the causes taking part in its etiology act for some time, either in an intermittent or a continuous manner.

We would mention here as conditions producing chronic catarrh, the various hurtful influences which have been mentioned in acute catarrh, and especially the lack of hygienic conditions, irregular 
feeding, the administration of indigestible, tainted, poor or laxative food, also coarse, muddy and sandy fodder. Anacker condemns the exclusive feeding of bran and finely cut straw, and sustains his opinion by calling attention to the frequency of chronic catarrh of the stomach in millers' horses.

Imperfect mastication and incomplete salivation of the food, in old horses, often induce chronic eatarrh of the stomach; at other periods of life it may be the consequence of diseases of the teeth and of irregular dental surfaces. Therefore, crushed oats have many advantages recommending them as horse food.

Anything producing a stasis of blood in the mucous membrane of the stomach or intestine ends sooner or later in chronic catarrh of these organs. We may particularly mention: repeated acute catarrhs; stagnant conditions of the portal circulation, provoked by chronic affections of the liver, or an alteration of the portal vein itself; also those produced in the posterior vena cava by lung or heart disease (valvular insufficiency, pulmonary emphysema, hepatization); blood-stasis in the intestinal walls, due to continued contraction when the peristaltic movements are under the influence of a reflex action (repeated colds); also intestinal hyperemia consecutive to the arterial emboli of verminous aneurism (Friedberger's embolic catarrh of the large intestine). ${ }^{1}$

In these cases the blood-stasis is not the direct cause of the catarrh, but it leads to organic alteration of the mucous membrane, which renders it less resistant to the various irritations to which it may be exposed. A persistent diarrhea often precedes chronic catarrh ; this is observed, for instance, when repeated colds influence the action of the intestine in relation to its peristaltic movements.

The presence of numerous entozoa in the stomach may also produce chronic catarrh of this organ. We may particularly point out: 1 . The gad-fly larvæ, which often produce serious digestive troubles (Cambron, Lessona, etc.), especially in young horses; the species living in the stomach are the Gastrophilus equi et pecorum; the Gastrophilus hoemorrhoidalis et nasalis is sometimes found there in innumerable quantities. The Spiroptera megastoma which is often found enclosed in the developed verminous cysts upon the line of union of the two mucous membranes of the stomach ; these cysts, which are usually purulent, vary in dimensions from the size 
of an acorn to that of an apple. (Concerning common intestinal worms in their relation to chronic intestinal catarrh, see the section on Helminthiasis: Ascaris megalocephala, Toenia mamillana, T. plicata, $T$. perfoliata.)

Symptomatic chronic gastro-intestinal catarrh is also determined by: ulcerations, dilatations, constrictions, displacements, new formations, and foreign bodies; and besides by calculi of the stomach and intestine, bezoars of animal or vegetable nature, abnormal accumulations of alimentary matters, and finally the diseases of the pancreas (Martens).

Among the numerous affections which may provoke gastrointestinal catarrh, let us particularly mention: Bright's disease, rhachitis, anemia, hydremia, leukemia, and chronic muscular rheumatism.

Pathological anatomy. 1. In chronic catarrh of the stomach, the alterations are generally located upon the mucous membrane of the right pouch, especially at the large curvature and in the neighborhood of the pylorus. We find there a thick and adherent layer of whitish-gray mucus, containing a considerable number of epithelial cells.

The coloration of the mucous membrane is variable and often shows nothing characteristic. It is usually of a dark or dirty brownish-red; quite frequently we observe a slaty pigmentation or spots of this color, giving the membrane a marble-like appearance. This pigmentation is produced through infiltration of black pigmented corpuscles, derived from the coloring matter of extravasated blood, or from small bloody collections due to capillary hemorrhages. In other cases the mucous membrane is of a uniform yellowish-gray, or pale, whitish, with a few much-distended bloodvessels upon its surface; sometimes this is regular and smooth, or, again, anfractuous, mammillated, or corrugated; polypous new formations are very rarely found in it (polypous gastritis ; polyposis).

Microscopic examination has shown us but one case of atrophy; hypertrophy is the rule; in the mammillated and polypous forms the thickening may be from three to five times greater than normal. The changed tissues are hard and inelastic, like cicatricial tissue; a sharp instrument can hardly make an incision in them; they creak under its action, the section surfaces are very rigid, and do not present the least retractility. The thickening of the submucous connective tissue and muscular hypertrophy, which is 
harder to the touch, is oftentimes perceptible to the naked eye. (For ulcerations happening in the course of chronic catarrh of the stomach, see Ulcers of the Stomach.)

In the exceptional cases where the catarrh is serious and of long standing, the mucous membrane of the pouch also presents alterations. Siedamgrotzky has found considerable thickening in it, fivefold the normal dimensions and extending over a surface equal to that of the hand; the surface of the mucous membrane was mammillated, covered with white and horny epithelial masses produced by irregular hypertrophy of the papillæ, and mainly by an abundant epithelial proliferation; the lesions of the right portion of the mucous membrane were nearly similar. We have ourselves made analogous observations. Histological alterations can only be detected by a very detailed study. At the outset they consist of an increase in volume of the glandular canals, which seems due to the infiltration of epithelial cells; soon proliferation of the inter-glandular and submucous connective tissue commences; gradually it causes an atrophy of the glandular tissue by pressure, also contraction and reduction of the canals, fatty degeneration, and destruction of the epithelial cells. Ziegler's researches have a tendency to demonstrate that atrophy of the glandular cells constitutes the fundamental alteration; hypertrophy of the connective tissue being looked upon as secondary and accessory. Friedberger has observed a case where the mucous membrane was indurated and atrophied; the epithelial covering was absent; the glandular canals were reduced and depressed, the inter-glandular connective tissue, on the contrary, was hypertrophied; the remaining glands had a less abundant epithelium than normal.

According to Bruckmüller, the lenticular projections and villous productions which are found on the mucous membrane are formed by hypertrophied papillæ, while the large pustules and the polypous formations are known to be caused by abuudant proliferation of the glandular tissue. Connective-tissue hyperplasia is quite often extended to the submucous tissue, even to the myolemma and the subserous tissue; in such instances the muscular fibres are atrophied. In cases of this kind there is often a more or less marked dilatation of the stomach. We may also observe hypertrophy of the muscular tissue, although this is quite rare.

2. The pathological anatomy of chronic catarrh of the intestine is almost identical with the same disease of the stomach. The 
mucous membrane is usually of a marked coloration; in some places it is marked with red-brown or slaty spots; in others it is pigmented and of the nature of an eel-skin ; its surface is covered with a thick exudate, which is grayish, mucous, or purulent, and sometimes granulous; its vessels are distended and varicose; the follicles are enlarged at first, later they become atrophied and disappear, giving the mucous membrane a perforated appearance. The intestinal contents are often very liquid, like curds, and hold certain flake-like masses in suspension. The mucous membrane rarely becomes thin (personal observation). We find it almost always thickened, hard, folded, and offering the same papillar and polypous proliferation as that of the stomach (see Dilatation and Atrophy of the Stomach).

Röll states that the follicles of the large intestine (especially in the cæcum and the large colon) take part in the inflammation. They project in the shape of small tumors of the size of a milletseed to a pea, are of soft consistence, and may become unfolded in forming follicular ulcerations, covering at times a considerable portion of the large intestine. Röll describes these ulcerations thus : they are of the size of a grain of juniper, or of a lentil, reaching as far as the submucous connective iissue, the edges of which are cut in shape of a point, and are infiltrated ; sometimes they are detached with a suppurating base. They may become confluent and have a small cicatrix; at times they persist, occasion diarrhea, intermittent colic, and in certain cases perforation of the intestine (see Ulcers of the Intestine).

Symptoms. The manifestations of the disease are easily understood, if one bears in mind the various anatomical alterations found in gastro-intestinal catarrh, especially atrophy of the pepsin gland of the stomach, fatty degeneration of the epithelium, hypersecretion of the mucous membrane, and irregular distribution of the blood.

1. When the catarrh has its particular seat in the stomach we first observe a change in the appetite, which diminishes or becomes capricious; aberrations of the taste, frequent gapings; the defecations are infrequent, and there is constipation ; the tongue is coated, the mouth dry and fuliginous. It is rare that we have a serious febrile reaction. These symptoms, which form the clinical picture at the beginning of the trouble, are subject to strange variations: the derangement of the appetite is always more marked when the 
animals are worked, especially when they are compelled to undertake violent exertions; in other circumstances they disappear entirely. The least mistake made in their alimentation may induce an aggravation of the general condition and intestinal pains. Periodical colics are frequently observed in chronic catarrh of the stomach and intestine.

In serious catarrhs and those of long duration we often observe strange sympathetic nervons complications, which were formerly included under the denomination "stomachal vertigo" (staggers). Besides unwillingness to work, which always exists, we observe a considerable nervous depression, as coma, stupefaction of the motor and sensory centres, or attacks of vertigo (gastric vertigo, abdominal vertigo). In order to explain these troubless it has been held that there was a transmission of the nervous irritations of the stomach to the brain, also cerebral anemia produced by bloodstasis in the walls of the digestive canal, and auto-infection by resorption of abnormal products formed in the stomach (Senator).

In his Traité de Médecine vétérinaire judiciaire, Gerlach points out the singular abuse formerly made of the term "stomachal vertigo." The disease described under this name by Spinola and Van Gemmeren corresponds without doubt to chronic lupinosis, and to most diseases which are included at the present day under the denomination of subacute encephalitis-as is proved by the cause to which the trouble is ascribed by these authors: the feeding of clover. There is quite a group of various morbid conditions which are made manifest by gastric troubles and nervous depression. We observe similar symptoms in ulcers of the stomach, in gastritis, in verminous affections, and especially in the condition called "Schweinberg's induration of the liver." In order not to confound these diseases with true vertigo, and on account of the reasons just mentioned, the expression stomachal vertigo ought to be dropped.

There are cases where persistent diarrhea forms the predominating symptom, permitting us to define the existence of chronic intestinal catarrh; we often observe also constipation and diarrhea alternating. These opposite conditions are almost always accompanied by flatulence and symptoms of anxiety returning periodically.

The urine is not of a constant character; its modifications are in accord with the degree and duration of the perverted appetite, with the alternations of diarrhea and costiveness, and also with the 
localization of the catarrh. In a few cases it is altogether normal, but ordinarily it is clearer and more transparent, as in acute intestinal catarrh. It is almost always of an alkaline reaction; we have often witnessed this fact, which is pointed out by Albrecht. Siedamgrotzky and Hofmeister ${ }^{1}$ have found it of acid reaction, which must without doubt be ascribed to the exacerbations of the chronic process, or to a special affection of the small intestine. The proportion of indican and phosphates is sometimes increased. In some cases we have observed albuminuria.

Albrecht has found crystals of oxalate of calcium in the urine of horses having intestinal catarrh for a long time, which disappeared with the chronic catarrh; he has wrongly considered this fact as a valuable data from a therapentic standpoint; these crystals belong, in fact, to the composition of normal urine, and their appearance in more considerable quantities is sometimes the result of trifling exterior conditions (such as standing for some time in any receptacle or vessel, etc.).

When the disease is old we find serious interference with nutrition. The animals lose flesh and perspire easily, even in the stable; the mucous membranes are pale and of a yellowish color ; the hair is dull and bristly; the pulse small, the contractions of the heart muscle are weak, irregular, and often intermittent. According to Röll, the existence of follicular ulceration is commonly produced by the symptoms just mentioned, but mainly by refractory diarrhea, or by constipation with meteorism, both accompanied by colics; at times symptoms of acute enteritis and fever complicate the trouble. However, as the Vienna professor observes, these symptoms are far from being characteristic of chronic follicular ulceration.

Duration and termination. The duration of catarrh varies with the causes which have provoked it, the possibility of removing them, and the extent and intensity of the disease. When it is caused by coarse, improper alimentation, it is easy to obtain a cure. When there are ulcerations of the mucous membrane, which are of themselves serious enough to maintain the catarrh, the disease is always of long duration. We see horses attacked by chronic gastro-intestinal catarrh continue to perform their work for years, but their strength and weight decrease progressively, and exacerbations are not rare when the morbid condition is thus prolonged. These animals are generally brought to the veterinarian at the time

1 Siedamgrotzky and Hofmeister: Sächs. Jahresber., 1875. 
of one of these exacerbations, for the disease characterized by these common symptoms almost always remain unobserved. There are cases where the weakness and emaciation progress much more rapidly; the patients become almost incapable of performing any service, and they die in a condition of marasmus in a relatively short time.

Chronic embolic catarrh, the course of which is often interrupted by attacks of colic, produces in the end circumscribed necrosis of the intestine and death (see Enteritis).

Treatment. The most important indications for the treatment of this disease are, in the main, the same as given for acute gastrointestinal catarrh. The cause has to be sought, and a proper dietetic regimen has to be observed; this is the basis of the rational treatment. Deteriorated or indigestible aliment must be avoided; only food of good quality should be given; it must be administered often and in small quantities at a time; the work of the animals must be carefully regulated; the dental surfaces must be attended to; old animals should receive crushed oats, etc. Unfortunately, in the majority of cases, circumstances render the application of these measures extremely difficult.

[The best results are to be obtained in these cases by a strict regulation of the diet and the administration of easily-digested focd. The oats should be prepared by scalding, boiling, or steaming, and allowing them to stand for twelve hours before feeding; the ration to consist of two parts of steamed oats, une part of bran, and one part of malted barley. Care must be taken to prevent the patient from obtaining such foud as would be irritating and indigestible. Therapeutic treatment by the administration of drugs is always unsatisfactory.-W. L. z.]

Medication occupies only a second place in the therapeutics of chronic catarrh. Hydrochloric acid in moderate doses (10 to 15 grammes) has rendered good service.

Alkalines play the principal part. We may give either table salt (sodium chloride) in small doses for a long time (half a tablespoonful at every meal), or carbonate or bicarbonate of soda in doses of 5 grammes. Sulphate of soda is equally good (30 to 50 grammes). We prescribe ordinarily these three agents in the shape of artificial Carlsbad salts :

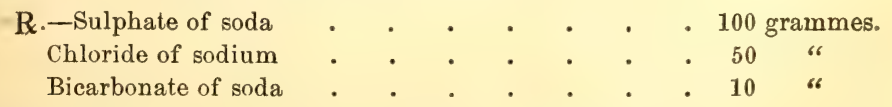

Make a watery solution. Dose, a dessertspoonful at each meal. 
We obtain from this several good results : the acids which are in excess are neutralized; secretion of the digestive fluids is aided by the presence of the sodium chloride; the abnormal fermentations are checked and the exudates dissolved; finally, the progress of the intestinal contents is promoted by the sulphate of soda.

The common stomachics can be used with success. Rhubarb (when economy does not exclude its use), gentian, aloes, ginger, etc., are advised, to regulate digestive activity. Pepsin (Johne) and apomorphine (Reindl, Lemle), deserve to be experimented with.

Temporary constipation may be relieved by a green diet and slops, to which must be added sulphate of soda or aloes; their action will be aided by cold-water, salt-water, or soap-water clysters.

Glycerin injections, per rectum, have been given lately with a syringe furnished with a long rubber tube (Rottner, Schindelka); Dr. Oidtmann's (of Maestricht) methods have been initated, and also Dr. Anacker's (of Château-Salins); the dose of glycerin for the horse is 5 to 10 grammes. Glycerin acts upon the rectum by its avidity for water; a sharp pricking is felt upon the mucous membrane, and the reflex excitement increases peristaltic movements and defecation. The experiments we have made with glycerin have demonstrated that it possesses properties similar to soap and water; its action is limited to the emptying of the rectum. When the arrest of the alimentary matters exists in a more forward portion of the intestinal canal, neither soap-water nor glycerin will overcome it.

The same tonics, astringents, and styptics have to be used in diarrhea as in acute catarrh; oak-bark, tannin, sulphate of iron, acetate of lead, nitrate of silver; they should be used moderately, otherwise there is risk of poisoning of the mucous membrane. The most reliable drug is opium. Styptics are best administered in the form of clysters; but they evidently only act upon the rectal mucous membrane; clyster of the colon and cæcum is not practicable in the horse (see Clysters in Colies).

\section{ACUTE GASTRO-INTESTINAL CATARRH OF THE OX.}

\section{Acute Dyspepsia: Acute Indigestion.}

General remarks on diseases of the stomach and intestine in ruminants. The positive information that we possess on dis- 
eases of the digestive canal in the ox is very incomplete. The paucity of our knowledge in this direction is of great disadvantage both to the practitioner and to the farmer, but especially to the former, for he is obliged to call many different pathological conditions by the common name of indigestion. We must therefore acknowledge the credit that is due to the veterinarians who, by endeavoring to define the symptoms of each, have tried to clear up the chaotic pathology of these affections. ${ }^{1}$

We must remark that the diagnosis of gastric affections is still more difficult in ruminants than in the horse and dog: this difference is easily understood if we think of the anatomical disposition and of the complex physiological actions of the stomach in ruminants, and also of the morbid sympathies of its several compartments, and consequently of the possibility of the simultaneous existence of different affections. All the circumstances combine in obscuring the clinical study of gastric diseases; the present condition of science, therefore, does not allow us to give an exact and detailed description. Moreover, it is doubtful if the question of diagnosis of these affections will make much progress in the future. Investigators find themselves halted by the similarity of symptoms, which, in most cases, do not offer anything characteristic.

We have accordingly adopted an essentially practical classification. We shall first study acute gastro-intestinal catarrh, then this affection in its chronic form; afterward taking up acute meteorism, chronic meteorism, overloading of the belly, catarrh of the abomasum, gastro-intestinal catarrh of young animals, and finally the digestive troubles produced by the presence of foreign bodies in the first three gastric compartments.

Physiological considerations concerning the digestive diseases of ruminants. 1. The first three compartments of the stomach of ruminants do not secrete any digestive fluid; they rather constitute a receptacle, in which the alimentary matters undergo physical modifications which render them more susceptible to the chemical phenomena of digestion in the true stomach. When the mechanical work is hindered on account of paresis of their walls, the digestion not only suffers, but the stomach and intestine become the seat of a certain degree of inflammation.

2. The movements of the rumen have a considerable influence

1 Harms: Deutsche Zeitschr. f. Thiermed., 1876. 
upon the course of digestion; normally, it performs two contractions per minute; its peristaltic movements are perceptible in the left flank; their diminution or suppuration provokes troubles of digestion.

3. Rumination is an absolute necessity for the performance of digestion. The average duration for each meal is one hour; each bolus requires about fifty seconds in order to undergo the second mastication, during which the jaws execute about sixty movements. In febrile affections, or when the animals make excessive efforts, and also in a number of morbid conditions, rumination is suppressed; this suppression occasions digestive disorders.

4. The paunch may be compared to a fermentation vat, in which there is constant formation of alcohol, acetic acid, butyric acid; cellulose and albumin ferment equally up to a certain point. Abnormal fermentations produce meteorism. The normal reaction of the contents of the paunch is alkaline; in dyspepsias it becomes acid.

5. The third stomach forms for the alimentary matters an apparatus of mechanical division furnished with appropriate innervation. Indigestion of the third stomach and consequent catarrh of the mucous membrane which lines it, take place under the influence of diverse circumstances: when there is paresis of this organ, or of the paunch; in every instance that rumination is suppressed and insalivation is performed in an incomplete manner; finally, when drinking-water is ingested in insufficient quantities.

6. The abomasum constitutes the so-called true stomaeh. All the diseases of the first three gastric compartments influence it. Its mucous membrane is irritated, and becomes inflamed through the contact of insufficiently prepared alimentary matters.

7. The reticulum is the principal motor of merycic rejection; paresis of its muscular wall prevents the process of rumination.

8. In ruminants, the food remains in the digestive canal for three or four days; after this lapse of time the largest quantity of digestible alimentary matter is ejected. Part of the food, however, remains in the belly and reticulum for eight days or more.

General considerations. We are liable to confound acute dyspepsia with acute gastro-intestinal catarrh, just as has happened in the horse. On account of the particular resistance of the mucous membrane of the first three gastric compartments, in the greater number of morbid conditions in which one of them is involved, 
clinical observation permits us to detect functional troubles before any manifest anatomical alterations exist. The most important of these functional disturbances is the suppression of rumination. But dyspepsia and catarrh depend upon the same causes, the latter often complicates the former, and there is no symptom that will enable us to make the distinction. Thus, contrary to custom, we shall describe dyspepsia as the first stage of acute gastro-intestinal catarrh.

In ruminants the diseases of the stomach are more frequent than those of the intestine; the paunch and third stomach are the gastric compartments most frequently affected. The intestine is very seldom the seat of a primary affection. Diarrhea is the principal symptom of the various alterations which may affect it.

Etiology. Not all animals are equally predisposed to acute gastro-intestinal catarrh. This predisposition varies according to the degree of activity of the digestive function, the constitution and temperament, pre-existing diseases, the age, etc.

The principal causes predisposing the animals to the disease are a continned feeding of poor, insipid food, slops and liquid foods given to the exclusion of all others, permanent stabling, a special diet inducing an increase of milk or fat production ; poor and narrow stalls in the neighborhood of large cities, where the animals are fed with all kinds of refuse. Let us add that the trouble is more common during the intermediate seasons of spring and fall than at any other time of the year.

The causes which determine it are:

1. Overloading of the paunch.

2. Cold, frosted, or frost-covered fodder ; the feeding on pastures before the proper season, or very late in the fall; very hot or cold drinks, and external colds.

3. Improper feeding; the abrupt change from dry to green food or from green to dry; new hay ingested to excess, especially if the animals drink a large quantity of water immediately after eating; also disproportion between dry and soft or liquid food, such as malt, grain, farinas, bran, roots, raw potatoes, etc.; the same is true when there are weeds in the food, such as leaves, vine-sprouts, turnip-leaves, hay-seed, sweepings; excess of straw as food, a continuous feeding of whole grain, rape cakes, and an exclusive fattening diet with dry feed. Straw cut very short has been condemned 
by many authors, but this theory has been discredited by Friedberger's experiences. ${ }^{1}$

4. The disease may also originate from soiled, decomposing, or mouldy alimentary matters; the remains of the kitchen in a more or less fermented condition, acid or fermented refuse of factories, muddy hay, etc.

5. Indigestible food, woody plants, green flax, foreign bodies of any kind, sand, and especially gravel; ingestion of the placenta; or insufficiently cooked bread may provoke catarrhal disease.

6. Excess of work, not allowing the animals the necessary rest to perform rumination. We must, however, remark here that oxen which are subject to hard work (by brewers, millers, and farmers) perform rumination during exercise.

Catarrhs of the digestive channels which complicate febrile diseases are caused by disturbed secretion, as in the horse. But they are complicated by cessation of rumination - an important etiological factor of gastro-intestinal catarrh.

Symptoms. 1. DyspePtiC PERIOD OF ACUTE GASTRO-INTESTINAL CATARRH. Though the animals still consume the food which is given to them, they do not eat it with the same avidity, and frequently they do not eat it entirely; they prefer fodder or green food. They refuse slops, but partake willingly of spring-water. They are depressed and cease to use the tongue to wipe their nostrils ; they lie down frequently, and remain in a recumbent position for a long time. Rumination is short, lazy, and interrupted. The movements of the paunch are performed with less energy. Defecation is retarded; the excrementitious matters may be normal or more or less hard. There is no fever, the extremities are warm, the mouth and nose damp and cool, the milk secretion is not sensibly diminished.

Sometimes this condition disappears rapidly after rest; at other times it persists for several days. If it lasts for more than four or five days, the true catarrhal condition follows it.

2. ACUTE Gastro-INTESTINAL CATARRH PROPERLY SO CALLED. This condition may either follow dyspepsia or may appear at the first onset. It is impossible to draw a typical picture of the condition, so that we must limit ourselves to explaining its symptoms briefly in their essential phases.

1 Friedberger: Münch. Jahresber., 1880. 
The animals keep away from the manger; the spinal column is arched upward, the limbs are gathered together. The hair is dull and bristly, especially at the head and along the dorso-lumbar region; the ears are hanging or flat. The temperature of the cutaneous surface is variable; the ears and horns are alternately cold and hot; the conjunctiva and other mucous membranes are injected; there are often chills and muscular tremblings. In certain patients the muzzle is also damp, but when wiped it is a considerable time before the moisture reappears upon it.

The temperature of the mouth is higher; its cavity is full of saliva and mucus; sometimes we observe ptyalism. The appetite is diminished or suppressed; thirst also undergoes the same changes. The rumination is stopped; still there are cases where it takes place, and then the merycic regurgitations are made in small boluses, which receive only twenty to thirty mastications; from time to time we notice fetid evacuations, vomituritions, and even true vomitings. The inflated abdomen is especially prominent on the left side and toward its inferior part; the left flank is also filled out. The contents of the paunch may be directly accessible to palpation through the flank wall; the hand feels a doughy, fairly consistent sensation. When the paunch is lifted by gases its contents can only be felt by exerting strong pressure upon it, which sometimes produces a dull bruit. Slight meteorism often appears. periodically. The movements of the paunch and its bruits are weakened; the contents of the rumen are somewhat lifted from time to time, but it never undergoes the normal movement of rotation (Harms). Pressure upon the abdomen (left flank and hypochondrium) is manifestly not painful.

Defecation is rare; the fecal matters are harder and of a darker color than normal, and often enveloped in a thin crust; we may find them ring-streaked and having a putrid odor ; they are sometimes covered with mucus, and form clammy and shiny masses. When diarrhea exists the excrements are mixed with alimentary matters that have not undergone merycic mastication, and which are not at all digested. In auscultating the right side of the abdomen we perceive rare or infrequent borborygmi; as a rule there are slight colics, indicated by anxiety, stampings, and kicks. which the animal directs toward the abdominal walls; also twitching of the tail, frequent and short decubitus, the decubito-costal position, and looking towards the flank, etc. In some patients. 
constipation is persistent. At the outset the urine has the color of white wine, and is of intense alkaline reaction; soon it is only passed in small quantities, its coloration becomes darker, and its reaction is acid. The lacteal secretion is often reduced one-half.

In the majority of cases we observe fever; the pulse is accelerated (an increase of ten to twenty pulsations or more per minute); it is small, the artery is full ; the rectal temperature rises, but this increase is not lasting or permanent; a temporary increase in temperature is not a reliable sign of the gravity of the disease. (Harms.)

In order to understand the pulse and temperature in the bovine species it will be necessary to remember that both are extremely variable, even during the normal condition. We must always examine comparatively for these symptoms, studying the phenomena presented by the sick animals with those of the healthy ones in the same stable.

The ox in a healthy condition has 45 to 50 pulsations per minute. According to Harms, Bendz, Prinz, Veith, and others, the normal number of pulsations alternates between 40 and 120 .

According to Krablee and Müller, the normal temperature of the ox is $38.8^{\circ}$ C. ; Siedamgrotzky has found it $38.9^{\circ}$; Zündel, $39^{\circ}$; Lydtin, $38.1^{\circ}$ to $38.5^{\circ}$.

Duration and termination. In slight cases-and these are the most numerous-improvement takes place between the fifth and eighth days; the movements of the paunch become more frequent and the bruits more distinct; the febrile manifestations are gradually alternated, rumination reappears, the appetite returns, defecation is more abundant, and lacteal secretion is re-established.

In catarrhs of some gravity the symptoms are more marked. The mouth is dry, hot, and fetid, the tongue coated; the ruminations and movements of the paunch cease entirely; all aliments are refused. In certain animals constipation persists, in others we notice a colliquative diarrhoa; the udder is flabby, and lacteal secretion almost nil; the movements become more and more heavy and difficult. The patients are much depressed, as if stupefied; when in decubitus they give vent to repeated groans; the look expresses suffering, the orbits sink, the inner angle of the eye becomes covered with an abundant mucus. The fever is intense, the temperature rises to $40^{\circ} \mathrm{C}$. and above; the pulsations are increased from thirty to forty beats per minute. The ears and horns 
are hot or cold, depending upon the movements of the animal; the muzzle is hot and dry. The respiration, which is accelerated at the beginning, becomes extremely painful and short. When acute catarrh assumes this grave form, it often becomes transformed into acute gastro-enteritis, terminating in death. There are also cases where it becomes milder and passes into a chronic state.

Differential diagnosis. When the symptoms of the disease are well defined, we can make a diagnosis by a careful examination of the animals. The course and duration of the disease are to be taken into consideration. It is difficult to accurately decide the portion of the gastro-intestinal canal which is especially affected; but to determine the localization of catarrh is not of great importance, for, in almost all cases, the inflammation rapidly extends to the annexes of the parts primarily attacked.

The accumulation of gas in the rumen may cause us to confound catarrh with meteorism, but in catarrh the tympanism is never so great as in common meteorism, and its periodic character will always allow us to recognize it. The possibility of the existence of a gastro-enteritis must be admitted when the symptoms denote an intense morbid process; the precise diagnosis in cases of this kind, however, is extremely difficult.

Prognosis. When the established symptoms are those of simple catarrh, the cure is almost certain. According to a table compiled by Hering, in 128 cases of "indigestion," killing was advised but five times, and even then without any absolute necessity of resorting to such extreme means. We must, however, always be careful in making a prognosis; as already stated, the disease may pass into a chronic state or may be complicated with gastro-enteritis.

Pathological anatomy. We rarely have occasion to make an autopsy on animals dead from gastro-intestinal catarrh ; and accordingly our knowledge concerning the pathological anatomy is still very restricted. Harms has found the mucous membranes thickened, uniformly red or spotted, striped; those of the true stomach and intestine were covered with an exudate rich in cells. The alterations due to the catarrh are especially remarkable in those parts of the intestines where the mucous membrane possesses large villi. In the third and second stomachs the lesions are ordinarily limited to certain lamellæ. The contents of the third stomach are sometimes much dried up and form blades, the central part of which is of a calcareous consistence. 
Treatment. Submit the animals to a severe diet; such is always the first indication to follow, and it will have to be enforced as long as rumination is interrupted.

With the object of stimulating the movements of the stomach, we give a few handfuls of fresh grass or food which is of easy digestion, and this regimen has to be maintained, even after the return of rumination; the rumen has thus time to get rid of the alimentary matters which are accumulated within it. In order to increase the thirst we administer drinks with plenty of salt. Whey, which some animals will readily take, is useful; if they refuse it, we must try to make them partake of as much water as possible, or of some farinaceous drink.

The movements of the stomach and intestine should be encouraged by dry frictions made upon the whole surface of the body and by massage of the rumen through the left flank; at times the rectum should be emptied, and soap- or salt-water clysters must be given.

When symptoms of dyspepsia exist we give hydrochloric acid in doses of 10 grammes in a bottle of water (four or five times a day). If there is constipation at the same time, and if defecations are infrequent, laxatives should be prescribed : sulphate of magnesia or Glauber salt in a mucilaginous excipient (flaxseed, mallow, fenugreek); we may also dissolve 750 grammes of sulphate of soda in two or three litres of mucilage prepared with 250 grammes of linseed; this drink should be administered in three doses, at intervals of two or three hours. We may also make them take these salts in a bitter decoction (gentian), or some aromatic bitter (calamus).

Tartar emetic and hellebore are advisable in cases where the peristaltic movements are wanting: these two agents are given in doses of 8 to 15 grammes. Tincture of white hellebore may also be used (same dose). Harms injects subcutaneously 10 to 12 centigrammes of veratrine in alcoholic solution of $1: 50$.

Modern authors recommend sulphate of eserine to counteract atony and paresis of the gastro-intestinal muscular fibre (Möller, Dickerhoff, Feser). This means would be more available were not the price of eserine so high. Feser administers it internally or by injection into the rumen, in doses of 2 to 5 decigrammes, in liquid solution, in small-sized oxen. In subcutaneous injections, the dose is 1 decigramme in $2 \mathrm{c.cm}$. of water. When no sensible effect is felt after five or six hours, the injection may be repeated. Hydrochlorate of pilocarpine has been overestimated; it is of little value 
when given to the ox. Its sialagogue action produces accessory effects which are nothing less than favorable. The same is the case when a combination of physostigmine and pilocarpine is used.

\section{ACUTE GASTRO-INTESTINAL CATARRH OF SHEEP.}

The etiology and symptomatology are almost the same as in the ox. Improper alimentation and colds are the principal causes of the disease. Gerlach has noticed catarrh in animals feeding in rich pastures ; Schick has seen it in sheep abundantly fed on raw potatoes.

The principal symptoms are: inappetence, disturbance of rumination, weakness, diarrhea, etc. ; constipation is rarely observed.

The weak constitution of the sheep exposes it much more than the ox to gastro-intestinal catarrh; and the mortality from this affection is quite considerable in this species. In a flock of 600 animals, Schick saw 60 sheep succumb in eight days; in a flock of 300 , Gerlach notes the death of 20 in four hours. The histological alterations are the same as those produced by drastic purgatives. The existence of the disease in a large number of animals of the same flock has made it resemble an infectious disease.

The use of the œsophageal sound or trocar may be necessary to counteract conditions which may arise during treatment.

\section{CHRONIC GASTRO-INTESTINAL CATARRH OF THE OX.}

Chronic Dyspepsia: Chronic Indigestion: Obstruction of the Third Stomach: Chronic Omasitis.

The disease which we are about to describe is ordinarily named "obstruction of the third stomach," a designation derived from the most characteristic alteration produced by it : the presence of dried alimentary matter in the third stomach. If the diagnosis of the chronic gastro-intestinal catarrh has seldom been made, it is because the disease is almost always designated under the name of "omasitis," or more commonly by "obstruction of the third stomach," a term which is incorrect for two reasons ; first, because it does not give a true idea of the nature of the disease; second, the obstruction of the third stomach is observed in a number of affections of the digestive apparatus which lead to cessation of rumination and to the suppression of the peristaltic movements of the 
stomach and intestine. Some have even gone so far as to apply this name to "bovine pest," because so-called obstruction of the third gastric compartment is often observed in this disease. The third stomach is exceptionally the seat of a true obstruction-that is to say, as far as presenting a material obstacle to the progress of the alimentary matter. Harms has observed but one case. We must, therefore, discard the old expression, "obstruction of the third stomach." Localized chronic catarrh has sometimes been found upon the mucous membrane of the third stomach; but, limited to this organ, it is extremely rare, if compared with the same affection relating to the rumen, the abomasum, or the intestine, and even catarrh of the stomach is in most cases but a complication of catarrh of the rumen. It would be absolutely illusory to attempt to describe separately catarrh of the rumen, of the reticulum, of the third stomach (omasum), of the rennet (abomasum), or of the intestine, and we have preferred to comprise them under one designation only, that of chronic gastro-intestinal catarrh.

Etiology. All causes apt to provoke acute catarrh may also determine chronic catarrh when acting in a lessened degree for a long time. This may also be the result of defective or neglected treatment in acute catarrh.

Among the most common causes which produce it are :

1. Imperfect mastication of the food due to the irregularity of the dental tables.

2. Adhesions of the paunch and reticulum consecutive to a circumscribed peritonitis.

3. Compression of the œesophagus by neoplasms interfering with rumination.

4. The tumors of the various gastric compartments (scirrhus, sarcoma of the abomasum, papillary vegetations of the orifice of communication of the reticulum with the third stomach, etc.).

5. Diaphragmatic hernia of the abomasum.

6. The foreign bodies introduced into the stomach or intestine (pointed bodies, ægagropilæ, stercoral balls, etc.); contractions of the intestine.

7. Blood-stasis in the intestinal mucous membrane, caused by chronic diseases of the liver, lungs, or heart.

8. Pressure of the gravid uterus upon the rumen, and weakness of the peristaltic movements of the rumen (Anacker).

Chronic gastro-intestinal catarrh may also be the result of deep- 
seated nutritive troubles and general diseases of slow evolution. It is quite common to see it during the course of tuberculosis.

Symptoms. The symptoms of chronic catarrh differ only from those of the acute form by their duration and their rebellious character. The trouble is specially characterized by remissions and frequent aggravations, which are to some extent periodic.

The general conditions are manifestly altered: the animals are low-spirited and feverish; the temperature of the body is irregularly distributed; the skin is dry and adheres to the subcutaneous connective tissue; the hair is dull and bristly : emaciation appears and increases with the duration of the trouble. Sometimes the patients lick any hard bodies within their reach. The buccal cavity shows the alterations which have been described as belonging to the acute catarrh.

The appetite, rumination, and the movements of the rumen are diminished or completely suppressed. The eructations are infrequent and fetid. The rumen is distended with alimentary matters; the pressure of the hand sweeping over the flank gives a unique sensation of resistance. Slight tympanites is almost always observed, which is at first only manifested after meals, but becomes permanent when the peristaltic movements are completely suppressed. In some animals we observe vomituritions and even vomitings (Mazoyer).

The defecations are infrequent; the excrements are ejected in black masses of the consistence of turf (often called "burnt" by the owners); they are covered with a thick, thread-like mucus, which is sometimes bloody, or with a muco-fibrinous exudate, and contain alimentary fragments which have escaped mastication or digestion. At times the fecal matters are soft and watery, at other times of acid odor or fetid-symptoms of grave lesions of the intestine. Generally we see constipation and diarrhea alternate.

Micturition is less abundant, but we know nothing exact about the reaction of the urine. The lacteal secretion is diminished or completely suppressed. We often observe complaints, groans, grinding of the teeth ; certain authors have insisted upon affirming the existence of painful sensations suffered by the animals when compressing the dorso-lumbar region or percussing the chest; we think, with Harms, that they rather form a manifestation of the inflammations of the gastric compartments (from foreign bodies or commencing peritonitis). If the disease is not going to end in a 
cure the symptoms become rapidly more acute. There is complete loss of appetite; the muzzle becomes dry and cracked, the fever is intense, the pulse small and weak, the heart-beatings are tumultuous; tympanites increases, the peristaltic movements are entirely suppressed, and, according to circumstances, there is a stubborn constipation or profuse diarrhea. The weakened condition becomes more marked every day; the exhausted animals remain in a decubital position and complain frequently; death results finally from marasmus.

Pathological anatomy. The alterations vary with the location, intensity, and duration of the affection.

The rumen is ordinarily distended by gases and dry alimentary matters; the epithelium can often be detached immediately after death, and Bruckmüller has found it much thickened. The mucous membrane is mostly of a uniform red, more or less dark, at times it presents numerous ecchymoses; a blackish or reddish-brown coloration can be observed upon portions of the mucous membrane, which is covered with large papillæ. According to Anacker, the papillæ are often destroyed. The reticulum contains a certain quantity of coarse, dried-up fodder; its mucous membrane presents lesions which are of the same nature as those of the paunch.

The third stomach is much distended and filled with alimentary matters; the dried masses interposed between the leaves of the organ are much compressed and very hard, and in some cases the epithelium is adherent to their surfaces immediately after death; at times the matter is coarse, or it may have undergone the second mastication and can be reduced to fine dust by the fingers. The mucous membrane of the leaves has a red, diffuse color, or is studded with ecchymoses; it may be inflamed, softened, and can be easily torn; at other times it is in a gangrenous condition, covered with dark hemorrhagic centres or bloody ulcerations. The abomasum ordinarily contains very little chyme mixed with food which is not entirely masticated; Bruckmüller has found a slaty pigmentation in the shape of streaks or irregular blots upon the mucous membrane; this membrane is often atrophied, smooth, and covered with a mucous exudate.

In the intestine we find the mucous membrane red or slatecolored and atrophied, with pitting as a result of the disappearance of Peyer's patches. In the duodenum, the intestinal contents are liquid, mucous, and colored by the bile. The large intestine con- 
tains stercoral matters which are quite dry and dark-colored; its mucous membrane is much coated. When chronic catarrh is complicated with phlegmonous enteritis or peritonitis, we observe alterations which are proper to the latter diseases.

The biliary vesicle as a rule is swollen by thickened bile. The liver presents various conditions of icterus by resorption.

All the secondary alterations-as those of the lungs, the brain, the right heart, and the venous system-are the results of blood stagnation.

Differential diagnosis. Chronic gastro-intestinal catarrh may be confounded with acute catarrh or with enteritis; the progress of the disease, however, gives one exact information. Chronic peritonitis caused by foreign bodies is hard to distinguish from chronic catarrh; but these two morbid conditions frequently coexist; one happens as a complication of the other. If in certain cases the diagnosis can be made by taking the evolution of the disease into consideration as well as the accidents accompanying it (such as symptoms of heart disease, or the existence of a foreign body that has perforated the stomach), there are others where the nature of the disease is only discovered at the autopsy. The intermittent swellings which are observed in chronic catarrh may lead us to diagnosticate a chronic or acute tympanites; but meteorism, whatever may be its form, does not produce the symptoms observed in catarrh.

Lastly, chronic gastro-intestinal catarrh may be confounded with contagious pleuro-pneumonia of slow evolution; here also we find emaciation, digestive and nutritive troubles, etc. However, the careful examination of the chest, and exploration of the abdominal cavities, permit us to make the diagnosis in the great majority of cases.

Prognosis. The prognosis varies with the intensity, extent, and duration of the catarrh, also with the nature of the causes which have produced it and the possibility of removing them. In general, it must be formulated with reserve. It is of a very serious nature when the disease lasts more than two or three weeks, when constipation persists obstinately for several days, also when the peristaltic movements and rumination have stopped altogether. In all these cases, killing the animal is often preferable to treatment.

Treatment. The number of recognized remedies is considerable. We shall only speak of the most useful. 
Most of the indications given in counteracting acute catarrh of the stomach and intestine apply also to the chronic form of the disease. The animals must always be subjected to a severe diet. In mild or dyspeptic cases, we prescribe hydrochloric acid in doses of 10 grammes, given several times a day. If there is any weakness of the three gastric compartments, without any true inflammatory alterations, we may give doses of essence of turpentine of 10 to 20 grammes two or three times a day in an infusion of aromatics; powder or tincture of white hellebore, in doses of 10 to 15 grammes, are also recommended. Veratrine must be given hypodermatically in doses of 0.05 to 0.1 gramme. As a cathartic, it is proper to use sulphate of soda ( $\frac{1}{2}$ kilo to 1 kilo) or artificial Carlsbad salts dissolved in a sufficient quantity of water, or aloes in doses of 30 to 60 grammes. In order to excite gastric activity, a subcutaneous injection of 10 centigrammes of sulphate of eserine may be given. In stubborn constipation we resort to tartar emetic (10 to 15 grammes), or calomel (3 to 6 grammes per day).

The caustic and toxic action of croton oil excludes it as a medicine; it is commonly replaced by 500 to 1000 grammes of castor oil. Here, as well as in all diseases of the ox, we must only use such medicinal agents as will not interfere with the use of the meat in case of failure.

The introduction of large quantities of water into the stomach and intestine has sometimes given good results, even in desperate cases. Höhner, Eber, and Deffke have obtained a cure by introducing lukewarm water directly into the rumen by means of a trocar and a funnel which fitted it. Deffke injects also fifty litres of lukewarm water within a few hours, and leaves the trocar in place. He praises the favorable effects obtained by water to which hydrochloric acid has been added (10 grammes to 15 litres of water), and also by infusion of aromatic substances (valerian root); he also advices not to delay the injections too long. The mucilaginous beverages, fresh linseed, and plain repeated clysters, etc., are also very useful means of treatment.

When the rumen is full of closely packed alimentary matters, and when the different indicated treatments have been without effect, there is one last resort-the incision of the rumen and extraction of part of its contents. 


\section{ACUTE METEORISM OF RUMINANTS.}

\section{Swelling: Meteorism: Tympanites.}

We designate by these expressions an affection due to the sudden and considerable production of gas in the first stomach of ruminants, which is characterized by enormous distention of the abdomen. This disease is seen quite often in the ox, more rarely in the sheep; but in the bovine species the disease often affects a large number of animals in the same flock.

Etiology. I. General causes. All foods which ferment rapidly and produce much gas may determine meteorism when introduced in sufficient quantities into the digestive canal. The cause of the trouble in meteorism is mostly due to the ingestion of green fodder, either on the pasture or in the stable. In animals kept constantly in the stable, the feeding with green or fermented food is usually the starting-point of tympanites. This explaius the frequency of the disease during the holidays, when the farmers have housed a supply of green food; also during stormy, or hot, damp weather, which favors fermentation. When the animals go to pasture the accident is especially to be feared, for the reason that they have been used to dry feed for a long time; the faster the grasses have grown the greater the danger. Experience has taught that meteorism is particularly common in years that have had a cold spring, with vegetation at first delayed, but becoming very active with the advent of warm weather (Spinola); the same observation has been made for those fertile plains where the soil is rich and vegetation luxuriant.

The animals are much exposed to it in the pastures when feeding on grass covered with heavy dews, or wet with rain, or covered with ice or frost; also when drinking much after meals, and especially when they swallow a large quantity of air while eating during rainy and windy weather (Spinola).

Without doubt many animals are specially predisposed to tympanites; a few mouthfuls of an easily fermenting food is enough to produce meteorism; the condition must then be ascribed to a weakness of the digestive organs or to some pathological condition which interferes with the eructations or the movements of the rumen. But these latter causes rather determine chronic meteorism. 
II. Special causes. The trouble has been ascribed chiefly to the following causes :

1. To the different clovers-mainly to the red variety found in the meadows-to lucerne and French grass; but, in general, this holds good for any kind of green food. It is generally admitted that clover is more dangerous after the application of plaster than when the grass has not been subjected to this form of manure. Ringuet has opposed this theory; but if the plaster of itself has no effect, it influences vegetation, and thus indirectly favors the development of meteorism.

2. The lentils, the vetches, separate or mixed with oats, buckwheat, and a few other grains.

3 . The gramineous plants of swampy and damp meadows', particularly Poa aquatica, Scirpus sylvaticus (Haubner, Siedamgrotzky), potato stems, cabbage leaves, rape, turnips, field mustard, and wild radishes.

4. The young sprouts and leaves of cultivated gramineous plants, etiolated plants, weeds and others, growing in corn or oat fields, and which the animals may eat immediately after harvest when pasturing in the stubble (some time after harvest, when the sun has exercised its salutary influence upon these growths, they are less injurious); also the young wheat sprouts which are at times very abundant a short time after harvest.

5. The fermented drinks, malt or malt buds, roots and tubercles, cotton dust, when the animals are not used to this food and when they take it in too large quantities.

6. Various toxic plants the absorption of which provokes more or less serious symptoms of meteorism (Conium maculatum, Cicuta virosa, Atropa belladonna, Taxus baccata, Veratrum album, Papaver rhoeas, Nicotiana tabacum); all ranunculaceous species; the fungi ; poisonings by meat or herring-brine, etc.

7. Further, the swallowing of air may produce a condition similar to acute tympanites (Weinmann). We observe such cases in nursing calves, when they swallow air with the milk while sucking.

8. Foreigu bodies, too, arrested in the osophagus and preventing eructation, are a cause of meteorism.

Pathological anatomy. The larger part of the contents of the rumen consists of gas. Carbonic acid is especially found in it when the animals are kept on a green diet, and carburet of hydrogen if they have consumed dry food. The quantitative and qualitative 
composition of this mixture varies in different cases of tympanites. Reiset has found the following composition in a cow which was meteorized, in a clover field, the animal having died two hours before :

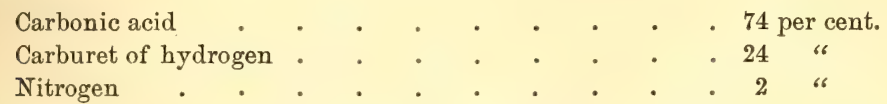

The same author has found 76 per cent. of carbonic acid in a meteorized sheep. Sulphuretted hydrogen does not appear in this analysis, which must be looked upon as pure chance, for this gas is one of the products of normal decomposition of albumen in the rumen.

The overdistention of the rumen may cause its rupture and sometimes that of the diaphragm; in such cases the alimentary matters and gases escape into the abdominal cavity, but the very rapid course of the disease does not allow peritonitis to develop.

The veins of the skin, those of the subcutaneous connective tissue, and the large venous trunks (jugular, etc.) are filled with a dark blood which becomes oxidized when in contact with air. The lungs are hyperemic and present sub-pleural and parenchymatous extravasations; at times we observe odema of the lungs. The parietal pleura is raised by injected blood, especially in the superior regions of the thorax. The right auricle and the right ventricle are greatly distended by the blood; the coronary veins are injected; the pericardium and the endocardium are marked with ecchymoses; we may exceptionally observe rupture of the right heart. The intestinal mucous membrane is congested; we observe hemorrhages into the submucous and subserous connective tissue. Anacker has pointed out laceration of the vessels of the spleen and the destruction of its tissue. The mucous membranes of the head, the cerebral meninges, and the sinuses are congested; sometimes we find apoplectic centres in the brain.

Symptoms. The most striking symptom is the sudden increase and the great volume of the abdomen; the left flank may extend so greatly as to become level with the dorsal spine; some cases are encountered where it even goes beyond this. The abdominal walls are much strained and very elastic; the percussion gives a clear tympanic, even metallic sound ; the normal bruit of the rumen has disappeared.

From the first onset the animals ordinarily cease to feed, and 
rumination is suspended; after several small defecations they make vain repeated expulsive efforts. The extremities are usually gathered under them, but at other times are much spread, as if to avoid falling; the back is arched, the tail high, the ears hanging, and the animals will only advance when pushed or when threatened by the whip.

With the increase of tympanites, the respiration is accelerated and becomes painful; we notice anxiety, excitement, stamping, congestion of the mucous membrane, conjunctiva, and especially of the sclerotic; the superficial veins, mainly those of the head and neck, as well as the mammary veins (Röll), are swollen and very salient; the eye is haggard, opened wide, and prominent ; the lower part of the ears, and the joints and flanks, are covered with sweat; the pulse becomes more and more accelerated, small, and soon imperceptible; the heart contractions are tumultuous, at times we notice slight attacks of cough.

The dyspnoea increases rapidly; the nostrils are dilated, the mouth is half open and the tongue hanging, the saliva runs on the ground ; the patients complain, groaning repeatedly ; there is nausea without any effectual eructations ; vomiting is very rare (Rijnders). Soon the anxiety becomes extreme, the extremities are cold, the mucous membranes cyanosed ; finally the animals become immovable and stupefied, sink to the ground, and succumb to convulsions.

Pathology. The pathology of these phenomena is very simple; the clinical picture of acute meteorism is the same as that of poisoning by carbonic acid, sometimes like that of cerebral apoplexy. The intoxication is partially determined by difficulty of breathing, in part also by absorption of carbonic acid, which takes place in the rumen, and by the passage of this gas into the blood in accordance with the laws of diffusion. The injection of the mucous membranes, cerebral hyperemia, and swelling of the veins, are due to the pressure exercised upon the large vessels contained in the abdominal cavity by the distended organs, and also to considerable hindrance experienced by the returning circulation; when this obstruction becomes intense, ecchymoses are produced, and cerebral apoplexy may supervene at any instant.

Course. The course of acute tympanites is always very rapid. If not remedied, it generally causes death within a few hours, sometimes in one hour, occasionally even in half an hour. When, by proper treatment, we succeed in evacuating the gas for the time, the 
cure is not always complete; there are cases where the food contained in the rumen continues to ferment, and the production of gas goes on from twelve to twenty-four hours, sometimes longer. On the other hand, a spontaneous cure may occur if there are eructations and abundant defecations.

Prognosis. The prognosis is always serious. It is formulated according to the cause and intensity of the disease, its more or less rapid evolution, the possibility of counteracting it immediately, and the number of meteorized animals.

Treatment. The indications of the prophylactic treatment are deduced from consideration of the etiology. The change from dry to green alimentation must be made gradually, with precaution, and not suddenly as is usually done. The organs of digestion must gradually become used to this food, in order to consume large quantities of green grass without danger.

Pastures growing a quantity of watery and poor vegetation must be avoided ; animals in lean condition must especially be kept from them.

If the animals are kept on green food in the stable, fermentation of the fodder may be avoided by keeping it in a cool place, or spreading it in thin layers; when such fodder is already heated, it must be given in small quantities and mixed with straw or hay. Lastly, all large farms and agricultural communities ought to keep the necessary instruments, and especially the œsophageal sound, always at hand and in working order.

As soon as the signs of meteorism are observed, the patients must be prevented from eating, and treated immediately. At first we must do our best to obtain the evacuation of the gases by the osophageal route. This indication can be performed in different ways, either by pulling the tongue out of the mouth, which provokes nausea and eructations, or by traction upon the commissures of the lips by means of a knotted straw rope; this gives rise to involuntary movements of the tongue which produce eructations and sometimes vomiting. A straw halter used like a snaffle and adjusted on the neck acts in the same way; some practitioners aim to make it more effective by coating it with pitch or wagon grease. Rouchon eulogizes the good effects of the movements called forth in ascending a hill; too rapid or violent movements, however, should be avoided, for they can certainly only increase the trouble.

Strong and prolonged pressures upon the left flank are sometimes 
sufficient to evacuate the gaseous contents of the rumen. Rychner considers this procedure the safest; it is also the easiest and the least expensive, and, we may add, the least dangerous of all. The renewal of the peristaltic movements can be distinctly felt under the hand, and eructations are soon produced. The existing centrifugal pressure upon the walls of the rumen expels all the blood from it, and consequently paralyzes the muscular fibres : in compressing a portion of the wall of the rumen, the blood rushes there, the movements reappear, are communicated to the neighboring parts, and soon the evacuation of gas takes place. Prolonged massage of the left flank or of the whole abdominal wall may empty the rumen entirely. Blows with the hand upon the left flank (Ferrari), the spiral ligature of the belly (Zwickel), cold water thrown upon the left flank, cold compresses, etc., act in a similar manner; but massage performed with both hands will always remain the simplest and most efficient means.

A large number of therapeutic agents have been recognized. Their value is generally inferior to that of mechanical means; they answer only in the cases where the latter are perfectly unsuccessful. Moreover, they have serious inconveniences: the water that ordinarily serves as the excipient favors fermentations, the liquid may enter the wrong way and pass into the trachea, and, above all, they prevent the use of the meat when, in case of unsuccessful treatment, the animals are slaughtered.

These therapeutic agents are sometimes stimulants, sometimes emetics, or absurbents, or antiferments. The most advisable is brandy ( $\frac{1}{4}$ or $\frac{1}{2}$ of a litre in double the quantity of cold water) or tincture of hellebore (10 to 20 grammes) mixed with brandy. The carminatives: infusions or decoctions of cumin seed, fennel seed, chamomile, valerian, aniseed, ginger, etc., have a less efficient influence.

Essence of turpentine (50 to 200 grammes) given well diluied in water, sometimes produces a relatively favorable action; but it is often insufficient. Asafoetida, ether, kerosene, camphor, etc., must be discarded entirely.

The absorbents are only of very doubtful value. Ammonia (10 to 30 grammes administered in thirty to fifty times as much water, at intervals of half an hour to two hours) has certainly done more harm than good; it has produced most of the mishaps relative to the wrong route taken by the liquids. This remedy, indeed, is 
more exciting than absorbent. The other absorbents-concentrated soap-water, or lye, potash, lime-water, etc.-are less dangerous. We must not, however, overlook the fact that absorbents reaching the rumen act under entirely different conditions from those obtained when we place one of these agents in a flask filled with carbonic acid gas, and then agitate the flask. The reactions do not take place in the same way in both cases; far from it.

The anti-ferments, which are of great advantage in chronic tympanites, are only advisable in slight cases of the acute form; for these cases we can use hyposulphate of soda (100 to 200 grammes) or chlorate of potassium (30 to 60 grammes), especially recommended by Marton, Brown, David, and others; Labarraque's liquor or hypochlorite of soda (50 grammes of the salt dissolved in half a litre of fresh water), hydrochloric acid, etc.

We must not lose too much time in trying these means; we ought to know the right moment at which to abandon them and make use of the osophageal sound or the trocar. Anxiety and dyspnœa often render the use of the sound extremely dangerous; at times also the obstruction does not permit its application. In such cases we must unhesitatingly puncture the rumen. By making a narrow incision in the skin, the introduction of the trocar is rendered much easier.

The plain round canula, without lateral openings, is preferable to any other. If the evacuation of gas happens too suddenly, vertigo may occur, also fainting or syncope through cerebral anemia provoked by the hyperemia of the organs of the abdominal cavity. The canula should not be removed too quickly; if fermentation should still continue, meteorism might again be produced. The instrument can safely remain in place for several hours. When asphyxia is imminent, and we are without a trocar, the puncture must be made with a knife, the blade of which should be absolutely firm.

When asphyxia is averted and the cure of the meteorism is obtained, the animals must be subjected to a severe diet for several days, for the accumulated matters in the paunch might act upon those introduced, and by producing abnormal fermentations cause a renewal of the meteorism.

The treatment of acute tympanites in the sheep is about the same as in the ox. Massage of the left flank and the raising of the anterior quarters are much to be preferred to the application of the œsophageal sound or puncture of the rumen (May). This latter operation is much more dangerous than in the ox. If we are 
compelled to use any therapeutic agents, we may give lime-water (one-fourth litre every ten or fifteen minutes); also sulphate of soda (in a dose of 15 grammes dissolved in a little water), and Groppe's remedy (a teaspoonful every quarter of an hour), or essence of turpentine (a half-tablespoonful in one-eighth litre of water), or kerosene oil (according to May, a half-tablespoonful in an equal quantity of water). Ammonia-water (a half-tablespoonful in one-fourth litre of cold water); we should make the same restrictions in its use as in the case of the ox.

When meteorism develops at the same time in a large number of animals of the same flock, a similar treatment would be too slow and too unhandy; in this case we advise removal of the flock at once and direct it toward a stream ; the animals should be immersed and thoroughly douched. There are some serious cases where the puncture of the rumen with the trocar or bistoury is the only means of averting death. ${ }^{1}$

In order to prevent the meat acquiring the disagreeable odor which the gases of the rumen might communicate to it, in the case of asphyxiated or slaughtered animals, it is advisable to open the abdominal cavity at once and to expose the intestinal masses fully.

Hurtrel d'Arboval has described a tympanites accompanied by very marked convulsive movements, which is said to be common in the Department of the Pyréneés-Orientales, where it is known under the name of falère. It is probable that it is a case of intoxication by poisonous plants.

In the pig, meteorism may happen after the ingestion of fermentable food, such as malts containing much leaven ${ }^{2}$ also after rapid ingestion of an excess of hot whey (Viborg), or during the course of certain poisons. As the administration of medicated liquids is accompanied with great dangers, we must always resort to the trocar (which is also recommended by Spinola). The subcutaneous injections of physostigmine may also be tried.

In the $d o g$ we have observed great meteorism in certain intoxications (enteritis from poisoning) and in contractions of the intestine.

1 In certain regions recourse is frequently made to an empirical treatment well known to shepherds and giving good results; as soon as the animals begin to swell they are given lard or butter (in doses of about 100 grammes for the ox and 20 grammes for the sheep). There are communities and farms where they are in the habit of burying here and there a pot containing lard in the fields where the animals are exposed to meteorism; this is a precaution which may be very useful.-N. D. T.

2 Eberhardt: Repertor., 1875. 


\section{CHRONIC METEORISM OF RUIMINANTS.}

Slow, chronic tympanites consists of a tenacious and periodic accumulation of gas, mainly of carburet of hydrogen, in the rumen; but this accumulation is less abundant than in tympanites. In a case observed by Erdmann the gaseous mixture was of the following composition :

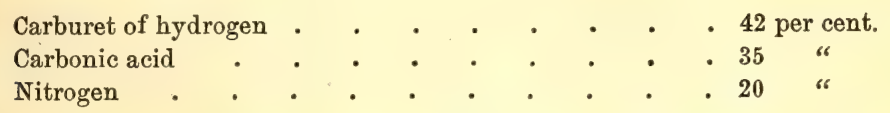

This composition varies with the ingested food, and these figures must not be considered absolute; they show, however, that carbonic acid is less abundant in chronic tympanites than in the acute form of this disease.

Etiology. Among the causes mentioned by authors, there are two very different ones which must be indicated. Chronic tympanites frequently is ouly a symptom of chronic catarrh of the stomach; it is caused by the arrest of the process of rumination, the activity of fermentation, and the suppression of the movements of the rumen. When catarrh exists, the least dietetic mistake, such as a too rapid ingurgitation of the food in adult animals, or the ingestion of a large quantity of milk in young ones, is apt to produce meteorism. It is evident that fodder which ferments easily favors its development, especially when given for any length of time. The following substances may also act in the same way : dry fodder when such aliment has been withheld for some time, hay cut too short, the farinas, etc.

All abnormal conditions which cause mechanical interference with rumination are causes of chronic meteorism. We must here especially mention the contractions of the osophagus (contractions by compression), due most frequently to tumors or to tuberculous degeneration of the mediastinal lymphatic ganglions. In fact, chronic tympanites is often related to tuberculosis. The tumors of the first three gastric compartments (polypi, fibromata, sarcomata, etc.), ægagropilæ, or stercoral balls may hinder eructations and produce meteorism. The adherence of the abdominal viscera to the abdominal walls interferes with their movements, causing stagnation of the alimentary matters and tympanites; these peritoneal adhesions are ordinarily due to sharp foreign bodies, to blows given upon the lower part of the abdomen, to repeated punctures of the 
rumen, etc. Diaphragmatic hernias with adhesion of the reticulum to the walls of the thoracic cavity and to the organs contained therein may also disturb rumination and eructations, and produce chronic meteorism (Lindenberg).

Symptoms. They are similar to those of chronic catarrh of the stomach. The principal symptom is the permanent or intermittent swelling of the left flank. Rumination is suppressed; the movements of the belly and its bruits are but slightly marked. The difficulties of respiration and the anxiety which is always observed in acute tympanites are ordinarily absent. Emaciation gradually advances in proportion to the progress of the disease.

Prognosis. The prognosis varies with the cause producing the affection. When meteorism is under the influence of gastric catarrh, it disappears with it; but if it is a consequence of one of the organic alterations mentioned above-displacement or abnormal adhesions of the large digestive compartments-it is incurable.

Treatment. Only where meteorism is symptomatic of chronic catarrh of the stomach should treatment be undertaken, and then it is similar to that of the latter affection.

A first important indication would be to establish proper alimentary diet. Besides, we must try to check fermentation by giving hydrochloric acid, hyposulphite of soda, etc., and also excite the movements of the stomach by means of tartar emetic, white hellebore, or essence of turpentine. As a last resource we may perform rumenotomy by means of an incision made upon the left flank. The swelling seldom attains such proportions as to necessitate puncture of the rumen. Absorbents procure but a temporary amelioration. They are powerless against fermentations and the inertia of the rumen.

[The hypodermatic administration of strychnine in small and repeated doses is of much value in overcoming the muscular inertia of the rumen in this disease. Emetine and apomorphine, eserine and pilocarpine may also be used with advantage in this form of partial muscular paralysis.-W. L. z.]

\section{OVERLOADING OF THE RUMEN IN THE OX.}

Etiology. The avidity of these animals for food to which they are not accustomed (grass, green clover, new hay, malt, various mashes, etc.), and the ingestion of excessive quantities of these 
alimentary matters after a prolonged abstinence, or the absorption of water of a very low temperature; are the principal causes of the overloading of the rumen.

Symptoms. These vary with the causal conditions, especially with the quantity and digestibility of the food ingested.

The animals keep away from their manger, arch the back, and expel normal excrements in small quantity and at short intervals; they will also move the tail, strike the abdomen with the hind feet, carry the head from one flank to the other, and complain; sometimes they lie down and almost immediately stand up again. The countenance is anxious and haggard ; the eyes are prominent and the conjunctiva is injected. At certain times we observe stupefaction; the animals are indifferent to everything happening around them.

Inappetence is complete and rumination has stopped; the thirst is often increased; we can observe ptyalism with motions of empty mastication, eructations, nauseas, and even true vomiting. The muzzle has a normal appearance.

The volume of the abdomen is increased, especially at the height of the left flank. The rumen is filled with packed alimentary matters; they are sometimes quite hard, but mostly soft. Percussion of the left flank gives a dull sound; later, it allows us to define the accumulation of a more or less considerable quantity of gas in the upper part of the rumen. Pressures upon the abdomen are painful, and the animal seeks to avoid them. The degree of fulness of the rumen can also be determined by rectal exploration. The peristaltic movements are lost or diminished; the hand applied upon the left flank is able to recognize the inertia of the walls of the rumen; the bruits of friction due to the activity of this organ are no longer perceptible; defecation is rare and not abundant, and the excrements are dark and hard.

The temperature is not increased and is regularly distributed; the pulse is accelerated and the artery distended. Respiration is not free; it becomes very painful in decubitus and when the animal descends a hill : these symptoms may lead us to suspect the existence of lung trouble.

Course. The course of the disease has nothing constant. When the rumen is too much overloaded death may happen by asphyxia or apoplexy, exactly in the same way as in acute meteorism. In other cases overloading is followed by a gastro-enteritis, but most frequently, especially when the fodder ingested is not too great in 
quantity, and is of easy digestion, it ends in resolution. This happens within a few hours in slight cases; the first symptoms of the return of digestion ordinarily appear in twenty-four to thirtysix hours. They are: return of the appetite and rumination, more active peristaltic movements, frequent eructations, and defecation. In three or four days the recovery is complete.

Differential diagnosis. The overloading of the rumen may be confounded with acute tympanites and with pelvic hernia.

Pelvic hernia is produced suddenly, and is mainly expressed by anxiety and restlessness, as in overloading of the rumen. Rectal exploration enables us to distinguish both ; the tension of the cord, which is characteristic in hernia, is not found in overloading of the rumen. In acute tympanites, which appears immediately after meals and is betrayed by a rapid enlargement of the belly, the rumen is distended by gases, while in overloading we perceive very distinctly the alimentary matters, which form a more or less consistent mass. In addition, respiratory and circulatory troubles are less annoying in this latter condition.

The diagnosis of overloading of the stomach from acute gastrointestinal catarrh is easy : the intensity of the symptoms and their sudden appearance are indications which are proper to overloading.

Treatment. In most cases we might limit ourselves to waiting; still it is advisable to subject the animals to diet and to walk them several times a day. When the affection has any gravity, we try to re-establish the peristaltic movements, by massage of the flank, and also by drastics and emetics. The most advisable therapeutic agents are: aloes, tartar emetic, or large doses of sulphate of soda, and white hellebore in powder or tincture $(10$ grammes of powder or 15 to 20 grammes of tincture). As a last resource, we may resort to incision of the flank and the cleansing of the rumen.

[In order to re-establish peristaltic movements in the rumen we may have recourse to hypodermatic medication; emetine and apomorphine in full doses, or eserine and pilocarpine may be very advantageously used after the gas has been removed with the trocar. -W. L. Z.]

NOTE ON "VACUITY OF THE RUMEN."

Voigtländer ${ }^{1}$ has described certain obscure morbid phenomena produced by emptiness of the rumen; he has observed this condi-

1 Voigtländer, Sächs. Jahresber., 1878-79. 
tion in cases in advanced gestation, after a long journey by railroad; before being shipped, the animals had been given a small quantity of grass, and had not received anything at all during the journey.

The symptoms observed much resembled those of vitulary fever. The animals were completely paralyzed, the head was moved from one side to the other; the respiration was accelerated, the eye sunk in the orbit, the pupil dilated, the countenance without expression, etc. The mortality was found to be considerable. At the autopsy the rumen was absolutely empty.

It would not be possible to affirm that the symptoms observed by Voigtländer were entirely dependent upon vacuity of the rumen. In certain acute and chronic diseases of the ox the rumen is sometimes entirely emptied without the appearance of the phenomena just mentioned. They are undoubtedly provoked by abdominal hyperemia and consecutive cerebral anemia-an explanation which does not agree with Franck's theory upon the pathology of puerperal eclampsia.

\section{CATARRH OF THE ABOMASUM AND DUODENUM IN THE OX.}

This disease seems to be frequent in the ox, but it is one that is very difficult to recognize during life. Basing his statistics on the examination of 600 animals which were killed for the market and which presented all the signs of health, Prietsch estimates that adult subjects of the bovine race are attacked by this affection in the proportion of 20 to 25 per cent. He has observed it in the acute, and at times in the chronic form. The symptoms assigned to it are vague, often contradictory. We shall limit ourselves to a very brief recapitulation of the observations referred to.

Saake has found this disease in growing bovines, in oxen and in cows, before and after calving.

At the onset, the symptoms are almost the same as in other gastric affections; we notice alternately an improvement and an aggravation of the disease; often there is an abnormal production of gas in the stomach. Notwithstanding a more or less marked periodic tympanites, the animals present all the signs of health. On auscultation of the rumen we hear a peculiar metallic noise, which is considered by Saake as pathognomonic of catarrh of the abomasum, being a symptom which is said never to exist in common tympanites. 
Later, an intense diarrhea sets in, and emaciation follows; then a jaundiced condition with tendency to œdema; the appetite is lacking or capricious, but there is no sign of "licking." The animals are sensitive to pressure upon the right hypochondrium. The course of the disease is very irregular, and often, after many months, it terminates in death. At the autopsy we find a considerable thickening of the mucous membrane and of the submucous connective tissue; this is the seat of an odematous infiltration in the neighborhood of the pylorus and in the duodenum; the mucous membrane, which is of a red catarrhal shade, may acquire a thickness of four centimetres; in the chronic form we notice follicular ulcerations in it. Tannin, in doses of 50 grammes a day, has been quite efficient.

Harms describes under the name of " catarrh of the abomasum and duodenum" a disease of the digestive apparatus appearing suddenly, and disappearing after a few days. This disease is distinguished from common stomachal catarrh by the icteric coloration of the conjunctiva, the preserved normal character of the fecal matters, the soundness of the functions of the rumen, even when the latter is full, and also by the sensitiveness of the region of the abomasum to pressure of the hand. This affection is said to be almost always accompanied by "licking." As lesions, we find, at the outset, an odematous swelling and hyperemia of the mucous membrane, which is covered with an abundant exudate. The autopsy made at an earlier period shows the mucous membrane folded and thickened. Harms has treated his patients by subjecting them to a dietetic regimen, giving forage of easy digestion, and administering Carlsbad salts and hydrochloric acid internally.

This author has also described a case of obstruction of the abomasum. ${ }^{1}$ In this observation the abomasum, which might at first sight have been mistaken for the rumen, contained forty-five litres of ruminated alimentary matters. During life frequent eructations had been observed, also an abnormal sensibility of the abomasum, salivation, vomiting, and obstruction of the rectum by fecal matters.

\section{GASTRO-INTESTINAL CATARRH OF YOUNG ANIMALS.}

By its etiology, its course and treatment, gastro-intestinal catarrh of young animals differs essentially from the same affection in

1 Harms: Hannov. Jahresbericht., 1879-80. 
adults. It has often been confounded with dysentery and described under this name; but it must be distinguished from it, and we have given it a special description.

Etiology. I. The causes of catarrh in sucklings are as follows:

1. The diseases of the mother. The constitutional morbid state, anemia, cachexia, affections of the digestive apparatus, and troubles of nutrition, act unfavorably upon the young; also the infectious diseases, aphthous fever, generalized tuberculosis, mammary tuberculosis, etc., are deadly for young animals; mammitis and plain congestion of the udder may also cause gastro-intestinal catarrh in the calf.

The alimentation of the mother has a considerable influence upon the condition of health of the young. Food which is too rich in nitrogenized matters (grains, legumina, clover), also food which possesses little nutritive properties, a poor quality of watery roots and green substances, spoiled roots and tubers and rancid oil-cakes, may all produce digestive troubles in sucklings. Several special qualities of milk and the alterations which it undergoes produce the disease easily: very fat or too watery, curdled, bitter or rancid milk, or milk containing too much or too little albumin or caseine, must be particularly mentioned. Certain medical substances mixed with this liquid (essential oil, resinous, vesicant or laxative substances) may irritate the gastro-intestinal mucous membrane. Excessive fatigue of the mother influences the quality of the milk and reacts upon the progeny.

[One of the most common causes of this disease among young animals on American farms is the careless and irregular manner in which they are fed. Kept for long periods without food while the mother is at work, they are allowed to take an unlimited quantity of milk furnished while the dam is overheated and fatigued. This is one of the reasons why so many of our animals are suffering from a chronic form of this disease. If the young animals are not nursing they are allowed to take an overabundance of milk or other food, thus producing indigestion of the abomasum, a common disease among calves. -W. L. z.]

2. Also when the young animals are fed irregularly, at the time their mothers are used as working beasts.

3. Also colds (cold or damp stables, draughts, and cold udder of the mother).

4. The retention of intestinal mucus of the fœtus (meconium), 
when the first milk (colostrum) is not used, the result of which will be constipation and a decomposition of the contents of the stomach, the products of which irritate the mucous membrane.

II. Among the circumstances capable of determining catarrh in weaned animals, we must specially point out:

1. Weaning when taking place under unfavorable conditions; alimentation with spoiled food or coarse forage, hard to digest, and with straw chopped too fine, etc.

2. The substitutes for milk, particularly those containing much starch. They act by producing acid fermentations (acetic, lactic, or butyric), which provoke irritation of the mucous membrane.

3. We observe it in cold and damp weather, especially when the animals go to pasture, and in the spring and fall; also from cold drinks.

4. The replacement and eruption of teeth, during which period the young chew and swallow the greatest variety of objects (Hamm).

5. Verminous affections of the digestive canal, mainly those due to the twisted Strongylus (verminous gastritis of the lamb), or also Toenia (in the same animal).

Finally, the development of the disease is favored by general predisposing causes: hereditary weakness of the digestive apparatus, affections of the mesenteric ganglion, etc., and also by extreme sensibility of the digestive organs in very young animals.

Pathological anatomy. The alterations found at the autopsy are nearly the same as those of gastro-intestinal catarrh in adults, and in cases where the morbid process has been very intense, the alterations are often found far from corresponding with the symptoms observed during life. In sucklings the stomach contains voluminous and dense clots of casein (the normal coagulation of the milk has often been taken for a pathological alteration); in weaned animals we find coarse alimentary matters which are more or less impacted. The mucous membrane of the stomach and intestine presents variable alterations, such as redness, tumefaction, follicular catarrh, ulcerations (in acute form); atrophy, thickening, pigmentation, etc. (in the chronic form); the mesenteric ganglions are ordinarily tumefied. The animals are emaciated and show all the signs of anemia and hydremia.

Symptoms. The manifestations of the initial period vary with the causes of the disease. Sometimes they appear suddenly; at other times they are outlined gradually, and do not attract attention 
for the first few days. In sucklings the principal symptomdiarrhea-is usually preceded by a slight anorexia, some depression, and weakness; in weaned animals, and especially when the disease is induced by the ingestion of very cold water or an external cold, it commences with fever and general troubles; the temperature of the body is irregularly distributed; the members are cold, the muzzle dry ; we notice a pronounced nervous depression, both sensory and motor. Diarrhea soon develops, the excrementitious matters become more and more liquid and clear-they are often expelled in a jet; then they become fetid, mucous, yellow or greenish-gray, spumous, and contain more or less fibrinous flakes or clots. They are of an acid, putrid odor; later they are streaked with blood or even bloody; they are rejected at any instant, soiling the posterior members, as well as the tail, and causing the hair around the anus, the perineum, and the buttocks to fall out. There is ordinarily tenesmus and slight colic; the animals have their backs arched and the legs gathered; the skin is dry and the hair bristly; the exhalations become fetid, and weakness is excessive.

We often find tympanites; gases are accumulated at certain times in the rumen and intestine, and produce a more or less intense swelling. This condition, which results from abnormal fermentations, may exist before, during, or after the diarrheic period.

In some cases death occurs after a lapse of time varying from a few days to several weeks. It is generally produced by exhaustion, anemia, or dropsy. Sometimes the disease is complicated by a catarrhal pneumonia; but this may possibly be a consequence of pulmonary atelectasia, quite frequent in weak newborn animals.

Prognosis. The prognosis must always be made with much care, taking into account the etiological considerations above indicated. The long duration of diarrhea and tympanites is always an unfavorable prognostic symptom.

Treatment. We must look for the cause of the trouble and suppress it or diminish its effects. In sucklings we ought mainly to look after the mother; very nutritious or very poor food should be avoided; also fatiguing work, as well as carrying the milk too long in the mammary glands. We may also select another nurse.

The feeding of the young animal should be regulated; the number of meals will have to be increased, diminishing the milk ration at the same time, which we should give in small quantities and at 
regular intervals. The calves must not be weaned before four or six weeks, and the lambs after three or four months only; weaning must be gradual, and heavy, irritating food must be avoided.

Diarrhea should be counteracted with roasted bread soups, browned flour, malt, coffee, raw eggs, gelatin-water, mucilage of marshmallow or gum, ginger seeds, poppy, almond milk, decoctions of hops, poppy-heads, etc. When the intestine is full of fermented or decomposing matter, a light laxative may be administered ; for the calf, two or three spooufuls of castor-oil or some other sweet oil ; 25 to 30 grammes of sulphate of soda or of magnesia in a mucilaginous decoction; for the lamb, one-fourth of these doses.

Carbonates of magnesia and soda have been recommended to counteract intestinal fermentations; also chalk, bone powder (a teaspoonful to a tablespoonful). Creosote could also be tried ( 3 to 5 drops for a calf, a half or one drop for a lamb, in a mucilaginous excipient); also creolin ( 2 to 5 grammes for the calf, 0.5 to 1 gramme for the lamb), salicylic acid ( 1 to 2 grammes for the calf, 0.25 to 0.50 gramme for the lamb), in an alcoholic solution; finally, resorcin and some other antiseptics.

In intense diarrheas, opium and rhubarb render good service. We give opium in doses of 0.2 to 1 gramme for the calf, and about 0.05 to 0.2 gramme for the lamb; opium tincture or Dover's powder may also be given (calf, 1 to 5 grammes; lamb, 0.5 to 1 gramme); also rhubarb root in decoction ( 2 to 4 grammes for the former and 0.5 to 1 gramme to the latter). Most of the styptics have also given good results. Lastly, it is also advisable to make astringent irrigations per rectum with a solution of alum or tannin at $1: 100$, of nitrate of silver or the lead salt at 1-2 per $100 .{ }^{1}$

1 Filliâtre has obtained excellent results with tar-water. In substance the treatment he recommends is: Pure vegetable tar, 150 grammes; boiling water, 6 litres. " Let the mixture become lukewarm, and give it in clysters of a third of a litre every halfhour; stop the treatment as soon as diarrhea ceases. The young calves had recovered the same evening, and drank their allowance of milk. The next day put one-fourth of tar-water into the milk; discontinue the treatment after two days."

Barinetti advises a treatment consisting of milk and ether clysters. He recommends two clysters a day with 300 grammes of milk, and a quantity of ether gradually extended from 5 to 12 or 15 grammes.-N. D. T. 


\section{GASTRIC DERANGEMENT CAUSED BY THE PRES- ENCE OF FOREIGN BODIES IN THE STOMACHS OF RUMINANTS.}

\section{Traumatic Indigestion: Traumatic Inflammation of the} Stomach and Diaphragm.

The bibliography of this subject is very rich, but has reference especially to traumatic carditis or pericarditis, which condition in reality constitutes a complication of those gastric affections which are here considered. The clinical picture of the traumatic lesions of the heart is much more precise than that of the morbid process which takes place in the gastric walls; thus special works treat almost exclusively of pericarditis. However, the facts related by Obich, Meyer, Harms, and Schmidt, and our own observations, permit us to give a general description of the troubles produced in the digestive organs and diaphragm.

We frequently detect foreign bodies in the stomachs of ruminants killed for the market. Bergemann found a carving-knife which had remained for three months in the stomach of a heifer, without causing the least local or general trouble. In most cases, however, they occasion grave disorders of the first gastric compartment and the diaphragm. Concerning their pathological importance, Schmidt has classified them in three groups :

1. Smooth foreign bodies (pebbles, stones, etc.).

2. Sharp foreign bodies perforating the walls of the stomach and remaining imbedded (large-headed nails, hairpins, etc.).

3. Sharp or pointed foreign bodies which perforate the walls of the stomach and travel into the annexed parts more or less far (needles, tacks, wire fragments).

Etiology. The frequency of foreign bodies in the stomach of ruminants results from the habits of the animals, as well as the mode of prehension and deglutition of foods. In proportion we find them more frequently in cows under the care of women than in stables which are looked after by men (Obich).

They are relatively frequent on small farms, where the greatest variety of kitchen refuse is given to the animals, also in stables owned by mechanics, especially shoemakers, or in the neighborhood of needle factories, and in stables where the ceiling or roof is of wood; they are more frequent among animals permanently stabled 
than in those living upon pastures. ${ }^{1}$ Harms has observed that the individuals occupying the head or end of a row are more often the carriers of such bodies than the others, and explains this fact by the accumulation of foreign bodies toward the ends of the troughs when they are accidentally deposited in them.

Anatomical alterations. As smooth foreign bodies we find the greatest variety of objects: pebbles, sand, wool-balls, leather-ware, straps, broken whip-handles, balls, pieces of money, clothing, etc., even the bodies of vipers and adders. All these materials may occasion catarrh and also true inflammation of the mucous membrane. If the rumen should contain any sand, it will generally form a compact mass, more or less hard; the mucous membrane itself may be incrusted by a thick layer of small gravel.

The sharp bodies most commonly found imbedded in the walls of the stomach are: nails with large heads, needles, hairpins, twisted wires, kitchen forks, etc. They are ordinarily found in the reticulum; the large size of the rumen and the regular disposition of its internal membrane explains clearly why their implantation is very rare in the walls of this compartment, while the irregularities of the mucous membrane of the reticulum easily hold them. Wherever the vulnerant body is fixed, the reticulum presents a circumscribed inflammation which hides a narrow fistulous canal, with thickened walls of a grayish or bluish-gray color, containing pus and alimentary matters. The peritoneal surface of the reticulum is covered with a fibrinous exudate or with a layer of proud flesh in the neighborhood of this lesion; it is adherent to the annexed organs and especially to the diaphragm; there may be abscess formation around the fistulous course, and the diaphragm is often lined with granulations. The rumen sometimes adheres to the abdominal walls and contains a large quantity of alimentary matters. The foreign body is found at times at the lower end of the fistulous tract, at other times it has left this to enter the stomach where we find it mixed with the food. The offending substance might be expelled with the excrementitious matter; this has sometimes taken place, and the erroneous belief has been entertained that the foreign body has been dissolved.

The sharp-pointed foreign bodies passing completely through the

1 Cosson, of Villeneuve-l'Archevêque, mentions that the neighborhood of knit-goods factories is equally dangerous to cows, on account of the scattering in the waters and highways of broken or defective needles taken out of the machines (Morot: Journ. de Lyon, 1890.)—N. D. T. 
walls of the stomach are: all kinds of needles, pieces of wire, broken nails, knives, scissors, forks, pieces of glass, of wood, vineshoots, etc. According to the direction taken by the foreign body we can also find, in addition to the alterations described as occurring in the reticulum and the diaphragm, symptoms of an extensive peritonitis, abscesses of the liver and spleen, adhesions between the reticulum and the liver, abscesses of the abdominal walls or of the chest, lobular pneumonias, pulmonary abscesses, adhesions between the lungs and the pleura, also pleurisy, etc. The foreign body may penetrate as far as the muscles of the arm and shoulder, may even reach the spinal cord, and leave indurations, adhesions, abscesses, and fistulæ in its course.

The alterations of the heart are described under Traumatic Pericarditis.

Symptoms. The symptoms disclosing the presence of smooth bodies in the stomach of ruminants consist principally in chronic or acute digestive troubles, produced by irritation of the mucous membrane. Krichels and Schmidt state that in incrustation of the mucous membrane by sand there is a continual mastication without deglutition, abundant salivation, complaining, and extreme weakness; the animals remain quiet, the back is arched, or they maintain a decubital position; the rumen is very resistant to palpation, and its peristaltic movements are suppressed. If death results, it will ordinarily occur between the ninth and seventeenth days; in some cases the disease takes the chronic form, and may persist for a long time.

When the foreign body is pointed, the symptoms are generally those of a traumatic inflammation of the stomach and diaphragm. Without any apparent cause, we observe sudden grave digestive troubles, colics, with stamping, kicking upon the abdomen, great agitation, etc.; at times these troubles simulate the symptoms of invagination, in other cases the symptomatic course begins with the ordinary signs of acute catarrh of the stomach. Later, we observe a series of periodic gastric complications of a very inconstant character. As a rule, chronic tympanites is the salient symptom, and it offers something very peculiar, inasmuch as all the remedies directed against it remain almost without effect. With the progress of the disease, emaciation and weakness become extreme, the countenance expresses anguish and pain, the various movements (mastication, lying down, getting up, and walking) 
are accompanied by evident signs of suffering and provoke complaints.

The animals are very sensitive to pressures made upon the reticulum; according to Ellenberger and Schaaf, the locality where the exploration ought to be made is situated upon the lower wall of the abdomen, somewhat to the left of the xiphoid appendix of the sternum; frequently, also, pressures upon the sternum, the thorax, the withers, or the rumen produce more or less severe suffering. In cases where the diaphragm is inflamed, percussion on the level of its peripheral edge is also painful ; we may observe circumscribed dulness in front of its line of insertion (abscess, granulous neoformations); its contractions at the time of inspiration or defecation, and also the opposite movement performed during expiration, are very painful to the patient. Thus defecation is performed as much as possible without abdominal muscular exertions; it takes place at infrequent intervals, and an increased accumulation of excrement in the rectum results from it. When the foreign body has injured an important vessel, blood may be seen to escape through the anus.

The sharpness of the foreign body very often enables it to perforate the walls of the stomach and penetrate into the peritoneal cavity, or into the diaphragm, sometimes the liver, etc.; and this is due to the contractions of the reticulum, to the peristaltic movements of the stomach, and to the action of the diaphragmatic muscle. The efforts of parturition help the progression of the foreign body to a great extent. In all these cases we observe, besides the described gastric symptoms, some manifestations answering to superadded alteration, which may be carditis, pneumonia, pleurisy, pneumothorax, enteritis, peritonitis, hepatitis, etc. The foreign body is sometimes directed against the skin; it provokes an inflammatory tumefaction under the integument, the meaning of which may be recognized immediately at this period of the disease; it is a very variable phlegmon in its dimensions, which occasionally attain the size of a man's head; its most frequent location is the lower part of the left hypochondrium; it may, however, appear on the right, or back of this region in the neighborhood of the umbilicus or mammary gland. This abscess is remarkable by its slight sensibility. It bursts suddenly and discharges a bloody pus mixed with alimentary matters and sometimes sand. The foreign body may be extruded at the same time. In following the fistulous 
tract with the sound we may penetrate as far as the stomach. Ordinarily the fistula heals only after some weeks, perhaps months; occasionally the cure is complete within a relatively short time; in some cases the patients are attacked by marasmus and succumb; finally, there are animals showing, so to speak, nothing abnormal either before or after abscess formation.

Differential diagnosis. The diagnosis is quite difficult; a careful examination of the patient, however, often permits us to detect important and significant symptoms.

If there is any possibility of confounding this affection with common catarrh of the stomach, the following indications may guide us and assist in eliminating the former condition: The absence of reactions when making compression of the insertion of the diaphragm, the region of the reticulum, etc., also the uniformity of the gastric troubles; etiological data that may be elicited by inquiry, and the effect of curative treatment prolonged for a certain time; further, we see a less marked emaciation in common catarrh of the stomach than in cases of traumatism of this organ.

We may be embarrassed in trying to establish a difference between the disease in question and a peritonitis or gastro-enteritis; but these latter affections are ordinarily distinguished by a more rapid march, a more intense fever, and more serious disturbance of nutrition. When the foreign body has penetrated into the lungs, it may produce symptoms of a chronic pulmonary affection which is often difficult to distinguish from pulmonary tuberculosis, except when former indigestions, an affection of the diaphragm, or absence of any bronchial catarrb, lead us to suspect the presence of a foreign body.

Contagious pleuro-pneumonia is recognized by its epizootic character, its typical course, and by the pneumonic symptoms which predominate; lastly, also to absence of serious disturbance in the direction of stomach and intestine.

Treatment. Measures of prophylaxis are indicated by the etiological considerations. Harms proposes to make small holes upon the opposite extremities of the crib, which are intended to gather the foreign bodies which are accidentally mixed in the forage. The efficiency of the treatment depends upon the time when the diagnosis has been made. Cases are not rare where treatment for stomachal catarrh has been first used; but as soon as we are convinced of the presence of a foreign body, we have simply the 
choice between killing for the market and the extraction of the offending material. The latter may be practised according to Obich's method. It consists in performing a vertical incision upon the left flank, through which the arm may penetrate into the rumen, and which has to be located about 8 to 10 centimetres from the transverse processes of the lumbar vertebræ; once in the reservoir, the hand searches for the foreign body by exploring especially the reticulum. We then apply Gély's or Jobert de Lamballe's suture upon the walls of the rumen, and the cutaneous incision is closed by an ordinary suture.

It is said that the operation is easily borne; it could even be attempted upon a cow in calf; the main point is to make an exact diagnosis. Obich, who has had occasion to perform it thirteen times, had but four successful cases.

The extraction of the foreign body is advisable every time the diagnosis is correct, provided the trouble is not too old; but, for reasons of economy, we generally prefer the slaughtering of the animal.

\section{GASTRO-INTESTINAL CATARRH OF CARNIVORA.}

Etiology. Overloading and catarrh of the stomach are much more frequent in the canine species than in the horse and ox. We can say that in the dog they occupy a place as important as all other diseases together. The cause of this extreme frequency of diseases of the stomach lies probably in the irregular alimentation to which this animal is subjected; the regular diet itself is often improper, and the very common habit of giving one meal only per day leads to a daily overloading of the stomach. Hunting dogs and nursing bitches are those most commonly affected, on account of their great need of nourishment. But it can be seen in all famished dogs, which literally stuff themselves with potatoes, bread, bones, etc.

The most common etiological agents of catarrh of the stomach are : all hard, irregular, heavy, spoiled, rotten, or fermented food; also foreign bodies used as playthings by puppies, and which are sometimes swallowed by them. We must also mention : decay of the teeth, a too rapid ingestion of the food, especially when it is too hot, and external colds after shearing or bathing.

Gastro-intestinal catarrh may be developed as a phenomenon of passing ailments and of several chronic affections-as ulcers of the 
stomach, intestinal worms, stagnation in the portal circulation, old heart affections, lung or liver trouble, Bright's disease, etc.

Symptoms. 1. Overloading of the Stomach. It provokes vomiting as a rule, then everything becomes regular again. When the matters contained in the stomach are not rejected, we observe serious symptoms; for instance, nauseas accompanied by abundant salivation and complete anorexia; the animals keep away from the food offered to them and seem disgusted, thirst is increased, pressure upon the distended stomach provokes complaints; the respiration is sensibly accelerated. The patients are depressed, of bad humor, or capricious and anxious; they often change position, lie down, stand up again, stamp the ground, complain, wag the tail, and are affected by colics. There is not the slightest fever.

2. Catarrh of the stomach. In the dog it is possible to distinguish catarrh of the stomach from that of the intestine; in the latter affection, vomiting is exceptional, while in the first it is almost constant. But we must not forget that vomiting is a symptom common to a great many affections, among which we must particularly mention : ulceration, cancer, inflammation of the stomach and intestine, dilatations, contractions, helminthiasis, peritonitis, inflammation of the diaphragm, the several diserses of the œsophagus, anginas, cough, uremia, septicemia, eclampsia, epilepsy, encephalitis, cerebral apoplexy, cerebral commotion, otorrhea, etc.

The gastric catarrh of carnivora is acute or chronic, febrile or apyretic. In mild cases we observe laziness for several days, also depression of spirits, a capricious appetite, and great thirst; then nauseas and vomitings appear. The vomited matters are at first chyme; later they become mucous, thread-like, and spumous, reddish or yellowish, and finally the patients reject bile almost pure, or mixed with altered gastric juice. The thirst is increased, and water ingested in excessive quantity causes renewed vomiting. Sometimes there is complete anorexia, at other times the appetite is only diminished; and again, we may see the alimentary matters vomited at the time when digestion has already begun. Defecation is delayed. The temperature rises to $39^{\circ}-39.5^{\circ} \mathrm{C}$; it is irregularly distributed; the nose is hot and dry ; the circulation is accelerated, and the number of pulsations exceeds the normal by twenty or more. The animals are depressed and remain lying down most of the time, but not on their usual litter ; they stretch themselves upon 
the floor of their kennels or seek cool places. Pressure upon the stomach is often very painful.

3. Catarrh of the intestine. In intestinal catarrh the appetite is less changed, the region of the stomach is not sensitive to pressure, and as a rule there is no vomiting. The most important symptom, which is always present, is diarrhea. Its characters vary with the degree of intensity of the disease. At the beginning there is a simple softening of the excrementitious matters, then they become serous, and finally bilious and bloody; but, in these intense conditions they are always frothy and fetid, and soil the parts adjacent to the anus. We detect tenesmus and violent expulsive efforts; in young patients we may also notice the appearance of prolapsus of the rectum. The urine contains the coloring matters of the bile (Frohner). The fever is always quite high, it may become very intense $\left(40^{\circ}\right.$ to $41^{\circ}$ C.). Icterus is a common complication of duodenal catarrh, and is much more frequent in the dog and cat than in the horse and ox.

Recovery is the common termination of the disease in adult dogs, but, in young or weak animals, severe diarrhea often persists and produces exhaustion and death.

Chronic catarrh is more common in the dog than is generally admitted - at least this is what the detection of the coloring matters of the bile in the urine tends to establish. It is ordinarily a termination of acute catarrh, but its prognosis is much more serious than that of the latter. When left to themselves, the animals lose flesh, become anemic, and succumb to marasmus.

Treatment. In most cases diet alone is sufficient to obtain a cure; it must be absolute and also be directed to the drink, especially water. When we judge it to have been' enforced for a sufficient time, the patients should be fed on raw meat given in small quantities at a time.

In overloading of the stomach by tainted or toxic food which has already produced a certain degree of irritation of the mucous membrane, an emetic is to be prescribed: this frequently cures catarrh of the stomach and prevents that of the intestine, which would unavoidably be developed otherwise, on account of the passage of irritating alimentary matters along the latter. Hydrochlorate of apomorphine must be used in preference to others, because it does not irritate the stomach $(3,5$, or 10 milligrammes in the dog and 20 to 25 milligrammes in the cat, in a $1: 100$ solution, by hypo- 
dermatic injection). The other emetics to be used are white hellebore (5 centigrammes to 2 decigrammes), also ipecac ( 5 decigrammes to 2 grammes), and tartar emetic (1 to 3 grammes in a sufficient quantity of water). For small pet dogs we can give wine of antimony in doses of a teaspoonful to a tablespoonful.

[Purgation should be produced by the administration morning and evening of calomel in doses of from 0.25 gramme to 0.50 gramme. From the first manifestation of icterus the administration of dilute nitro-muriatic acid in from two- to five-drop doses has given very satisfactory results.-W. L. z.]

If there is loss of appetite we must resort to the stomachics. The most favorable are: hydrochloric acid (5 grammes in 250 grammes of water, to be given in tea- or tablespoonful doses; pepsin (hydrochloric acid and pepsin, of each 5 grammes in 250 grammes of water); bitters (tincture of gentian 1 gramme, hydrochloric acid 5 grammes, water 300 grammes; in tea- or tablespoonful doses); and tincture of rhubarb, in water or wine, in same doses, two or three times a day.

For diarrhea we should give the proper dietetic remedies: dry food, toasted bread soups, rice and barley soups, and hydrotherapic compresses on the abdomen; we may also give good red wine in tea- or tablespoonful doses, and plain opium tincture, in doses of 20 to 60 drops in mucilaginous mixture, or Dover's powder from 5 centigrammes to 2 grammes. For obstinate diarrhea we should employ nitrate of silver in doses of 0.01 to 0.05 gramme in a solution of distilled water, or in the shape of pills. Before resorting to this medication we may try astringent clysters (alum, tannin, sulphate of iron and sulphate of copper at 1-2 per 100), the advantage of which we have often observed.

Constipation, which is usually not of a tenacious character, should be counteracted by means of calomel in doses of 0.03 to 0.1 gramme; this agent has the advantage of disinfecting the digestive canal.

If there are vomitings we should prescribe ice or iced substances for delicate animals, or small doses of opium, bromide of potassium or hydrate of chloral. Subnitrate of bismuth has sometimes been useful. The same is the case with creolin, creosote, and iodine tincture.

The abnormal fermentations are remedied by calomel $(0.03$ to 0.1 gramme), creolin ( 1 to 2 grammes), and naphthalin ( 0.5 to 2 grammes). 
For convalescents we must avoid heavy food which is difficult to digest.

Ancient authors, from having seen constipated dogs eat grass, thought that material to be necessary in the treatment of constipation, attributing to this aberration of the taste the value of an instinctive medication. Some have gone so far as to grant to couch-grass (Triticum repens) certain specific properties. We look upon the act of grass-eating as a caprice of the taste, or kind of play; we have never noticed it in sick dogs; on the contrary, it is common in healthy animals, in which it provokes diarrhea much rather than vomiting.

\section{CONSTIPATION IN THE DOG.}

Etiology. This affection, which is very frequent, consists essentially either in a mechanical obstacle preventing defecation or in insufficient peristaltic movements. Its principal causes are :

1. Heavy food not containing any exciting principles; also food composed exclusively of bones, bread, leguminous plants, and flour pap ; dry alimentation.

2. Forced idleness or insufficient exercise, as in house-dogs or those tied by a chain.

3. Also advanced age, which weakens the peristaltic movements as a result of atrophy of the mucous membrane and the muscular fibre of the intestine.

4. Chronic intestinal catarrh, which deprives the mucous membrane of its sensibility by covering it with a mucous exudate and causing its atrophy. In this affection the reflex movements of the intestine are lost and the intestinal secretions diminished, a dual condition favoring constipation.

5. Mechanical obstacles opposing the progress of the intestinal contents. We must especially mention here ; coprostasis, constriction of the intestine by compression (tumors, hypertrophy of the prostate, abscesses); also dilatation and paralysis of the intestine, hemorrhoidal nodes, tumefaction of the anal glands, and compression of the anus by agglutinated and packed hair, preventing the exit of fecal matters (false constipation).

6. Constipation is a symptom of a large number of general febrile diseases in which the secretion of intestinal fluids is interfered with; it accompanies also most of the chronic or asthenic 
affections. It is often seen to complicate acute gastro-intestinal catarrh, icterus-a disease in which the bile no longer exerts its excitant action upon the intestinal walls; peritonitis and enteritis -affections in the course of which costiveness happens through œdema or paralysis of the intestinal muscular fibre; also the diseases of the diaphragm and abdominal walls; or it is produced by the suppression of abdominal contractions. It appears also during the course of or after paraplegia and cerebral affections: it is then the expression of disease affecting the intestinal innervation. Lastly, it may be determined by abundant perspiration, by very active lactation, and causes of a similar nature.

Symptoms. The principal symptom is the difficulty, scarcity, or suppression of defecation. The animals often make violent expulsive efforts which are manifestly painful; in general, these efforts are without result, but in some cases there is an expulsion of small, dry dung, of earthy color, of penetrating fetid odor, and containing undigested bone fragments, sometimes covered with mucus and blood.

Some patients will at first continue to eat, but later there is complete anorexia for whole weeks. The peristaltic movements are much less active. The abdomen is sometimes voluminous and hard; at other times distended with gas. In making an exploration we find a kind of hard voluminous roller, formed by the rectum stuffed with excrement. This organ, thus distended, may touch the lower abdominal wall and advance as far as the sternum. Any manipulations performed upon the abdomen are very painful to the animal. The circumference of the anus is the seat of an inflammatory tumefaction, and it is sometimes soiled with yellowish or grayish fecal matters. In false constipation the hair in the neighborhood of the anus becomes agglutinated by the excrementitious matters and offers a mechanical obstruction to their exit. Rectal exploration, which is always painful for the anirnals, shows an increase of temperature, dryness, and swelling of the mucous membrane, and the presence of hardened excrements or bones in the rectal cavity. There are cases where the obstructed and distended rectum is difficult or even impossible to recognize through the abdominal walls. The matters contained in the anterior portion of the intestine in front of the hardened mass sometimes break through and are ejected in the shape of a diarrheic fetid liquid, more or less mixed with gas. Occasionally we notice the appearance of colicky symptoms. 
In complete obstruction of the intestine we frequently observe vomiting.

The fever is never intense, but at an advanced period of the affection we may detect great nervous depression. The movements are slow and lazy; the males urinate without lifting the leg. Lastly, the tail is carried straight in a particular direction; this sign in most cases permits us to make the diagnosis from a distance.

Duration. The duration of the disease varies from a few days to five or six weeks; it is, on an average, from eight to fourteen days. When it lasts over a month the mucous membrane becomes irritated by the excrements, and we may note the appearance, as complications of enteritis, of necrosis of the mucous membrane, and of peritonitis by perforation. The disease sometimes leaves behind it organic alterations, such as cicatricial retractions and intestinal diverticula, which may themselves in their turn cause constipation.

The rectal obstruction may be confounded with an abdominal tumor; but the particular form of the dilatation being outlined, as well as its consistence, its direction, and the course of the disease, a minute examination always permits us to recognize it. People ignorant of veterinary medicine often believe in the existence of constipation when defecation is suppressed on account of prolonged abstinence.

Constipation generally ends in a cure. Its prognosis is not serious; however, at the beginning of the disease, and when it has lasted for some time, the prognosis must be reserved.

Treatment. In the great majority of cases treatment must be dietetic-mechanical or medicinal. Surgical treatment may be indicated in exceptional cases (such as puncture, incision, or enterotomy), in abscess, hypertrophy of the prostate, or foreign bodies in the intestine.

We must suppress all food that is heavy and voluminous (as bread, bones, potatoes, etc.); at first the animals may even be subjected to an absolute diet of fresh water only. Later, it is advisable to sustain them with milk, beef-tea very salt, and a little lean meat without bones. The peristaltic movements should be stimulated by frequent walks.

Among the mechanical means, we must first mention lukewarm water clysters, given with a rubber tube. Cold-water injections are particularly advisable in intestinal paresis. Glycerin injections, 
advised for human beings, are inefficient in the dog. ${ }^{1}$ The action of the clysters is completed by massage of the rectum through the abdominal wall, or by a frequent rectal exploration with extraction of the accessible fecal matters.

Purgatives are generally favorable. We give castor oil in doses of from 20 to 50 grammes and calomel in doses of 0.05 to 0.1 gramme, with sugar as the excipient; subcutaneous injections of sulphate of eserine in proportions of 0.001 to 0.003 have been advised; in obstinate cases and when the intestine is not the seat of an inflammatory affection, we can resort to croton oil (castor oil 10 to 20 grammes, croton oil 1 to 5 drops). These purgatives act perfectly in many cases, but we must not trust them too much; the most powerful drastics are sometimes unable to move the enclosed excrementitious mass.

In order to avoid a return of constipation, the cured animals must be subjected to a special diet. The same precautions should be taken for pet dogs and for those having intestinal diverticulæ.

[Constipation in dogs is often of such a character that the fecal masses can only be removed by mechanical means (curette). In old dogs the prostate gland is very frequently the cause of the trouble; in this case the curette may be inadvisable; we then must have recourse to continuous inter-rectal irrigation daily for half an hour, accompanied by massage of the abdominal walls. In this way we may succeed in removing all of the fecal mass from the intestine in from two to three weeks. Constipation may persist in dogs for very long periods-in one case coming under my notice it was of over ninety days' duration.-W. L. z.]

\section{FOREIGN BODIES SWALLOWED BY THE DOG.}

Etiology. Accidents caused by the deglutition of foreign bodies in the food are frequent in the dog, much more rare in the cat. These accidents deserve a special mention, first, because it is often possible to make the diagnosis intra vitam, and further because the symptoms that they provoke have sometimes been confounded with those of hydrophobia. A certain number of these cases occasion gastro-intestinal hemorrhage or perforations followed by fistulæall purely surgical accidents; others produce special phenomena which will be studied in the section on Peritonitis by Perforation;

1 Hanson: Wiener medicin. Zeitung, 1879. 
again, under a third heading may be classified some which are remarkable by their growing into the intestine, and which are more or less obscure. Accidents of this nature produce gastric symptoms and are ordinarily complicated with hemorrhages or with necrosis of the mucous membrane. We shall discuss them in this article.

Among the substances swallowed by dogs we often find bones, pebbles, lead or iron balls, glass marbles, currency, buttous, corks, chestnuts, strings, etc. The animals usually swallow these objects in playing or carrying to their masters, more rarely in taking their food. We have seen a dog which had swallowed a projectile measuring 4 centimetres in diameter and weighing 250 grammes (8 ounces).

Symptoms. As long as the foreign body floats freely in the stomach its presence is generally indicated by symptoms of an acute or chronic gastro-intestinal affection. Often, however, it does not provoke any abnormal phenomena. Nichoux has seen a dog which carried a silver five-franc piece and a large two-cent copper for two years without showing the least inconvenience. ${ }^{1}$

When the foreign body obstructs the opening of the intestinal canal the symptomatic picture becomes definite. At the outset there is no fever, but marked nervous depression is often seen; the animals remain lying, eat little or refuse all food, and drink with avidity; they are soon affected with vomitings, especially after meals or ingestion of water. These vomitings are sometimes performed with extreme violence, and then mucous, bilious, and excrementitious matters are rejected; icterus appears quite frequently; defecation is either suppressed or is reduced to a minimum. We may observe rabiform symptoms; the dog is cross and very excitable; he has often a tendency to bite and to destroy objects which he can seize; generally he runs from man. The fever appears and increases with variable rapidity; anorexia is complete and emaciation is striking. There are cases where the trouble is overwhelming; the animals are apathetic, remain almost always recumbent; the countenance is anxious, the fever increases rapidly, the pulse is small, and death takes place during deep somnolence.

Diagnosis. The course of the disease and the information

1 We have observed a dog swallowing spinning-tops, and carrying them in the stomach for eleven months without any sign of distress, but finally one of the two tops entered the duodenum, and, being there held fast, superinduced grave symptoms and death. At the autopsy the other top was found free in the stomach.-N. D. T. 
obtained by palpation of the abdomen are of much more value in diagnosis than the symptoms, we have just described. The exploration of the stomach must be performed while the animal is kept standing on two feet, the hands of the examiner being applied upon the lower abdominal wall immediately above the sternum. The exploration of the intestine is made with the hands applied upon the lateral walls of the belly when the anima? is in a standing or dorso-decubital position.

Duration. The duration of the affection is extremely variable. Death happens at times within a few days, at other times after several weeks, sometimes after months; we may see successive periods of amelioration and aggravation, corresponding with the migrations of the obstruction in the intestinal canal.

Pathological Anatomy. The foreign body may become enclosed in the pyloric opening; then we find the right cavity of the stomach dilated and the corresponding muscular fibre much hypertrophied (in a medium-sized dog we have found it one centimetre thick); the intestine is almost always empty. When the object is stopped in the small intestine the part of this organ which is above the obstructed point is much dilated and hypertrophied; the posterior portion, on the contrary, is contracted, atrophied, and devoid of food. In the neighborhood of the obstruction the mucous membrane is the seat of an inflammatory tumefaction or of a necrotic eschar presenting a starchy appearance; often the muscular layer and the peritoneum itself have participated in the morbid process. In that portion of the intestine where the foreign body has travelled it has left certain marks, such as ecchymoses, ulcerations, cicatrices, pigmentary infiltration ; ordinarily its arrest is not due to its extraordinary size, but to the irregularity of its surface; the muscular fibre becomes tired and ends by being stricken with paresis.

Treatment. Emetics, purgatives, and the forced ingestion of a large quantity of water are so many recognized remedies, but they have no successful results to their credit. If the animals continue to eat, very copious and extensive alimentation (with bread, potatoes, etc.), by dilating the digestive tube, may form a wider passage to the arrested body, and thus determine its evacuation. At all events we must not count too much upon such a result, and surgical extraction will always remain the true and proper means of treatment. The favorable results obtained by Félizet, Siedamgrotzky, and others (laparotomy, incision of the stomach and intestine. 
extraction of the foreign body, intestinal suture, then simple suture of the external wound), must encourage practitioners in undertaking this operation. ${ }^{1}$

\section{GASTRO-INTESTINAL CATARRH OF THE PIG.}

Etiology. Gastro-intestinal catarrh of the pig is derived from the same causes as that of the dog. Being an omnivorous animal, the pig is often given food of bad quality, which is spoiled or poorly prepared, which may determine gastro-intestinal catarrh in them as well as in our other domestic animals. At certain seasons the disease takes an epizootic form; it has been defined under the name of "infectious gastritis." As described by Busch, this affection is frequent during rainy years and in all pigs going to the fields. It has been ascribed to dampness, colds, and to the neglect to which these animals are subjected ; also to the ingestion of excessive quantities of snails, worms, etc.

Symptoms. The principal symptoms are inappetence, vomitings, colics, constipation or diarrhea, a more or less intense fever, the chilled condition of the extremities-mainly of the ears-dryness and increased heat of the groin, the injected condition of the mucous membranes; the walk is painful and the tail hanging. If the patients are kept inside they remain constantly lying down; they seek the corners and bury themselves in the litter.

The affection ends in resolution or continues in a chronic form. In this latter case it may lead to marasmus and death.

Treatment. This is the same as for the dog ; we must, however, select certain ways of giving the medicines in preference to others, and use larger doses. In the pig drenches are very dangerous; they often produce pneumonia through a foreign body. We must give the therapeutic agents as much as possible in the food, or in an electuary form; also in clysters, or in hypodermatic injections.

In cases of overloading of the stomach we must, prior to any other treatment, administer an emetic. We have often resorted to veratrine $(0.03$ gramme dissolved in $3 \mathrm{c.cm}$. of alcohol, with equal parts of water) in hypodermatic injections; vomiting is produced within an hour. According to Feser the hydrochlorate of apomorphine is without effect. It is hard to administer white hellebore

1 Félizet has twice practised successfully the operation of extraction of a foreign body (a cork) which obstructed the intestine (Revue Vét., 1877).—N. D. T. 
powder (the dose is 0.5 to 2 grammes) or root of ipecac ( 1 to 3 grammes); we might avoid this inconvenience by giving clysters with the decoction or infusion of white hellebore ( 2 grammes in 50 grammes of water).

If there is any constipation it is advisable to prescribe calomel ( 1 to 4 grammes), and give repeated clysters. Diarrhea may be opposed by various styptics and opium (1 to 2 grammes).

Finaliy, the animals must be subjected to diet (complete abstinence), or they must be given liquid and cooling nourishment (milk, farinaceous drinks, soups, etc.).

\section{GASTRO-INTESTINAL CATARRH, CONSTIPATION, AND DIARRHEA OF POULTRY.}

In order to describe these three morbid conditions we have drawn a great deal from Zürn's work.

1. The common gastro-intestinal CATARRH AND DiarRHEA of poultry must be distinguished from infectious diseases accompanying diarrhea, especially chicken cholera and intestinal tuberculosis ; they must not be mistaken for certain intense inflammations of a toxic nature. We find them as a rule in subjects of improved or imported races, in the spring, and during the moulting period of young birds.

Etiology. Among the causes that are responsible for this condition may be mentioned overloading of the stomach, ingestion of heavy or tainted food, cold, damp coops, and ice-water. The diarrhea is sometimes symptomatic of helminthiasis.

Symptoms. The principal symptoms are inappetence, erectness of the feathers, depression and the downcast condition of the birds; also paresis of the crop, which is voluminous and empties slowly; the expulsion of soft, clay-like, whitish excrements, which become gradually more liquid-greenish-and are irritating to the neighborhood of the cloaca and to this organ itself, the mucous membrane of which becomes inflamed; the feathers surrounding it are stuck or glued together; the thirst is increased, the weakness and emaciation are accentuated, and the diarrhea becomes more and more liquid. Soon death occurs from exhaustion or from intestinal hemorrhage.

In an autopsy we find the alterations of desquamative catarrh, redness, tumefaction, epithelial degeneration ; or those of enteritis. 
Treatment. This is first of all hygienic. The patients must be placed in an aviary or in a room of mild temperature.

In order to obtain this, Zürn advises to cover the floor of the stable with a thick layer of horse manure. It is preferable to heat the place and to keep it perfectly clean. We should give food of easy digestion, and preferably cooked food (millet, rice, etc.). In order to check the diarrhea, we may use roasted barley, rice-water, old biscuit, hemp-seed, poppy-seed, chocolate (for parrots) bread soaked in cognac, red wine (a tea- or tablespoonful with grain, biscuit, etc., which must be macerated in it), or mucilaginous decoctions-Zürn advises decoctions of pearled oats (15 grammes per litre of water)-linseed, roots of marshmallow (15 grammes per 200 grammes of water), to be given in a dose of one large spoonful.

We can also give a drink composed of $1: 100$ of sulphate of iron, a decoction of oak-bark, opium (5 to 10 drops of tincture of opium for large birds, according to Zürn), also nitrate of silver (1 to 2 teaspoonfuls of a solution of $1: 100$ advised by the same author).

For indigestion, Zürn recommends small particles of garlic, also pills made of pepper and butter, a decoction of peppermint, or calamus $(2: 60)$, given three times daily in doses of a tablespoonful for the chicken, and a teaspoonful for the pigeon.

2. Constipation in poultry may follow diarrhea or may be the result of the presence of intestinal worms or foreign bodies in the stomach or intestine (pebbles, feathers, indigestible bodies in general), also from an accumulation of thickened fecal matters in the cloaca, or from weakness of the digestive canal, etc.

Zürn advises to counteract constipation of the chicken by green food, soft and salted prepared food, and that of the pigeon with oat soups. The mucilaginous and fat bodies, oils and cold-water clysters administered with the siphon are also very desirable remedies. In small birds we can introduce the well-oiled blunt or button sound into the rectum. The mechanical extraction of fecal matters accumulated in the rectum and cloaca is also advised. Among the purgatives we must select castor oil (two large spoonfuls for a hen) also senna ( 1 to 2 grammes of powder at one time, or 4 grammes of crushed leaves soaked for six hours in $200 \mathrm{c.cm}$. of fresh water, to be given a teaspoonful at a time till the appearance of a laxative effect), also rhubarb (0.4 to 0.6 gramme with honey, in pills).

For small fancy birds we may use the tincture of rhubarb, per drop, in the drinking-water. 


\section{COLICS OF THE HORSE.}

UNDER the name of colics (enteralgia, intestinal pain) we designate a whole group of pathological conditions of the stomach and intestine in which the peristaltic movements of these organs diminish in activity or cease to be performed, and which are expressed by an ensemble of fairly uniform symptoms, but particularly by more or less intense suffering.

The expression "colic" does not indicate a unique and welldefined affection; it is applied, on the contrary, to a large number of morbid processes, the clinical symptom of which is abdominal pain ; it is a very comprehensive generic expression-similar to the words heaves, hydropsia, icterus-which ought to be used only in the domain of general pathology. Nevertheless, in order to avoid almost insurmountable difficulties, and also errors which would be of frequent occurrence if we wished to specify all the affections of the stomach and intestine that may be observed, we continue therefore to use it in its broad and general acceptation. In practice it is even applied to the painful affections of different viscera contained in the abdominal cavity-kidney, bladder, liver, uterus, etc. (false colics), and also to pains happening during the course of certain general diseases-petechial fever, anthrax, ete.

Although this expression is undoubtedly vague and is wanting in the precision to be desired, it will nevertheless be used for a long time to come in works upon special pathology, and that for several reasons: first, it would not be without inconvenience to abandon an old nomenclature which is universally accepted in ordinary everyday language; and, further, it has the advantage of designating, in regard to the horse, an entire category of affections, not observed so frequently in any other animal, and of which it is sometimes extremely difficult, if not impossible, to make the differential diagnosis. In a large number of cases, indeed, the practitioner is obliged to be satisfied with ascertaining the presence of abdominal pain-with making the diagnosis of "colic."

History. All authors, from the earliest times, who have written upon diseases of animals mention colic of the horse and advise different remedies to combat it.

In the first century of our era Columella describes three forms 
of colic. Eumelus (third century) advises the treatment of colics: with nitrate of potash. Vegetius, Apsyrte, and Hierocles, in the fourth and fifth centuries, gave a tolerably exact symptomatology of colics. Vegetius considers the pains of the belly, those of the ileum, of the colon, and of the bladder, as so many special colics.

In the thirteenth century Jordanus Ruffus makes a difference between gaseous or windy colics and those produced by overloading with alimentary matters. Three centuries later Vegetius Renatus and Joannes Ruellius give a brief description of colics.

The eighteenth century witnessed the production of Ehrmann's, Sander's, and Weber's works, and the beginning of the nineteenth those of Ribbe, Tennecker, and Brunswig. Among the modern authors who have particularly written upon colics must be mentioned Ullrich, Legrain, Bollinger, and Friedberger.

Frequency. Colics are the most frequent of all internal diseases of the horse. Bollinger's statistics have shown that among one hundred horses attacked by any internal diseases forty are affected by colics (or 7549 colics in 18,984 patients). The same author estimates that in Bavaria, upon every 400,000 animals forming the equine population of that province, 40,000 are attacked by colics every year.

The average mortality, according to Bollinger, is 13 per cent. (in 12,857 cases, 1625 fatal cases). In gathering 26,253 cases of colics, most of them mentioned in the different clinical reports, we have found 2832 fatal cases - that is to say, a mortality of 10.7 per cent., a proportion differing very little from that indicated by Bollinger; these findings, taken together, result in an average mortality of 12 per cent.

According to Bollinger, the mortality caused by colics would represent 40 per cent. of the general death-rate.

In the various German veterinary schools the mortality is stated thus :

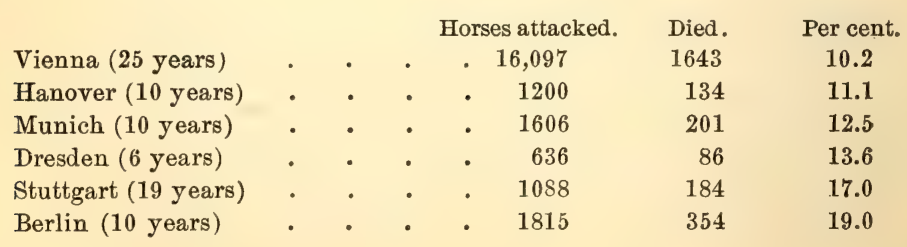

In the Prussian army the average mortality for the years 1880 to 1886 was $11 \frac{1}{4}$ per cent. 
General causes of colics. 1. Predisposition. The special proneness of the horse to colic is owing to the particular anatomical disposition of the stomach and intestines. The small dimensions of the stomach, the oblique insertion of the osophagus upon this viscera, the presence of membranous folds, the fulness of the left pouch, are peculiarities which render vomiting extremely difficult in this animal ; thus the contents of the stomach, if solid or gaseous, cannot be evacuated through the oesophagus. The length of the mesentery, the great volume and mobility of the large abdominal reservoirs, favor the changes of relation of these organs and the accumulation of alimentary matters in their interior, and accordingly these are very frequent accidents.

We must also point out the extreme sensibility of the terminal fibres of the intestinal and peritoneal nerves, which become easily stimulated under the influence of numerous trivial causes.

Finally, the frequent aneurism of the first colic artery is again an important etiological factor; by producing thrombi or emboli of the intestinal arteries it plays a very important rôle in the pathology of colics.

2. Colds. At times the action of cold is felt upon the surface of the body; it is particularly to be feared at the time of shedding the coat and during damp days, especially when winds from seaward and rain follow a long dry spell. This action is produced directly upon the mucous membrane when the animals ingest very cold drinks, or food covered with frost or ice. Adam has noticed frequent colics in horses after drinking water at a temperature of $5^{\circ} \mathrm{C}$.; the same water at the temperature of $7^{\circ} \mathrm{C}$. was harmless.

3. Overloading the stomach. This happens generally when the horses eat too fast, when the various alimentary substances which compose the ration are given them too rapidly; also when the food is not in proportion to the work performed (on resting days, Sundays, etc.). It is also produced when the animals consume heavy alimentary matters which are hard to digest, such as grains (rye), hay cut too short, fresh hay and oats, raw potatoes, etc., and especially when mastication is insufficient.

Immediate work after meals is a frequent cause of colic.

4. Dirty alimentary matters. Muddy hay and grass covered with sand, dust, or mould; also straw, oats, and bread covered with mould; dirty drinking-water containing earthy matters.

5. Abundant production of gas in the stomach after the ingestion 
of new grain or young or mouldy grass. Let us particularly mention red clover, lucerne, sainfoin (fenugreek), buckwheat, grass, and mainly gramineous plants of damp meadows, leguminous grains which absorb much water, all kinds of new grain; ${ }^{1}$ also turnips, potato-stems, etc. Fermentation is accelerated by the ingestion of a large quantity of water immediately after meals. Another cause to be mentioned here is the deglutition of air.

6. Retention and hardening of excrementitious matters-coprostasis. Besides the constipations which are under the influence of intestinal catarrh, arrest of matters contained in the digestive canal is produced after prolonged inactivity of the animal; after alimentation with long or short straw; with hard, woody fodder; poor aliment, which is deprived of all stimulating principles; or food that is too dry (flour, bran, crushed oats). The alimentary matters may cease to move forward in a weakened, dilated, or paralyzed intestine.

7. Calculi and concretions which are formed in the stomach or intestine, and the accumulation of sand or earth in these organs. This latter cause has especially been observed in army horses in the field, but it can also be seen in animals going to pasture. Calculi rich in phosphate of magnesia are particularly observed in millers' horses, which consume large quantities of bran.

8. Intestinal worms, especially when formed in balls.

9. Constrictions of the intestine due to compression, torsion, or invagination and strangling of this organ.

10. Prolonged abstinence, which causes the colic of abstinence common in army horses in the field.

11. Unusual movements, mainly rolling on the back when the animals are cast. Kohne has seen volvulus happen in a horse which had been thrown in order to undergo a surgical operation, and which had been turned several times. In slaughter-houses we frequently observe volvulus in pigs and calves that have been unloaded in a brutal way. It would, however, be unreasonable to try to prevent a horse attacked by colic from rolling; observation teaches us that horses at liberty roll with impunity; moreover, recent volvulus may be cured spontaneously by rolling.

1 Vuibert affirms that well-ripened and well-harvested oats never produce colic. If, in the country, the horses are often attacked by colic at the time when they are given new oats, it is because they have been deprived of this food for months, their provisions being frequently exhausted by June. Based upon a twenty-five-years' experience, Vuibert maintains that this deviation of diet is the great cause of colics, which are attributed to ingestion of unseasoned oats. - N. D. T. 
12. More or less intense colics accompany the various alterations of intestinal structure: catarrh, simple or croupous inflammation, intoxications, ulcerations, etc. (see the articles on these affections).

The etiology of false colics will be defined in connection with our study of the respective diseases by which they are produced.

Symptoms. All colics, no matter what the cause may be, are marked at the beginning by phenomena expressing sudden painmost frequently without the slightest precursory sign. Harnessed or mounted horses slow their gait, stagger behind, stop, are nervous, scratch the ground, stamp, become restless, bend their legs halfway, and lie down if not prevented from so doing. If the animals are in the stable we can see certain prodromes ; anxiety, depression, keeping away from the manger; then come agitation, stampings, complaints; the animals abruptly carry one of the hind legs against the abdomen, they look at their flanks, sway the tail, bend the front legs, gather the hind legs under the body, arch the spinal column, stretch the head, neck, and shoulders; finally they lie down slowly and carefully; sometimes, after having bent their members in order to take a decubital posture, they come back at once to the standing position; in other cases they let themselves fall or even throw themselves upon the ground. At times they perform unconscious movements of extreme violence; then, again, they remain as if overcome by a deep somnolence; sometimes they remain for a long time in a dorsal decubitus, the legs gathered toward the body. The pains are not continuous; we can observe remissions, during which the animals stand up again, and are relatively quiet and look for nourishment; but this improvement is only temporary.

If we examine the patients attentively we find an irregular distribution of temperature: the ears and extremities are cold; often we observe sweating spots upon the base of the ears, on the sides of the neck and shoulder, on the ribs and flank; the sweat may trickle to the ground; in some cases the wet regions are hot, in others cold. The pituitary gland is generally more or less colored, but the injection of the mucous membrane is not constant. The buccal cavity is dry, its mucous membrane is hot, injected, and catarrhal, or pale and cold, as if it had been washed. The abdomen is often enlarged, at other times empty and tucked up. The peristaltic movements are ordinarily weak or even completely suppressed, and the bruit of the borborygmi has disappeared; there are cases, however, in which we perceive loud noises having a 
metallic timbre. As a rule, defecation is infrequent, and the most violent repulsive efforts are followed by the discharge of a few dry masses, which are hard, brownish, and covered with mucus or with a bloody coating; sometimes, however, the excrements are soft, diarrheic, and of acid odor; gas frequently escapes per rectum; exceptionally some necrotic portions of the intestine may be expelled with the fecal matters.

On exploration of the rectum we may find it packed with dung or entirely empty; its mucous membrane is hot and tumefied; the neighboring intestinal pockets are distended with gas or alimentary matters, the bladder may be empty or filled with urine. There are cases where the lumen of the rectum is increased; in other instances it is so diminished that the finger can hardly penetrate it. In the stallion, rectal exploration may enable us to detect a strangulated inguinal hernia, and in alı animals it permits us to recognize the presence of fecal balls or of an intestinal calculus. When the large colon is much distended by gas the fleshy longitudinal bands of the walls are like stretched and hard ropes to the touch.

The animals brace themselves frequently in order to urinate; they hang the penis from its sheath and expel a few drops of clear urine, or perhaps containing coloring matters (we must beware of confounding this condition with hemoglobinuria); according to the special case, the urine may be alkaline, neutral, or acid; it may contain albumin. The respiration is ordinarily painful; sometimes there is dyspnœea. At the beginning the pulse is not modified; generally it is much accelerated, and we may count 50,60 , or 100 pulsations, or more, per minute; the pulse is small, the artery is distended, the contractions of the heart are hard and tumultuous. Immediately following the exacerbation of colic the temperature is normal, or almost so, but its course is most variable; it often reaches a very high mark, whilst death will sometimes take place without any serious febrile reaction having been noticed.

The general sensibility is more or less diminished, and the habits with which the animals may be affected are no longer noticed.

In very intense colics we observe certain strange symptoms. The patients bend the front legs and kneel down, the posterior quarters remaining high; they will lie in the position of the sphinx or that of the dog. We notice nauseas, regurgitations, sometimes vomitings; then alimentary matters are rejected through the nostrils. The intensity of the pains may provoke rabiform attacks; 
the horse rears, sneezes, groans, utters distressing cries, opens his mouth, grinds his teeth, bites the manger or some part of his body, and sometimes attacks man; in other cases, where the pains are less violent, he pushes himself against the wall and performs queer movements; the head is also shaken or swings like a pendulum. When the cases end fatally the weakened patients lean against the walls, tremble, and are subject to convulsive movements; soon they are covered with a cold and profuse perspiration, the pulse is falling, intermittent, imperceptible ; the heart's action is precipitate, irregular, tumultuous; the eye is haggard, the pupil dilated, the lower lip hanging; the anus, wide open, runs with a brownish fluid. Finally, without any further resistance, the animals stagger, fall, and soon die. At this point, just before they succumb, some animals will begin to neigh, others willingly partake of fresh water. Often the pains cease, but they may possibly continue up to the last moment; death takes place either during coma or in an attack of violent suffering.

Course, duration, and complications. The course of colics is ordinarily extremely acute. Sometimes they last but a few minutes; in most cases they persist for several hours; when they last more than from twenty-four to thirty-six hours they are often fatal. They may, however, take a subacute course, or even become chronic and last for weeks, with remissions; this is the course taken by colic from constipation.

As a complication of colics, we may mention the dry crusts which are formed upon the salient points of the body, and which are produced by compression of the skin at the time of decubitus; wounds, tendinous and ligamentous distentions, fractures, internal lesions, etc., determined by unusual movements on the part of the patients. Unskilful administration of drinks may result in traumatic pneumonia. There are cases where vomited alimentary matters fall into the trachea and likewise cause pnemuonic accidents, which almost always end in gangrene and death. Drinks which are too hot, and also caustic remedies (croton oil, emetics), which are not given sufficiently well diluted, will produce either superficial or deep lesions of stomatitis and pharyngitis. We may also find similar alterations produced by the application of pepper and salt upon the vaginal mucous membrane of mares-a very popular means of treatment with empirics. Unskilful manipulations in the rectal cavity may also produce colic by provoking 
inflammation of the mucous membrane or even laceration of the organ.

Prognosis. The prognosis must be reserved at the outset, for it is impossible to formulate it with certainty. Colics appearing most benign (rheumatic colic) may be complicated by volvulus; and, according to a general belief, the gravity of a case of colic may vary from one moment to another in the same animal. We can therefore only express our opinions with some reserve.

Improvement is made apparent by diminution of the pain, by more regular distribution of the temperature of the body, the cessation of the sweats, the improvement of the pulse, the reappearance of peristaltic movements, an abundant emission of excrements and gas ; also by the lessening of fever, and return of the appetite and thirst.

Among the unfavorable symptoms which may induce us to fear gastro-enteritis, peritonitis, rupture of the stomach, the intestine, or the diaphragm, we must point out: an accelerated, hard, small, evasive pulse ; the elevation of temperature, intensity of pains, cold and profuse sweats, chilled condition of the extremities, alteration of the physiognomy, persistence of constipation, complete suppression of peristaltic movements, swelling, dyspnœa, and vomiting.

General differential diagnosis. Colics must be distinguished from abdominal affections having their seat in organs other than the stomach and intestine. We will now glance at the morbid conditions with which they may be confounded.

1. Rheumatic hemoglobinuria. Its sudden appearance after a cold, the intensity of the suffering it produces, the cessation of peristaltic movements, also disturbance of the functions of defecation, micturition, and sweating, the dyspnœa and disturbance of the circulatory apparatus which accompany it, make its symptomatic picture very similar to that of ordinary colic. But, in these latter cases, paresis or paralysis of the hind quarters, so characteristic in rheumatic hemoglobinuria, do not exist; we do not observe any painful myositis, there is no hemoglobin free in the blood, and dissolution of this liquid is not appreciable. Finally, in colics, the horses lie down and stand up again frequently, while in rheumatic hemoglobinuria the act of standing is almost always impossible.

2. The pains of parturition are expressed by agitation, stampings, complaints, and by frequent decubitus of short duration, etc. -symptoms common to colics and to parturition. However, the 
examination of the genital organs, of the teats, and of the abdomen, the course of the symptoms and their periodicity, permit us in all cases to make a correct diagnosis.

3. Retention of urine, produced by paralysis of the bladder (detrusor) by calculi, tumors, etc., is often taken for true colics by the non-professional. In the latter, the pressure exerted upon the bladder by the distended intestines provokes efforts at micturition without the bladder being full. Catheterization of the bladder or rectal exploration always gives us very accurate information. We have also to point out that retention of the urine is extremely rare in the horse, and that the function of the digestive apparatus is not at all influenced.

4. Cystitis. In this affection the modifications of the urine (the presence of epithelial cells, pus-cells, phosphatic crystals), also the sensibility of the bladder to the pressure of the rectum, dysuria, and the integrity of the gastro-intestinal canal, constitute facts sufficient to establish the diagnosis.

5. Metritis. Information gained as to parturition or abortion, the muco-purulent discharge through the vulva, the sensitiveness of the uterus, with the persistence of these symptoms, are sufficiently characteristic of metritis.

6. Changes of position of the uterus (torsion, prolapsus, inversion, and constriction). Attentive examination of the patient permits us to recognize these conditions. Only partial or incomplete changes in the shape or position of the uterus could be mistaken for colics.

7. Strangulated hernias (abdominal, umbilical, or inguinal) always produce very violent colic. The first two are expressed by evident objective symptoms, and it is only necessary to make a comparative examination of the scrotum, the inguinal canal and ring in stallions, or even geldings, in order to immediately recognize the entanglement of an intestinal loop in the vaginal sheath.

8. Peritonitis is hard to distinguish from colic. Like the latter, it sometimes assumes a rheumatic character, and then the diagnosis is very embarrassing. It is otherwise with peritonitis succeeding a surgical operation (laparotomy, castration, herniotomy), or when it occurs consecutively to nephritis, cystitis, or metritis. When it complicates colic, we may recognize or at least suspect it by taking into account the intensity of the fever, the character of the pulse, which is always accelerated and small, also the weakness and more or less marked prostration of the patients. 
9. Nephritis is very clearly indicated by the presence of epithelial cells, leucocytes, and red corpuscles in the urine; also of granular and hyaline casts, and of more or less albumin. Moreover, nephritic colics are dull, slightly intense; the symptoms are often only an exaggeration of the sensibility of the lumbo-renal region and stiffness of the gait.

10. Acute hepatitis, extremely rare in the horse, is ordinarily accompanied by a certain anxiety and slight colic, due undoubtedly. to phlegmasia of the peritoneal covering of the liver. The diagnosis of this affection is only possible in cases where icteric complication exists.

11. Intestinal catarrh, enteritis, and ulcerations give rise to "inflammatory colics" which are often difficult to diagnosticate from true colic. In intestinal catarrh the abdominal pains constitute only a secondary phenomenon; the predominating symptom is diarrhea. The duration of the affection and the knowledge of its cause (ingestion of acrid toxic substances, etc.) are the most useful data for establishing the diagnosis. Often the intensity of the fever, the abundance and persistence of the diarrhea, which at times is bloody, and also the retraction of the abdomen, enable us to dispense with everything relating to past conditions. Ulcerations of the stomach or of the intestine are very hard to recognize; they are distinguished from true colic by their chronic course and by the repeated hemorrhages which they produce.

12. Certain febrile diseases are accompanied by pains (and often they are quite acute) which may be taken for true colic on a superficial examination; such are encephalitis, pleurisy, pneumonia, petechial fever, typhoid fever, founder, arthritis, and more rarely thrombosis of the branches of the posterior aorta. It is sufficient to recognize the possibility of these errors in order to avoid them.

Pathological anatomy. The alterations determined by colics vary with the nature of the causes which have produced them. According to one of Bollinger's statistics, 50 to 60 per cent. of the fatal cases of colic are due to modified relations or to mechanical obstructions, and 40 to 50 per cent. are caused by inflammatory affections of the intestine or peritoneum.

Concerning the frequency of volvulus observed upon the different portions of the intestinal canal, the indicated figures are far from agreeing. According to the statistics of the Munich and Berlin schools, the change of relations of the colon would be more frequent 
than those of the small intestine. Schütze ${ }^{1}$ has related 62 intestinal rotations-performed according to the axis of the organ; 56 were located upon the colon and only 13 upon the small intestine (18861888). It is ordinarily the left portion of the colon which is the seat of the rotation. At the Vienna school they have observed a greater frequency of anomalies of position of the small intestine; out of 618 autopsies upon horses that had died of colic, 27 per cent. showed this complication. Change of relations of the rectum or of the stomach is very rare. The twisted portions of the intestine are anemic, and they are pale in color, but on the level of the folds the intestinal walls are tumefied and infiltrated with blood; the mucous membrane is dark red and softened; the intestinal contents are bloody; the mesentery is the seat of hemorrhagic infarctions.

Accumulations of alimentary matters, of calculus and concretions, accompanied by catarrhal inflammation, hemorrhage, diphtheria, or necrosis of the mucous membrane, are also alterations which are quite frequently found. These irritating bodies are especially met with either at the union of the duodenum with the cæcum or of the floating colon with the rectum, or, finally and most frequently, in the cæcum. The colon is sometimes strangulated by a portion of the small intestine; in certain cases this latter is twisted, interlaced, and knotted; it has been found enclosed by the omentum, by lipoma, or by some mesenteric bands. Certain parts of the intestine may be compressed by others which are in a very distended condition. Strangury of the intestine in the inguinal canal has been seen, also in an old fissure of the diaphragm, in Winslow's hiatus, in lacerations of the omentum, in the mesentery, the broad ligament, or between old peritoneal adhesions. Invaginations are not very rare; the most frequent is that of the small intestine in the cæcum.

In a great number of animals we find a rupture of the stomach, of the intestine, or of the diaphragm. Rupture of the stomach generally takes place in the great curvature; it measures often three-fourths of its length; it is almost always rectilinear and situated at the point where the serous membrane which covers the stomach joins in order to form the omentum ; the edges of the rupture are tumefied, infiltrated with blood, festooned, and irregular; the solution of continuity is larger upon the. serous than on the

1 Schütze (unpublished communication). 
mucous membrane; at the point of rupture the layers of the gastric wall are separated by alimentary matter. The contents of the stomach are covered with blood, and a portion has fallen into the peritoneal cavity. Ruptures of the intestine occur at various points, but always in the regions where fecal matter has accumulated. They may involve the mucous membrane only, in which case the fecal matters have penetrated to a greater or less distance under this membrane, detaching it; at other times the muscular coat is torn at the point of mesenteric insertion, and part of the intestinal contents is engaged between the laminæ of its organ of suspension. The lacerations of the diaphragm present very irregular jagged lips, between which one or more organs of the abdominal cavity have passed.

The intestinal mucous membrane may be the seat of a simple hyperemia or of a catarrhal, croupous, diphtheric, hemorrhagic, or necrotic inflammation. In quite a large number of cases we find the whole intestinal wall tumefied, soft, and friable, the subserous and submucous tissue infiltrated with blood, and the contents of the intestine more or less bloody; on the peritoneum we detect inflammatory alterations of variable intensity, from sero-fibrinous exudation to purulent or gangrenous phlegmasia; the mesentery is covered with hemorrhagic infarcts; the mesenteric ganglions are tumefied and infiltrated with blood. The first artery of the coliac plexus is frequently altered by an aneurism containing the larvæ of Strongylus armatus; the arteries of the intestine then become obstructed in many cases by the thrombi consecutive to the emboli emanating from the aneurism.

We find also as accessory alterations: hyperemia of the organs of the abdominal cavity or of the lung, pulmonary odema, hypostatic pneumonia, hemorrhages of the pleura and meninges, parenchymatous degeneration of the heart, the kidneys, the liver, and the spleen. The blood is dark, viscous, tar-like, and rich in bacteria.

General treatment. Horses attacked by colic must be placed in a roomy and closed place, with a floor covered with a good litter, or in a box stall with proper arrangements to prevent the patients hurting themselves while making unusual movements. In order to produce intestinal reflex and to obtain a regular distribution of the blood, the whole cutaneous surface should be rubbed with straw or a piece of cloth. These frictions may be rendered more efficient by means of essence of turpentine or spirits of camphor, applied upon 
the surface of the body. When the weather allows it, it is advisable to walk the animals; this is particularly to be recommended in cases of chronic colic. Very active movements, a rapid gait, as the trot or galop, are harmful. In violent colics the animals must be left free in a box, and we should not persist in making them walk.

Rectal exploration must always be performed, either to empty the organ or to obtain information with a view of defining the diagnosis.

In order to soften the fecal matters and to encourage the peristaltic movements, we may use clysters, which are administered with a syringe or a clyster-pipe. The infusions should be made by means of Hegar's infusion funnel as improved by Damman.

Injections of glycerin in the rectum, at first advised for men (Oidtmann and Anacker) have been used in animals (Schindelka, Kattner, etc.) in a dose of 3 to 5 grammes, but their action is purely local, similar to that of soap ; they are not able to excite the peristaltic movements of the intestine generally.

The introduction of large quantities of water into the intestinal canal is a therapeutic remedy which is now quite old in veterinary art. $^{1} \quad$ The efficiency of these monster injections has been greatly exaggerated. As much as fifty litres of water have been injected into the intestine per rectum. It is not possible to use more than ten litres with any favorable result, as anything exceeding this quantity is immediately rejected. ${ }^{2}$ In the acute and febrile colics we should give, by preference, injections of cold water; lukewarm water may be used in cases where the intestinal troubles have a tendency to become chronic. Cold or warm compresses on the abdomen may be used at the same time as the injections.

When the animals roll moderately, the movements they execute are without danger and sometimes they are of advantage; they may, indeed, actually cause the correction of certain kinds of volvulus. It is otherwise when they throw themselves down violently, as though the intensity of the pain made them lose the instinct of self-preservation ; such violent movements may produce rupture of the stomach or intestine, when these organs are distended. In such cases we may use with advantage a subcutaneous injection of 0.3 to 0.5 gramme of hydrochlorate of morphine in a watery solution of $4: 100$. It would be imprudent to give larger doses (one

1 Lustig: Hannover. Jahresber., 1885.

2 Puitz: Puitzische Zeitschrift, 1876. 
gramme, for instance), which might produce a great excitement, also some tendency to forward movements, and even to attacks of vertigo.

When there is any swelling the intestine should be punctured at once. Puncture, when peformed according to the rules of surgery and with the proper antiseptic precautions, hardly ever produces unfavorable consequences.

Absorbents given in drinks (ammonia, lime-water, liver of sulphur, hypochlorite of sodia) are of very questionable value.

Bleeding is useful in some cases, mainly when a cerebral or pulmonary congestion is feared.

If defecation is delayed through suppression of peristaltic movements, we may resort to the remedies advised for counteracting the constipation that accompanies intestinal catarrh. Sulphate of physostigmine has given excellent results ; this agent, recommended by Dieckerhoff, is administered in subcutaneous injections in a dose of 0.08 to 0.12 gramme dissolved in 5 to $10 \mathrm{c}$. cm. of distilled water, or in a $1: 1000$ sublimate solution.

Physostigmine must be used with discretion. It is contra-indicated by spasmodic colics because of its tetanic and convulsant action; it is also forbidden in colics produced by overloading and in persistent constipation on account of the danger of rupture of the stomach or intestine (Rögener, Klemm, Wilhelm, Rust). ${ }^{1}$

As the administration of eserine favors the production of pulmonary oedema (Friedberger), it is prudent not to resort to it when an accident exists in the neighborhood of the lungs. Ellenberger has proposed to associate eserine ( 0.1 gramme) with pilocarpine ( 0.2 gramme), this latter having the effect of exciting the intestinal secretions and of liquefying the intestinal contents. Bass also recommends this compound.

In order to combat certain colics we have used for a very long time sulphate of soda (200 to 300 grammes) and tartar emetic (4 to 8 grammes), given together or separately; the following electuary has also been recommended: tartar emetic, 6 grammes; sulphate of soda, 250 grammes; fennel-seed and marshmallow-root flour,

1 We have several times seen harmful effects from sulphate of eserine administered in doses of 0.10 to 0.12 gramme in cases of colic from overloading of the stomach, whether accompanied or not by swelling. But this remedy may be of service if used in small and repeated doses (0.03, 004 , or 0.05 gramme) at intervals of one, two, or three hours, according to the course of the colic.-N. D. T. 
q. s.; aloes, 30 grammes, in a bolus with green soap; also calomel 4 to 8 grammes), castor oil in full dose (100 to 500 grammes), and, in very serious cases, croton oil ( 15 to 20 drops in a mucilaginous decoction: these are the medicines generally used in the treatment of colics.

A well-known fact, and one which has been frequently mentioned in the annual reports of the Munich school, is that colics are cured in a great number of cases without any intervention. Whilst this fact is given from observation, yet we are not to conclude that most of these affections ought to be treated on the expectant plan.

Some authors recommend certain remedies as specifics, to the exclusion of all others. Lemke strongly advises morphine, Adam extols cold-water injections, and Luelfing advocates the mixture of aloes, sulphate of soda, and fenugreek. All the so-called specific means are inefficient. We shall see in the following sections that the different varieties of colic require special treatments.

The remedies which are used should be administered preferably in the shape of an electuary or bolus. Drinks are very dangerous when in the hands of unskilled persons (producing pneumonia from foreign bodies). It is wise to use them as little as possible, and to be satisfied with croton-oil emulsions.

\section{SPECIAL COLICS.}

At different periods the attempt has been made to distinguish a certain number of varieties in colics, by taking as a basis either the causes which produce these troubles or the nature of the process by which they are characterized. Thus the older authors have described several kinds of colics. Spinola rejects all the different proposed divisions, under the pretext of not being practical enough; he is satisfied to make a synthetic study of colics under the title of Constipation. But if the question of the differential diagnosis of these affections is yet one of the most obscure in veterinary pathology, we must admit that a good classification established in this chaos may serve as a guide to the practitioner, and permit him to formulate more rational therapeutic indications, also to institute special treatment more appropriate to the morbid conditions which may come under his observation.

We shall lay aside all the more or less arbitrary divisions adopted 
up to date. For colics resulting from indigestion, we refer to he article on Gastro-intestinal Catarrh; for inflammatory colics, to Gastro-enteritis; and for toxic colics, to the description of the poisonings in the course of which they are observed.

We have decided upon the following classification:

I. Spasmodic colics.

II. Colics from overloading.

III. Thrombo-embolic colics.

IV. Colics through intestinal occlusion.

V. Gaseous colics.

VI. Verminous colics.

\section{Spasmodic Colics.}

SIMPLE, NERVOUS, AND RHEUMATIC COLICS; ALSO COLICS "À FRIGORE."

Nature. Under the denomination of spasmodic colics we designate abdominal pains produced by internal or external colds, and which consist essentially in a painful contraction of the intestine. The horse being much exposed to colds is particularly subject to colics. They are relatively frequent in horses of well-bred races, also in stud and army horses-which are favored by an almost perfect hygiene and alimentation.

Intestinal changes being excluded, we must conclude that the pain is produced by compression of the nerve terminals at the time of the intestinal contractions; some consider the condition as being exclusively of a rheumatic nature. In colics produced by cold (à frigore) the sufferings are very violent, but of short duration; they appear suddenly and disappear with the same rapidity, often returning in the form of successive attacks. These particular types of abdominal pain, associated with continued peristaltic movements and defecation, allow us, in most cases, to make the diagnosis of nervous colics. There is generally diarrhea; we hear loud and frequent borborygmi, and the metallic sounds called "spasmic bruits" are more marked than in other colics. These abnormal sounds, similar to those made by a drop of water falling upon a very thin metallic plate, are due to the unequal tension of the gases in the different parts of the intestine, the establishment of equilibrium being performed in an almost explosive manner.

Course. The course of these colics is ordinarily rapid and 
their termination favorable. As a rule they last but a few hours, sometimes only a quarter of an hour or half an hour. When lasting more than twelve hours some very serious complications may step in. Among these, volvulus is the most common.

Differential diagnosis. It is sometimes difficult to distinguish nervous colics from thrombo-embolic colics. Bollinger thinks it not always possible to establish the difference. However, the diagnosis can be based upon the history, the etiological data, and the appearance of colic after a cold.

Treatment. We must resort to anti-spasmodics. Generally we use morphine. This agent is a true specific for nervous colics, which oftentimes disappear shortly after its administration. Morphine not only puts a stop to the painful contractions of the intestine, but has also the advantage of preventing volvulus. Hydrochlorate of morphine is given in dosts of 0.3 to 0.5 gramme in subcutaneous injections; we generally use a 4:100 solution. Sometimes the colics cease almost immediately ; it is rarely necessary to resort to a second injection.

The other anæsthetics or narcotics (belladonna, hyoscyamus, aconite, ether, chloroform) have become useless since the very favorable results of morphine have been established.

The hot beverages prepared with chamomile, peppermint, valerian, and elder, possess a certain anti-spasmodic action, due to the essential oils contained in these plants, and which, like camphor and asafoetida, act through evaporation in the digestive canal. But all these drinks must give place to morphine, which produces much more rapid effects and which can be given without any danger. Eserine is absolutely counter-indicated.

Externally, we should make energetic frictions with essence of turpentine and spirits of camphor $(1: 10-20)$. The patients should be kept warm. Cold compresses and cold injections only increase the contractions; hot infusions, on the contrary, exercise a very salutary influence by their quieting and anti-spasmodic action. Rest is preferable to exercise.

\section{Colics from Overloading.}

Symptoms. We recognize colic from overloading of the stomach by the length of duration of the pain, by the frequency of the attacks, and the rapid and considerable aggravation. The respiration is laborious, the abdomen swollen; the animals take unusual 
and strange positions; they are often found in the dog's sitting attitude, undoubtedly because this position decreases the pressure exercised upon the diaphragm and the lungs by the distended stomach. We notice eructations, nauseas, regurgitations, and even vomitings; these are so many phenomena characteristic of overloading. The disease ends almost always by rupture of the stomach or diaphragm-accidents which are announced by the cessation of the pain and by general symptoms of collapse.

Diagnosis. The diagnosis is sometimes difficult. To a great extent we should be guided especially by the fact of the ingestion of a considerable quantity of food; for vomiting, the most important symptom of the overloading of the stomach, may be provoked by other pathological conditions of that organ and of the intestine. These considerations apply equally to the diaguosis of rupture of the stomach and of the diaphragm.

Prognosis. The prognosis is grave. The horse does not possess the faculty of vomiting, and the unusual movements performed by the patients may at any moment terminate in rupture of the stomach or diaphragm.

Treatment. The drastic purgatives are powerless against absolute overloading. Eserine causes contractions of the gastric walls; but in using it we may provoke rupture of this organ. The cleansing of the stomach by means of the sound has been studiously experimented with by Damman: the agitation of the animals is found to render this method impracticable. We are therefore limited to the use of morphine injections in order to counteract the accidents which may result from ungovernable movements, and also to facilitate the evacuation of the stomach contents through the pylorus; and, in addition, to cleansing the intestine with injections and infusions.

ADDENDA. Among the accidents liable to occur in the course of colics by overloading of the stomach, vomiting and rupture of that organ or of the diaphragm deserve particular mention.

1. Vomiting. This is due to the considerable distention of the gastric walls and to paralysis of the cardiac orifice. When it happens the symptoms observed are as follows :

The muscles of the abdomen and of the neck and shoulders are the seat of convulsive contractions, the lowered head is kept near the chest; at the same time there is a discharge by the nose and mouth of a yellowish-green spumous liquid, of chymous odor, and 
of the same consistence as the ingested fodder. Its quantity varies from a few spoonfuls to the contents of a stable bucket or more. The animals perspire abundantly, the members are gathered under the body and the eye is haggard and fixed. Soon after vomiting the patients are very weak; they tremble and stagger; some animals are affected by violent coughing-spells. Sometimes the phenomena do not go to the extreme of vomiting; we simply observe salivation, nausea, and regurgitation.

Formerly vomiting was considered a fatal symptom, ${ }^{1}$ but strict observation of the facts teaches that this is erroneous. In some cases, indeed, it has a clearly favorable action, in others it has no harmful consequence. If any reason exists for regarding it as a grave prognostic symptom, it is because considerable distention of the stomach is always liable to cause rupture of the walls of that organ. Vomiting is sometimes a symptom of other affections of the gastro-intestinal canal, such as ulcerations, constrictions of the intestine, or peritonitis and even lesions of the diaphragm.

[Vomiting is also a symptom of dilatation of the osophagus (jabot), and of spasmodic constriction of this organ due to the presence of a foreign body (choke), from which horses sometimes recover. Complete rupture of the stomach often takes place without symptoms of vomiting. But when true vomiting occurs-that is, ejection of the contents of the stomach-as a complication of colic from overloading, it is almost invariably an indication of rupture of the organ, and may be looked upon as a fatal prognostic symptom; exceptions to this rule are very rare.-W. L. z.]

2. Rupture of the stomach. This may be the result of an extreme distention taking place by gradual enlargement, or it may be the effect exerted by a sudden fall (chute) on the stomach filled to excess with alimentary matters; sometimes also it is due to spasmodic constrictions of the gastric walls, when the patients make efforts in order to vomit and the cardiac orifice remains closed. ${ }^{2}$

Concerning the relations existing between vomiting and rupture of the stomach, erroneous opinions have existed for a long time; it was thought that vomiting was not the cause, but indeed the consequence of the rupture. But it would seem illogical, certainly, to conceive that the alimentary matters would pass through the cardiac orifice, which is so narrow, while a wide opening of escape is actually formed for them toward the abdominal cavity. The

1 Kolb: Adam's Wochenschr., 1882.

2 Rabe: Hannov. Jahresber., 1875. 
rupture can only become a cause of vomiting when it does not bear upon the entire thickness of the gastric walls, if it be limited to one of the membranes-to the muscular coat, for instance, the serous being intact. There must, too, be paralysis of the cardiac opening at the same time. We will later on speak of rupture of the stomach when its walls are ulcerated, or in cases of colics of constipation.

In most cases it is characterized by a collapse which appears suddenly when the colics are most intense; the pain seems to have disappeared, the abdominal bruits become almost imperceptible, the pulse is small, filiform, much accelerated ; the extremities are chilled, the body is covered with a cold and profuse perspiration, the temperature falls below the normal and ascends abruptly, the animals are stupefied and trembling; sometimes they neigh, they take strange positions and gather their limbs under them; the eye is anxious and haggard; we observe dyspnoea; the walk is embar. rassed and staggering; often a thick mucus, of a yellow-greenish color, escapes from the mouth and nose (Gielen).

The symptoms of rupture of the intestine are almost the same as those of rupture of the stomach. Ordinarily it is preceded by eructations, regurgitations, or vomitings.

Some horses have been seen to perform their work for a few hours after having had the stomach torn and while suffering (Schmolke). Death often follows rapidly; it may be anticipated for days when the laceration is not great, or when an organ, for instance, the omentum, has lodged itself upon and occludes the rent in the stomach-wall, thus preventing escape of the alimentary matters through its lips; such cases as the last mentioned, however, are very rare.

3. Rupture of the diaphragm. The cause of this accident is recognized as being the pressure exercised upon the diaphragmatic wall by the much-distended stomach, a pressure which is aggravated in its action by falls and violent movements on the part of the animal. Chronic inflammation of the diaphragm and the various alterations of this organ give a predisposition to rupture. We may find engaged in the diaphragmatic opening: the stomach, the small intestine, and the colon or omentum. When the condition is an old one these organs are united to the walls of the pectoral cavity; often the hernial portion of the intestine becomes strangulated.

The symptoms are always alarming. We observe an intense 
dyspnoa which may lead to asphyxia. The animals have attacks of violent colic, they neigh from pain and take abnormal positions, especially that similar to the sitting position of the dog. They sweat profusely, the respiration is snoring, and at times we hear a slight abortive cough. When a portion of the intestine has penetrated into the pectoral cavity, percussion of the thorax often gives a very clear tympanic sound, and on auscultation we perceive borborygmi replacing the respiratory murmur, which has disappeared up to a certain height. These latter symptoms are sufficient to confirm the diagnosis; they are wanting when the laceration has not been followed by a hernia, or when it is not voluminous, or, again, when it occupies the floor of the chest.

The course of the phenomena provoked by the tearing of the diaphragm is ordinarily very rapid. Some animals apparently recover after having been affected by remittent colics for several weeks; in others the colics reappear interruptedly and at intervals more or less close; in others, again, they are produced by the slightest efforts, especially by the descent of a hill; and some no longer daring to lie down. Within a variable time dyspnœa becomes persistent and the patients are overcome by marasmus.

\section{Thrombo-embolic Colics.}

History. For more than half a century veterinarians considered verminous aneurisms of the greater mesenteric artery as a determining cause of colics. Rigot (1829), and Schutt (1835) noticed these lesions in horses that had died from colics; Bruckmüller (1852) very clearly proves verminous aneurism to be the cause of numerous colics; Prehr (1855) relates a case of obliteration of the first mesenteric artery; H. Bouley (1856) considered the bloody excrements and intermittent colics as symptoms indicating arterial obliteration. Kohne (1861), in a case observed by him, thought that obstruction of the greater mesenteric artery induced as a consequence paresis of the large intestine, and especially of the cæcum; Hahn (1862) ascribes to aneurism of the greater mesenteric some intermittent colics detected in a horse; Cornevin (1869) describes a good case of colic provoked by the obliteration of the arteries of the intestine and the cæcum.

This brief historical sketch shows that the relation of cause and effect existing between aneurism of the mesenteric trunk and cer- 
tain colics was observed and affirmed before Bollinger's day. ${ }^{1}$ But we cannot deny to the Munich professor the credit of having established the frequency of intestinal thrombi and having increased our knowledge concerning those affections. ${ }^{2}$

Pathological anAtomy of Verminous ANEURism of THE GREAT MESENTERIC ARTERY. The researches of Hering (1830) demonstrate the frequency of this aneurism in the horse; horses which are not affected, says this author, are rarer than those having several such aneurisms. The statistics of Bruckmüller, Röll, Bollinger, etc., establish that 90 to 94 per cent. of the subjects of the equine species are affected by this disease, which increases in frequency as the animals increase in age. In post-mortem examinations made upon 85 anatomical subjects, Ellenberger found 84 with verminous aneurism.

Aneurism may be found at the age of six months; but in the newly-born and during the first months of life it does not exist.

Aneurisms are more common upon the anterior mesenteric arteries than upon the posterior; it is exceptional to detect any upon the posterior aorta.

They form dilatations which are ordinarily regular, oval, fusiform or pyriform, at times irregular, and may attain the size of a man's head and a length of thirty centimetres or more. The walls of the vessel are thickened, especially the medium layer and the peritoneal coating; the internal layer presents the greatest variety of inflammatory and degenerative alterations ; tumefaction, fatty degeneration, sclerosis, calcification, ossification, loss of substance, etc. The contents are represented by a stratified thrombus, abundantly supplied with vessels and adhering to the wall; it may completely obstruct the lumen of the aneurism and advance more or less into the arterial divisions, even into the aorta ; at times it is very soft,

1 Bollinger: Die Kolik der Pferde und das Wurmaneurysma der Eingeweiderarterien, 1870.

2 The veterinarians and French authors who have written on colics during this century have described, under the name of red colics, cutting colics, intestinal apoplexy, enterorrhagia, and subacute enteritis, various colics which set in suddenly without prodromes, are charatcerized by violent intestinal pains, and present at autopsy intense hyperemia of a more or less extended intestinal tract. Very free bleedings, revulsive frictions, sedative drenches (opium, camphor, asafœida), and walking exercise are the measures generally preferred for combating such conditions. These colics have had many and very dissimilar causes assigned to them. Their pathology is yet very imperfectly understood; but, in many cases, they are certainly provoked by embolic or thrombotic occlusions of the vessels.-N. D. T. 
friable, and puriform. In the centre of this clot we find the Strongylus armata (the average number of parasites is nine, according to Bollinger), which is the specific agent in these grave alterations; chronic traumatic endarteritis causes them by producing in its turn thrombus and aneurism.

Verminous aneurism in its Relation to colics. Aneurism not only occasions nutritive troubles in the intestinal walls and favors the development of colic through its hindrance to the course of the blood, but the horse which is affected by it is constantly exposed to a triple danger: at times the thrombus ends by obstructing the opening of the great mesenteric artery, though this is rare; at other times it is prolonged more or less into one of the branches of this trunk; then, again, we see a part of the clot becoming detached from the principal mass, carried along by the blood, and arrested in an intestinal artery which it completely occludes. In all these cases, when the collateral circulation is not established in a sufficient degree to keep up the blood circulation in the anemic territory, we soon observe grave disorders, which have been studied and experimented upon by Virchow, Cohn, Panum, Cohnheim, Litten, and others. The arterial occlusion causes stagnation and reflex of the venous blood, which is rapidly accompanied by a sero-bloody transudation in the intestinal tube and in the substances of its walls (sero-hemorrhagic infarct). This infiltration immediately causes paralysis of the intestine, and this in turn leads to stagnation of the intestinal contents, which ferment and give off an abundance of gases. It may also cause volvulus and invagination, accidents due to the activity of movement of the healthy intestinal loops, which pass into the interior of the paralyzed portion, or envelop it. We have frequently observed rotation of the left portion of the large colon upon its axis - that is to say, of the free portion of this organ. Now, of the different intestinal compartments, it is here that emboli and thrombi are most frequently observed; we must therefore, it would seem, admit that arterial anemia leads to change of relations of the intestine by the disturbances which it occasions. However, we do not think that rotation of the colon can be produced simply by the violence of the movements acting in the neighboring intestinal divisions.

Among the other complications of paralysis of the intestine are to be mentioned ruptures of this organ, of the stomach and dia- 
phragm, and also enteritis. The anti-peristaltic movements originating in the intestinal portion anterior to the paralyzed segment sometimes fill the stomach to such an extent that it is almost bursting; the abundant production of gas in the intestine may produce tearing of the walls of this organ or of the diaphragm, through the enormous pressure exerted upon it.

The anatomical construction, especially of the anterior mesenteric plexus singularly favors the development of embolic colics. This artery issues from the posterior aorta in the shape of a large trunk from ten to twelve centimetres in length; immediately after its origin it gives off-first, the superior colic artery; secondly, posterior and exactly opposite to the preceding, from seventeen to twenty divisions to the small intestine. From this point the remainder of the vessel receives the name of ileo-creal-colic artery, and it is this which is ordinarily the seat of aneurism. The ileo-cæcalcolic artery is divided into four branches: the anterior, the largest; the inferior colic artery, which is the termination of the common trunk; then in succession are given off the superior cæcal artery, then the inferior, and lastly the ileo-cæcal.

As a general rule, the ileo-cæcal-colic artery is not completely obstructed by the aneurismal thrombus; its four terminal branches still distribute the full amount of blood to those tissues into which they ramify, so that no serious trouble is apparent. On the contrary, however, when the obstruction is complete, the cæcum receives absolutely no blood; this is not the case with the colon, because of the anastomosis which exists between the superior "left" and the inferior "right" colic arteries. The obliteration of one of these latter arteries cannot produce fatal thrombo-embolic colics; they are only both obliterated when the aneurism originates in the great mesenteric. Then we may observe emboli in the small intestine, the arteries of which do not ordinarily take part in the aneurism. The sudden obliteration of a single colic or cæcal artery may determine light and transient colics.

When these fragments become detached from the aneurismal thrombus they are carried generally into the inferior arterial branches, and especially into the right colic. The obliteration of a single cæcal artery is not fatal, because of the anastomosis which exists between the two cæcal arteries, and also because the cæcum is supplied by a small arterial branch coming from the inferior colic artery. 
The arteries of the small intestine anastomose near the viscera; an embolus in a single branch is never a fatal accident.

Fatal alterations. When at the autopsy of animals that have died from these colics we find emboli, thrombi, a rupture of the stomach or intestine, an enteritis or peritonitis, there can be no doubt as to the nature of the disease. But embolic colics may be observed which terminate in death after twelve, eighteen, or twentyfour hours, before these grave gastro-intestinal alterations have had time to develop; then the intestine is usually much distended with gas or obstructed. In this case death may have been produced by various causes :

1. Through strong compression exerted for some time upon the diaphragm, with cedema of the lungs and asphyxia. The symptoms of asphyxia are frequently observed.

2. Through an elevation of the arterial tension occasioning cerebral and pulmonary apoplexy. This increase of tension is due to the pressure exerted by the distended abdominal viscera upon the large vascular trunks.

3. Through carbonic acid intoxication. This takes place according to the laws of diffusion, as soon as the amount of the gas present in the blood is less in quantity than in the contents of the digestive organs.

4. Through the passage of septic products into the blood. They may be of a chemical nature (intoxication), or may consist of germ elements (infection). Semmer ${ }^{1}$ has often found the latter in gaseous colics. The possibility of blood-infection, when a portion of the small intestine is paralyzed or necrotic, is unquestionable. Proof of this is found in the alterations presented by the liver. This organ is a true filter where the septic products collect; immediately after death, cadaveric putrefaction not having yet set in, we find it already, in a very advanced condition of septic decomposition. The chemical poisons are not yet well understood so far as their nature is concerned; Bollinger states that it is principally due to the action of sulphuretted hydrogen (an assertion which is frequently disproved by the cadaveric rigidity) and butyric acid.

5. Through cardiac syncope, as a consequence of the violence of intestinal pains or of intoxication through phenol, which is produced in greater abundance by abnormal intestinal fermentation.

1 Semmer: Virchow's Archiv, 1877. 
Tereg ${ }^{1}$ asserts that the production of phenol is rather hindered than increased.

In most cases death happens through septic infection, through pulmonary odema, or through carbonic acid intoxication.

Symptoms and course. The ccurse of thrombo-embolic colic is far from being uniform. We may recognize the following modifications :

1. Acute course ending in recovery. The colies belonging to the first group come and go without any definite exterior cause; they have much similarity with colics of a rheumatic nature, for they are extremely painful. Their duration is short. They are probably due to partial emboli of the intestinal arteries (from a colic or cæcal artery). They disappear gradually as soon as the circulation is re-established through the anastomoses. As it is very difficult to make the differential diagnosis, these colics are often confounded with other affections of the intestine. Sometimes, however, the reaction of the urine may guide us: in acute embolic colics it is alkaline, in colics due to enteritis it is acid. They are not due to the action of cold; this fact enables us, in some instances, to distinguish it from nervous colics.

2. Acute course with fatal end. To this group belong all emboli producing changes in the relations and situation of the intestine. The colics thus provoked appear without appreciable external cause. The pains are sometimes insignificant, contrary to what we observe in most of these affections (Lustig). This fact is explained by the sudden paralysis of the anemic intestinal compartment. Usually the symptoms are similar to those of constipation colics produced by changed relations of the abdominal organs. They have nothing characteristic. Death occurs through tearing of the stomach or intestine or through septic infection.

3. Chronic course with a relatively favorable termination. Chronic embolic intestinal catarrh. Colies of this variety are produced through collateral hyperemia consecutive to arterial obliteration. This congestion may persist for quite a long time (as in blood-stasis of the portal system of the liver) and occasion chronic intestinal catarrh (embolic intestinal catarrh).

The course of the disease is ordinarily as follows: When the first attack is over, the temperature and the number of pulsations 
return to a normal figure, but the peristaltic movements are interfered with, defecation infrequent, the appetite is diminished, and the general condition is not not entirely satisfactory. After twentyfour or forty-eight hours the fever reappears, with or without being accompanied by slight colics. This condition may last for days and even for weeks (chronic colic); Friedberger has seen it persist for three weeks. In some cases, embolic intestinal catarrh ends in recovery, but it often leads to marasmus and cachexia, if death does not supervene through septic infection.

4. Chronic course with production of hemorrhagic thrombo-embolic enteritis (intestinal hemorrhagic infarct). The pathology of this hemorrhagic enteritis is very simple; the embolic obstruction of the small intestinal arteries causes, in circumscribed regions of the mucous membrane, those disturbances of circulation previously described -arterial anemia, blood-stasis, hemorrhagic infarct, and necrosis.

The principal symptoms are the lessening of the appetite or complete inappetence, increase of thirst, scarcity of defecation in spite of the persistence of the peristaltic movements; the balls of dung, which are small and dry at the start, become soft, doughy, and later bloody and fetid; the urine is acid, rich in phosphates and in albumin ; the fever is intense and persistent, $40^{\circ} \mathrm{C}$. or higher, pulsations from 60 to 100 , the pulse being small; the general weakness increases; the animal loses flesh, the abdomen is contracted; at certain moments we detect coma and stupefaction. After meals we observe an aggravation of the general condition and of the colic. In some cases we observe remarkable febrile paroxysms (muscular trembling, chills, coldness of the extremities, paleness of the mucous membranes, and accelerated respiration, which is painful and rattling; there are also tumultuous beatings of the heart, and considerable increase of the rectal temperature.

The duration of hemorrhagic enteritis varies from a few days to several weeks. Its most common termination is death, which may occur from septic infection, or through peritonitis consecutive to perforation of the intestine, or by exhaustion. Recovery, which is extremely rare, may, however, be obtained after elimination of the sloughs, through cicatrization of the lesion. There are cases where transformation into ulcers takes place. The convalescence is always of long duration.

Autopsy reveals characteristic alterations. The mucous membrane of the stomach is swollen, injected, ecchymosed, and dotted 
with small blood-clots, which are dark and attached to the thrombi of the vessels of this membrane and to the submucous connective tissue. The small intestine contains a bloody liquid; its wall is stained with the coloring principles of the blood, which give it a dark-red shade. The same alterations are found in the large intestine, the walls of which show a marked thickening-three or four times greater than normal. They consist essentially in a serohemorrhagic infiltration, particularly marked on the level of the mesenteric insertion. Where the hemorrhagic infarction is thickest the mucous membrane is necrotic and forms merely a crust of the consistence of rotten wood, around which the serous infiltration is often enormous. At the base of this crust we ordinarily find a thrombosed intestinal artery, and in dissecting this toward its origin we come to a verminous aneurism. Gelatinous infiltrations are met with at numerous places in the abdominal cavity (especially at the points of attachment of the mesentery), and the peritoneal sac contains a considerable quantity of sero-sanguineous liquid. Often we observe all the lesions of septic infection.

Treatment. The predominating symptoms must be fought. We must try to prevent those complications to which intestinal paralysis predisposes, particularly coprostasis, which of itself may provoke a local phlegmasia and intestinal gangrene. The evacuants are therefore useful; tartar emetic, calomel, and alkaline laxatives are particularly favorable. Eserine must not be used; the abrupt and violent contractions that it might provoke in those portions of the intestines remaining sound may occasion a volvulus or an invagination. Frictions and walking exercise are to be recommended. The rectum should be emptied frequently with the hand or by means of water injections.

The treatment of hemorrhagic thrombo-embolic gastro-enteritis is confounded with that of common gastro-enteritis. A good diet is of the greatest importance for the patients in convalescence. In necrosis of the mucous membrane we should give calomel. In order to combat septic fever we must resort to the various antipyretics. Violent drastics (emetics, aloes, and croton oil) must be strictly excluded as agents in treatment.

Bollinger's theory upon the close relations of verminous aneurism with the colics, which has just been mentioned, has numerous supporters, also many enemies. Gerlach ${ }^{1}$ denies absolutely all relation

1 Gerlach : Gerichtl. Thierheilkde., 1872. 
between aneurism and colics. Anacker ${ }^{1}$ and $Z_{\ddot{3} r n^{2}}$ admit the possibility of thrombotic or embolic colics, but they disagree with Bollinger in reference to their frequency. Röll, ${ }^{3}$ on the contrary, would like to extend this pathogeny of colic to all cases where abdominal pains appear suddenly and without precise apparent cause, making, however, certain restrictions, and in this he is supported by the Munich professor. In our opinion the existence of thromboembolic colic cannot be the subject of doubt; we even believe these accidents to be quite frequent, but we think that Bollinger has gone too far in incriminating the thrombi of intestinal arteries as the cause of half the fatal cases of colic, and in considering as being of a thrombo-embolic nature any colic appearing suddenly and without appreciable cause.

\section{Colics through Intestinal Occlusion.}

Under this title we classify colics due to any kind of obstacle hindering the circulation of intestinal matters. Looking at the causes producing them, we can admit the following varieties :

1. Colics due to the accumulation and hardening of alimentary or excrementitious matter in the cæcum, the colon, and the rectum (coprostasis);

2. Colics provoked by gastric or intestinal calculi;

3. Colics determined by changes of relation of the intestine;

4. Colics of invagination ;

5. Colics produced by neoformations which diminish the calibre of the intestine;

6. Colics due to constriction of the intestine;

7. Colics provoked by the dilatation or paralysis of the intestine.

1. COLICS DUE TO THE ACCUMULATION AND HARDENING OF ALIMENTARY OR EXCREMENTITIOUS MATTER IN THE CACUM, THE COLON, AND THE RECTUM (COPROSTASIS): COLICS OF CONSTIPATION.

Etiology. The causes of these colics have been mentioned in the article on General Colics. We must mainly look for them in the diet-for instance, in cases of ingestion of a large quantity of

1 Anacker: Specielle Pathol. u. Therap.

2 Zürn: Thierarzt, 1871.

3 Röll : Handbuch der Pathol. u. Therapie. 
hay cut too short (straw colics), also from considerable quantities of wooden stems, of rye straw, from hard and ligneous clover, from bearded wheat husks or from buckwheat, from bean stems, and in general from food rich in cellulose, a substance which the horse digests with difficulty. We also blame those alimentary matters which are not stimulating enough; again, the abrupt change from a green to a dry diet, or the reverse. On the other hand, insufficient exercise when the animals are receiving abundant nourishment favors coprostasis. Foals during the first days following their birth frequently have colics due to accumulation of fotal meconium in the intestine.

Symptoms. The symptoms of these colics are usually not well marked. The pains are moderate; the animals remain down ordinarily; at certain times they complain; defecation is rare, and the fecal masses are hard and dry, sometimes coated. In a rectal exploration, during which the patients make violent expulsive efforts, the intestine is found filled with hard stercoral matters. The pressure exerted by the latter upon the bladder provokes efforts of micturition; the animals frequently stretch themselves to urinate. The abdomen is more or less inflated; the intestinal reservoirs are distended by gases. Vomiting is rare. In many horses the constipation may persist for several days without producing any symptoms of colic (the same as in the dog), but after it has lasted for some time, abdominal pains supervene.

Pathological anatomy. The alterations consist in modifications of the contents of the stomach and of its walls. From the start the alimentary matters dry up on account of the continual resorption of the fluids, and then play the part of irritating foreign bodies. The mucous membrane is soon invaded by a catarrhal inflammation accompanied by numerous ecchymoses (hemorrhagic catarrh); later, the inflammatory process may assume the character of a "diphtheria" or necrosis. According to Ernest, diphtheritic inflammation of the mucous membrane starts with dots or spots of a grayish, yellowish-gray, or bluish color. Small bodies of a clear shade appear upon the inflamed mucous membrane; they are particularly noticed on special points of this membrane (papillary and follicular diphtheria) the surface of which is covered with exfoliated cells. These lesions are due to necrosis of the superficial layer of the mucous membrane; they are, as a rule, followed by small ulceratious which form a more or less rapid cicatrix ; in some cases 
they are complicated by progressive necrotic destruction of the muscular wall and end in perforation of the intestine.

Among the accidents of intestinal obstruction must be pointed out rupture of the stomach and intestine. The arrest of fecal matter in the cæcum is followed by fatal lacerations of this organ, and when this takes place treatment is entirely useless. Ruptures of the colon and rectum are relatively frequent. Obstruction of the terminal portion of the small intestine is followed in half the cases by tearing of the stomach; the anti-peristaltic movements cause accumulation of the intestinal contents in the stomach, producing its rupture, except when vomiting relieves the overdistended condition of the organ. The neighborhood of the ileo-cæcal valve is the most frequent seat of intestinal obstruction.

Duration. In general, the duration of these colics is quite long; it varies from a few days to two, three, six, and seven weeks (chronic colics); the remissions, properly so called, that are observed in thrombo-embolic colics are missing in this form of colic. When rupture of the stomach occurs, it induces the whole symptomatic ensemble which has been described in the article on Rupture of the Stomach.

Treatment. Evacuants, laxatives, eserine, clysters, and tobaccosmoke; massage of the abdomen, cold compresses, rectal injection of large quantities of cold water, moderate exercise, and a rigid diet-such are the means to be employed against colics due to intestinal obstruction.

\section{COLICS PROVOKED BY CALCULI OR BY FOREIGN BODIES RE- MAINING IN THE INTESTINAL CANAL.}

Nature. The bibliography of this variety of colic is relatively rich. This is explained if we consider that any calculous affection detected at the autopsy strikes the observer, and decides him to record it. Nevertheless, calculous colics are in reality relatively rare. They are usually observed in millers' or bakers' horses, which consume a large quantity of bran and meal. Gurlt states that bran contains 1 to 2.5 per cent. of phosphate of magnesia, which constitutes the principal element of calculi. Dusts coming from the millstone, and which are mixed with the bran, have only a secondary influence in the formation of calculi.

The "sand colics" happening after ingestion of sand and gravel, when the animals drink in small rivulets, or when they are fed on 
muddy food; also, that observed in famished army horses, and in animals addicted to the habit of eating earth, are classified in the same group.

Deglutition of hair during shedding may cause balls and concretions (ægagropilæ), which produce disorders similar to those occasioned by calculi., Other foreign bodies swallowed (pieces of cloth, needles, etc.) produce these accidents more rarely.

Intestinal calculi, properly speaking, consist principally in a deposit of ammonio-magnesium phosphate (90 per cent., as stated by Fürstenberg), to which are added lime phosphates, phosphates of magnesia and silica, the different chlorides, some traces of iron, and organic matters (mucus, epithelium, alimentary substances). They are formed at the expense of the phosphate of magnesia, with which the bran is particularly loaded, and which is added to the ammoniacal compounds of the intestinal contents. These originate from the atmosphere of the stables, and are introduced into the digestive canal by the intermediary of the drinking-water. A phosphate of insoluble base is thus formed in the stomach. The starting-point of the precipitation is generally a foreign body, a particle of sand or oats, around which the basic phosphate deposits are continually accumulating.

Gastric calculi are intestinal calculi brought back into the stomach by the anti-peristaltic movements. They could not be formed in the stomach itself, on account of the acid reaction of its contents, and because of the short time the alimentary matters remain there.

Calculi are ordinarily formed in the large intestine, especially in the larger curve of the colon, more rarely in the cæcum. In 900 autopsies, Colin found calculi 23 times in the large curvature of the colon, and only once in the cæcum.

Local alterations produced by the various intestinal concretions are the same as those which complicate the obstruction through alimentary stagnation.

The precise diagnosis is to be drawn from the process of evacuation of these products, or from their direct detection through rectal exploration. In horses consuming large quantities of bran for a long period, and which suffer frequently from colic (millers' horses), we must always consider the probable existence of calculi, notwithstanding the frequency of colic from overloading of the stomach in these animals. 
Symptoms. The symptoms of calculous colic are similar to those of intestinal obstruction from alimentary stagnation. Calculi are found in animals which do not experience any trouble that would indicate their presence, these concretions remaining for quite a long period, until one of them grows into the intestine itself. At other times the calculi produce frequent colics of a periodic character. During an attack we may observe intermissions or abatements corresponding with the various stages of a calculus, or with the passage of several calculi together into a narrow portion of the intestine. These symptoms are ordinarily more intense than in colies from coprostasis. More frequently in the latter than in the former type of colic we see rupture of the stomach or intestine, and vomiting. The colics of calculi are also distinguished from those due to accumulation of alimentary matters in one of the intestinal reservoirs by their more rapid course-although their duration may last quite a long time under certain circumstancesand also by their more serious prognosis.

Treatment. This is the same as that of coprostasis. In desperate cases laparotomy may be attempted. Félizet ${ }^{1}$ has practised it with success. By incising the flank and the intestine he was able to extract a calculus of the size of a child's head. Trasbot has proposed to distend the intestine, and to facilitate the progress of the calculus, by provoking an abundant escape of carbonic acid gas by means of a mixture of bicarbonate of soda and tartaric acid administered per rectum.

\section{COLICS DUE TO CHANGES OF RELATION OF THE INTESTINE:} VOLVULUS.

Changes of relation of the intestine generally consist of a volvulus (rotation of the organ upon its axis), and are most frequently observed upon the colon and small intestine. The changes of relation of the cæcum are rather inflections and elbow formations, created by the displacement of the point backward and downward, or of the base upward and forward. Volvulus of the rectum is very rare. The knots are formed when the loops of the small intestine become entwined with other parts of the same viscera, or of the colon or rectum. The omentum may also surround and smother the small intestine. Hernias observed at the natural or accidental

1 Félizet: Revue Vét., 1877. 
openings of the peritoneal cavity, inguinal ring (even in geldings), Winslow's hiatus, old lacerations of the diaphragm, ruptures of the omentum and of the mesentery, openings existing between old adhesions, or between the detached ureter and the upper wall of the abdominal cavity, etc. Röll has found 51 cases of internal hernia in 140 horses that had died of colic-a proportion of 36 per cent.

Etiology. The causes of these anomalies are most obscure. There are some which are related to the thrombo-embolic processes described previously; others are produced by the unequal weight or the volume of the solid, liquid, or gaseous contents of the various portions of the intestine; here the volvulus operates according to the laws of gravity, the heaviest intestinal loops.taking a depending position in the abdominal cavity. The frequent and forcible peristaltic contractions which accompany certain colics may determine changes in the relations of the intestine.

Finally, rolling and all unusual and violent movements, which the horses are apt to perform, produce a certain influence in the generation of volvulus, knots, and hernias. Cold drinks have been erroneously given as a cause.

Symptoms. There is nothing very characteristic. At first they are most variable: sometimes the intestinal obstruction is revealed with the abruptness of a strangulated hernia, at other times, on the contrary, its symptoms develop slowly. Vomitings are observed in some instances which persist for several days; in other cases they are absent. The abnormal positions, such as the dorsal decubitus, the intensity of sufferings, the symptoms of a rupture of the stomach or intestine, and the infrequency or impossibility of the act of defecation, are manifestations which have been specially recorded in the article upon Strangulations and Lacerations, but these are also seen in most colics of impaction. The duration of the affection varies according to the complete or incomplete intestinal obstruction; it may persist as long as a week. In complete occlusion death is generally produced within twenty-four hours. These colics are sometimes intermittent, periodical; they disappear suddenly, then reappear in the same way at variable intervals. These conditions are observed when an intestinal loop is incarcerated at certain moments and free again at others; the colics of short duration which are called frappeurs de nuit (night-strikers). belong perhaps to this group. ${ }^{1}$ 
Diagnosis. We can only formulate it when we have to deal with a strangulated inguinal hernia, or with a volvulus of the rectum; nevertheless, torsions of the colon and cæcum may sometimes be recognized. Failure of the remedies used, and especially of injections of physostigmine, help to clear up the diagnosis.

[A symptom which may be considered as almost diagnostic of this condition is the continual up-and-down swinging of the head and neck known as incensing; this, together with a peculiarly drawn countenance, should be given special significance from a diagnostic point of view.-W. L. z.]

Treatment. Treatment is rarely effective, except in strangulated, inguinal, umbilical, or abdominal hernias. These latter are reduced either by taxis or by a regular surgical operation. By manipulation through the rectum we may sometimes destroy the the connective-tissue adhesions producing the strangulation. Some success has been obtained by placing the animal in different positions more or less unusual; also by rolling or making them ascend or descend a steep hill, etc. Evacuants and mercury have been used, but almost always unsuccessfully. Laparotomy and reduction of the accident constitute a dernier ressort in cases in which the diagnosis is established with enough certainty.

\section{COLICS DUE TO INVAgINATION.}

Nature. Invagination consists in the penetration of a portion of the intestine into that which is immediately next to it, either above or below. Most frequently it is formed by the small intestine being invaginated into the cæcum, and this upon a considerable scale, or by the same organ being invaginated in itself, more rarely by the entire cæcum being engaged in the colon (Leblanc, Caussé, Hermkes, Colin, Cartwright, Bagge, Stohrer, V. Ow, etc. We meet with quite a number of cases where the point of the cæcum is engaged in the median portion of this bowel. The invagination seems to be caused by paralysis of the peripheral portion of the intestine, of the invaginating segment, or by violent peristaltic movements, which produce engagement of the central portion of the invaginated segment.

Symptoms. They are similar to those produced by the changed relations of these organs, but ordinarily they persist for a longer time, for in invagination the whole lumen of the intestine is not necessarily obstructed. In a case observed by $\mathrm{V}$. Ow, diarrhea 
occurred on the tenth day, corresponding with an apparent amelioration, while death only took place on the twenty-seventh day. Even invagination of the cæcum in the colon seems to be compatible with life. ${ }^{1}$ In very rare cases recovery may be produced through elimination of the invaginated portion and the joining of the two intestinal ends (Hartmann).

Diagnosis. This can only be established with certainty in cases where the cylindrical intestinal scab resulting from the invaginated segment is evacuated with the excrements.

Treatment. It should consist in the use of remedies calculated to obtain and maintain the liquefaction of the intestinal contents, in order to facilitate its progress through the contracted portion of the intestine. For this purpose we may give any kind of purgative, and also frequent and abundant simple or purgative clysters.

\section{COLICS PRODUCED BY NEOFORMATIONS WHICH DIMINISH THE INTESTINAL CALIBRE.}

Neoformations of the digestive canal are quite rare, and they give rise to colic only exceptionally. Their nature is extremely variable. Krieger observed a polypus of the stomach which obstructed and dilated the duodenum till it rendered its rupture imminent. In the small intestine fibroma has been formed (Magri), also myxosarcoma (Friedberger), myoma with smooth fibres, and the submucous lipoma. Sarcomatous or cancerous neoformations (Brusasco) have been met with mainly in the large intestine. The mesentery sometimes contains lipomata which may compress the intestine or cause volvulus. ${ }^{2}$

Ordinarily the intestinal contents are arrested and accumulate in front of the portion constricted by the neoformation; it then produces dilatation and compensatory thickening of the abdominal walls. The surface of the tumors developed in them is frequently ulcerated toward the intestinal canal.

Symptoms. Persistent constipation and intermittent pains, the intensity of which increases gradually, constitute the principal symptom of these colics, which may last for years, and end in a fatal intestinal obstruction.

1 G. Colin: Recueil Vét., 1850.

2 We must associate with these colics the same affection produced by abscess of the intestinal walls or by phlegmons developing in the connective tissue of the pelvis and compressing the rectum. Martin (of Brienne), Palat, Bringard, Lardet, Le Berre, and a few other practitioners have reported such instances.-N. D. T. 
Treatment. In the great majority of cases the treatment is purely palliative. We merely liquefy the intestinal contents through the administration of purgatives. When these neoformations are accessible to the hand introduced in the rectum (we have ourselves observed some of these cases), it is advisable to empty this bowel several times a day. If pedunculated, they may be removed either by torsion or by means of an écraseur. ${ }^{1}$

\section{COLICS DUE TO CONSTRICTION OF THE INTESTINE.}

Etiology. In our domestic animals the small intestine is most frequently the seat of constrictions. They are due to the consequent formation of cicatrices when there has been loss of tissue of the mucous membrane in cases of enteritis, helminthiasis, etc., or where there has been a hernia or invagination. The opening of the intestinal canal may be reduced to such an extent as hardly to permit the introduction of the finger. Immediately in front of the contraction we notice a more or less extended dilatation.

Symptoms. In general the symptoms are those of colics superinduced by intestinal obstruction. Sometimes death occurs rapidly, at other times-and this is the most common-the contraction provokes intermittent colics, appearing at irregular intervals. In spite of the retained appetite, the patients lose flesh and die in a condition of marasmus.

1 Professor Mauri has related a very interesting observation of a valvular constriction of the rectum cured through partial resection of the constricted portion. The subject of this observation, an Anglo-Norman horse, seven years old, was affected by intermittent colics for one month, when put under treatment at the Veterinary School of Toulouse. He had preserved his appetite, but at certain times he was downcast : he would stretch himself frequently, pass small quantities of urine, look at his flank, prepare himself for defecation, and make violent and repeated efforts which were unsuccessful; he would also scratch the ground, or lie down, seeming to be relieved by assuming the sterno-costal decubitus. Rectal exploration showed a very marked constriction at twelve centimetres from the ánus, in front of which there was a large reservoir formed by the distended rectum, which was filled with packed fecal masses, the extraction of which was difficult. This artificial evacuation having been performed, the rectum became normal, the animal showed a very manifest and immediate relief, and the colics stopped for several hours. But with reaccumulation of the excrements the symptoms returned. At the time the animal made these efforts, there could be seen at first a considerable dilatation of the anus; then, during the contraction of the abdominal muscles, the fecal mass became packed against the valve and carried it forcibly backward. Notwithstanding the greatest efforts, no excrements could be rejected. A definite cure was obtained through the resection of the valvula with the écraseur. Macroscopic and microscopic examination of this wall showed that it was formed of smooth muscular fibre covered with normal mucous membrane.-N. D. T. 
Treatment. We can only treat with any success those contractions of the rectum which are accessible to the hand. We must try to obtain enlargement of the bowel by means of a frequènt introduction of the arm. The contractions located upon other parts of the digestive canal, especially those of the small intestine, cannot be the subject of any useful intervention.

\section{COLICS DUE TO DILATATION AND PARESIS OF THE INTESTINE.}

Etiology. Dilatations of the intestine are ordinarily secondary, and produced by pre-existing contractions (neoformations, constrictions, coprostasis, calculi, invagination, etc.). We find these immediately in front of these obstacles. Dilatation is more rarely due to paresis of the intestinal wall, consecutive to its extreme distention, produced by gases or alimentary matters, or indirectly through embolic obstruction of the intestinal arteries. Circumscribed dilatations of the intestinal canal are generally the consequence of partial ruptures. We do not yet know anything definite about the relations which seem to exist between certain dilatations of the gastro-intestinal walls and chronic gastro-intestinal catarrh, or the nutritive troubles related thereto. At all exents, the walls of the dilated intestinal portion are much hypertrophied.

The dilatation of the stomach may be due to pyloric contraction, but also to a contraction located more or less upon the intestine; it is produced passively through stagnation of the food.

Symptoms. The intestinal dilations and paresis which are the most important from a practical point of view are those located upon the rectum. They determine chronic, intermittent, and tenacious colics, which may end in intestinal rupture.

The rectum is filled with packed fecal matters, the extraction of which puts an end to the colic. There are cases where these matters produce a compression upon the pelvic portion of the urethra, the consequence of which is the retention of urine in the bladder, the dilatation of this organ and hypertrophy of its walls (Friedberger).

In considerable dilatation of the stomach the pressure exerted by that organ upon the diaphragm may produce grave dyspnoic symptoms during the course of the ailment. Leisering has described a case of dilatation of the stomach in which the great curve between the cardia and the pylorus measured 130 centimetres, and the small one 90 centimetres. 
Treatment. The treatment is as inefficient as that of colics through constriction of the intestine. In cases of dilatation and paralysis of the rectum the principal indication is to empty this bowel from time to time with the hand. A radical cure cannot.be obtained.

\section{Gaseous Colics: Flatulent Colic.}

Etiology. Gaseous colics are generally due to the ingestion of especially fermentable food, which subject has been treated in the article upon General Colics. They are also quite frequently observed in horses that have the habit of wind-sucking, or deglutition of air. According to Haubner and Siedamgrotzky, they are sometimes produced when the animals are driven at a sharp gait against the wind.

The gases which predominate in the bowels are carburetted hydrogen and carbonic acid. An analysis by Pinner ${ }^{1}$ gives them as in the proportion of 49 per cent. of carburetted hydrogen, 8 per cent. of carbonic acid, and 42 per cent. of nitrogen. He has also demonstrated the presence of sulphuretted and of phosphoretted hydrogen gas.

Symptoms. The rapid swelling of the belly constitutes the characteristic symptom of these colics. The abdominal walls are distended; percussion of the flank renders a very clear tympanic sound, sometimes metallic. In auscultation, we hear loud borborygmi. With the increase of swelling, dyspnœa occurs, also anxiety and restlessness; the mucous membranes are of a darkbluish or red shade, the jugulars swell up, the heart-beats become tumultuous, the walk is painful, and the animals stagger in their hind quarters. Cure is indicated by the expulsion of considerable quantities of intestinal gas, and by a lessening of the tympanites. Death may occur, either through excessive pulmonary compression or odema, or through carbonic intoxication, through apoplexy, or, lastly, through rupture of the stomach or intestine.

Diagnosis. This is quite easy. The tympanites which is sometimes observed during the course of other colics (thrombo-embolic, or from occlusion, etc.) is distinguished from that of gaseous colics by its slow appearance, its progressive development, and its long duration.

Treatment. Puncture of the cæcum, to be made at the right

1 Pinner: Archiv f. Thierheilkde., Bd. i. 
time, is the principal indication. The operation is practised in the right flank, in the centre of the triangle formed by the external angle of the ilium, the transverse processes of the lumbar vertebra, and the last rib. The skin should be shaved and disinfected; we next make a narrow buttonhole by means of the lancet or the fleam, and the puncture is then made by directing the point of the trocar toward the left elbow. After evacuation of the gases, the cutaneous wound is closed with collodion or a piece of court-plaster. It may happen that the left flank is more prominent than the right; the puncture may then be performed on the left side.

The puncture through the rectum, recommended long ago by Chabert, Aubry, etc., and quite recently by Föringer, must be rejected, even if the swelling seems localized to those viscera which it is impossible to reach through the cutaneous puncture. In the first place, the operation through the rectum cannot be made under antiseptic precautions; besides, we run the risk of a colo-rectal fistula.

In cases of tympanites of medium intensity, sulphate of eserine administered by subcutaneous injections ( 0.1 gramme in 5 to 10 grammes of water) has given excellent results; but when the viscera are much distended, this agent is dangerous; it may, by its violent action, produce an intestinal rupture.

Ether clysters (15 to 20 grammes of ether in 1 litre of water) pretty commonly used in France, are also useful in the treatment of flatulent and spasmodic colics (Cagny, Heu, Zorn).

It is always of advantage to resort to cold injections, also to cold compresses, massage of the abdomen, etc., in order to induce the evacuation of gas, but it will be wise not to lose too much time with these remedies. Other agents recognized by different authors are of little value. Absorbents are not to be advised; some of them, like ammonia and liver of sulphur, for instance, increase the quantity of gas by adding ammonia and sulphuric acid; others, such as lime-water, do not retain their alkaline condition any farther than the stomach.

\section{Verminous Colics.}

Etiology. We must particularly mention the following intestinal parasites which produce colics : Ascaris megalocephala; Tcenia plicata, T.mammillana, T. perfoliata ; Spiroptera megastoma; Sclerostoma tetracantha; Gastrophilus equi, and Gastrophilus hoemor- 
rhoidalis. The action of these parasites is very variable; they obstruct either the opening of the intestine by forming balls or knots (round-worms and tænia), or they determine traumatic accidents, inflammation, ulceration, and even perforation of the gastric or intestinal walls (gastrophilus larvæ). However, these latter lesions are generally limited to the mucous membrane.

Symptoms. Verminous colics are especially common in young animals. Their symptoms offer nothing very particular. Usually they are not well marked, and appear in paroxysms. In irritable mares the hemorrhoidal gastrophile fixed upon the rectal mucous membrane may determine violent pains, accompanied by rabiform manifestations (Eletti). These colics generally take a chronic course; we observe conditions of simple digestive troubles and of more or less intense suffering. But if the stomach is perforated, or if the intestinal obstruction is complete, the colics become subacute and the patients die rapidly.

In general, the prognosis of verminous colies is not very serious. They provoke death only in exceptional cases, and their cure is easily obtained as soon as their nature is revealed.

Treatment consists in the administration of vermifuges. Tartar emetic in a solution of water, in doses of 12 to 15 grammes daily, arsenous acid ( 2 to 4 grammes), essence of turpentine (80 to 150 grammes), with bitters, and the sulphate of soda, are the most advisable agents. The gastrophile larvæ which are located in the rectum may be detached by hand or checked by means of injections of soap-water, or a mild solution of petroleum, benzine, or creosote.

\section{COLICS OF THE OX.}

\section{COLICS DUE TO INVAGINATION.}

Etiology and symptoms. Intestinal invagination is the most frequent cause of colics in the ox. It is much more common in this animal than in the horse. It is ascribed to colds and to violent efforts on ground which has an irregular surface, which causes disturbance of the peristaltic movements.

Invagination is sometimes latent during some hours following its production; the complications which arise may develop slowly; but cases of this kind are rare. Ordinarily its symptoms are quite expressive; the animals are suddenly affected by more or less 
violent colics, without any previous indigestion. These attacks persist from six to twelve hours, then disappear abruptly and entirely, as if cured. But the depression and broken-down condition, as well as inappetence, cessation of rumination, diarrhea, or the expulsion of small quantities of dry fecal matter, indicate the persistence of the trouble. The constipation becomes obstinate and does not yield to any purgative. Under the action of violent expulsive efforts on the part of the animal, thick mucus, often mixed with dark blood, escapes through the anus. The peristaltic movements are completely suppressed, and the abdomen becomes gradually distended by the accumulation in the intestine of gases which are formed there in abundance. On palpation we may detect an intense sensitiveness of certain regions. The pulse is small and accelerated, but the temperature is hardly increased; sometimes it is even lower than normal. The body is cold, the patients are apathetic and in a comatose condition. We can recognize the invagination by rectal exploration; the hand perceives a cylindrical enlargement, more or less extended, along the course of the intestine.

Course. This is generally slow, and death is, with very rare exceptions, the inevitable termination of the affection; it takes place in a calm manner, without struggles; ordinarily from the sixth to the ninth day, rarely before the fourth or after the fifteenth.

Recovery is exceptional ; it may be produced by the elimination of the invaginated segment and the peritoneal union of the ends. In a few cases we have observed the expulsion of a cylindrical slough through the anus.

Diagnosis. Sudden colics followed by an apparent calm, persistent constipation, the expulsion of bloody mucus from the anus, arrest of intestinal bruits, sensibility of the abdomen, especially marked upon the right side, the existence upon the intestine of an elongated tumefäction, also the duration of the disease and its progressive aggravation, are facts sufficiently helping to establish the diagnosis. Let us point out that the left kidney holds a relatively low position in the abdominal cavity; when making rectal exploration, we must avoid taking it for an abnormal production.

Treatment. The treatment must be exclusively surgical. Purgatives, exercise upon irregular ground, etc., are very unreliable means. In order to find the lesion, we must make a laparotomy 
in the right flank. Invagination, which is often difficult to discover in pregnant animals, may be reduced by taxis (Degive). When attempts to loosen the intestine are unsuccessful we must make a resection, and then join the ends, serous to serous, by means of the intestinal suture (Meyer, Taccoen).

\section{COLICS DUE TO INTESTINAL STRANGULATION.}

Etiology. In the ox, intestinal strangulation may take place through a perforation in the omentum, mesentery, diaphragm, the broad ligament, etc., but it is especially found as a complication of pelvic hernia, which has for its cause the tearing of the testicular cords during castration. In fact, rupture of the testicular cords may be the cause of strangulation acting through a variable mechanism, as follows: In some instances the traction upon the cords results in a tearing of the peritoneal fold which is located at the entrance of the pelvis-an intestinal loop becomes engaged in the opening thus produced and is strangulated there; in other instances, the end of the testicular cord, which has been torn, enters the abdominal cavity and entangles a portion of the intestine, which it crowds more or less closely; lastly, the end of the testicular cord becomes joined to the abdominal wall, and in this case the intestine may also be strangulated in the funicular-peritoneal cleft thus formed.

Symptoms. The symptoms and duration of the disease are nearly the same as in invagination. Upon the anterior border of the pelvis is formed a soft, painful tumor of the size of an apple or the fist; this, together with the extended condition of the testicular cord, constitutes very precise diagnostic information.

Treatment. This is exclusively surgical. In some cases the hernia may be reduced by rectal taxis. When this latter method is insufficient, the cause of strangulation must be removed by rupturing the cord by the hand introduced into the rectum or through an incision in the flank.

\section{OTHER COLICS OF THE OX.}

We may also observe in the ox colics produced by overloading of the stomach, by cold, by intestinal obstruction (tumors, fecal matters, ægagropilæ), or by ingestion of the placenta in females just delivered. But, ordinarily, these colics are not so serious as those 
caused by invagination or intestinal strangulation. Volvulus is: very rare in oxen.

According to the nature and degree of intensity of the abdominal pains, the animals are restless, stamp, strike the belly with the hind legs, sway the tail, look at the flank, etc., or they become agitated, roll, and assume the position of dorsal decubitus, indicating their sufferings by plaintive moans.

Treatment. The treatment consists, as in the horse, in the use of eserine (0.1 gramme) laxatives (sulphate of soda, 500 to 1000 grammes; aloes, 45 to 60 grammes ; croton oil, 15 to 25 drops), and also injections. It will be of advantage to make dry frictions upon the abdominal cavity.

\section{COLICS OF THE DOG.}

The dog is quite frequently affected by abdominal pains which are entitled to be called colics. They may be caused by colds ; also through the accumulation of hardened alimentary matters in some parts of the intestine, as, for instance, bones, pebbles, or any kind of foreign bodies; through intestinal worms; through invagination, etc. The Toenia echinococcus quite frequently provokes colics, and Röll has observed rabiform symptoms during their course.

Dogs attacked by colic are agitated, run, lie down, gather themselves in a ball, complain, etc. These symptoms are often but little marked, and they very rarely continue for any length of time.

The treatment should vary with the cause of colic. In order to relieve the pains we may use Dover's powder (0.5 to 2 grammes); also tincture of opium (20 to $40 \mathrm{drops}$ ) in a mucilaginous mixture. We must give injections and apply hydrotherapic compresses on the stomach. If invagination is detected we must make a laparotomy in order to reduce it. ${ }^{2}$

${ }^{1}$ In the dog the pains produced by muscular rheumatism are often taken for colic. -N. D. A.

2 Biot has related an interesting observation of an invagination which was treated with success by laparotomy. The subject was a spaniel seven or eight months old. He was affected by colic for eight days, was constantly moving about, lying down and standing up again, without appearing to be at all relieved; the posture of sternal decubitus was the one he preferred; he would assume it by letting himself fall abruptly upon the sternum and abdomen, the posterior members stretched under the abdomen and the anterior extended in front of him. Symptoms of icterus, with bilious vomiting, appeared on the eighth day; on palpation of the abdomen the author distinctly 


\section{COLICS OF THE PIG.}

Etiology and symptoms. Colics of the pig are ordinarily due to overloading through heavy food of bad quality; also through bran, fermentable food, such as malt, etc. Colics may also be produced through colds, intestinal worms-especially through the Ascaris lumbricoides and the giant Echinorrhynchus. Phlegmasia of the stomach and intestine, and the invaginations, which are quite frequent in the pig, are also accompanied by colic.

The animals are restless and agitated; they refuse their food, lie down, complain, and cry with the pain. They often stretch themselves upon the ground, and are affected with convulsions and tetanic attacks.

Treatment. We must administer purgatives and quieting electuaries, give injections, and make repeated dry frictions upon the abdomen. The patients will have to be placed in a warm place covered with an abundant litter.

\section{ULCERS OF THE STOMACH AND INTESTINE.}

In our domestic animals ulcers of the stomach and intestine rarely constitute any essential lesions independent of other acute affections. They are most frequently developed during the course of certain diseases (hydrophobia, bovine pest, petechial fever, dysentery, gastro-enteritis, and gastro-intestinal catarrh); in some cases they are caused by foreign bodies or by parasites (gastrophile larvæ, spiroptera, etc.); finally, they are observed in poisoning by irritating or caustic substances. Considered from their pathological

felt a lengthy tumor, measuring from ten to twelve centimetres in length, which was movable in all directions, and of such sensibility to exploration, even when practised delicately, as to evoke cries of pain. Biot diagnosticated invagination, and decided to operate immediately. He performed laparotomy upon the left side, and with the right index finger brought out an invagination of the small intestine, the circumference of which was seventeen centimetres. The reduction could not be obtained by traction upon the invaginated part; there were some adhesions which had to be severed with the bistoury. There was but a slight hemorrhage, which soon stopped. The intestine was put back into the abdomen, and the lips of the flank wound brought together with a cross stitch, which was covered up with a slight emplastic coating of melted pitch. From the third day the wound was in an excellent condition for cicatrization, and the recovery was complete within a couple of weeks (Recueil Vét., 1879. -N. D. T. 
point of view and from the troubles they provoke, we must recognize the following two varieties:

1. Ulcers of inflammatory origin (catarrhal ulcerations and hemorrhagic erosions).

2. "Peptic" ulcers of the stomach or duodenum, which are round or perforating (peptische Geschwüre).

Etiology and pathology. 1. UlCERS OF INFLAMMATORY ORIGIN are developed when the phlegmatic infiltration is intense, or when it is accompanied by hemorrhages in the substance of the mucous membrane. As the inflammatory process weakens ti:? ulcers appear. In the stomach they may be found at various points, while in the intestine they are located in preference upon the lymph follicles (follicular ulcers). Whilst the principal condition of their development is the existence of acute catarrh of the mucous membrane they may nevertheless occur after wounds or partial necrosis determined by insignificant causes.

2. The "PePtic" UlCer (ulcus rotundum) is only seen upon the surfaces bathed by the acid gastric juice-that is to say, in the stomach and duodenum. Its genesis is quite different from that of inflammatory ulcers : it is developed by a true gastric auto-digestion. The causes of this circumscribed gastro-malacia produce local circulatory troubles, which prevent the alkaline blood from neutralizing the acid gastric juice, and the mucous membrane is then exposed to the destructive influence of this fluid. The following are among the most important causes: thrombi and emboli of the arterioles of the mucous membrane; the atheromatous, fatty, fibrous, and amyloid degenerations of the muscular walls, and also the vascular spasm. In some cases the peptic ulcer is the consequence of an exaggerated acidity of the gastric juice. If left to its action, the slightest solutions of continuity of the mucous membrane are transformed into ulcers, while in ordinary conditions they form a cicatrix very rapidly. The general anemia and the venous hyperemia of the gastric mucous membrane act in the same manner; the weakened membrane, insufficiently irrigated by the arterial alkaline blood, cannot resist the contact of the acid gastric juice. ${ }^{1}$ But in the generality of cases a complex process determines the formation of peptic ulcer.

1 This theory, according to which the gastric juice is considered the principal factor in the production of ulcer of the stomach, has lost many adherents since the reports of chronic ulcers of the œsophagus in man.-N. D. T. 
If these two varieties of stomachal and intestinal ulcerations sometimes give rise to particular symptoms which permit us to distinguish one from the other, yet it is by no means easy to do so, and the observations on record testify that the two kinds have frequently been confounded. Thus there is a neceesity for a more precise anatomical description of gastric ulcer than that hitherto outlined by different authors.

At the Berlin slaughter-house R. Ostertag found in the calf six cases of peptic ulcer of the abomasum; in several the stomach was perforated, and he could detect an intense catarrh of the mucous membrane in all. The macroscopic and microscopic examination indicated that none of these cases were of thrombotic origin. The form of the ulcers, however, showed clearly that the process had its starting-point in vascular troubles. Ostertag, while pointing out that the pathology of stomachal ulcers is as yet little known, estimates their close relation to catarrh and to nutritive disturbances produced by this cause.

Cohnheim asserts that the numerous anastomoses existing in the gastric mucous membrane are sufficient to prevent thrombi and ulceration. According to him, the circulatory troubles could not by themselves produce a true persisting ulcer, for in cases where local artificial thrombi produce ulcers, the latter heal very quickly.

Introduction of a large proportion of acid into the stomach has given negative results. In man it has been established that ulcers of the stomach often correspond to a diminution of acidity of the gastric juice. These few facts are sufficient to bring out the vague points of our actual knowledge upon the pathology of peptic ulcer.

Pathological anatomy. Catarrhal ulcerations of the stomach are developed from the erosions which accompany intense mucous hyperemias and phlegmasias complicated by hemorrhage. These erosions are ordinarily observed in the right compartment of the stomach, especially in the neighborhood of the pylorus; they appear in the shape of small solutions of continuity of the superficial layer of the mucous membrane, are round, with sharp and soft edges, with an irregular and bloody ground. In the intestine the catarrhal ulcerations are developed preferably at the expense of the follicular ulcers; these latter are themselves the result of an abundant cellular infiltration of the lymphatic follicles, ending in molecular gangrene of the affected tissue. At the outset they 
constitute small crateriform cavities, due to loss of substance. In the end they may acquire dimensions varying from the diameter of a ten-cent silver piece to that of a small saucer. The necrobiotic process affects successively the submucous connective tissue, the muscular tunic, and sometimes also the serous. More or less abundant hemorrhages are produced through the destroyed bloodvessels, peritoneal adhesions are formed upon the level of the ulcerations, and in some cases these lead to a complete perforation of the intestine.

Near lesions in which the destructive process is well marked there may be found others showing a tendency to healing; others, again, are in a more or less advanced stage of repair, and we may even detect star-shaped cicatrices resulting from healed ulcerations. The great losses of substance are never entirely repaired, their edges become thickened and form a swelling of a cartilaginous consistence; the submucous connective tissue and the destroyed muscles are replaced by a fibrous neoformation, and the neighboring mucous membrane shows streaked folds. We also observe all the alterations of chronic catarrh.

Peptic ulcers have rarely been observed. They are found exclusively in the stomach and duodenum, which localization is sufficient to establish the difference between these and catarrhal ulcerations. Their form is regular, round or elliptic; the edges are smooth and cut sharp, as if stamped out; their depth is variable. They may become quite healed, leaving a radiating cicatrix; they often produce hemorrhages or peritoneal adhesions; sometimes they end in perforation of the gastric or intestinal walls.

Among the cases of ulcers described in our special literature, those of Siedamgrotzky, Grad, and Zippelius were of a catarrhal hemorrhagic nature; Bruckmüller, Roloff, and Fröhner have studied round peptic ulcers; the lesions described by Gotteswinter and Münich were probably also peptic ulcers. In a case related by Roloff, an obliterated arteriole ended in ulceration.

Symptoms. It is impossible to distinguish the clinical differences of these two varieties of ulcers, the existence of which is demonstrated by the pathological anatomy. Indeed, the symptoms are obscure, and are confounded with those of chronic gastrointestinal catarrh. Siedamgrotzky has only observed emaciation and a progressive weakening. Grad and Zippelius have noticed symptoms of chronic catarrh of the abomasum of the ox; also 
chronic tympanites, constipation, disturbances of nutrition, and cachexia, etc. Sometimes, however, we observe special symptoms, which permit us to diagnosticate or at least to suspect an ulcer. These are: repeated vomitings, colics, hematemesis, and bloody excrements. In the horse we observe principally nutritive changes, emaciation, periodic colics appearing some time after meals (Siedamgrotzky, Münich), and even vomiting of blood (Gilis). Weiskopf has several times seen in this animal perforation of the stomach caused by gastric ulcers. In the ox Gotteswinter recognized ulcer of the stomach through anemic symptoms, also through gastric troubles and the expulsion of black excrements resembling tar. The autopsy confirmed his diagnosis.

The duration of the disease is somewhat long. While recovery may be possible, the prognosis of a gastric ulcer is, in general, very grave. Its ordinary termination is death, which happeus either as the result of hemorrhage, or peritonitis consecutive to perforation of the stomach or intestine, or through weakness and marasmus.

Differential diagnosis. The symptoms determined by ulcers of the stomach and intestine are often attributed to chronic gastrointestinal catarrh-an error which is very excusable if we think of the close relations existing between these affections and the similarity of their manifestations. In the ox, even when ulcers of the abomasum are accompanied by hemorrhages and marked by vomiting and the expulsion of bloody excrements, it is not always possible to distinguish them from the morbid condition which is the result of fixation of a foreign body in the walls of the stomach. We may, however, be guided by the symptoms furnished by exploration of the region of the reticulum: the sensibility of this - portion, which is increased when a foreign body is fixed in the abdominal walls, is normal in cases of ulcer of the stomach.

Treatment. The treatment is essentially dietetic. The gastric and intestinal ulcerations must be freed from all irritations which might be produced by coarse and indigestible food. Zippelius recommends us to feed the oxen on liquid or cooked food, and avoid at the same time all those fermenting easily, such as brewery waste, for instance. The horses must be kept on flour and bran slops; the dog should be given milk, cooked eggs, and hacked meat. The therapeutic agent deserving preference is artificial Carlsbad salt: it neutralizes part of the acidity of the gastric juice, it also disinfects the stomach and assists the passage of ingested matters from 
the stomach into the intestine. The following composition may be prescribed: Sulphate of soda 80 grammes, chloride of sodium 20 grammes, bicarbonate of soda 5 grammes. For the ox the doses are 100 grammes, for the horse 50 grammes, and for the dog 2 to 5 grammes. Hydrochloric acid is not at all advisable. Zippelius observes that ulcer of the stomach constitutes perhaps that grave form of indigestion in which hydrochloric acid acts as a true poison. In obstinate cases it will be proper to use subnitrate of bismuth (0.1 to 0.5 gramme for the $\mathrm{dog}$, as an antiseptic), or else nitrate of silver. We may counteract intestinal hemorrhages with styptics (tannin, iron, lead preparations). Narcotics will have to be used to fight persistent vomiting of the dog (opium, hydrate of chloral, bromide of potassium), also creosote, creolin, tincture of iodine, iced drinks, or hypodermatic injections of small doses of morphine and cocaine together.

\section{GASTRIC AND INTESTINAL HEMORRHAGES.}

These hemorrhages result from various processes, the nature of which is often difficult to determine. While they are always secondary and constitute only a symptom of another affection, we have thought it proper to devote another article specially to these troubles.

Etiology. Gastric and intestinal hemorrhages are generally due to mechanical or chemical lesions of the mucous membrane. Sharp foreign bodies (bony splinters, fish bones, acicular and coniferous leaves, etc.), also intestinal parasites (the gastrophile larvæ, the echinococcus, and the giant echinorrhynchus), caustics (non-dissolved emetics), besides commotion of the stomach through a blow upon the hypochondrium, falls, and violent exertions may determine them. As seen in the preceding article, in some cases they are the result of the opening of bloodvessels produced by an ulceration of the mucous membrane. They may be seen during the course of acute gastro-enteritis, in diseases of dogs, and in general in all the infectious diseases which are accompanied by a serious venous hyperemia of the stomach and intestine (anthrax fever, petechial fever of the horse, septicemia, septicemic vitulary fever of the cow). ${ }^{1}$

1 As causes of these hemorrhages we must also mention the venous stases produced in the gastro-intestinal mucous membrane through changes of relation of the intestine, cardiac valvular insufficiency, emphysema and pulmonary sclerosis, cirrhosis of the liver, and thrombosis of the portal system. 
Rectal hemorrhages are almost always produced by hemorrhoids. These diffuse or varicose dilatations of the superficial and deep hemorrhoidal veins are usually found in the dog and pig, rarely in the horse and ox. They originate from an obstructed return circulation (heart, lung, or liver diseases) ; the hemorrhoidal veins, especially those most distant from the heart, give way under the bloodstasis. There seems to be a congenital predisposition in the pig favoring the development of hemorrhoids. Spinola attributes it to an overabundant supply of food which is too rich and too irritating, also insufficiency of exercise and abuse of drastic purgatives.

In the ox we may observe intestinal hemorrhages during the evolution of enzootic gastro enteritis. This affection (formerly designated under the name of sang de lombes; Rücken, Lendenblut) has been regarded by Ryschner as the cause of hemorrhoids, and Spinola has sustained this opinion. Haubner and Siedamgrotzky have considered it an inflammation of the rectal mucous membrane, determined by a too exciting nourishment, or by hard work, necessitating excessive efforts. The sang de lombes is therefore only a hemorrhagic proctitis (inflammation of the anus and rectum).

Vogel has related a very curious case of gastric hemorrhage in a dog. An aneurism of the abdominal aorta, after having become united to the gastric walls, opened into the cavity of the stomach and caused death in a few seconds. ${ }^{1}$

Symptoms. The principal symptom of gastric hemorrhage is hematosis. At intervals more or less close we observe the expulsion from the anus of a mixture of fecal matters and blood-clots. The intestinal hemorrhage is marked by bloody defecations. If the blood-discharge takes place in the stomach or intestine, we observe, in addition, all the symptoms of internal hemorrhages or those of chronic anemia-a condition which is soon accompanied by hydremia and serous transudation in the splanchnic cavities.

Hemorrhoids of the dog are indicated by painful and rare defecation, and by rectal hemorrhages, which are ordinarily scant. The neighborhood of the anus is frequently the seat of inflammation. In introducing the finger into the rectum-a very painful operation for the animal-we feel the irregularity of the mucous

1 Labat and Cadéac have mentioned a case of the same kind observed in a mare which, during the last days of her life, presented symptoms of colic and ejected bloody excrements. At the autopsy they found an aneurism of the aorta opening into the floating colon (Revue Vét., 1884).-N. D. T. 
membrane; it may also be covered by pediculated vegetations, which at times protrude through the anus. The animals make frequent expulsive efforts or drag themselves upon their seat, the posterior members stretched under the body; the gait is stiff, painful; the dog is often cross; at certain moments we observe a sexual excitement, and even rabiform symptoms. Rectal prolapsus is not rare; the mucous membrane which covers the ruptured mass is very vascular, sometimes torn. Later, the varicose vegetations may become thrombosed, suppurate, and lead to death through septicemia or pyemia.

In a horse, Utz observed slight colics related to hemorrhoidal vegetations of the size of a nut; these colics were accompanied by anal hemorrhages and by a periodic expulsion of dark bloody clots. Anemia and hydremia appeared gradually ; finally, bloody perspiration was observed, and the animal succumbed rapidly.

In cases of sang de lombes (rectal hemorrhagic inflammation) of the ox, Haubner and Siedamgrotzky have noted the following symptoms: a febrile condition occurring suddenly, a stiff walk, sensitiveness of the lumbar and croupal region, frequent expulsive movements, rare and scant defecations, dry excrements mixed with blood; high temperature; anal hemorrhages and tumefaction of the rectal mucous membrane. The disease ends generally in a cure within three, four, or five days ; but when the inflammation is very intense it may cause death.

Differential diagnosis. It is relatively easy to recognize gastro-intestinal hemorrhages due to gastro-enteritis, from a general disease like rectal inflammation or a hemorrhoidal ectasia; but in some instances we are much embarrassed in distinguishing hemorrhages caused by ulcers, by mechanical hyperemias of the mucous membrane, or through the attachment of foreign bodies to it. The symptomatology may then guide the practitioner.

When liquid or coagulated blood is rejected through the mouth and nostrils, it is important to determine its source, in order to know if the hlood comes from the lungs or from the stomach. Hematemesis is recognized through gastric troubles which precede, accompany, or follow it, also through bloody excrements, nauseas, and vomitings, and lastly by the character of the rejected blood, which is more or less coagulated, of a black color, similar to coffeegrounds, and which has an acid reaction. In hemoptysis, on the contrary, the blood is vermilion-red, spumous, and of alkaline reac- 
tion; besides, there are always respiratory troubles present, and mainly cough. In cases where the blood coming from the lungs is swallowed and then rejected, and in those where the nauseas of the hematemesis are accompanied by coughing-spells and from laryngeal irritation, the diagnosis is often very difficult. Spinola insists upon a liability to confound hematemesis with the vomiting of blood which has been ingested in too large a quantity.

In the dog, abscess of the anal glands has sometimes been mistaken for hemorrhoids. ${ }^{1}$

Treatment. The agents most frequently used are: cold water, iced drinks, astringents, also alum, tannin, sulphate of iron, a solution of perchloride of iron ( 3 to 5 drops, in pills, for the dog), salts of lead, nitrate of silver, opium, and ergot. Heavy and irritating alimentary matters must be avoided and constipation prevented by the administration of light purgatives and increasing regular moderate work. Hemorrhages due to persistent intestinal hyperemia, produced by a serious chronic alteration, resist all treatment. Those produced by vascular rupture of the rectal mucous membrane may be combated by astringent injections. If they originate from hemorrhoidal vegetations which are accessible to the hand, we may have recourse to surgical means-ligature, cauterization, etc. Rectal hemorrhages of the ox yield ordinarily to styptic solutions administered in clysters.

\section{GASTRO-ENTERITIS.}

The gastro-intestinal mucous membrane may be the seat of phlegmasia of a very variable nature. We will here dwell only upon primitive and essential gastro-enteritis, laying aside those developing secondarily during the course of numerous affections-as bovine pest, influenza, hydrophobia, petechial fever, also in changes of relation of the intestine, etc. According to their localization, these inflammations have received different denominations pointing particularly to the specially affected intestinal compartment; we thus distinguish gastritis, duodenitis, ileitis, typhlitis, colitis, and proctitis; practically, however, this distinction is almost always deceptive. It is preferable to classify them according to the character or intensity of the phlegmasia, and to recognize parenchymatous, phleg-

1 Eiselen: Repertor., 1841. 
monous, purulent, hemorrhagic, croupous, diphtheritic, and caseous gastro-enteritis, etc. In its most benign form, gastro-enteritis is confounded with gastro-intestinal catarrh.

An etiological division would be the most rational; we may describe a simple gastro-enteritis, produced by thermo-mechanical irritatious; then the toxic and infectious (dysentery), embolic, tuberculous, and mycotic (due to fungi), etc. The division into diffuse, circumscribed, sporadic, and infectious, as also into acute and chronic gastro-enteritis, is by far too general.

From a clinical point of view it is best to be satisfied with a classification based upon the degree of intensity and the nature or etiology of the affection. Consequently, we adopt the following division :

1. Simple gastro-intestinal catarrh (non-toxic) of the different domestic animals ;

2. Croupous gastro-enteritis of the ox ;

3. Mycotic or infectious gastro-enteritis ;

4. Toxic gastro-enteritis.

Caseous gastro-enteritis of the pig will be treated in the article on Tuberculosis, and dysentery will be placed among the infectious diseases under the name of infectious enteritis. This classification seems to us the most logical and also the most interesting, scientifically as well as practically.

\section{Simple Gastro-Enteritis.}

Etiology. The causes of simple, ordinary, non-toxic gastroenteritis are identical with those of acute gastro-intestinal catarrh, only they act more intensely. Among these causes we must especially mention the action of cold in all its forms, sudden colds when the body is in a perspiring condition, the ingestion of very cold water, of frosted or frost-covered fodder, etc.; also burns of the stomach through very hot drinks or infusions, and also hot milk, in calves (Read); irritating alimentary matters, foreign bodies, and intestinal worms (Spiroptera megastoma in the horse, Spiroptera sanguinolenta in the dog, Spiroptera strongylina in the pig, etc. The Ascari found in the fodder have also been considered as a cause by quite a number of authors, but through error without doubt, for they are found in all the different kinds of hay. Simple gastro-enteritis is sometimes a complication of certain colics.

Pathological anatomy. The alterations of gastro-enteritis 
differ only from acute gastro-intestinal catarrh through their greater intensity and by the character of the phlegmasia, which is parenchymatous. But in certain cases the catarrhal inflammation ends by involving the mucous membrane in its whole thickness, and determines lesions which are difficult to connect with gastroenteritis or acute gastro-intestinal catarrh. However, such cases are but rarely met with in practice. As a general rule, to gastroenteritis belongs a more marked congestion, often going as far as hemorrhage; the superficial desquamative process is replaced by a parenchymatous, phlegmonous, or purulent infiltration of the mucous membrane, or even by its mortification.

In the stomach we find a diffuse or circumscribed redness, taking various shades from a light to a very dark red; the mucous membrane, which is much tumefied and folded, is covered with ecchymoses or blackish spots, varying in size. Its surface is sometimes smooth and coated with a mucous exudate, which may be of a whitish, grayish, or bloody color, at times marked with superficial ulcerations, with sharp, regular edges, and the base of which is sometimes covered with a blood-clot (hemorrhagic erosions.) These are especially found upon the folds close to the pylorus (particularly in the stomach of dogs and in the abomasum of the ox). They are produced by hemorrhagic infiltration and the consecutive necrosis of the mucous membrane. The surface of this membrane is also dotted with small spherical nodes formed by the pepsin glands, which are tumefied and affected by fatty degeneration. The submucous connective tissue is the seat of a gelatinous or purulent infiltration.

The intestinal mucous membrane is distinctly hyperemic; its redness is diffuse or spread in small dots, stars, nets, in spots or blotches. It is tumefied, infiltrated with liquid, and softened; at times it presents but a purulent mass, whilst in other instances it is covered with dry crusts of bad appearance. The latter are more or less adherent according to the depth of the furrow of separation which is formed at their periphery ; their complete elimination may determine perforation of the viscera. In some regions the epithelium of the mucous membrane is often detached from a large surface; the papilı are hypertrophied and projecting; if the section is placed in water they float manifestly and give the mucous membrane a velvety look. The solitary follicles are sometimes intact, sometimes swollen and surrounded with a red areola; they predominate in the shape of small pimples of the size of a pin-head; 
the agglomerated follicles constitute enlarged swellings of irregular surface. When these cellulo-purulent infiltrations are disintegrated follicular ulcerations are formed in their place, infundibuliform in the case of the solitary follicles, which are net-like or areolar-shaped at Peyer's patches.

The submucous connective tissue is ordinarily injected or odematous; we sometimes find a gelatinous or hemorrhagic infiltration which makes the mucous membrane appear considerably thickened. In the cæcum and colon of the horse this membrane may have acquired the thickness of two or three inches. In some cases the muscular wall is simply infiltrated with serum, in others it is partially destroyed by the ulcerations. The serous membrane is injected in varying degrees, and is covered up by an exudate. The intestinal contents are of a variable consistence; they may be watery, serous, mucous, purulent, transparent, of a milky appearance, or grayish, tinted red, mixed with epithelial flakes, with fibrinous, bloody, or purulent clots; sometimes they are almost exclusively composed of blood. The mesenteric ganglions are tumefied; on section their tissue presents a marbled infiltration. The liver and spleen are more or less markedly hyperemic.

symptoms. The manifestations of -sporadic gastro-enteritis succeed those of acute gastro-intestinal catarrh or are developed from the onset. As soon as the disease is well established the appetite is suppressed; the pig and dog vomit frequently, the horse and ox are affected by nausea. The buccal mucous membrane is injected, dry, and rough ; thirst is intense, the ingestion of liquids by the dog is ordinarily followed by vomiting. In all animals we detect persistent colics, and at times rabiform symptoms in the dog. Palpation of the stomach and abdomen is painful ; the abdominal walls are distended, with or without swelling. The peristaltic movements are suppressed. At the beginning constipation is obstinate; the patients make frequent and violent expulsive efforts; the excrementitious matters are hard, of the shape of large chestnuts, and covered with mucus and blood; later they become liquid, they are sometımes mixed with pure blood (hemorrhagic enteritis); in some exceptional cases they contain sloughs which are of a fetid odor. In very serious cases a short time before death constipation is replaced by an abundant diarrhea, and paralysis of the anal sphincter is added to the intestinal complication. The urine contains albumin; that of the herbivora is acid (phosphates). 
The intense gastric phlegmasias are accompanied by a sharp fever, which is marked by a small, hard, accelerated pulse and a considerable elevation of the temperature, also by injection of the mucous membranes, by a chilled condition of the extremities, and, lastly, by profuse perspiration. In the horse the heart beats 80 to 100 times per minute; the rectal temperature oscillates between $40^{\circ}$ and $41^{\circ}$. When death approaches the temperature becomes lower and may fall to the normal figure. As a rule, the general sensibility is diminished; in some animals, however, we observe restlessness, anxiety, spasms, and convulsions; but stupefaction and collapse soon follow the excited condition; the beast staggers, lies down, or falls exhausted. The colics cease toward the end of life. Death occurs in coma.

Course. In the horse the course of simple gastro-enteritis is always rapid; it rarely persists longer than a few days. The chronic form, which lasts several weeks, is divided into periods of exacerbation and ends in death. This is the form described by Haubner and Siedamgrotzky; it is probably only a thromboembolic enteritis.

Prognosis. It is a very serious and fatal disease in the majority of cases. Moderate colics, light fever, the strong pulse which is but little accelerated, and easy expulsion of the excrements, are favorable prognostic symptoms. An intense fever, small and evasive pulse, dyspnœa, obstinate constipation, the sudden cessation of pains, collapse, and profuse diarrhea are, on the contrary, unfavorable prognostic signs. Death may be caused by various accidents, but it is ordinarily provoked by intestinal necrosis with consecutive septic infection, either through pulmonary odema, intestinal hemorrhage, or cardiac syncope.

Differential diagnosis. 1. In order to distinguish gastroenteritis from gastro-intestinal catarrh we must depend upon the intensity of the symptoms. The first will be recognized by its high fever, the weakness of the heart, the general alarming condition, the intense colics, and the obstinate constipation followed by bloody evacuations.

2. It is harder to distinguish subacute gastro-enteritis designated under the name of "inflammatory colics" from other varieties of colic; sometimes the diagnosis is absolutely impossible during life. As special symptoms of this variety of gastro-enteritis, we may quote: high fever, a small and accelerated pulse, colics without 
remissions, a serious general condition, an obstinate constipation, sensitiveness of the abdomen to palpation. The symptomatology may enlighten the practitioner. In some instances the diagnosis can be established by exclusion.

3. A differential diagnosis of the various forms of gastro-enteritis is often very difficult to make. We have as a guide in toxic gastroenteritis the history and certain special symptoms; in mycotic enteritis we have the cerebral symptoms and a very rapid course; in croupous enteritis we observe the rejection per rectum of fibrinous membranes and the slow course; and in dysentery we see an epizootic character in the disease, and also very bloody ejections.

4. In order to distinguish essential gastro-intestinal catarrh from that accompanying hydrophobia, anthrax, bovine pest, etc., it is only necessary to make a careful examination of the patients; in doubtful cases we must wait for an evolution of the disease. It is especially in the dog that we meet with difficulties; gastroenteritis quite frequently starts with rabiform symptoms in this animal.

Treatment. The first thing to be done is the regulation and care of the diet; it should start with complete abstinence, then easily digested food may be given gradually. We must not use very cold water, as it provokes vomiting in the dog; we must give only such quantity as is strictly necessary. Circulation and the cutaneous functions must be stimulated by dry or exciting frictions (camphorated alcohol, essence of turpentine, oil of mustard) and we should also apply hydrotherapeutic compresses upon the abdomen. Internally, we must prescribe emollients and sedatives, mucilaginous decoctions, and oil emulsions; linseed mucilages, fresh linseed oil, barley mucilage, a solution of gum with opium, opium tincture, etc. Violent colics will have to be combated by hypodermatic injections of morphine. As purgatives, we should preferably use laxatives, castor-oil, sulphates of soda or magnesia, also calomel, alone or mixed with opium.

We give below a few very excellent formulas:

For the horse: Powdered opium, 10 grammes ; protochloride of mercury, 2 grammes; marshmallow powder, 100 grammes; distilled water, q. s. To be given as an electuary at one time.

For the ox: Decoction of linseed (100 grammes in 750 grammes of water); linseed oil, 250 grammes.

For the dog: Tincture of opium, 2 to 5 grammes; mucilage of 
gum arabic, 10 grammes; distilled water, 10 grammes. To be given in doses of a tablespoonful.

If there is great weakness and collapse, we may use the following ingredients with advantage: camphor, ether, wine, coffee, caffeine, or hyoscyamus.

\section{Croupous Enteritis: Intestinal Croup.}

Croupous enteritis is more frequent in animals of the bovine race than our literature seems to indicate. It is rare in the horse, still more uncommon in carnivorous animals, and altogether exceptional in sheep. It is a sporadic disease. In the ox it has always a chronic course; in other animals it may be observed in the acute and subacute forms.

Etiology. Its causes are as yet imperfectly understood. The influence of colds has mainly been charged with being the cause of the trouble. The disease is particularly frequent in the spring and fall. Irritating and indigestible alimentary matters have also been accused of having a bad influence, and besides these, hay containing a large number of aromatic plants ; finally, the ingestion of too large quantities of husk may also do harm. It has sometimes been observed in the ox after the administration of certain medicinal agents, drastic purgatives, camphorated alcohol, etc. (Schwanefeldt). In animals of the bovine race, mainly in young subjects which are well nourished, and also in cows in a pregnant condition, there seems to exist a constitutional predisposition to intestinal croup-a predisposition which is said to result from the richness of the blood in albumin and fibrin (Anacker), and which is apparent in other diseases, especially in pulmonary phlegmasia, through the abundance of plastic exudations. The lymphatic condition of the ox has perhaps a certain etiological influence. Constipation, and the accumulation of excrementitious matter in the posterior portions of the intestines of the horse; tapeworms in the dog and cat (Eberth, Bruckmüller) may also provoke croupous enteritis; but its cause often remains undetermined.

Pathological anatomy. The alterations are located in the small intestine and colon in the ox ; in the horse they are principally observed in the small intestine. The mucous membrane presents catarrhal or true inflammatory lesions; it is covered, to a greater or less extent, by croupous membrane, which can easily be removed, and under which the tissue of the mucous membrane is 
found injected, soft, friable, and infiltrated with blood or pus. In the ox the croupous membranes are at times disposed in layers more or less thick, at other times they are tubular or cylindrical; they may be mixed with or free from alimentary matters; finally, they are sometimes found stratified, formed by several superposed layers or by tubes fitting into one another, and between which are found alimentary matters. Their length and calibre are most variable; some of them may measure as much as thirty feet. Microscopic study of these membranes has demonstrated their amorphous structure. They are formed of a fibrinous substance, containing epithelial cells undergoing fatty degeneration and numerous white globules. The intestinal contents are exclusively formed of croupous membranes, or of a mixture of these membranes and of fetid liquid of bad aspect. The tube-shaped glands of the mucous membrane are filled up with a fibrinous exudate.

Symptoms. In the ox the manifestations of intestinal croup are much less pronounced than in those of other forms of enteritis. The characteristic symptom of the disease-the expulsion of the croupous membranes-appears after a certain period. At the onset we observe symptoms of intestinal catarrh or of chronic enteritis; disturbance of the appetite and rumination, light colics, constipation, and a moderate fever. Within a few days diarrhea sets in; the patients expel very liquid excrements of a grayish color and fetid, containing fibrinous portions, which are infiltrated with blood or pus. This diarrhea almost always leads to an improvement, and the case then gradually shapes itself toward a cure. The ordinary duration of the affection is from six to eight days. Festal observed a croupous enteritis in the ox which started with colic, an obstinate constipation, intense fever, and great depression; the pains then ceased, and croupous neoformations were expelled, while constipation persisted. On the sixth or seventh day a fetid diarrhea followed the costiveness. In the majority of cases the disease takes the course of recovery; in others the symptoms become aggravated, an extreme weakness sets in, and a subacute peritonitis takes off the affected animal. The duration of this affection varies from eight to twenty days.

In the horse croupous enteritis is often announced by symptoms of chronic colic; Prietsch has detected rabiform phenomena. The colics are sometimes absent, and the disease develops with all the appearances of a febrile intestinal affection; there are expulsions 
of soft fecal matters, which are fetid and intermixed with croupous masses of a febrile, flaky, membranous, tubular, or cylindrical form. In the other species the symptoms of intestinal croup are the same as those of simple enteritis, but are less well marked.

Prognosis. This is not of the same gravity in all the animal species. In the ox it is relatively benign. Cases where the croupous exudate succeeds in completely obstructing an intestinal loop are quite exceptional. In other animals, especially the horse, the prognosis is more serious, and the practitioner should be careful to exercise some precaution in this respect. According to Grimm, croupous enteritis is said to be frequently complicated by rheumatism.

Differential diagnosis. Croupous neoformations-the only characteristic symptom of the affection-are frequently taken by non-professionals for snakes, tapeworms, or the intestines of small animals that have been swallowed, and even for the patient's own intestinal loops. The grayish-white color of these productions, their amorphous structure and homogeneous consistence, also the absence of traces of mesenteric insertion, etc., permit us to recognize their nature at once, and to distinguish them, whatever their form may be, from an intestinal loop.

In the horse we must not confound the mucous coating of the dung (covered dungs), which is frequently observed in catarrhal proctitis and ordinary constipation, with the false membrane of intestinal croup. In carnivorous animals the excrements sometimes contain organic matters which are incompletely digested, and these have a certain resemblance to the croupous exudate.

Treatment. The treatment is nearly the same as that of simple enteritis. However, liquefacients and dissolvents, mainly the alkaline carbonates and sulphates should be preferred to emollients. In the dog especially, these salts administered in clysters (in a solution of $1: 100$ of sea-salt or other soda or potash salt) are of much advantage.

\section{Mycotic or Infectious Gastro-enteritis.}

INFECTIOUS, SEPTIC, SEPTIFORM, AND TYPHOID GASTRO-ENTERITIS : INTESTINAL MYCOSIS : INTESTINAL TYPHUS : INTOXICATIONS FROM FUNGI, AND FROM MEAT AND SAUSAGES.

The different designations here given have been applied to morbid conditions bearing a resemblance to one another that is 
sufficiently close to induce us to combine them under the title of mycotic gastro-enteritis. All have the same origin and pathology : they are produced by injurious matters, the action of which is first felt upon the digestive canal, and, secondly, upon the different organic apparatus. The active principle which they engender probably operates through a double mechanism: first, through infection, or multiplication of the micro-organisms which they contain; secondly, through a septic or putrid intoxication, in which certain chemical compounds generated by microbic activity constitute the principal pathogenic factor. The latter have received the name of "septines," "ptomaines," and "cadaveric alkaloids." Mycotic gastro-enteritis can thus be defined: An intestinal affection accompanied by general disturbances, produced through the invasion of pathogenic micro-organisms and through the resorption of poisonous ptomaines. It possesses features similar to infectious diseases and gastro-enteritis produced by poisoning, between which it constitutes a kind of intermediary morbid condition. We could, in fact, connect the poisonings produced by meat with septicemia or putrid infection - that is to say, with infectious diseases - and the fungi intoxications with the poisonings produced by acrid narcotics.

The serious cerebral symptoms and the rapidity of the course of mycotic gastro-enteritis have caused it to be designated by the name of "intestinal typhus." Some authors have classified it with the abdominal typhus of man - a relation which is absolutely arbitrary, for there does not exist any similarity between these two diseases.

We shall describe in succession mycotic gastro-enteritis in carnivorous and omnivorous animals, and also in poultry, which is produced by the ingestion of decomposed meats, and at the same time that variety occurring in herbivorous animals after ingestion of fungous plants (moulds, rust, or black rust).

Ptomaines. We designate under the name of ptomaines all organic bases of microbic origin (Brieger). These bases are not only produced in the cadaver, but there are some which are formed in the living organism; these have been given the more restricted denomination of leucomaines. In the majority of infectious diseases (anthrax, hydrophobia, etc.), the lencomaines produced by microbic waste products determine by their poisonous action the principal symptoms of these diseases.

According to Hoppe-Seyler, the ptomaines are formed when the excess of oxygen is insufficient or hindered. Under physiological 
conditions such poisons can only be produced in the digestive tube (indol, skatol). Recent researches have demonstrated that the ptomaines differ but little or not at all from vegetable alkaloids (strychnine, atropine, muscarine, morphine, curarine); the chemical composition and effects of these products are also very similar.

We propose to give here a brief review, or rather an enumeration, of the principal ptomaines, taking Robert's classification as a guide $:^{1}$

First group. Bases approaching the ammoniacal: methylamine, dimethylamine, trimethylamine (contained in herring-brine); ethylamine, diethylamine, triethylamine, ethylendiamine, dimethylethylendiamine (putricine), and its isomere saprine, pentamethylendiamine (cadaverine). All thesealkaloids produce spasms like ammonia itself.

Second group. Ptomaines of the muscarine type and counting trimethylamine among their decomposition products. They are: Muscarine, choline, neurine, and a few others. It is an interesting fact that muscarine $\left(\mathrm{C}_{5} \mathrm{H}_{15} \mathrm{NO}_{3}\right)$ is found alike in poisonous fungi and in the production of cadaveric decomposition (Brieger).

Third group. Ptomaines having as a type guanidine, and including: Methylguanidine, creatine, creatinine, and other waste products of the organism. These alkaloids are therefore in reality leucomaines.

Fourth group. It includes: Guanine (the ptomaine of guano and leucomaine of pancreatic decomposition), xanthine, hypoxanthine, methylxanthine, carmine, adenine, and others coming close to uric acid.

Fifth group. Amide acids: Glycocoll, leucine, tyrosine, betaine, sarcosine.

Sixth group. Pyridine bases: Pyridine, picoline, lutidine, collidine, parvoline, corindine.

Seventh group. Aromatic bases: Indol, skatol.

Eighth group. Aromatics containing no oxygen: Phenic acid, cresol, and a few others.

Ninth group. Includes: Mydine and myatoxine, extracts from cadavers ; tetanine, obtained from cultures of the traumatic tetanus bacillus, also typhotoxine, produced from cultures of the typhoid fever bacillus, and mytilotoxine, found in poisonous mussel; perhaps also lupinotoxine, and several others.

1 Robert: Compendium der praktischen Toxicologie, 1887. 
Tenth group. Comprising some of the most interesting products of decomposition: 1, Schmiedeberger and Bergmann's sepsine, the most common ptomaine and principal agent in septic gastro-enteritis; 2 , ptomatocurarine, a cadaveric alkaloid which paralyzes the terminal motor plaques, exactly like curara (paralytic vitulary fever); 3 , ptomato-atropine or atropine of the cadaver, which plays an important rôle in poisoning from tainted sausages, and produces phenomena identical with those observed in intoxication through atropine, dilatation of the pupil and impaired vision included ; 4, mydaline, possessing the properties of atropine and muscarine; 5, ptomatomorphine, whose properties are similar to those of morphine (Panum) ; 6, ptomatodigitaline, which, according to Selmi, provokes all the symptoms of digitalis poisoning in the animals. The anthrax alkaloid is also classified in this group; Hoffa extracted it from bacteria cultures. This basis has been extracted by the StasOtto process; in animals in which it is injected it produces, after a short period of excitement, somnolence and a bloody diarrhea, followed by death. Numerous ecchymoses are found at the postmortem upon the pericardium and peritoneum; the blood is black, and devoid of microbes.

MYCOTIC GASTRO-ENTERITIS OF CARNIVOROUS AND OMNIVOROUS

ANIMALS, AND OF POULTRY, PROVOKED BY INGESTION OF DECOMPOSED MEAT.

This gastro-enteritis offers the greatest similarity to the poisoning produced by tainted meat in human beings. It is probable that this was the affection which has been described under the name "typhus of dogs" (Hertwig, Bruckmüller), and also as the "typhus of pigs."

Etiology. As a rule, it is caused by meat in a more or less advanced state of decomposition, or by sausage, and sometimes by old herring-brine. Shepherd dogs are the most frequently affected; it has also been observed in poultry after the ingestion of putrid meat (Rosenkranz). The specific agents of the disease are the micro-organisms contained in meat in the process of decomposition, and the ptomaines which they generate. The history is rarely precise enough to enlighten the veterinarian, and in most cases the observed conditions are ascribed to an intoxication through mineral poisons or " rough on rats."

Symptoms. They are similar to those of poisoning through 
brine. We see a diarrhea appearing suddenly, and almost always becoming bloody; there are vomitings, the thirst is great, and the fever intense $\left(40^{\circ}\right.$ to $42^{\circ} \mathrm{C}$.), the weakness is extreme. Death, which is almost the constant termination of the affection, occurs more or less rapidly, sometimes within a few hours, mostly in less than a day.

These are exactly the symptoms observed by Panum in the dog after an intravenous injection of the poisons (0.012 gramme) extracted from decomposed meat. At the autopsy of these subjects of experiment he has found lesions of hemorrhagic enteritis, and has noticed a very rapid decomposition of the cadavers. ${ }^{1} \quad$ Susceptibility to the mycotic poison varies greatly in the different carnivorous animals. Experimental results related by various authors testify to that effect. Semmer has made dogs and cats eat the meat of a horse that had died from septicemia, without producing any trouble in these animals, but three pigs died after the ingestion of the same meat. Colin has seen slight diarrheas in similar experiments. Lemke produced a very serious diarrhea in three young dogs, to which he gave meat tainted by anthrax, while an older dog did not show any marked discomfort.

Pathological anatomy. The contents of the stomach and intestines are composed of fetid meat partially digested; the mucous membrane is inflamed, both the solitary and the agminated follicles as well as the mesenteric ganglions, are tumefied; the intestinal contents are bloody, of a chocolate color and of a semi-liquid consistence. The blood is greatly altered; the liver is obstructed and it is soon affected by putrid decomposition; the spleen is tumefied and filled with hemorrhagic points; the myocardium is very friable. Siedamgrotzky has found small immobile bacteria in the blood, which were distinguished from the bacteridia by their greater diameter and the absence of spores.

In man, the accidents of poison through meat and sausages have long been considered to be of similar nature to anthrax ; therefore, the expressions "intestinal mycosis" and "intestinal anthrax" have been used indiscriminately, but Bollinger has shown that these accidents are septic inflammations of the intestinal mucous membrane, due to mycotic poisons. We must distinguish between those poisons which are ectogenous or formed post-mortem, and those which are endogenous or formed intra vitam.

1 Panum: Virchow's Archiv, Bd. ix. 
1. The principal ectogenic toxic agents are the sausage poisons, those of fish, and some few from meat. They contain bacteria which do not resist boiling. The pathology of the disturbances which they create consists in a true infection. But these bacteria produce ptomaines which are not destroyed by cooking (mixed infection). Haupt, in studying (1887) poisonings produced by tainted meats at Chemnitz, found a microbe (Proteus mirabilis) which he considered as the pathogenic agent in these accidents. In poisonous sausages and in the intestinal canal of the pig the meat of which had been used in their making, Nauwerk detected a bacillus of putrefaction remarkable for its extreme activity. The different poisons are undoubtedly produced by special microorganisms generating ptomaines, the chemical composition of which, as well as their effects, may be very different. These, however, vary with the origin and composition of the meat, the mode in which it is prepared, its curing, also the temperature and the degree of putrefaction, etc.

The clinical picture of poisoning through sausages (botulism, allantiasis) has a peculiar significance. Paralysis of certain groups of muscles are added to the symptoms of gastro-enteritis (diarrhea, vomitings, weakness, stupefaction, collapse); the muscles of the eye are affected (ptosis or lowering of the upper eyelid) also those of the larynx, pharynx (dysphagia) and of the intestine (constipation), etc.

Similar paralysis is observed in the course of a large number of infectious diseases, such as hydrophobia, diphtheria, and vitulary fever of the cow. They must be considered as toxic accidents; we can, indeed, occasion these phenomena experimentally by the injection of certain ptomaines (ptomatocurarine).

2. The intoxications due to endogenic poisons are produced by meat coming from diseased animals. Bollinger has described these troubles in the human race by the generic expression of intestinal sepsis. Most poisonings from meat belong to this class. Here, again, the pathogenic activity being a function of germs and their wasted products-ptomaines-cooking can only relieve the meat of part of its toxic property. The meats which cause these poisonings most frequently come from cows affected by puerperal septicemia, or from calves slaughtered during the course of pyemic polyarthritis. Suppurating nephritis, septic diseases of lungs and liver, enteritis, peritonitis, and mastitis, also communicate toxic properties 
to the meat. Meats of healthy appearance and of an almost normal taste sometimes produce poisoning. These peculiarities are explained as follows: the meats come either from animals killed at the beginning of some serious disease, or they have undergone a certain degree of alteration after having been killed.

The symptoms induced by these poisonings are those of septic gastro-enteritis. They are the same in animals as in human beings: vomiting, diarrhea, colics, intense fever, extreme weakness, depression of sensibility, and collapse. In man they have sometimes a great resemblance to those of cholera and abdominal typhus, and this has caused meat poisons to be considered as one of the causes of this latter affection. Whilst it may be true that the symptomatology of typhus can be produced by other agents than the bacillus of this disease, this one nevertheless constitutes a specific morbid entity. All the attempts to inoculate the typhus of man upon animals have failed, while the action of meat poisons are very quickly felt by animals.

In most epidemics of poisonings by tainted meat observed in the human race, accidents of the same nature have been observed in dogs, cats, pigs, and poultry. ${ }^{1}$

MYCOTIC GASTRO-ENTERITIS OF HERBIVOROUS ANIMALS PRODUCED BY THE INGESTION OF PLANTS COVERED WITH FUNGI (MOULDINESS, RUST, OR BLACK RUST): FUNGOUS POISONINGS.

Etiology. Under the names of intestinal typhus, typhoid gastroenteritis, etc., there has been mentioned, in herbivorous animals, a group of morbid conditions which are really poisonings, or which are at least similar with those we have just studied in carnivorous animals. Frauenholz has described an epizootic of this kind, observed upon oxen fed on spoiled beets from a sugar factory; Schleg has seen the same accidents in the cow, and Rey in the horse, following alimentation of fermented or spoiled potatoes. Microbic and ptomaine infection of the blood is very distinct from the defined gastric and cerebral symptoms, as well as from the complications roccurring in most cases.

Veterinary publications contain a great number of observations on poisonings due to mouldiness and fungi of food which are in-

I Huber: Archiv der Heilkunde, Bd. xix. 
gested by domestic animals. These parasites can be classified into four groups :

1. Moulds of every description. Those of the genera Mucor, Aspergillus, and Penicillium, are especially interesting. Inoffensive of themselves, they induce the decomposition of alimentary matters (bread, flour, hay, oats, straw), and produce ptomaines.

2. Rusts (uredo), the most important of which is the Puccinia graminis. Frank has experimented upon the rabbit with rust, and has produced gastric troubles, vertigo, and convulsions.

3. Black rusts (ustilago). From a clinical point of view the most important are the Tilletia caries (Albrecht, Adam, Koch, Herele, etc.); we have besides the Ustilago carbo and the Ustilago maydis. Of all fungi these produce the most marked alterations upon the intestinal mucous membrane, undoubtedly through the medium of ptomaines formed in the plant as a waste product of these parasites. The researches of Brefeld have demonstrated that black rusts are microphytes which are akin to the yeast plants, these latter being only more advanced states of development of the same fungi. Thus, wheat rust would be transformed into yeast when located upon a damp soil-in the animal organism, for instance.

4. Yeasts. These agents of alcoholic fermentation (malts), as just seen, would only constitute the last stage of development of mould, according to Brefeld and other authors.

Symptoms. The disease takes the appearance of an infectious morbid condition. It appears suddenly, and generally attacks several animals at a time. The diseased subjects refuse food and drinks; they are depressed, restless, and are affected with abdominal pains, which are marked by cries, bellowings, and groans. The peristaltic movements are suppressed. There is a persistent constipation at the start, followed by a profuse diarrhea, which is fetid and sometimes bloody. Some animals are slightly inflated; pressure upon the abdomen produces pain ; the tongue and pharyngeal muscles are paralyzed, especially in poisoning by the Tilletia caries, which is also said to produce ptyalism, a continual empty or false mastication, and cough.

These symptoms are always accompanied by intense fever, a strong acceleration of the circulation with tumultuous cardiac pulsations (100 and more per minute in the ox), also chills, chilled condition of the extremities, etc. At the same time we observe a 
great weakness; the animals are in a condition of somnolence and stupor, remain continually down, or, if they are standing, they are hardly able to hold themselves, they stagger, and end by falling. Nervous phenomena may be observed, spasms, grinding of the teeth, and trembling. In some cases the wide-opened anus allows liquid excrements to escape; in others there is tenesmus. When the poisoning is produced by mould we see also symptoms of nephritis or cystitis (polyuria of the horse). Abortion is a frequent complication of the conditions produced by Ustilago maydis.

The duration of the trouble varies from one day to one or two weeks. Fatal apoplexies are not rare.

Pathological anatomy. The mucous membrane of the stomach and intestine is tumefied, infiltrated, and dotted with ecchymoses; that of the small intestine is sometimes the color of soot (eel-skin); the contents of the intestine are watery, mucous, or bloody; the lymphatic ganglions are softened and areolated. When the disease has lasted longer, gangrenous spots can be detected, or even intestinal perforations and a suppurating peritonitis. The blood is somewhat dark and pitchy; it stains a dark-red all the tissues with which it is in contact. The liver is hypertrophied; when the disease has taken a slow course it is found to be very soft and affected by fatty degeneration; it quickly undergoes putrefaction. The lungs, heart, the meninges and the brain are hyperemic and ecchymosed. The flesh is sodden; after being cooked it spreads a repulsive odor, and its flavor is disagreeable; the fat is gelatiniform.

Differential diagnosis. Infectious gastro-enteritis may be confounded with several diseases. It has to be especially differentiated:

1. From toxic gastro-enteritis, which has a similar course and is accompanied by a general serious condition. The diagnosis can only be based upon etiological considerations.

2. From bovine pest. This is characterized by an intense inflammatory process of all the mucous membranes (digestive, respiratory, ocular, and genital), by erosions of the buccal and vulvar mucous membranes, by its extreme contagiousness, its course and rapid extension, and at the autopsy by typical alterations.

3. From anthrax. This is recognized by its rapid course and by the detection of a micro-organism differing from the other bacilli that may be found in intestinal mucus; it is motionless, clearly outlined on its extremities, and forms spores outside of the organism. 
It appears in the shape of a very small articulated rod, which is very thin, of an average length of 0.007 millimetre, giving very characteristic cultures and reproducing anthrax by inoculation.

4. From dysentery. This infections gastro-enteritis is accompanied by diarrhea from the beginning; it is ordinarily of long duration, and it has all the appearance of an infectious contagious disease.

5. From subacute encephalitis of the horse and cerebro-spinal meningitis of the ox. In these two diseases the cerebral disturbances are absent.

6. From hydrophobia. The diagnosis must be based upon the history and the predominance of intestinal symptoms in mycotic gastro-enteritis.

7. From influenza. This disease will be recognized by special symptoms which are produced in the eye, and by its contagiousness. In isolated cases this distinction is sometimes very difficult.

Mycotic gastro-enteritis is more uncommon in the horse than in the ox and pig - a difference which is very probably due to the diet of these animals. But its existence in the horse is not to be doubted: the febrile diseases accompanied by grave cerebral symptoms, and described under the name of "infectious gastro-intestinal catarrh," are but mycotic gastro-enteritis consecutive to the ingestion of spoiled food.

Gerlach has described in the horse a typical gastro-enteritis accompanied by colics, constipation, considerable acceleration of the circulation, with weakening of the pulse, grinding of the teeth, etc., and which is distinct from ordinary colics, from anthrax, and from the gastro-bilious form of influenza. He has ascribed this morbid condition to ingestion of new oats not well dried, and to tainted straw given instead of hay.

Derache made several observations of the same kind of disease, which is sporadic, designated under the name of "typhus," but which has nothing in common with this trouble, nor with petechial fever. As a cause, he has attributed it to alimentation with mouldy, dusty oats or hay. Generally the disease affected several animals in the same stable. The principal symptoms were the abruptness of the attack, paresis, weakness of the pulse, paucity of defecation, then diarrhea and glairy evacuations. The course was rapid, and death happened suddenly. At the autopsy were found very characteristic alterations, which recalled those of abdominal 
typhus of man ; injection of the intestinal mucous membrane, with large ecchymoses, tumefaction, and purulent infiltration of the solitary follicles and Peyer's patches; also ulcers and scars which might affect the whole thickness of the intestinal walls; the contents of the intestine were liquid, pitchy, purulent; the muscles were discolored, the liver soft and friable; the heart had a cooked appearance; the uncoagulated blood contained bacilli; the volume of the spleen was not increased; there were no anthrax tumefactions to be detected in the subcutaneous and submucous connective tissue ; the cadaver passed rapidly into a condition of putrefaction. All attempts to inoculate the disease (even by intravenous injection) were unsuccessful.

By its anatomo-pathological characters this disease resembles abdominal typhus of man very closely, but the identity of these two morbid conditions cannot be admitted. No fact observed absolutely demonstrates it, and all attempts at inoculation of typhus (calf, pig) tried by Bollinger, Obermeier, Lebert, and others, have failed. Abdominal typhus is unknown among our animals.

Treatment. In the dog, at the outset, emetics; later on, purgatives, especially calomel, which last is preferred for the horse, on account of its disinfecting action (formation of sublimate). In ruminants we should use saline purgatives. In the larger number of cases tonic medication and stimulants (wine, alcohol, camphor) are indicated. The same general treatment is to be adopted as in uncomplicated gastro-enteritis.

\section{Toxic Gastro-enteritis.}

Toxic gastro-enteritis can be produced by numerous poisons, which are classified in two groups:

1. Acrid poisons;

2. Acrid narcotic poisons.

A knowledge of the different poisons is very important for the practitioner; therefore, instead of giving a general description of toxic gastro-enteritis, we have preferred to examine the clinical effects of the principal poisons. We can give here but a brief description of the different intoxications. As to the details, we must refer to special works, and notably to the Traité de Médécine vétérinaire judiciaire of Gerlach.

The poisonings from narcotics have been added to the others from practical considerations. 
The diagnosis of poisonings which are accompanied by gastroenteritis is not always easy; their symptoms are frequently like those of true gastro-enteritis. In general, we must especially take into account:

1. The history, which is ordinarily very incomplete;

2. The sudden invasion, the rapid course, and sudden death;

3. The appearance of the symptoms during or immediately after meals ;

4. The enzootic character of the disease;

5. The gastric and mucous complications;

6. The special symptomatic phenomena of certain poisonings and anatomical lesions;

7. The physical, chemical, and physiological analysis.

\section{TOXIC GASTRO-ENTERITIS PRODUCED BY ACRID POISONS.}

\section{Poisoning by Caustic Alkalies (Quicklime, Ammonia, Lye).}

Symptoms. Salivation, dysphagia, diarrhea, groans, cough (in poisoning through ammonia), dyspnœa, throwing out of membranous masses of a croupous appearance, muscular weakness, collapse.

Pathological anatomy. Croupous stomatitis and pharyngitis, croupous laryngitis (when volatile alkali is involved), tracheitis, bronchitis, pneumonia; intense inflammation, black or dark-red coloration, gelatinous infiltration, and corrosion of the gastric mucous membrane.

Treatment. Diluted acids, and especially vinegar; opium, and mucilaginous preparations. Poisoning by quicklime may be produced when the stables are disinfected by this agent. Ammonia provokes more or less serious accidents when given in a form too concentrated (in tympanites of the ox, for instance) when used to increase the action of setons, or when it is applied upon the tongue of the ox as a therapeutic agent (Steinzunge). Lye is sometimes lapped by thirsty dogs.

\section{Poisoning by Caustic Acids (Sulphuric Acid).}

Symptoms. They are the same as in ulcerous stomatitis and in subacute gastro-enteritis : tumefied, red, cauterized lips; much inflamed buccal mucous membrane; violent colics, suppressed peristaltic movements; frequent and small pulse; rapid death. 
Pathological anatomy. There is a cauterization of the digestive mucous membrane from the mouth to the stomach, tumefaction of the tongue and cheeks, etc.

Treatment. Diluted alkalies; soap-water, lime-water, lye of soda or potash, calcined magnesia. Stimulants.

Poisoning through caustic acids is very rare in our domestic animals; in a case mentioned by Gerlach the accident was caused by straw from oats which had been used as a packing of sulphuric acid flasks; in another case, related by Johne, sulphuric acid had been mixed with malt. ${ }^{1}$ But exterior cauterizations are quite frequent, particularly in the dog.

\section{Poisoning by Sea-salt, Meat-brine, Herring-brine, etc.}

Symptoms. I. In poisoning from sec-salt the predominating symptoms are those of gastro-enteritis accompanied by paralytic conditions; diminution of appetite; a dry, hot, red mouth; great thirst, vomiting, colics, watery diarrhea, frequent micturition, great muscular weakness, a staggering gait, falls, a more or less extended paralysis, paraplegia in the horse; stupefaction, dilatation of the pupil, trembling; spasms in the pig; heart weakness, small, slight pulse; dyspnœa. [It is not uncommon to see epileptiform convulsions preceding the paralysis in the horse and dog.-W. L. z.]

Death occurs from the sixth to the forty-eighth hour. When the disease lasts longer it is complicated by anemia; in the ox we frequently observe symptoms of chronic enteritis.

Ir. In poisoning from brine, we observe as well all the symptoms of a severe nervous excitation and spasms: grinding of the teeth, trismus, epileptiform convulsions, opisthotonos, pleurosthotonos; rotation of the eyes (nystagmus), convulsive shutting of the eyes, dilatation of the pupil, blindness; extended shock, vertigo, the sitting position of the dog, symptoms of immobility, stupefaction and insensibility; in cows, abortion and prolapsus of the uterus are frequent accidents.

1 The Veterinary Review for 1877 contains a very interesting note from Abadie (of Nantes) relative to poisonings of animals of the bovine species, produced by sulphuric acid. Two quacks of the Ancenis district poisoned stabled cows by means of this agent whenever they could gain entrance to a stable; they afterward called to see the affected beasts, pronounced them incurable, bought such animals for almost nothing, and sent the meat to the market of Nantes. In a few months they caused a damage of more than 25,000 francs in the six communities where they operated. -N. D. T. 
The course is very rapid; death occurs between the sixth and twelfth hours.

Pathological anatomy. The lesions of acute gastro-enteritis are particularly marked in the abomasum of the ruminant. The blood is liquid and vermilion in color; ecchymoses are found in the various organs. The mucous membrane of the bladder is red. Inflammatory alterations exist in the different intestinal compartments, especially when the poisoning is due to brine. We also find cerebral hyperemia as well as serous exudation into the cerebral ventricles and meninges.

Treatment. Abundant drinks : mucilages, oils, and stimulants (ether in subcutaneous injections, camphor, wine, etc.); also narcotics, when the excitement is very pronounced.

Drewien recommends hydrate of chloral and oil $(2: 60)$ for pigs that have been poisoned with brine.

In our domestic animals chloride of sodium is frequently used as a condiment and as a remedy in cases of digestive troubles; thus the poisonings caused by these salts are very frequent.

Death is due to gastro-enteritis and to the paralyzing action of the sodium. Fatal doses: for the ox, 3 to 6 kilogrammes; for the horse, 1 to 1.50 kilogrammes; for the pig, 250 grammes; for the dog, 3.7 per kilogramme of body-weight.

Intoxications from brine are also very common; they are especially observed in hogs fed with all kinds of salted kitchen waste; they are sometimes determined by the injection of food prepared in barrels that have been used in the transportation of herrings, or through the administration of herring-brine used to check a gastrointestinal affection. The water of salted or smoked meat, found in pork-butchers' shops, may produce poisoning in young dogs and pigs. The difference of action which exists between sea-salt and brine depends mainly upon the nitrate which is added to the latter; but the cerebral symptom caused by the brine can only be explained by the intervention of a specific nareotic poison acting upon the cerebellum and medulla oblongata, and which belongs to the ptomaine group (see Mycotic Enteritis).

\section{Poisoning by Sulphate of Soda.}

Symptoms. Colics, intense thirst, watery diarrhea, disappearance of peristaltic movements, paralytic condition, and increasing weakness. Death within a few days. 
Treatment. This is the same as that for poisoning by sea-salt.

The ox, relatively speaking, will stand well large doses of sulphate of soda; we have seen a heifer which ingested 5.5 kilogrammes within four days, without any inconvenience. But, if given on an empty stomach and in excessive quantity (2.5 to 3 kilogrammes), this salt may produce death.

\section{Poisoning by Nitre (Nitrates of Potash and Soda).}

Symptoms. Those of gastro-enteritis : colics, diarrhea, meteorism, vomitings, salivation, nauseas; lowering of the general temperature; weakness, staggering gait, falls, stupefaction, tremblings ; tumultuous and palpitating heart; polyuria. Sometimes death is apoplectiform, we may also observe at times spasms, rotation of the eyes, etc.

Course. It is generally very rapid; the animals may succumb within a few minutes. Usually the disease lasts from half an hour to several hours, exceptionally more than twelve hours.

Pathological anatomy. Hemorrhagic gastro-enteritis; cherryred, purple, or red-brown coloration of the mucous membrane of the stomach (abomasum), and the small intestine; superficial ulcerations of this membrane; red-brown liquid intestinal contents; hyperemia of the abdominal viscera; inflammation and ecchymoses of the kidneys and of the mucous membrane of the bladder. The blood is liquid, vermilion in color, or otherwise of bad aspect. ${ }^{1}$

Treatment. Emollients, narcotics, or stimulants.

Poisoning with the nitrates is odinarily the consequence of the confusion of this substance with sea-salt or sulphate of soda. But the animals may also be poisoned by licking walls covered with nitrate; by drinking from receptacles that have served in the washing of nitrate bags, and occasionally from Chilean nitrate used as manure.

1 The lesions of poisoning from nitrate of soda are almost similar to those of intoxication from malt; this resemblance has given rise to the supposition that la maladie de la pulpe was caused by nitrates which exists in great abundance in turnip roots (Gassend). But experiments, the results of which are as yet unpublished, have demonstrated that the injurious properties of pulp do not depend upon the salts it may contain (Arloing). About fifteen years ago, poisonings from nitrate of soda were very frequent in animals of the ovine and bovine species; they were caused by the water which had been used to wash manure-sacks: the ingestion of this liquid produced almost always a fatal gastro-enteritis. Nowadays, on farms, this washing is made in tubs, placed on the manure heap; thus nothing is lost from the manure, and the animals are protected (Rossignol, communicated note). 
The principal effects of the nitrates of potash and of soda are due to the nitric acid element; but potassium nitrate acts more quickly than sodium nitrate on account of the potassium element. (Fatal dose of nitrate of potassium in the dog, 0.02 per kilogramme of body-weight; that of nitrate of soda in the rabbit, per kilogramme of body-weight, is 3.9 grammes.)

The toxic property of Chilean nitrate is not at all due to impurities that it may contain. Pure nitrate of soda is prone to act more violently than impure nitrate employed as a chemical agent in composts (Fröhner). It has been advanced that these two salts introduced into the organism are reduced to nitrites, and that the oxygen becomes free and exerts a toxic action upon the blood; but the accuracy of this theory is not yet demonstrated.

The intensity of the effects of these agents is in inverse ratio to the fulness of the stomach.

\section{Poisoning by Tartar Emetic.}

Symptoms. Ulcerated stomatitis, salivation, vomitings, colics, diarrhea ; vertigo, trembling, spasms, paralysis ; small, hard, often imperceptible pulse; accelerated respiration; pneumonia. Rapid death.

Pathological anatomy. There is an inflammation and cauterization of the mucous membrane throughout the entire length of the digestive canal, and mainly in the stomach; pulmonary hyperemia, desquamation of the epithelium of the bronchial tubes, hemorrhagic infarct, pneumonia.

Treatment. Tannin (decoction of oak-bark, ink); also oils, mucilages, narcotic substances, etc.; ice pills for the dog.

Poisonings from tartar emetic are sometimes caused by the administration of an excessive dose of this agent, at other times by its too great concentration. It ought to be a rule not to give tartar emetic except in a very weak solution. The toxic action of this drug is partly due to the potassium (paralysis of the heart), as well as to antimony (fatty infiltration of the organs). Its principal antidote is tannin, which, with the oxide of antimony, forms an insoluble compound. Fatal dose for horse and ox, 25 to 30 grammes; for the pig, 6 to 8 grammes; for the dog and cat (except when there is vomiting), 0.2 to 0.5 gramme. 


\section{Arsenical Poisoning. (a.) Acute Intoxication.}

Symptoms. These are similar to those of gastro-enteritis: vomitings, salivation, intermittent colics, constipation, meteorism (in the ox), fetid diarrhea, and bloody excrements.

In ruminants we sometimes observe a painful tumefaction of the inferior abdominal region, formation of abscess and fistula of the abomasum, and even hernia of this organ; fistula of the reticulum (Detroye) is rare. To these signs are added nervous troubles: paralysis, staggering walk, trembling, weakness, stupidity, dilatation of the pupil, etc. The pulse is very much accelerated, small and hardly perceptible, the temperature of the body is irregularly distributed; the respiration is often accelerated and painful. There is albuminuria.

When the poison has penetrated through an exterior wound, the region is much tumefied. Arsenical baths produce dermatitis of variable intensity, accompanied by the symptoms which have just been mentioned.

The duration of this intoxication varies from a few hours to several days.

Pathological anatomy. Corrosive gastro-enteritis with redness, tumefaction, ecchymoses, erosions, and ulcerations of the mucous membrane of the stomach, and especially of the abomasum. Sometimes we detect the perforation of the abomasum and of the abdominal wall. Fatty degeneration of the different parenchymatous organs: liver, loins, heart, and brain. Numerous ecchymoses upon the endocardium.

Treatment. The principal and officinal antidote is the liquor of sulphate of iron, 100 parts ; water, 200 parts; add calcined magnesia and water, 250 parts; hydrate of the oxide of iron ${ }^{1}$ forms with the arsenic an insoluble combination.

For the dog, the dose of this antidote is a tablespoonful every fifteen minutes; for pigs and small ruminants several tablespoonfuls; for the horse and ox, a quarter of a litre to one litre. A simple aqueous solution of calcined magnesia $(1: 20)$ also produces

1 The hydrate of iron can also be obtained in the following manner: Dissolve 100 grammes of common suiphate of iron in 400 grammes of water, then add 20 grammes of concentrated sulphuric acid; boil the mixture in a stone jar, and add nitric acid as long as any red vapors are seen. Let the preparation cool, then dilute it with thirty times its weight of water, and precipitate with carbonate of soda.-N. D. T. 
an insoluble arsenical combination. Dose, to be given every quarter of an hour: for the dog, 0.5 to 1 gramme; for the horse, 5 to 10 grammes; for the ox, 10 to 20 grammes. When these preparations are not at hand, other ferruginous preparations may be used: sulphate of iron, rust-water, etc., also lime- or sugarwater, white of egg, and mucilages. Oil is counter-indicated.

The acute arsenical poisonings are by far the most frequent; they are seen in all domestic animals. Pure arsenic, its preparations, the yellow arsenic and red arsenic, arsenical paintings (arsenate of copper and Scheele's green), Fowler's solution, the arsenical vapors escaping from high furnaces where minerals are reduced which contain arsenic, poisons thrown around to kill rats, arsenical baths used for treatment of sheep mange, arsenous acid used as a caustic -these agents are the principal causes of arsenical poisoning. The intensity of the general effects depends upon the condition of the arsenical preparation which has produced the poisoning. Thus, dissolved arsenous acid produces general effects rather than local accidents; the contrary takes place when this agent is administered in a solid condition. The perforation of the abomasum and of the abdominal wall in the ox is due to the accumulation in the stomach of arsenous acid administered in powder or in small pills.

For the horse and the small ruminants the fatal dose is 10 to 15 grammes; for the ox, from 15 to 30 grammes; for the pig, 1 gramme; for the dog, from 0.1 to 0.2 gramme.

Fine pulverized arsenous acid put upon an external wound is fatal to the horse in doses of 2 grammes; to the sheep, 0.2 gramme; to the dog, 0.02 gramme. As a caustic it is much less dangerous.

\section{(b.) Chronic Arsenical Poisoning of the Ox (Disease due to Vapors of High Furnaces).}

Symptoms. They are : chronic diarrhea, cough; dry adherent skin, epidermic desquamation; muscular atrophy beginning with the hind quarters, weakness, paresis, paraplegia; emaciation and cachexia ; arrest of lacteal secretion, abortion, non-delivery, difficult fecundation (Haubner).

Pathological anatomy. There is a considerable emaciation of the cadaver, hydremia; sloughs, ulcerations, cicatrices of the abomasum, and more rarely of the rumen; redness, ecchymoses superficial inflammation, swelling and softening of the gastro- 
intestinal mucous membrane (Franze); ulcerations of the respiratory mucous membrane (Siedamgrotzky). Caseous or tuberculous broncho-pneumonia as a secondary lesion.

Treatment. Prophylactic. Be careful to discard all fodder which might be covered with arsenical dust. Treat symptomatically the diarrhea, as well as the emaciation and weakness.

The disease produced by vapors from high furnaces in the neighborhood of Freiberg must be considered as a chronic arsenical intoxication, judging from the description given of it by Haubner. The smoke of these furnaces is mainly formed by sulphuric acid and arsenous acid, with which are associated small quantities of lead and zinc vapors and traces of earthy matter. Lead and zinc cannot play any poisonous rôle here ; on the other hand, sulphuric acid does not exist in the dust which covers all plants in the environs and occasions poisoning. The poisoning under consideration, therefore, can only be produced by arsenic ; besides, arsenic is found in all the organs of animals which succumb to the intoxication. Pneumonia through inhalation of foreign bodies, described by Haubner and Siedamgrotzky under the name of "caseous pneumonia induced by fumes from high furnaces," is nothing ${ }^{1}$ but a true tuberculous broncho-pneumonia, in which the inhalation of dusts from the furnaces acts as a predisposing cause, favoring the penetration of the respiratory mucous membrane by Koch's bacilli.

The ingestion of considerable quantities of arsenical dust may also produce acute poisoning.

The "acid disease" (anemia and osteomalacia), attributed by Haubner to sulphuric acid contained in the smoke of furnaces, has nothing in common with chronic arsenical poisoning.

\section{Poisoning by Phosphorus.}

Symptoms. They are : colics, vomitings, complaints, icterus, albuminuria, dysphagia, tumefaction of the tongue; paresis, trembling; acceleration of the pulse and respiration, fever ; hemorrhages when the disease has a tendency to become chronic. The inhalation of phosphorus vapors produces cough, dyspnœa, and subcutaneous emphysema upon the neck, shoulders, and thorax, also 
pulmonary emphysema. In poultry we observe weakness, intense thirst, diarrhea, and choreic movements in walking. The duration of this poisoning varies from a few hours to several days. Within five or six days an apparent amelioration is frequently seen. Among a certain number of cows that have resisted a slight poisoning, Schindelka has noticed a complete suppression of lacteal secretion.

Pathological anatomy. The lesions are similar to those of acute poisoning; gastro-enteritis, sometimes stomatitis and pharyngitis, glandular gastritis (degeneration of the gastric glands); fatty degeneration of most of the organs, especially of the liver, heart, kidneys (epithelium), and of the muscles; bronchitis in cases of vapor inhalation; ecchymoses and hemorrhagic centres in the different viscera; black, non-coagulated blood; phosphorescence of the gastric and intestinal contents, with development of vapors of a characteristic garlic odor.

Treatment. Milk and fatty alimentary matters should be prescribed; as an emetic, sulphate of copper (to cause the formation of insoluble phosphate of copper); old essence of turpentine, rich in oxygen, administered in a large quantity of mucilage (from oxidation of the phosphoric acid, which is innocuous; it is given in the following doses : for the horse, 50 to 100 grammes ; for the ox, 100 to 200 grammes; for the pig, 25 to 50 grammes; for the dog, 5 to 10 grammes; and for the chicken, 5 to 10 drops. Also stimulants. Oil must be discarded, as it would dissolve the phosphorus and render it more soluble.

Phosphoric acid poisoning is observed in all domestic animals, including poultry. It always take an acute form. Chronic poisonings similar to those described in the human race (necrosis of the bones, mainly of the lower maxillary) have never been seen in our domestic animals.

This intoxication is ordinarily produced by the ingestion of poisons which have been prepared for the destruction of rats or mice, or phosphorus from matches, more rarely through inhalation of phosphoric vapors. ${ }^{1} \quad$ As is the case with all poisons, the effects of phosphorus are more rapidly developed and more accentuated when the stomach is empty. Death is due to the ozonization of

1 Phosphorus paste is indeed the most common cause of this form of poisoning, because it is readily taken by the animals. The dog, however, on account of the facility of vomiting, rarely succumbs after the ingestion of this substance.-L. T. 
the oxygen of the body through the phosphorus, and also through consecutive decomposition of the albumin of the parenchyma. Fatal doses: for the horse and the ox, 0.5 to 2 grammes; for the pig, 0.15 to 0.38 gramme; for the $\mathrm{dog}, 0.05$ to 0.1 gramme; for the chicken, 0.025 gramme.

\section{Mercurial Poisoning (Mercurialism).}

Symptoms. Besides the local caustic effects we observe the following manifestations :

1. Ptyalism, ulcerous stomatitis, fetid breath, softening of the gums, shrinking of the gums from the teeth in ruminants;

2. Symptoms of gastro-intestinal catarrh, profuse diarrhea, expulsion of greenish-gray excrements, which are liquid, bloody, and of putrid or cadaveric odor ;

3. Cough, purulent nasal discharge, broncho-blennorrhea, broncho-pneumonia, painful dyspnoa, complaints, sometimes epistaxis ;

4. Cutaneous eczema, and mainly the impetiginous or squamous forms, accompanied by an intense pruritus. The animals scratch themselves constantly; the hair falls out, herpes are formed which suppurate, scabs, vesicles, and pustules, resembling those of smallpox, and reproducing a vesico-pustulous eczema through inoculation; the skin and subcutaneous connective tissue become thickened. These alterations are generally located in the neighborhood of the eyes, the muzzle, anus, teats, and vulva, also upon the fetlock and folds of the articulations.

5. Weakness, paresis, apathy, stupefaction, vertigo, mercurial trembling, paralysis of certain muscles, emaciation, and death in coma; convulsions are very rare.

6. The temperature is normal or scarcely increased; the pulse is very accelerated and weak; hemorrhages are produced in different organs, and especially upon the nasal, pulmonary, intestinal, and uterine mucous membrane (abortion). Death may occur through internal hemorrhage.

Course. The course is very rapid in young animals, and when the stomach or intestine has been affected by the poison. Poisoning through sublimate sometimes causes death within a few hours and without producing symptoms of mercurialism. The duration of acute mercurial intoxication varies from ten to fourteen days. It may be chronic and persist for weeks and even months. Its symptomatic picture is ordinarily incomplete. 
Lesions of generalized MERCURIALisM. The skin and the subcutaneous connective tissue are quite anemic; the latter is the seat of a serous infiltration and of bloody suffusion in the neighborhood of the eczematous spots; the muscles, which are pale, soft, and of cooked appearance, are filled with ecchymoses and infiltrated with a gelatinous serum. The blood is black, mud-like, uncoagulated. The mucous membrane of the digestive canal is inflamed, ulcerous stomatitis (not constant), there are also ecchymotic blots or a diffuse redness, hemorrhagic erosions and ulcers in the stomach and in the abomasum, especially upon the salient points of the mucous membrane; an œdematous tumefaction is also found there; the submucous connective tissue is infiltrated; the sub-peritoneal connective tissue is œedematous, and dotted with ecchymoses. The liver is tumefied and anemic; the kidneys are infiltrated, anemic, and ecchymosed.

We also find alterations of rhinitis, laryngitis, trachitis, catarrhal bronchitis, and sometimes even croupous inflammation of the larynx and trachea. The infiltrated lung shows hemorrhagic centres on its sections, or small spots of broncho-pneumonia, or even abscesses; the bronchial and mediastinal ganglions are tumefied and infiltrated. We observe sub-pleural ecchymoses, degeneration of the myocardium, interstitial hemorrhages into the cardiac muscle and under its two serous coverings. The arachnoid is raised here and there by discharged blood; the cerebral substance is softened, watery, and shiny; its superficial layer contains small ecchymotic points.

Treatment. We must administer the sulphur compounds in order to form insoluble sulphate of mercury : flowers of sulphur, sulphate of potassium; iron filings and sulphate of iron; iodide of potassium. Symptomatic medication appropriate to gastro-enteritis, stomatitis, bronchitis, eczema, etc.

[In the treatment of poisoning with corrosive sublimate Orfila recommends the free use of the whites of eggs beaten up with water. The albumen forms an insoluble and comparatively innocent compound with the corrosive sublimate. It is, however, asserted by M. Lassaigne that this compound of albumen and corrosive sublimate when recently precipitated is soluble in acid and alkaline liquids and in solutions of the chlorides of potassium, sodium, and calcium, also in an excess of albumen, whether introduced into the stomach or previously existing there. It is, therefore, important 
when administering this antidote to evacuate the stomach before the new compounds can be dissolved.

-W. L. z.]

The principal causes of mercurialism belong to the imprudent use of mercurial salve which is in use as an antiphlogistic and parasiticide, also from calomel as a purgative, and from the sublimate bath for mange, also through its use in uterine irrigations or its internal administration. It is more rarely produced by red ointment or by poisons intended for rats. It is also observed in the vicinity of the factories in districts producing mercury (Idria); in such instances it is due to mercurial vapor inhalation.

The sublimate produces especially a corrosive gastro-enteritis, which may cause death before mercurialism has had time to appear. Fatal dose: for the ox, 4 to 8 grammes; for the horse, 8 to 10 grammes; and for the dog, 0.25 to 0.50 gramme.

Calomel in large doses is a violent poison for ruminants : 8 to 10 grammes of calomel produce grave symptoms of poisoning in the ox: a profuse obstinate diarrhea and mercurialism. We must avoid large doses of calomel, or not give it at all to this class of animals. This susceptibility of the ruminants to calomel is perhaps explained by the prolonged stay (three to four days) of the toxic agent in the digestive canal, and its more abundant transformation into sublimate. It is undoubtedly in the same manner that medium doses of calomel act in producing symptoms of poisoning in dogs suffering from serious constipation. ${ }^{1}$

Mercurial ointment is very dangerous for ruminants; it becomes so much more so when it is old and when a fatty acid is combined with a suboxide of mercury, which is very easily reabsorbed. In the ox an application of thirty grammes of mercurial ointment produces very marked symptoms of poisoning: eczema, stomatitis, diarrhea, respiratory troubles, general weakness, and internal hemorrhages.

The dog and the horse are much less sensitive than the ox to the action of mercurial ointment.

[Frequent instances have been brought to my notice of the great danger in the use of mercurial preparations in the dog. This danger exists not only in the absorbing power of the skin, but also in

1 The recent researches of Adam have demonstrated that the transformation of calomel into sublimate in the organism is very small, when even alkaline chlorides are present. - N. D. T. 
the almost absolute impossibility of preventing licking. It is, therefore, inadvisable to make mercurial applications to the skin of these animals. The horse appears to be far less sensitive than are other animals to the action of this drug when it is locally applied.-W. L. z.]

Mercury penetrates into the canals of the sebaceous glands in the shape of fatty suboxide salts of mercury, and may be absorbed. Another entrance for it is found in the lungs, into which the mercurial vapors penetrate. These produce, first, through their local action upon the mucous membrane of the respiratory organs (transformation into sublimate), symptoms of bronchitis and pneumonia, to which are soon added the manifestations of generalized mercurialism.

Sometimes mercurial poisoning is observed in a more or less large number of animals of the same stable, and takes thus an epizootic character.

\section{Lead Poisoning (Saturnism, Plumbism).}

I. Symptoms of acute poisoning.

They are those of gastro-enteritis : anxiety, salivation, vomiting, intestinal pains, obstinate constipation, meteorism in ruminants, fetid diarrhea, arrest of secretions (urine and milk). We observe, besides, nervous troubles : convulsive movements of the head, neck, and shoulders, saturnine trembling, trismus, epileptiform attacks, spells of vertigo, furious delirium, convulsions, choreic contractions; weakness, drowsiness, complete or partial paralysis, paraplegia (lead-paralysis), anæsthesia (lead-anæsthesia). The pulse is hard and evasive, often slow ; the respiration is difficult and dyspnœic.

II. Symptoms of Chronic Poisoning.

Lead herpes and cachexia, colics, troubles of locomotion (saturnine arthralgia and lead-rheumatism); the cerebral symptoms similar to acute poisoning (saturnine encephalopathy), epileptiform attacks (saturnine eclampsia); wheezing in horses and sterility in the goat; ulcerous stomatitis (blue line on the gums), and chronic nephritis (atrophied kidneys). The experimental researches of Ellenberger and Hofmeister have shown that there is a diminution of urea, a disappearance of hippuric acid, and albuminuria in the serious forms of saturnism.

Pathological anatomy. Paleness and retraction of the intestinal tube; upon the mucous membrane there is a circumscribed 
redness, inflammation, scarification, ulcerations, and gray or blackish coloration, due to the presence of metallic lead; thickness of this membrane in the chronic form; hydrocephalus and hydrorhachis ; the kidneys are atrophied. In apoplectic death no essential alterations are found. Ellenberger and Hofmeister have experimented upon sheep. They have produced a chronic diarrhea, fatty degeneration of the liver, turbid tumefaction of the hepatic and renal cells, diffused nephritis, and special degenerations of the renal epithelium. Mayer has observed fatty degeneration of the intestinal glands and a connective tissue proliferation in the neighborhood of the intestinal ganglions, with degeneration of the latter (cause of saturnine colics). Paralysis, amaurosis, and saturnine arthralgia are also the consequences of the inflammation and of hyperplasia of the connective tissue.

Treatment. Sulphuric acid should be given (in a mucilage or in drinking-water), or sulphates of soda, potash, or magnesia, with the object of forming insoluble sulphate of lead. Emetics, purgatives; white of egg, milk, mucilage. We can also use iodide of potassium, which favors the elimination of the lead. Symptomatic medication: morphia, chloral hydrate, for combating saturnine colics and eclampsia, etc.

Saturnism or plumbism is developed after the ingestion of lead shot (ox), pieces of lead, also lead vapors or dust, and fertilizers rich in this substance. This latter cause often generates enzootics of saturnism which are observed in the neighborhood of lead-producing establishments, and of factories where lead is used, or in localities which are situated along watercourses containing this substance (the Innerst in Hanover, the Bleibach, "lead river," in the Province Rhénane, etc.). Paints containing lead salts, lead acetate, sugar of lead, may also produce this poisoning. Lead exerts its physiological action upon the nervous system particularly, and not on the muscles. Oxen and poultry are very sensitive to it; the animals resisting its effect most successfully are the horse and dog. The cerebral symptoms of saturnism may induce one to suspect epilepsy or meningitis.

\section{Copper Poisoning (Cuprism).}

\section{ACute Poisoning.}

Symptoms. The symptoms are the same as those of gastroenteritis : vomiting of greenish matter; inappetence, colics, diarrhea. 
Later are observed weakness, trembling, convulsions, anæsthesia; small, evasive pulse, somewhat slow ; accelerated respiration.

Autopsy. The lesions observed are those of acute gastroenteritis.

Treatment. White of egg, mucilage, milk, iron filings, porphyrized iron, sulphur, calcined magnesia, may be used, as well as narcotics.

II. Chronic poisoning. (Produced upon the sheep for experimental purposes by Ellenberger and Hofmeister.)

Symptoms. We see albuminuria, icterus, hemoglobinuria, and later hematuria. There is great muscular weakness and emaciation; momentary constipation, derangement of the appetite and rumination; also diarrhea toward the end of life. Death occurs in convulsions.

Autopsy. Methemoglobinemia : the blood is dark, there are granules of hematin in the renal canals, also in the parenchymatous structure of the liver and spleen, etc. Parenchymatous hemorrhagic nephritis. Fatty degeneration and icteric coloration of the hepatic cells. Icterus, granular condition of the striated muscular fibres and myocardium; the spleen is enlarged; we observe also intestinal catarrh and pulmonary œedema.

These poisonings, which are very rare in our animals, are produced by damp food that has been kept in copper vessels, more rarely through sulphate of copper, copper alum, acetate of copper (verdigris), oxide or carbonate of copper, Egyptian ointment, paints with a copper base, etc. They are never produced by metallic copper. The copper salts are deposited and accumulate in the different organs, especially in the liver. The essential alteration of chronic copper poisoning is the destruction of red blood-corpuscles, the same as in poisoning through chloride of potassium (Johne, Ellenberger, and Hofmeister).

\section{Poisoning by Zinc.}

Symptoms. Vomitings, colic, diarrhea, weakness, and paresis; later anemia and cachexia.

Auŕopsy. There is anemia or atrophy of the intestinal mucous. membrane, gastro-enteritis in the shape of inflammatory centres and circumscribed erosions.

Treatment. We give white of egg, mucilage, sugar-water, sulphur, and calcined magnesia. 
This form of poisoning is very rare; it has, however, been observed, complicated with lead and arsenical poisoning, in the ox, in the pig, and in poultry, in the neighborhood of zinc works (Galmeiwasser), and likewise within the sphere of ore furnaces. The chemical toxic combinations are oxide, sulphate, carbonate and silicate of zinc.

\section{Poisoning by Carbolic Acid.}

Symptoms. Diminution of appetite, salivation, vomitings, diarrhea, slight colics, arched back ; dark urine, of a dirty greenishbrown color (hydroquinone), containing albumin, and diffusing a carbolic odor. Paresis, and paralysis of the hind quarters, or of the whole body, sudden falls, trembling, anxiety, clonic and tonic convulsions, stupor, coma, collapse. Lowering of the temperature, acceleration of the pulse, painful irregular respiration. Nephritis (presence of white and red blood-corpuscles in the urine, tube-casts). Local cauterizations when the poisoning is produced by concentrated solutions.

Pathological anatomy. Hemorrhagic gastro-enteritis, scarification of the mucous membrane; fatty degeneration of the liver and kidneys; parenchymatous degeneration of the heart; parenchymatous nephritis; blood thick, black and non-coagulated; pulmonary and cerebral hyperemia, œedema of the vascular coat of the brain, serous exudation in the cerebral ventricles, carbolic odor exhaled by all organs.

Treatment. We prescribe sulphates, and preferably Glauber salt, which transforms phenic (carbolic) acid into phenylsulphuric acid, which does not possess any toxic property;

Milk of lime, stimulants: ether, camphor, alcohol, coffee, and wine.

Poisonings through carbolic acid have been noticed since this agent has been used as an antiseptic. Frictions of carbolic acid, creosote, tar, and of impure wood vinegar, used for parasites, cutaneous affections, etc., also the bath in which these drugs are put, may determine intoxications of this character either by cutaneous absorption or through ingestion when the animals lick themselves. The cat is the most susceptible animal to carbolic acid: doses of 0.5 to 1 gramme are sufficient to kill it. The fatal dose for the dog is from 2 to 5 grammes. Munk has given a horse 100 grammes of carbolic acid at one time and 500 grammes within a week, without producing death. 
Besides the caustic action it exerts upon the mucous membrane and the skin, carbolic acid is also a nerve poison; the symptoms which it produces in the domestic animals prove this. In human beings convulsions are almost always wanting, which is a remarkable fact.

\section{Poisoning by Acetic Acid.}

Symptoms. Inappetence, colic, diarrhea, staggering gait, stupor, convulsions, collapse; acceleration of the respiration and circulation.

Treatment. Alkalines and stimulants.

This intoxication is very rare; it has only been observed after ingestion of very acid malt (change of alcoholic fermentation to the acetic form).

\section{Poisoning by Cantharides.}

Symptoms. Salivation, stomatitis, dysphagia, vomiting, colics, dysentery, tenesmus ; frequent micturition, strangury ; blood, albumin, and cylindrical epithelial cells in the urine; genital excitation, redness of the vaginal mucous membrane. General weakness, convulsions, falls. Accelerated pulse, which is light, evasive, almost imperceptible. Also conjunctivitis, but in some cases only.

AUTOPSY. The lesions of stomatitis, pharyngitis, laryngitis, acute gastro-enteritis, nephritis, and cystitis are found.

Treatment. Mucilaginous drinks and opium are advisable, in order to counteract gastro-enteritis. Stimulants-camphor is to be preferred to all others. Oily preparations should be proscribed.

Frictions that are too extended or concentrated, too large doses used as an aphrodisiac, and ingestion of cantharides with the food, form the principal causes of this intoxication. Fatal dose for the ox and the horse, 25 to 35 grammes; for the dog, 0.5 to 1 gramme. Whether poisoning is the result of cutaneous absorption, or ingestion of the cantharides, nephritis or gastro-enteritis predominates in the morbid changes. In experiments made upon the horse by Friedberger, 50 grammes of cantharides ointment applied upon the skin had no toxic effect, while 250 grammes of powder, administered internally, produced symptoms of intoxication within four hours.

\section{Poisoning by Croton Oil.}

Symptoms. Subacute gastro-enteritis with choleraic diarrhea; general weakness, exhaustion, evasive pulse. Death in one, two, or three days. 
Autopsy. Lesions of corrosive gastro-enteritis; sometimes stomatitis and pharyngitis.

Treatment. Mucilages and opium are to be given; later stimulants must be used.

Poisoning may be produced by croton seed as well as by the oil. It is rare to see poisoning produced by cutaneous absorption; this most frequently results from errors made by administering the drug in too large doses. Fatal doses : for the horse, 20 drops ; for the ox, 40 drops ; for the sheep, 15 drops ; for the dog, 5 to 10 drops.

\section{Poisoning by Euphorbia (Euphorbia lathyris).}

Symptoms. They are colic, constipation, abundant and bloody diarrhea; hematuria, tympanites; the pulse is light, the heart tumultuous. We observe stupor in the ox and sheep.

Autopsy. Lesions of acute gastro-enteritis.

Treatment. Symptomatic.

18. Poisoning by Mercurialis annua et perennis.

Symptoms. Gastro-enteritis, hematuria, strangury, sensitive condition of the region of the kidneys; accelerated respiration, tremblings; small, accelerated pulse.

In one case the symptoms appeared ten minutes after the ingestion of the plant.

Autopsy. We find lesions of gastro-enteritis and nephritis.

Treatment. Symptomatic.

H. Schulz has made experiments in feeding pigs and rabbits with Mercurialis perennis; none of these animals died. The urine showed a red coloration, but this was not due to coloring matters of the blood. The urinary secretion was considerably increased, the bladder much distended (paralysis of the bladder), and its neck was spasmodically contracted. The intestinal functions were diminished.

19. Poisoning by Daffodils (Narcissus poeticus et N. pseudonarcissus).

Symptoms. Intense gastro-enteritis, abundant diarrhea; stupor and weakness; convulsions and spasms.

Autopsy. Lesions of gastro-enteritis.

Treatment. Symptomatic.

The stem and bulbs are poisonous. 


\section{Poisoning by Laurel (Nerium oleander).}

Symptoms. Gastro-enteritis, abundant diarrhea, bloody excrements, colic; weakness, muscular tremblings ; polyuria ; small pulse, tumultuous heart-action.

Autopsy. Lesions of acute gastro-enteritis, with yellowishbrown coloration of the mucous membrane.

Treatment. Symptomatic.

Oleandrine and nereine, alkaloids extracted from rose laurels, have the same action as digitalis.

\section{Poisoning by Rape Cake containing Oil of Mustard.}

Symptoms. Chronic enteritis, meteorism, constipation, diarrhea, bloody excrements; suppression of the lacteal secretion. Cerebral excitement; forward movements, vertigo (Anacker). Abortion.

Autopsy. Lesions of hemorrhagic enteritis.

Treatment. Symptomatic.

Poisoning from rape cake is at times observed in the $o x$; it is due to an irritating oil, similar to oil of mustard, which is formed when the cakes are damp, and which is very dangerous to young animals. The rape cakes are harmless when in a dry condition and after having been cleaned of their oil by means of carbon bisulphide or by prolonged cooking.

\section{Poisoning by Pepper.}

Symptoms. They are those of gastro-enteritis (in the pig).

Autopsy. We detect an inflammation of the mucous membrane of the stomach and intestine.

Treatment. Symptomatic.

\section{Poisoning by Acorns.}

Symptoms. Digestive troubles. In serious cases gastro-enteritis with obstinate constipation; later, diarrhea, tenesmus, dysentery, bloody discharge through the rectum, and extreme weakness. According to Simonds and Brown, the manifestations of poisoning by acorns are said to have a great similarity to those of bovine pest.

Autopsy. Lesions of intense gastro-enteritis, especially will marked in the third stomach ; and excoriations of the buccal mucous membrane. 
Treatment. Symptomatic.

The poisonings from this source detected in England during the years from 1868 to 1879 were observed in all the domestic animals except the pig. The green acorn showed itself to be extremely dangerous.

\section{Poisoning by Aloes.}

Symptoms. There are colics and profuse watery diarrhea; weakness; the pulse is imperceptible. Death occurs within two to five days.

Autopsy. Again we see lesions of gastro-enteritis. In cases where the poisoning has lasted for a long time, the alterations of enteritis are not observed, but only emptiness and paleness of the intestine.

Treatment. We must make use of drugs that will arrest secretion and soothe the irritated mucous membrane.

The main causes of these poisonings are the administration of excessive or repeated doses at too close intervals, also colds or violent efforts after the administration of aloes.

\section{Poisoning by Mezereum-Garou Bark (Daphne gnidium).}

Symptoms. We observe stomatitis, salivation, colics, vomitings, diarrhea, exhaustion, and a small and weak pulse.

Autopsy. The same lesions of gastro-enteritis are revealed.

Treatment. Symptomatic. The toxic factor is an acrid resin called mezereon.

26. Poisoning by Wild Horse-radish (Cochlearia armoracia).

Symptoms. We see violent colics.

Autopsy. A gastro-enteritis, with gelatinous infiltration of the walls of the stomach, is well marked.

Treatment. Symptomatic.

27. Poisoning by Swallow-wort (Asclepias vincetoxicum).

Symptoms. As a rule, polyuria, strangury, general weakness, and cachexia.

Autopsy. Lesions of nephritis and cystitis.

28. Poisoning by Cotton-seed Meal (Baumwollsaatmehl).

Etiology. This form of poisoning, described lately by Völker, Stein, Gautier, Werner, Emmerling, Stutzer, Bongartz, and others, 
seems to be the result of a chemical agent, rather than from special fungi.

Symptoms. Young animals are most frequently affected. Tympanites, diarrhea, dysentery, and hematuria are to be observed. The duration of the disease is very variable. ${ }^{1}$

Autopsy. In the acute form we detect a hemorrhagic gastroenteritis, tumefaction of the liver and kidneys, pulmonary odema, also exudations into the serous cavities; the urine is dark-red, the spleen is not enlarged. In the chronic course we see cachexia and dropsies, sometimes a parenchymatous nephritis.

Treatment. Symptomatic. Prophylactic.

\section{Poisoning by Turpentine Plants (Enzootic Gastro-enteritis; Disease of the Forest).}

Etiology. This intoxication is produced by the ingestion of young stems of conifera or acrid plants, also through astringent or irritating vegetation (brush, cowberry, myrtle, rush, and alder bush, etc.). It therefore produces an essential form of poisoning from turpentine.

It is especially observed in the spring. It is the disease described by Chabert (1787) under the name enzootic gastro-enteritis, and which has been ascribed by him to the ingestion of young willow. It was on account of its former frequency, and its connection with various poisons, that it received the improper name of enzootic gastro-enteritis.

$\mathrm{W}$ illows have gradually become scarcer in the woods nowadays, and the disease shows a tendency to disappear. Following Delafond's example, we have classified it in the group of gastro-enteritis produced by poisoning.

Symptoms. Chronic gastro-enteritis, disturbed appetite, colics, constipation, infrequent defecation, dry excrement coated with blood and mucus; more or less intense fever; besides diarrhea, emaciation and weakness. Irritation and inflammation of the kidneys and bladder; hematuria, ischuria, strangury, and sensitive condition of the region of the loins. As a rule, it lasts several weeks.

1 Rossignol has proven that cotton-seed cakes, recommended for milch cows, are poisonous for young animals, especially for lambs at the age of three or four months. Symptoms of peritonitis and ascites are seen, the animals weaken rapidly, and die in an advanced state of cachexia. In Egypt it has been observed that these cakes do not produce any poisoning when they are completely decorticated. The toxic principle seems to be located in the brownish pellicle which covers the cotton-seed.-N. D. T. 
Autopsy. The autopsy shows great emaciation; also inflammatory alterations of the stomach (abomasum) and of the intestine; besides degenerative alterations of the kidneys; enlargement of the liver and the spleen. Finally, lesions of asphyxia.

Treatment. We apply prophylaxis and treatment as for common gastro-enteritis, such as mucilages, cutaneous frictions, hydrotherapeutic compresses, etc. When the weakness increases we give stimulants. We should combat nephritis with tannin and sugar of lead. (See Acute Nephritis.)

Enzootic gastro-enteritis is commonly designated under the name of hematuria. We shall thus return to its consideration in the articles on Hematuria and Hemoglobinuria. We only wish to point out gastro-enteritis as an affection consecutive to the ingestion of resinous and terebinthine plants.

II. TOXIC GASTRO-ENTERITIS PRODUCED BY ACRID NARCOTICS. (ACRIA NARCOTICA.)

\section{Poisoning by Colchicum-Meadow Saffron (Colchicum autumnale).}

Symptoms. They are : acute gastro-enteritis, inappetence, vomiting, colic, violent diarrhea, sometimes bloody; meteorism, dysphagia (Dentler); hematuria, polyuria. We see also apathy, a more or less deep somnolence, weakness which is especially pronounced in the hind quarters, trembling, staggering walk, falls, difficulty or impossibility of rising. The pulse is small, imperceptible; the horse is subject to periodic cardiac palpitations (Friedberger); the extremities are also chilled, and there is excessive sweating, the mucous membrane is of a livid coloration; the pupil is dilated; the breathing accelerated. Death occurs within one to three days.

AUTOPSy. Hemorrhagic gastro-enteritis is present; numerous ecchymoses; the blood is black, thick, non-coagulated; the liver undergoes fatty degeneration (in one case on record).

Treatment. We prescribe tannin, astringents, opium; emollients. We perform rumenotomy in the ox. Symptomatic treatment of the gastro-enteritis and of the paralytic condition.

Poisonings through meadow saffron are quite frequent (ox, horse, sheep, pig). They are seen at the time of ripening of the plant (in 
May and June). Certain kinds of hay may be found containing half their weight of meadow saffron.

This plant exerts a local inflammatory action (upon the stomach and intestine), and a paralyzing action upon the central nervous system.

\section{Poisoning by Tobacco (Nicotiana).}

Symptoms. They are regurgitations, nauseas, vomitings, ptyalism, colic, tympanites, diarrhea (intestinal tetanus), polyuria. Also great muscular weakness, trembling, falls, with inability to get up again, paralysis, clonic and toxic contractions of the striated muscles, opisthotonos, diaphragmatic spasms, contractions of the muscles of the eye, and protrusion of the pupillary body. Stupor and coma. Accelerated beating of the heart, of a violent and tumultuous nature; chilling of the extremities ; difficult respiration.

Course. The course is mostly rapid, and the animals succumb in less than a day. When the tobacco has penetrated through the skin the intoxication is subacute, and death may happen within an hour. The convalescence may last from eight to fifteen days.

Autopsy. We find, generally, hemorrhagic gastro-enteritis (which is absent in endermic poisoning); also sub-pleural and subperitoneal ecchymoses, etc. The blood is dark and non-coagulated; and pulmonary and cerebral hyperemia is present.

Treatment. We give tannin (it precipitates the nicotine). Also stimulants; black coffee. We perform rumenotomy upon the ox.

Nicotine, which is an acrid narcotic alkaloid of the tobacco plant, is very dangerous for ruminants. The poisonings may be produced by nicotine baths (in mangy sheep), or lotions made of decoction or infusion of tobacco. The effects of nicotine are especially intense when this factor reaches the organism through the epidermis. Fatal doses of tobacco leaves: for horse and ox, 300 to 500 grammes; for the goat and the sheep, 30 grammes. The half-withered leaves are the most dangerous.

\section{Poisoning by Yew Leaves (Taxus baccata).}

Symptoms. As usual, we observe weakness, staggering, falls, convulsions; apoplectiform death from fifteen minutes to an hour. More rarely the disease lasts from several hours to several days; we then observe symptoms of gastro-enteritis : nauseas, foaming salivation, vomitings, tympanites, constipation, polyuria, stupor, 
staggerings, falls, trembling, spasms-the last being often epileptiform. The pulse is slow and small; the extremities cold.

Autopsy. Gastro-enteritis (inconstant) is present; also cerebral and odemic hyperemia; the blood is dark and non-coagulated.

Treatment. This consists of purgatives and a symptomatic treatment. Also rumenotomy in the ox. Generally, the rapid course of the poisoning renders all intervention impossible.

The toxic property of the yew tree is well known; its action depends upon a narcotic principle isolated by Marmé-the so-called taxine. The effects of this alkaloid are more pronounced when the stomach is empty than when it is full; the organism seems to become accustomed to it after a certain length of time, and the immunity enjoyed by some animals to the toxic effects otherwise resulting from the ingestion of yew leaves is thus explained. In practice the poisoning is always produced through a direct ingestion of the leaves.

Fatal doses : for the horse about 200 grammes of leaves; for the pig, 75 grammes ; for chickens, 30 grammes. The caustic action exerted upon the digestive mucous membrane seems to depend upon formic acid.

\section{Poisoning by Digitalis.}

Symptoms. We observe salivation, nauseas, vomitings, colic, and diarrhea. The heart-action is at first slow, later accelerated; there are palpitations, cardiac sounds of a metallic resonance; the pulse is small, irregular, and dicrotic, and is imperceptible toward the end of life. Polyuria, albuminuria, and staggering exist. The excitement is intense; later we see stupor, weakness, and staggering, paralysis of the lower lip, contraction of the pupil, and spasms.

Autopsy. Gastro-enteritis, endocarditis, myocarditis ; the heart arrested in diastole; evidences of suffocation (hyperemia and hypostasis in the lungs, ecchymoses and liquid blood).

Treatment. There is no specific antidote. The treatment is symptomatic-sedative or stimulating. Tannin may be tried. The principal stimulant is camphor.

Poisoning by digitalis is produced by the ingestion of the plant, or by very large therapeutic doses. The horse is particularly sensitive to its action. Fatal doses of dry leaves : for the horse, 25 grammes; for the dog, 5 grammes. The official extract of digitalis is very inconstant in its effects. 


\section{Poisoning by Flax (Linum usitatissimum).}

Symptoms. Violent colics, meteorism, diarrhea, staggering, trembling, cardiac palpitations, and death in convulsions.

Autopsy. Lesions of gastro-enteritis; evidences of asphyxia.

Treatment. Tannin, purgatives; the treatment must be symptomatic.

Flax, which is ordinarily innocuous, sometimes causes poisoning in the pig, sheep, cow, and in poultry; this toxicity is due to an acrid narcotic poison (linine) contained in the seed capsuleswhich may also be formed in all sickly etiolated plants. After having thrown flax into small rivulets, Gerlach has seen fish overcome by coma and death.

\section{Poisoning by Box Leaves (Buxus sempervirens).}

Symptoms. Vomitings, colic, diarrhea, coma, and vertigo alternately. Death occurs rapidly in convulsions.

Autopsy. Gastro-enteritis and asphyxia.

Treatment. Symptomatic. Tannin is advisable.

The box tree, which belongs to the family Euphorbiaceo, contains an alkaloid identical with berberine and very closely related to taxine, called buxine. It is a narcotic and a paralyzant of the nervous centres. The leaves give rise, in the horse and pig, to an intoxication which is rapidly fatal.

7. Poisoning by Black, Green, and Fetid Hellebore (Helleborus niger, $H$. viridis, $H$. foetidus).

Symptoms. They are vomitings, colic, bloody diarrhea, coma, stupefaction, weakness; death during convulsions.

Autopsy. Lesions of gastro-enteritis and asphyxia.

Treatment. Symptomatic. The stimulant to be given is camphor.

The animals may be poisoned by ingesting hellebore growing in the wild or cultivated state, or as ornamental plants. The poisoning is also produced by the hellebore troche or by setons strengthened with the powder of this plant. Hellebore contains two glucosides: helleborein and helleborin.

\section{Poisoning by Veratrum.}

Symptoms. They are nearly the same as in intoxication through hellebore, except for the reduced intensity of the gastro-enteritis, 
which, indeed, is entirely absent when hellebore is used externally. The principal symptoms are: nausea, violent vomitings, salivation, colic; clonic and tonic (even tetanic) contractions; intense excitation, and later paralysis.

Autopsy. Lesions of asphyxia.

Treatment. Symptomatic.

Poisoning by veratrine is almost always the consequence of blunders occurring in the use of this agent or plants containing it. Poisoning may be observed after the preparation of lotions with a decoction of rhizome of veratrum. Veratrine is extracted from the seeds of a melanthacea, the Veratrum Sabadilla officinalis. The white hellebore contains several active principles : first, pseudojervine ; second, jervine ; third, veratralbine; fourth, veratrine (very small amount). Official veratrine is a mixture of the various alkaloids, but mainly of cevadine (principal alkaloid), veratrine, and cevadilline.

9. Poisoning by Crow-foot (Ranunculus acris, $R$. arvensis, $R$. sceleratus).

Symptoms. They are nauseas, vomitings, salivation, colic, diarrhea, albuminuria; staggering, stupefaction, trembling, falls, apoplectiform death.

Autopsy. Lesions of gastro-enteritis and of asphyxia.

Treatment. Symptomatic. Tannin is advisable.

10. Poisoning by Aconite (Aconitum napellus).

Symptoms. Stomatitis, salivation, nauseas, vomitings, grinding of the teeth, diarrhea, violent colic, meteorism; weakness, paralysis, trembling; mydriasis, loss of consciousness, falls. Death occurs within a few hours, during convulsions.

Autopsy. Lesions of gastro-enteritis ; cerebral hyperemia.

Treatment. Symptomatic. Stimulants, especially camphor ; tannin.

Poisoning by aconite is observed in the goat, and is due to Aconitum napellus. Toxic alkaloid: aconitine.

11. Poisoning by Poisonous Hemlock: Water Hemlock: Cowbane (Cicuta virosa).

Symptoms. Violent gastro-enteritis ; vertigo, stupefaction, staggering gait or immobility, weakness, paralysis; muscular spasms 
of an epileptic nature, tumultuous heart-action. Death in convulsions within twenty-four to forty-eight hours.

Autopsy. Lesions of gastro-enteritis, asphyxia, cerebral œdema. Treatment. Tannin, narcotics, chloral hydrate.

Hemlock poison is an alkaloid, obtained by Böhn and designated by the name of cicutine; it is a very violent spasmodic.

\section{Poisoning by the American Water Hemlock (Conium maculatum).}

Symptoms. Salivation, nauseas, stupefaction, staggering, paralysis, convulsions, and rapid death.

Autopsy. Lesions of gastro-enteritis.

Treatment. Tannin, stimulants, and camphor.

13. Poisoning by the Small Hemlock (Ethusa cynapium).

Symptoms. Salivation, nauseas, stupefaction, paralysis, and convulsions.

Autopsy. Shows gastro-enteritis.

Treatment. The same agents as for the large hemlock.

This plant is much less dangerous to our domestic animals than the other hemlocks; Harley thinks that many authors have confounded it with the great hemlock in the cases of poisoning which they have mentioned.

\section{Poisoning by Field Poppy (Papaver Rhoeas).}

Symptoms. They are: ptyalism, colic, constipation, tympanites, bloody diarrhea; rabiform attacks in the ox, bellowings, grinding of the teeth, aggressive tendency, butting with horns, biting, violent and wild movements; epileptiform spasms, falls, collapse, somnolence, staggering, and genital excitement. The duration of these attacks is ordinarily about seven hours. Their termination is rarely fatal.

Autopsy. Lesions of gastro-enteritis, nephritis, and asphyxia.

Treatment. Tannin. Cold douches upon the head. We must combat the somnolence with stimulants-for instance, with ether, camphor, etc.

This poisoning is produced by an acrid narcotic agent contained mainly in the tops of green poppies (in June and July); it has, therefore, nothing in common with opium poisoning, which, besides, is very exceptional in our animals. Intoxication by the Papaver somniferum (rare) is expressed by nearly the same symptoms. 
Gerlach has observed a chronic poisoning in the horse, caused by Papaver rhoeas; after ingestion the cut straw was found to contain a large quantity of poppies. The symptoms were especially marked by anæsthesia and immobility.

\section{Poisoning by Fennel Seed (Agrostemma githago).}

Symptoms. Colic, regurgitation, vomitings, salivation, dysphagia, diarrhea ; paralysis, trembling, dizziness, and dyspnœa.

Autopsy. Lesions of gastro-enteritis. Hyperemia of spinal cord and brain; softening of the medullar substance; evidences of asphyxia.

Treatment. Change of diet. Purgatives and stimulants.

Lately we have observed poisoning produced by the mixture of fennel seed with flour or bran; these accidents have been observed in the pig, calf, and cow. Tabourin has described numerous poisonings observed in sucking calves which had received flour containing fennel dust. According to Malaport, the acrid narcotic poison of fennel-githagin-presents characters similar to saponin. For the dog 16 grammes of fennel flour is fatal. Fennel may also produce a chronic poisoning.

\section{Various Poisonings by Acrid Narcotic Plants.}

We have observed gastric and nervous troubles produced by rhododendron, stramonium, staphysagria seed, Aristolochia clematitis (upon the cow), ${ }^{1}$ the leaning chevril, belladonna (paralysis, dilatation of the pupil, gastric troubles), and also by the Ailanthus glandulosus.

\section{Poisoning by Ergot of Rye: Ergotism.}

Symptoms. (a) Acute poisoning. Gastro-enteritis, sometimes having a certain similarity to bovine pest; salivation, vomitings, colic, diarrhea ; ulcerous stomatitis similar to that of aphthous fever. In bearing females, parturition pains, abortion, and prolapsus of the uterus. Vertigo, stupefaction, anæsthesia, paralysis, dilatation of the pupil, spasm of the flexors (spasm of ergotism).

(b) Chronic intoxication. Gangrene of the extremities (gangrenous ergotism); sterility, enzootic abortion (Heusinger).

Autopsy. Lesions of gastro-enteritis.

1 Schwarz: Adam's Wochenschr., 1887.

2 Curaven-Cachiu: Annal. de Bruxelles, 1885. 
Treatment. Tannin; vaso-dilatators and anti-spasmodics: chloral hydrate and morphine.

Ergot of rye (the persistent mycelium of Claviceps purpurea) contains, according to Robert, cornutine, sphacelic acid, and ergotinic acid (which has no effect upon the uterus).

The poisonings which it produces, especially chronic poisonings, are rarer in domestic animals than in the human race. They have, nevertheless, been observed in the pig, the horse, the ox, the sheep, and in poultry.

\section{Poisoning by Iodoform.}

Symptoms. 1. The acute poisoning is announced by slight gastric disturbances (loss of appetite, vomiting, constipation), also by somnolence, with coma and stupefaction, alternating with convulsions. Dogs sometimes show from the outset an intense excitement and rabiform symptoms. We see, in addition, a considerable lowering of the temperature, disturbances of circulation, small accelerated pulse, tumultuous heart, dyspnœa, oliguria, albuminuria.

2. Chronic poisoning has the appearance and course of chronic iodism ; emaciation, atrophy of the glands and mainly of the teats, iodic eczema, catarrh of the mucous membranes, and especially of the pituitary (iodic coryza), also of the conjunctiva, of the mucous membrane of the larynx and of the bronchial tubes.

Pathological anatomy. We see a fatty degeneration of the liver, the myocardium, the striated muscles, and in some cases glomerulo-nephritis.

Treatment. The first indication is to remove the poison from the stomach by the use of emetics. There is no specific antidote; large doses of starch may be tried. The treatment is symptomatic, with stimulants.

Cases of poisoning by iodoform have been observed in the dog. As a rule, they are the consequence of the licking of wounds covered with iodoform powder. According to Poljokow, the fatal dose for warm-blooded animals is 0.5 to 1.5 gramme per kilogramme of body-weight. When the drug is absorbed through a wound the toxic dose will be less. 


\section{LUPINOSIS OF THE SHEEP: ACUTE ICTERUS: ACUTE YELLOW ATROPHY OF THE LIVER.}

Historical. Isolated cases of lupinosis were seen about 1860 by Wienands, Liebscher, and Güttlich. The first observations of lupinosis taking an enzootic course date from 1872. They became more and more frequent till 1875. At that date, at the request of the Prussian Minister of Agriculture, researches were begun in the veterinary schools of Berlin and of Hanover, in order to determine the nature of the disease.

The regions where lupinosis made its first ravages were those whose soil is particularly favorable to the culture of the lupin : in Pomerania, in the provinces of Posen and Brandenburg, western Prussia, and Silesia; later it reached Hanover, and finally all northern Germany. It is unknown in southern Germany.

The losses to agriculture caused by lupinosis are considerable. Often one-half or three-fourths of the flocks attacked die. In some districts of Pomerania its annnal mortality amounts to several thousands of sheep. In the Bulletin of the Royal College of Social Economy of Prussia for February 3,1880,Von Below-Saleske asserts an annual loss of 14,000 sheep, and consequently 13,000 lambs, in an ovine population of 240,000 head in one Pomeranian district alone.

Lupinosis may also attack the goat, the horse, the ox, and the stag. The dog contracts it experimentally. The rabbit is refractory to it.

Etiology. The race, the age, and individual characteristics are almost without influence in the development of lupinosis. It has, however, been observed that the lambs and mothers furnished a larger percentage than the rams and wethers-a fact which is easily explained by their stronger constitution. The yellow lupin (Lupinus luteus) is the most toxic of all the different lupins.

The nature of the toxic agent found in the lupin has given rise to several hypotheses :

1. The farmers have ascribed it to the exhaustion of the soil. Weakened by too numerous crops, it was said to be unable to produce anything but weak and sickly plants, in which a certain toxic principle was developed. But the study of this theory does not confirm it: lupins growing upon virgin ground may determine 
lupinosis, and, on the other hand, this affection has never been seen on some farms where the lupin has been cultivated for many years.

2. It has been asserted that lupinosis was produced by very fine toxic dust deposited upon the lupin; but Dammann (1876) has demonstrated the worthlessness of this theory.

3. Veterinarians supposed that under the influence of exclusive lupin-alimentation there was formed an excess of albuminoid matters in the blood, causing the manifestations of lupinosis. This doctrine, like the preceding, is not upheld by a close examination of facts. Sometimes but very small quantities of lupin are required to cause the disease, and it has been observed on several farms that certain portions of the field produce exclusively hurtful lupins, while those harvested in other places are altogether harmless.

4. The toxic action of lupins was attributed to fungi (Zürn). But this theory again is in opposition to the facts. It has against it, first, the absence of fungi in the anatomical alterations found at the autopsy of animals that have succumbed to lupinosis. On the other hand, it has been demonstrated by experience that lupins covered with mould and partially decomposed often remain quite innocuous, while plants which seem to be perfectly sound produce lupinosis. Moreover, it has been found that the upper layers of lupin kept in stacks in the open air are harmless, while the lower layers were found to be toxic-a fact which does not point in favor of the fungous nature of the affection. Finally, attempted transmission of lupinosis by inoculation has not succeeded. The harmful principle of lupins may be extracted with a solution of soda (2-3 per cent.), and the extract thus obtained does not contain any fungi. In Lemke's and Arnold's experience, the toxic lupins caused lupinosis after having been in rectified alcohol during four weeks; the ingestion of the alcoholic extract did not produce any morbid phenomena. If there were really any pathogenic fungi, the alcohol must have killed them.

5. The theory attributing lupinosis to the action of the alkaloids of the lupin has decided experimental facts against it. The researches of Brümmer and Krocker ${ }^{1}$ have demonstrated that the toxic lupins frequently contain fewer alkaloids than the inoffensive ones. On the other hand, Liebscher has established that the anatomical symptoms and alterations in lupinosis differ essentially

1 Brümmer and Krocker: Landwirthschaftl. Jahrbüch, 1880. 
from those found in the ease of intoxications by alkaloids, which act as nervous poisons. Finally, Arnold has produced lupinosis with lupins stripped of their vegetable alkaloids.

These results have led to the admission that the pathogenic agent of toxic lupins must be a chemical poison which is not classified. The empirical controversy concerning the non-inoculability of the superficial layers of the stacks kept in the open air also indicates that the rain has washed away the poison. The intensity of the disease is generally in relation mainly with the quantity of lupin ingested; however, the various lupins may show different toxic properties. By experiments we may produce, at will, an acute or chronic lupinosis by varying the dose of toxic extract. Kühn has proposed the expression ictrogene in order to denominate the toxic agent of lupins. Arnold and Schneidemühl designate it as lupinotoxine, which is preferable to the former term, the signification of which is correct for some only of the cases of lupinosis.

Properties of Lupinotoxine. Of slight solubility in pure water (Kühn), lupinotoxine is very soluble in alkaline water (Arnold, Roloff). It resists dry heat, and a temperature of $100^{\circ}$ C. prolonged for three hours (Kühn). Steam acting for several hours under the pressure of one to one and a half atmosphere weakens the poison, and at the pressure of two atmospheres destroys it completely.

Preparation of Lupinotoxine. Lupinotoxine is especially abundant in the fruit, in the husk as well as in the grain. The following is the mode of preparation, according to Arnold and Schneidemühl: The finely pulverized plant is macerated for two hours in a solution of soda $\left(1 \frac{1}{2}: 100\right)$ at $40^{\circ}$ to $50^{\circ} \mathrm{C}$. The filtered liquid is concentrated in a water-bath at $60^{\circ} \mathrm{C}$., then add acetic acid and filter again; treat it then with a solution of acetate of lead; the filtered solution is then saturated by hyposulphuric acid, then it is concentrated at a temperature of $50^{\circ} \mathrm{C}$., and, finally, mixed with fifteen times its volume of alcohol at $98^{\circ}$. Within twenty-four hours a precipitate will be formed (a brown resinous matter) which is dried and dissolved in water, then treated with acetate of lead and ammonia ; the precipitate is washed with distilled ammonia water, with alcohol and ether, then put in water and decomposed by means of hydrosulphuric acid. After that we filter, concentrate at $70^{\circ} \mathrm{C}$., and add ten times its volume in alcohol at $98^{\circ}$. 
Nothing positive is known about the mode of formation of lupinotoxine. It is generally admitted that the poison is a product of transformation, a result of germination on the surface of the plant of a certain micro-organism, which would thus become the indirect cause of lupinosis.

Anatomical alteriations. Parenchymatous inflammation of the internal organs, and mainly of the liver, kidneys, etc.; also lesions of icterus and of a "chronic hemorrhagic diathesis."

1. The alterations of the liver are those of simple acute hepatitis. Its volume is normal or increased. This depends upon the condition of the organ, whether or not it has undergone fatty degeneration; the hepatic cells are the seat of granular change and of a turbid albuminoid tumefaction; then they are affected by fatty degeneration; their volume is increased, and they present, toward their periphery, albuminous granulation and fat-corpuscles. The degree of fatty infiltration of the liver seems to depend upon the obesity of the animals. Schneidemühl admits that, as in poisoning by phosphorus, the fat is transported from the subcutaneous connective tissue into the liver. This organ is soft and friable. At this stage of softening, during which most animals succumb, an atrophic change follows (acute yellow atrophy of the liver, Schütz) which is characterized by the resorption of the liquid contents of the hepatic cells. The liver shrinks considerably; according to Schütz, the atrophic period lasts a couple of weeks; but it is not certain, it is even doubtful, whether this hepatic alteration is identical with the acute yellow atrophy of the human race.

In chronic lupinosis, the alterations of the liver consist mainly in a chronic interstitial hepatitis, with hypertrophy of the interlobular connective tissue; the liver is small, hard, uneven, very irregular on its surface; we then detect the consecutive lesions of blood stagnation in the portal vein (ascites, odematous tumefaction of the spleen and of the intestinal mucous membrane, etc.).

2. Icterus shows itself in the first place in the liver, which becomes of a lemon-yellow, yellowish, or reddish shade. It is hepatogenic, which is due to the catarrh of the bile canals; we observe sometimes the icteric coloration of certain organs or tissues which are more or less distant from the liver; for instance, upon the subcutaneous connective tissue, the skin of the belly, the omentum, upon the mesenteric, etc. The gall-bladder is often much distended by the bile; its mucous membrane is red and tumefied. 
3. The kidneys show parenchymatous alterations similar to those of the liver, but less marked. We see a parenchymatous nephritis, turbid tumefaction of the epithelial cells of the renal canals, the existence of cylindrical exudates in Henle's tubes, tubuli uriniferi, etc. Finally, we find in the bladder, which is almost empty, alterations showing catarrhal cystitis.

4. In the digestive apparatus we observe the icteric coloration of the mucous membrane, a catarrhal parenchymatous gastritis (glandular), especially marked in the abomasum, an inflammatory redness with hemorrhages in the small intestine, with catarrhal lesions of the entire intestinal canal.

5. The myocardium is pale and friable; the blood contained in the heart and the large veins is dark and thick; when exposed to the air it coagulates and returns to its vermilion color. In most organs (intestine, skin, subcutaneous connective tissue, peritoneum, uterus, meninges, etc.) there exist capillary hemorrhage.

6. The cadavers are, as a rule, emaciated. The muscles are of a gray-yellowish hue; their fibres are granulous, they have undergone fatty degeneration, they have sometimes lost their transverse striation. The carcass decomposes rapidly.

Symptoms. The first symptom of lupinosis is loss of appetite. The sheep, even the animal so accustomed to consume lupins, only eats the toxic plants after some hesitation; he will frequently go hungry rather than touch them; later he refuses all food. The temperature rises; it may be found as high as $40^{\circ}$ or $41^{\circ} \mathrm{C}$. and more; the thermic curve shows remissions ; hyperthermia is sometimes observed the day following the ingestion of toxic lupin; a short time before death the temperature falls considerably. The pulse is accelerated; we may count as many as 130 pulsations and more per minute.

Icterus appears sometimes on the second or third day, at other times only on the fifth or sixth ; its invasion is sudden, or, on the contrary, slow and gradual-in these cases the yellowish coloration is always first apparent in the eye. In certain cases this is altogether absent; it is, therefore, not a constant symptom of lupinosis.

Within a few days, and often from the very beginning of the trouble, we detect weakness and stupefaction; the gait is stiff; the animals remain recumbent for a long time, and rise up only with a great deal of trouble; they do not defend themselves when touched. 
There sometimes exists a physical and psychical depression which is quite marked; the patients hold the head low and lean it against the surrounding objects, or extend it when in a decubital position. They show a tendency to lateral and forward movements, and also backward action. In some cases they are very excitable and become scared at the least noise. They grind the teeth; the jaws are agitated or spasmodically contracted (Schütz). Kotelmann has observed trismus from the third day of the attack in twenty-two sheep affected with lupinosis.

At the beginning defecation is rare and the excrements hard; they are coated with a yellowish mucus, which afterward becomes pitchy dark-brown from the blood mixed with it; diarrhea may set in later.

Micturition is frequent and in small quantity; the urine is of a yellowish color ; it contains ordinarily the coloring matter of bile, bilious acids, and albumin. Epithelial, renal, and vesical cells are found in it, also round cells and renal casts of different kinds.

Respiration, which is regular at the start, becomes accelerated and painful (100 and more per minute). In some sheep we may observe a nasal mucous or spumo-bloody discharge.

The average duration of the disease is from four to five days, but death may happen in twenty-four to forty-eight hours; it is caused by the rapid emaciation and extreme weakness of the patients.

When the affection is likely to end in a cure, the symptoms become lighter, and disappear more or less rapidly. Sometimes the improvement is only apparent, and the disease ends in cachexia.

Chronic lupinosis, produced by a prolonged use of moderately toxic plants, ordinarily develops without icterus, with the clinical symptoms of anemia and cachexia. Roloff has often detected catarrh of the nasal and conjunctival mucous membrane; in some cases cutaneous phlegmasia occurs, also scabs upon the lips and ears. Zürn mentions an inflammatory tumefaction of certain parts of the head (eyelids, lips, ears), with exudation of a yellowish liquid and the formation of crusts.

Prognosis. The prognosis is very serious. In cases where the disease is produced by lupin the toxic property of which is relatively weak, and when consumption of this plant is immediately suppressed, we may hope for a cure; but acute cases of lupinosis almost always terminate in death. 
Treatment. This must be mainly prophylactic, for there is no specific antidote.

When it is impossible to completely exclude the lupin from the food we must seek by experimentation to find out the fields which produce the toxic lupin, in order to avoid the troubles produced by them, we may

1. Mix these plants with good food-in the proportion of 1:6-10, for instance-and preferably to old animals.

2. Put the toxic fodder in small heaps, and leave it exposed to the action of rain, which washes the poison from the superficial layers (Dammann).

3. Macerate the toxic lupins for forty-eight hours in a solution of soda of $1: 100$, renewing the dissolving liquid from time to time.

4. Subject the lupins for two hours to the action of steam under a pressure of two atmospheres at least (Kühn, Roloff).

When the poisoning has already appeared and is indicated by its special symptoms, we must avoid all alkaline liquids, and above all, alkaline water. A very advisable method is to add a small quantity of some acid to the drinking-water, in order to render the lupinotoxine insoluble. We advise, besides, the evacuants, which are susceptible of producing as rapid an expulsion as possible of the poison contained in the digestive canal; but here, again, sulphate of soda must not be administered, oily substances and brewers' grains should be used (Dammann). The meat from diseased animals can be consumed; we need only reject that of animals that had reached the last stage of the poisoning (Roloff).

From an anatomical and symptomatological point of view lupinosis of the horse is identical with that of the sheep. It is usually due to the presence of lupin seed in the oats or to the use of lupin stubble as litter. Generally we detect gastro-enteritis, which is more intense than in the sheep. The animals refuse drinks and food, even the best oats and good meadow hay. The general sensitiveness is weakened; sometimes we observe a very marked cerebral depression: the head is rested upon the manger, the animal grinds the teeth; the head is carried low, the gait is uncertain and staggering. The fever, which is ordinarily slight, may become very intense $\left(40.4^{\circ} \mathrm{C}\right.$., Butzert), the heart beats sixty times and more per minute (Kobel has noticed-undoubtedly at a more advanced period-thirty-two to thirty-six pulsations only). The respiration is quiet and accelerated; we have counted thirty-six to 
forty respirations per minute (Butzert); the icterus is more or less marked; defecation is rare; the dungs, which are small, are covered with a mucus exhaling a putrid odor. The micturitions are frequent and very small. These symptoms persist without great modification during about one week, then recovery is complete after sixteen to twenty-one days. No case of death has yet been reported. As accessory and inconstant manifestations, we must mention (after Butzert): slight colics, a thick orange-colored nasal discharge; circumscribed scabs (of the size of a silver dollar) of the lingual mucous membrane, mummification (dry gangrene) of the skin of the forehead, a cutaneous inflammation with exudation of a yellowish liquid, also formation of scabs and elimination of the epidermis, an inflammation observed upon the lower lip as well as on the pasterns, and which is accompanied by an oedematous tumefaction of the limbs coming up as far as the knee and hock.

ADDENDA. The symptoms assigned to lupinosis may be produced by other diseases which are very little known, but which are not due to the ingestion of lupin. One of these affectious which most resembles lupinosis has been observed, by Haubner, upon sheep which were fed on malted potatoes, and described by this author under the name of "malignant icterus" or "typhic hepatitis." 1 The reading of the monogram of the Dresden professor gives the impression of a perfect identity of these two diseases, and in the last edition of Haubner's book they are described in one chapter by Siedamgrotzky. The "hepatic typhus of the horse" studied by Sander ${ }^{2}$ recalls in every point acute lupinosis, and yet this affection is attributed to the injurious action of the inundated pastures. Reinemann, Jansen ${ }^{3}$ and several other practitioners assert that pea, bean, and vetch-stubble sometimes produce a condition which is similar to lupinosis.

The clinical picture of chronic lupinosis recalls equally that of induration of the liver or "Sweinsberg disease" (chronic hepatitis).

Finally, the morbid condition known under the name of "clover disease" has much similarity with lupinosis; we have therefore mentioned it with this latter trouble.

1 Haubner: Landwirthschaftl. Thierheilkde.

2 Sander: Hering's Pathologie, 1858.

\&einemann and Jansen : Preuss. Mittheil., 1880-81. 


\section{TRIFOLIOSIS (CLOVER DISEASE) OF THE HORSE.}

\section{(Kleekrankheit).}

Etiology. Authors are unanimous in recognizing that hybrid clover is the only hurtful clover plant. The pathogenic properties of this plant have been known for a long time (Delafond). They are, perhaps, due to mould (Erysiphe communis?), which exerts its irritating action directly upon the gastro-intestinal mucous membrane, or which produces the formation of a toxic substance which is like that of which we have spoken when referring to lupinosis, and which acts particularly upon the liver and the brain.

Symptoms. Clover disease is sometimes expressed by symptoms which are localized upon the skin and the buccal mucous membrane, at other times by manifestations showing the existence of grave lesions of the internal organs. In the first case we observe a tumefaction of the face and an intense stomatitis, accompanied by ulcers of the skin and of the mucous membrane of the lips, also a desquamation of the epithelium of the tongue, etc. (Lübicke, Hackbarth). Similar alterations are found in the regions of the cutaneous integument covered with lanugo (as on the face, the legs, etc.). The skin becomes yellowish on these places, is covered with vesicles, and acquires an extreme sensitiveness and slowly becomes converted into an eschar (the same processes as are observed in lupinosis). Among the general symptoms resembling lupinosis that are produced by the exclusive ingestion of clover, let us particularly point out: the icteric coloration of the buccal mucous membrane and of the conjunctiva (Aubry, Uebele, ${ }^{1}$ Zipperlen), attacks of colics, great weakness, drowsiness alternating with an intense nervous excitement (convulsions, rabiform symptoms, epileptiform attacks), the staggering gait, paretic accidents such as amaurosis, paralysis of the pharynx, and hemiplegia (Aubry, Uebele).

This latter form of the disease, which is frequently observed as an enzootic, and which resembles subacute encephalitis, is almost always rapidly fatal.

Treatment is rarely followed by success. We must at once stop the feeding of clover, and then resort to symptomatic medication. 


\section{PURELY NARCOTIC POISONINGS (NEUROTICS).}

\section{Poisoning by Strychnine.}

Symptoms. They are those of toxic tetanus : intense and general tetanic convulsions, the duration of which varies from a few seconds to several minutes; the neck and shoulders, spinal column, limbs, and tail are extended and stiff; there is hyperæsthesia; dyspnœic condition during the attacks; death occurs through asphyxia. The course is very rapid; the average time in dogs is from five to six hours, and if this time is exceeded there is no longer any danger of death. A very important symptom of diagnosis in small animals is the recovery from tetanic attacks as soon as they are placed in a standing position (Gerlach).

Autopsy. Lesions characteristic of asphyxia. There are no material anatomical alterations, which is similar to the conditions found in tetanus.

Treatment. The best antidote is chloral hydrate ( 2 to 10 grammes for the dog). Repeated anæsthesia with chloroform is also of great advantage.

Injections of morphine, bromide of potassium administered internally, tannin given in a decoction of coffee or tea, are also advisable means. At the outset we should prescribe an emetic. Finally, artificial respiration may become necessary.

Poisoning by strychnine is most frequently observed in the dog after the ingestion of poisons intended to kill rats, but they may be detected in subjects of our different domestic species as a consequence of errors in doses or of mistakes in the administration of remedies. The most sensitive of all animals is the dog (the fatal dose is 0.005 to 0.020 gramme); next comes the horse (the fatal dose is 0.1 to 0.3 gramme), then the ox (fatal dose, 0.3 to 0.4 gramme), the pig (which may be killed by a dose of 0.05 gramme), and lastly chickens.

\section{Poisoning by Beech-tree Fruit.}

Symptoms. There are very violent colics (intestinal tetanus); tonic and also clonic spasms which recall the tetanus produced by strychnine, with symptoms of paralysis; a staggering gait, falls, inability to rise. The course is very rapid, death being produced by asphyxia within a few hours. 
Autopsy. Lesions of asphyxia.

Treatment. Tannin, chloral hydrate, and morphine.

The poisonings were formerly quite frequent; they are produced by a narcotic poison (medullar) contained in the residue left over after the pressure of beech-tree fruit. The animal most sensitive to this agent is the horse, in which three pounds of this cake produces a fatal intoxication; among the subjects of our other domestic species the poisonings are more rare.

\section{Poisoning by Solanum (Nightshade).}

Symptoms. In the ox : staggering gait, paralysis, slow respiration, sometimes sudden falls, and death within a few minutes; in other cases death is delayed for a few hours, sometimes even one or two days.

Autopsy. The alterations have nothing characteristic. Symptoms of gastro-enteritis, which have sometimes been observed, are not related to poisoning by solanum, but to the irritating action exerted upon the intestinal mucous membrane by some tainted food.

Treatment. Tannin and stimulants.

Poisonings by solanum are especially observed in the ox and the goat, after the feeding of potatoes in process of germination, potato stems or their fruit.

\section{Poisoning by Hydrocyanic Acid.}

Symptoms. Respiration is accelerated and difficult; restlessness and anxiety, a staggering gait, paralysis, trembling; convulsions, epileptiform contractions, vomiting, colic, diarrhea, collapse.

Autopsy. In sudden intoxication, the blood is light red; when the disease lasts longer it is dark brown, and is of an odor similar to bitter almonds; symptoms of asphyxia.

Treatment. We prescribe hydrated oxide of iron (see Arsenical Poisoning), which is a direct antidote, on account of the formation of the harmless ferrocyanide. Bleeding, artificial respiration, and stimulants are advisable.

Poisonings by pure hydrocyanic acid are unknown in domestic animals, but we have observed in most species poisonings by plants or fruit containing prussic acid: prunes, plums, and cherry stones; laurel leaves (Prunus lauro-cerasus), also from the grape-cherry tree (Prunus padus), from the common peach tree (Persica vulgaris), and by bitter almonds, etc. Poultry are very sensitive to the action 
of hydrocyanic acid. Death occurs by asphyxia, paralysis of the brain and of the respiratory centre; it is preceded by convulsions.

\section{Poisoning by Furrow-weed (Lolium temulentum; Darnel).}

Symptoms. In the horse we see colics and symptoms of immobility ; in the ox, loss of consciousness and general convulsions.

Autopsy. Slight gastro-enteritis; cerebral and medullar hyperemia.

Treatment. Tannin and stimulants.

Intoxications by furrow-grass are very rare; some cases have been observed in the horse. The symptoms appear only after the ingestion of large quantities of this plant.

\section{Alcohol Poisonings (Alcoholism).}

Symptoms. Excitement and anxiety ; rabiform manifestations in the ox; redness of the mucous membrane; tumultuous beatings of the heart, full and accelerated pulse. Later, dizziness, staggering, loss of consciousness, paretic condition, collapse. The end is often fatal. Abortion (Möbius) and genital excitation (Genée).

AUTOPSY. There are no constant alterations. The organs have an alcoholic odor. There is cerebral hyperemia and at times ventricular dropsy.

Treatment. Tonics : coffee, ether, camphor, ammonia, carbonate of ammonia. Cold head douches.

The common alcohol poisonings are only observed in our animals after the ingestion of too large doses of some kind of alcoholic drink prescribed as a stimulant or an antipyretic. But intoxications by malt and alcoholic malts are not rare. The mode of action of these substances is complex : alcohol acts upon the nervous centres after its absorption, and the fermented matters irritate the gastro-intestinal mucous membrane, consequently we observe gastric troubles, tympanites, etc. In the pig, alcoholism is often accompanied by epileptiform convulsions. Spinola has observed a case of chronic alcoholism in a distiller's dog.

7. Poisoning by Horse-tail Grass, Scouring Rush, Shave-grass (Equisetum palustre, E. limosum, E. arvense): Mal d'ébriéte.

Symptoms. At the beginning there is anxiety and excitation, without cerebral disturbance; later, unsteadiness of motions, staggering gait, and finally paralysis of the hind quarters, falls, general 
paralysis, complete insensibility to various external stimulants, loss of consciousness, coma, acceleration of the pulse; normal appetite; nutritive changes, and diabetes when the disease lasts for a certain time. The evolution is sometimes very rapid (death occurring within a few hours), or it may be subacute (lasting two to eight days), or chronic (duration of several weeks).

In the ox, an obstinate diarrhea and symptoms of paralysis predominate when the animals have ingested shave-grass in large quantities; but when the feeding of these plants has been continued for some time, cachexia, hydremia, and paralytic weakness gradually appear.

Autopsy. Hyperemia, œedema of the nervous centres, and especially of the cerebellum (Schmitt). Ventricular and arachnoidal dropsy, also hyperemia when the disease is old. In some cases we see inflammatory alterations of the mucous membrane of the stomach and intestine.

Treatment. A change of diet, purgatives, stimulants, camphor; also derivative applications along the vertebral column.

According to Viborg, "Equisetum disease," also called mal d'ébriété, was regarded as a serious disease by the ancients (Pliny). Spinola ${ }^{1}$ considers the Equisetum palustre as one of the causes of vertigo. This plant contains a narcotic poison which is still unknown, but which reminds us of the effects of alcohol. The indications of authors about the degree of toxicity of the different shave-grasses, and upon the susceptibility of the various animal species (horse, ox, sheep) are quite contradictory; nevertheless, it seems to be evident that the toxic property of shave-grasses, as well as that of lupins, is influenced by the soil, climate, mode of preservation, etc. Certain authors affirm that the equiseta are inoffensive when mixed with other fodder, which gives rise to the supposition that the organism becomes gradually used to them, and acquires a kind of immunity by consuming small quantities of these plants. They have also found that hay containing much shavegrass becomes innocuous six months after the harvest, or after being washed by rain. Schwartz has communicated to us the following curious information : The Equisetum arvense grows abundantly on the banks of the Vistula and upon the two highlands separated by this stream ; it is considered a dangerous grass, designated under the name of Heermoos, and it enters frequently-to

1 Spinola: Handbuch der spec. Pathologie. 
the extent of one-half-into the composition of hay : under these conditions such hay ought to be entirely rejected as a food for cows. Bovine animals take it only with disgust; when they are compelled to feed on it they lose flesh, are overcome by ptyalism, the teeth loosen (the edge of the gums becomes the seat of a very intense inflammation), and if the use of this hay is continued, the teeth become detached and drop out. It is, however, a very strange fact, that horses will eat this dangerous hay with avidity, and not feel any discomfort from it; it is therefore reserved for the horses -in the royal stables it is accepted as good food. Equisetum palustre is regarded as an excellent food for the cow ; it is designated under the name of cow moss (Kühmoos).

We are entirely ignorant of the conditions imparting the toxic property to shave-grass; the poison is perhaps produced by parasites, as in lupinosis. Aquatic glyceria (Glyceria aquatica) and the common reed (Phragmites communis) seem to act in the same way as the shave-grass. ${ }^{1}$

\section{Poisoning by Oxide of Carbon.}

Symptoms. Vertigo, stupor, loss of consciousness, asphyxia.

AUTOPsy. The blood is vermilion red; there are lesions of asphyxia.

Treatment. Fresh air, stimulants, cold douches, bleeding.

Poisonings by carbonic acid and by illuminating gas present the same clinical symptoms. Poisonings by ether and chloroform (the latter being extremely dangerous in the cat) are accompanied by symptoms of similar lesions.

\section{Poisoning by Lathyrus Cicera (Chick-pea).}

Symptoms. In the horse: anxiety and laryngeal whistling; paralysis, weakness of the lumbar region, and asphyxia.

Autopsy. There is not any characteristic alteration.

Treatment. Change of diet; tracheotomy.

\section{Poisoning by Viper-bite (Vipera berus et V. aspis).}

Symptoms. Weakness, anxiety, tremblings, dyspnœa, acceleration of the pulse, which becomes almost imperceptible toward the end of life. The course is very rapid. There is local congestion

1 Morro, Köpke: Arch. f. wiss. u. prakt. Thierheilkunde, 1855. 
(ordinarily in the limbs and head), which extends toward the trunk.

Autopsy. We see local inflammation. The blood is dark and liquid; the other alterations are not constant.

Treatment. Potential or actual cauterization of the wound. Prevent, if it is still possible, the absorption of the blood by applying a rubber ligature. Externally and internally ammonia; also hypodermatic injections of carbolic acid $(3: 100)$, or also cresol. Internally stimulants: large doses of alcohol, ammonia flavored with aniseed. ${ }^{1}$

Clichy, ${ }^{2}$ Lies, ${ }^{3}$ Fünfstück, ${ }^{4}$ Bramstedt, ${ }^{5}$ and other practitioners have observed upon the horse poisonings produced by bee-stings. The symptoms presented quite a resemblance with those produced by viper-bites.

In a number of dogs bitten by vipers, Reindl-Aibling has obtained excellent results with subcutaneous injections of carbolic acid. ${ }^{6}$

\section{INTESTINAL HELMINTHIASIS.}

The gastro-intestinal canal in our domestic animals often contains worms, which happily are not dangerous in the majority of cases.

Their noxious action depends upon their number, and upon the species to which they belong. Some produce symptoms of much gravity through the intestinal alterations which they produce, and through their entrance into organs where they are not usually observed (ascarides in the ductus choledochus, Röll).

When they exist in large numbers, they may cause troubles through irritation or traumatism of the mucous membrane (Toenia Echinococcus), through the obstruction which they offer to the progress of alimentary matters, or through a complete obstruction of the intestine (Ascaris megalocephala); on the other hand, the

1 I have obtained several successful results in treating the engorgement of these bites; by repeated frictions with ammonia liniment; giving ammonia and wine internally.-L. T.

2 Clichy: Recueil Vét., 1853.

3 Lies: Adam's Wochenschrift, 1875.

4 Fünfstück: Sächs. Jahresber., 1886.

5 Bramstedt: Archiv. f. wiss. u. prakt. Theirheilkunde, 1885.

6 Kaufmann recommends counteracting viper-bites with hypodermatic injections of permanganate of potash (in solutions of $1: 100$ ) and internal administration of milk containing a little alcohol and a few drops of ammonia.-N. D. T. 
absorption of materials necessary for their nutrition does not fail to exert a debilitating influence upon the affected animals.

The principal symptom observed in animals whose intestines cortain many worms are as follows:

1. Emaciation and disturbances of nutrition (anemia and cachexia);

2. More or less intense gastro-intestinal catarrh and intestinal hemorrhages ;

3. Constipation and its consequences; colics;

4. Nervous phenomena of very numerous forms and degrees.

But these symptoms have nothing characteristic ; they are sometimes observed without any helminthiasis being noticed, and in many animals they are wanting, even when the intestine contains numerous parasites. However, they acquire a genuine importance when accompanied by the expulsion of worms. In this latter case we must avoid making a diagnosis of helminthiasis before having made a complete examination of the patient, for the phenomena detected may be related to other affections. Facts which are extremely common show that intestinal worms sometimes exist in large numbers, and yet the animals have all the appearances of perfect health.

The course of helminthiasis depends upon the species and quantity of the parasites. Its termination is sometimes fatal in the case of young animals, in which it readily produces anemia and cachexia.

The prophylaxis is only possible, in the present state of our knowledge, for a very restricted number of helminths (Toenia echinococcus, T. Coenurus, Trichina spiralis); in most cases we cannot even suspect the presence of eggs or larvæ in the food or in the water.

When there is any reason to suspect the existence of helminthiasis we may insure the diagnosis by using anthelmintics. As soon as it is clearly established, we must institute a treatment which will vary according to the species of the parasite, and also that of the animal which constitutes its host.

\section{Plathelminths (Tapeworms).}

The most important species in our domestic animals are:

$A$. In the DOG: 1 . Tonia cucumerina, which is the most frequent; according to Melnikow, its larva is found in the Pulex 
and Trichodectes (flea and louse) of the dog. ${ }^{1}$ 2. Toenia serrata, which comes from the Cysticercus pisciformis of the hare and the rabbit (liver, lungs, peritoneum). 3. Toenia marginata, produced by the Cysticercus tenuicolli of ruminants and pigs. 4. Toenia Coenurus, produced by the Coenurus cerebralis of ruminants. 5 . Toenia Echinococcus, produced by the Echinococcus polymorphus of herbivorous animals and pigs. 6. Bothriocephalus, very rare, found by Krabbe in Iceland and in Greenland. ${ }^{2}$

$B$. In the SHeEP: 1. Tcenia expansa (which may reach a length of 60 centimetres), the cause of epizootic helminthiasis of lambs, and of many other species imperfectly known.

C. In the HORSE: 1. Tonia perfoliata, the most frequent. 2. Toenia plicata. 3. Toenia mamillana, very rare (common, however, in France), and only one centimetre long.

D. In the ox: 1. Toenia expansa. 2. Toenia denticulata. 3. Tonia alba.

$E$. In the GOAT: Toenia expansa.

F. In the CAT: 1. Toenia crassicollis. 2. Toenia elliptica. 3. Bothriocephalus latus.

G. In POUltry: 1. Toenia infundibuliformis (chicken and duck). 2. Toenia lanceolata (goose and duck). 3. Toenia cuneata. 4. Toenia proglottina. 5. Tonia Cesticillus. 6. Toenia tetragona (chicken). 7. Toenia cantaniana (turkey). 8. Tcenia crassula (pigeon). 9. Toenia fasciata. 10. Tonia setigera (goose). 11. Tcenia trilineata. 12. Toenia coronula. 13. Tonia anatina. 14. Tonia gracilis. 15. Toenia sinuosa. 16. Toenia megalops. 17. Toinia conica. 18. Toenia imbutiformis (duck). 19. Bsthriocephalus longicollis (chicken).

Natural history. The Tænias or Cestodes belong to the group of Plathelminths. They form colonies (strobila), the different portions of which are formed at the expense of the scolex (head), which is the head of the whole colony. The ripe animalculi are designated under the name of proglottis. The head possesses a retractile trunk (rostellum), and it fixes itself upon the mucous membrane by means of tentacles and hooks.

Each segment is formed by an external muscular layer possessing

1 Melnikow : Archiv für Naturgesch., 1869.

2 The larva of the large Bothriocephalus (a species which is quite common in central Europe) lives in various fishes.-N. D. T. 
a cuticula, and also by an internal layer containing the genital apparatus and the vascular trunks. The genital apparatus is hermaphrodite; it is represented by the uterus, ovary, vagina, and vulva, on one side, and by the testicles, the deferent canals, and the cirrus, on the other side. The genital orifice is almost always located upon one of the edges. The eggs, which are very abundant in the uterus, possess a very hard membranous shell (embryophore) covering a globular embryo, which is clear, and provided with four or six hooks; they are extremely resistant, but dryness kills them. When these eggs reach the stomach of a vertebrate, an embryo escapes which perforates the gastric wall and settles in the numerous organs, becoming encysted and losing the hooks; thus forming the "vesicular worm," or cysticercus. If this is introduced by any kind of mechanism into the digestive canal of a host favorable to its development, its scolex (head) becomes free as a result of the digestion of the vesicle; by means of its hooks it attaches itself to the intestinal mucous membrane, and is developed, producing a new colony.

The knowledge of the transformations of ribbon-worms, establishing this capital fact, that the cysticerci form but a preliminary stage of the toenias, is due to the experimental researches of Van Beneden, Küchenmeister, V. Siebold, Leuckart, Haubner, and a few others.

The opinion of Mégnin, ${ }^{1}$ according to which the tænias armés would only represent a stage of development of the tænias inerme, has been denied by various authors (Moniez and Railliet; Zürn). Mégnin affirms that the tænias armés and tænias inerme are produced by the same cystic larva. The Toenia perfoliata of the horse, for instance, would not be anything else than the Toenia Echinococcus of the dog arrived at its last period of evolution.

Anatomical lesions of the intestinal mucous membrane produced by ribbon-worms. When the worms are not numerous, the alterations frequently are but slightly marked, they are catarrhal only. In other cases, on the contrary, we find intense inflammatory lesions. On the intestinal mucous membrane of the dog, Schieferdecker has detected regular tunnels in each of which was located a cucumerine tænia. These canals were formed at the expense of hypertrophied intestinal villi encysting the worm by

1 Mégnin: Bullet. Soc. cent. Vét., 1879. 
being soldered over it, and forming an arch covered with thick layers of desquamated epithelial cells. In their neighborhood Lieberkühn's glands were shortened and bevelled; here and there was observed a hypertrophy of the intertubular connective tissue. Such alterations lead forcibly to functional disturbances, as much through obstruction to absorption resulting in papillar hypertrophy as to the diminution of intestinal secretions consecutive to atrophy of the glands.

In the dog, intense anatomical alterations are sometimes produced by the Tcenia Echinococcus. These parasites, which are extremely small, are fixed by the hundred thousand upon the mucous membrane; they often produce intestinal hemorrhages and a fatal enteritis. Other ribbon-worms completely obstruct the lumen of the intestinal canal, and may produce invagination.

In poultry, the main alterations which are detected upon the intestinal mucous membrane are: hyperemia, infiltration, desquamative catarrh, with abundant secretion of a reddish-yellow or purulent mucus.

In the horse, Mégnin has observed perforation of the small intestine, with the formation of diverticuli and cysts, produced by the Toenia perfoliata.

We owe to Schöne some interesting statistics on the entozoa of the dog:

1. The relative frequency of tænias, and entozoa in general, in the canine races, is established by the following figures:

In hunting dogs examined, 52.94 per cent. carried entozoa (Toenia serrata was found most frequently).

Butcher dogs gave a proportion of 66.66 per cent. (the most common entozoon, Tonia marginata).

Watch dogs, 40.44 per cent. (the most common entozoon in this breed, Toenia cucumerina).

Coach dogs, 72.22 per cent. (the entozoon found in almost all cases was the Toenia marginata).

Shepherd dogs, 57.14 per cent. (they were affected by Toenia marginata, T. serrata, and $T$. cucumerina; these are the only ones in which the Toenia Coenurus has been detected, 7.14 per cent.).

Pet dogs, 70.37 per cent. (36 per cent. were affected by Toenia marginata and T. cucumerina, and 15.74 per cent. by T. serrata.

2. Ascaris marginata has been found most frequently (42 per cent.) in pet dogs. 
3. Uncinaria trigonocephala has been found exclusively in pet dogs (5.2 per cent.).

4. Echinococcus polymorphus, exclusively upon coach dogs $(5.5$ per cent.).

5. Cysticercus cellulosce, also exclusively upon coach dogs $(5.5$ per cent.).

6. Hemistoma alatum, exclusively upon coach dogs (5.5 per cent.).

7. Pentastoma toenioides (Linguatula rhinaria) has been detected most frequently in shepherd dogs (21.42 per cent).

According to Zschokke's researches made upon 177 dogs, 60, or 34 per cent. were affected by tænias: thirty-eight carried Toenia cucumerina, nine Toenia marginata, seven Tcenia Echinococcus, three Tcenia serrata, and three Toenia Coenurus.

Symptoms. In the great majority of cases the tænias do not produce any appreciable symptom.

When they cause some disturbances they are ordinarily those of chronic intestinal catarrh, accompanied by particular manifestations, varying according to the species. The animals in which we most frequently detect accidents caused by entozoa are the $\operatorname{dog}$, lamb, and chickens.

1. The tænias are very frequent in the $d o g$, but cases where they produce disturbances of a general nature are, as a rule, quite rare. They are most frequently found in butcher, shepherd, and hunting dogs, also in pups of all breeds. The symptoms regularly produced by these parasites are those of chronic intestinal catarrh. The appetite is very capricious; at certain times we observe a true hunger-disease, nevertheless the condition of the body is reduced; young dogs are often restless; they wag the tail, run, and snap toward the abdomen with their teeth, lie down frequently, etc.

In very irritable dogs which carry large numbers of tænias, and mainly Toenia Echinococcus and T. cucumerina, we detect at times rabiform symptoms, which were formerly responsible for the belief that there was a close connection between helminthiasis and hydrophobia. The manifestations of this "pseudo-lyssa" are vertigo, convulsions, epileptiform attacks, paralysis, a tendency to biting, alteration of the voice, paralysis of the lower jaw, great weakness, and stupefaction (Leisering has produced all these symptoms in his experiments upon dogs by making them ingest echinococci). In general, these rabiform manifestations are determined by the Toenia Echinococcus; we have also observed them with the Toenia cucu- 
merina. They are probably due to the intense pains produced by enteritis. By accumulating in certain places, the tænias may produce a complete obstruction of the intestine, and death.

Lesbre has observed a very remarkable and unique case of auto-infection of the dog through Tcenia serrata (Cysticercus pisciformis-in the brain?); as symptoms he especially noticed nervous troubles, grinding of the teeth, and unusual movements.

The larva of the Toenia cucumerina, found by Melnikow in the trichodecte of the $\mathrm{dog}$, has received the name of Cryptocystis Trichodectidis. The dogs would be infected by ingesting trichodectes. But something which is at least very strange is the fact th the dogs with cucumerine tænias are but very rarely affected with trichodectes; we have hardly ever found any, and think that this is not the usual mode of infection. ${ }^{1}$

2. In the ovine species the Tonia expansa may produce an epizootic helminthiasis. Epizootic tæniasis of lambs is frequent during wet summers in subjects which are kept in swampy pastures. Very young sheep are particularly predisposed to contract Toenia expansa; in older ones its development is favored by debilitating diseases and dysentery (Spinola). The Tcenia expansa is remarkable by its extremely rapid growth. Spinola has found worms ten metres long in lambs four weeks old; this assertion has led to the erroneous acceptance of the idea of an intra-uterine infection.

The symptoms of epizootic tæniasis are in no way different from those of cachexia; the patients show all the signs of anemia; the skin and conjunctiva are pale, the wool is clear, deprived of fat, and may be easily pulled out, etc. Later we observe the manifestations of digestive troubles. The appetite becomes capricious, and rumination is no longer regularly performed; the young animals are thin and develop slowly; they are weak, they do not try to escape when an attempt is made to catch them, and they drag behind the flock. There is ordinarily constipation; at certain times we observe restlessness, anxiety; the animals lie down and rise again immediately ; they arch the back, lift the tail, and make useless efforts at defecation; we can detect a slight swelling. When cachexia is advanced, diarrhea occurs, and the weakness is soon extreme. Arrived at this stage, the disease is absolutely incurable,

1 In fact, the recent researches of Grassi have shown that it is more frequently the dog's flea which plays the rôle of intermediary.-N. D. T. 
and the animals soon perish, in an exhausted condition and with marasmus.

3. In poultry, which carry at times a great number of species of tænia, helminthiasis is announced either by gastric troubles, and a mucous or bloody diarrhea, or by a simple wasting away, the appetite being preserved. The bird seems depressed; the feathers are dull, standing out, and are often alive with different kinds of parasites. Periodical epileptiform attacks may be seen. The appetite diminishes slowly, and later disappears entirely; the birds become weak, emaciated, and die. The trouble sometimes take an epiornithic ${ }^{1}$ character, as observed in the pheasant by Friedberger.

The tænias of other animals (horses, oxen, goats, and cats) very rarely produce a so-called true morbid condition. Mégnin has related two cases of peritonitis by perforation produced by the Toenia perfoliata.

In the Black Forest, in 1874, we observed in the cat an epizootic produced by the Tcenia crassicollis, and at the same time the almost complete disappearance of the field mouse has been noticed-it was killed by the Cysticercus fasciolaris. The sick cats became emaciated and died rapidly. ${ }^{2}$

As a probable cause of intestinal perforation in the cat, Perroncito points out wounds of the mucous membrane, produced by the hooks on the head of the Toenia crassicollis. In several cats which had died from gastritis, Zschokke has found the Toenia crassicollis in the stomach, while in the healthy cats he has always found it in the small intestine, and mainly in the jejunum. He has looked upon the presence of the parasite in the stomach as the cause of death.

Diagnosis. In our domestic animals the existence of tænia can only be recognized by the expulsion of segments or by the autopsy (frequently thus in the sheep). The parasites are ordinarily rejected with the excrements; in the dog they are often seen on the surface of the dung; sometimes they reach the margin of the anus, progressing slowly through the hair of the region, and soon dry up and form yellowish or whitish strings, more or less flattened. Expulsion by vomiting is more rare. The segments sometimes irritate the rectal mucous membrane, and produce a pruritus which the

$\left[{ }^{1} \mathrm{I}\right.$ have used this word in preference to epizootic and epidemic, the words used in the text.-w. L. z.]

${ }^{2}$ Lydtin; Mittheil. über das badische Véterinärwesen, Carlsruhe, 1882. 
animals try to allay by rubbing the anus, the tail, and the perineum against hard objects, or by dragging themselves on their seat; but these symptoms exist also in other diseases. The abnormal dilatation of the pupil, considered by some authors as a pathognomonic symptom of helminthiasis, has no symptomatic value. $^{1}$

Zürn suspects the presence of intestinal worms in chickens when they show a great avidity for water.

Concerning the differential diagnosis of the various tænias which may be located in an animal, and also their zoölogical determination, we have to refer the reader to the treatises on parasites. We here restrict ourselves to giving the principal macroscopic characters of the most common tænias known in the dog.

1. Toenia cucumerina. Often in ball shape, in rings having the form of a gourd - that is to say, a long ellipse-and possessing a genital opening upon each side of the free edge; maximum width, 0.002 millimetre; length, from 0.05 millimetre to 0.10 millimetre (rarely 0.20 millimetre); quite thick; the eggs of a reddish shade, in heaps or in cocoon.

2. Toenia serrata. Quadrangular rings, joined by rough edges (saw edges), and provided with a genital opening, alternately on the right and the left upon the free edge; the rings are very long (up to 0.013 millimetre) and wide (about 0.005 millimetre); total length, 0.50 to 1 metre.

3. Toenia marginata. Quadrangular rings with regular joints, often wider than long, with undulated edges, and with alternating lateral genital opening. Total length, 2 to 5 metres. This is the widest and the longest tænia of the dog.

4. Tcenia Conurus. White quadrangular rings; the latter are oblong, and very long relatively to their width. Total length, 0.50 to 1 metre.

5. Tonia Echinococcus. Formed by three or four rings, and 0.004 to 0.006 millimetre long; the last segment contains the eggs. The rings are white, elliptical, and may be confounded with the intestinal villosities.

Treatment. The tæniafuge remedies are very numerous. In order to act properly they must be given in relatively large doses; in addition, their administration must be completed by the admin-

I This dilatation of the pupil is significant in some cases.-L. T. 
istration of a purgative, except when the anthelmintic possesses laxative properties. The detached scolex (head) may become fixed anew upon the mucous membrane.

The tæniafuge preparations ought to find the digestive canal prepared by a fast of twenty-four hours, and by clysters. They should be given on an empty stomach. It is always prudent to hold a second dose ready for the dog, in case the first one should be vomited.

1. Against tænias affecting the dog we use especially, etherized extract of the male fern ( 2 to 8 grammes in capsules or pills, with powder of fern rhizome); we may also give the latter fresh, in doses of 10 to 25 grammes. Fern extract is incontestably one of our best tæniafuges. Kamala, in doses of 2 to 12 grammes, is both anthelmintic and purgative. We use also the bark of pomegranate root (50 to 150 grammes macerated in a litre of water for twenty-four hours, and reduced by boiling); also the flowers of the kousso (Brayera) (5 to 25 grammes in milk); renewing the dose at the end of an hour. (Koussin, 5 grammes in 50 grammes of castor oil, has not given us any satisfactory results.) Pulverized areca nut was recommended by Hauly as early as 1862 , and lately it has come back into favor; it is given in the dose of 8 to 20 grammes, put into fresh butter, and administered twice with an interval of one to two hours; if there is no evacuation after two or three hours we give 10 to 30 grammes of castor oil. The tænias and nematode worms are ejected after two to six hours. The areca nut is more frequently vomited than most of the other anthelmintics. We use more rarely pumpkin seeds (25 to 30 seeds) dried and pulverized, completing their action by the administration of a laxative (Zürn), the fruit of the Embelia Ribes (Harris), and the black oxide of copper, advised by Hager ( 0.05 gramme three times per day for several consecutive days). Chloroform and cresol are much more efficient than these latter agents.

2. In cases of epizootic tæniasis we must first remove the lambs from the suspected pastures. According to Hackbarth, the tænias only become really efficient where the worms have attained a certain development and when the animals present serious troubles produced by parasites. We may use all the anthelmintics indicated for the dog, and in the same doses. Among the modern remedies, the most efficient are the picrate of potash $(0.6$ to 1.25 gramme, in pills). and koussin (0.12 gramme). Philippi prefers 
extract of male fern, in doses of from 2 to 4 grammes, or kamala (5 to 6 grammes), with the administration of castor oil three hours after the tæniafuge potion has been taken.

Formerly veterinarians used the worm cakes of Spinola (male fern, absinthe, and wagon grease, of each 1 kilogramme; kitchen salt, $\frac{1}{2}$ kilogramme ; flour and water q. s.), and Chabert's empyreumatic oil, given per teaspoonful, with 0.2 to 0.3 gramme of tartar emetic.

3. For poultry Zürn recommends pulverized areca nut, in doses of 2 to 3 grammes, in pills, and a purgative. This agent seems to be the best anthelmintic for the different species of birds; after its administration, turkeys sometimes exhibit a certain excitement. Pulverized rhizome of male fern (in doses of 1 to 4 grammes) is much inferior to the areca nut.

In other domestic animals we may use the same tæniafuges as for the $\operatorname{dog}$ and the sheep.

For the horse, it is proper to resort to the drugs mentioned in relation with the nematode worms (tartar emetic, arsenic, essence of turpentine, etc.), or to the areca nut, two tablespoonfuls after each meal (Fuchs).

\section{Ascarides.}

The principal ascarides of our domestic animals are:

a. In the horse and donkey: Ascaris megalocephala;

b. In the dog: Ascaris marginata and Acaris mystax;

c. In the cat: Ascaris mystax;

d. In the ox and the pig: Ascaris lumbricoides (vituli and suilla);

e. In poultry: Heterakis (formerly Ascaris) inflexa and vesicularis, in the chicken, etc.; Heterakis maculosa, in the pigeon; Heterakis dispar, in the goose.

Anatomical alterations of the intestinal mucous membrane produced by ascarides. Ascarides often exist by thousands in the digestive canal of animals. Through their armed mouth they produce deeper wounds in the intestinal mucous membrane than do the tænias; at times, though rarely, they perforate the intestinal walls and thus produce peritonitis. At the autopsy of dogs that have died from disorders produced by ascarides we find the mucous membrane tumefied and catarrhal; it shows numerous little round darkish spots, due to the special action of the worms, and on the level of which are noticed a central ulcerative 
depression, surrounded by a salient zone; in addition is observed an intense hemorrhagic enteritis; less frequently we see signs of furrows or caverns with ulcerated edges, which are irregular and thick-a process which sometimes extends itself to all the layers of the intestinal wall. The parasites may form compact balls, which obstruct the passage of the intestinal canal completely, and at times give rise to an invagination.

In the horse, Zürn has described two cases of intestinal perforation produced by the Ascaris megalocephala at the point of mesenteric attachment. Where the intestinal wall offers the least resistance we detect small round openings, surrounded with thick edges, which are cicatricial ; these openings lead to caverns which are furrowed between the two thickened layers of the mesentery, and contain pus and chyme, as well as ascarides. The opening of these intra-mesenteric abscesses has resulted in a purulent peritonitis. Generali has found an ascaris in the pancreatic canal which was tumefied and thickened; the gland itself was not altered.

In chickens, the intestinal mucous membrane is at times the seat of a catarrhal phlegmasia, sometimes it is much inflammed, ulcerated, and covered with a thick and purulent exudate.

Symptoms. They do not differ essentially from those produced by the tænias. We observe disturbances of digestion and nutrition, diarrhea, constipation, tympanites, etc., a more or less marked emaciation, nervous manifestations, and pruritus; the animals endeavor to allay the itching by scratching or rubbing themselves.

1. In the horse we observe troubles without any clear significance: diarrhea and constipation, intermittent colies, and when the trouble is old a more or less marked emaciation. The worms may produce death by obstruction or perforation of the intestine.

In some subjects we observe symptoms which are quite special. In a mare Fréminet detected a very evident tetanic condition (trismus, a special posture being taken by the horse), produced by a reflex, and which disappeared after the administration of an anthelmintic. Damitz has observed a particular spinal affection; paraplegic, which yielded to the administration of anthelmintics.

2. In the $d o g$ the symptoms are identical with those produced by the tænias. In this animal and in the cat fatal intestinal perforations are quite common; the manifestations which they produce are similar to those of certain poisonings, and often they are only recognized at the autopsy. 
3. Chickens and pigeons are often infected in an epiornithic ${ }^{1}$ manner by the Heterakis. The affection is characterized by diarrhea, an intense thirst, weakness and paresis, apathy, emaciation, pectoral atrophy, weakness of the wings, which are hanging, by ruffling and falling out of the feathers, etc. (Unterberger). Cases of sudden death are not rare. We often observe in the pigeon a true epiornithic ${ }^{2}$ due to Heterakis maculosa. As many as 500 of these parasites have been found in the intestine of a single pigeon. Heterakis inflexa causes the same symptoms in the chicken (it may go astray in the oviduct and penetrate into the egg).

In other animal species the ascarides do not offer any clinical interest. At the autopsy of a calf which had shown curious symptoms during life (swelling up and clonic movements of the jaws), Deschamps found in the intestines fifteen litres of ascarides.

Treatment. The anthelmintics which we may use with advantage against the nematode worms in general, and the ascarides in particular, are the following :

1. In the horse: tartar emetic in a dose of 15 to 20 grammes in drinks or electuary, to be administered with bitters, in three or four doses at intervals of three hours; arsenous acid in a dose of 2 to 3 grammes, with bitters and purgatives, in pills (pulverized arsenous acid, 2 to 5 grammes; pulverized aloes, 30 grammes; powder made of absinthe stems, 20 grammes ; marshmallow powder and distilled water, q. s. for two pills); essence of turpentine in a dose of 100 to 200 grammes in emulsion; fetid animal oil, 20 to 30 grammes; benzine, 50 to 100 grammes; "semen contra vermes," 100 to 200 grammes, with tartar emetic and alkalines in electuary; rhizome of male fern, essence of tansy ( 40 to 50 drops), absinthe, gentian, asafoetida, creolin (50 to 150 grammes). Formerly we used also the powder made of the seed of the Strychnos potatorum, but this agent is dangerous. As a preparatory measure to the anthelmintic treatment we give sugar beets and turnips.

2. In the $d o g$, the drug to which we should give the preference is santonin, in a dose of 0.05 to 0.2 gramme, given with sugar, and afterward a purging with castor oil. We have besides the extract of male fern, the picrate of potash, creolin, etc. A vulgar remedy is the decoction of garlic in milk, per os or per anum.

[ ${ }^{1}$ Used in preference to the word epizootic.-W. L. z.]

[ ${ }^{2}$ Used instead of epidemic, as in the original.-W. L. z.] 
3. In poultry, Zürn recommends the areca nut, in the dose of 3 grammes for the chicken and 1 gramme for the pigeon.

\section{Strongylus.}

The species found in the intestines of animals are:

a. In the HORSE : Sclerostoma armatum and tetracanthum.

b. In the SHEEP and the GOAT: Strongylus contortus (strongylus of the abomasum), Sclerostoma hypostomum, Uncinaria cernua, Strongylus filicollis, and Esophagostoma venulosum.

c. In the ox: Uncinaria radiata, Esophagostoma inflatum, and Strongylus ventricosus.

$d$. In the DOG: Uncinaria trigonocephala and U. stenocephala.

$e$. In the CAT: Uncinaria trigonocephala.

$f$. In the PIG: Sclerostoma dentatum.

g. In POULTRY: Strongylus tenuis and nodularis (goose), and Strongylus pergracilis (grouse).

\section{(A.) SCleRostoma OF THE HORSE.}

Natural history. 1. Strongylus armatus manifests itself in the equine organism under two forms: first, in the larval state, deprived of genital organs-in aneurism of the anterior mesenteric artery (see Thrombo-embolic Colics); second, in the state of the adult worm-in the cæcum and colon. Expelled with the excrements, the ripe eggs are developed in water and mud, and produce rhabditiform embryos.

When these latter are introduced into the intestines of the horse with the water, they penetrate partially into the large mesenteric artery, and mainly into the trunk; there they undergo several metamorphoses, they develop their genital organs, then they return into the large intestine, where they become fixed through the medium of their head, which is in the form of a trephine.

The Sclerostoma tetracanthus becomes encysted in the submucous connective tissue of the intestine; when arrived at an adult age, and provided with genital organs, they emigrate into the inside of the digestive canal.

Anatomical alterations of the intestine. 1. The sclerostoma of the armed form produces at its point of attachment upon the mucous membrane a small reddish-blue blot, which is often taken for an ecchymosis; but on microscopical examination we notice in the centre of this spot a worm of a reddish color, one-fourth of 
a millimetre to twelve millimetres long; in addition, we always detect a slight enteritis.

2. The S. tetracantha, which often exists in larger numbers in the colt, produce a hemorrhagic enteritis, with small submucous purulent yellowish pimples, the centre of which is the seat of larvæ of the sclerostoma; quite often the mucous membrane is marked with small cicatrices at this point.

Symptoms. The sclerostomas al ways cause trouble when they exist in any considerable quantity in the intestine. Generally they produce an inflammatory condition of the mucous membrane of the large intestine, and sometimes fatal colics. We may see violent expulsive efforts, and a liquid diarrhea which is also bloody. If we make a rectal exploration, the arm is often covered with a great quantity of small sclerostomas. An incident has been related of strongylosis, with extreme emaciation, in an old mare; and another with intense diarrhea, in a colt. In a case reported by Hill, the parasite, in emigrating to the surface of the brain, had caused a meningitis complicated with hydrocephalus, which was marked by symptoms of immobility; in others it had invaded the genital organs and produced sclerosis and atrophy of the testicles (Railliet). In two horses, Kilt found adult sclerostomas in the peritoneum.

Treatment. The same indications would be adapted to strongylus as in cases of ascarides, if the difficulty of the diagnosis did not render it visionary in the majority of cases. According to Spooner, the encysted larvæ of the Sclerostoma tetracanthum would seem to be very hard to expel.

\section{(B.) Strongylosis of THE ABOMASUM OF SHeEP.}

(Strongylus contortus.)

The round worms that we find in the stomach of the sheep and of the goat, which exist often in large numbers in lambs, determine a disease known under the name of strongylus of the abomasum or "red verminous gastritis" (rothe Magenwurmseuche). In the spring and summer this disease appears in an epizootic form, and its economic importance equals that of epizootic tænia; it is frequent in localities where stagnant waters are to be found-that is to say, wherever verminous bronchitis exerts its ravages (Strongylus Filaria). Gerlach, who detected in several regions the coexistence of these two affections, has searched the etiological and pathogenic reports which exist concerning them. 
Autopsy. We find the mucous membrane of the abomasum covered with strongyli and presenting all the symptoms of gastritis or chronic catarrh. We must remark here, that the strongylus is quickly destroyed, digested, in the cadaver; it is therefore difficult to find them a certain time after death, and as the autopsy is the only way to determine the diagnosis, it is advisable to make it as soon as possible.

Symptoms. They are similar to those of tæniasis. We observe the symptoms of anemia, chlorosis, pernicious anemia, with "poikilocytosis" 1 (Wernicke), gastric derangements, diarrhea, progressive emaciation, finally an extreme weakness and cachexia.

Treatment. Chabot's empyreumatic oil and picrate of potash (Rabe) in a dose of 0.1 to 0.3 gramme are the most efficient agents.

The other species of strongylus are of secondary importance. In sheep, the Sclerostoma hypostomum may produce digestive troubles, simulating slight colies. At the autopsy we have found the small intestine full of small punctiform hemorrhages; the intestinal contents were of chocolate-brown color. In the dog, the Uncinaria trigonocephala sometimes produces a serious enteritis. Grassi has described in the cat an affection produced by this parasite, and marked by an intense diarrhea, obstinate vomitings, emaciation, weakness, anemia, etc. In the pig, and in poultry, the symptoms are nearly similar.

\section{Uncinariosis: Pernicious Anemia of Hunting-hounds.}

Under the terms Pernicious anemia of hunting-dogs, Nose-bleed, Ankylostomiasis, Uncinariosis, we designate a parasitic disease of the dog, caused by Dochmius trigonocephala (Uncinaria trigonocephala, Rud.), complicated by Uncinaria stenocephala (Raill.), and characterized by manifestations which, until recently, have been ascribed to essential anemia and cachexia.

It is only found in hunting-dogs, and is especially frequent in those living in packs. The age, constitution, and nature have no influence upon its development. The animals contract it by lapping water from certain pools, or trenches within the kennel, which contain the larvæ of the worms.

1 Alteration of the blood characterized by a mixture with the red blood-corpuscles of reddish-yellow cells, isolated from one another and larger than normal globules, and some of which are spherical, others leaf-shaped, scutiform, pyriform, or claviform (Neumann).-N. D. T. 
Symptoms. At its initial period, the duration of which is very variable, as it depends upon the resisting power of the subjects and the conditions in which they are kept, pernicious anemia is only marked by two symptoms which are common to a great many affections: by weakness and emaciation, which become gradually more marked, notwithstanding the preservation of the appetite. The dogs are depressed and have lost speed, are less active, and less ardent during the hunt. The hair is brittle, the body is covered with squames (scales), it frequently presents redness and erythematous spots; the nose is tumefied, split, rugous, excoriated; a muco-purulent or bloody discharge runs out of both nostrils. When the disease is left to itself other symptoms appear, the most striking of which are epistaxis and the obstruction of the limbs. The blood running out of the nostrils is sometimes dark-red, at other times clear, rosy, but always more or less spumous; the attacks of epistaxis are separated by intervals which may vary from a few days to a few weeks; the bleeding is often quite abundant; some dogs lose a decilitre of blood or more at each hemorrhage; during these intervals the discharge is muco-purulent or bloody. The microscopic examination of the blood drawn from a superficial vein permits us to recognize a great diminution in the number of red globules and a certain degree of leucocytosis ; there are cases where anemia is rapidly produced, and is accompanied by nervous phenomena which are manifested by spells. The obstruction of the limbs is odematous and indolent; at first it is intermittent, then permanent; its proportions increase with the progress of the affection. Often the excrements are almost normal ; at other times we see diarrhea. In the last phase of the disease the appetite becomes irregular, capricious, and then disappears; the subjects, which are much emaciated and anemic, remain constantly in a recumbent position, and complain when compelled to rise or change their position; the skin, which is partially depilated, is covered with red spots and excoriations, and upon the legs it is at times the seat of a sero-sanguinolent sweating; we may also observe wounds of an ulcerous aspect or having gangrenous centres. The diarrhea is persistent, and it is often followed by dysentery during the last stage. Reduced, so to speak, to a skeleton, the patients soon die. As a rule, death takes place in coma, more rarely in an attack of convulsions.

The duration of the disease varies from a few months to a year. 
Once established in a pack, uncinariosis may exercise its ravages for years. Almost all animals, if not all, are successively affected.

Pathological anatomy. At the autopsy of dogs which have perished from pernicious anemia, or of those killed during an advanced period of the disease, we find, besides the ordinary alterations of cachexia, lesions related to uncinariosis. These are ordinarily located in the small intestine and the cæcum. The mucous membrane is considerably thickened and marbled with large red-colored spots; the villi are increased five times in size, and are injected with blood corpuscles which are arrested in the bloodvessels and crowded and packed together. These alterations of the mucous membrane are at first observed in the duodenum, and spread themselves later to the jejunum and to the ileum. In the parts which are relatively healthy we see on the mucous membrane a large number of punctiform hemorrhages, little islets formed by small drops of blood half coagulated, and in the centre or side of which we find one or more filiform wormsthe uncinaria-0.01 to 0.015 millimetre long, whitish or marked with a black longitudinal line. The number is more or less considerable in proportion to the size of the healthy mucous membrane. While the parasites are always very numerous in dogs recently affected, we can hardly find any in the ileum of animals whieh have been sick for a long time. They seem to leave successively the parts of the intestinal mucous membrane in which inflammation is developed under the action of their bites; thus is explained the progressive extension of the lesions of the duodenum and of the ileum. ${ }^{1}$ We find frequently the depressed trichocephalus in the cæcum (T. depressiusculus, Rud.). This latter parasite producing an intense inflammation of the cæcal mucous membrane, it was thought that it took an important part in the development of the anemia in whole packs (Mégnin). The experiments of Railliet have not confirmed this hypothesis. ${ }^{2}$

Diagnosis. When uncinariosis exists for a long time in a pack of hounds its diagnosis does not offer any difficulty, but when it first makes its appearance, and if we are not used to it, it may be taken for essential anemia. This, however, is much more rare than the former, and it affects simultaneously the greater number of animals in the pack. In doubtful cases the diagnosis may be estab- 
lished by means of the treatment. The administration of some anthelmintics, very efficient indeed in parasitic anemia, do not give any good result in others. The autopsy of one of the first cases which succumbs permits us to establish clearly the nature of the disease. It should not be confounded with the morbid condition produced by the Pentastoma or Linguatula. If these parasites occasion at times epistaxis, the general condition of the animals which have remained healthy permits us to exclude parasitic anemia.

Treatment. As soon as the disease has made its appearance in a pack the treatment must be prophylactic and curative. The prophylaxis comprises the following means: Keep the kennel in a perfect state of cleanliness ; make frequent and thorough washings, in order to scatter and wash away the eggs of the parasites, which exist in large numbers in the excrements of dogs which are affected; also prevent the beasts from lapping the water running in the trenches of the kennel or of neighboring pools; it is also advisable to give them boiled water, or at least water which comes from a source protected from matters dejected by the patients; finally, distribute the food in pails or other recipients, and be careful not to throw it upon the ground, where it may become soiled by the dejections. Isolation of the patients is an excellent measure; it ought always to be applied when the situation of the locality permits.

The curative treatment comprises the administration of anthelmintic agents powerful enough to kill the worms. Mégnin has advised kamala in a dose of 3 to 4 grammes; it is of very great advantage to associate calomel ( 0.5 gramme) and arsenous acid ( 0.005 to 0.01 gramme) with this remedy. The aqueous extract of male fern, which has given many good results in the treatment. of anemia in young animals (Perroncito), is also an advisable agent. It may be given in doses of 3 to 6 grammes.

[Trasbot recommends as a treatment in this form of helminthiasis, and one that has given him great success, the administration of etherized extract of male fern, 4 to 8 grammes, tincture of male fern, 20 to 40 grammes, given twice, with an interval of four days, purge with castor oil, allow the patients an interval of quiet for four days, and then repeat the treatment.-W. L. z.]

Finally, it is necessary to prescribe a tonic regimen; a nutritious alimentation composed mainly of meat, hacked meat, milk prepara- 
tions; cod-liver oil, gentian, wine, and ferruginous preparations. (See Pernicious Anemia.)

\section{Oxyuris.}

Among the varieties of oxyuris in our animals we must mention:

$a$. In the horse: Oxyuris curvula and Oxyuris mastigodes.

b. In the dog: Oxyuris vermicularis.

The worms, on leaving the large intestine, fix themselves upon the rectal mucous membrane, irritate it, and produce proctitis. The pruritus occasioned by this latter is a cause of continued rubbing; many horses, which are tormented by these parasites, have a rat tail. The diagnosis is established either by the presence of the worms in the excrements or upon the exploring arm, or it may be by the finding of the eggs in the scabs surrounding the tail or in the anal cavity.

Quite recently Friedberger has found upon several horses the Oxyuris mastigodes, a species that has also been seen by Nitzsch upon a horse which for a certain time consumed dandelion (Leontodon taraxacum).

This oxyuris, which is undoubtedly not very rare, is distinguished from the Oxyuris curvula by its greater dimensions, by the size of its tail, which is very thin (the female measures 13 centimetres, and has a tail $10 \frac{1}{2}$ centimetres long), and also by the eggs, which are very glutinous. ${ }^{1}$

We combat the oxyuris with vinegar injections, weak sublimate solutions ( 0.5 to 1 per cent.), or with soap-water. The administration of vermifuges is not necessary.

\section{Giant Echinorrhynchus of the Pig.}

According to Schneider's researches, we must admit that the larvæ of the echinorrhynchus are ingested by the pig with the white worms, and perhaps also with the June bugs. This latter mode of infection has been seen in man.

Autopsy. The anatomical alterations of the mucous membrane of the small intestine are considerable. The echinorrhynchus. with the help of his armed trunk, penetrates deeply into the mucous membrane, and causes serious inflammatory alterations.

1 According to Railliet, Oxyuris mastigodes is but a form of Oxyuris curvula.-N. D x. 
sometimes perforation of the intestine, and a consecutive peritonitis. The intestine has its serous surface pearled; its walls contain purulent centres of the size of a hemp-seed, which is surrounded by a red zone. In making the incision we find, at the level of these points, circumscribed inflammatory spots having the echinorrhynchus' head as a centre; at their periphery the mucous membrane is red and thickened. Sometimes the inflammatory process leads to an ulcerative destruction, and the loss of substance extends as far as the serous membrane. In other places the mucous membrane is slatish-gray, thick, and covered with exudate (Kocoureck).

Symptoms. Loss of appetite, constipation, restlessness; the animals scratch, dig, and carry the muzzle or the teeth against the abdomen; the emaciation increases; there are convulsions, epileptiform spasms; in young pigs death may occur in three or four days. The disease assumes quite frequently an epizootic form.

Treatment. Prophylaxis: destruction of the June bugs or permanent stalling of the pigs. The internal treatment requires the administration of various anthelmintics. Kocoureck has obtained good results from the use of essence of turpentine (per teaspoonful), followed by a purgative (sulphate of magnesia, 10 grammes; aloes, 5 grammes).

\section{Various other Intestinal Parasites.}

(A.) Trematodes : Amphistoma conicum, in the rumen of the ox, the sheep, the goat. Generally it is inoffensive; in Australia, however, it is said to cause great losses in the flocks.

Hemistoma alatum, in the small intestine of the dog.

Gastrodiscus polymastos, in the large intestine of the horse, mule, or ass, in Egypt (Sonsino), in Senegal (Sarciron), and in Guadeloupe (Couzin).

Distoma echinatum (duck, goose), oxycephalum, ovatum (chicken, goose), lineare, dilatatum, pellucidum, armatum, commutatum. (chicken), cuneatum (peacock).

Monostoma mutabile (goose), attenuatum (goose), verrucosum (chicken, goose, duck).

In poultry the trematodes produce similar symptoms to those of round-worms and tænias. The treatment is the same as for the latter.

(B.) SpIropteras: Spiroptera megastoma and microstoma, in the stomach of the horse. S. megastoma produces, in the cardiac 
portion of the stomach, tumors from the size of a bean to that of a nut, provided with an opening and able to cause gastritis, colic, etc.

Spiroptera sanguinolenta, in the dog's stomach, where it produces inflammatory centres and gastritis.

Spiroptera strongylina, in the pig's stomach.

Spiroptera scutata, under the œsophageal mucous membrane of the ox, sheep, and pig.

(C.) Gnathostoma : Gnathostoma hispidum, in the pig's stomach.

(D.) Trichosoma: Trichosoma tenuissimum, in the pigeon's intestine, where it is often found in considerable quantities (as many as 1830 parasites); it causes an intense catarrh, a hemorrhagic chronic enteritis, emaciation, anemia, etc.

(E.) Trichocephalus: Trichocephalus affinis, in the ox, the sheep, and the goat; T. depressiusculus, in the dog; T. crenatus, in the pig. All are harmless.

(Concerning the symptoms produced by the gastrophile larvæ, see Verminous Colics of the Horse.)

\section{DISEASES OF THE LIVER.}

\section{ICTERUS : JAUNDICE.}

ICTERUS does not constitute a special morbid entity ; it is merely a symptom common to many pathological conditions.

We have long ago distinguished :

1. Catarrhal icterus, due to catarrh of the biliary canals.

2. Hepatogenic icterus, which appears as an epiphenomenon of the liver diseases (congestion, parenchymatous and interstitial hepatitis, atrophy, amyloid degeneration, fatty degeneration, carcinoma, distomiasis, echinococcus of the liver; thrombosis of the portal vein, etc.).

3. Hematogenic icterus, which is a third and very different form, considered from the standpoint of their pathogenesis may be distinguished as "icterus of stasis," "icterus of absorption," and "icterus of retention," also "mechanical icterus"-all, without exception, are associated with a liver trouble or with an obstacle preventing the discharge of the bile, while the hematogenic icterus is due to a decomposition of the blood produced during the course of infectious 
or septic diseases, septicemia, contagious pneumonia of the horse, typhoid fever, anthrax, etc.

Catarrhal icterus is the only one which can be looked upon as a special disease; the bilious poison constitutes its principal phenomenon. In the other two the icteric symptom is a secondary matter, and may even be wanting.

Etiology of catarrhal icterus. Catarrhal icterus, which is rare in the horse, is especially frequent in the dog. It most cases it is but a complication of the duodenal or gastro-duodenal catarrh, determined by the inflammatory obstruction of the ductus choledochus : the bile, not being able to escape any longer into the intestine, penetrates into the lymphatic system of the liver, and thence passes into the blood.

The causes of catarrhal icterus are confounded with those of gastro-duodenal catarrh. The most common are: the irregularities of meals, overloading of the stomach, ingestion of tainted food, and irritations of a mechanical nature, thermics or chemicals, infections of the mucous membrane of the small intestine, and blood stagnation consecutive to heart disease.

Anatomical alterations. The so-called catarrhal alterations are circumscribed to the intestinal portion of the ductus choledochus, and more especially marked toward its entrance, which is often obstructed by the inflammatory swelling. The biliary canals are very much dilated (in the dog they may present the volume of a finger); the gall-bladder is much distended by the bile, and is only emptied with difficulty through pressure; sometimes the thickened bile forms a grumous, friable mass ; the finest hepatic canaliculi are the seat of a bilious injection, which is clearly defined, and the ductus choledochus may be obliterated by an exudate for a considerable part of its length. The liver is voluminous, much impregnated with bile, and anemic ; the hepatic cells are pigmented or atrophied (cirrhosis), according to the more or less extended duration of the disease.

All organs, with the exception of the cartilages, the peripheral nerves, the white substance of the brain, the spinal cord, and the horny tissue, are more or less colored yellow.

The cadaver is anemic. The number of white globules is increased; the volume of the red globules is very variable (Siedamgrotzky). We find constantly a fatty degeneration of the myocardium, the kidneys are anemic, the epithelium of the canalicula urinifera is the seat of a fatty degeneration with yellowish-brown pigmentary granu- 
lations (Siedamgrotzky). Finally, we find hemorrhages in the different organs and mainly in the mucous membranes.

Symptoms. At the outset the symptoms of gastro-duodenal catarrh form the base of the clinical picture of catarrhal icterus. This affection is preceded by a disturbed digestive function, vomitings, and often diarrhea; the tongue is coated, and the thirst very intense, etc. Soon the characteristic symptom of icterus appears. Before all other tissue, the sclerotic, conjunctiva, and eyelids present a yellow color with quite different shades, depending on the intensity of the affection; this tint also appears later upon other visible mucous membranes, and mainly upon that of the mouth, skin (dog and sheep), in the urine, perspiration, milk, etc. According to the case, the urine is of a lemon-yellow, dark brownishyellow, or greenish-yellow, due to the coloring matters of the bile; these may be extracted by stirring with chloroform. Their detection is ordinarily made by Gmelin's method, with the help of diluted nitric acid, which is gradually added to the urine; the green tint of the mixture disappears and is replaced by blue, violet, and red. We can also apply Fleisch's method: the urine is mixed with a concentrated solution of Chilean nitrate, then is added concentrated sulphuric acid, which produces the same changes of color as by the preceding method. Besides the coloring matters of the bile, the urine contains albumin (very often in small quantity), pigmentary granulations, fat-drops, yellow-colored epithelial cells, short renal cylinders much pigmented, and biliary acids. In the dog, biliary matters may exist in the urine without any yellow coloring of the mucous membrane; we have pointed out this fact in relation with gastro-duodenal catarrh. In the horse, on the coutrary, in numerous cases of icterus, the urine does not seem to contain the coloring matters of the bile.

According to Latschenberger, Gmelin's method would be insufficient in demonstrating the presence of coloring matters of the bile in the urine of the horse; there is said to be formed a brown or darkish zone masking the characteristic modifications of the color. Latschenberger dilutes the urine, adds a solution of baryta, lets it stand for twelve to twenty-four hours, decants the dark supernatant liquid and submits it to Gmelin's test. Often, in proceeding this way, the chromoscopic reaction is but little marked.

While the introduction of the coloring matter of the lile into the blood determines the yellowish tint of the organs, the resorption of 
biliary acids produces serious symptoms. First of all, these acids slacken cardiac activity, and lower the temperature by several degrees (to $36^{\circ}, 35^{\circ}$, or $32^{\circ} \mathrm{C}$.) ; through their influence upon the nervous centres they produce great weakness, stupefaction, and, in the horse, pronounced symptoms of immobility. The cessation of the biliary flow into the intestine leads to another series of troubles. Constipation occurs, which is due to a want of stimulation of the mucous membrane of the intestine through the bile, and to a diminished secretion of intestinal liquids. The excrements are of a clearer tint, and often have an unbearable odor; in dogs fed on meat they become gray or clay-colored, a modification which is due to the circumstance of the absence of bile, which under normal conditions saponifies the fat contained in the stomach. The increase in size of the liver is but very rarely observed in the horse.

Course. The course of catarrhal icterus is very variable according to the species. It usually lasts a few weeks. In the dog it often causes death by a complete obstruction of the ductus choledochus; in some cases (serious icterus, cholemia), it is complicated by a "hemorrhagic diathesis."

Treatment. This is identical with that of gastro-duodenal catarrh. We may prescribe a strict dietetic regimen (meat for the dog, green fodder, turnips, etc, for the horse) and the administration of salts, among which the artificial Carlsbad salts are the most advisable (see page 89 ). In the $\mathrm{dog}$, the following preparation may be used with advantage: Artificial Carlsbad salts, 10 gaammes; distilled water, 150 grammes. Dose, three tablespoonfuls daily.

We may also use calomel, castor oil, tincture of rhubarb; we should avoid the drastics, which increase the tumefaction of the duodenal mucous membrane. We have tried to diminish the latter by giving astringents (tannin in red wine); but the efficiency of these agents is at least very doubtful.

[Trasbot says that derivation by means of a plaster applied upon the belly is of much advantage; bleeding is often useful at the commencement; with the revulsion, calomel in small doses $(0.25$ to 0.30 gramme, morning and evening), and rice-water mixed with milk have given him more cures than all other means. From personal experience $I$ have found that the administration of blue mass and podophyllin in small doses three or four times a day, aided with dilute nitro-muriatic acid, in three- to five-drop doses three 
times daily, will usually cure the most serious cases in from one week to ten days.-W. L. z.]

Certain mechanical manipulations have given quite a large number of cures. Among these manipulations, we must notice: pressure exerted upon the abdominal walls in order to remove the bile which has accumulated in the vesicle, cold injections, and faradization of the liver through the abdominal walls-a remedy used with success by Siedamgrotzky. To counteract weakness, somnolence, and coma, which happen in serious cases and threaten a fatal termination, we must employ stimulants (camphor, coffee, ether, etc.). ${ }^{1}$

Icterus of newborn animals does not seem to be very rare in our domestic species. Hartmann has seen, in Babolna, colts born icteric or which became such after five or six days; he ascribes this affection to phlebitis of the cord, accompanied with an alteration of the liver. But constipation (non-elimination of the meconium) and insufficient blood-pressure may produce icterus in the newborn. According to Hartmann, the prognosis is very serious ; most patients are carried away in a few days by an exhausting diarrhea. This author advises the use of alkalines and rhubarb. In the slaughter-houses we can often find icterus in the calf.

The serious icterus (cholemia), very frequent in the dog, is ordinarily due to the complete obstruction of the ductus choledochus by a mucous plug, or to an invagination of the duodenum, more rarely to the pressure exerted upon the biliary canal by the tumefied spleen (De Jong) or by neoformations of the pancreas (Friedberger). But sometimes it is the consequence of poisoning by moulds, phosphorus, or sea-salt (tainted meat, herring-brine). Its course is extremely rapid. Death is preceded by a deep collapse, and a lowering of the temperature. The jaundice is much marked;

1 Rancilla, taking as a starting-point the theory that icterus of the dog is almost always produced by an intestinal obstruction, advises the following treatment: 1. Make the patient swallow three lead balls of a variable calibre, according to the size of the animal (16 Lefaucheux calibre for a medium sized dog; calibre 12 for a large dog), and administer, after the swallowing of each ball, a small dose of castor oil. 2. Then walk the animal, or if he is too weak, take him from time to time by the front paws and shake him slightly, and make him perform a few "dancing steps." 3. For one or two days give exclusively a slight decoction of linseed as a drink (5 grammes per litre of water). Notwithstanding the publication of some favorable results obtained by the use of this treatment, we continue to combat icterus in the dog by ordinary means, especially calomel administered in small doses until it purges. -N. D. T. 
the urine is of a dark lemon-yellow color; the skin of the inferior abdominal wall also becomes of a yellow tint. At an advanced period we may observe hemorrhages upon the principal mucous membranes.

\section{HYPEREMIA OF THE LIVER.}

Etiology. Congestion of the liver is active and passive. Its causes are very numerous. The active hyperemia is produced normally at the time of the digestion, and abnormally in the various diseases of this function, following the ingestion of an excessive quantity of food, especially in subjects not having enough exercise (fat dogs); after the administration of tainted, irritating food, or food covered with mould at the onset of hepatitis. It may also be determined by violent exertion, or by the great heat of summer; it is frequent in warm, damp, and swampy countries. The low and poorly-ventilated stables have been accused, but without sufficient proof. Passive congestion is often produced by an insufficiency of the cardiac mitral valve, by chronic affections of the lungs (pulmonary emphysema, chronic intestinal alterations, compressions of the lungs in hydrothorax, etc.). In other cases it is observed as a secondary phenomenon of the infectious diseases; also of inflammatory diseases of the intestinal canal, of cachectic conditions, and of the parasitic diseases of the liver.

Pathological anatomy. The congested liver is tumefied; its coloration is darkish; and it has a relatively firm resistance; the centre of the lobules is dark, whilst the periphery is of a light tint. When hyperemia persists for some time the particular alteration designated by the expression "nutmeg liver" is created; dark parts alternate with grayish portions and give the liver a bronzed aspect, which is similar to that of nutmeg; histologically, this lesion is produced by the dilatation of the central hepatic veins, determining, consecutively, compression, fatty degeneration, pigmentation, and finally the atrophy of the hepatic cells (passive congestion). In the course of chronic hyperemias of the liver, hemorrhages sometimes take place in the hepatic parenchyma and give it a particular pigmentation (Anacker). According to this author, this form of pigmentation of the liver is found quite commonly in animals sent to the slaughter-house; in others it is a peculiarity of the race, or a kind of melanemia.

Symptoms. These are very vague, and in consequence the 
disease is recognized in but the smallest number of the eases ; it is almost always confounded with some other affection, and mainly with gastro-intestinal catarrh. As diagnostic symptoms of the disease-although frequently wanting-may be looked for, in the dog, the sensitive condition of the region of the liver (left and right hypochondria), and a slight icterus due to the compression of the biliary canals by the distended hepatic veins. The serious hyperemias may be complicated by lacerations of the liver and by rapidly fatal hemorrhages.

Treatment. We regulate the diet, walk the patients, or leave them at liberty in a box stall, and also administer laxatives-artificial Carlsbad salts in preference to all others: such are its principal indications.

In our animals the diagnosis during life of diseases of the liver is extremely difficult; ordinarily it is made only upon the dissectingtable. The numerous errors of diagnosis committed in these cases are easily explained, if we reflect that the symptoms are usually very vague and are common to other affections, also that palpation of the suffering organ is almost an impossibility, and that its percussion is impracticable on account of the quadrupedal attitude of the animals.

\section{RUPTURE OF THE LIVER: HEPATIC APOPLEXY.}

Etiology. Laceration of the liver with a partial destruction of the hepatic parenchyma and hemorrhage into the peritoneum are ordinarily the effects of an intense mechanical action. The principal causes are: falling, uncontrollable movements performed by animals affected with colics, contusions of the region of the hypochondria, and violent exertions (Friend).

In the great majority of cases, these mechanical influences only rupture the liver, when this organ is predisposed to it by some alterations of its parenchyma or of its vessels. Among the alterations we must cite, in the first place, the amyloid degeneration. Lately, various authors (Caparini, Johne, Rabe) have insisted upon the relation of amyloid degeneration to rupture of the liver, which is said to be produced because of a greater friability of the tissue of the organ, together with a lessened resistance on the part of its vessels. Fatty degeneration (Siedamgrotzky and others) and inflammatory or hyperemic diseases (Zündel, Julian) act after the 
same manner. According to La Notte, a too substantial alimentation would also produce a fatty degeneration of the liver, and thus predispose it to rupture. Sometimes hepatic hemorrhages occur as a consequence of embolism of the hepatic artery (Wright) or from obstruction of the hepatic veins (hemorrhagic infarctus), and during the course of serious infectious diseases or poisonings (anthrax, phosphoric poisoning), which lead to parenchymatous hepatitis. Finally, they may be connected with some malformations of the liver : with tubercle, melanoma, or carcinoma (Bruckmüller).

Pathological anatomy. The alterations are usually very simple. In superficial hemorrhages the hepatic tissue is softened and transformed into a thick dark-red mass. The serous membrane often forms upon the surface of the liver soft pustules of a vesicular appearance and which contain blood. In the horse, in the case of abdominal hemorrhage, the fissure through which the blood has passed into the peritoneal cavity almost always exists upon the convex side and near the inferior border of the liver; these edges are covered with coagulated blood. We may find the hepatic parenchyma stuffed with numerous little hemorrhagic centres, the contents of which are composed of a mixture of blood and brokendown hepatic tissue. In case of hemorrhagic infarctus, well-defined cuneiform infiltrations are formed. The microscopic examination reveals all the degenerative alterations mentioned in the preceding chapter. Limited hemorrhages of the hepatic parenchyma may be the starting-point of abscess of the liver.

Symptoms. Sometimes they appear suddenly, without prodromes, at other times they are preceded by manifestations of the predisposing hepatic disease: icterus, constipation, pain on palpation of the hypochondrium, etc. In general, we observe all the symptoms of internal hemorrhage. We notice especially a very pronounced paleness, cold sweats, chilling of the extremities; the pulse and beatings of the heart are weak, almost imperceptible; the animals stagger and lie down, or fall exhausted; the eye rolls, the pupil is dilated; there is amaurosis, sudden blindness, etc. Death occurs very rapidly - as a rule, within a few hours. The small hemorrhages may become reabsorbed if they are not complicated by a fatal disease.

Benjamin, in a horse which had been kicked, three days before, in the region of the hypochondrium, found polyuria which continued 
up to death. At the autopsy he found the left lobe of the liver thickened, friable, and containing an abscess.

Treatment. No matter what means are used, the treatment is almost always useless. We may prescribe absolute rest, and the administration of astringents : ergot of rye and sugar of lead. If weakness is very great, we must use stimulants (camphor, ether, alcohol, etc.).

\section{PARENCHYMATOUS HEPATITIS.}

Parenchymatous hepatitis, inflammation of the hepatic cells, has been observed in the horse, the ox, the pig, the dog, and the sheep. But few examples of it have been mentioned, undoubtedly because the affection is very hard to diagnosticate intra vitam.

Etiology. Its causes are the same as those of congestion of the liver (ingestion of tainted food, mechanical lesions, excessive heat, infectious diseases).

Pathological anatomy. The principal alterations are: the increase in size of the liver, the disappearance of its sharp edges, its soft and friable consistence, its pale yellow color, of a clay-like aspect, perihepatitis, the union of the hepatic lobes one to another, and of the liver to the diaphragm, the augmentation in volume of the lobules, and the granular aspect of the ruptured surface; a turbid tumefaction, granular and fatty degeneration, with pigmentary infiltration of the hepatic cells, and hyperemia of the interlobular connective tissue. In some cases we find numerous small hemorrhagic centres in the hepatic parenchyma, and under the serous membrane; sometimes we find quite extensive blood collections with threatened rupture of the organ.

Symptoms. The symptoms of parenchymatous hepatitis are only distinguished from those of congestion of the liver by their intensity. As a rule they are obscure. With icterus and the symptoms of a febrile gastro-intestinal disease, there have been observed, in the horse, pains at the time of defecation (Leisering); these pains are probably due to the compression of the liver by the diaphragm and the abdominal walls. Palpation of the region of the liver, in small animals, produces also more or less marked suffering.

Treatment. We administer antiphlogistics, salts; the regimen should be dietetic. 
Acute yellow atrophy of the liver is but a parenchymatous hepatitis with a very rapid course. We observe it most frequently in lupinosis, in the pathological anatomy of which it constitutes a feature that is always present (see Lupinosis). The hepatic typhus, described by Haubner, Franzen, and others, consists also of an acute yellow atrophy, produced by alimentation with malt or with grasses from swampy meadows. In septicemia, in phosphoric poisoning, and in many infectious diseases, the parenchymatous hepatitis is frequently transformed into acute atrophy; the liver has decreased in size, it is softened, its parenchyma is yellow in color; the hepatic cells form but a fatty granular detritus. Besides we find an intense icterus of the liver (acute yellow atrophy); in some places, however, there exists a pronounced hyperemia (acute red atrophy). In the other organs we almost always find hemorrhages. The indications are those of lupinosis (gastric troubles, icterus, cerebral symptoms). Adam has observed, in the horse, anorexia, a benign icterus, a brownish-red coloration of the urine; later on, weakness, uncertainty of movements, staggering gait, trembling, diminution of the pulse-rate ; finally an intense icterus, with symptoms of immobility, and acceleration of the pulse-all this in the space of twenty-four or forty-eight hours.

Suppurating hepatitis (abscess of the liver). Abscesses of the liver are not very rare in our domestic animals. Suppurating hepatitis is but a form of parenchymatous hepatitis. It is due to different causes: 1 . Embolism of the sub-hepatic veins or branches of the hepatic artery (pyemia, acute infectious diseases. 2. Thrombosis of the ramifications of the portal system or of the umbilical vein (omphalo-phlebitis). 3. The irritating action directly exerted upon the liver by foreign bodies (food, sand, parasites) coming from the stomach, the intestine, or the blood. ${ }^{1}$ 4. Mechan-

\footnotetext{
1 Mégnin has related an interesting incident of fatal hepatic hemorrhage caused by the penetration into the liver of barbs of the flat or double-edged barley (Hordeum distichum). The horse which was the subject succumbed in ten minutes, presenting all the indications of internal hemorrhage. At the autopsy was found in the abdomen a discharge of blood of eight to ten litres; the posterior part of the liver presented an anfractuous cavity, exactly at the point of introduction of the portal vein; this cavity was surrounded by a large zone three or four centimetres wide in which the hepatic tissue was red-brown and much softened; in the region which surrounded this zone we observed a large number of brownish-red spots, having the appearance of hemorrhagic centres, and in the middle of each existed a small filiform, rigid and hard body. In the anfractuosities of the rupture a great many of these bodies were found, which had become the cause of its softening and tearing through their intro-
} 
ical lesions, hepatic calculi, the propagation into the liver of a phlegmasia which is first localized in the intestine, et. Abscesses exist at times in large numbers in the hepatic parenchyma (pyemia); they are mostly isolated, very rarely confluent; all are surrounded with a zone of consolidated hepatic tissue of a dark-brown coloration. We find almost always cuneiform hemorrhagic infarct with a central softening.

The principal symptoms of hepatic metatastic abscesses are an intense fever, which is persistent and irregular, chills, digestive troubles, and icterus.

\section{INTERSTITIAL CHRONIC HEPATITIS: CIRRHOSIS OF THE LIVER.}

\section{Chronic Induration of the Liver: Schweinsberg Disease.}

Chronic interstitial hepatitis is mainly formed of a slow inflammation of the connective tissue of the liver with consecutive compression and atrophy of the hepatic cells. It is mostly seen in the dog. In the horse it is known under the name of Schweinsberg disease. It is also found in the ox and the pig, but more rarely.

Etiology. The causes of this disease are as yet unknown. In the case of the horse it has been ascribed to swampy districts and to irritating plants (the latter being believed to act upon the liver of animals in the same way as alcohol upon the human liver). Bruckmüller has found it in a horse affected by a very evident pulmonary emphysema. In the dog, cirrhosis of the liver, which is quite frequent, coexists almost always with valvular defects of the heart (cardiac liver).

Pathological anatomy. According to the phase of the morbid process, we find in the liver hyperplasia, increase in volume (hypertrophic cirrhosis, or reduction (atrophic cirrhosis). Its consistence is firm ; it has often acquired the resistance of leather or cartilage;

duction into the liver. The hepatic territory in which they were found corresponded with the left cul-de-sac of the stomach. The author states that they had penetrated into the gastric mucous membrane, through the peristaltic contractions of the stomach, then into the muscular wall and the serous membrane, and finally into the liver, where they stopped, as no further influence that would cause progression was exerted upon them. We must notice that if these foreign bodies had reached the liver by way of the ductus choledochus or the portal vein they would have been found disseminated in the whole hepatic parenchyma mass. (Bull. Soc. centr. Vét., 1875.) -N. D. T. 
it creaks under the sharp knife. When cirrhosis is old, the surface of the liver is granulated and irregular, sometimes covered with new membranes; the serous membrane is thickened, whitish, and opaque. At the beginning the color of the liver is nutmeg-brown; later it turns light yellow, and the organ becomes anemic. On section we find a fibrinous network formed by the connective hyperplasia, a network the principal traces of which circumscribe more or less large fields of the atrophied hepatic parenchyma, whilst its ramifications separate the various hepatic lobules. From a histological point of view we observe, at the beginning of the process, a cellular infiltration in the connective tissue of the portal spaces; later the proliferation of the intra- and extra-lobular connective tissue commences. ${ }^{1}$ With these alterations of the liver we find also a passive hyperemia and a chronic catarrh of the stomach and intestine, ascites, anasarca, congestion of the meninges, and hemorrhages in different organs. In Schweinsberg disease there exists a manifest gastric dilatation.

Symptoms. They appear very slowly. In most cases the disease starts insensibly with digestive troubles; constipation alternates with diarrhea, and emaciation becomes gradually apparent. To these symptoms are added a slight icterus, the sensitive condition of the region of the liver to palpation, and, in the dog, ascites and anasarca. In the horse we observe, in addition, a special sensitiveness, weakness, periodic colics, and symptoms of immobility. The causes of cerebral troubles are obscure; they may be attributed either to the retention of biliary matters in the blood (acholia) or to a complication of chronic pachymeningitis. At the start the trouble is apyretic; later, a febrile reaction sets in which is more or less marked $\left(39^{\circ}\right.$ to $39.5^{\circ} \mathrm{C}$.). Its duration varies from a few months to several years.

Treatment. The treatment of chronic hepatitis is purely palliative. It is based on the use of alkalines (Carlsbad salts) and diu-

1 In our domestic animals, but mainly in the dog, we may recognize three different varieties of cirrhosis found in man : 1. Atrophic cirrhosis (Laennec)-annular, multilobular, and extra-lobular, of venous origin, which starts with a phlebitis of the small portal vessels, and in which the liver is atrophied, deformed, granular, and the hepatic cells altered or destroyed. 2. Hypertrophic cirrhosis (Hanot)-insular, monolobular, extra- and intra-lobular, which starts with an angeiocholitis of the small biliary canals, and in which the liver is very voluminous and the hepatic cells are either intact or hypertrophied. 3. Mixed or intermediary cirrhosis (Dieulafoy), in which we find added some elementary lesions belonging to atrophic and hypertrophic cirrhosis.-N. D. T. 
retics. It is also necessary to regulate the alimentation and give especially green food. The so-called Schweinsberg disease is a particular form of chronic interstitial hepatitis, similar to that observed in lupinosis. It was first recognized in Schweinberg, in the valley of the Ohm (Hesse), and later in the valleys of Glon and Zusam (Bavaria), as well as in other regions. It seems to depend upon telluric conditions and on the particular constitution and properties of the plants which are consumed by the animals. It appears in the form of a persistent local enzootic in districts which are exposed to frequent freshets and in countries where the soil is turfy and swampy. It is unknown upon the high lands and on farms located at a certain height. It is admitted that it is produced by irritating substances, which act slowly but constantly upon the liver (like alcohol or lupinotoxine). Its intimate causes are hardly known. In the Glon Valley the alimentation of clover has been generally considered as the principal cause of its development. It has been stated that it more frequently affects horses between the eighth and tenth year, but more recent observations seem to weaken this assertion. At Schweinsberg the disease has been the cause of the death of all the horses of several stables. At first the symptoms are very vague, and often unperceived; in other cases they are confounded with those of chronic gastro-intestinal catarrh. The appetite is capricious; the animals gape often, and are depressed or affected by abdominal pains. These manipulations may persist without special modification for a period varying from several weeks to a few months. Then, in most instances, we have the manifestation of icterus and symptoms of immobility (a staggering gait, leaning of the head upon the manger, tottering, etc.), to which are added attacks of colic, constipation, and fever. There is often absolute anorexia. The emaciation increases with the persistence of the disease. From time to time a passing improvement may be observed; but soon the symptoms grow aggravated, the limbs œdematous, and death follows from exhaustion. Some cases have been related where death occurred within three or four days; the duration of the affection varies generally from a few weeks to nine months. At times death happens abruptly during the course of the colics, through the rupture of the stomach (Friedberger):

At the autopsy we find alterations in the liver which consist mainly of an inflammatory hyperplasia of the interstitial and interlobular connective tissue (Bonnet), with centres where the hepatic 
cells are affected by fatty degeneration. In addition, we may see a considerable gastric dilatation and lesions of catarrh (a slaty coloration and hypertrophy of the gastro-intestinal mucous membrane). All treatments directed against the Schweinsberg disease have been unsuccessful. In the country where it exists the owners of horses know its fatal course perfectly well; they therefore get rid of their animals at the beginning of the trouble, when the stock is yet in good condition, and have preserved enough vigor to be exposed for sale.

\section{AMYLOID DEGENERATION OF THE LIVER.}

Amyloid degeneration of the liver has been found alone or coexisting with degeneration of the kidneys, the spleen, or the intestine. It has been found in the horse, the dog, the ox, the sheep, the rabbit, and in poultry.

Etiology. In our domestic animals the principal causes of this affection are cachectic conditions, chronic inflammations, and suppurations of long duration. In 50 per cent. of the cases of amyloid degeneration of the liver observed by Rabe in the horse, the alteration took place under the influence of pleurisy, pericarditis, or chronic peritonitis. This author has also found it in cases of hydrothorax and muscular suppuration. Caparini has found it in exudative pleurisy, orchitis, phlebitis, and cachexia. Bruckmüller has found it in oxen affected by chronic nephritis. It may finally depend upon the alimentary regimen and the conditions under which the animals have been kept: it is under these circumstances that Bruckmüller has observed it in animals which consumed malt, and Werner saw it in lambs fed largely upon oats and kept in confined stables.

From these facts it results that amyloid degeneration is due to the same causes in animals as in human beings. The following causes are especially suspected of producing the trouble in man : chronic pulmonary phthisis, intestinal tuberculosis, suppurations of long duration, syphilis, broncho-ectasia, dysenteric intestinal ulcerations, pyelitis, fistulæ, and ulcerated carcinoma.

Pathological anatomy. The liver which has undergone amyloid degeneration is hypertrophied, its edges are thick and rounded; in the horse, contrary to what exists in man, its consistence is soft and clammy; its substance is very friable; it is anemic, of a 
brownish-gray color; sometimes the serous membrane is covered with plastic exudations.

The amyloid centres may be distributed regularly or irregularly; in the chicken they are found in the shape of small disseminated spots (Leisering). Microscopical examination shows that the walls of the hepatic capillary and mainly the interlobular vessels are thickened, homogeneous, and transparent. The hepatic cells exceptionally participate in the amyloid degeneration (Johne); on the other hand, they are always found atrophied and infiltrated with fat. Outside of the liver we often find amyloid degeneration in the spleen, the kidneys, and the intestine (Bruckmüller, Rivolta, Rabe). Amyloid degeneration may be revealed through chemical process; Lugol's solution of iodine colors the hepatic substance a red brown or mahogany red; if diluted sulphuric acid is then used, we obtain a tint varying from bluish gray to violet blue; methylviolet gives a red aniline color.

Symptoms. They are very vague. We may suspect the disease when tumefaction of the liver is established, or albuminuria (amyloid kidney) and symptoms of cachexia. The icterus is far from being constant. Rupture of the liver, which is accompanied by internal hemorrhagic symptoms, is quite a common complication.

Treatment. It is almost always insufficient. It has to be applied according to the causes which have determined the hepatic lesion. In cases where amyloid degeneration was the consequence of an improper diet, Werner has obtained favorable results by having the food modified.

\section{CARCINOMA OF THE LIVER.}

Pathological anatomy. Carcinoma of the liver is either primary or secondary, It is quite often found in old fat dogs ; it is rarer in the horse and other domestic animals. The liver is hypertrophied; its surface is uneven and irregular; the organ is filled with miliary nodules, and more voluminous neoformations reaching fometimes the size of the fist; they are sometimes isolated, at other times confluent or gathered in series, usually of a soft consistence and a clear yellow color. These neoplasms are often richly vascularized; the centre is softened or calcified. When carcinoma of the liver is secondary we find tumors in the portal system, in the spleen, kidneys, pancreas, omentum, lungs, etc. 
Symptoms. In the $\operatorname{dog}$ and the cat we may suspect the existence of hepatic carcinoma when palpitation of the hepatic region reveals an irregular and rough surface, or when external tumors exist (especially common upon the teats). Among the morbid phenomena which may accompany them we must mention : in the dog, ascites (due to carcinomatous peritonitis or to cirrhosis); in the horse, colic, also caused by peritonitis (Röll). We may observe symptoms of an internal hemorrhage consecutive to softening and rupture of carcinomatous tumors, which frequently contain hemorrhagic centres

[Muscular emaciation is so marked and constant in this disease that it may almost be given pathognomonic value; this condition is especially marked upon the masseters and temporo-maxillaris muscles, giving to the patient a physiognomy that is altogether special, and which may be called a cancerous cachexia.-W. L. Z.]

\section{BILIARY CALCULUS: BILIARY LITHIASIS.}

Biliary calculi of our domestic animals but rarely give rise to serious troubles (calculous colics), contrary to what is observed in man-in whom, by the way, these calculi are much more frequent than in animals. It is in the ox and the dog that they are mostly found. They have also been found at times in the horse, the pig, and the cat.

Etiology. This morbid condition is caused by penetration of alimentary particles into the biliary canals; catarrh of these canals; some hepatic disease influencing the secretion of the bile; stagnation, condensation, decomposition of the bile, and the precipitation of its soluble elements (cholesterin, calcite of bilirubin). These are the principal causes capable of producing the formation of biliary calculi. They may be found in the gall-bladder and in the biliary canals. The smallest calculi vary in size from a poppy seed to a pea ; they are generally of a round shape, oval, or cylindric; their surfaces are often abraded by attrition. Birbaum has found as many as four hundred in one horse. The largest may be ten centimetres long, and have a diameter of four centimetres; those contained in the gall-bladder are pyriform; those found in the canals are cylindric; oftentimes they considerably dilate the duct containing them.

Symptoms. Biliary calculi produce colics, which appear sud- 
denly and persist for a few days, together with icterus, diarrhea, or constipation (Birnbaum). They also frequently produce troubles of digestion. ${ }^{1}$

Diagnosis. The diagnosis may be formulated by taking as the basis the coexistence of colics and icterus.

Treatment. We ought to give Carlsbad salts and alkalines, regulate the diet, and give the patients moderate exercise.

\section{PARASITIC DISEASES OF THE LIVER. (LIVER FLUKE.)}

\section{Distoma Hepaticum.}

This disease is frequent in the sheep, quite common in the pig, much rarer in the ox. Distomiasis is produced by two species of parasites, which are found isolated at times, or simultaneously, in the biliary vesicle and canals.

1. The hepatic distoma (Distoma hepaticum), one centimetre and a half to four centimetres long, one-half to one centimetre wide, of an oval or oblong shape. It is found in the sheep, the ox, the goat, and the pig, and more rarely in the horse, the donkey, and the cat.

2. The lanceolated distoma (Distoma lanceolatum) is smaller, $\frac{1}{2}$ to 1 centimetre long, 1 to 3 millimetres wide, lanceolated or linguiform. It is more particularly observed in the sheep and the ox, but is also found in the goat and the pig.

Natural history. The distomas are plathelminths of the Trematode group, which, in order to reach a complete state of development, undergo very complicated metamorphoses; the studies of Leuckart and Thomas have thrown some light upon those of hepatic distoma.

The eggs, provided with an operculum, are expelled with the excrements, when the temperature and humidity are favorable; within four to six weeks an embryo is hatched with a ciliated

1 Chassing has found a case of tearing of the gall-bladder produced by calculus arrested in the ductus choledochus. The ox which was the subject of this observation had shown symptoms of indigestion: colics and meteorism, pulsations 70, respirations 50 ; the mouth was hot and dry, the extremities cold. At the autopsy an acute peritonitis was found, which was consecutive to the rupture of the gall-bladder, and at the mouth of the ductus choledochus an encysted calculus, absolutely preventing the discharge of bile.-N. D. T. 
cuticular membrane which is provided with a sting at its anterior pole. This ciliated embryo penetrates into the respiratory cavities of some mollusks, and mainly of the Limnoea peregra and L. truncatula (Leuckart). According to Thomas, it is especially the latter which shelters the embryo. Of about half a centimetre in length, very common, and very resistant, provided with a spiralshaped shell, living rather on the ground than in the water, it would be more likely for the embryo to penetrate it than the Limncea peregra. This author believes that the ciliated larvæ do not penetrate directly into the respiratory cavities, but that they reach the mollusks by perforating the wall of the body and the intestine, or by ingestion.

Within fourteen days in summer time; from three to four weeks in winter, the embryo is transformed into a sporocyst provided with germinative cells which are developed in their length in order to form the redies (cercaria sacs); these latter, in undergoing a new metamorphosis, give birth-directly according to Leuckart, indirectly according to Thomas, who describes female radies-to the cercaria, small microscopic beings provided with a tail, which live in liberty in the water. A single distoma egg gives birth to nearly 1000 cercaria.

Leuckart states that the sheep ingests the cercaria either by swallowing the limnæa with the food, or by drinking water in which they are suspended, or through the intermediary of a new host (mollusk, worm, etc.), in whose body the tailed cercaria are said to penetrate, and where they could be preserved for more than two years. Quite recently Thomas has demonstrated in a decisive way that the cercaria leave their host's body (redie or mollusk) in order to swim in the water and to fix themselves upon aquatic plants or damp meadow grass. They lose the tail, become ball-shaped and surrounded with a clammy mass which gives them a cyst of a diameter of two or three millimetres, and of "snowy whiteness;" they preserve their vitality for several weeks. The sheep ingest them in browsing the grass to which they are adhering. Spinola had already established by experiments that healthy sheep contract distomiasis when they are made to ingest limnæa containing cercaria, picked up in the infested pastures.

Distribution of distomiasis. The disease has been known from time immemorial. It appears in the ancient Germanic jurisprudence as a "vice setting aside a contract of sale." It is de- 
scribed by Gentilis Arnulphus toward the middle of the sixteenth century.

It makes numerous victims during damp years (snail years), which are particularly favorable to the distoma and limnæa; then we observe distomiasis in an enzootic or even panzootic form; it is rare during dry years. This observed fact testifies to the enzootic character of the affection in regions which are periodically under water (as the Narenta district in Dalmatia), as well as in the flat regions of northern Germany, the meadows of which are damp and swampy. In Holstein there is, so to speak, hardly an animal killed which is not infected by the parasite (Leuckart).

Among the most remarkable distoma years, we may mention $1753,1816,1817$, and 1854 . The disease ravaged whole provinces at these periods. In Germany the decennial period, 1850 to 1860 , was particularly inauspicious. For France, Davaine points out nine distoma years in the first half of this century. In 1812 distomiasis destroyed 300,000 sheep in the vicinity of Arles; in 1873 it carried off one-third of the ovine population of Alsace-Lorraine, occasioning thus, according to Zündel, a loss of 1,150,000 francs; in 1862 it was seen in Ireland with marked virulence, where 60 per cent. of the sheep died. The number of victims which it secures yearly in England is estimated to be 1,000,000. During the year 1876 Slavonia lost 40 per cent. of its cattle by distomiasis. In Brazil, in the single district of Tondil, it occasioned the death of 100,000 sheep in the space of eight months.

According to Krabbe, the hepatic fluke-worm does not exist in Iceland, while it is very common in the Faroe Islands-a fact which is evidently in close relation with the nature of the mollusks of these countries.

The sheep are ordinarily infested when they are brought into damp, swampy, water-covered, turfy pastures, etc. The water-pools which are to be found in such places are dangerous. Formerly the shepherd accused a plant, the Lysimachia nummularia ("fluke-worm grass"), which certainly does not possess any influence upon the development of distomiasis. Lambs, and in general animals with a weak constitution, like fine wool merinos, are the subjects which are most exposed to contract it ; it is from these that the disease secures the greatest number of victims. It is not absolutely necessary for the animals to go to the pasture in order to be stricken by it; infection sometimes takes place in the sheep-fold, either through 
the consumption of green fodder, or through the ingestion of water containing cercaria. The disease may be contracted very rapidly. Many animals left in the fold on account of indisposition, or of some other affection, or which have been carried by the shepherd because they were very lame (Hahn), have been observed to escape distomiasis, while the whole remainder of the flock became infested.

From time to time it is observed in the hare, the stag, and mainly in the deer; these beasts lose flesh, become greatly emaciated and die of marasmus.

Ingestion and emigration of the hepatic fluke-worm (Distoma). The infection of the flocks takes place during summer and fall, up to the first frosts (Gerlach); the summer's heat is very favorable to the hatching of the eggs, but the night frosts do not destroy the larvæ of the distoma (Friedberger), and the animals may contract the disease during the last months of the year, even in December.

As we have already stated, this infection may take a very rapid course : a quarter or half an hour's grazing in a meadow which is invaded by these parasites is often sufficient. Sometimes successive invasions are observed upon the same animals, or it may appear in spells upon some groups of animals of the same flock.

We have three theories before us concerning the mode of introduction of the fluke-worms into the liver :

1. The fluke-worms are said to reach the liver through the portal system. According to Heller, it seems, indeed, to be in this way that the invasion of the liver by the embryo of echinococcus is produced; this opinion, however, concerning distomiasis is not based upon any fact.

2. The embryos having become free by the digestion of their covering membrane, would pass through the stomach and intestine, and penetrate into the liver through its peritoneal coating, and would finally settle in the biliary canals (Gerlach, Spinola, May). Friedberger points out that the frequent existence of perihepatitis is far from being a sure proof of the correctness of this hypothesis; the inflammation of the serous membrane which surrounds the liver may just as well be produced by a centrifugal emigration of the fluke-worms, by their sub-peritoneal progression, and by the perforation of the serous membrane from the inside outward. It is not rare to find a fluke-worm's head protruding from the liver surface. 
3. The fluke-worms pass directly from the duodenum into the ductus choledochus (Leuckart). This hypothesis has the most probabilities in its favor. According to Leuckart, the progress of the hepatic worms into the liver proceeds as follows: the suction apparatus serves to dilate the narrowest biliary canals; this alternate function of suction produces successively the stretching and contracting of the anterior part of the body, and determines the progress of the parasites, while the scaly appendices of the cuticula prevent its retrogradation. The lanceolated fluke-worm does not possess any of these points upon the surface of the cuticula, but its progression is favored by its tenuity.

If most of the fluke-worms which have invaded the liver remain in the biliary canals, there are some which perforate the walls of these canals, penetrating into the hepatic parenchyma, which they destroy and furrow ; they perforate Glisson's capsule as well as the serous membrane which covers it, and produce a perihepatitis or a peritonitis; others penetrate into the ramifications of the portal system, where the fluke-worms cause endophlebitis, thrombosis, and embolism; others finally penetrate into the central hepatic veins, and thence to the most distant parts of the body. After having crossed the right heart, the parasites reach the lung, where they produce hemorrhagic centres, also caverns with bloody contents and pseudo-tubercles (Gurlt, Rivolta, Hedley, Mégnin, Schell, etc.). It is especially in the ox that cysts and pulmonary tubercles are found, which are of the size of a hazel-nut, a chestnut, or a hen's egg, with thick walls, which are calcified and contain the parasite, swimming in a brownish viscous liquid. Morot has found these parasites in the slaughter-houses of Troyes upon animals of the bovine species, in a proportion of 4 per cent.; they contained one or two fluke-worms. From these cysts the parasites pass into the pulmonary veins (Friedberger), and are carried along by the strong current of the circulation in the various organs; thus Drosse ${ }^{1}$ found some in the skin of a cow's belly, and Morro in the intercostal muscles. Hertwig found some in the diaphragm and the pharyngeal muscles of the pig. In man peripheral abscesses with fluke-worms have been found upon the sole of the foot (Giesker), upon the neck (Harris), behind the ear (Fox), and in the region of the right hypochondrium (Dionis). The opinion of Leuckart, according to which the worms would have reached these

1 Drosse: Preuss. Mittheil., 1855-56. 
points by starting from the vena cava, is not admissible. In the first stage of distomiasis we observe sometimes apoplectic death, due undoubtedly to cerebral embolism produced by young flukeworms which have penetrated into the arterial circulation (Gerlach).

The fluke-worms begin to leave the liver at the time of reaching sexual maturity, a condition which occurs within about three weeks, as stated by Leuckart. Gerlach has asserted that emigration takes place from nine to twelve months after ingestion-that is to say, toward the months of June or July. Pech and Friedberger have found in the fall and winter numerous fluke-worms in the gallbladder and in the duodenum (in the large intestine they are digested very quickly; they are, therefore, never detected in that organ). According to Thomas, the duration of the fluke-worm's life varies from one to several years (six years in one case).

Pathological anatomy. The main alterations are found in the liver; they differ mainly according to the period in which they are observed. In most cases the left lobe is first affected (Falk). The liver is more or less hypertrophied; its weight may have doubled or trebled; its edges are rounded and its coloration has changed; the appearance of the latter is at times very close to porphyry; upon a yellow, gray, or dirty yellowish-brown ground we observe dark red or dark reddish-brown spots, which are round, linear, or ramified, and which are irregularly distributed. The surface of the liver is coarse, irregular, covered with filiform ' or membranous neoformations, which sometimes join the liver to the diaphragm very closely (perihepatitis). We observe quite often, in the capsule, openings of the diameter of a pin-head or a milletseed, through which trickles a bloody dirty matter, or which is filled with a worm's head. When performing an incision upon their surface in the liver we often find, immediately below the capsule, a more or less considerable quantity of young fluke-worms located in grooves or in sinuous passages. The lymphatic ganglions which are located upon the hilum of the liver are tumefied and œdematous.

On section we see variable alterations of the biliary canals and hepatic parenchyma ; there are many lacunar, chambered spaces of a very irregular form, and full of a clammy, bloody, red-brownish, or grayish matter, formed of red globules and leucocytes, also of hepatic cells having undergone fatty degeneration, broken-down tissue, and fluke-worms in an imperfectly developed stage. The 
biliary canals have ordinarily trebled or quadrupled in size; in the ox they may reach the diameter of the thumb, which gives the liver a "vesiculous" or eystic appearance. In the dilated biliary canals we find, with a red mucous bile, fluke-worms and their eggs in more or less large quantities ; in the sheep's liver the number of parasites may increase to one thousand. The mucous membrane of the canals, which is constantly irritated by the stinging cuticula of the large fluke-worms, becomes the seat of a hemorrhagic mucous or purulent catarrh; later on it is considerably thickened by a connective tissue neoformation; it may become incrusted with phosphate of lime. The biliary canals are then transformed into hard columns, which are very visible to the naked eye; they are often prominent upon the surface of the liver. At the same time the hepatic parenchyma becomes more and more atrophied, hard, and creaks under the sharp instrument; its surface is irregularly embossed (atrophy of the liver, cirrhosis). In the ramifications of the portal system we may find thrombosis produced by fluke-worms floating free within the bloodvessels. The gall-bladder contains, as a rule, turbid and dirty bile, isolated fluke-worms, and eggs in considerable quantity.

At a more advanced phase of the disease we find almost always ascites, with isolated fluke-worms in the peritoneum, as well as a thickening of the pleura and pericardium. The carcasses are much emaciated, the fat has entirely disappeared, the muscles are pale and flabby; the blood is thin and watery; the connective tissue is infiltrated with oedema (anemia and hydremia).

Symptoms. The symptoms of distomiasis have nothing characteristic; at the start, and often during several weeks, there does not exist any morbid manifestation; after one or two months, where the trouble has acquired a certain gravity, we observe symptoms of cachexia, serious disturbances of nutrition, and hydremia (the "sheep rot"); but all these phenomena are common to other diseases. The animals lose flesh very rapidly; the mucous membranes are pale, as if washed; the wool is dry, dull, and drops off very easily; the eyelids, the larynx, and the belly become odematous. In the ox the hair appears dull and brittle, the skin is dry and sticky. Gastric complications are added to these symptoms. The appetite is capricious or wanting, rumination is suspended, and constipation alternates with diarrhea ; the urine becomes acid. The sick sheep are very weak, they remain behind 
the flock, and do not avoid being caught; the cul-de-sac of the conjunctiva becomes filled with mucus; the temperature presents abrupt variations; the obstruction to the return circulation produces ascites, bronchial catarrh, etc. In the ox the disturbing symptoms appear very late and the disease rarely ends in death. When slaughtered, we may see considerable hepatic lesions without any manifestation of their existence by any kind of symptom during life.

Formerly hepatic hypertrophy, detected through the abdominal walls, and the icteric coloration of the mucous membranes and skin, were looked upon as very important manifestations from a diagnostic point of view; but these two symptoms are oftener wanting than present. We have never seen icterus in this disease, and this is also the experience of Gerlach. Concerning the sensitive condition of the liver and the tumefaction of this organ advancing behind the posterior edge of the last rib, these are two phenomena which can only be perceived during the first stage of the disease; when this is advanced they are seen no longer. A fact which insures the diagnosis is the presence of fluke-worm eggs in the excrements (oval-shaped eggs, with opercula); but in the large majority of cases the existence of distomiasis is positively established by the autopsy.

It is not unusual to fiad small numbers of fluke-worms in animals appearing perfectly healthy.

Course. The course of distomiasis is mainly chronic. Death through cerebral apoplexy (cerebral embolism) is exceptional.

We may, with Gerlach, accept four stages of the disease : 1 . The invasion, soon followed by traumatic hepatitis, by inflammatory tumefaction of the liver; it occurs during the latter end of autumn; it is almost always disregarded during life. 2. Anemia, which appears from six weeks to two months after the opening of the pasture; it is mainly observed from September to November. 3. Cachexia, which starts three months after the ingestion of the fluke-worm's eggs ; it is the ultimate stage of the disease; the liver becomes atrophied and odemas are formed; this period sets in toward the month of January. 4. The emigration of the flukeworms, which takes place in May and June, as stated by Gerlach, but which may start much sooner, from the moment when the parasites have reached sexual maturity - that is to say, about three weeks after the ingestion of the eggs. 
When the distomas invade the liver in large numbers the disease often takes a more rapid course. In well-nourished adult animals the symptoms of anemia and cachexia may disappear with time, even after years, and an apparent cure is not a rare thing; but there are always persisting lesions of the liver. In general, the prognosis of advanced distomiasis is very unfavorable. It is in winter and the beginning of spring that the trouble acquires its maximum gravity.

Cadéac has related a case of hepatic distomiasis in a female donkey. The biliary canals were dilated in the shape of blisters. The animal showed a marked emaciation, with a very pronounced paleness of the mucous membranes. At the autopsy were found, besides the alterations of the liver, dropsies in the peritoneum, the pleura, and the pericardium.

Treatment. The treatment must be prophylactic. We must avoid taking the sheep into infected pastures, and destroy the flukeworm eggs: such are its two principal indications. The first is the shepherd's business; he should keep the flocks away from doubtful pastures as soon as the damp weather sets in; according to an old custom, after St. John's day. This precaution is particularly important for the lambs and wethers. Besides, the animals should be given an alimentation keeping them in good condition. Observation has taught that the more resistance they possess, the more easily they escape infection.

In order to destroy the fluke-worm eggs and to prevent their dissemination, several means have been recommended. Thomas has advised to kill the sick animals at the time when the meat may still be used for consumption; to destroy the liver or feed it to the dogs after having it well cooked; to drive the animals into dry pastures, where their excrements will also be carried; finally, to drain the wet pastures and spread salt or chalk on them.

In order to kill the cercaria contained in the stomach and intestine, rock-salt should be left within reach of the flock. This agent, which the animals lick constantly, may replace the bitters, aromatics, and astringents used formerly. ${ }^{1}$

1 Branches of the oak, elm, and yoke-elm-cut in July or August, and dried with their leaves, and given in the sheepfold before the starting of the sheep for their pasture-constitute an excellent preventive means. Those of the juniper and of all the aromatic trees are equally useful. Buds of the sea-pine, dried and mixed with bran, have a true curative value. I have many times obtained remarkably favorable results from these various treatments in Sologne.-L. T. 
When in a more or less extended radius it is impossible to check the trouble, the raising of sheep there ought to be abandoned. As distomiasis may be imported by sick sheep into countries where it had never been seen, Leuckart advises, in the interest of the buyer, to make a microscopic examination of the excrement and see that it does not contain any fluke-worm eggs. Thomas admits the possibility of infection of the pastures by the hare and the stag.

The time allowed for the contest of a buyer's contract is fourteen days in Bavaria, in the grand duchy of Baden, in Hohenzollern, and in Würtemberg (where the affection is known under the name of rot, dropsy); it is twenty-eight days in Hesse, thirty in Saxony, two months in Austria, fifteen days in Bâle (Switzerland), thirtyone days in Schaffhausen (Switzerland), and forty-two days in the district of Frankfort-on-the-Main.

\section{ADDENDUM.-DISTOMIASIS OF THE COMMON CRAWFISH.}

(Distomiasis Astacina.)

The "crawfish pest," which has appeared during the last few years in the watercourses of central Europe, has been principally attributed ${ }^{1}$ to the ingestion of the larvæ of Distoma cirrigerum. As symptoms (in man) we notice an upright and stiff walk, the inability of those affected to defend themselves, the tumefaction of the anal opening, some anæsthesia and irritability, convulsions, and paralysis. At the autopsy we find numerous larvæ encysted in the muscles.

This distomiasis must not be confounded with other diseases prevailing in the crawfish. (See Poisonings: Mycosis Astacina; Toxic mycosis caused by ptomaines).

\section{Echinococcosis.}

This disease is produced by the ingestion of the Echinococcus polymorphus (formerly E. veterinorum), larva of the Toenia Echinococcus of the dog. It is particularly frequent in the ox, less common in the sheep, the goat, and the pig, and much rarer in the horse. It is also found in man, in the wild ruminants (stag, chamois, antelope), in the monkey, the felines, in the turkey, etc. The echinococci have been found in almost all the organs; if they

' Harz: Zeitschr. f. Thiermed., Bd. vii. 
take up their abode by preference in the liver or in the lungs, they nevertheless do not spare the heart, the spleen, the kidneys, the muscular system, the œsophagus, the reticulum, the omentum, the subserous connective tissue, the eye, the brain, the muscles or even the bones.

Echinococcus is quite prevalent. According to Bollinger, in southern Germany it is the most common infectious disease in the ruminants, after tuberculosis and distomiasis. Schmidt estimates that it causes a yearly loss of about 200,000 francs. Its distribution corresponds with that of the dog. In India, where dogs are very numerous, 70 per cent. of the cattle carry the echinococcus.

Natural history. The echinococci or hydatids are developed after the ingestion of the eggs of the tænia mixed with the food or with drinking-water, etc. The vesicle may be seen under the four following forms: 1 . Common fertile echinococcus, consisting of a capsule filled with a watery liquid and containing a more or less large number of heads enclosed in the proligerous cysts; this is the most frequent. 2. Compound echinococcus, formed by a mother cyst containing daughter cysts (secondary), or even tertiary cysts, developed either inside (endogens), or outside (exogens) of the mother cyst. This form is also relatively quite frequent. 3. Multilocular echinococcus. These are objectively similar neoformations to cancer or to an agglomeration of tubercles; on section they show lacunar spaces; they seem to be produced by the exaggerated development of an echinococcus deprived of a capsule, having perforated this membrane. They are found in the hepatic canals; sometimes they acquire the hardness of stone. This form is very rare. 4. Acephalocyst, which is but a headless vesicle, and is consequently sterile. This later form is quite common.

The embryo reach the liver through the biliary canals or through the portal vein. Their development is slow : after four weeks the vesicle is 1 millimetre in length; in two months it is 1.5 millimetre; toward the fifth month it reaches the size of a nut. The wall of the vesicle is of a gelatinous consistence, and is composed of a double membrane-an external cuticle, which is very thick and of lamellar structure (hydatic membrane) and an internal relatively thin (parenchyma or germinal membrane). This vesicle is enclosed in a cyst which belongs directly to the host, and is shown to be formed of connective tissue, of thin muscular fibre and vessels; its dimensions vary from the size of a pea to that of a man's head; 
it is filled with a serous liquid, which is transparent and does not contain any albumin; some are found to contain as much as five kilogrammes of this liquid. The scolices of young tænias are formed at the expense of the proligerous vesicles originating from the germinal membrane; each vesicle may contain as many as thirty-four scolices, therefore one echinococcus alone may contain as many as a thousand.

Pathological anatomy. The liver is ordinarily hypertrophied; its volume may be increased fivefold or even tenfold. It presents an irregular surface, the salient parts of which correspond with the echinococcus (more than a thousand have been found in one liver). Its weight is increased in proportion to its volume. In the ox Perroncito found a liver affected by ecchinococeus which weighed 158 French pounds (livres), while the weight of the normal organ is ten pounds. In the pig the liver may weigh fifty pounds (Perroncito has found one of the enormous weight of 111 French pounds; the average weight of this animal's liver is four pounds (livres). The serous membrane which covers the organ is often thickened and joined to the neighboring organs, the diaphragm and the intestine (perihepatitis). On section, the liver is found to be filled with a number of caverns, between which the parenchyma remains in the shape of small islands or ribbons of variable dimensions. When the echinococei die their contents are transformed into a yellow, fatty, pasty material, which sometimes seems to be composed exclusively of lime; pus may be found in it, more rarely blood. When they have reached this state their nature may still be recognized through a microscopic examination; we find hooks in the contents of the vesicles.

In the lung, the echinococci are of a size varying from that of a pea to a man's fist; the surface of the lobes is uneven ; the parenchyma is compressed; often it keeps its normal appearance in some points, while in other places it is very hard to the touch. Uhse compares the lung affected by the echinococcus to a "bag full of potatoes." The lung of the ox may acquire a weight of fifty livres, its normal weight is about six livres. When the hydatids die the cysts become filled with a detritus which gives them a caseous tuberculous aspect.

In most cases the heart contains but one vesicle, which is mostly located at the inferior extremity of the median septum near the point; it may bud either toward the ventricle or the pericardium. 
In the bones, mainly at the lower maxillary, the echinococcus often produces exuberant tumors; they have been found in the medullar cavity of the tibia and femur (Perrin, Morot).

In the pig, echinococci are not rare in the muscular system; Lemke found eighteen specimens in one pound of the meat.

Symptoms. They differ according to the affected organs, but as a rule they have nothing characteristic.

1. Among the principal symptoms of hepatic echinococcosis we must point out digestive troubles, a progressive emaciation and marasmus. On percussion we find a more or less marked increase in the size of the liver (normal dulness upon the right side, on the surface of the last three or four ribs); the region of the hypochondrium is painful to palpation; the rectal exploration sometimes permits us to establish the existence of embossments or even fluctuating tumors upon the surface of the liver. But when the echinococci are not numerous they are not marked by any serious manifestation; we even possess observations in which the parasites had caused a considerable increase in the volume of the liver without producing any functional disturbance.

Ordinarily the thermometer does not record the slightest fever; this symptom appears only with complications, or during the last period of the disease. The opening of the superficial vesicles in the peritoneum may be followed by a fatal peritonitis.

2. Pulmonary echinococcosis resembling tuberculosis producing symptoms of phthisis: cachexia, the disappearance of the subcutaneous connective tissue (skin closely adherent to the bones), etc.; respiration becomes gradually more painful ; the dyspnœa appears suddenly at times when the echinococci exist in large numbers in the two pulmonary lobes; the inspiration is then abrupt, and a weak, hard cough is to be heard. On percussion we find a dull tympanic sound or a bruit to be compared to that of a cracked pot. On auscultation the vesicular murmur has disappeared from the regions where the modifications of resonance are found; it is replaced by strange bruits, and according to Harms, by a gurgling noise (which is also produced when compressing a lung containing echinococci, and to which this author attributes a pathognomonic significaance); Schmidt also points out a particular metallic sound. If percussion of the chest is somewhat violent the animals try to avoid it and complain; this manifestation of pain is accentuated when pressure is made upon the last ribs. In most cases there is 
no fever, and the appetite, as also the lacteal secretion, is kept up for a long time. The opening of a cyst in the pectoral cavity may lead to a fatal pleurisy.

3. Echinococcus cysts in the heart sometimes produce apoplectiform death through their rupture (Harms, Haussmann, Rivolta, and others).

4. Echinococcus in the muscles produces, as a rule, lameness; Johne has found a cyst in the psoas of the horse.

Prognosis. The prognosis is grave when one important organ contains a large number of these cysts; in such a case they may produce death. The presence of only one in the heart is a very dangerous lesion. It is possible that these cysts become calcified or caseated, and it has been seen that the presence of numerous hydatids in the lung and the liver is perfectly compatible with all the appearances of health. Nevertheless, when we succeed in establishing a certain or nearly conclusive diagnosis, the most advisable procedure, and the most practical, will be the slaughtering of the animal.

Differential diagnosis. The increase in the size of the liver shown by an abnormal dulness, the sensitive condition of the right hypochondrium, and the finding of hepatic embossments by means of rectal exploration, are symptoms sufficient to establish the diagnosis. In taking only the first two into account, we may confound echinococcosis of the liver with the condition of gestation, or of hydrometria, pyometria, uterine and ovarian tumors, abdominal gestation, etc.; but these various morbid conditions are easily recognized by rectal exploration. Concerning ascites, the signs obtained by percussion, and the modifications which take place in the local symptoms by the single fact of dorsal decubitus, characterize it sufficiently. The differential diagnosis from cancerous tumors of the liver or a considerably developed hydronephrosis is more embarrassing; these affections, however, are rare.

Echinococcosis of the lung must be differentiated from tuberculosis. According to Harms, the diagnostic symptoms would be the following: 1 . In echinococcosis the cough is often absent, or is very slight, while in tuberculosis it is never wanting and is usually severe. 2. The gurgling noise is pathognomonic of the presence of the echinococcus in the lung. 3. In echinococcosis the respiration is more frequent, more painful; the dyspnœea increases rapidly ; it is not in proportion to the general condition of the subjects, which is often excellent. 4. The friction bruits are always 
wanting in echinococcosis, while they are frequently heard in tuberculosis. 5. In echinococcosis it is at times possible to recognize an increase in the volume of the liver. Finally, tuberculosis in the lymphatic ganglions participate very generally in the morbid process, and there exists at times a tuberculous mammitis.

Treatment is absolutely illusory. We could, it is true, try puncture of the cysts, the same as in man, but it is far preferable to sacrifice the patients.

The prophylaxis is of the highest importance : destroy the organs affected by the echinococcus (a measure which ought to be obligatory); free the dogs from the Toenia Echinococcus; reduce the number of dogs by raising the tax-such are the principal means which ought to be resorted to.

AdDEndum. Echinococcosis is not very rare in the human race. In central Germany it causes quite a high mortality: 5 out of each 1000 deaths are placed to its credit. In Iceland, 5 to 10 per cent. of the population perish from this disease. Its symptoms are very slow in developing. Hepatic echinococcosis is marked by a fluctuating tumor, which is soft, irregular, with a sensitiveness to pressure upon the right hypochondrium; in addition, we observe dyspnœa, bronchial catarrh, icterus, and sometimes abscess formation in the liver. When the lung is affected the cysts may be ejected with the expectorations-a valuable indication, enabling us to define the diagnosis. In cases where the kidney is affected it is found to be tumefied and painful, and the urine often contains isolated hooks. At times echinococci are developed in the brain and produce special nervous symptoms. The disease ends in death; its average duration is about five years.

\section{Coccidiosis : Psorospermosis.}

Coccidia produce true enzootics in the rabbit. They have also been found in the dog, calf, pig, sheep, birds, amphibia, arthropoda, and the mollusks. In poultry it is not rare to find them upon the mucous membrane of the cephalic region. We shall return to this fact in treating upon diphtheric stomatitis. (See Infectious Diseases.)

Natural history. The oviform psorosperms of the rabbit's liver were discovered by Hake in 1839, and attributed to gregarina by Lieberkühn in 1856. These are parasitic protozoa living in colonies (hence the name of gregarina, from grex, gregis, meaning 
"flock"), and essentially constituted by protoplasm containing a nueleus, the whole destined to become encysted.

According to Leuckart, the term psorosperms is incorrect as a denomination for these organisms, which belong to the group of sporozoa; this appellation is indeed extended to the entire mother gregarina, inside of which the so-called psorosperms-that is to say, the spores-are only developed later. Leuckart designates the sporozoa in question under the name of coccidia. We recognize one principal species, the Coccidium oviforme, which inhabits the liver, and a secondary species, Coccidium perforans, which lives in the intestine.

These coccidia represent («ccording to Leuckart), in their young age, parasites without covering except the epithelial cells of the biliary canals and intestine; having arrived at the adult age, these worms become surrounded with a solid shell; they then leave the inside of their host, reaching the open air, and if deposited in a damp and dark place (stables, barns, etc.), they are slowly developed. Their globular contents are transformed into four spores of oval shape, each representing a small stick of hyaline, having the shape of a $\mathrm{C}$, the concavity of each little stick containing a remnant of the granular mass of the spore; the four elements (falciform corpuscles) fill the inside of the coccidium. ${ }^{1}$ In the stomach of a new host, into which they enter with the food, they become free, each little stick being transformed into a naked amoeboid cell of the size of a white blood-corpuscle, which penetrates, either into the intestinal epithelium (Coccidium perforans), or into the hepatic epithelium through the biliary canals (Coccidium oviforme). Once inside of the epithelial cell, the gregarina is surrounded by a cuticle and increases to double its primitive size; it is then oviform, with a microscopic opening at its pointed extremity, which renders it similar to the eggs of some entozoa, with which, indeed, it has been confounded.

Pathological anatomy. In coccidiosis of the rabbit the liver is more or less increased in volume; sometimes it is bosselated upon its surface. Its parenchyma is filled with white-yellowish cysts, of the size of a millet seed, of a bean, or of a hazel-nut, and which are sometimes found in such numbers that the interposed hepatic tissue is found to be completely hypertrophied. These cysts,

1 According to Balbiani, each of these four falciform corpuscles is in reality composed of two associated corpuscles, disposed tête-bêche.-N. D. T. 
precisely similar to tubercles or small tumors, contain a yellow, thick, creamy, clotted, or caseous mass, which upon microscopic examination is shown to be constituted by encysted oviform coccidia, epithelial cells that have undergone fatty degeneration, free nucleus, fat-drops, etc. ; they are separated from the hepatic parenchyma by a thick connective-tissue shell, composed of numerous nuclei and fibres concentrically disposed; they are developed at the expense of several parallel biliary canals; they are, indeed, confluent and dilated biliary canals (Leuckart).

The coccidia of the intestinal canal have been found in the rabbit, dog, sheep, pig, cat, chicken, etc. They are imprisoned in epithelial cells; they are found isolated or displaced in groups forming little whitish spots, standing in relief upon the mucous membrane, which they often cover up to some extent as a pseudo-membrane. It is in the intestinal villi that the largest number are found ; they are there in the shape of small white points, and overrun also Lieberkühn's glands. The prosence of coccidia occasions destruction of the epithelium, infiltration of the mucous membrane, even its ulceration, and inflammation of the submucous connective tissue.

Symptoms. Coccidiosis of the rabbit, which frequently destroys whole warrens, is ususlly snnounced, according to Roloff, by a diminution of the appetite, by icterus, weakness, emaciation, an exhausting diarrhea, tympanites, a staggering or uncertain gait, etc. Death takes place in convulsions. Very often, however, no really appreciable symptoms are observed, notwithstanding the existence of a large number of coccidia; but in these instances the organic molecular transformations seem to be profoundly altered, for, according to Cohnheim, irritation of the fourth ventricle does not produce diabetes in a rabbit affected with coccidiosis.

In calves that have just been weaned we may observe a fatal dysentery produced by intestinal coccidiosis (Pröger). Rivolta has found rabiform attacks in the dog caused by the invasion of the intestinal mucous membrane by coccidia (?).

Differential diagnosis. Hake, who discovered these parasites, mistook them for pus corpuscles; Lang considered them as neoformations. A frequent error is to confound the encysted coccidia with the eggs of the linguatulas, tænias, and nematodes. They have not always been distinguished from the utricles of Miescher (sarcosporidia); we shall, however, return to this subject in the article on Diseases of Muscles. We shall also examine further the 
laryngeal psorospermosis of the goat, observed by $\mathrm{V}$ on Niederhäusern, Dammann, and others.

Treatment. The treatment of coccidiosis is the same as for the preceding diseases; we must practise prophylaxis before everything else. Separate the healthy from the sick animals, and disinfect the stables. Internally we may use mercurial antiseptics; calomel might, perhaps, give favorable results.

A few authors (Zschokke, ${ }^{1}$ Semmer, ${ }^{2}$ etc., have found in the liver of pigs cysticerei of the Tcenia marginata. ${ }^{3}$

\section{DISEASES OF THE PANCREAS.}

Diseases of the pancreas are extremely rare in our animals, and generally they are only discovered at the autopsy. They remain unnoticed, because, as a rule, they are neither accompanied by any physical symptoms nor by any rational phenomena. This quiet evolution of the morbid conditions of the pancreas is easily understood when we take into account the deep location of this organ, protected by the sub-lumbar arch and the mass of abdominal viscera, and if we remember its physiological function, shared by other glands of the digestive tract. Serious troubles may, however, be produced, affecting also neighboring organs, especially when complicated by alterations of the liver.

\section{PANCREATITIS.}

Inflammation of the pancreas is acute or chronic. The principal symptoms which are ascribed to it are : a depressed condition, a more or less apparent febrile disturbance, general emaciation, and

1 Zschokke: Schweizer Archiv, 1887.

2 Semmer: Deutsche Zeitschr. für Thiermed., 1885.

3 Mégnin has observed in the horse a case of chronic hepatitis which was determined by the Sclerostoma armatum. The median lobe of the liver was transformed into a true fibro-plastic tumor, in which the hepatic tissue had completely disappeared; it was marked with small blood-cysts, all containing the worms, and, in addition, nodes containing embryos of the same worm more or less developed. The parasite was also found in the small vessels. (Lourdel and Mégnin : Bull. Soo. Vét., 1884.) -N. D. T. 
the expulsion of fatty excrementitious matters. In the ox, the pig, the dog, and the cat, the pancreatic canal connecting with the intestine is at a point somewhat distant from the ductus communis, and icteric complication is not observed, which is to be expected in the horse, the sheep, and the goat, animals in which the ductus communis and pancreatic canals discharge into the intestine by a common opening (Vater's ampulla). In these latter species the inflamed dilated pancreatic canal may compress the ductus choledochus, plug it up, and prevent the discharge of the bile.

Mégnin and Nocard mention a case of this kind in a horse, whose history was that he was without ambition, had the habit of becoming quickly tired, and had been eating lazily for several weeks when first seen by them. The soft condition, weakness, falling off of the appetite, constipation, the yellow coloration of the mucous membranes and of the integument, becoming more apparent as emaciation advanced, induced a suspicion of biliary calculus.

The animal died about two months after the commencement of the disease. At the autopsy were found, as essential lesions, an enormous dilatation of the pancreatic canals, obstructed by a whitish obstacle, of a grumous, albuminous nature, which compressed the ductus choledochus at a certain distance and obstructed the discharge of bile; finally, also, a slight induration of the gland, the parenchyma of which was whiter, firmer, and more resistant than in the normal condition, the excretory canals presenting lesions of catarrhal inflammation.

Neoplasms of the pancreas are sometimes primary, but more frequently they are secondary. Nocard has described a primary epithelial tumor of the head of the pancreas in a dog. The animal had suffered for six weeks when brought to Alfort's clinic; he was very weak and in an extremely emaciated condition; all the integuments were colored yellow-the conjunctiva, sclerotic, buccal, anal, and vulvar mucous membranes were of a well-marked saffron tint; the urine was thick, oily, and dark yellow; there was besides quite an abundant abdominal accumulation. The diagnosis was made that the icterus was produced by tumors hindering the abdominal circulation and obliterating the ductus choledochus. The patient died about two months after the appearance of the first symptoms. At the autopsy a whitish tumor was found upon the sub-lumbar arch, which was the size of the fist, irregularly rounded, embussed, and strongly attached to the surrounding organs, and 
involving the head of the pancreas in its mass, together with the portal vein, the upper extremity of the ductus choledochus, and the duodenum. In the liver a large number of small whitish tumors were found, from the size of a millet seed to that of a cherry, which formed part of the hepatic tissue. Microscopic examination of the principal neoplasms and of the tumors of the liver showed lobulated epithelioma.

Several similar observations have been related by other authors, mainly by Friedberger.

\section{DISEASES OF THE SPLEEN.}

DISEASES of the spleen are not more frequent in our domestic animals than in the human race. Most of the pathological works do not even mention it. The obscure functions of this organ, and its deep location, which removes it from the influence of external agents, give a good and sufficient reason for the extreme rarity of primary diseases of this organ. The alterations which are found in it are almost always under the influence of a general morbid condition, which is diathetic or infectious. We find, however, in the veterinary journals a few observations relative to congestion, inflammation, and rupture of the spleen.

\section{SPLENIC CONGESTIONS.}

They are either active or passive. Active congestions of the spleen have been observed in all our animals, but particularly in the ox, the sheep, and the dog. Their etiology is little known. The main causes which have been assigned are: the sudden plethora resulting from a very rich alimentation, when animals used to poor feeding are suddenly given a large quantity of food, or placed in abundant and watery pastures; also, the feeding of pulp, the overloading of the rumen, excess of work, and damp heat.

Passive congestions, which are caused by some obstruction to the splenic vein, the portal vein, or the posterior vena cava, are so much the more intense as the interference to the circulation is most 
localized, and when the obstacle to the blood current is situated nearer to the spleen.

The clinical symptoms of these congestions are always very vague. Generally we find more or less intense colics. In some instances, however, the tumefaction of the spleen may be recognized by palpation and percussion of the left hypochondrium; there is even sometimes a certain swelling of this region and of the flank, without any apparent meteorism (Gellé).

At the autopsy we find an increase in the size of the spleen; its weight and dimensions are, as a rule, doubled or trebled; in a case observed by Gurlt upon an animal of the bovine race, it weighed seventeen kilogrammes. The splenic tissue is dark red or marble colored; some apoplectic centres may be found in it; its consistence is much weakened according to the more or less intense congestion.

The ordinary duration of the disease is from six to twelve hours. It ends in resolution or laceration of the organ. This latter, which is exceptional, is indicated by the violence of the abdominal pains, the obliteration of the pulse, the discoloration of the mucous membranes, and by cold sweats and tremblings; death occurs rapidly.

We must be careful not to mistake for a true disease of the spleen the splenic congestion occurring in some infectious diseases, notably in anthrax and in septic and typhoid affections.

Bleeding, refrigerant applications upon the hypochondrium, and revulsive frictions upon the extremities, are the principal means to which we should resort.

\section{SPLENITIS.}

The phlegmasias of the spleen are located in the perisplenic tissues or in the splenic parenchyma itself.

Perisplenitis is altogether assimilable to perihepatitis. Sometimes it is limited to the peritoneal serous membrane, at other times it concerns this and the capsular membrane at the same time. Any pathological process located in the neighborhood of the spleen will reach these membranes in the end. It is either the consequence of a splenic affection, or of phlegmasia of the neighboring organ, or, again, of a simple prolonged abnormal compression exerted upon the spleen. At all events, it causes thickening of the peritoneal fold and of the subjacent capsule; often also it results in the 
forming of adhesions between the peritoneal folds which come in contact. Fixations of the spleen to the abdominal wall, the intestine, and liver, are thus produced.

Splenitis is of internal or traumatic origin. Cruzel claims to have observed it quite frequently in the ox, in the acute, subacute, and chronic forms.

It may be provoked by direct causes, by contusions and wounds penetrating the left hypochondrium; at times it is produced under the influence of a general disease; Cruzel ascribes it especially to the extreme sanguine temperament of the oxen belonging to the working races, excessive efforts, over-driving, cold and damp weather, and long feeding upon very nutritious food. In certain instances its cause eludes all investigation.

Its symptoms, which are always undefined, can be thus described: tumefaction of the spleen, abnormal sensibility and sometimes rising of the left hypochondrium, an intense pain produced by percussion of the region; inappetence, constipation, nausea, vomitings, fever of slight or of intense reaction, finally various traumatic lesionscontusion, contused wounds, deep wounds-when the disease is of external origin.

The following are the symptoms given by Cruzel as pathognomonic of splenitis of the ox: Chills which are more or less marked at the beginning of the disease; uneasy respiration; tension, rising (soulevement) of the left flank-differing from the swelling of ordinary meteorism, as it appears to be determined by the driving back of the spleen. The sound rendered by percussion is dull, similar to that resulting from a blow upon a soft body offering a certain resistance. Cruzel declares that he has never made a mistake since his attention was called to this rising of the flank.

Resolution is the most common ending of splenitis. Even when this has been determined by a traumatism, the cure is regular if peritonitis is not added to the primary affection. But in cases where the spleen is directly affected by a vulnerating body, a hemorrhage takes place in the peritoneum, and death may occur rapidly. Splenitis may also end in suppuration or gangrene. The symptoms of these complications, masked by an intense fever, are difficult to understand. We see paroxysms alternate with remissions of a variable duration, and percussion denotes an intense sensibility of the left hypochondrium. The splenic abscesses are mostly discharged into the peritoneum and determine fatal accidents; they 
may also open into the intestine, the stomach, or even externally; they rarely become encysted in the splenic parenchyma.

If the anatomical alterations of splenitis have been neglected up to the present time, the study of pyemic infarcts of the spleen, in their different stages of evolution, has permitted us to recognize that inflammation follows the same course in this organ as in the other viscera. It is marked there by hyperemia and by softening through a kind of hepatization due to hypergenesis of the elements of the splenic pulp, and to the thickening, induration, and softening of the fibrous trabeculæ which constitute the frame of the organ. When splenitis has ended in suppuration the section presents numerous small purulent centres, or more or less voluminous abscesses. We may detect disorders produced by the opening of the abscess into the peritoneum. Rarely are found old splenitic lesions or cicatricial spots marking the disappearance of the abscesses and the resorption of the pus.

We would advise opposing the splenitis by bloodletting, refrigerants, revulsives or vesicants applied upon the left hypochondrium. Cruzel recommends combating acute and subacute splenitis of the ox by the following means: free bleeding, which is to be repeated when indicated-that is to say, if resolution is not sufficiently prompt; cold affusions upon the left hypochondrium ; drinks, and mild clysters.

\section{RUPTURE OF THE SPLEEN.}

Rupture of the spleen is ordinarily the result of a penetrating traumatism or of some external violence, which action is felt upon the left hypochondrium. In some cases, especially in the ox, there seems to be a possibility of its origin in a sudden and intense congestion of the organ; the parenchyma and capsule which surround it would give way under the pressure of the blood, and this would be discharged into the peritoneal cavity (Cruzel, Lafosse). But ruptures of the spleen are almost always of a traumatic nature, and coexist with other lesions of the same kind, involving certain abdominal organs, mainly the liver.

Ruptures may be produced on any part of the spleen; whether single or multiple, superficial or deep, complete or incomplete, they are very variable in their shape and extent. Sometimes the splenic capsule resists and forms embossments which are due to the blood 
which is extravasated in the regular tissue of the spleen. When the complete ruptures are recent they are found to be covered with a bluod clot and their lips are infiltrated with blood; later, if death has not followed, these lips form a cicatrix and become hardened. In old horses it is not unusual to find marks of former lacerations which are limited to the spleen.

The symptoms of rupture of the spleen are those of internal hemorrhage. There are cases which are almost foudroyant; the animals show all the symptoms of extreme anxiety and die within a few minutes. Most frequently death does not take place before a few hours, and a local pain may be detected, also a gradual discoloration of the mucous membranes, a chilling of the extremities and all the symptoms of serious hemorrhages. When the accident is consecutive to a traumatism, there are also traces either of a contusion or a penetrating wound of the splenic region.

The patient ought to be placed in absolute immobility and protected from any exciting causes, refrigerant applications are to be made upon the hypochondrium, drinks with an addition of perchloride of iron are to be given internally, or if the pains are great, hypodermatic injections of morphine should be given: such are the means to apply in order to arrest the splenic hemorrhages. Any traumatic lesions which may exist may be treated by antiseptics.

Tuberculous alterations of the spleen are quite common in all the species, but especially in birds, where the spleen affected by tubercle may acquire a considerable volume (see Tuberculosis).

Tumons of the spleen are rarely primitive. We find mainly cancer, lymphadenoma, melanotic sarcoma (in white horses), serous and hydatic cysts in this organ.

Parasites of the spleen do not, as a rule, produce troubles. The most common are the echinococcus (in cattle), the cysticercus (in the pig), and linguatula in the dog. 


\section{DISEASES OF THE PERITONEUM.}

\section{ACUTE PERITONITIS.}

Division. According to the point of view from which we consider peritonitis, we may recognize in it numerous and very different varieties. Clinically it presents the acute, chronic, circumscribed, or diffused forms; according to its origin it is primitive, idiopathic or secondary, traumatic, tuberculous, sarcomatous, carcinomatous, pyemic, metastatic, septic, etc. ; anatomy distinguishes the dry, exudative serous, sero-fibrinous, purulent, hemorrhagic, ichorous peritonitis, etc. If considered as to pathogenesis, peritonitis is almost always secondary, and produced by wounds of the serous membrane or by pathological processes which develop in a neighboring organ and finally reach this membrane. The existence of primitive peritonitis $\grave{a}$ frigore cannot be doubted (rheumatismal peritonitis), but it is extremely rare in the horse, in which animal rheumatismal colics are, however, quite frequent.

Affected animals. Acute peritonitis is observed in all animals, including poultry. Females are more frequently affected than males, a difference undoubtedly due to the more extended and intimate relations existing in the former between the genital organs and the peritoneum. It is more frequent in the cow than in the mare, first on account of the circumstances surrounding gestation, the puerperal condition, and its complications-phenomena which are repeated more often in the former-but also because of hepatic distomiasis and tuberculosis, affections which are more frequent in the bovine race and are often complicated by peritonitis. The sensitiveness of the peritoneum varies considerably with the different species of animals. The horse is the animal whose peritoneum is the most sensitive; then come the ruminants (sheep, goat, and ox), then the carnivora (dog, eat), the omnivora (pig), and lastly the birds.

Etiology. The causes of acute peritonitis are:

1. Wounds penetrating the abdomen, accidental solutions of continuity, surgical traumatism-the laparotomies made in practising various operations (herniotomy, puncture of the intestine or of the peritoneum, incision of the rumen, enterotomy, castration of females, 
etc.). In all these cases the determining cause of the inflammation is the penetration into the peritoneum of irritating germs which are suspended in the atmosphere, or the use of unclean instruments, soiled with inflammatory, septic, or putrid matters. The simple entrance of air through the inguinal canal at the time of castration of a stallion may produce a fatal peritonitis under certain conditions (Hering).

2. Ruptures or perforations of the organs covered by the peritoneum and the invasion of irritating bodies into the abdominal cavity. Here we must particularly mention ruptures of the stomach, intestine, bladder, liver and spleen, etc.; also the perforation of the gastric or intestinal walls by ulcerations, foreign bodies, packed stercoral matters, and worms; the tearing of the uterus at the time of parturition, the perforation of the capsule of the liver by flukeworms, the opening of an abscess or echinococcus cyst into the peritoneum, the perforation of the intestine consecutive to hemorrhagic infarctus, or necrosis determined by vascular thrombosis, the laceration of the rectum when exploration is practised unskilfully. (Duvieusart has observed this accident "with appearances of an enzootic character" within the working district of a quack.)

3. Propagation of a localized inflammation by contiguity of tissue to one of the organs contained in the abdomen or tc the walls of this cavity; contusion of the abdomen, affections of the stomach or intestine, violent inflammation of the gastro-intestinal mucous membranes, ulcerations or partial tearings, etc., changes of relation of the intestine (volvulus, invagination, strangulated hernia), inflammatory disease of the liver, kidneys, bladder, and uterus (metritis, parametritis, perimetritis), also of the ovaries, testicles, and testicular cord. In rare cases the peritoneum may burst as a complication of pleurisy.

Metastatic peritonitis, originating during the course of pyemia and a few other general diseases, is little known in animals.

Pathological anatomy. In recent acute diffused peritonitis we find the peritoneum hyperemic and filled with small ecchymoses. The redness is more or less intense, sometimes nearly uniform, and at other times disposed in ramified tracts; it varies in color from rose to brick-red or scarlet; these colorations are changed later into a gray-red tint. As a rule, the inflammatory process is not equally advanced in the whole extent of the serous membrane, and the starting-point of the inflammation may be easily recognized. Some- 
times the peritoneum is covered with an exudate masking these lesions.

The serous membrane loses its transparence from the first; it hecomes dull, takes a velvety appearance, and may easily be torn or detached from the organs which it covers. Within a few days, newly formed vessels are to be recognized, and it becomes filled with an exudate that is very variable in character as well as in abundance; according to the case, it may be covered with a thin coagulated fibrinous layer forming a transparent membrane which may be detached from the serous membrane by the slightest traction, where it forms a thick, clear or dark-yellow layer, similar to the croupous membranes; at times it forms adhesions between the two folds of the peritoneum (mesentery) and the organs covered by it (fibrinous or dry peritonitis). In serous or sero-fibrinous peritonitis, the abdominal cavity contains a more or less considerable quantity (as much as forty litres in the horse and the ox), of a yellowish, reddish-yellow, or greenish-yellow liquid. This liquid exudate is mainly formed of serum, fibrinous or purulent flakes, and of cells undergoing destruction; when it contains a large proportion of red corpuscles, these latter will impart to it their coloration. The red exudate is frequent in the horse (sero-fibrinous peritonitis complicated with hemorrhage). If the peritonitis is due to a perforation of the intestine consecutive to a disease of this organ, the exudate, which is ichorous, has a very fetid odor (ichorous peritonitis); ordinarily it is mixed with alimentary matters; in peritonitis caused by rupture of the bladder it exhales a strong urinous odor. In the dog and cat it is often purulent (purulent peritonitis), and forms a thin creamy covering upon the peritoneum, or again becomes collected in the abdominal cavity in the shape of a turbid flaky liquid with a fetid odor (mainly after the opening of the abscess).

When the peritonitis runs its course the exudates may be entirely or partially reabsorbed, but their disappearance requires quite a long time, as the cellular and fibrinons elements must first undergo fatty degeneration and become disintegrated into small detritus. After this reabsorption, some adhesions will frequently persist between the two folds of the peritoneum (adhesive peritonitis); new connective tissue membranes and ligaments are formed which often produce contractions, dilatations, or atrophy of the organs of the abdominal cavity by undergoing cicatricial retraction. When the 
reabsorption of the exudate is incomplete and if the leucocytes continue to emigrate, the liquid takes a purulent or muco-purulent aspect. In rare cases, when partial adhesions and formations of walls are established, more or less considerable portions of the exudate may become enclosed, they are gradually condensed, caseated, and ultimately undergo calcareous infiltration.

In peritonitis of long duration the intestine participates in the morbid process; its walls become oedematous, thickened, and very friable; they are struck by paralysis; this produces an obstinate constipation, with an accumulation of gas in the intestines, and disorders which are the consequence of it. With time catarrhal inflammation develops in the intestinal mucous membrane.

Finally, the peritoneal exudate may, through its abundance, cause disturbances, either by acting mechanically by a pressure upon the diaphragm and the abdominal viscera, or by producing infiltration and softening of these organs. The serous infiltration of the diaphragm causes paralysis of this muscle and thus leads to dyspnœa.

Acute circumscribed peritonitis is accompanied by the same special alterations, but they are confined to certain regions of the peritoneal membrane (intestine, liver, uterus, ovaries). Local peritonitis is developed as a result of narrow wounds which intersect the serous membrane (puncture), and in inflammation of the organs which are covered by this membrane (perimetritis, perihepatitis, etc.). Phlegmasia remains circumscribed when the irritating products are isolated soon enough, either by a union of the inflamed folds or by encysting. These mild forms of circumscribed fibrinous peritonitis are somewhat frequent in our animals, mainly in the horse; in fact, it is not rare to find remains of old exudates at the autopsies.

Symptoms of acute diffused peritonitis, particularly in the horse. The clinical picture of peritonitis does not possess anything expressive, and above all nothing typical; the numerous variations which are observed in it are due to its frequent secondary nature; the symptoms of the pre-existing morbid condition are dominant. In cases where peritonitis follows closely the development of a primary lesion, it is very difficult to distinguish the manifestations belonging to this, from inflammation of the abdominal serous membrane; as a rule, the primitive disease (intestinal changes of relation, for example) produce troubles of great intensity, which obscure the symptoms of peritoneal phlegmasia to a great 
extent. The course of the different forms of peritonitis is far from being uniform; some develop slowly, whilst others are accompanied by very alarming symptoms from the beginning; in certain instances it will lead to death within such a short time that it is impossible to recognize the special symptoms. It is thus that in peritonitis by perforation, the blood becoming very rapidly loaded with infectious products, the manifestations of the septic infection masks absolutely those of peritoneal inflammation.

The most important symptoms of peritonitis are :

1. Acute pains, which are marked by more or less intense colics. They are often severe and continuous at the beginning of the affection: later they are remittent or intermittent. We often see traumatic peritonitis starting from the testicular cord (peritonitis of castration) giving rise to colics which appear violent from the onset, and are marked by grinding of the teeth, groans, convulsive extensions of the extremities, and costo-dorsal decubitus; the animals, which are much depressed, keep their heads in the litter, without moving; sweating spells are apparent upon various parts or on the whole surface of the body, etc. If the disease runs through these different phases, these manifestations of pain diminish in intensity or present a series of passive remissions and exacerbations. In other cases the sufferings are particularly slight during the whole course of the affection; they produce only frequent decubitus, complaints, and a certain agitation; the animals scratch the soil, look at the flank, sway the tail, etc. Finally, in some instances the pain seems to be absolutely wanting.

2. The fever is generally acute. In diffused peritonitis we have almost constantly observed a temperature of $41^{\circ}$ to $42^{\circ} \mathrm{C}$. In peritonitis by perforation, fever starts with chills, and it may rapidly become intense. But, in general, the thermic curve of peritonitis is very erratic; the fever has an irregular course, it is either intermittent or remittent. In septic peritonitis the temperature at times is nearly normal.

The circulation is always much accelerated, and the number of pulsations does not at all correspond to the temperature; 60 to 130 per minute may be counted. From the onset the pulse is slow and hard; as the disease progresses it grows gradually weaker, and is almost imperceptible in some patients; the artery is filiform and contracted; parallel with the changes of the pulse, the contractions of the heart become increasingly stronger and more tumultuous; 
the mucous membranes are red or cyanosed; the temperature of the surface of the body is unequally distributed, the extremities are cold.

3. Retraction and sensitiveness of the abdomen. From the onset the belly is often tucked up; it is sensitive to the pressure of the hand, which the animals seek to avoid, and which produces complaints. Sometimes hyperæsthesia of the abdominal parts is at first localized; it is extended and generalized with the diffusion of the peritoneal phlegmasia; in serious peritonitis it disappears at a more or less advanced stage. In horses whose abdomen is excessively large, palpation is difficult, and the increase of sensitiveness is only slightly marked. On the other hand, there are very irritable and ticklish animals which hardly allow anyone to touch their abdominal walls, even when the peritoneum is absolutely healthy.

In numerous cases we recognize at a certain period of the affection, often shortly after the beginning, a sudden increase in the volume of the abdomen. The flanks are lifted by gases which distend the intestines affected by paresis; this meteorism sometimes becomes disturbing. In small animals the augmentation in volume of the abdomen is mainly marked inferiorly and posteriorly; percussion and auscultation reveal the symptoms of ascites in these regions.

The peristaltic movements of the intestine, which are still perceptible at the beginning of the affection, are completely suppressed later (paralysis of the intestine due to odematous infiltration of its muscular system); it results that defecation is painful, delayed, rare, and even entirely wanting, notwithstanding the violent and repeated expulsive exertions made by the animals. The rectum is distended by small dry and coated dungs. Toward the end of the disease an abundant and persistent diarrhea often develops.

Micturition shows constant troubles. As a rule, we sbserve a frequent desire to urinate; the animals stand extended and make slight exertions to expel the urine. When pericystitis exists there may be retention of urine for several days (paresis of the bladder); besides, micturition, like defecation, is rendered painful by the contraction of the abdominal muscles. The urine is ordinarily discharged in small quantities; the prolonged inappetence renders it acid, its specific gravity increases, and it contains albumin (in one instance we found a large number of bacilli in it when death was approaching).

4. Anorexia. It may be sudden or be preceded for a few days 
by a simple diminution of appetite ; later we always observe complete anorexia. In the horse (as in man) we have seen eructations and vomiting (Röll); these are phenomena which must be ascribed to an affection of the gastric serous membrane and to paralysis of the constrictor muscle of the cardiac orifice.

5. Acceleration of the respiration. Sometimes the respirations are short and superficial, at other times deep and painful ; 20 to 70 per minute may be counted; it is not unusual to recognize a serious dyspnœa, the causes of which are: paresis of the diaphragm, due to inflammation of its peritoneal covering, the compression of the pectoral cavity by the exudate, and at certain times by tympanites, painful contraction of the diaphragm and abdominal walls ; lastly, circulatory troubles consecutive to the diminution of cardiac activity. The respiration may be exclusively pectoral, on account of the sensitiveness of the diaphragm and abdominal walls (considerable rising and lowering of the ribs, and activity of the intercostal muscles). When the diaphragm participates in the inflammation, we observe a kind of painful hiccough (singultus), which produces abrupt respiratory movements and shaking of the whole body, and along the insertion of this muscle an abnormal sensitiveness easy to find by palpation (Anacker).

6. The general condition is ordinarily disturbing. As soon as the colics have disappeared we observe a profound apathy, sleepiness, and great weakness. The patients seem to fear the least movement, and hold the head low or lcaning against the walls of the stall; they are absolutely indifferent to anything going on around them. Being very weak and staggering, they are subject to partial tremblings and convuisions; novertheless, they generally keep a standing position (we have seen a horse maintain this position until death occurred); they become stiff, immobile, and it is hard to displace them; in some instances they neigh a short time before succumbing. When exhausted they sink upon the ground, and the fatal termination is close at hand.

Course. In the majority of instances acute peritonitis ends in death; this is produced either through septic infection or by heart weakness (and its paralysis), sometimes by asphyxia. There is peritonitis by perforation, which may take the patient off within twelve hours, but oftener in twenty to thirty hours. The disease may follow a slower course and last from eight to fourteen days, become gradually attenuated, and pass into the chronic state. 
Diagnosis. The diagnosis of acute peritonitis is often very difficult, and sometimes impossible in our large animals. More or less violent abdominal pains are common to peritonitis and to most of the affections which concern the digestive organs, especially to colics and enteritis; recognition of the peritoneal exudate alone enables the practitioner to make the diagnosis with certainty. Acceleration of the respiration and circulation might induce us to diagnosticate the existence of pneumonia, pleurisy, or endocarditis, but exploration of the organs contained in the thoracic cavity suffices to eliminate these affections. The distinction between the peritoneal exudate and other morbid productions developing in the abdominal cavity will be given in the article on Ascites (differential diagnosis).

ACUte PERItonitis of the ox. Acute peritonitis of the ox is shown by such vague symptoms that it is almost always extremely difficult to recognize it. All authors agree upon this point. Recognition of the serous exudate in the abdominal cavity can only be made in the minority of cases, as palpation rarely produces manifestations of pain. The characters of the pulse are not of any true diagnostic value, as in the horse, for even in a normal condition the pulse of the ox is subject to numerous variations. The same is true of tympanites, a phenomenon common to a great many affections. In general, the principal symptoms are: increase in size of the abdomen, symptoms marking dull pains, slight trembling of the abdominal walls, chills, difficulty of movement, constipation alternating with moderate diarrhea, and tympanites. It lasts from a few days to several weeks, often ending in death. (According to Bagge and others, the mortality is about 50 per cent.)

ACUTE PERITONitis of THE DOG. In small animals, and particularly in the dog, the possibility of making a complete exploration of the abdomen permits us to easily recognize peritonitis. The trouble is marked by signs of pain (groans, howls); there is a sensitiveness of the abdomen to palpation, an exudate exists in the abdominal cavity ; there is constipation, meteorism, stiffness of the hind quarters, complaints during defecation, dyspnœa, fever, etc. A very marked tympanites is frequently seen in the cat.

ACUte Peritonitis of Poultry. It may be caused by perforation of the stomach or intestine (foreign bodies, ulcerations), through a rupture of the oviduct, by the parasites of the abdominal cavity (echinorrhynchi, acari), or by operations upon the abdomen 
(caponizing). According to Zürn, its principal symptoms are weakness, fever, inappetence, pain, and complaints produced by the exertions necessitated by defecation and by the pressure exerted upon the abdominal walls.

Treatment. The treatment of acute peritonitis comprises local means and internal medication. In small animals the peritoneal inflammation may be combated by the application of cold or ice compresses upon the abdominal walls. Mercurial ointment, much used in human medicine, is dangerous, on account of possible accidents of poisoning; it must only be used for the horse, and might here perhaps be replaced with advantage by essence of turpentine. When a liquid exudate is accumulated in large quantity in the peritoneal cavity, it is advisable to cause its evacuation by the trocar. In suppurated peritonitis we must perform laparotomy and remove the exudate, and then proceed to a careful washing of the serous membrane with a weak solution of sublimate, carbolic acid, salicylic and boric acids, or cresol $(1: 400)$. The insignificant toxic property of the latter ought to give it the preference. Ultimately it is proper to use Lugol's solution of iodine by injections: we have never seen it produce disturbing accidents.

Internal treatment consisted formerly in the administration of antiphlogistics and purgatives (emetic, nitre, sulphate of soda). These latter are more injurious than useful ; they increase the peristaltic movements of the intestine, which causes extension of the exudate upon the whole surface of the serous membrane; we ought, therefore, following the example of physicians, to relinquish these internal remedies. Calomel, however, may be used as a disinfectant of the digestive canal. We generally gire opium in relatively high doses (opium in powder: for the horse, 10 grammes; for the ox, 15 grammes; for the dog, 0.1 to 0.5 gramme), either to reduce the pains or slacken the peristaltic contractions. If this agent produces constipation, it should be counteracted by tepid injections. When the quantity of peritoneal liquid is considerable, diuretics may be tried (digitalis, squill, strophanthus, caffeine, juniper seeds, essence of turpentine, a solution of the acetate of potash, etc.), or sialagogues (pilocarpine).

In cases where peritonitis is secondary we must combat the primitive trouble when peritoneal inflammation is but an epiphenomenon (disinfection of the uterus in metritis, of the bladder in cystitis, etc. ; herniotnmy in strangled hernia ; also puncture of ripe 
abscesses in the thickness of the abdominal walls, etc.). When weakness is excessive it is advisable to resort to subcutaneous injections of camphor.

\section{CHRONIC PERITONITIS.}

Etiology. Generalized chronic peritonitis is much less frequent than acute peritonitis; it is most often observed in the ox. Sometimes it succeeds acute peritonitis, at other times it arises directly. Chronic inflammation of the peritoneum in the ox is almost always determined by foreign bodies of the stomach ; in the dog it is ordinarily the consequence of repeated punctures made to counteract ascites; it may complicate the phlegmasic processes which develop in the different organs covered by the peritoneum in all animals (uterus, kidney, liver, etc.). Finally, it is sometimes a simple accident of an infectious or diathetic disease; it is thus that chronic peritonitis is found to be sarcomatous, carcinomatous (the first in the ox and pig, the second in the horse). We shall treat this trouble according to its different phases.

Pathological anatomy. In most cases the anatomical alterations of chronic peritonitis are localized upon one part of the serous membrane (perihepatitis, perimetritis, etc ); they are mainly formed by a hyperplasia of the peritoneal connective tissue, which frequently produces vegetations, considerable thickenings of this membrane, or true fibrinous pouches surrounding certain organs; these neoformations form sometimes tendinous bands or callous, cartilaginous, or bony spots. Besides these lesions we find adhesion of the organs one to another, or to the abdominal walls-a condition which is accompanied by tractions, compressions, or contractions.

In this "deforming" peritonitis, which is the most typical, and in tuberculous and infectious peritonitis (caseous enteritis of the pig), the joined intestinal loops form balls, the interstices of which are ordinarily filled with a purulent, gelatinous, oily mass, an exudate, which later undergoes a caseous transformation and calcareous infiltration. ${ }^{1}$ Chronic peritonitis with abundant liquid exudate is rare. Friedberger has, however, found an abundant liquid exudate in the sarcomatous peritonitis of the horse.

Symptoms. In general, the symptoms are similar to those of acute peritonitis, but their intensity is much less ; thus the diagnosis

1 Steiner: Magazin, 1836 (observation upon the ox). 
of chronic peritonitis is extremely difficult, except when following the acute form. Its course is slow and its evolution obscure. In the ox, colics and sensitiveness of the abdominal parts are wanting; the only characteristic symptom is the progressive and considerable increase in the size of the abdomen, while the appetite is lessened from day to day and diarrhea persists. The inferior abdominal wall may later become the seat of an oedematous infiltration. Friedberger has observed identical symptoms in the horse, which were complicated by intermittent colics of short duration, a certain sensitiveness of the flank, acceleration and weakness of the pulse, or a fever of medium intensity. In the dog it is not always possible to distinguish chronic peritonitis from ascites (see Ascites).

Treatment. It is the same as that of ascites. We must administer diuretics, laxatives, and practise paracentesis. At the start, when the exudate is yet relatively "dry," we may use hot compresses upon the abdomen with advantage (Priessnitz's compresses); internally, we ought to give iodine (iodide of potassium, or of iron). Puncture of the abdomen is completed by a flushing of the peritoneum. For that purpose we use generally the following preparation:

$$
\begin{aligned}
& \text { R.-Iodine . . . . . . . . . } 1 \text { gramme. } \\
& \text { Iodide of potassium . . . . . . . } 5 \text { grammes. } \\
& \text { Distilled water . . . . . . . . . } 500 \text { " }
\end{aligned}
$$

\section{ASCITES: ABDOMINAL DROPSY.}

Ascites is dropsy of the peritoneum. It is an exudation of a noninflammatory liquid, which may be observed in all our domestic animals, including also poultry.

Very severe ascites is mostly found in the dog, and particularly upon young or very old animals; but we may also see it in the sheep, ox, or goat, and more rarely in the horse, in which animal Woodger has only observed it once in twenty-two years. We have ourselves only seen it very exceptionally in this animal. The rarity of ascites in this species must be attributed to the condition under which the animals live, to the work they perform, which necessitates energetic muscular efforts, also to suitable and regulated alimentation-circumstances which are against the production of blood-stasis and the transudations which are its consequences.

Etiology and pathology. Ascites is not a pathologic entity; it forms but a common symptom of numerous affections. 
I. Chronic diseases of the heart, lungs, kidneys, of the portal system and mesenteric ganglions, which determine sometimes general circulatory troubles (heart, lung, kidney), at other times local circulatory troubles (liver, portal vein, mesenteric ganglions); it is the venous blood and mainly the lymph which, hindered in their course, determine the transudations (passive ascites):

1. Concerning heart disease, we must especially mention here: valvular insufficiencies of long standing, chronic pericarditis, and epicarditis with abundant serous exudation or adhesion of the two pericardiac layers (cardiac symphysis); hydro-pericarditis.

2. Among the lung diseases we have emphysema and the interstitial processes with sclerosis and consecutive atrophy (tuberculosis).

3. In kidney diseases we may mention interstitial nephritis (Bright's disease) and the degenerative processes.

4. The liver troubles are: interstitial hepatitis leading to cirrhosis (in distomiasis and echinococcosis) and the neoplasms (adenomas, carcinomas, etc.).

5. Concerning affections of the portal system : compression of the portal vein by tumors and thrombi.

6. We have among the alterations of the lymphatic ganglions: caseous degeneration and atrophy (infectious enteritis of the young horse).

In all these cases, if the discharge of the blood in the capillaries and the veins is obstructed, or if any kind of an obstacle is opposed to the circulation of the lymph, there is at first a stage with increase of the intra-vascular pressure, to which is soon added a greater permeability of the vascular walls due to a modification of the endothelium. These conditions suffice to explain the passage of the blood through the vascular walls. When the object which obstructs the circulation resides in the portal system, the capillaries and veins of the peritoneum, as well as the roots of the portal vein, are particularly congested.

II. Hydremia - that is to say, the predominance of serum in the blood-and mainly of albumin (hydremic or cachectic ascites). Hydremia is idiopathic or symptomatic; it may be developed without any morbid change, or may appear consecutively to chronic affections and to exhausting diseases (watery and verminous cachexia in the sheep and the ox). The transudation is here the effect of an alteration of the vascular walls: ascites never exists alone; with 
it we find dropsies of the pleura, of the pericardium, and subcutaneous œdemas.

III. Tumors-carcinomatous, sarcomatous, or tuberculous-of the peritoneum, as well as cured peritonitis, are also charged with causing ascites. In these cases true exudation is meant rather than transudation, and chronic peritonitis rather than ascites. However, the development of true ascites under the influence of these pathological conditions is not to be doubted. In advanced tuberculosis of the cow we have several times recognized a very pronounced ascites, which was certainly produced by tuberculous neoformations, there being no trace of inflammatory alterations upon the peritoneum. On the other hand, we have observed in a dog ten years old a generalized peritoneal carcinomatous condition which involved all the abdominal organs, without any trace of ascites.

Pathological anatomy. The abdominal cavity often contains a considerable quantity of liquid. In the horse as much as 150 litres may be found; Brusasco has abstracted 107 litres at one time. In the dog, Hordt was able to draw 18 litres. The liquid of ascites is more or less similar to blood-serum; at times it is clear and yellow tinted or opalescent, slightly turbid; then again it is of a greenish-yellow tint due to epithelial residue in the course of granulo-fatty degeneration; in some cases it is colored red by red blood-corpuscles, in others we find small fibrinous flakes suspended in its mass. Its specific weight is relatively not very high (1012 in man, and we have observed the same density in the horse). Albumin is ordinarily found in but a small proportion; in the dog we have found 3.5 per cent.

Alterations of the peritoneal serous membrane vary with the age of the trouble. This membrane is at times hardly infiltrated; it is oftener tumefied, thickened, bleached, desquamated, and its endothelium is the seat of fatty degeneration. According to Ziegler, a formative irritation is developed, which becomes gradually infiltrated with cells. When the process has lasted a long time, it is accompanied by connective-tissue neoformations, a considerable thickening of the serous membrane, and at times by partial adhesions of the peritoneal folds.

Under the influence of prolonged accumulation of the transudate the various organs of the abdominal cavity become pale, anemic, and atrophied; the intestine is frequently retracted, the diaphragm is softened and much pushed forward; the capacity of the pectoral 
cavity is diminished and the lung compressed. Finally, we find other accessory alterations, causes or effects of ascites (alterations of the heart, lungs, liver, etc.; anemia, hydremia, cachexia).

Symptoms. The principal symptom of ascites is the increase in the size of the belly, becoming gradually more marked, but sometimes taking place rapidly; it is especially manifested in the dependent portions of the abdomen; the flanks become sunken, the spinous processes are more prominent, and they appear to become saddle-backed. In small animals the umbilical region is much protruded. In the ox, the sheep (recently shorn), the dog, and the cat, the abdomen forms a salient swelling back of the hypochondria. The development of the belly, the saddle-back, sunken flank, and general emaciation form quite a characteristic symptom-complex.

The abdominal walls are flaccid above, and more or less distended below; we perceive fluctuation on palpation. When applying the hand upon one side of the abdomen and giving slight blows upon the opposite side with the other hand, or if practised by the aid of an assistant, we perceive swinging and undulating movements. The same sensation is given by exploration of the abdomen through the rectal walls. Spinola advises holding the hand in a vertical position like an oar and having an assistant exert pressure upon the opposite side. In applying the ear upon one side of the belly while an assistant strikes slight blows upon the opposite region, we perceive a chopping noise produced by waves of liquid, which are broken against the abdominal wall. According to Spinola, this same noise could be caused in small animals by abrupt blows upon the body. We must not mistake for ascites a condition of repletion in the stomach or intestine by water ingested in excess.

In the regions where the ascitic serum is accumulated, percussion gives a dull sound, unless it is performed with too much force, or the liquid layer is too thin, or excessive pressure exerted upon the area percussed expels the peritoneal liquid. The dulness found in the inferior region of the abdomen is limited above, following a horizontal line, above which we find a tympanic resonance. The displacement of the dulness depending upon the different attitudes of the patient is a very important symptom. When small animals are kept in a posterior bipedal position we are surprised at the prominence of the abdomen immediately above the symphysis; at the surface of this prominence percussion shows dulness which is also limited upward, following a horizontal line. When placed in an 
anterior bipedal position, the serosity accumulates above the diaphragm and the dulness starts at the xiphoid cartilage; the pressure exerted upon the diaphragm by the liquid influences the respiration, which may become rapidly dyspnœic; the animals are restless, agitated, and endeavor to return to the quadrupedal position.

The breathing is accelerated and painful, phenomena due to the crowding forward of the diaphragm and the reduced mobility of the abdominal walls, which are more or less distended; the accessory muscles of respiration come very actively into play; sometimes we observe dyspnœa, the breathing becomes "suction-like," and is accompanied by convulsive movements of the alæ of the nostrils.

Not the slightest fever is observed, unless produced by some complication. The pulse is almost constantly accelerated and small ; toward the end of life it is often evasive ; the heart is very excitable and its movements are tumultuous. The mucous membranes become increasingly pale; in the sheep the conjunctiva is infiltrated with liquid and the skin is very pale. The hair is dull and bristly, and the extremities cold. Dogs prefer to remain in a sitting position, the fore legs extended; their movements are slow and seem to be performed against their will. They become tired quickly, and finally remain down constantly. Paresis of the vesical sphincter produces incontinence of urine.

If the disease follows its regular course the appetite diminishes gradually, and then disappears. In the ox, rumination and the peristaltic movements take place but irregularly. In the dog, the appetite is not impaired for some time, but it is capricious; the animals vomit from time to time; we see alternations of constipation and diarrhea, sometimes of meteorism; the quantity of urine is often increased at the beginning of the trouble, later it is much lessened. The lacteal secretion is reduced, and the symptoms of a serious digestive trouble become more clearly defined.

Course. The course of ascites is essentially chronic. Organic consumption progresses slowly; œdema appears upon the sheath, the teats, the dependent portion of the belly, upon the legs, etc., as much from the effect of the pressure exerted upon the large venous trunks of the abdomen by the ascitic fluid as by the slowly developed hydremia. The animals become gradually weaker and more miserable; a time comes when they are unable to rise; their eyes sink, dyspnoea increases (in the ox, according to Eberhardt, nauseas and vomitings are observed); a profuse diarrhea appears, and death 
happens as a consequence of pulmonary or cardiac paralysis, or by the progress of marasmus.

Diagnosis. The diagnosis is shown by the information furnished by the inspection, palpation, percussion, and auscultation of the abdomen. In order to insure it in the dog we may make an exploratory puncture; this is of advantage in cases where the liquid is not abundant and where the trouble is not shown by any wellmarked symptom. The possibility of making a complete examination upon small animals renders the diagnosis much simpler in them than upon those of our large domestic species.

It is often very difficult to ascertain the cause of ascites, but it is very important from the standpoint of prognosis. Hydremia with a tendency to dropsy may be affirmed if cutaneous oedema and transudations are found in serous membranes other than in the peritoneum. Heart and lung diseases as well as interstitial chronic hepatitis, which are frequent causes of ascites, often remain undetected; their diagnosis is quite delicate. When ascites is symptomatic of atrophic cirrhosis there are usually two symptoms wanting which belong to this: they are the impossibility of recognizing the reduced size of the liver and the absence of icterus. In affections of the liver accompanied by an increase in the size of this organ or alterations concerning its surface, and which are appreciable by exploration (echinococcosis, tumors, etc.), ascites may be related to its true cause. In hepatic ascites the urine generally contains coloring matters of the bile; but this symptom is of little value. In the dog, a simple gastro-intestinal catarrh produces this urinary anomaly, and cases of enormous hepatic lesion are not rare (for instance, in distomiasis), without any appearance of coloring matters of the bile in the urine. In this animal the etiology of ascites is rendered still more embarrassing on account of the frequency of dual factors in its causation; indeed, it is not unusual to find the coexistence of valvular insufficiency and hepatic cirrhosis. Concerning peritoneal tumors, it is exceptional to recognize these through palpation.

In those animals in which the peculiar condition of the organism may be confounded with ascites we must specially mention :

1. Peritonitis. This disease is accompanied by fever, profound disturbance of the general condition, manifestations of pain, abnormal sensibility to the touch; the specific weight of the peritoneal fluid obtained by the exploratory puncture is higher than in ascites. 
2. Gestation. Manual exploration enables us to recognize it; in addition, the general condition is not much altered; notwithstanding the great distention of the belly, the abdomen is rather dilated on one side only.

3. Obesity. Old dogs affected by valvular alteration generally have very short respiration; obesity may mislead the practitioner in such cases. The differential diagnosis has to be established by auscultation of the heart and an exploratory puncture (examination and palpation of the abdomen gives insufficient information).

4. Hydrometria, pyometria, dropsy of foetal membranes (mainly in the cow), ovarian cysts, abdominal tumors (in a two-year-old foal Cunningham found a tumor weighing 236 livres), renal cysts, etc. All these morbid productions may be recognized by a rectal or vaginal exploration, by an exploratory puncture, or by their resistance to palpation and percussion practised with the animal in different positions. While in ascites the symptoms deduced by percussion differ according to the attitude in which the patients are placed, they do not at all vary in abdominal or ovarian cysts, etc. The diagnosis between ascites and coprostasis is always very simple.

5. Paralysis of the bladder and its abnormal distention. Palpation will enable us to recognize a spherical swinging tumor in the vesical region; percussion does not show any displacement of dulness, no matter in what position the patients are placed. The characters of the urine and the data furnished by catheterization rarely leave the practitioner in doubt, and exploratory puncture insures the diagnosis absolutely.

6. Rupture of the bladder with accumulation of urine in the abdominal cavity. This accident is quite frequently observed in the ox, being produced in this animal by urethral calculus. It is indicated by colics, stoppage of micturition, emptiness of the bladder, urinary perspiration, a more or less acute febrile reaction, and collapse.

Prognosis. The prognosis of ascites is almost always very serious. The animals are generally only presented to the veterinarian when it is no longer possible to combat the causal trouble with any efficiency, and alterations of the blood already exist (hydremia). However, our personal observations prove that ascites is not equally persistent at all ages; in young subjects cure may be obtained in many cases, and in some instances it has been seen to disappear spontaneously in young dogs. The prognosis of chronic peritonitis, 
which has doubtless been frequently mistaken for this disease, especially in the equine race, is of less gravity than that of ascites.

Treatment. The indications are subordinate to the nature of the particular disease by which the abdominal dropsy is produceddiseases of the heart, lungs, liver, etc. These morbid conditions, however, even when recognized, frequently resist the agents directed against them, and we become restricted to the use of palliative means. The alimentation of the patients should be rich in albumin, and we must try to stop the peritoneal transudation by using purgatives and diuretics; sialagogues and diaphoretics may also be tried.

As a diuretic, digitalis associated with juniper berries and acetate of potash is especially recommended. We may prescribe as follows for the dog:

$$
\begin{aligned}
& \left.\begin{array}{l}
\text { R.-Infusion of leaves of digitalis - } \\
\text { Acetate of potash liquor }{ }^{1}
\end{array}\right\} \text { a } \bar{a} \\
& \begin{array}{l}
\text { Juniper berries } \\
\text { S.-One to two tablespoonfuls daily. }
\end{array}
\end{aligned}
$$

We administer also squill and sulphate of soda, caffeine, in the shape of sodo-benzoate of caffeine (for the dog the dose is 0.5 to 2 grammes; for the horse, 5 to 10 grammes), tincture of strophanthus (for the dog, 10 to 25 drops; for the horse, 10 to 25 grammes). Purgatives are of less value than diuretics; nnder their continued use the organism becomes debilitated; gamboge, however, has frequently been used for the dog, also croton oil and jalap. Subcutaneous injections of pilocarpine may be tried in all animals; it is given in small doses (great caution is required, especially in cases of heart troubles): 0.20 gramme for the horse; 0.30 gramme for the ox; 0.01 gramme for the dog. The administration of sublimate ( 0.1 to 1 gramme), formerly much used for dogs, is absolutely worthless and is dangerous.

Surgical intervention-puncture of the abdomen-should be reserved for the cases where the fluid, having become too abundant, presses upon the diaphragm to such an extent as to cause dyspnoa and threaten asphyxia. In all other circumstances paracentesis is of no value; the obstacle to the circulation remaining, the abdomen becomes again rapidly filled, and the blood, at the expense of which this new transudation is made, becomes poorer, especially in al-

1 This liquor, recognized by the Russian and German Pharmacopœias, is an aqueous solution of the acetate of potash by weight 1 to $18 .-$ N. D. T. 
bumin. In addition, it is not without some danger; whilst inoffensive by itself in most cases, it may lead to a fatal result when the animals make violent motions during the operation and try to escape it, and when the abdomen is emptied too rapidly, life may also be cut short by syncope or cerebral paralysis.

In young dogs paracentesis will often give good results; we have frequently obtained a cure after one puncture only.

Injection of Lugol's solution of iodine as an accompaniment to the operation is not to be made in cases where the ascites is symptomatic of a degenerative alteration of the peritoneum.

Hemorrhage of the peritoneum. Peritoneal hemorrhage is never primary. It is caused either by a traumatic lesion of the abdominal walls, or of one of the abdominal organs, or by a general affection (anthrax, bovine pest, purpura homorrhagica). Under the title of hemorrhagic irritation of the peritoneum, the liver, and the spleen, Roche Lubin ${ }^{1}$ has described an affection which he observed in lambs in an enzootic form, in the Department of Aveyron, and which Labat looked upon as an enzootic of purpura homorrhagica.

Neoplasms. Tumors of the peritoneum are primary (fibroma, lipoma, cysts, dermoid cysts), or secondary (tubercles, cancers, melanoma). They are developed upon the true serous membrane or upon its appendages (mesentery, omentum). Whether solitary or multiple, isolated or confluent, they are very variable in their physical characters. Under the name of foreign bodies of the peritoneum, round or ovoid neoformations have been designated, which are free in the peritoneal cavity (Arloing) or fixed upon the serous membrane by fibrinous ligaments or by a pedicle which may be thirty centimetres long (Goubaux). Mostly developed under the peritoneum, they raise this membrane, which soon forms a complete envelope and becomes prolonged in the form of a pedicle; when this becomes used up through the friction which it suffers from contact with the intestine, the body which it sustained becomes free in the abdomen (Delafond). These neoplasms are formed by an external connective or fibrinous tissue covering and by a central adipose, fibrous, caseous, or calcareous mass. Those possessing long pedicles may become twisted around the intestine and cause its strangulation.

Parasites. The parasites which are found on the peritoneum belong to the class of helminths, or worms. They are:

1 Roche Lubin: Recueil Vét., 1848. 
1. In the SOLIPEDES: Cysticercus fistularis, Filaria equina or $F$. papillosa, and Sclerostoma equinum.

2. In the ruminants: Cysticercus tenuicollis, Filaria cervina or F. labiato-papillosa, also F. terebra.

3. In the PIG: Cysticercus cellulosce tenuicollis, Cysticercus tenuicollis, Stephanurus dentatus, or Sclerostoma pinguicola.

4. In the DOG and CAT: Cysticercus Bailleti and Echinococcus veterinorum or $E$. polymorphus.

5. In the RABBIT: Cysticercus pisiformis. 


\section{SECTION II.}

\section{DISEASES OF THE URINARY APPARATUS.}

\section{IMPORTANCE OF URINALYSIS IN MORBID CONDI- TIONS, PARTICULARLY IN AFFECTIONS OF THE KIDNEYS.}

The examination of the urine in the different pathological conditions of the kidneys furnishes information quite as precise as that which is required by auscultation and percussion in lung and heart diseases. ${ }^{1}$ Without analysis of the urine, the diagnosis of most affections of the uropoietic system is impossible. The study of the pathology and therapeutics of the renal affections must be preceded by a short description of abuormal matters occurring in the urine, which are particularly important from a diagnostic point of view. We shall describe, with some detail, the following: albuminuria, hematuria, hemoglobinuria, the presence in the urine of tube-casts, epithelial cells, white corpuscles, fatty corpuscles, micrococci, etc. We shall take advantage of this circumstance in order to explain methodically and as briefly as possible interesting facts which can be drawn from analysis of the urine in a large number of diseases.

We find very complete information upon this question in the Manuel du Diagnostic microscopique et chimique des Maladies des Animaux domestiques, of Siedamgrotzky and Hofmeister, ${ }^{2}$ and in the treatise l' Etude de l' Urine, by Salkowski and Leube (1882).

Historical sketch on urinalysis. The analysis of urine was known in very ancient times, it having been undertaken, then

1 Analyses of urines will never render, in veterinary practice, such services as pointed out by the authors. In order to obtain indications of any value from these analyses, and to be able to institute comparisons between them, they must be made upon a sample obtained from the whole urine passed during twenty-four hours, and have relation to the quantity of liquid furnished during that time. Everybody knows that it is difficult, under ordinary conditions, to collect the urine of animals.-(Vignardou.)

${ }^{2}$ French translation by Wehenkel and Ch. Siegen. Brussels, 1881 ; 2d edition, 1884. (336) 
as now, with the object of making the diagnosis of diseases. Physicians in India knew sugar diabetes and several other conditions giving rise to anomalies of the human urine. Hippocrates (400 years B. c.), mentions the icteric coloration of urine. Celsius (contemporary with Christ) mentions numerous urinary sediments, and ascribes to them prognostic symptoms. Theophilus (600 A.D.) wrote a treatise upon the analysis of the urine, and John Actnarius (thirteenth century) has left a work of seven volumes upon the same subject. At the end of the last century more than two hundred large works were in existence upon the subject of the examination of urine.

Given up to quackery during the obscure period of the middle ages, urinalysis took a new start in the eighteenth century, under the influence of the school of Paracelsus, as a consequence of the great progress in chemistry. Dekkers discovered albumin in 1726; Cutugno demonstrated the presence of this substance in the urine by boiling, in the year 1776 ; the other matters found in it under various conditions were successfully discovered at later periods. After the use of the microscope became established in our medical studies, toward the middle of the present century, the diagnosis of diseases of the kidney attained great precision.

In veterinary medicine, Haubner was the first who undertook the examination of urines. In the domestic animals this study is much more complicated than in the human race, on account of the differences existing in the urines of the different animal species, and notably between the urines of herbivorous and those of carnivorous species.

\section{(A.) Chemical Analysis of the Urine.}

This comprises the search by chemical processes for the matters contained in the urine; such are: albumin in its various forms, hemoglobin, the coloring matters of the bile, sugar, leucin, cystin, tyrosin, etc. But the normal matters which are contained in the urine may be present in abnormal quantity (augmentation or diminution); such as: water, urea, uric acid, hippuric acid, biliary acids, phenol, indican, creatin, xanthin, catechin, the sulphonic acids, the coloring matters of the urine, mucus, and finally saltschlorides of sodium, potassium, and ammonium, the phosphates of lime, magnesia, soda, potash, and iron; also, the sulphates, carbonates (of lime), oxalates, etc. 
We shall only treat of such matters the recognition of which is important from a practical standpoint.

I. Albuminuria. Ancient theories upon the PATHology of ALbUMinuria. Until recent years an increase of the blood pressure in the kidney has been considered by a great number of authors as necessarily the cause of albuminuria. It was thought that albumin - a colloid substance, in opposition to crystalloids (salts, sugar, etc.) - could only pass through the animal tissues by an increase of the pressure of filtration (Malpighian bodies). But this doctrine has against it this physiological fact that albumin will pass through animal membranes as easily as many other substances. The process of nutrition should be rendered impossible if this property of albumin were wanting.

In 1879 Runeberg, relying upon physical experiments (filtration of albuminous solutions through dead animal membranes), created a new theory on the pathology of albuminuria. He looks upon the lowering of the normal pressure in the glomeruli as the cause of albuminuria. An objection which has been made against Runeberg's theory is that the dead animal membranes and the membranes of the living organism may act differently under albuminoid solutions, and that some chemical phenomena, such as the appearance of ascites through the increase of the pressure in the portal vein, or the escape of albuminous serum into the pulmonary alveoli by the increase of blood pressure in the lung (pulmonary œdema), testify absolutely against it (Senator). Ribbert and Cohnheim afterward proposed two different theories-both, however, untenable : in the first, the capillaries of the glomeruli, and in the second, the epithelium of these capillaries, would have the property, when in a normal state, of retaining the albumin, while all the other capillaries and epithelium would be passed by this substance. Heidenhain attributes to the epithelial cells of the glomeruli secreting properties; he makes true glandular cells out of those having the function of urinary secretion, while it would retain the albumin. According to Heidenhain's theory of secretion, albuminuria is produced when the epithelium of the glomeruli is altered or destroyed.

Generally admitted up to date, this theory has been successfully combated by Senator. ${ }^{1}$ This author points out that the epithelial cells of the glomeruli-flat cells, which are poor in protoplasm-

1 Senator: Berliner klin. Wochenschr., 1885. 
possess all the characters of the endothelial cells of the serous membranes, and that it is rash to attribute secreting properties to them; also that in the foetus and the newborn, whose cube-shaped glomerular epithelial cells are richer in protoplasm and quite similar to the glandular cells, the urine is albuminous to a normal degree. Finally, quite a number of clinical and experimental facts have been observed which contradict Heidenhain's theory.

Pathology of albuminuria according to Senator. Contrary to the generally admitted opinion and that of the majority of physiologists, Von Wittich, Henle, and Küss had maintained that the product of filtration in the glomeruli is albuminous. Senator ${ }^{1}$ gives reasons of a purely physical kind in order to explain the absence of albumin in the normal urine: the pressure in the capillary glomeruli, he says, is stronger than in any other part of the body; much liquid and little albumin, therefore, passes through their walls; the product of filtration is here poorer in albumin than the other products of transudation-than the cerebro-spinal liquid, for instance, which only contains traces; on the other hand, the secretion of the epithelium of the renal canaliculi also helps to dilute the quantity of albumin contained in the liquid that has left the glomeruli. All these circumstances together make it clear that the proportion of albumin contained in the urine is so small that it would not be possible to put it in evidence by means of our reactions.

But this albuminous solution may become concentrated-in other words, albuminuria may be produced-when one or several of the four factors coming into play in the filtration begin to act, viz.: the pressure of filtration, the constitution of the membrane, the composition of the blood, and the temperature.

1. Modifications of the pressure of filtration. Increase of the arterial pressure alone lessens the relative proportion and increases the absolute quantity of albumin in the product ; but when the urine becomes less watery (by violent muscular efforts, or perspiration, etc.), the relative quantity of albumin must necessarily increase: there is then a certain degree of albuminuria. The effects of venous stagnation are nearly the same as those of augmentation of arterial pressure: the distended veins compress the uriniferous canaliculi ; they produce urinary stagnation and a renal odema, which is accompanied by an albuminous transudation through the interstitial capillaries

1 Senator: Die Albuminurie im gesunden und kranken Zustande, 1882. 
and by a lessening of the pressure of filtration in the glomeruli. It is probable that there is here, in addition, an intervention of troubles of nutrition and physiological functions of the membranes. The diminution of arterial pressure weakens the transudation in the glomeruli and increases consequently the relative quantity of albumin. The urinary stagnation produces an oedema which is more marked than blood stagnation, and, like this, it results in a diminution of the filtrating pressure; the quantity of albumin is therefore augmented, and again there is albuminuria.

2. Alterations of the filtering membrane. These are: inflammatory lesions, fatty degeneration, turbid tumefaction, amyloid degeneration. These are the principal alterations which may affect the walls of the glomeruli and their epithelial covering. All these morbid processes also favor the passage of albumin in the urine. A small proportion of albumin is added to that derived from the altered or destroyed cells, the largest part of which is dissolved.

3. Blood alterations. Albuminuria appears as soon as the proportion of albumin increases in the blood (through a too active regeneration, accelerated organic metamorphosis, considerable losses by sudation or diarrhea, infusion of white of egg, the setting free of the albumin of the blood-corpuscle, etc.).

4. Elevation of the temperature (fever) produces albuminuria by increasing the relative quantity of albumin filtered and by causing alteration in the membrane of the filter.

Clinical importance and proof of albuminuria. Albuminuria was formerly looked upon as a pathological entity, but the preceding considerations show that in general it constitutes but a symptom which is common to the most diverse diseases. Among these we must particularly mention :

1. Diseases of the kidneys. (Acute and chronic nephritis, fatty and amyloid degeneration). Albuminuria is never wanting in these affections; it is the result of the greater permeability of the inflamed tissues.

2. Febrile and acute infectious diseases. (Influenza, pneumonia, contagious pneumonia, etc.) The causes are: alterations of the renal tissue, troubles of circulation in the kidneys, abnormal composition of the blood, elevation of the temperature; these influences act concurrently.

3. Passive hyperemia of the kidneys. Such are the valvular lesions of the heart, pulmonary emphysema and induration (heaves), 
and obstacles to the circulation in the posterior vena cava. Causes: a diminution of the filtering pressure in the kidneys, also lessening of the arterial pressure (cardiac weakness), and alterations of the filtering membrane.

4. Certain blood diseases. (Anemia, leukemia, diabetes, etc.) Causes: alterations or decomposition of the blood.

5. Nervous affections. (Epilepsy, tetanus, diseases of the spinal cord.) The cause is derived from circulatory troubles in the kidneys.

So-called physiological albuminuria has been found in man in ten to twenty per cent. of the examined cases; it is ascribed to violent muscular efforts, to colds, to food rich in albumin, also to emotion, etc. No special study has as yet been made upon this subject in our domestic animals. We must, however, mention one of Fröhner's tables, according to which he found but two cases of slightly albuminous urine in a total of fifty healthy horses examined for this condition.

Albuminuria of gestation, which is quite frequent in the human race, has been observed by Franke in cows at an advanced period of pregnancy. We have made a fruitless effort to demonstrate the presence of albumin in gestating cows, mares, and bitches. Pflug and Gross have not succeeded any better. ${ }^{1}$

Albuminuria of newly-born animals, appearing in the first days following birth, has only been established in the child; nothing like it has ever been seen in our young animals. Senator ascribes it to post-partum blood pressure, to the destruction of a considerable number of blood corpuscles, and to the abstraction of water by the transudation which takes place through the skin and lungs.

Examination FOR ALBUMin IN URINe. The urine may contain several of the varieties of albumin contained in the blood; in the majority of cases we find in it the albumin and globulin of the serum ; more rarely propeptone (hemialbumose) and peptone. We have not to consider here albumin contained in urine when this is more or less mixed with blood or pus. Among the numerous methods which enable us to recognize the presence of albumin in urine the following are to be recommended :

1. Add nitric acid to cold urine: when it contains albumin, a precipitate or a manifest turbidity of the liquid sets in, according to the case. The precipitate of nitrate of urea, which is sometimes

1 Pflug and Gross (unpublished communication). 
formed when the urine contains much urea, is rapidly dissolved by the action of heat.

2. Add to urine which is acidulated by acetic acid an equal volume of a saturated solution of sulphate of soda or of magnesia, and make it boil (Hoppe-Seyler's reaction). The precipitate or the turbidity which is formed may contain, besides albumin, sulphate of lime (due to the large quantity of lime in the horse's urine), also administered alkaloids-quinine, for example, etc. The microscopic examination will always enable us to recognize the substances easily.

3. Urine which is slightly acidulated by acetic acid is mixed with a concentrated solution of ferrocyanide of potassium. When the urine is much concentrated, it must first be diluted with distilled water. A precipitate which is formed around drops of the reagent falling into the urine, indicates the presence of albumin.

4. Boil acid urine, or if alkaline the urine must be slightly acidulated by means of acetic acid. If the precipitate which is the result is not dissolved by nitric acid, it is formed of albumin. By proceeding in this way we can recognize the albumin of the serum and globulin, but not propeptone, which does not coagulate.

In doubtful cases the urine must be examined by employing several of these processes successively.

Among the other urine reagents, we must also mention metaphosphoric acid (Hindenlang), picric acid (Johnson), mercurial iodide of potassium (the urine is previously rendered acid by means of citric acid-Geissler's reaction paper), citric acid tablets, or tablets of the ferrocyanide of potassium (Pavy), carbolized water (Méhn), tannin solution (Almén), trichloracetic acid (Raabe), chlorides of mercury and sodium (Fürbringer), formo-chloride of gold (Axenfeld).

Treatment of albuminuria. The symptoms of albuminuria are generally not treated as such; an exception must be made, however, for the cases where the loss of albumin becomes considerable and endangers life.

The most important indication is the regulation of the diet. According to Penzoldt, ${ }^{1}$ a meat diet increased the quantity of albumin in an albuminuric dog; it was reduced with farinaceous foods, especially bread. The regimen in albuminuria should therefore consist of non-nitrogenized food (fats, hydrate of carbon, gela-

1 Penzoldt: Verhandl. des Congress f. innere Med., Wiesbaden, 1885. 
tine). However, from Oertel's ${ }^{1}$. observations, we notice results showing that exclusively nitrogenized food does not produce the elimination of albumin through the kidney. In the present state of veterinary science it is advisable to start with a diet which increases the quantity of albumin in the blood, and consequently to make the patients ingest many albuminoids. The effects of remedies which might be tried are little known. Fröhner has observed a lessening of albumin in the horse by means of digitalis and tincture of strophanthus; this effect is explained by the rising of arterial pressure and the depletion of the hyperemic kidneys.

II. Hemoglobinuria. The appearance of free hemoglobin in the urine (almost always methemoglobin, more rarely hemoglobin or oxyhemoglobin) may accompany the various affections in which the principal morbid phenomenon is hemoglobinemia (nethemoglobinemia), that is to say, the addition of dissolved hemoglobin to the blood. But, as hemoglobin has a double source in the organismthe red blood-corpuscles on one side and the striated muscles (coloring matter of the muscle) on the other, we must distinguish two principal groups of diseases which are accompanied by hemoglobinemia and hemoglobinuria :

a. Alterations of the blood: toxemic hemoglobinemia (or hematogene) and hemoglobinuria (Bollinger).

b. Muscular alterations : rheumatismal hemoglobinemia (or myogene) and hemoglobinuria (Fröhner).

Toxemic hemoglobinemia and hemoglobinuria. The dissolution of the red blood-corpuscles and the setting free of hemoglobin are sometimes produced by true chemical poisons (properly so-called toxemic hemoglobinemia), at other times by infectious matters (infectious hemoglobinemia), and very probably by mieroorganisms.

a. Among the chemical agents producing hemoglobinemia when they are introduced into the blood, we must mention: distilled water, glycerin, the gallates, sulphuric acid, hydrochloric acid, arsenate of hydrogen, iodine nitrobenzol, nitrate of amyl, nitrate of soda, pyrogallic acid, paraldehyde, chlorate of potassium, copper, edible mushrooms, etc. The transfusion of blood coming from an animal of another species, extended burns (facts observed by H. Bouley and others upon animals), fatty embolism, etc., act in the same way.

1 Oertel : Handb. der allgem. Therap. der Kreislaufsstörung, 1885. 
$b$. We must admit the intervention of a specific agent in order to explain the hemoglobinuria which accompanies certain serious diseases, such as influenza, septicemia, etc. A considerable number of cases of hematuria of the ox must undoubtedly be classified in this group (miasmatic influences, similar to malaria in the human race?), which must also include a certain proportion of cases of hematuria in the horse (Schwarze Harnwinde) (auto-infection ?).

Rheumatismal hemoglobinemia and Hemoglobinuria. When the striated muscles are exposed to violent irritations, phenomena of decomposition are produced in their substance, in the course of which the coloring matter of the muscle, which is identical with hemoglobin, becomes free and passes into the blood. Among these irritations, external cold plays a most important part; it increases in a reflex manner the organic transformations (Fröhner). The very great majority of cases of hemoglobinuria in the horse are related to no other cause; in the red urine of the ox, also, a predominant pathogenic influence seems to be exercised by cold. In man, hemoglobinuria may be determined by violent exercise and rapid walking.

Proof of reduced hemoglobin or methemoglobin in urine is obtained by spectroscopic examination-one line of absorption only between $\mathrm{D}$ and $\mathrm{E}$, and sometimes another line between $\mathrm{C}$ and $\mathrm{D}$; besides, the urine is brown, with a varnish appearance; it contains much albumin, and the red corpuscles are wanting.

We must also mention the examination made with tincture of guaiacum and essence of turpentine (Almén).

III. Coloring matters of the bile in the urine. The presence of bilirubin and biliverdin in the urine always constitutes a pathological phenomenon; contrary to what was formerly asserted, coloring matters of the bile are not found in the urine of healthy animals of our different species, but they are found in it in the following morbid conditions :

1. In catarrhal icterus (either hepatogenic or mechanical, icterus by resorption or passive icterus). The cause of this icterus is the stagnation of the bile in the liver and its passage into the blood; this is favored by the obstruction of the biliary canals or their inflammatory tumefaction.

2. Acute affections of the liver (more rarely chronic affections of this organ, in which the biliary secretion is completely exhausted).

3. Such diseases as are accompanied by a diminution of the vas- 
cular pressure in the liver (chronic affections of the heart with lowering of the arterial pressure). The activity of the biliary secretion being regulated by the vis à tergo of the arterial blood, when the blood pressure is lowered (in intense pain, for instance), the bile is subjected to a true resorption and passes into the blood.

4. Intestinal catarrhs of the dog. This cause explains the frequency of the appearance of the coloring matters of the bile in the dog's urine. Here the pathology of hemoglobinuria is complex. There exists sometimes an inflammatory obstruction of the ductus choledochus; in other cases the diarrhea does not give the coloring matters of the bile time to become decomposed as in the normal state; they may thus pass as they are from the intestine into the blood, to be eliminated with the urine. (When normal they are transformed into hydrobilirubin or urobilin, and are eliminated in the shape of coloring matters found in the urine.)

5. Certain alterations of the blood, which seem to be able to create the coloring matters of the bile at the expense of the hemoglobin (hematogenic icterus).

The biliary acids, contrary to coloring matters, constitute elements of the normal composition of the urine; they are found in quite large quantity in cholemia and choluria; these are cardiac poisons (reduction of the pulse in icterus). Every urine gives a weak reaction of gallic acid (Pettenkofer's test) - that is to say, a purple coloration when treated with cane sugar or concentrated sulphuric acid.

Tests for coloring matters of the bile in the urine. 1. By means of Gmelin's reaction, dilute fuming nitric acid gives successively the following characteristic colors: green, yellow, red, violet, blue. 2. According to Brück, the same combination of colors is obtained by adding pure nitric acid and sulphuric acid. 3. In adding a concentrated solution of Chilean nitrate we see again the same color transformations (Fleischl). According to Latschenberger, it is difficult to demonstrate the coloring matters of the bile in the urine of the horse. This author advises to dilute the urine with water, then to add a solution of baryta and let it rest from twelve to twentyfour hours, decant, and finally submit the precipitate to Gmelin's test.

IV. Grape sugar. The transient existence of this sugar in urine is called glycosuria or mellituria; this condition when persistent and chronic bears the name of sugar diabetes (diabetes mel- 
litus). The demonstration of sugar in the urine may be made in different ways :

1. By four reduction tests. a. Trommer's test: by means of carbonate of soda and a solution of sulphate of copper (Fehling's liquor); by heating, a red coloration is produced, due to the formation of peroxide of copper. $b$. Böttger's method: the urine, containing soda, must be boiled (carbonate of soda or Seignette salts are advised by Almén), on adding a pinch of subnitrate of bismuth a black coloration is obtained, due to the reduced metallic bismuth. c. Salkowski's method: heat the urine with an ammoniacal solution of chloride of silver; we obtain a black precipitate of reduced silver. $d$. Indigo test: heat the urine with soda and indigo-blue carmine, a yellow coloration is produced as a consequence of the reduction of blue or yellow indigo.

As reagents for grape sugar, we must also mention :

1. Picric acid, sulphodiazobenzolic acid (Penzoldt), sugar of lead (Rubner), acetate of lead, phenylhydrazin and acetate of soda (of Jaksch).

2. The fermentation test : urine which contains grape sugar gives carbonic acid and alcohol when yeast is added to it; the respective quantity of these two products permits us to easily obtain the quantity of sugar.

3. The optical method, or polarization : grape sugar produces a deviation to the right of the polarization ray.

4. Moor's test : the urine becomes brown when it is boiled with carbonate of potash (caramel formation at the expense of grape sugar).

V. Indican. Indican (indoxylo-sulphate of potash) comes from indol, which is formed in the small intestine by pancreatic digestion; in minute proportion it forms a normal principle of the urine. The excretion of indican by the urines is increased in all affections which are accompanied by paresis of the intestine, abnormal fermentations, and in the conditions of consumption and inanition. The normal quantity of indican in the horse's urine is 0.15 gramme per litre; in the urine of the dog fed on meat there is found about 0.01 gramme; in a dog the small intestine of which was ligated, Jaffe has found 0.043 gramme of indican per litre of urine.

The presence of indican is revealed by Jaffe's method (for the horse's urine the operation is easy): we add to the urine an equal volume of commercial hydrochloric acid and containing a few drops 
of a calcium hypochlorite solution; the indican is transformed into indigo-blue (blue coloration); the indigo-blue may be extracted by means of chloroform, and its quantity determined by the colorimetric method or by means of the scale.

VI. Chlorides. Normal urine contains chlorides the quantity of which diminishes in certain diseases: in febrile affections and exudative inflammations of the internal organs, particularly in those of the serous membranes; the proportion of these salts diminishes also in the dropsies of the large splanchnic cavities (the chlorides pass in these instances into the transudation products and are consequently less abundant in the urine). The increase of the chlorides of the urine form thus a favorable prognostic symptom in these different morbid states (resorption of the pathological liquids and elimination through the urine).

The quantitative analysis is practised by subjecting cold urine to the action of nitrate of silver, after having first deprived it of albumin, and acidulating with nitric acid. The simple comparison of the precipitates (chlorides of silver) obtained for several days successively, permits us to conclude as to an augmentation or a diminution of chlorides.

\section{(B.) Physical Examination of Urine.}

I. Color. In a normal condition, the urine shows various tints, due to diverse and trivial causes. It is very pale in polyuria, sugar diabetes, chronic nephritis, during convalescence from febrile diseases, etc.; it is very dark, on the contrary, when its quantity is much reduced during fever ("loaded urine"); it is of an intense yellow in icterus and liver diseases; it is red in hemoglobinuria and hematuria, after the ingestion of rhubarb, senna, mercurialis annua, and "semen contra" (santonica), etc.; it is dark green in tar and carbolic acid poisonings, after administration of thallin, etc.; finally it is grayish, of bad appearance, in the septic diseases.

II. Quantity. It is diminished in diarrhea, in the diseases accompanied by more or less abundant exudations, in an intense fever or lowering of the blood pressure by weakness of the heart, and in acute nephritis, etc.; it is increased in polyuria, sugar diabetes and diabetes insipidus, renal hyperemia, interstitial chronic nephritis, during a convalescence, in febrile crisis, after the resorption of exudates and transudates, and after the administration of diuretics. 
III. Specific gravity. The specific gravity of normal urine in most of our domestic animals is 1040. It rises in sugar diabetes and in fever, and diminishes in polyuria, interstitial nephritis, febrile crises, and diabetes insipidus.

IV. Reaction. In a normal state the urine is alkaline in herbivorous animals (carbonate of lime); it is acid in the carnivorous (acid phosphates).

The urine of herbivorous animals becomes acid (phosphated):

1. When the herbivorous animals become carnivorous (through abstinence, alimentation with powdered meat, milk diet of the newborn.

2. In diseases of the digestive apparatus, mainly in intestinal catarrh (see this affection). Fever does not at all influence the reaction of the urine.

The urine of carnivorous animals becomes alkaline:

1. When the food is wholly composed of vegetable substances.

2. Immediately after meals.

3. Through prolonged administration of alkalines.

4. In catarrh of the bladder (ammoniacal fermentation of the urine).

V. Microscopic examination. This enables us to recognize the presence of red corpuscles and leucocytes, tube-casts, renal. and vesical epithelia; also crystals of abnormal forms, fat-drops, bacteria, etc.

1. Hematuria. Hematuria, or true urinating of blood, must be clearly separated from hemoglobinuria, an affection in which the urine is colored red by the hemoglobin derived from the blood and muscles. These two morbid states were for a long time confounded, and even until quite recently a deep obscurity hid the nature of these affections of the horse, and of the ox, which had the pathological symptom "passage of the urine" (see Hemoglobinemia of the $\mathrm{Ox}$ ). This confusion is so much more to be regretted, inasmuch as the distinction between hematuria and hemoglobinuria is rendered very easy by microscopic search for red corpuscles.

Hematuria, like albuminuria, does not constitute a pathological entity, but only a symptom which is common to the very numerous affections of the organs of the uropoietic system; the diagnosis "hematuria," therefore, does not not mean anything at all. Among. its causes we must mention : 
a. Wounds of the kidneys, occasioned by a contusion of the lumbar region or a serious fall, by crushing (in the dog), by excessive efforts, by penetration of ingested foreign bodies into the kidneys, etc.

b. Hemorrhagic nephritis. Cases of acute nephritis are quite frequently accompanied by a slight hematuria in their initial period; the same is the case in renal phlegmasia produced by acrid principles, animal or vegetable (young coniferous sprouts, cantharides, mayworm, mercurialis, white bryony, acrid diuretics, etc.); also by some mushrooms (rusts, anthrax, moulds, ergot of rye), and by metallic poisons (mercury, phosphorus, etc.). The most important of these hematurias is that happening quite frequently in the ox after the ingestion of acrid plants (see Poisonings by Turpentine Plants).

c. Renal hyperemia, in an active or passive state. It is to this cause probably that we must ascribe the hematurias of gestation observed in the cow (Hering), especially among pure-bred Durhams, and which generally happens immediately before or after parturition. The ingestion of water which is too cold (Ryschner), the feeding of green oats or rye (Hering), a too abrupt change from a poor to a rich and abundant alimentation (observed in Auvergne by Hurtel d'Arboval upon almost all the animals purchased in poor condition, and in Normandy, England, and northern Germany by other observers) are so many predisposing causes to the production of renal hyperemia. Albert has seen hematuria as a symptom of pneumonia from a foreign body; the red urine was here undoubtedly the expression of septic infection or pyemia.

d. Pyelitis, particularly the suppurating form of this affection, and the presence of the giant eustrongylus in the pelvis of the kidney.

e. Cystitis: the acute and hemorrhagic inflammation of the vesical mucous membrane, and the chronic cystitis occasioned by vascular dilatations, may accompany hematuria.

$f$. Vesical calculi, wounds, vesical tumors (cancer, polypus, tuberculous or glanderous infiltrations); also ulcers of the mucous membrane.

g. Uiethritis, ulcers, tumors, and calculi of the urethra.

h. Prostatitis and concretions of the prostate.

$i$. Acute infectious diseases, during the course of which a "hemorrhagic diathesis" is developed (anthrax, pyemia, septicemia, contagious pneumonia of the horse, variola, canine distemper, petechial fever, etc.). 
$k$. The constitutional diseases, and especially hemophilia, leukemia, pernicious anemia, etc.

l. Filaria of the blood (Lange) producing vascular lesions in the kidney.

The most salient characters of hematuria are the red or redbrown coloration of the urine, the red precipitate which is formed in it and the microscopical evidence of the red corpuscles; the coexistence of these three symptoms is necessary in order to make the diagnosis of hematuria. We have already seen how hematuria can be distinguished from hemoglobinuria; we must add that the presence in the urine of a few red corpuscles, which are insufficient to give this liquid the red tint, ought not to be qualified as hematuria in ordinary description.

A very delicate question which is often most difficult to solve is: Where does the blood come from? Does it emanate from the kidneys, the bladder, or the urethra? We have a thoroughly established series of symptoms serving as a starting-point for the differential diagnosis, but, as shown by Hering, the practical value of these symptoms is open to doubt. In general, we must take into account the following facts :

The cylinders of coagulated blood are characteristic of renal hemorrhage, for they can only be formed in the renal canaliculi. When the hemorrhage comes from the kidneys, the urine and the blood are intimately mixed, the color of the liquid varies from a brownish red to a brown-black, the quantity of red corpuscles is small, and the fibrinous clots are colored red (the first two characters are quite often wanting). The symptoms of nephritis, the presence in the urine of the epithelium of the kidney or of albuminous cylinders (casts), etc., permit us to decide as to the renal origin of the hemorrhage.

In vesical hemorrhages the blood is eliminated in the shape of more or less voluminous clots ; the animals have frequent desire of passing urine (pollakiuria). The color of the blood is clear; the red corpuscles are well preserved, the fibrinous clots are colored red. The abundance of the vesical epithelium is a valuable symptom for the establishment of a diagnosis.

In urethral hemorrhage the blood runs off, drop by drop, during the intervals of micturition.

We must not confound hematuria with the hemorrhages of menstruation, metrorrhagia, and with the abnormal colorations of the 
uxine due to the medicinal agents (rhubarb, senna, tar) or biliary coloring matters, etc.

It has been maintained that it is sometimes impossible to make the distinction between hematuria and hemaglobinuria, on the ground that the red corpuscles might leave their coloring matters in the urine in which they are held ; this assertion is weakened by Franck's observations. ${ }^{1}$ This author has established the integrity of the corpuscles in alkaline and acid urine eight days after micturition; it is only at the time when the urine becomes ammoniacal, in consequence of putrid fermentation, that the red corpuscles are destroyed. In very concentrated urine the corpuscles become starshaped.

The prognosis and treatment of hematuria vary with the disease of which it is the expression. (See Nephritis, Cystitis, Vesical Hemorrhage, etc.)

2. URINARY CYLINDERS (CASTS). These are generally tubuliform productions, more rarely full cylinders, formed by an albuminoid substance (albuminous eylinders) or by carbonate of lime, with mucus as the fundamental substance (calcareous cylinders). They are derived from the renal canaliculi.

The albuminous cylinders are sometimes hyalin, with a transparent and homogeneous fundamental substance, at other times granulous (fat, etc.). In certain cases we find cylinders called "epithelial," which are formed by the desquamated epithelium of the renal canaliculi or by morsels of exudate covered with epithelial cells; in others the cylinders have a waxy aspect, and are formed by a colloid mass, which is of high refraction. The albuminous cylinders, especially the granulous and epithelial cylinders, indicate nephritis most of the time; in the horse, in the course of hemoglobinuria, some are found which well deserve the name of hemoglobin cylinders. The hyaline cylinders are seen in albuminuria, the febrile diseases, and passive congestion of the kidney.

3. Epithelial cells. The short, cylindrical, pigmented cells (yellow cells being absent), and also the round cells, which are rich in protoplasm (renal epithelium), indicate an affection of the kidney; the pavement epithelial cells indicate vesical, urethral, or vaginal catarrh; but these elements have a diagnostic value only when found in large numbers.

1 Franck: Adam's Wochenschrift, 1873. 
4. Leucocytes. The pus corpuscles which are found in the urine may proceed from nephritis, pyelitis, cystitis, or urethritis. We must, therefore, be guided mainly by the other symptoms, and particularly by the presence of urinary cylinders, by cylindric or pavement epithelium, etc.

5. BaCteria, FAtTy DRops, Basic Phosphate CRYstals, ETC. All these bodies are abnormal elements or accessories of the urine. The bacteria indicate a general septic infection, abscess formation in the kidneys, vesical catarrh, or a putrid decomposition of the urine; the small fatty drops are sometimes found in the dog in a normal condition, in other cases they indicate a fatty degeneration of the renal epithelium. Friedberger has found cholesterin crystals in a dog which had a catarrh of the bladder caused by a tumor having undergone atheromatous degeneration. The appearance of basic phosphate crystals in fresh urine (coffin-lid shaped) indicates cystitis.

\section{CONGESTION OF THE KIDNEYS.}

Congestion or hyperemia of the kidneys is active or passive. Sometimes it forms but a passing phenomenon, which is symptomatic of several diseases, in which case it ought not to be considered as a true pathological condition.

Etiology. In general, the causes of active congestion are those of acute nephritis, which, in its first stage, is especially characterized by a severe hyperemia of the renal tissue. It is developed by the action exerted upon the kidney, by acrid alimentary matters (anemone, adonis, asclepias, damp or mouldy hay and oats); this etiological influence explains why, in the spring, we often notice the appearance of active renal hyperemia with its main symptom, polyuria, in an. enzootic state. It is sometimes determined by dirty, stagnant drinking-water from swamps, pools, etc. Any abdominal hyperemia may induce congestion of the kidney leading in certain conditions to hematuria. (See Hematuria.) It is also a phenomenon of most of the infectious diseases (influenza, contagious pneumonia, hydrophobia, etc.).

Passive congestion accompanies the general blood stagnations related to heart or lung diseases (valvular alterations, pulmonary emphysema, induration of the lungs, pleural exudates); it is also developed when the course of the blood in the posterior vena cava is obstructed by the presence of aneurisms, of thrombosis, or of 
tumors, or by the abnormal accumulation of food and gas in the intestinal canal. Sometimes it is produced by thrombosis or compression of the renal vein or artery (extension of a thrombus from an aneurism of the great mesenteric).

Pathological anatomy. In the two forms of congestion of the kidney the latter is tumefied, softened, and colored a deep red. In the active congestion especially, the kidney is infiltrated and very soft (parenchymatous oedema); the surface of the organ is at times cyanosed; a slight traction exerted upon the capsule is sufficient to detach it. Active hyperemia determines mainly the aceumulation of the blood in the vessels of the cortical substance; the passive, congestion in the veins of the medullar substance.

When the passive congestion persists for a long time, the organ takes a dark purple coloration, and the proliferation of the interstitial connective tissue gives it a much firmer consistence (cyanosed induration); at the same time the epithelium of the urinary canaliculi is subject to regressive alterations; it is affected by fatty degeneration. These lesions may lead to hemorrhages and nephritis.

Symptoms. In active renal hyperemia we observe polyuria; the urine is clear, transparent, its specific weight is diminished; its quantity. may increase from five litres (a common normal amount in the horse) to twenty-five litres and more; its specific gravity, which is 1040, may be lowered to 1005 or 1001 (polyuria is a form of diabetes insipidus). The animals hold the back slightly arched, the gait is stiff; no serious febrile reaction is to be observed.

In passive congestion the quantity of urine is lessened and albuminuria is present, often even hematuria; we find at times hyaline cylinders in the urine. A certain weakness of the posterior quarters may be observed. The affection is chronic and apyretic.

Treatment. The active congestion must be combated by a strong counter-irritant upon the intestine.

The treatment of passive congestion is identical with that of the causal disease. It is against this latter that active means must be directed.

\section{Nephritis.}

The chapter on nephritis is the most incomplete of the entire veterinary pathology. This weak point of our medicine depends on two principal causes: the very short time during which the practice of analyzing the urine of our animals has been in vogue, 
and the insufficiency of the anatomico-pathological and microscopic studies related to the diseases of the kidney-studies which have not kept pace with those of similar diseases in human medicine, in which important acquisitions upon nephritis have been recently made (Ribbert, Friedländer, Fürbinger, Aufrecht, Ziegler, Nauwerk). As long as special researches are not forthcoming to fill the void that we have pointed out, veterinary pathology will have to be guided by the discoveries made in man. To explain the pathological anatomy of nephritis we have put Ziegler's work in requisition. ${ }^{1}$

The division which it is proper to establish in nephritis is one of the most delicate questions affecting this disease. In taking the etiology or the anatomical characters as a basis of classification, we may recognize numerous varieties of inflammation of the kidney, but they do not answer to the requirements of practice. From an etiological point of view, we might consider such forms of nephritis as infectious, bacterial, mycotic, toxic, traumatic, rheumatismal, metastatic, embolic, etc. According to the anatomical region of the kidney which is first affected, we formerly distinguished a parenchymatous nephritis (an affection of the renal epithelium) and an interstitial nephritis (inflammation of the vascular apparatus and of the interstitial connective tissue)-forms to which modern authors have added glomerular nephritis-that is to say, a localization of phlegmasia upon the Malpighian glomeruli. Nephritis may also be divided into acute or chronic, diffused or circumseribed forms.

If among these numerous forms of nephritis we take those answering to the actual state of veterinary pathology, we arrive at the following clinical division :

1. Acute nephritis, formerly "acute Bright's disease." It may be of infectious, rheumatismal, toxic, or traumatic nature; sometimes it affects principally the glomeruli, at other times it appears under the form of an acute diffused nephritis.

2. Chronic nephritis: "chronic Bright's disease" of the older school. It comprises the chronic parenchymatous interstitial nephritis and the terminal period of this latter-that is to say, atrophy of the kidney (small granulous kidney).

3. Purulent nephritis, a consequence of pyemia or of suppurating pyelitis which has extended to the renal parenchyma.

1 Ziegler: Lehrbuch der pathol. Anatomie. 
Principal Clinical forms of nephritis in man. (Signification of the term "Bright's disease.") In man, excluding suppurating nephritis, we distinguish three principal forms of nephritis:

1. Acute nephritis, which is generally related to an infectious disease and usually quickly terminates in cure or death; it very rarely passes into a chronic state. The principal symptoms are: polyuria, slight albuminuria, very high specific gravity of the urine, hyaline cylinders, renal epithelium, white and red corpuscles in the urine.

2. Chronic parenchymatous nephritis, which often leads to death or ends in atrophy of the kidney. Its evolution is very slow. Its principal symptoms are : oliguria, albuminuria, increase of the specific gravity of the urine; cylinders, white corpuscles, epithelial cells in the course of fatty degeneration, various forms of detritus, more rarely red corpuscles in the urine; tendency to dropsy. It is characterized anatomically by a much marked epithelial desquamation.

3. Renal induration, granulous kidney, atrophied kidney, sclerosed kidney. It is often developed at the expense of chronic parenchymatous nephritis; its evolution is very slow and obscure. Its principal symptoms are: polyuria, slight albuminuria, a lessening of the specific weight of the urine, infrequency of germ elements, absence of dropsy, hypertrophy of the heart. It is characterized anatomically by an interstitial connective tissue proliferation.

We generally designate by the expression "Bright's disease" (from the name of the celebrated kidney pathologist, R. Bright ${ }^{1}$ ) the ensemble of the renal affections accompanied by albuminuria (Bright, Leyden, etc.); some authors give the appellation to the true nephritis by adding to it amyloid degeneration of the kidney (Virchow, Aufrecht, Wagner, etc.); Krebs reserves it for primary interstitial nephritis. In view of this diversity, it seems to us that this earlier designation ought to be completely abandoned and replaced by a terminology based upon the actual pathological anatomy. In veterinary pathology it has given rise to deplorable confusion on the subject of hemoglobinuria of the horse. ${ }^{2}$

1 Bright: Reports of Medical Cases, London, 1827.

2 Bright's disease corresponds to a perfectly defined state from a clinical point of view-being characterized by a persistent albuminuria, dropsies, and a lesion of the kidneys; but the progress of pathological histology has established the plurality of these anatomical varieties. At the present day we recognize the following forms: 1. Chronic parenchymatous, diffuse or epithelial nephritis, determining the condition of 


\section{Acute Nephritis.}

Etiology. Acute nephritis is observed in all the different domestic species, but particularly the horse, ox, and dog, in which animals it is produced by the most varied causes. ${ }^{1} \quad$ It may succeed congestion of the kidney or be determined by traumatic influences, such as blows and injuries upon the back (Reboul), falls, violent efforts while pulling, too abrupt turns performed by saddle-horses while at a rapid gait, etc. It is at times produced by the action of cold: the intimate physiological connection which exists between the skin and the kidneys, and the bad effects of the suppression of cutaneous functions, sufficiently explain the origin of nephritis à frigore.

[The action of cold when associated with great nervous excitement, as fear of steam, etc., may frequently be the cause of an acute nephritis. I have seen several unquestionable examples of this, one occurring in my own driving horse, which had been tied where he could hear escaping steam, of which he was very much afraid. After being exposed to this influence for over half an hour on a cold day there developed an acute nephritis with diarrhea and loss of appetite.-W. L. z.]

It also happens frequently in the course of infectious diseases ; it is then the result of renal elimination of irritating products. Benign infectious nephritis leads only to turbid tumefaction and to fatty degeneration of the renal epithelium. The septic and pyemic processes, such as puerperal septicemia of the cow, typhoid fever of the horse, and also, but more rarely, contagious pneumonia, often induce acute infections nephritis. We have seen a horse which was recovering from contagious pneumonia die from a double intense acute nephritis. In a case of serious angina of a horse, Friedberger has seen acute nephritis and septic infection occur; Siedam-

the kidney known under the name of large white kidney. 2. Chronic interstitial or sclerous nephritis, which affects particularly the connective tissue and the vessels, and which leads to the small red contracted or atrophied kidney. 3. Mixed nephritis, in which the epithelia, the connective tissue, and the vessels are affected simultaneously. -N. D. T.

1 Parenchymatous nephritis has been observed among birds by Larcher. In certain cases the inflamed kidney is smooth, its capsule thin and easy to detach, and the subjacent tissue, the tint of which is dark yellow, is strewn with small bloody spots; in others the surface of the organ is dented, irregular, rough to the touch, and the opaque and thickened capsule strongly adheres to the subjacent tissue, the tint of which is pale yellow, and the consistence firmer than in the normal state.-N. D. T. 
grotzky has observed a fatal subacute nephritis as a complication of bronchitis; Rivolta has described a case of subacute bacillary nephritis. (Concerning glanderous or tuberculous nephritis, see Glanders and Tuberculosis.)

This pathology is also that of toxic nephritis (metallic and organic poisons), which are ordinarily produced by the internal use or a too extended application of cantharides, essence of turpentine, croton oil, squill, etc. Numerous clinical facts coming within this group have been described under the name of "hematuria" of the ox. The following agents also possess an inflammatory action upon the kidneys : tar, carbolic acid, iodoform, phosphorus, arsenic, lead, mercury, etc.; also moulds, rust, fungi, charbon ; certain insects, such as snails, cabbage-lice (Neubert); raw potatoes ingested to excess (Schick), and certain varieties of cotton-seed flour.

Secondary nephritis may occur by propagation of the inflammation in cystitis or pyelitis, etc. We must also specially mention nephritis produced by hemoglobin infarctus, which appears in the course of hemoglobinemia ("black urine") of the horse. Formerly it was wrongly considered as the primary affection.

Pathological anatomy. The macroscopic alterations vary with the anatomical form of the disease. Sometimes one kidney only is diseased, at other times both are affected. The augmentation of volume may reach double or treble the normal dimensions. The renal tissue is soft, sometimes as if reduced to pulp (inflammatory œdema, hemoglobin infarctus); its tint is yellowish, marked with red spots, or with a uniform diffuse red; hemorrhagic infiltrations may be observed in it. When the substance of the kidney undergoes fatty degeneration it is pale gray or even whitish; these abnormal colorations coexist at times and give the organ a particular marbled aspect. On section, a thick creamy liquid often runs out; the glomeruli are very prominent; the renal capsule may ordinarily be detached with the greatest ease. In the pelvis we find a thick urine, gelatinous or bloody. There are cases (nephritis of the glomeruli, circumscribed nephritis) where the surface of the kidney does not show anything abnormal.

The microscopic alterations differ considerably according to the location of the process. In human medicine we distinguish the following anatomical forms:

1. Acute nephritis of the glomeruli. The epithelium of the glomeruli and of the capsules is tumefied, desquamated; the capsules 
are filled with an inflammatory liquid, which is albuminous and coagulates by ebulition, also by the action of alcohol, etc., and forms a sickle-shaped zone surrounding the glomeruli; the capillary walls are hyaline and tumefied; these canals may be thrombosed by white corpuscles or by epithelial cells undergoing proliferation. The other parts of the kidney are but little or not at all altered.

2. Diffused acute nephritis. With the infiltration and thickening of the interstitial connective tissue by a liquid fibrinous exudate we observe compression of the vessels, tumefaction, softening, fatty degeneration, desquamation of the epithelium of the urinary canaliculi, and the appearance within them of hyaline cylinders. The glomeruli are intact.

3. Nephritis circumscribed to small centres. It is characterized by circumscribed cellular infiltration from the cortical substance and from the neighborhood of the veins; the epithelium of the glomeruli and of the uriniferous canaliculi is generally intact, but hyaline cylinders may be formed inside of these. This form of nephritis, which is the most frequent, is often accompanied by circumscribed indurations, abscesses, cavities, and cicatricial spots.

4. Purulent nephritis. This is the result of an intense cellular infiltration in the neighborhood of the veins or in the glomerular capsules; it is recognized by the presence of purulent centres which are rounded or stretched in bands and surrounded by a red zone. It is developed after the penetration of micro-organisms into the glomerular loops ; these become obstructed and necrotic ; the inflammation starts at their periphery, and the neighboring epithelium becomes desquamated. Under the name of "embolic nephritis," Friedberger has described a case of this kind which was observed in the horse. White corpuscles and micrococci sometimes penetrate in very large numbers into the inside of the uriniferous canaliculi and completely obstruct them (see the case of bacterial nephritis of the ox, related by Dammann). The small abscesses thus formed may become confluent and constitute a large purulent centre, and even transform the kidney into a true sac filled with pus, an alteration which is observed quite frequently upon the ox (see Purulent Nephritis). In man, in cases of diphtheria, we find accumulations of micrococci in the uriniferous canaliculi (Letzerich).

The so-called "parenchymatous" degeneration of the kidney is 
often connected with inflammation. Like this, it is developed under the influence of infectious or toxic principles exercising their action upon the renal epithelium. According to its stage we distinguish: 1. Turbid tumefaction (granulous trouble). 2. Fatty degeneration. 3. "Hydropic" degeneration. 4. Necrosis of the renal epithelium.

Symptoms. The most important and often the only manifestations of nephritis (in the course of infectious diseases, for instance) are furnished by the urine. Its quantity is diminished; it is thickened, albuminous (from 0.3 to 1 per cent. of albumin in man); its specific gravity is increased ; it is often turbid and also of abnormal color; occasionally we observe hematuria.

With the microscope we see a more or less large number of cylinders in the urine, which are partially or totally covered with cells, with leucocytes and red corpuscles; we discover, besides, numerous epithelial cells within, also white or red corpuscles, according to the desquamative, interstitial, or hemorrhagic character taken by the nephritis. Micturition is painful; the urine often runs off drop by drop only, notwithstanding the violent efforts made by the patients (strangury, dysuria). In serious cases the urinary secretion may be completely suppressed; Funk has observed an anuria of five days in a cow, and Friedberger one of seven days in a horse. This latter symptom is due to the obstruction of the uriniferous canaliculi by fibrinous cylinders and to the diminution of excretion in the diseased organ. The lumbar region is very sensitive to the pressure of the hand. In a rectal exploration we always find the bladder empty; the kidneys seem to have increased in size; they are sensitive to pressure. At the beginning of the disease we often observe renal colics; the back is arched, the gait stiff and staggering, rising is very painful; the animals remain almost constantly standing, with their legs outstretched or gathered under the body. In unilateral nephritis, the leg of the diseased side is oftentimes dragged during the walk, or it covers less ground than the corresponding limb on the opposite side. In males, one of the testicles is frequently higher (Röll).

From the beginning, the appetite may be suppressed; in the dog vomiting is not unusual, and is habitually followed by constipation, and this later on alternates with diarrhea. The elevation of the temperature is moderate as long as there are no uremic complications; the pulse, strong and hard at the start, becomes afterward 
frequent and weak. Cardiac hypertrophy produced a few weeks after the beginning of nephritis-a phenomenon quite frequent in the human race-is unknown in domestic animals. We have observed neither pulmonary disease (bronchitis, pneumonia), nor disease of the pericardium (pericarditis), etc., which occur in the human species in the course of nephritis. But we can recognize an odematous tumefaction of the subcutancous connective tissue-on the head, chest, belly, scrotum, and upon the articulations (De Cesare)and dropsies of the splanchnic cavities.

When nephritis is about to end in death, and in all very serious cases, the clinical tableau which has just been sketched is complicated by the symptoms of uremia, especially vertigo, spasms, and dyspnnea. In a cow suffering from double nephritis, Pflug observed, a few hours before death, eclampsia, opisthotonos, and a deep coma during the intervals of the attacks. In a horse with bilateral acute diffused nephritis we have ourselves observed, twelve hours before death, a deep alteration of the general sensibility, a great weakness, staggering, abundant perspiration, muscular contractions, and a considerable elevation of temperature (about $42^{\circ}$ C.).

True uremia is very rare in our various domestic species, except in the dog. In this animal, in cases of urinary calculus, of urethral contraction, and paralysis of the bladder, vomitings are quite frequently observed, also paretic weakness, coma, a lowering of the temperature, and even epileptiform attacks; these are uremic symptoms. In the sheep, Dammann has seen an alternation of epileptiform contractions with a soporific state.

Concerning the nature of uremia we must refer to treatises upon general pathology. We shall only say that it consists of an accumulation, in blood and tissues, of the principles of urine, above all of urea and potash salts. The theory of Traube, according to which uremia would be the consequence of a cerebral œedema, and that of Frerichs, who considers as the cause of uremia the transformation of urea in the blood into carbonate of ammonia, have not been confirmed by decisive experiments.

The principal symptoms of uremia in man are: cephalalgia, somnolence, anxiety, delirium, nausea, vomitings, restlessness, slight convulsions and serious eclampsia (epileptic habit), these phenomena are often followed by amaurosis or uremic deafness (central troubles). In addition are observed hiccough, diarrhea, the elimination of urea 
through the cutaneous surface, pruritus, slowing and later acceleration of the pulse, a temperature which is above or below the normal, dyspnœa, etc.

Cuurse. The termination of acute nephritis is defined to be from the first to the second week. Rarely the animals succumb within a few days.

Prognosis. The number of fatal cases is nearly equal to the cures. The development of uremic symptoms considerably aggravates the gravity of the prognosis : death is generally produced by cerebral or pulmonary œedema. The passage to a chronic state is exceptional.

Differential diagnosis. Acute nephritis is often confounded with the inflammatory conditions of other organs of the abdominal cavity : with peritonitis, colics, enteritis, cystitis, metritis, etc. These errors are only possible when we neglect to make the examination of the urine, which permits us in all cases to affirm the diagnosis of nephritis. Besides, the symptoms furnished by the exploration of the bladder, of the uterus, etc., may guide the practitioner.

Treatment. We should prescribe absolute rest and avoid all irritating food or medicines (frictions of essence of turpentine, and cantharides ointment on the back). For the dog we should recommend a milk diet, which has sufficiently shown its value in human medicine. The depletion of the kidneys must be rendered more easy by means of diaphoretics and laxatives; diuretics and derivatives locally in the shape of wet, tepid compresses.

In large animals we must produce sudorific action by repeated and energetic rubbings upon the surface of the whole body, also by warm blankets, wet, tepid compresses (Priessnitz's) applied upon the trunk, etc.; we may also resort to sialogogues, to subcutaneous injections of hydrochlorate of pilocarpine (for the horse, 0.20 gramme; the ox, 0.50 gramme; the $\operatorname{dog}, 0.005$ to 0.02 gramme); in small animals (dog and pig) the cutaneous perspiration is sometimes induced by warm baths, the temperature of which is gradually brought to that of the body; warm compresses may also be used.

Among the purgatives, drastics have the most favorable action cabeuse they draw a large quantity of water from the organism (calomel, aloes, infusion of senna for the dog); we must avoid those irritating the kidney. The same indication obtains for the diuretics, among which we should only employ the alkalines, mainly acetate 
of soda. When the heart weakness becomes alarming, we must resort to digitalis, to caffeine, etc.

The eclamptic attacks must be combated with bromide of potassium, chloral hydrate, and chloroform inhalations.

Concerning astringents or styptics (tannin, sugar of lead, sulphate of iron, etc.), very much used formerly against acute nephritis, whilst theoretically we attribute to them a constrictive action upon the vessels, and consequently an antiphlogistic effect, their practical utility remains to be demonstrated. We have often used cinchona bark (as an astringent and antifebrile agent), and we have obtained encouraging results from it.

\section{Chronic Nephritis.}

Etiology. Sometimes chronic nephritis is developed from the onset; at other times, but less frequently, it follows acute nephritis. Its causes are still less known in our animals than in man. They seem to consist especially of irritations of a chemical or parasitic nature exerted directly upon the renal tissue. Among the chemical irritants we must particularly mention copper and lead salts, which, according to Ellenberger and Hofmeister, give rise in the course of time to special inflammatory alterations of the tissue of the kidney (similarly to the action of alcohol and uric acid in the human subject). As noxious agents of a parasitic nature, we must mention, in the first place, the bacilli of glanders, then certain microbes which after having penetrated the bloodvessels are eliminated with the urine; these, in passing through the kidneys, set up in them a chronic phlegmasia. It is thus that we can explain the frequency of the disease in the cow-the kidneys of this animal, after parturition, being exposed to the action of septic substances coming from the uterus. In the horse, intestinal and laryngeal ulcerations, etc. (Lustig), also bronchial catarrh accompanied by bronchiectasis and emphysema (Fröhner) constitute so many portals of entry for the microbic agents which give rise to this condition. In man, we often observe chronic nephritis in the course of infectious diseases of slow evolution, also chronic endocarditis, etc. Chronic nephritis of the horse is sometimes of embolic nature; it is then related to an aneurism with thrombosis of the anterior mesenteric artery and of the renal arteries (Lustig). In old animals, as in aged human beings, the senile vascular alterations (parenchymatous degeneration) seem to exercise a real influence upon the generation of certain chronic 
inflammatory processes of the kidney. That repeated colds may cause chronic nephritis is not positively demonstrated.

Pathological anatomy. Formerly there were recognized two quite distinct principal forms of chronic nephritis-parenchymatous nephritis and interstitial nephritis, but modern researches have established the unity of the inflammatory process; they have shown that, in chronic nephritis, the so-called renal parenchyma (the epithelial cells) and the interstitial tissues are inflamed at the same time in very different degrees. If in human pathology we still preserve the division into chronic parenchymatous nephritis and interstitial nephritis or renal atrophy, it is because this distinction is of utility from a clinical point of view. It is generally admitted that atrophy represents the last stage of chronic parenchymatous nephritis. In our animals nothing is known as to the existence of a non-inflammatory renal atrophy, so that we shall continue to consider chronic parenchymatous nephritis and chronic interstitial indurative or atrophic nephritis as two successive degrees of renal phlegmasia. Although these two morbid states may offer certain anatomical and special clinical characters, yet they represent only stages of development of one and the same process, sclerous nephritis forming the ultimate state of parenchymatous nephritis.

1. Stage of chronic parenchymatous nephritis. The histological alterations vary considerably according to the degree of intensity of the inflammation, the abundance, localization, or diffusion of the epithelial alterations, and the inflammatory œedema. In certain cases we find the epithelium of the glomeruli tumefied and in the course of fatty degeneration or desquamated on the inside of the capsules ; the epithelium of the uriniferous canaliculi is tumefied, turbid, degenerated, necrosed, desquamated, and its canals are full of epithelial cylinders. In inflammatory œdema of the kidney, on the contrary, we observe a cellular infiltration especially marked in the neighborhood of the veins; the uriniferous canaliculi and Bowman's capsules are filled with white corpuscles of an inflammatory exudate (exudative or hyaline casts); we may also recognize red corpuscles in more or less large numbers in it. In one case we have found a gelatinous infiltration of the connective tissue surrounding the openings of the uriniferous canaliculi.

By macroscopic examination the kidney appears little or not at all altered in many cases; but frequently also alterations of structure impress certain modifications of form or coloration upon it. We 
designate under the names of "large white kidney, large fatty kidney, fatty inflammatory kidney," a moderate tumefaction of the organ with slightly marked fatty degeneration of the epithelium ; the cortical substance is then a pale-gray color, filled with stripes or white spots (adiposity), and of very soft consistence; in the calf we have found these points to have a lardaceous appearance. When the inflammatory œedema is prominent and masks the characters of fatty degeneration of the epithelium, we have what is called "large spotted kidney." Here the organ is much tumefied; upon section, the thickness of the cortical substance appears considerably augmented; it is softened, infiltrated, and of reddish-gray color. When the glomeruli are principally affected, and when the migration of the red globules is profuse, we say that there is chronic nephritis of the glomeruli or chronic hemorrhagic nephritis.

2. Stage of sclerous, interstitial, ANd indurative NEPHRITIS. It is characterized by hyperplasia, connective tissue induration, and atrophy of the true glandular parenchyma. The inflammatory process is marked at the outset by a cellular infiltration of the interstitial connective tissue, and later by hypertrophy of this tissue, the compression of the capillaries which are located in it, and their obliteration. The epithelial alterations are not very apparent; the glomeruli are isolated, either by capsular thickening or by tumefaction of the vascular walls, which frequently undergo hyaline degeneration. The atrophy of the glomeruli ultimately involves that of the uriniferous canaliculi.

At a more advanced period, after months or years, there are formed in the structure of the kidney cicatricial centres which contract the surface of the organ (contracted kidney, granular kidney, granular atrophy). ${ }^{1}$ The kidney becomes reduced more and more (atrophied and indurated kidney); the small projections

1 Three principal theories have been advanced on the pathogenesis of the small granular kidney. According to the first, the alterations of the renal epithelium constitute the earlier part of the process, and the interstitial connective tissue lesion is consecutive. According to the second (Traube), it is in the interstitial connective tissue that the sclerous process originates, this giving rise secondarily to the modifications of the glandular epithelium, and finally atrophy of the kidney. According to the third (Peter, Lancereaux, Gull and Sutton), the lesions of the kidney are but a localization of generalized arterio-sclerosis, an affection characterized by inflammatory alterations of the small vessels, often also by atheroma of the more voluminous vessels-alterations which are said to determine sclerosis and hemorrhages in the parenchymatous organs; in the kidney they would constitute the initial process of interstitial nephritis (Dieulafoy).-N. D. T. 
which are not indurated take a grayish coloration (fatty degeneration); the capsule can no longer be detached from the cortical substance, to which it is intimately united by fibrous bands. The surface of the kidney is sometimes lobulated. The renal tissue itself is hard and creaks under the knife; the cortical substance is greatly reduced in thickness; it is furrowed with fibrous bands surrounding atrophied glomeruli and uriniferous canaliculi, these bands being separated by small islands of renal tissue, which are nearly normal in character. The kidney may also be reduced to half its original size. In the ox we have constantly found the two kidneys affected in an almost identical manner.

Symptoms. In most cases chronic nephritis is developed very slowly and without giving rise to any very manifest symptoms; the animals are therefore only presented to the veterinarian at a very advanced period of the disease.

It is first indicated by the lessening of the appetite and a gradual weakening; but these phenomena, which are common to a number of morbid affections, are very insufficient to enable us to recognize a determinate organic affection. Later, odemas are formed upon the members, in the pectoral and lower abdominal regions, etc. This tendency to dropsy characterizes the parenchymatous form; it should decide the practitioner to make the examination of the urine, which alone is able to give precise information upon the nature of the disease: this liquid is emitted in a smaller quantity; its specific gravity is increased; it is very albuminous, contains casts, epithelial cells, fatty degenerations, and at times also red corpuscles. In the examination of the circulatory apparatus we find the artery distended, the pulse hard, the cardiac impulse violent, often perceptible on both sides, the heart bruits stronger, and sometimes the zone of cardiac dulness more extended; these are so many symptoms of hypertrophy of the heart. The rising of the rectal temperature is moderate or medium; the thirst is ordinarily very great. At this period, if the intensity of the disease increases rapidly, uremia steps in and death may occur in a few days.

When interstitial sclerotic nephritis is prominent the clinical picture is modified. We observe a considerable lowering of the specific gravity of the urine, which falls to 1025 , sometimes to 1015 (in the horse); in advanced atrophy the specific gravity falls to 1010, and even 1001 ; the urine, which is very watery, is emitted in a large quantity (polyuria), it contains but little albumin, casts, and epi- 
thelial cells. The odemas often diminish gradually, then disappear entirely. The modifications of the urine and the resorption of the transudates are results of the increased activity of the heart and elevation of arterial pressure.

As long as the cardiac activity compensates the renal alteration the affection remains stationary ; this condition mạy last for years, but sooner or later the period of fatigue appears, with atony of the heart. The pulse becomes more feeble and irregular: blood stagnations are produced; we observe the appearance of bronchial and gastro-intestinal catarrh, extended œedema, symptoms of heaves, cardiac palpitations, vertigo, pneumonia, pleurisy, pericarditis, and hemorrhages; the quantity of urine is reduced, its specific gravity increases, and it contains albumin. Death occurs through acute or chronic uremia.

Treatment. The treatment of chronic nephritis is nearly the same as that of acute nephritis; but the chances of success are even less than in the acute form. In chronic nephritis we should seek to influence the general nutrition, to combat anemia, and to relieve vascular pressure in cases of cardiac insufficiency. It is essential to administer cardiac tonics and also diuretics: digitalis, caffeine, strophanthus, etc.

[The mercurial preparations exert a very powerful influence upon connective tissue indurations. The iodide of potassium also exerts a similar influence, whilst arsenous acid diminishes arterial tension. These are all valuable agents in the treatment of this disease, either alone or in combination in the form of Donovan's solution. Spirits of turpentine in small and continued doses often gives good results. -W. L. z.]

If there is any œdema or dropsy pilocarpine may be used.

Several hypotheses have been advanced on the pathology of cardiac hypertrophy which appears during the course of nephritis. ${ }^{1}$

1 The pathology of cardiac hypertrophy occurring in the course of interstitial nephritis of the human race is variously interpreted by different authors. By some hypertrophy of the heart is considered tributary to the renal lesion; by others, the cardiac and renal lesions are regarded as independent but to be traced back to a common cause (Dieulafoy). Upon the first hypothesis the cardiac hypertrophy is explained either by an excess of arterial tension (Bright, Potain, Senator), or by this influence together with the vascular alterations of the kidney (Traube). The second hypothesis asserts that the cardiac and renal lesions are produced simultaneously by arterio-sclerosis. The sclerous thickening of the walls of a number of small vessels of the parenchyma would hinder the general circulation, elevate the arterial tension and produce hypertrophy of the heart, necessitating increased activity. - N. D. T. 
1. According to Traube, the blood-pressure is increased by the retention of a certain quantity of water in the vascular system when the urine is eliminated in small quantity, and by the circulatory troubles which give rise to the renal alterations; this elevation of the blood-pressure would lead to compensating cardiac hypertrophy. 2. To this mechanical theory Senator offers in opposition the chemical theory, according to which the increase of the vascular pressure would be due to the retention of urinary waste products in the blood, and mainly of urea. Notwithstanding the numerous discussions which have taken place upon this subject, these two hypotheses are still sub judice, and it is very hard to tell which expresses the truth.

\section{Purulent Nephritis.}

Etiology. The pathogenic agents of purulent nephritis may reach the kidney in two different ways : at times they are conveyed by the blood, at other times they enter it by ascending the ureter. 1. In the course of septicemia, of pyemia, endocarditis, pulmonary gangrene, pneumonia, grave pharyngitis, etc., the blood may produce, within the tissue of the kidney, infectious emboli originating from circumscribed inflammatory, purulent, or ichorous centres. (The non-infectious emboli, those originating from a verminous aneurism, for instance, do not produce any purulent phlegmasia, but an ordinary chronic nephritis with infarctus and cicatricial centres.) In the ox purulent nephritis most frequently takes the form of renal abscess. Infectious and septic nephritis are complications which are quite common in cases of dystocia. 2. The phlegmasia, at first limited to the bladder, may, by continuity of tissue, be propagated to the renal pelvis, and thence to the kidney (pyelonephritis).

Pathological anatomy. In purulent metastatic nephritis we find the renal parenchyma strewn with yellowish-gray spots, from the size of a pin-head to a pea, which represent embolic abscesses which are formed of white corpuscles, softened tissue, and micrococci, which are particularly abundant in the centre of the lesion. These abscesses, which seldom acquire large dimensions, are determined by microbic emboli, sometimes a local necrosis, and always have an intense inflammatory reaction in their neighborhood.

The pyelonephritic abscesses are developed at the expense of purulent tracts which are disposed parallel to the excreting canals. 
of the kidneys. Their dimensions vary from that of a hazel-nut to that of an apple; sometimes they become confluent, and may even transform the kidney into a purulent pouch (pyonephrosis); the renal tissue is completely destroyed, and the thickened capsule of the organ constitutes a kind of cystic membrane. Such purulent cysts have been found weighing thirty-five livres and more. The pus is sometimes gathered in layers which are more or less thick, and are located under the capsule of the kidney (Bruckmüller); it may work its way into the perirenal connective tissue (paranephritic abscess).

Symptoms. The symptoms of purulent nephritis not only have nothing characteristic, but they are always masked by the primary affection (pyemia, pyelitis, etc.). Very often we observe but general or rational symptoms, such as emaciation, derangement of the appetite, increase of thirst, weakness, dull hair, etc. In some cases, however, the urine presents characters which clear up the diagnosis; it is fetid, thick, gelatinous, mixed with pus and tube-casts; blood has also been found in it, as well as mortified renal tissue (Leisering); in the cow the tail is often soiled with pus or mucus (Buhler). Later we may observe hemorrhage, embolic pneumonia (Voigtländer), pyemia, etc.; but the course is slow as a rule, and the renal alterations are only recognized at the autopsy. In the ox, by palpating the kidney per rectum, we may sometimes recognize fluctuation, also purulent cyst of the organ.

Treatment. The treatment of purulent nephritis is essentially surgical. It consists of the puncture of the abscess through the flank or extirpation of the kidney by laparotomy. Munich has attempted this latter operation upon a cow. Notwithstanding that it has not been followed by success, it must nevertheless be recommended, for the ox is not very sensitive to the operative manipulations affecting the abdominal cavity, and the slaughter of the animal remains as a last resource should an accident happen which leaves little hope of a cure.

\section{AMYLOID DEGENERATION OF THE RIDNEY.}

Amyloid degeneration of the kidney existing as an essential affection, isolated, has been but rarely observed; generally this degeneration involves several organs at the same time. Rabe has, however, observed this condition in three dogs, and Gerlach (1865) in an ox. 
In the dog, the anatomical alterations of the kidney are: the waxy consistence, paleness, dryness, and anemia of the cortical parenchyma, increase in size and hyaline tumefaction of the glomeruli, and finally the presence, in the loops of the uriniferous canaliculi, of homogenous casts of dull appearance and but little refraction.

Subjected to the test of methyl-violet, the affected tissue becomes of an intense purple; treated with an iodated aqueous solution, it becomes mahogany red; this latter coloration is transformed into steel blue under the influence of sulphuric acid.

The symptoms observed by Rabe were subcutaneous œdema, especially marked upon the legs; also ascites, albuminuria and uremic accidents: vomitings, paralytic weakness, coma, lowering of the general temperature. Cardiac hypertropy has not been observed.

Concerning the treatment of the amyloid kidney, it is the same as that of chronic nephritis, with which it is always confounded during life.

Tumors of the kidney are rare. In the dog and cat, they may be recognized through abdominal palpation. We have found in - the kidney : carcinoma, sarcoma, melanoma, adenomyoma, sarcomyoma, and rhabdomyoma. In the majority of cases the diagnosis is not made except at the autopsy; during life we only observe hematuria, sensitiveness of the renal region, and troubles of the urinary secretion. The treatment would consist in extracting the diseased kidney.

\section{ADDENDA TO THE DISEASES OF THE KIDNEYS.}

\section{Hemoglobinemia of the Horse.}

CONGESTION OF THE KIDNEYS, AZOTURIA, BLACK STRANGURY, HEMOGLOBINURIA, CONGESTION OF THE SPINAL CORD.

Hemoglobinemia, as indicated by the expression itself, is not a disease of the kidney. Under the influence of its various causes, a secondary nephritis may accompany it, but this fact does not bring it closer to the renal affections. Strictly, it ought to be described in the article on Diseases of the Muscles. Our reason for studying it here is because the practitioner usually seeks "black strangury" among the "diseases of the kidney," and because one of its important symptoms-hemoglobinuria - is related to this group ; moreover, we have decided not to separate this affection of the horse 
from "hematuria" of the ox, in which we may recognize as a cause hemoglobenemia as well as nephritis.

History. Hemoglobinemia in the horse is one of the oldest diseases mentioned; it has been designated under the names: black strangury, spinal typhus, lumbar typhus, renal typhus, spinorenal congestion, epizootic paraplegia, nervous syncope, acute Bright's disease, brachial and lumbar neuropathia, lumbago (Kreüzverschlag, Windrehe), azoturia, etc. In the first third of this century, after the appearance of veterinary journals, hemoglobinemia was still described under different names. In those places where apparently it did not exist it was doubtless confounded with other affections. In Austria and Denmark this confusion has continued till the latest period. It was first studied in Bavaria by Hofer, Adam, Frick, Kolb, Franzissi, Gierer, Ableitner, etc.; then in Würtemberg, northern Germany, France, and Holland.

The most diverse opinions have been given on the nature of this disease. It has been looked upon as the expression of one of the following pathological processes :

1. Nephritis, from which comes the name acute Bright's disease (Hofer, Hering, Bruckmüller, Pflug, Zündel).

2. Myelitis or congestion of the spinal cord (Haubner, Friedberger, Csokor, Trasbot).

3. Decomposition of the blood, produced by septic and infectious matters coming from tainted food (Kolb, Spinola, Vogel, Bollinger, and others). Bollinger thinks that hemoglobinemia of the horse consists of an auto-intoxication in which a noxious agent (ferment, waste products) is formed under the influence of exercise or cold; this toxic agent would act upon the globules, destroy them, and thus produce hemoglobinemia.

4. Dissolution of the red corpuscles by the products of increased retrogressive metamorphoses in the muscles, especially by the urea and the extractive matters which accompany it (Siedamgrotzky and Hofmeister).

5. Rheumatismal affection of the muscles of the croup and loins, with inflammation, œdema, degeneration, etc. (Weinmann, Lechleuthner, veterinarians of northern Germany, and others).

Etiology and pathogenesis. We ought to consider black strangury of the horse as an indirect myositis produced by cold-an affection in which the coloring matter of the muscles, identical with hemoglobin, is dissolved and passes into the blood. The expres- 
sion "rheumatismal hemoglobinemia" may be applied perfectly well to almost all cases that we meet in practice. We are not going so far as to deny the existence of hemoglobinemia either "infectious" or "toxic" in the horse, but it is indisputable that examples of it are most rare and are least important.

The principal cause of hemoglobinemia of the horse is the action of cold. This etiological factor is admitted by the great majority of observers. According to Göring, the disease may be experimentally produced by cold. An abnormal sensitiveness to the action of cold is acquired by a rest of several days in a hot, badly ventilated, and damp stable, and the animals are specially predisposed to the disease if during the time of inactivity they have been given full rations. Hemoglobinemia appears especially after holidays (Monday disease, Easter disease, Whitsuntide disease). The predisposition is acquired also by forced rest from lameness. The high temperature of the stables acts by rendering the organism less resistant to cold. Observation teaches us that it most frequently affects the best stabled horses, when the stables are warm, rather than those located in defective stables belonging to poor owners. In most cases, indeed, the appearance of the disease may be explained by the hygienic conditions of the stables. Heavy draught horses are particularly exposed to it

Hemoglobinemia is of myogenic or non-hematogenic origin. The action of cold in the freeing of hemoglobin will be explained briefly. Physiology teaches that nutritive metamorphoses increase in the muscles when the sensitive nerves of the skin are irritated. Every time that intense impressions are felt upon the integument the organic changes become active in the muscular system; a decomposition of organic albumin may result from it-that is to say, of the muscular substance itself. Consecutively to these degenerative alterations of the muscles, the products of transformation, mainly the coloring matter of the muscular fibre, which is identical with hemoglobin, pass into the blood. The effects of the cutaneous irritation are especially intense after a long stay in a hot atmosphere. The hind quarters being most exposed and the least protected, and the croup and lumbar muscles being in more active function than any of the others, we can understand that these effects are felt much more upon the hind quarters than upon any other region.

There are cases where the influence of cold seems to be wanting; they are generally considered as facts establishing the existence of 
an "infectious hemoglobinemia;" but this view does not rest on any sound basis, it is purely hypothetic (development of ferments, stable miasms, etc.). Besides, the horse may be affected by a cold in the stable.

We have based our theory upon the following facts: 1 . The action of the cold is looked upon as the main cause of the disease by almost all authors. Of course, we do not refer to a cold determining a dissolution of red corpuscles such as that which is produced when the blood is subjected to experimental coagulation. 2 . It results from Lassar and Nassaroff's experiments ${ }^{1}$ upon animals, that abrupt colds determine parenchymatous degeneration of the muscles and their discoloration. 3. We observe in man a disease $\grave{a}$ frigore which is well known as "paroxysmal or winter hemoglobinuria," which, according to Rosenbach, may be produced experimentally by a cold foot-bath, the symptoms of which are quite similar to those of hemoglobinemia in the horse. 4. Schindelka has shown quite recently, by the hemometric method, that the blood of subjects affected by rheumatismal hemoglobinemia is richer in hemoglobin than that of the healthy horse. In order to explain this singular statement, he admits that a coloring principle is added to the blood which is identical with hemoglobin and coming from elsewhere; and this principle can only be the coloring matter of the muscles. 5. Concerning the other theories advanced upon the nature of black strangury, there are none which can stand the test of discussion: a. Nephritis constitutes but a symptom, and one which is often wanting, even at the autopsy. $b$. Myelitis has never been found at the autopsy, and the observed symptoms do not belong to this affection. $\quad c$. The theory of infection and decomposition of the blood, outside of a few isolated and obscure cases, has no positive foundation; the disease appears in animals fed with the very best food; its symptoms, especially the absence of hyperthermia, indicate that we have not to do with an infectious morbid state; the examination of the blood and its inoculation have constantly given negative results; finally, true contagion has never been established. $d$. The increase of urea and extractive matters of the blood are observed in uremia without determining hemoglobinemia, and in several cases we have found a decrease of urea in the urine; Schindelka has made the same remark upon three horses.

The opinion admitted formerly that the disease does not affect

I Lassar and Nassaroff: Virchow's Archiv, Bd. xxic. u. xxc. 
horses fed on roots hardly deserves any refutation; we refer to this because of its pretended geographical localization. At the present time it is known in all the countries of Europe.

The obscure hypothesis formulated by Dieckerhoff reminds us of certain old methods; it is not based upon any scientific fact. According to this author, in horses that are well fed and left standing in the stable for several days, the nutritive matters would accumulate in the blood and the albuminoid substances would become decomposed there and generate a principle possessing specific toxic properties.

Like ourselves, Winkler recognizes two distinct forms of black strangury: a "rheumatismal" and a "toxemic" form; but we doubt whether the differential diagnosis of these two morbid states is so easy as is indicated by this author.

Pathological anatomy. The principal alterations are found in the muscles and in the blood.

1. The muscles of the croup are particularly affected; they are pale, tumefied, cedematous. The microscope shows a granulous alteration, an irregular segmentation, of hyaline aspect, and the loss of the transverse striation-degenerative lesions due to the inflammation of the muscular fibres. Between these we sometimes find small hemorrhagic centres. These alterations, which are more or less accentuated according to the case, often exist in a great number of muscles; concerning their frequency, Siedamgrotzky classifies them in the following order: (1) psoas, long adductor, and posterior part of the other adductors of the thigh, ilio-spinal, pectoral ; (2) anterior straight of the thigh (crural triceps); (3) abductors and anterior portion of the adductors of the thigh, great dorsal.

2. The blood appears much altered, its color is darker; it is noncoagulated, is tar-like, with a varnish gloss. Taken from the living animal, it gives, after coagulation, a reddish serum (due to dissolved hemoglobin; this alteration is not lasting, which depends perhaps on the elimination of hemoglobin. According to the researches of Siedamgrotzky and Hofmeister, the blood is very rich in urea and in extractive matters-that is to say, in the waste products of the combustion due to muscular activity. Lastly, on microscopic examination we find here and there a considerable quantity of hematoidin crystals. We may observe hemorrhages of various organs (spleen, kidneys, etc.).

Besides these constant essential alterations, others exist which 
concern certain internal organs. When hemoglobin of muscular origin reaches the blood, the organism destroys or eliminates it through the emunctories in which it is deposited. The spleen, which has gathered the scoriæ of the red corpuscles, is obstructed ("spodogenic" tumefaction, Ponfick); the liver is also more or less tumefied; the red marrow of the bones is infiltrated and colored black. These lesions of the marrow, observed in long bones (femur, humerus, radius, tibia), must not be considered, contrary to Dieckerhoff's opinion, as signs of osteomyelitis; this author ascribes these to "the irritating influence of the hemoglobinic serum," but the injection of a considerable quantity of serum (obtained from eight litres of blood) did not give rise to any trouble in a horse used for experimental purposes (Dieckerhoff). To ascribe the conditions observed during the course of the disease to osteomyelitis is a fanciful hypothesis, for the alterations in the bone-marrow are often entirely wanting. According to Ponfick, hemoglobinuria is set up only when the quantity of free hemoglobin circulating in the blood reaches more than one-sixtieth of the hemoglobin mass of the body. When this condition is realized, hemoglobin infarctus is formed in the kidney, which is soon accompanied by an acute parenchymatous nephritis.

Symptoms. They consist of troubles of locomotion which appear within a quarter to half an hour, during exercise generally, as stated above, in horses that have been resting for several days in a place where ventilation has been neglected, having at the same time received working rations. In benign cases, one might think that the horse was suddenly affected by rheumatism; the hind quarters are stiff and distended, there is sometimes lameness in an anterior or posterior nember; in addition, perspiration is also observed upon certain regions. In the graver cases, the animals stagger behind and get along with difficulty, the walk being uncertain and painful; the angles of the joints remain open, the members seem contracted, and the toes are dragged upon the ground; at times we observe spasms like flexion of the fetlock; the animals tremble, perspire, stand with difficulty upon their posterior members, and even sink without being able to raise themselves again without help; excitement and anxiety are very marked, there is dyspnoea, and the body is covered with perspiration. The animals make useless efforts to return to the standing position and have to be brought back to the stable by means of a vehicle. 
On palpation of the paralyzed hind quarters we find the muscles of the croup hard, distended, and sometimes painful; the skin is warmer and has lost its softness (œdematous infiltration of the subcutaneous connective tissue and derma). The sensitiveness to exploration by touch, pin-punctures, electric currents, is diminished or abolished.

Paralysis exists at times in other parts of the body : in the front quarters, upon a posterior member, upon the muscles of the shoulder, upon the superior muscles of the neck and shoulders, etc.; but recorded facts of this kind are quite rare. Upon ten horses observed by $\mathrm{H}$. Bouley the left posterior leg was always paralyzed first. Lippold has seen an extreme extension of the posterior members; the ergot was touching the ground, the sole of the foot was much held up, and the shoes visible in front when supported. In some cases the tail is high and stiff. We have several times observed upon the muscular walls of the abdomen contractions which produced cries of pain.

The second important symptom is hemoglobinuria. Formerly it was looked upon as constant and pathognomonic; but numerous observations establish that urine does not contain hemoglobin in benign cases, nor in those where the disease is abortive; this shows that the old denomination "hemoglobinuria" is improper. Urine which contains hemoglobin or methemoglobin is of a dark-red color, ruby red, dirty brown, or inky black, shades which are due to hemoglobin; later, if there is a nephritic complication, the urine becomes albuminous, it contains cylinders, leucocytes, a few red corpuscles, and sometimes abundant débris of desquamated renal epithelium ; it is often rich in urea, and in extractive matters which are similar to this in composition, but poor in hippuric acid. As for its reaction, contrary to what has been advanced by some authors, it remains alkaline in benign cases and even in the grave form; in twenty-five cases which were very carefully observed, five of which were fatal, and which all deserved the name of severe, we have found the urine acid but once ( 4 per cent.); this reaction was due to a large proportion of phosphates. Its specific gravity does not generally undergo any modification. Let us add that the characters of the urine are far from possessing positive diagnostic value in all cases.

The temperature rarely increases, even in the grave form. It is irregularly distributed and the extremities are cold. In twenty- 
five patients we have found it normal in twenty cases ( 80 per cent.); in four cases it rose above $39^{\circ}$; in one case it was $40^{\circ}$. Engel has also observed the absence of fever in four cases. The pulse is generally accelerated; in the twenty-five cases already quoted four times (16 per cent.) the pulse was found normal ; in five cases (20 per cent.) we have counted fifty to sixty pulsations, and in sixteen others (64 per cent.), a still higher figure. The number of pulsations is quite often from seventy to eighty and over; the pulse is hard and rapid. The visible mucous membranes are red and dull (a sign of blood dissolution). When the animals remain down for a certain time, the blood is soon much altered.

Regarding the digestive apparatus we notice, as a rule, a diminution or complete suppression of peristaltic movements, but it is rare to observe other alarming symptoms; the appetite is generally preserved. The stopping of the peristaltic contractions explains the retention of the urine in the bladder; this is much distended; it can be emptied by rectal pressure. The respiratory troubles which are observed at times during the course of the disease have been described before.

The psychological state remains normal, except in cases of nephritic complication. We have once observed uremic complications which were marked by a depression of the general sensitiveness and by epileptiform cramps. The cerebral troubles and the symptoms of immobility which have been seen are perhaps explained in this way. All these phenomena are but secondary and accessory manifestations of the trouble.

Course and duration. To speak generally, hemoglobinemia has an acute or subacute evolution. When ending in a cure this may take place within a few hours (benign or abortive cases) or in a few days, generally on the third or fourth day ; often it is complete, and the various functions come back entirely to their former condition, the paralytic symptoms and red coloration of the urine disappear, the animal gets up and recovers entire freedom of movement. Recurrent cases are not rare; the same horse may be affected several times in the same year, even on the same days for several years. The affection would seem to create a predisposition to rather than immunity from the disease. (Paroxysmal hemoglobinemia of man behaves in the same manner, and has been called periodic intermittent hemoglobinemia.)

When serious hemoglobinemia ends in a cure, a paralytic condi- 
tion of the extremities often persists. At times it is a bilateral paresis of the hind quarters, which lasts for weeks, months, or years (a slowness of the posterior members in walking, a staggering, uncertain gait), at times the paralysis is unilateral, localized in certain muscular groups in which progressive atrophy occurs. After hemoglobinemia we have seen a case of chronic spinal meningitis, with paresis of the hind quarters, bladder, and intestine; at the autopsy we found an inflammation of the meninges of the posterior portion of the cord.

When the disease is going to end fatally the paretic phenomena become more and more accentuated ; there is soon a complete paralysis; the restlessness of the animal increases, the respiration is dyspnœic; scabs appear, produced by the decubitus; the senses become dull, and death occurs by carbonic intoxication (pulmonary hypostasis), cardiac or uremic syncope.

Prognosis. The prognosis varies according to the degree of intensity of the morbid forces, but on account of the frequency of the exacerbations it must be formulated with reserve.

If the animals are no longer able to rise, and especially when it is impossible to keep them standing by means of a suspensory apparatus (complete paralysis) there is little hope of recovery.

The mortality varies according to the race, the climate, the individual disposition, the degree of intensity of the affection, etc.; sometimes almost all the patients get well, at other times there is a long uninterrupted course of reverses. Thus the statistics established by various authors are far from agreeing. Bay has a proportion of 70 per cent. of unsuccessful cases (368 were observed); Stockfleth says 50 per cent. ; H. Bouley, 60 per cent.; Grimm, 40 per cent. In the Stuttgart clinic we have found it to be 40 per cent., and at Munich it is only 20 per cent. In general the mortality is from 20 to 40 per eent., and in cases of confirmed paralysis, from 50 to 70 per cent.

Differential diagnosis. Hemoglobinemia may be confounded with a whole series of diseases, the most important of which are:

1. Colics, especially the rheumatic forms produced by colds. They are marked by intense pains, the suppression of peristaltic movements and frequent decubitus. The history-mainly the lengthy stay in the stable-will direct the practitioner; the examination of the croupal muscles, the composition of the urine, the inability of the animals to rise, etc., permit us to make the differ- 
ential diagnosis between hemoglobinemia and rheumatic colies. (See Differential Diagnosis of Colics.)

2. Muscular rheumatism. In hemoglobinemia the affected muscles are the seat of serious alterations, and their coloring matter passes into the blood-phenomena which are always wanting in rheumatism. When the characters of hemoglobinemia are not clearly marked, if several animals of the same state are affected simultaneously, we may still make the diagnosis of "black strangury" if determined by a rheumatic affection of the hind quarters. The history, the hemoglobinuria, also sudden appearance of paresis or paralysis, are sufficient to establish the diagnosis in most cases. In addition to this, extended muscular rheumatism, without hemoglobinemia, is extremely rare in the horse.

3. Thrombosis of the posterior aorta and of its terminal branches (iliac arteries, arteries of the pelvis, crural arteries). This condition sometimes occasions manifestations similar to those of "black strangury," and the differential diagnosis may be difficult at the outset; a rectal exploration, however, informs us at once as to the nature of the trouble. Moreover, in obliteration of the aorta, the symptoms are very rapidly dissipated to make place for a complete recovery, and they reappear quite as abruptly without any apparent cause; lastly, they may be produced artificially by forced movements.

4. Spinal apoplexy. Spinal paralysis and paresis of the hind quarters which are of an inflammatory or traumatic nature may be confounded with hemoglobinemia. The traumatic origin and slow development of spinal meningitis, the possible existence of primary morbid centres for the emboli in cases of apoplexy, are very important points helping to establish the diagnosis. The composition of the urine almost always gives very precise information.

5. Tetanus. This may be confounded with hemoglobinemia on account of the hardness and tension of the muscles of the croup; but comparison of the symptoms of both diseases renders the distinction easy, even in cases where hemoglobinemia is accompanied by trismus.

Nephritis, bacterial anthrax, and petechial fever. Careful examination of the animals permits us also to establish a difference between hemoglobinemia and these affections.

Treatment. Prophylactic means have especially to be applied. When the horse is left resting for twenty-four or forty-eight hours 
or longer, the food ration has to be diminished and the animal must be given a little exercise in the open air every day; the organism will thus remain accustomed to the outside temperature. The ventilation of the stable should be arranged in such a way as to avoid draughts, and it must be seen to that the temperature remains moderate. In observing these indications, one can almost surely counteract hemoglobinemia.

When the disease has set in, we must above all things give the animal rest, by placing it in a quadrupedal position, supporting it if necessary in a suspensory apparatus ; permanent decubitus is acccompanied by pulmonary hypostasis, and rapidly leads to death. But the suspended animal ought to be watched; if its limbs are inert, it may be asphyxiated in a few seconds. Every time the dissolution of the blood is such as to determine a severe dyspnœa and a considerable acceleration of the heart's action, it is of advantage, according to our observations, to bleed copiously; when the blood is very thick, and runs off with difficulty, bilateral bleeding is indicated. It often happens that paresis of the bladder produces retention of urine; we must then try to empty it by pressure of the hand flattened in the rectum, also by catheter.

With the object of provoking the elimination of hemoglobin through the kidneys, we must make the patient ingest as much water as possible and excite the cutaneous function by dry frictions, or by stimulating frictions of camphorated alcohol, essence of turpentine, etc. The suppressed peristaltic movements should be induced by evacuants, laxatives, and even by drastics. Sulphate of physostigmine is recommended on account of its easy administration and the promptness and safety of its effects; it is injected (dissolved in water or in a sublimate solution of $1: 1000$ ), in a dose of 0.08 to 0.12 gramme, under the skin of neck or shoulders.

The dietetic treatment consists in giving farinaceous drinks, clear slops, carrots, green fodder, or good hay. We must avoid heating food, especially oats. Convalescing animals must be gradually accustomed to service.

Utz has described a subacute form of hemoglobinemia. The symptoms, instead of being apoplectiform, would evolve slowly, so that the animals would only appear sick after the third or fourth day. This author also finds a tumefaction of the masseters accompanied by a difficulty of mastication (trismus). In one case Friedberger has also observed a very pronounced trismus. 
Infectious hemoglobinemia described by a few authors is expressed by symptoms which are similar to those of mycotic enteritis or certain poisonings. It seems to us to be related to these latter affections.

\section{Hemoglobinemia of the Ox and Other Ruminants.}

\section{HEMATURIA, PASSING OF RED URINE, PASSING OF BLOOD.}

The disease of the ox described under the name of hematuria, red water, red urine, red passage, May disease, pasture disease, red disease, etc., is one of the most obscure of veterinary pathology. The indications given by authors upon its causes and manifestations differ considerably and are even contradictory. We have done our best to make a clinical and anatomical study of it, and to make it as exact as possible. We must first separate from the collective expression "hematuria," enzootic gastro-enteritis and its complication, the hemorrhagic nephritis accompanied by hematuria. Of this we have already spoken in the article upon Poisonings. We have decided to use the term "hemoglobinemia," after a thorough examination of the bibliography of the subject, combined with our personal observations; we have found positive proof that the passing of blood with urine is not hematuria, but hemoglobinuria. Hemoglobinuria of the ox must be related to the hemoglobinemia of the horse; numerous other facts confirm the similarity existing between them. We consider this morbid state to be complex, and to consist essentially either in a decomposition of the blood produced by toxic and infectious agents (toxic and infectious hemoglobinemia), or in a myogenic (that is to say, rheumatismal) hemoglobinemia.

Affected animals. Hemoglobinuria is most frequently observed in an epizootic found in oxen which live in freedom in pastures; more rarely in a sporadic state in animals which are kept in the stable. The sheep and goat are also affected. This trouble, which was known by the Greeks and Romans, has been found in all the countries in Europe; but with the progress of agriculture it has become much less frequent, and has a tendency to disappear. Nowadays it is only found in the region of the Alps and in other mountainous countries, but also in some of the plains of northern Europe. Like the "licking-disease," it exists permanently in some localities ; some "red-water farms" are known; they are generally located in valleys which are surrounded by alder (elecampane) forests. 
It is particularly common in females, milch cows, and young subjects; the males are but rarely affected by it; native cattle are much more resistant than the imported animals; an individual predisposition is indisputable: it is not rare to see some cows affected twice during the same year-in the spring and fall; others are troubled several times during the same season. A disease which is mainly apparent in the springtime, it may make serious inroads on flocks which have been kept in close quarters and poorly fed during the winter, when such flocks are led to pasture while the weather is still cold and the vegetation hardly started. It is enzootic in cold pastures which are swept by the wind, exposed to the north, poor, swampy, and turfy; ordinarily it shows itself toward the end of the first, or in the beginning of the second, week of pasturing; when started too early, it is quite common to see the disease appear almost immediately, and to remain active till the middle of the summer. According to Gerlach, it may also be seen eight or ten days after the abandonment of the pasture regimen. It must be mentioned that not only is it seen in the spring, but sometimes in full summer after a cold rainy spell. It is said to exist in the Alps only in those localities where the soil is schistous in character; where the soil is calcareous the farms are said to be free from the disease (Lechner).

Etiology. The majority of authors look upon passing of blood in the ox as the consequence of a decomposition of the blood (Stockfleth, Gerlach, Spinola, May, Cauvet, Wieners, Pichon, Friedberger, etc.). Quite recently it has been attributed by Lechner to a toxic affection of the kidney. We have separated renal hematuria from hemoglobinuria, and agree with the first-mentioned view. The alteration of the blood in this disease seems to be hemoglobinemia ; the presence of red globules in the urine has not been established by any authentic observation. Notwithstanding that we do not know the real causes of this hemoglobinemia, however, the results to be obtained from the examination of the reported observations on this subject indicate that we have two principal etiological factors: either toxic or infectious products ingested with the food effect decomposition of the red blood-corpuscles, or the phenomena are brought about by the action of cold, which undoubtedly acts here as we have indicated in dealing with the pathogenesis of hemoglobinemia of the horse.

1. The etiological influences of a toxic or infectious kind are said 
to be due to a peculiar or defective composition of the soil, and to a defective constitution of the grazing animals, according to the statements of Stockfleth, Gerlach, Spinola, and others. Spinola considers it due to an excess of vegetable acids, and Stockfleth to a poison similar to sulphuretted hydrogen. Both of these opinions are hypothetical, but have this fact in their favor, that the disease is often closely dependent upon certain pastures to which the animals are driven; besides, it has been observed that the grass or hay coming from these pastures and fed in the stable causes red urine. These alimentary substances first produce a diarrhea, then a hemorrhagic gastro-enteritis, and later, after the introduction of their active principles into the blood, its decomposition is the result.

Quite a number of authors, and among them Stockfleth, have witnessed the manifestation of this disease in the ox after the ingestion of spoiled food (turnips and their leaves, malt, mouldy fodder); the facts which they have observed tend to establish the mycotic origin of hemoglobinemia. Lastly, the frequency of the disease in swampy and turfy regions, which has been recognized by a large number of practitioners, warrant us in admitting that it is produced by a malarial agent, undoubtedly by a microbe. ${ }^{1}$

Those poisonous plants called "bloody urine plants," produce

1 Babes has established the parasitic nature and the transmissibility of hemoglobin which affects the bovine race in Roumania. It is especially observed in the low and swampy districts. It occurs every year and is propagated in a more or less extended radius, where it often does considerable harm. It produces a yearly mortality of 30,000 to 35,000 animals. Its main symptoms are: prostration, suppression of the appetite, difficulty of gait, an intense fever, the red coloration of urine (which generally contains albumin and hemoglobin), constipation and diarrhea with tenesmus. Toward the end of the disease, the animals remain down continually, the fever becomes intense, and the urine dark-red or blackish; we observe, too, watering of the eyes, muscular tremblings, and subcutaneous odema. At the autopsy of the animals that have died of hemoglobinemia, the author has found in the blood and tissues certain rounded, shiny micro-organisms, forming frequently diplococci ; in the blood they are free or attached to the red corpuscles, or even contained in their interior. By inoculating rabbits, in the subcutaneous connective tissue or in a vein, with blood of an animal that has died from hemoglobinuria, or with cultures of the microbe of this disease, we produce in these animals a special morbid state, the manifestations of which appear ordinarily from the eighth to the eleventh day, and which becomes fatal within one or two days. At the autopsy of these rabbits we recognize in the blood, and especially in that of the kidneys, the microbes of the hemoglobinuria of the ox. The inoculations when made on animals of the bovine race give variable results according to the dose of blood or injected cultures. Small injections cause only a febrile state without hemoglobinuria; but in using larger doses we may produce hemoglobinuria with all its usual symptomatic complications, and sometimes death.-N. D. T. 
hematuria rather than hemoglobinuria ; among these we must especially mention : the coniferous buds, crowfoot, rush, water pepper, mercurialis, euphorbia, hemlock, etc. Several observations establish very clearly the pathogenic influence of the alder (elecampane) (Alnus alpina, A. viridis, A. incana, A. alnobetula). It is doubtful whether sedges, bulrushes, flowering rushes, and the horse-tails grasses are able to produce hematuria, as stated by Spinola.

2. The theory of the rheumatismal nature (rheumatismal or myogenic hemoglobinemia) of this disease has many adherents. Among the facts invoked in its favor, we must first mention the frequency of the disease in the spring, when the bovines that have been kept in stables are led to cold and damp pastures, and its infrequency when pasturing of the animals is delayed. At the stable, the passing of blood may be produced by the ingestion of icy fountain water (Ryschner). It appears sometimes when the animals drink at very cold mountain springs (Zündel). In Gallicia, says Röll, it exists mainly in the cold and mountainous regious. Dotter declares himself clearly in support of the rheumatismal nature of the disease. Utz, ${ }^{1}$ who has observed it in the stable, attributes it especially to chilliness during the cold spells. According to Hink, "rouge de pâturage," which is very common in the Black Forest, is a hemoglobinuria similar to black strangury of the horse. Frequent upon young subjects, at the time they are led to pasture for the first time, it would be produced by cold, by rapid ingestion of cold or damp fodder, and also by violent and unaccustomed exertions. In an establishment "for the cure of disease by milk diet," Saur ${ }^{2}$ saw a milch cow which was well fed, overcome with general rheumatism and blood emission after a simple cold. All these facts and the analogy of certain clinical symptoms observed in hemoglobinuria of the ox with that of the horse (stiffness of the hind quarters, sensitiveness to pressure of the lumbar region), indicate, in our opinion, a very close relationship between these two diseases.

Symptoms. Most modern authors have confounded the symptoms of renal hematuria with those of true hemoglobinuria. We have been obliged to guide ourselves according to the descriptions of older authors (Spinola, Stockfleth, Gerlach), which are quite different from those found in some of the more modern publications.

According to these pathologists, the symptoms consist principally

1 Utz : Bad. Mittheil., 1884.

2 Saur (communicated). 
in stiffness of the hind quarters, sensitiveness of the lumbar region to pressure by the hand, and the excretion of a more or less redcolored urine, but which does not contain any red globules. The disease commences as a rule with diarrhea, which is sometimes bloody; the appetite is often preserved or hardly altercd; we observe a general weakness and a slight increase of the temperature. After six to twelve hours the urine becomes of a pale-red color, which turns more and more dark, till it resembles tar. It is rich in albumin and hemoglobin; micturition renders it foamy and the ebullition transforms it into a brownish, gelatinous or doughy mass. It frequently deposits a turbid sediment, which sometimes, in the sheep, colors the wool red in the neighborhood of the excreting orifice of the urine (urine spots). According to Reuter, the reaction of the urine is acid for the first three or four days; later it becomes alkaline (ammoniacal odor of the urine). It is singular that the disease can be evolved without a red coloration of the urine (Stockfleth); but this fact makes complete the similarity of the disease with hemoglobinemia of the horse. The quantity of urine, likewise modified, is increased from the start ; micturition is frequent (pollakiuria) and painful (strangury); toward the third day the diarrhea gives place to a persistent constipation which is often accompanied by meteorism. The temperature rises to $41.8^{\circ}$ and even higher (Reuter).

To these symptoms motor troubles of the hind quarters are soon added: staggering and heavy gait, pains on lying down and rising, an arched back, very marked sensitiveness of the lumbar and croupionic regions; we may observe convulsions, also spasms of the posterior members, and at the time of micturition vermiform movements of the perineum throughout the length of the urethra. Weakness of the posterior extremities gradually increases, and soon it is impossible for the patients to rise. At times, when the affection has reached its last stage, odematous tumefactions are observed upon the head, neck, chest, etc. (Reuter); the mucous membranes are injected or colored with a yellow red; this icterus may become generalized (Gerlach). Death is announced by the increasing acceleration and weakness of the pulse, by the depression of the subjects, and lowering of the temperature; it takes place from exhaustion often at a time when the urine has recovered its normal color. According to Stockfleth it is sometimes caused by a secondary pneumonia. 
Course. The disease takes both the acute and subacute forms ; in this latter (apoplectic form), the animals may perish in a few hours (Reuter). When the causes exert their influence for a short time only the disease is generally benign. In most cases it ends in recovery, but generally it is very capricious. Its average duration is from ten to fourteen days. Upon 79 patients observed by Stockfleth, 23 died ; in 330 cases, Krabbe lost 38, that is to say, 8 per cent. A first attack does not confer immunity. Reuter has seen the same cows affected three times during the one season. According to this author in certain communities where the disease affects 30 to 40 per cent. of the bovines it has been found necessary to restrict the raising of these animals as much as possible. ${ }^{1}$

Pathological anatomy. The anatomical alterations are often insignificant. Most cadavers are anemic; the blood is very liquid, of a red cherry or inky black color; according to Gerlach, the eliminated serum, after the coagulation of the blood, is colored red and is rich in hemoglobin. Icterus is sometimes found generalized. The organic lesions are wanting, except those of acute intestinal catarrh and hemorrhagic enteritis, which exist almost constantly; the kidneys are ordinarily normal; the volume of the spleen is sometimes considerably increased. According to Spinola, the muscles are pale and flaccid; the same statement has also been made by Hink. Reuter has also found the muscles pale, as if cooked; at certain places, especially upon the neck, shoulder, and forelegs there were sero-bloody or gelatinous infiltrations. There is a striking resemblance between these alterations and those of rheumatismal hemoglobinemia of the horse.

Differential diagnosis. Hemoglobinuria of the ox may be confounded either with enzootic enteritis or with several affections of the kidneys and bladder accompanied with hematuria. In order to establish the diagnosis it is sufficient to make an examination of

1 According to Eloire, hematuria observed in cows fattening in the pastures of Thierache is expressed by the following symptoms :

At first, hindrance of the movements, stiffness of the loins, prolonged decubitus, difficulty in rising, frequent and marked micturition, foamy and slightly rusty urine. During this first period the appetite is preserved, the hair glossy, and the patients still gain in flesh. Gradually the symptoms grow more apparent; the urine becomes bloody, often there is an expulsion of intense red blood, similar to arterial blood; the loins are much arched and the limbs gathered together; there are often colics with prolonged decubitus; the appetite is capricious, the hair becomes bristly, the skin dirty; the mucous membranes become pale, the conjunctiva finally becoming absolutely bloodless. Death generally occurs from exhaustion.-N. D. T. 
the urine. Anthrax may also be complicated by hematuria and hemoglobinuria, but the general course of the disease, with the examination of the blood, etc., enables the practitioner to express himself with certainty. In morbid conditions of the liver and in hematogenic icterus, whilst yellow-brown coloration of the urine might lead us to suspect the presence of hemoglobin in this fluid, the demonstration of the coloring matters of the bile indicates these affections. $^{1}$

Treatment. Prophylaxis is of primary importance. We must not lead the animals to the pasture before the favorable season, and must avoid crowding in the stables. The beasts should get dry food daily before leaving the stable. Agricultural improvements, with the tendency to create native and more resisting races of animals, are means which may lessen considerably the frequency of hemoglobinuria.

As soon as the disease has made its appearance the herd ought to be kept in the stable. The trouble itself is very hard to reach; we are limited in the practice of symptomatic medicine. The constipation of the early stages may be combated by laxatives, and diarrhea by styptics (sulphate of iron, 15 to 25 grammes; alum; 15 to 25 grammes; tannin, 15 to 25 grammes; sugar of lead, 2 to 5 grammes; opium, 10 to 20 grammes, etc.); we may also try pulverized iron ( 2 to 5 grammes). Bleeding must be proscribed, especially when the disease has existed for some time and when anemia is much marked (Stockfleth, Gerlach). In serious cases we might try blood transfusion (Stockfleth).

Hemoglobinemia of other animals. Hemoglobinemia has been observed in small ruminants, in the mule (Arloing), in the dog after a cold (Saur), in the zebra (Johne), and in the pig. In a pig which was transported a distance of eight kilometres when the temperature was $25^{\circ}$ to $27^{\circ}$ below zero, the urine became albuminous and dark; the bacon showed a yellow tint after the slaughtering of the beast. ${ }^{2}$

1 Dealers who suspect the existence of hematuria in subjects exposed for sale examine for the disease in the following manner: With the back of the index finger they rub successively upward and downward that part of the perineum which touches the vulva. Under the influence of this kind of masturbation, which must act upon the clitoris, the animal stretches itself and passes water. By the color of the rejected liquid they judge at once whether or not the cow is affected by hematuria (Eloire).N. D. T.

2 Utz: Bad. Mittheil., 1884. 
Colds also seem to play an important rôle in the development of hemoglobinuria in the subjects of our other domestic species.

\section{Inflammation of the Pelvis of the Kidney: Pyelitis.}

Pyelitis presents much more interest for the pathological anatomist than for the clinician. It is almost always secondary, consecutive to other diseases of the urinary tract (nephritis, cystitis, etc.) ; moreover, intra-vitam diagnosis is only possible in the minority of cases. We shall thus be limited to a brief description of this disease.

Etiology. Inflammation of the pelvis occurs ordinarily by an extension of nephritis or cystitis to the mucous membrane of the kidney pelvis (pyelo-cystitis and pyelo-nephritis). It is quite frequently produced in the course of infectious diseases and poisonings, when the infections or toxic matters which are eliminated by the kidneys exert an inflammatory action upon this mucous membrane (glanders, tuberculosis, acid diuretics). Foreign bodies, renal calculi, or sand and sedimentary deposits (renal lithiasis) occasion at times a more or less intense inflammation of the mucous membrane of the renal pelvis (calculous pyelitis); according to Bruckmüller, the peculiar sediment which exists in the normal urine of some horses may act in the same way. ${ }^{1}$ The presence of the giant eustron. gylus in the renal pelvis (dog, horse, and ox), or stagnation of the urine due to a mechanical or dynamic retention in the pelvis, and its consecutive decomposition, are also causes of pyelitis. When urinary stagnation is prolonged, the kidneys become atrophied and its substance undergoes "cystic rarefaction" (hydronephrosis).

Pathological anatomy. According to the causes, degree, and duration of the inflammation, we may distinguish simple, acute, or chronic, purulent, croupous, diphtheritic, or calculous pyelitis. In simple acute pyelitis the mucous membrane is tumefied, red, sometimes ecchymosed, and covered with a mucous exudate which is rich in desquamated epithelial cells; in the suppurating form this exudate is almost exclusively composed of white corpuscles. Renal calculi produce circumscribed inflammatory centres upon the mucous membrane, which become necrotic and are transformed into ulcers covered

1 Urinary deposits are not rare among birds. They may be found in the cloaca, the ureters, and kidneys; in these two latter organs they appear in the shape of concretions or whitish striæ. (Dr. Larcher: Mélanges de Pathologie comparée, 1878.) -N. D. T. 
with a croupous exudate, or become incrusted with sediments which render the membrane very rigid ("incrustation catarrh.") According to Bruckmüller, chronic catarrh, quite frequent in the horse, is marked by a brownish coloration, pigmentation, thickening, and ulceration of the mucous membrane by the infiltration of the submucous connective tissue and the dilatation of the renal pelvis. Soon nephritis and cystitis complicate pyelitis; the purulent form especially is easily complicated by suppurating nephritis and pyonephrosis.

Symptoms. Pyelitis being almost always accompanied by nephritis and cystitis, the symptoms are necessarily very vague; it would be impossible to define the diagnosis with certainty except in cases where microscopic examiuation of the urine shows the cylindrical epithelium characteristic of the lower layers of the renal pelvis (dentate cells which resemble the eminences on the molar teeth.)

In the ox it is at times possible to recognize distention of the kidney pelvis through a rectal exploration. According to the nature and form of the pyelitis we find pus corpuscles in the urine; also bloody or fibrinous clots, necrotic mucous tissue, earthy sedimentary masses, and even eggs of strongyli (Adacco), when the renal pelvis contains the giant eustrongylus. The other symptoms vary with the nature of the primary affection; it is thus that the renal calculi produce colics and dysuria (renal colics). Prolonged retention of urine is always complicated by uremia.

Treatment. The treatment is identical with that of the afiection which has produced the pyelitis; alkalines may be used, also antiseptics and astringents: boric acid, salicylic acid, creolin, and the various substances containing gallic acid.

\section{Retention of the Urine.}

Retention of the urine, also called anuria, dysuria, urinous colic, etc., consists of the accumulation of urine in the bladder, the ureters, and the renal pelvis. It is accompanied by distention, then by inflammation of these organs, and may occasion mechanical atrophy of the renal substance, hydronephrosis, uremia, and peritonitis through rupture of the bladder. It does not constitute, so to speak, a true disease, but only a symptom of many affections. It is quite frequently observed in the ox, the dog, the horse, and the sheep; in all the different species it is much more common in entire or castrated males than in females. 
Etiology. The causes of retention of the urine are the following:

1. The prevention of micturition by foreign bodies in the bladder and urethra: ureteral and vesical calculi, sediments in the S-curved penis in the ox and ram, a fragment of broken catheter; bloody or fibrinous clots, diphtheric or croupous membranes detached from the vesical mucous membranes, polypous tumors producing ocelusion of the urethral canal, etc.; foreign bodies introduced intentionally into this duct: straws, broom sprig, etc.

2. Compression and obturation of the urethra or of the neck of the bladder by neoformations developed in the neighboring organs (prostate, uterus, ovaries, the bladder itself-leiomyoma, also by the accumulation of excrements in the rectum, displacements of the uterus, gestation; also by contractions of the urethra, by constrictions, inflammation, or tumefaction of the walls of the canal, by a fracture of the penis or of the penic bone, paraphimosis (strangling of the glans penis by a tumefied prepuce) or phimosis (abnormal narrowing or displacement of the opening of the prepuce).

3. Paresis or paralysis of the bladder in consequence of croupous, diphtheric, or phlegmonous cystitis, pericystitis, affections of the spinal cord (traumatisms, spinal meningitis, apoplexy, etc.); a general weakness; infrequent defecation (in horses suffering from a cerebral affection, or in those which have made a prolonged journey); a reflex paralysis of the muscular wall of the bladder, occurring either during colics or in the course of rheumatismal hemoglobinemia of the horse, or of certain general serious morbid states (vitulary fever, etc.).

4. Tetanic contraction of the vesical sphincter caused by the action of cold or by cystitis, by foreign bodies in the bladder; reflex spasms in colics, tetanus, etc.

The enumerated causes in the first two groups just established produce mechanical retention of the urine, and the others dynamic retention. This latter alone enters into the domain of internal medicine.

The urinary calculi, which are especially important from a surgical standpoint, have a triple origin : often they are formed by the precipitation of urinary salts around foreign bodies in this liquid (clots, spermatozoa, etc.); at other times they are due to the excess of such salts in the urine-a phenomenon due to the influence of a 
calcareous drinking-water, or food too rich in lime, salts, and magnesia (bran), or also in protein (formation of uric acid), etc. ; sometimes they are the result of a formation of insoluble ammoniacomagnesium phosphate (tribasic phosphate) at the time of the urinary stagnation, accompanied by a decomposition of the urine in the bladder (retention of urine, cystitis).

As to their chemical composition the urinary calculi of herbivorous animals are generally carbonates (more rarely oxalates and silicates); in carnivorous animals we observe principally the urates (sometimes the oxalates and cystin calculi); in the omnivora we find oxalates and carbonates. Lastly, in all animals we may recognize phosphatic calculi (ammoniaco-magnesium phosphate) when the urine is retained in the bladder and becomes decomposed there.

Symptoms. The retention of urine is shown either by the absolute suppression of micturition (ischuria, anuria), or by incomplete and painful micturitions, or again by the running off of the urine drop by drop (dysuria, oliguria, and strangury). In very sensitive animals (horse and $\operatorname{dog}$ ) the accumulation of urine in the bladder rapidly produces symptoms of pain and alarm, while in the "indolent ruminants," particularly the ox, days may pass before the appearance of fairly characteristic symptoms. The horse and $\operatorname{dog}$ present from the very first symptoms of colic (urinous colics): they stretch themselves frequently as if trying to effect micturition, the posterior members are spread, the penis is out of the sheath, etc. In the ox and the sheep these colics are almost always wanting and pass unobserved; but we notice peristaltic movements along the perineum; the litter is less damp than usual, and the hair surrounding the opening of the sheath is dry. Abdominal or rectal palpation enables us to recognize the fulness of the bladder, except when this organ is already ruptured; we may also be able to recognize the obstacle preventing the flow of the urine (calculus, neoformation, etc.): the minute examination of the urethral canal and the catheterizing of the bladder sometimes furnish very precise information. The animals are sad and apathetic, the appetite has disappeared, the pulse is accelerated; at certain moments there are profuse sweating spells.

When at this stage, if the obstacle to the flow of the urine is not removed, the symptoms are aggravated: or the bladder becomes ruptured, the urine runs off into the abdominal cavity and pro- 
duces a peritonitis ; or a eystitis is developed which is accompanied by nephritis or hydronephrosis and uremia. ${ }^{1}$

In the first case the pains cease suddenly. In making a rectal exploration we find the bladder completely empty. The escape of the urine into the abdominal cavity is soon indicated by very expressive symptoms; the urinary excretion is suppressed, the circulation is accelerated, the temperature increases, the general condition becomes more serious, there are chills and general muscular tremblings, the expirations and cutaneous perspiration spread a urinous odor, the abdominal cavity becomes more and more filled. The animals are taken off by a diffuse peritonitis which is complicated by uremia. Death ensues very rapidly in the horse and dog, preceded by ischuria which is from one to three days old; it often occurs a few hours after the tearing of the bladder. In the ox, on the contrary, it only takes place after eight, fourteen, or even eighteen days (Haubner-Siedamgrotzky). According to Stor, Cruzel, and others, the patients may survive from four to five weeks after rupture of the bladder. (Some practitioners-Jacobi and Huth among others-speak of having observed in the ox a healing after the confirmed rupture of the bladder ; such assertions, however, seem to us more than doubtful).

In the second case, death is preceded by symptoms of gangrenous cystitis, acute nephritis, and uremia ; this termination is very rare.

Differential diagnosis. Retention of the urine may be confounded with ordinary colics, in which affections micturition is often suspended and on rectal exploration a distention of the bladder is observed ; the diagnosis, however, may be made by an attentive examination of the patients. While the true colics are mainly associated with symptoms of an intestinal affection, the retention of urine is accompanied by manifestations indicating a vesical alteration: a continual desire to urinate, a stretched position, unsheathing of the penis, and great agitation of the tail, etc., ischuria

1 At the autopsy of a cow which Eloire believed to be affected by "hematuria" was found a remarkable example of double hydronephrosis produced by polypus or fungi of the bladder. These tumors, which are developed at the surface of the terminal openings of the ureters, produced an incomplete occlusion of these openings. In preventing the escape of the urine they had little by little created an enormous dilatation of the ureters and the kidneys. The ureters had acquired a circumference of sixteen to twenty centimetres, according to the points of measurement. The kidneys measured thirty centimetres in length, fifteen in width, and ten in thickness. The pelvis, which was considerably enlarged, was filled with purulent urine.-N. D. T. 
or persistent strangury, sensitiveness of the bladder to pressure, etc. Rheumatismal hemoglobinemia of the horse, simple cystitis, nephritis, peritonitis, the pains of parturition, cannot at all embarrass the practitioner; in all these circumstances the troubles of micturition are not lasting, and the bladder is not distended.

Treatment. The treatment of mechanical retention is purely surgical (urethrotomy, cystotomy, puncture, circumcision, etc.).

In case of the so-called dynamic retention, which is generally produced by a paralysis of the bladder, we must try to empty this by rectal or abdominal pressure or by catheterization. We may also try to produce micturition as a reflex, by irritating the opening of the urethral canal, or by frictions along the perineum. Paralysis is much more frequent than spasm.

In the case of spasm of the sphincter it is proper to make repeated frictions upon the perineum, with essence of turpentine, oil of anise, cumin, fennel, chamomile; internally we may also administer these agents, or morphine, belladonna, hydrate of chloral, etc. Confirmed paralysis of the bladder must be combated by strychnine, physostigmine, and electricity.

Incontinence of urine, an affection which is the opposite of retention, is but a common phenomenon of various diseases, especially the morbid states of the spinal cord. It is almost always due to paralysis of the vesical sphincter, exceptionally to muscular spasm of the bladder (in cystitis). It may also have as causes weakness of the sphincter (in very old animals), its paresis (cystitis), its section (cystotomy), or its destruction (by tumors), the distention of the bladder by calculi, or of the urethral bulb by polypus, etc. Sometimes it follows prolonged retention of the urine. Its treatment varies with the nature of the causal affection. We must combat paralysis of the vesical sphincter by strychnine, electricity, cold injections into the bladder, and toning up of the constitution.

\section{Catarrh and Inflammation of the Bladder: Cystitis.}

Catarrh and inflammation of the bladder, like the other affections of this organ, belong to the domain of surgery. They are, however, in close relation with diseases of the kidneys: their symptoms are quite similar to those of the latter, their treatment requiring mainly internal medication, and retention of the urine, if prolonged but a little, leads constantly to cystitis. We have therefore thought best to describe it briefly here. 
Etiology. The cause of cystitis is especially due to irritation of the vesical mucous membrane by toxic or infectious matters arising. from the blood, and which are eliminated with the urine (cantharides, essence of turpentine, fungi). That which is produced during the course of serious infectious diseases belongs to this group. It may also be occasioned by a common cold, by an inflammation of the surrounding tissues (nephritis, pyelitis, urethritis, vaginitis, peritonitis, metritis, etc.), which has extended to the bladder by contiguity or continuity of tissues, by causes of a tranmatic order (wounds and contusious of the mucous membrane, vesical calculi, catheterization), or by local infection (unclean sound). Retention of urine in the bladder (frequent in pet dogs), compression of the urethra, constrictions, contractions (paralysis of the muscular system), spasm of the canal, etc., easily produce a catarrhal or narcotic cystitis in consequence of the decomposition of the urine and the action of the septic matters upon the vesical mucous membrane.

[The action of cold upon trophic nerves and prolonged retention of urine are influences which, combined, often produce this disease. In cases in which house dogs, unaccustomed to the action of cold, are for some reason exposed to it, as for instance in railway travel, when confined in close quarters, they will retain their urine for quite long periods, and thus frequently results in an attack of cystitis.—W. L. Z.]

Pathological anatomy. The alterations vary with the degree of inflammation. Cystitis may adopt the following forms: the $c a$ tarrhal, purulent, croupous, diphtheric, necrotic, hemorrhagic, chronic, hyperplastic, with a varicose dilatation of the vessels, phlegmasic, with submucous or pericystic abscess, or even perforation of the bladder, and lastly, it may be incrusted, the urinary salts being deposited upon the mucous membrane. In the common catarrhal form the mucous membrane is tumefied, red, covered with an exudate composed of mucus, epithelial cells, and isolated white corpuscles; in the purulent form this exudate is exclusively composed of white corpuscles. Chronic cystitis is characterized by thickening of the mucous membrane, by warty vegetations of that membrane, varicose distention of its vessels, or calcareous incrustation (frequent in the mare and ox). In croupous and diphtheric forms the mucous membrane is sometimes covered by a necrotic membranous exudate, at other times it is strewn with ulcerations produced by the elimination of the sloughs. 
Symptoms. We observe frequent micturitions, which are scant and painful; the urine sometimes runs off drop by drop; in the horse the testicles are drawn upward and the penis is in an almost constant half-erected condition; the female animals make expulsive exertions, the lips of the vulva are constantly opening and closing, the clitoris is turgescent. Slight colics are added to these symptoms. Palpation of the bladder through the abdominal walls (in small animals) or by rectum is painful. According to the degree of the phlegmasia, we recognize a more or less intense fever; the appetite is diminished or completely suppressed. The urine has undergone important modifications; it contains abnormal elements coming from the inflamed mucous membrane: desquamated pavement epithelium, white corpuscles, mucus, albumin, crystals of ammoniaco-magnesium phosphate when it has undergone some decomposition. In carnivorous animals its reaction becomes neutral or alkaline. In purulent cystitis it deposits a more or less thick mucous or flaky sediment, at times only purulent, to which are added necrotic crusts in the "croupo-diphtheric" form, and red corpuscles in the hemorrhagic form. Chronic cystitis will often pass unobserved, especially in the ox ; the examination of the urine is then of the greatest importance.

Treatment. It consists in the internal administration of agents which are capalle of modifying advantageously the inflammatory condition of the mucous membrane : boric acid, salicylic acid, chlorate of potash or creosote. In a medium-sized dog we give this latter medicine in a dose of 0.15 gramme per day in three pills. In chronic cystitis we must resort to resinous substances like turpentine, aloes, etc. But the treatment of cystitis comprises also indications of a surgical kind. We have to practise cleansing of the bladder by means of antiseptics : solutions of creosote, $\frac{1}{2}: 100$; carbolic acid, boric acid, chloride of zinc, chlorate of potash, 1-3:100; sublimate, $1-5: 100$; nitrate of silver, $\frac{1}{2}-2: 100$.

\section{Vesical Hematuria.}

Etiology. The causes of this affection are: wounds of the vesical mucous membrane by calculi; contusions and injuries of the bladder caused by a fall or by a fracture of the pelvis, etc.; hemorrhagic cystitis produced by the ingestion of irritating agents, chronic cystitis with varicose dilatation of the veins; finally the 
very vascular neoformations in process of purulent or putrid destruction (papilloma, sarcoma, or more rarely carcinoma).

Symptoms. The principal symptom is the addition to the urine of a variable proportion of blood (hematuria), which colors it a light or dark red, and forms a reddish sediment, composed of red globules and fibrinous clots. In certain cases the quantity of the blood increases with exercise; the excreted liquid may be almost pure blood. The decomposition of this liquid in the bladder leads to catarrhal cystitis, which in the end is accompanied by its symptomatic complications. Anemia and general weakness become accentuated little by little. The disease sometimes ends in a fatal hemorrhage.

Treatment must be first surgical : cleansing of the bladder with a solution of perchloride of iron, of creosote, alum, tannin, etc. Internally, we must administer ergot of rye, tannin, or sugar of lead. We also advise counteracting the decomposition of the blood in the bladder by the administration of boric acid, salicylic acid, chlorate of potash, etc.; also by antiseptic urethral injections.

Tumors of the bladder, mainly epithelial neoplasms, may sometimes be recognized through palpation, when the attention is attracted by the difficulties of micturition, hematuria, and the symptoms of cystitis Against such alterations any intervention would be illusory. ${ }^{1}$

1 In a cow presenting for several days symptoms of nephritis and retention of urine, colic, frequent exertions of micturition, dysuria, and hematuria, Mollereau found the bladder almost empty, and was not able to perform catheterism, the sound being stopped by a tumor which appeared to be developed upon the vesical neck. At the autopsy he recognized that the neoplasm was of the size of a hen's egg, firm and hard at its base and soft and friable toward its surface. Microscopic examinination showed that it was formed by lobules of pavement epithelial cells and of connective tissue. (Bull. Soc. centr. Vét., 1890.)-N. D. T. 


\section{SECTION III.}

\section{DISEASES OF THE GENITAL ORGANS.}

\section{PARTURITION FEVER: PUERPERAL FEVER: VITU- LARY FEVER: SEPTICEMIA: PUERPERAL ECLAMPSIA.}

ANCIENT AND MODERN THEORIES UPON THE NATURE OF VITULARY FEVER. Acute post-partum disease, which is observed in all domestic species, and especially in the cow, have at all times attracted the attention of veterinarians. They have been designated by expressions which are as numerous as they are various : vitulary fever, milk fever, parturition fever, puerperal fever; inflammatory, septicemic, nervous, paralytic, and tympanic forms of vitulary fever; septicemia, puerperal eclampsia, etc. But the symptons mentioned by various authors are far from being uniform; the symptomatic characters assigned to vitulary fever are, on the contrary, very complex and polymorphic; sometimes the morbid phenomena are especially characterized by an intense fever, at other times by general paralysis, and it is not rare to observe cases in which these dominating phenomena are associated. Hering dwells upon this point: the monographs of various authors, he says, "seem to refer to so many different diseases." Thus very divergent opinions have been advanced upon the nature and significance of the phenomena produced by these affections. Most authors recognize several varieties of vitulary fever (the inflammatory, septicemic, and nervous or paralytic forms). Others, and among them Spinola, describe a principal form of this disease which is characterized by paralysis; as to the forms grouped under the collective name of "inflammatory forms of vitulary fever," they clearly have to be separated from true vitulary fever and classified among "diseases of the uterus."

In later times writers have generally indorsed Franck's theory. There is a tendency to admit two forms which differ from vitulary (396) 
fever in their nature, their anatomical alterations, and their symptoms :

1. Puerperal fever, puerperal septicemia, or the inflammatory form of vitulary fever of the ancients, which is also looked upon as a phlegmonous or "diphtheric" nephritis, a gangrene of the uterus, or a metro-peritonitis. Produced by septic matters, it is especially characterized by acute inflammation of the vagina and uterus, and it leads to a general septic infection.

2. Puerperal eclampsia, the "nervous or paralytic form" of the ancients, also designated quite improperly by such expressions as vitulary fever, fever of parturition, milk fever, etc. According to Franck it would essentially consist of a cerebral congestion, accompanied by œdema and encephalic anemia, a condition occasioned by the too abrupt retraction of the uterus, and made apparent by a deep nervous depression, general paralytic symptoms, and more rarely by convulsions.

The theory of Franck on the pathology of the paralytic form of parturient fever has been combated by Schmidt and Mühlheim.

These latter authors consider paralytic vitulary fever as the result of a poisoning produced by the resorption of an agent similar to the sausage or meat poisons; this agent is said to be the product of a special decomposition (different from putrid decomposition) of the lochia excluded from the air by rapid occlusion of the neck of the uterus (os uteri); paralytic vitulary fever would thus be an accident similar to those known in hygiene under the name " botulism."

The tonic symptoms would consist essentially in paralysis or paresis of the striated muscular fibre of the extremities, of the trunk, the tongue, the covering of the palate, the œsophagus, the larynx, the superior eyelid (ptosis), etc., and of the unstriped muscular fibres of the intestine and bloodvessels, while any anatomical macroscopic alteration would be wanting.

The other theories or hypotheses upon the nature of vitulary fever have merely an historical interest.

1. The old theory (Bentele, Born, Allemanni, and others) considered milk fever as a lacteal metastasis. Certain authors are believed to have observed this in the kidneys (milky urine), in the abdominal cavity (milky peritoneal contents), in the lung (lactiform nasal discharge), etc. But these lesions and symptoms belong evidently to metastatic nephritis, to peritonitis, or to pneumonia occurring in the course of pyemia. 
The expression "milk fever" has singularly helped to confuse the terminology of the fever of parturition. Some thought to designate in this way the inflammatory form of the affection, the others the paralytic form, and others again a disease differing in its main features from both. Quite recently Flusser still persisted in distinguishing simple milk fever from other forms of vitulary fever. But this milk fever is certainly nothing but an inflammatory affection of the mammary gland, accompanied by febrile symptoms ; it has, therefore, no relation with parturition fever. Besides, in normal parturition the temperature is increased from $\frac{1}{2}^{\circ}$ to $1^{\circ}$; in the bitch we have found it $39.5^{\circ} \mathrm{C}$. The expression, milk fever, ought, therefore, to be dropped altogether.

2. The theory of Harms, according to which vitulary fever would be the result of the introduction of air into the bloodvessels (aëremia) at the time of parturition, with consecutive cerebral anemia, is too hypothetical, and too unlikely, to be accepted. The gaseous globules found in the cerebral vessels are the result of blood decomposition; when the autopsy of the subjects which succumb to vitulary fever is made immediately after death, it is not rare to find it wanting; the paralytic form of vitulary fever is usually observed after a relatively easy parturition, when the uterus returns quickly to its former position, and its neck is closed rapidly; finally, the veins of the uterine mucous membrane are the seat of a positive pressure and not of a negative tension (absorbing force).

Again, how are the ante-partum cases of vitulary fever to be explained, as well as those in which the affection is produced but twelve hours or more after parturition?

3. The authors who have localized the disease in the lumbar spinal cord (Friend and Garreau) have evidently confounded it with paraplegia or paretic weakness of the hind quarters (festliegen). The assimilation of vitulary fever to the typhoid diseases (Köhn) does not even deserve any refutation.

Criticism of Franck's theory upon the pathology of PARALYTIC VITULARY FEVER. The division of vitulary fever into the septicemic and paralytic forms must be considered as a great advance attained in the domain of puerperal pathology. We approve this conception fully. But Franck's theory of the pathogenesis of the paralysis of parturition cannot withstand serious discussion; this complex disease cannot be explained by simple 
troubles of circulation. First of all, the expression eclampsia, adopted by Franck, is not suitable. Eclampsia is a form of spasm; it consists essentially of convulsions or true epileptiform attacks; from a certain point of view it might be considered as an acute epilepsy. In veterinary practice it is only observed in nursing bitches, and clinically it constitutes a morbid state which is quite the opposite of the paralytic vitulary fever of the cow. (See Eclampsia of the Dog in the section on Nervous Diseases.) Puerperal eclampsia in woman is also marked by convulsive attacks which last a few minutes and are separated by comatose intervals. These attacks are most frequently seen in primipara immediately after labor; at times they are dependent upon a uremic state (nephritis). On the other hand, paralytic vitulary fever consists essentially in a paralysis the duration of which is often quite long, and which happens ordinarily a few days after delivery, and most frequently in cows which have already had several parturitions. (According to Haycock, the predisposition to the disease is said to be more marked at the fifth calving.)

The pathology of parturient fever, as explained by Franck, does not seem to us to be sustainable. Anemia and encephalic oedema are not only marked by different symptoms from those of parturient fever, but these morbid states lead rapidly to death. If we admit that vitulary fever is dependent upon cerebral œedema it is not possible to explain the sudden resolution quite frequently observed in this affection, nor the cases happening ante-partum. This theory is especially weakened, however, by the fact that an interval of several days sometimes exists between the parturition and the onset of the disease, whereas the circulatory troubles, if any are produced, should appear immediately after delivery.

The insufficiency of Franck's doctrine has led us to seek another explanation of paralytic vitulary fever. We have drawn upon the Schmidt-Mühlheim theory, of which we have accepted the principle, as pointed out in the first edition of this work. If our opinion-. to which negative facts have been advanced in opposition-deserved many criticisms, yet it is as good, if not better, than all the diverse statements which have been put forth in its stead. At the present time we do not think we ought to modify it; rather we would point out that recent discoveries as well as certain monographs (Bass, Stube) strengthen and confirm its accuracy. Experimental poisonings by ptomaines determine symptoms of striking resemblance to 
those of paralytic vitulary fever, which is a favorable fact for the theory of auto-poisoning by the ptomaines, considered as an essential cause of paralytic vitulary fever. In botulism and allantiasis (see Mycotic Enteritis) we observe upon certain muscular groups, especially the pharynx and the eye, characteristic paralysis (dysphagia, ptosis), which recall all the symptoms of paralytic vitulary fever. They are due to cadaveric alkaloids: ptomatropine, found recently in toxic food (sausage, fish, ham, salt beef, corned beef); ptomacurarine, a most common alkaloid, the effects of which upon the organism are absolutely the same as those of curara and which give rise, like the latter, to paralysis of certain muscular groups; mytilotoxine, etc. All these alkaloids are only formed when not exposed to the air (Hoppe-Seyler); the uterus thus constitutes one of the most favorable locations for their development, but this is not equally rapid in all cases, and we can understand that parturition fever sometimes appears only a few days after delivery. The objection that poisonings by ptomaines are developed more rapidly than the paralysis of parturition is without value, for in this latter affection the production of ptomaines implies of necessity a certain lapse of time. The interval which passes between the time of parturition and the appearance of the discase represents, so to speak, the period of incubation. The abrupt disappearance of the paralytic symptoms sometimes observed is naturally explained by the rapid elimination of the poisons. Concerning the ante-partum cases of vitulary fever, they would be accounted for by the formation of ptomaines in the uterus, before the epoch of parturition, favored by an anticipative relaxation of its neck - a phenomenon to be distinguished from the dilatation produced during the first period of labor. The explanation of this delicate point is impossible on Franck's theory.

Division of Vitulary fever. The division of this affection into puerperal septicemia and paresis of parturition is insufficient in order to classify all the facts met with in practice. These two forms may appear independently, but they are sometimes associated; the complex or mixed cases are far from being scarce. Thus we have thought of establishing the three following morbid types of vitulary fever :

1. Puerperal septicemia.

2. Paresis of parturition (poisonings by the ptomaines).

3. Puerperal septicemia complicated with paresis. 
In order to justify this division, we advance the following physiological and etiological considerations :

1. The healthy uterine mucous membrane is favorably situated, anatomically and physiologically, for the absorption of the dissolved chemical principles, and consequently of toxic substances (Ellenberger); it possesses the same properties to a more marked degree when modified by the puerperal condition; it is irrigated by a larger number of blood and lymphatic vessels. In herbivorous animals, contrary to what exists in the carnivora, the uterine mucous membrane does not show any post-partum wound; often it does not present any door of entrance to the corpuscular, mycotic, or bacillary elements, but it may absorb the dissolved noxious products. In these animals ptomaine poisoning may take place through the intact uterine mucous membrane.

2 . The uterine and vaginal wounds which are produced accidentally during parturition or normally by the elimination of the maternal placenta, in the carnivorous and omnivorous animals, are portals of entrance for toxic matters and the germ elements (bacteria). Septic poisoning and infection may thus occur, isolated or simultaneously.

3. The uterus and vagina represent natural culture-chambers for the micro-organisms conveyed by the atmosphere, or by the hands or instruments of the obstetrician, and which come either from the local walls or from sick animals in the neighborhood. The utero-vaginal mucous membrane, covered with lochia and placental detritus, constitutes a very favorable culture-ground for these noxious agents, and, when air has access to the uterus, may easily produce septic infection, putrid poisoning, and pyemia. The phenomena of decomposition, which take place when protected from the air, result in the production of ptomaines or leucomaines.

4. The micro-organisms which come into play here, and the poisons which they generate, have nothing very particular, so far as their pathological rôle is concerned and the manifestations that they produce. In fact the same agents are the etiological factors of a number of infectious diseases (malignant oedema, phlegmon, erysipelas, septicemia, putrid poisoning, botulism and allantiasis, mycotic enteritis). Vitulary fever differs only from these latter affections by the localization of the process; it consists of an infection or intoxication starting from the uterus and related to parturition, while the preceding morbid conditions have their starting-point 
in other organs: skin, subcutaneous connective tissue, digestive mucous membrane, etc.

5. In the various general diseases which are consecutive to the resorption of soluble toxic substances, the uterus may be found absolutely intact, as is the case in paresis of parturition. Even in acute septicemia having for its starting-point wounds of the uterine mucous membrane, the disease may develop with such rapidity that at the autopsy the tissues of the womb are found almost unchanged.

In the majority of cases, however, the septic matters determine at their point of entrance a phlegmonous or necrotic inflammation, which sometimes extends over the whole womb and adjacent organs. Among the wounds of the vulva, vagina, and uterus, the most dangerous are those located upon the floor of these organs and in permanent contact with the infectious liquid; they are very often found covered with necrotic or "diphtheric" scabs, and accompanied by lesions of phlegmonous metritis, perimetritis, parametritis, or peritonitis.

When these ulcerations are located upon the lips of the vulva they may become complicated within a few hours by an enormous œdematous tumefaction, which is propagated to the cutaneous integument, and which simulates malignant odema. The great analogy which exists objectively between this latter and symptomatic anthrax ${ }^{1}$ has led to the erroneous belief of the identity of septic vitulary fever and anthrax (Himmelstoss).

6. We need only call to mind the septic form of vitulary fever, the septic origin of which is acute metritis itself immediately related to parturition, to have all the phlegmonous, "diphtheric," and necrotic forms of metritis. All the other varieties of this disease (endometritis, acute or chronic, etc.) enter into the group of true organic diseases of the uterus. They are described in works on surgery and obstetrics.

\section{Puerperal Septicemia (Septic or Pyemic Form of Vitulary Fever).}

Puerperal septicemia is observed in all our domestic species. It is frequent in carnivorous animals, rare in the cow ; its recognized cause is at times the inoculation of septic matters coming from parts of the placenta, and which are in process of putrid decomposition,

1 Kitt: Münch. Jahresber., 1883-84. 
also from a dead and decomposed foetus, etc. (auto-infection), or introduced by the obstetrician's hand, or by instruments, sutures, etc.; at other times an infection by neighboring animals which are affected by vitulary fever, non-delivery, or abortion. The infectious agents enter through wounds on the mucous membrane produced through brutal or ignorant interference in difficult or complicated parturition (fœetal dystocia, embryotomy, premature violent mechanical separation of the foetal envelopes).

The infectious process requires a certain time for its accomplishment; ordinarily the disease appears on the third day following parturition.

Pathological anatomy. Except in some rare cases of subacute evolution in which no local inflammatory alteration has had time to be produced, we find upon the mucous membrane of the vagina, the os uteri, and the womb so-called puerperal ulcerations; they occupy the place of primitive wounds, and are also covered by a necrotic or "diphtheric" exudate of bad aspect, or by granulations. These ulcerations are surrounded by an odematous tumefaction, which sometimes extend to the vagina or the interual side of the thighs. The other regions of the utero-vaginal mucous membrane are invaded by a phlegmonous inflammation, which, in carnivorous animals, often ends in a "diphtheric" eschar (colpitis or vaginitis and phlegmonous or croupous endometritis); in this variety the mucous membrane is of a dirty-brown or greenish-black color, covered with ulcerations and coated with an ichorous and fetid muco-pus; in certain places it is transformed into a pultaceous, necrotic matter, immediately upon the mucous membrane, which is laid bare by the elimination of gangrenous spots.

The uterus is not contracted, its walls are friable, softened, and infiltrated by a serous or purulent liquid; the lymphatic vessels and the thrombosed veins are filled with pus. Under the microscope we find the lymphatic interstices of the muscles filled with colonies of micrococci (Letzerich); the muscular fibres are affected with turbid tumefaction or with fatty degeneration. There are at times abscesses formed in the muscular tissue (phlegmonous metritis). The connective tissue is also odematous, infiltrated with pus, filled with small abscesses (phlegmonous parametritis).

Later, the peritoneal coating of the uterus, the pelvis, and at times the whole peritoneum, show lesions of serous, purulent, or putrid inflammation (perimetritis, pelvi-metritis, diffuse peritonitis). 
When the process is thus advanced we generally find the alterations of septicemia and pyemia: purulent pleurisy, pericarditis, ulcerous endocarditis, meningitis, arthritis, hemorrhagic gastroenteritis, metastic pneumonia and nephritis, and abscesses of the mediastinum (Stohrer, Viborg), of the subcutaneous and intermuscular connective tissue, of the soft intra-ungulate parts ; tumefaction and degeneration of the spleen, of the liver, the myocardium, the mesenteric ganglions, and the lymphatic ganglions generally; thrombosis of the vessels of the lungs, of the liver, of the kidneys; ecchymoses in all the organs and especially under the endocardium, badly coagulated blood, viscous, tar-like, soaking the tissues; the red corpuscles are destroyed; there is a rapid decomposition of the cadavers with considerable production of gas.

Symptoms. Cases of general septic infection, whether of acute or subacute evolution, are rare. The disease starts, as a rule, with the phenomena of inflammation of the genital passages. The mucous membrane of the vulva and of the vagina is red, tumefied, hot, and covered with croupous ulcerations and putrid exudates; these ulcerations are ordinarily grouped around the urinary meatus; sometimes the odematous tumefaction of the vulva is extended upon the internal side of the thighs; by abdominal palpation performed upon the right side, or on rectal exploration, the uterus appears tumefied and sensitive.

The animals seem to feel intense pain, they are restless, sway the tail, arch the back, look at the flank, stamp, lie down, contract the abdominal walls; they make violent expulsive exertions, which result only in the expulsion of a fetid, ichorous, foul-looking liquid; they stretch themselves as if effecting micturition; this is painful, and the urine comes only drop by drop.

The fever, which is always great, is expressed by chills and an elevation of temperature, which, if taken in the rectum, is $40^{\circ}$, $41^{\circ}$, or even $42^{\circ}$; the pulse beats 80,100 , or as much as 120 times per minute, it is small, filiform, often imperceptible; the muzzle is dry; the extremities (limbs, horns, ears) are sometimes hot, at other times cold.

The animals refuse food and drink; the lacteal secretion is exhausted; we observe constipation and slight meteorism. The general sensitiveness becomes dulled, weakness increases, the movements of the hind quarters are difficult, the animals soon stretch themselves upon the ground; they remain continually down, and we 
notice the appearance of lesions produced by a persistent decubitus. At that period the weakness, the insensibility, and stupefaction give to this affection a great similarity to the paresis of parturition, from which it is only distinguished by the high temperature, the local inflammatory alterations, and the absence of ocular, œsophageal, or laryngeal paralysis, etc.

The disease may run three different courses: 1 . The preceding symptoms increase in intensity, and death, which is announced by an abundant diarrhea, ordinarily occurs on the third or fourth day, but sometimes on the fifth or sixth ; more rarely it is apoplectiform, and then occurs within twenty-four or forty-eight hours. 2. The inflammatory phenomena as well as the fever disappear, and complete cure takes place from the eighth to the fourteenth day. 3 . The affection passes into a chronic state. When this latter termination takes place the emaciation is seen to become gradually accentuated, also the exhaustion, cachexia, marasmus, and hectic fever. Chronic endometritis is accompanied by a purulent discharge and leads to sterility, or is complicated by metastases in the lung, the joints, or elsewhere, and by chronic nephritis, with their usual symptomatic complications. If the animal organism is resistant, these metastatic inflammations may be produced several times-they return at variable intervals. When the disease has lasted for some time the "hemorrhagic diathesis" appears; it is marked by hematuria, hemoptysis, enterorrhagia, etc. We have several times seen a considerable number of ecchymoses upon the pituitary, as in petechial fever of the horse. Contamine has related similar observations.

The mortality from puerperal septicemia is from 50 to 70 per cent.

Symptoms of PARTURITION FEVER IN THE SOW. In this animal the observed symptoms are rather related to the paralytic form of parturition fever of the cow than to puerperal septicemia. The disease consists probably of a complicated poisoning from septic infection, but it is generally benign. It is found most frequently in the half-bred English races and in subjects which are in a well-fed condition. As a rule, it appears after an easy parturition and when the fotal membranes are not eliminated for several days. According to Harz, it is marked by the following symptoms: the appetite is lessened, the lacteal secretion is exhausted; the gait is uncertain and staggering, and the animals lie down frequently and 
give expression to their pain by low groans; they avoid all movements of the head and extremities, which gives the impression of a paralysis; they are apathetic, the look is dull and sad, the eyelids are half shut, there is sometimes a rolling of the eyeball. The temperature is irregularly distributed; in the rectum it is from $40^{\circ}$ to $41.5^{\circ}$, and the pulse beats as much as 140 times per minute; the hair is rough, the skin is hard and dry, and behind the ears and upon the legs is of a red tint, more or less dark; we observe trembling of certain muscular groups. The vulva and the mucous membrane are dark-red and slightly tumefied; there is a whitish vaginal discharge, which is mucous, thick, and not very abundant. The respiratory movements, which are from 20 to 40 per minute, are accompanied by a slight complaint. The peristaltic movements are suppressed, and there is constipation.

The course is rapid. The cure is produced, as a rule, after one or two days, more rarely toward the fifth day. In some cases the disease is complicated by weakness or paresis of the hind quarters and by interstitial mastitis.

Symptoms OF PARTURITION FEVER IN THE SHEEP AND GOAT. The patients are downcast, they hold the head low and refuse food; the abdomen swells and becomes sensitive, the back is arched; we observe colics which are similar to the pains of parturition, and also violent expulsive exertions (Sturm). The muscles are red, the temperature rises to $41^{\circ}$, later great weakness and stupefaction occur. The external genital organs are more or less tumefied; the vaginal mucous membrane presents dark-red stripes and ulcerations. The duration of the affection varies from a few hours to a day or more; ordinarily all the affected animals perish (Deigendesch, Schleg). Very likely most of the affections of the sheep designated as gangrene of the uterus are but varieties of parturition fever.

Symptoms of parturition fever in the bitch. The disease is announced by the symptoms of septic metritis : tumefaction of the genital parts, fetid vaginal discharge, of foul appearance; high fever $\left(41^{\circ}\right.$ or more), inappetence, permanent decubitus, a sleepy and comatose state. The course is rapid; at the end of twelve to twenty-four hours there is either an amelioration or a fatal ending, and considerable lowering of the temperature. When recovery takes place we very often see chronic endometritis persist, as in the cow. 
Symptoms of Parturition Fever in the MaRe. In this female the principal manifestations of the disease again enter into the eategory of septicemia. We observe especially the high state and irregular distribution of the temperature, the acceleration and irregularity of the pulse; chills, inappetence, and the abolition of peristaltic movements, colic, stupefaction, finally inflammatory alterations of the genital organs. The course is always rapid.

Treatment. The principal indication is the disinfection of the uterus and vagina. Carbolized water ( 1 to 5 per cent.), very much used formerly, may be replaced with advantage by cresylic acid in $\frac{1}{2}$ or 1 per cent. solution. This latter agent is not toxic, is more efficient than carbolic acid, and produces the almost immediate disappearance of putrid odor which comes from the genital organs. Carbolic acid incompletely dissolved may have a corrosive action upon the utero-vaginal mucous membrane. The sublimate injections $(1: 1000)$ are only proper for carnivorous animals; for the herbivora, and especially the cow, they are very dangerous, in spite of what some have stated concerning this question, and by the irritation produced they cause violent expulsive movements. Iodoform, which is toxic and is also insoluble in water, is not advisable.

In administering ergot of rye, we may produce contractions of the uterine walls and lessen the resorption of septic matters. Laxative purgatives are indicated with the object of favoring elimination of the infectious products through the intestinal mucous membrane. Cutaneous frictions act in the same way. Fever and weakness have to be combated by means of antipyretics and stimulants.

Prophylactic measures are of the highest importance. They comprise : a rigorous asepsis of the obstetrician's hands and instruments; the isolating of females about to be delivered, and their removal from other animnls in a less advanced stage of gestation, especially from those having aborted lately or which are affected by a chronic vaginal discharge, or by diseases of the skin accompanied by suppuration, etc.; the necessary precautions in order to prevent the accidental transmission by men having charge of the patients, through the utensils, food, drink, sponges, etc.; in the disinfection of the stables, the stalls, etc., with chloride of lime, cresylic or carbolic acid, and the other disinfectants; finally a properly regulated ventilation of the premises.

The question of consumption of the meat coming from animals having been killed during parturition fever is important, and it is 
not always easy to solve it. The meat must be rejected when coming from animals affected by any septic trouble of the uterus which has become generalized, for it might give rise in man to mycotic or septic enteritis or other poisoning by the substances it contains (botulism). We must proceed in the same way in all cases where the paresis of parturition is complicated by a septic infection. In. simple forms of paralytic parturition fever the meat is less dangerous; nevertheless, the frequency of the serious alterations which accompany these, and the similarity of the paretic symptoms observed in man, in cases of botulism, with those of parturition fever, are facts which, in our opinion, call for the greatest reserve concerning the use of the flesh of animals affected with this disease as alimentation for human beings. So far, it has not been demonstrated that such toxic results have been produced, but nevertheless the carcasses of such animals ought not to be considered available for consumption.

\section{Paresis of Parturition (Toxic or Paralytic Form of Parturition Fever).}

Paresis of parturition is particularly observed in the cow : the absence of a maternal placenta, of a natural uterine wound after parturition, constitute the evident cause of the frequency of this disease in the bovine race: the uterine mucous membrane, which is generally intact, allows only dissolved toxic matters to penetrate. The same is the case with the goat, which is quite frequently affected by this form of parturition fever. It is generally found in the sow. Good milch cows, which are well fed, great eaters, fat, and subjected to a liberal regimen before parturition, and which are kept in permanent stabling, are much exposed to the affection; this predisposition becomes very evident from the frequency of the disease among our good races (the Holland and other flat country races), and by its infrequency in animals kept on pasture, as well as in those of countries where little milk is produced. It is remarkable that paresis of parturition is mostly observed after rapid and easy calving; it is very rare in primipara; in these, calving is always laborious and the uterus is contraeted more quickly and with more power than in ulterior calvings. The cow is generally affected at the third, fourth, and fiftli delivery (Haycock; statistics of Bavarian veterinarians). The most numerous cases coincide with great heat spells of summer and abrupt changes of tempera- 
ture. The etiological rôle which a few authors have ascribed to the action of cold is not demonstrated by facts. Ordinarily the disease develops in from twenty-four to forty-eight hours after parturition; very seldom after the third day, more rarely at the time of delivery; there are, however, isolated observations of ante-partum parturition fever. It has never been recognized before the starting of milk secretion (Müller, Söhngen, Albrecht, Brüller). We must point out that in natural parturition there is always quite a long interval between the relaxation of the os uteri and the expulsion of the foetus, and the cases of ante-partum vitulary fever are immediately produced after relaxtion of the os ; this latter phenomenon is important from a pathological point of view, for it explains how certain processes of decomposition may take place in the gravid uterus.

Franck makes the statement that there exists in the ox a disease identical with that of parturient fever in the cow ; but we question whether the authors who have mentioned such examples have not had to do with other diseases, especially with affections of the spinal cord or of the brain, or with poisonings.

Pathological anatomy. This presents nothing, or almost nothing, characteristic. Ordinarily the uterus is greatly retracted, without the existence of any wound or inflammatory alteration of the mucous membrane. Especially noticeable is the irregular distribution of the blood in the organism; the abdominal viscera are the seat of a much more intense hyperemia than the other organs. In numerous cases the brain does not offer anything abnormal; sometimes, however, the meninges are congested and filled with ecchymoses; we may also notice internal or external hydrocephalus by transudation of a slightly yellowish liquid; finally, we often find the brain markedly anemic, odematized, friable, gelatin-like, and with its cortical substance colored yellow. The buccal, nasal, and pharyngeal cavities are ordinarily filled with alimentary matters; the desiccation of the contents of the third stomach, which was formerly considered to be characteristic of the affection, is found in most diseases accompanied by anorexia or adipsia; it is, therefore, but an alteration altogether accessory. As a rule, the other organs are normal; hyperemia of the sympathetic mentioned by Binz, and the presence of air in the bloodvessels (Harms), are lesions devoid of any importance.

Symptoms. The affection commences almost always with a slight anxiety; the animals stamp and make expulsive exertions; 
they strike the abdomen with the hind legs. More rarely it is announced by symptoms of cerebral irritation: the patients bellow, mount into their manger, and show all the symptoms of a more or less intense agitation; we may observe epileptiform convulsions, convulsive contractions of the muscles of the face and neck, and grinding of the teeth. After this initial period, which often passes unobserved, the characteristic paralytic manifestations succeed one another rapidly. The forelegs are bent; the general weakness increases; the animals seem exhausted, they stagger, fall upon the ground, and often attempt to rise, exerting themselves without success; as soon as the paresis of the hind quarters has invaded the whole body, they stretch themselves upon their litter exhausted the legs half bent or in an extended straight and stiff position. It is generally in this condition that the veterinarian finds the patients; besides sleepiness, apathy, and decubitus, other symptoms attract his attention: the head is inclined laterally, it rests frequently upon the costal wall, and if we endeavor to straighten it, it falls back to the former position. The upper eyelid covers the ocular globe (ptosis); cornea dull, sometimes vague, as in an animal in agony, it is dry, non-sensitive, and in some cases wrinkled and furrowed; the pupil is dilated as in amaurosis; the lacrymal duct is full of pus, the eye is sunk in the orbit. The tongue is paralyzed, flabby, and hanging; as long as the salivary secretion continues it runs in long threads upon the ground, the paralysis of the pharynx and œesophagus no longer permitting deglutition. Respiration is painful, rattling and sometimes whistling (paralysis of the muscles of the larynx); the nostrils are greatly dilated. At the examination of the digestive apparatus we observe at first dysphagia, and later a complete incapacity of deglutition, the cessation of the peristaltic movements, constipation and meteorism, phenomena which are produced by paresis of the muscular wall of the intestine, a paresis which withstands the most energetic purgatives (even physostigmine). Micturition is suspended (paralysis of the bladder); the urine is albuminous; Nocard has constantly found sugar in it, sometimes in a proportion of forty grammes per litre. The lacteal secretion is exhausted; the udder is flabby and wrinkled. As regards the circulatory apparatus we have first as a constant symptom the irregular distribution of temperature; the horns, ears, and feet are cold. The pulse is small, often imperceptible; the number of pulsations is from 50 to 70 per minute, later we can count as many 
as 120. Concerning the hyperthermia, authors give contradictory indications ; the greatest number, with Franck, consider as a characteristic symptom of septic parturition fever the regular decrease of the temperature, which may fall to $35^{\circ}$ and even below. During the first few days we have almost constantly found a temperature of $39^{\circ}$ to $40^{\circ} \mathrm{C}$. In eight cases very carefully observed from a thermometric point of view, Engel-Weingarten ${ }^{1}$ found in seven instances a temperature of $39.1^{\circ}$ to $40^{\circ}$; in three of these cases the disease was only one day old, in three others it had already set in for forty-eight hours, in the last it was the fourth day of the trouble. It seems that in paresis of parturition the same thermometric peculiarities are observed as in the nervous form of distemper of dogs (Staupe): the temperature, high at the start, falls below the normal; this last phenomenon is attributed to the loss of a greater quantity of heat (vascular paralysis) and to the reduction of calorification in the paralyzed muscles. Paresis of parturition is never accompanied by any considerable hyperthermia; when the temperature rises above $40^{\circ}$ to $41^{\circ}$ it indicates complications of septic infection.

Course. The disease, as a rule, advances rapidly ; its termination is sometimes observed at the end of twelve to eighteen days. When a cure is going to occur, improvement becomes marked as early as the second or third day: the animals move about, sometimes they succeed in resuming the standing position; the appetite, defecation, micturition, and lacteal secretion reappear successively; the temperature rises again and is distributed regularly. Recovery is complete at the end of two to five days; it is rare to see paresis of the hind quarters persist.

When the end is to be fatal, we observe the symptoms of paralysis of the heart and brain, anxiety, agitation, convulsions; death supervenes in one to three days. Sometimes it is immediately preceded by a profuse diarrhea. We may observe apoplectiform death, which occurs shortly after the start of the affection. Finally, the condition is often complicated by a pneumonia caused by foreign bodies (food or therapeutic agents which have "gone the wrong way"). This pneumonia, the duration of which is from four to six days, invariably ends in death.

The toxic or paralytic form of vitulary fever produces an average

1 Engel-Weingarten (communication). 
mortality of 40 to 50 per cent., but the figures mentioned by authors are quite different (Hering and Franck have found 40 per cent.; Köhne, 25 to 50 per cent.; Saint-Cyr, 45 per cent.; Stockfleth, 50 per cent. ; the statistics of Bavarian veterinarians give 48 per cent.). The convalescing animals succumb in the proportion of about 10 per cent. to pneumonia by foreign bodies. The disease returns quite frequently.

Differential diagnosis. The paresis of parturition being closely related to delivery, could not be confounded with other morbid states in gestation or parturition. It must be distinguished from ante- or post-partum "paraplegic weakness" (festliegen). This disease, which sometimes complicates the first, consists in a lumbar weakness occurring ordinarily a few weeks before parturition; it resists all treatment, disappearing of itself as soon as the puerperium: is at an end.

We must be careful not to mistake for paralysis of parturition the passive paraplegic symptoms which are consecutive to pain or injury of the lumbar plexus and the nerves which arise from it. In these affections the general state is not at all troubled, the temperature is normal, the appetite continues, and the paralysis is always localized. The symptoms of cerebral depression which one observes generally in puerperal septicemia some time before death may be easily distinguished, at least in most cases, from paresis of parturition.

Treatment. The prophylactic indications must not be neglected. Daily exercise is particularly recommended, also the reduction of the ration and the administration of mild laxatives (sulphate of soda) some time before parturition, the maintenance of perfect conditions of temperature and ventilation. We should avoid all causes of cold. For stabled animals, in which the disease is frequently observed, Schmidt-Mühlheim advises a cleansing of the uterus after parturition, with carbolic or boric solutions; we should resort to it even when the delivery has taken a normal course without any intervention. These antiseptic washings must be followed by the introduction into the uterus of a small quantity of iodoform powder; for that purpose we use a glass tube attached to a rubber bellows (a very simple apparatus which is readily constructed). According to some authors, iodoform is said to oppose the development of specific toxic agents. The solution of cresol in $\frac{1}{2}$ or 1 per cent. seems to us to be preferable to carbolized water. 
When the disease exists, the treatment must especially consist in combating the more alarming symptoms : the paralysis of the voluntary muscles, that of the muscles with unstriped fibres, and the cerebral depression. It would be illusory to attempt to reach the cause. We must as much as possible avoid giving any medicinal agents through the mouth on account of the danger of having them go the wrong way, and pneumonia from foreign bodies result. The administration by hypodermatic injection is much preferable.

1. We should counteract the general paralysis and the nervous depression with stimulants, administered in preference in subcutaneous injections: veratrine ( 0.1 to 0.2 gramme), caffeine (5 grammes), spirits of camphor (an injection of 5 to 10 grammes every hour), ether ( 2 to 5 grammes per hour) nitrate of strychnine (0.02 to 0.05 gramme per dose), sulphate of eserine, which excites also the intestinal peristaltic movements $(0.1$ to 0.3 gramme per dose, dissolved in 2 to 5 grammes of plain water, or of a sublimate solution $1: 1000)$. But eserine, like other remedies, is often absolutely inefficient.

The principal stimulants administered internally are: wine (which is also a valuable analeptic), essence of turpentine, carbonate of ammonia, acetate of ammonia, brandy and diluted alcohol, which many practitioners use in doses large enough to produce drunkenness. Instead of giving these medicines in drinks, it is more advisable to administer them in the shape of clysters. The most used external stimulants are cutaneous frictions with essence of turpentine, camphorated alcohol, oil of mustard, croton oil, diluted ammonia, etc. ; and frictions of stibiated ointment $(1: 4)$, cold and warm compresses, cold douches upon the head, the application of Mayor's hammer along the spinal cord, taking care to cover the skin with a woollen cloth, etc. Some veterinarians have used electricity with advantage. Bleeding has been found useful in some cases and valueless in others.

2. We should use drastics to combat the eessation of peristaltic movements of the intestine, those which likewise act as derivatives upon the digestive canal; such as eserine, tartar emetic (10 to 15 grammes per dose), croton oil (20 to 25 drops in a mucilaginous decoction), aloes (40 to 50 grammes), sulphate of soda (500 to 1000 grammes), etc. Injections or a rectal exploration frequently repeated, and the manual extraction of fecal matters, have a similar action to that of these agents. 
3. Spasms and general excitement should be combated by morphine injections, injections of hydrate of chloral, inhalations of chloroform, etc. The antispasmodic drinks (chamomile decoctions, valerian, fennel, anise, cumin, etc.), must be avoided on account of the dangers which are incurred by their administration.

[The disease is one which causes very great loss to our owners of dairy stock, taking off as it does the best and most valuable animals ; the question of prevention, therefore, offers far more interest to our stock-owners and veterinarians than does that of cure. Bleeding these animals a few days prior to parturition, taking a liberal quantity of blood, will prove absolutely effective as a preventive and will not be productive of the slightest harm to either the foetus or the mother. It is also one of the best curative measures at our disposal.-W. L. z.]

\section{Puerperal Septicemia Complicated by Paralysis.}

At an advanced period of the septic form of parturition fever it is quite common to see the disease becoming complicated by poisoning. The special literature on this subject contains quite a number of these cases, which have contributed toward altering the opinions formerly expressed upon the nature of parturition fever. But the dual character of the final affection was recognized very soon. In ending the description of "inflammatory" vitulary fever, Hering in his Pathology says: "Then a great weakness occurs and also paralysis affecting principally the hind quarters ; this state represents the paralytic form of vitulary fever!"

This ensemble of pathological phenomena, no matter how complicated they seem, is nevertheless explained by the duality in the nature of the uterine infectious matters; some, purely toxic, are absorbed when the uterus is intact; the others, septic, reach the organism by penetrating the tissue through wounds on the surface of the uterus. According to the predominance of absorption of one or the other, the manifestations are very different. There are cases in which we observe symptoms of the paresis of parturition together with the anatomical alterations of septic inflammation of the uterine mucous membrane. We could, by synthetic study, demonstrate this assertion and support our opinion upon the possible synchronism of the septic and paralytic forms of the disease, but we prefor to report here one of our observations, which affords valuable aid toward deciding the pending question. We refer to a clinical case in which, 
during life, we could but make the diagnosis of paralytic vitulary fever, while at the autopsy were shown the alterations of phlegmonous septic endometritis.

In the beginning of the year 1878 , a milkman at M- possessed for some months a cow which had rapidly gained in flesh, in consequence of a liberal alimentation. On January 20th this cow dropped a calf of extraordinary size; the parturition, however, proceeded very rapidly, without any obstetrical intervention, and the labor was over at the end of a few hours. The owner did not admit the possibility of a cold.

On January 21st, twenty-four hours after parturition, the first symptoms of the disease were observed: the cow declining all food; there was anxiety, restlessness, decubitus ; the hind quarters were soon paralyzed; the animal in vain exerted constant movements with the forelegs in order to return to the standing position. The head was violently thrown from one side to the other, and at certain moments we observed convulsive paroxysms separated by periods of coma. Defecation and micturition were suppressed; the lacteal secretion continued.

On January $22 d$ the cow was down, the legs, neck and shoulders, and the head extended, but from time to time the latter was brought upon the left costal wall; in trying to straighten it, it would immediately return to its first position; the movements of the neck and shoulders were elastic, similar to those of a spring. The ears, the base of the horns, and the extremities were cold, the skin of the trunk was dry and of moderate temperature. The muzzle was damp and cool, the heart beat 104 times per minute, the pulse was irregular, small, evasive, the rectal temperature was $39.5^{\circ}$. Sometimes the respiration (twenty per minute) was loud and rattling; at other times it was very feeble and silent, to such an extent that in examining it alone we might have doubted the presence of life. A spumous saliva escaped from the mouth; the lower lip hung down; the peristaltic movements were suppressed, the abdomen was empty and hollow; the rectum was filled with small excrementitious masses of the size of a chestnut, of green-brownish color, with yellowgrayish false membranes, rendering these very smooth and shiny; the bladder was full, the udder normal, giving milk at every teat; when once drawn, the milk would run out of itself. A piece of the placenta protruded from the vulva; the visible genital mucous membrane was covered with ecchymoses, without being specially 
tumefied. The cow was quiet, she tried to rise at long intervals, and exerted repeated movements of the head and front members; the posterior members were inert. The conjunctiva was slighly injected, the face without expression, as if frozen, the cornea had lost its brilliancy; the pupil could not be examined. On the afternoon of this same day the animal was killed by bleeding.

The autopsy was made eighteen hours after slaughtering. As visceral lesions, we observed the friability and the grayish-white color of the kidneys affected by fatty degeneration, as well as paleness and flaccidity of the cardiac muscle. The coagulated blood did not offer any alteration. The consistence of the cerebral substance was normal; the medullar substance was colored a dirty-yellow gray (cadaveric alteration). Most of the bloodvessels were empty, and contained gas at certain places. In the vagina was found a little semi-liquid matter of a chocolate color, and upon the vulva submucous ecchymoses. The fold of the os was the seat of an odematous tumefaction; in the neighborhood of its internal orifice there was a loss of substance of the size of a silver dollar; the uterine mucous membrane showed several others of smaller size; the bottom of these erosions was grayish and of bad aspect. The uterus still contained one-third of the placenta, adhering to six cotyledons, and a liquid similar to that found in the vagina, but not at all fetid. The uterine mucous membrane and the subjacent counective tissue were the seat of an odematous tumefaction and of a gelatinous yellowish infiltration; at certain places this tumefaction reached the thickness of one and a half centimeters.

\section{EXALTATION OF THE GENESIC INSTINCT: NYMPHOMANIA : SATYRIASIS.}

The morbid exaltation of the genital functions is designated in our domestic animals by the expressions lascivity, bellowing disease, monthly heat, ovarian tic (Mutterkoller), testicular tic (Samenkoller), etc., and also under the improper name of hysteria. The cows which are affected by it are said to be "bullers." The term satyriasis applies specially to males, and that of nymphomania to females. It is most often observed in the cow, mare, ewe, and male dog; the other males-the stallion, the bull, and the he-goat - are but very rarely affected by it; it is exceptional in the pig. The causes of the frequency of nymphomania in the cow are stable- 
life and the artificial regimen to which this female is generally subjected, the habit she possesses of conceiving at determined periods, and the diseases of the genital organs produced by repeated parturition, tuberculosis, etc. The expression "exaggeration of the genesic instinct" implies already a first degree of this anomaly. No precise demarcation can be fixed between the normal genesic instinct and its morbid exaggeration.

Etiology. The exaltation of the genesic instinct does not constitute a pathologic state in the true sense of the word, but only a symptom which may depend upon various causes.

1. It is more frequent in old than in young cows. The animals which are subjected to fattening (Uebelen) and those which, toward the age of four or five years, are kept in a too fat condition (Rychner), are particularly exposed to it.

2. Over-stimulating alimentation; this is accused in all species; rye, barley, and leguminous food are thus designated under the name heating food; insufficient work, and stabling almost permanent.

3. Nymphomania of the cow usually depends upon alterations of the genital organs, such as inflammation, cystoid degeneration, hypertrophy, dropsy, cancerous tumors, sarcomatous and tuberculous ovaries. The frequency of the exaltation of the genesic sense in tuberculosis has led to the designation of this vice under the name of French disease, monthly heat, "lascivious disease," etc. But tuberculosis only produces lascivity when the genital organs, and especially the ovaries, are affected. Schmidt has performed the autopsy of ten cows which were affected by this vice without finding any lesion explaining nymphomania. The diseases of the uterus which prevent conception (chronic vaginitis, chronic endometritis, obstruction of the os uteri, tumors, changes of relation, atrophy and hypertrophy of the uterus) may determine it.

4. In the equine race the monorchids and cryptorchids are generally affected by satyriasis.

5. Repeated genital excitement in the entire horse (a stabling in the neighborhood of heated mares), the continual excitement of young mares by the stallion, and onanism in the latter, produce at times satyriasis and lasting nymphomania.

6. The exaggeration of the genesic sense is also observed in the vesiculous affection of the genital organs of the horse (benign mal de coit, horsepox), in syphilis, in hydrophobia of the dog. Finally, 
it may be a manifestation of medullar affections, of certain gastric states, and of abdominal plethora (Haubner-Siedamgrotzky).

The identity formerly admitted between nymphomania of the cow and hysteria of woman is not at all established. Hysteria is a general neurosis, especially cerebral, which often has no relation with troubles of the genital organs; and even when it is due to organic alterations of the uterus (catarrh, changes of relation, tumors, etc.), it is very rarely accompanied by genesic hyperexcitation. The main symptoms of hysteria are: melancholia, hallucinations, hyperæsthesia, convulsions, anæsthesia, paralysis, etc.

symptoms. A. In the cow. In this female nymphomania begins by heat which returns regularly every three weeks, and by the want of conception in spite of repeated copulations. The patients, which are anxious, restless, agitated, feel an irresistible desire to satisfy their genesic ardor. They try to come near other cows, and mount these and even bulls. The degree of intensity of the vice varies with the individuals: in some cows the symptoms are only slightly marked; in others, in which they are much more accentuated and violent, they characterize the morbid condition designated bellowing disease. This latter anomaly seems to be much more frequent in some countries than in others; according to Schmidt-Kettwig, it is expressed by the following symptoms :

The cows show great anxiety, the look is fixed, and the eye shiny ; they are restless, agitated, and try to mount other animals; they rub their horns against the pillars of the stable and the manger; at times they scatter their litter, dig the soil, and go on their knees, etc. ; at other times they scratch the floor of the premises violently with their forefeet. At the same time they utter bellows very similar to those of the bull; these are low at the start, later very loud, extended, and frequent, which take place night and day, but especially in the morning or when strangers enter the stable. The patients have often a savage demeanor; when approached they become furious and break their chains in going through uncontrollable movements; at times they attack man. Among the other symptoms we must also mention the diminution of the lacteal secretion, the curdling of the milk when boiling, the depression which takes place upon each side of the tail, as in an advanced stage of gestation, and which is due to the relaxation of the ischiatic ligaments. The cow acquires gradually the exterior appearance of the bull, the muscles of the neck increase materially 
in size (Vorberg); finally emaciation sets in, also weakness, and sometimes cachexia. ${ }^{1}$

$B$. In the mare. We observe in her also at the start unusual heats, which are remarkable for their frequency and the loud manner in which they are expressed. We notice an unusual restlessness, anxiety of expression, and vivacity of the eye; the mare neighs frequently; she extends her body and makes exertions of micturition; the lips of the vulva are opened and closed successively; the clitoris is turgescent; the urine, which is ejected in small quantity, is mixed with mucus, which renders it viscous and gives it a yellowish-white aspect. Conception only rarely takes place, notwithstanding repeated coitus, and when happening by hazard abortion soon occurs.

Nymphomaniac mares are ticklish and excitable; when the shafts, reins, traces, the leg or spur happen to touch the hindquarters we notice symptoms which have been the cause of the name "urinating mares": there are also piercing cries, a salient condition of the clitoris, emision of a few drops of urine, wagging of the tail, reaction against the rider's foot, a lowering of the croup, kicking, propensity to biting; these animals often become very dangerous (see Immobility). Soon we can see aberration of the appetite, emaciation, convulsive paroxysms, and maniac excitement. In a mare affected by nymphomania, Eletti has observed rabiform symptoms, tetanic attacks, grinding of teeth, dysphagia, contractions of the abdominal muscles, cardiac palpitations, etc. (this case, which is quite exceptional, presents a certain analogy with the hysteria of woman). When the disease exists for a long time the general sensitiveness becomes depressed, a state which is similar to immobility ("ovarian tic") appears; at times there are periodical exacerbations which correspond with the periods of heat. While in certain cases the described symptoms disappear for a time after copulations, they lead, however, in the end to serious trouble of the general system, which is due to the constant excited condition of the animals.

1 In a memorial addressed recently to the Société centrale de Médecine Vétérinaire, Champagne (veterinarian at Montmirail) called attention to the exterior characters of the cows which constantly return to heat, and particularly, first, to the disposition of the posterior edge of the sacro-sciatic ligament, which is concave, hard, and distended; second, to the depth and unevenness of the depressions of the posterior part of the pelvis on each side of the base of the tail.-N. D. T. 
C. In male animals. Satyriasis is shown by frequent erections, which are nearly permanent in certain subjects (priapism, corda venerea), also by restlessness and excitement, by neighing or continual bellowings, by unusual movements at the sight of females, and by the violent exertions made by the animals to perform the act of copulation. Sometimes, when this genesic ardor is not satisfied, the excitement is increased and may become rabiform; the bull and the horse may even pursue man, and especially women (Spinola). Later the animals become downcast, refuse their food, grow more and more emaciated; some observers have noticed accidents of medullar origin, which were characterized by uncertainty of movements and staggering. The trouble may end in a fatal cachexia. In the entire horse we have recognized a state similar to immobility, and designated it under the name of "testicular tic." According to Röll, this condition is especially observed in old stallions retired from the stud. (The expression "testicular tic" has served to designate a great many different morbid conditions; certain authors have applied it to the rabiform symptoms which appear at times in the course of satyriasis; others, to a permanent cerebral depression which must be likened, from a comprehensive point of view, to immobility ; finally, some seem to have used it to express viciousness accompanying satyriasis.)

Satyriasis produces also attempts at masturbation : the horse and donkey strike the erected penis against the abdominal wall; the bull, the dog, and the ram rub the organ against the abdomen or between the front legs when gathered; often the dog tries to ejaculate by mounting other animals or taking hold of a man's leg with his forelegs.

Independently of satyriasis, onanism in male animals is not very rare; it is observed to a certain extent as vitium animi-the same title as in man. That which well demonstrates that it has no relation with the morbid exaltation of the genesic instinct is the fact that many stallions and bulls upon which it has been seen did not undergo any excitement when near females (Prangé, Spinola). The $\operatorname{dog}$ and the ram are the animals masturbating most frequently; but this aberration is also observed in the horse and the bull. Its consequences vary considerably according to the degree of the vice. Sometimes we see troubles which are hardly perceptible; in other cases the animals become more and more lazy; they lose flesh, perspire easily and become tired on the least exertion; the general 
sensitivenass is dulled; afterward we note the occurrence of paralytic symptoms produced by an alteration of the spinal cord, as diminution of virility, and lastly impotence, cachexia, and apoplectiform attacks. Spinola has found the lumbar marrow softened over an extent of five inches. Exercise, fatiguing work, moderate food, and coitus are the principal means to which we may resort. If they remain inefficient we must perform castration. Prangé cured a stallion by having him completely immersed in cold running water, two hours daily, for about ten days.

Treatment. The cases offering the greatest chance of cure are those where the disease is the result of over-stimulating alimentation or of insufficient exercise. The patients must be subjected to a debilitating diet (green fodder), and they must be tired out by hard or prolonged work; the males should be separated from the females, or used for reproduction. ${ }^{1}$ Bleeding may also be resorted to, or we may produce a derivation upon the digestive mucous membrane by means of purgatives (sulphate of soda in the ox, aloes and tartar emetic for the horse). Cold lotions and baths are also efficient.

In order to lower the genesic ardor we should use hydrate of chloral (horse and ox, 20 to 50 grammes in drinks), bromides of potassium and sodium, or subcutaneous injections of morphine $(0.2$ to 0.5 gramme in the ox and the horse).

The process recognized by Eloire, consisting in the introduction of a foreign body into the uterus, a ball of lead for example, should be tried, but generally the only efficient treatment is castration. In male animals and in the cow, the consequences of this operation are almost always very simple; in the mare a fatal peritonitis is to be feared, and it must not be attempted till all other means have failed. ${ }^{2}$

1 I have followed the case of a horse for eight years, which took to the habit of masturbating when five years old, and became epileptic. The attacks, which at first were rare, became of almost daily occurrence. I castrated him seven years ago. He had an attack about six weeks after the operation, and a second, which was quite mild, about six months later. Since that time he has not shown anything abnormal. I see him from time to time.-L. T.

2 Castration of the mare per vagina, performed with the help of antiseptic precautions, is not so dangerous as it has generally been regarded up to the present time. In the last three years we have castrated nine mares, and none has succumbed. One showed (from the sixth to the twelfth day) somewhat serious symptoms, which we ascribe to a local peritonitis; in the eight others no special febrile reaction was observed. We perform the operation in the following manner: The animal is placed in stocks, put under the influence of hydrochlorate of morphine and ether; the rectum is emptied, then the base of the tail, the anus, perineum, vagina, vulva, and buttocks are 
In mares and cows affected by ovarian cysts, their crushing has been recommended with the hand through the rectal walls (Zangger, Utz, etc.). Utz claims to have cured 90 per cent. of his patients in that way. After castration or crushing of the cysts, anxiety or excitement may continue if there is any uterine trouble (tuberculosis of the uterus, etc.). Then we ought to decide as quickly as possible as to sacrificing such animals as are in a sufficiently healthy condition to permit the consumption of the meat. For the cow we must not wait too long to see the effects of the different treatments which have just been indicated. We cannot rely upon the effects of internal medication.

[Operative interference in mares affected with nymphomania requires very serious consideration when it is a question of ovariotomy. This is due to the fact that the danger from peritonitis is very great, so that few practitioners are willing to recommend it, and few owners will assume the risk of operation, preferring to sell the animals for something rather than lose the whole value of the beast. Before undertaking such a serious operation, I would advise having recourse to extirpation of the clitoris, which may be removed with the knife, scissors, or thermo-cautery. My operations have always been done with the knife, but Dr. Hollingsworth reports a case with the thermo-cautery as very satisfactory. The patient should be cast, the parts thoroughly cleansed and cocainized by subcutaneous injection, and a classical operation performed. The operative procedure is simple, the dangers almost nil, and the results in the majority of cases are very satisfactory. The small percentage of cases which do not yield to this treatment may, if thought advisable, be treated by oöphorectomy, but many of them after recovery from the operation will still be found to have nymphomania. -W. L. Z.]

disinfected with sublimate solution of $1: 1000$. The operator's hands and arms should be washed with soap and water, then dipped in the sublimate solution. We use but two instruments-a bistoury with a hidden blade and an écraseur; they are to be immersed in an antiseptic solution. With the bistoury we make upon the upper part of the vagina, above the os, a simple puncture. When the instrument is withdrawn and the arm introduced again into the vagina, we put the index finger into the wound, then successively the middle finger and the thumb, and, spreading the fingers as much as possible according to the vaginal axis, we tear the wall of this organ, till the hand can penetrate into the abdominal cavity. We then practise ablation of the ovaries, which is very easy to perform with the help of Chassaignac's long écraseur. The only subsequent care consists in antiseptic washings of the vulva and of the posterior part of the vagina, when this is possible.-N. D. T. 


\section{WEAKENING OF THE GENESIC INSTINCT.}

Diminution of the genesic instinct, as far as it is a pathological phenomenon, is especially observed in females, particularly in the cow and the heifer; it is rare in males. This anomaly, which has a certain importance from a zootechnical standpoint, exists in varying degrees: in most cases the desire for copulation is rarely felt, or it is little marked, or lasts but a very short time, etc. We may, however, meet with a complete abolition of the genesic instinct; as a rule, this is transient, a character which distinguishes it from sterility.

Etiology. It comprises various causes, among which we must specially mention :

1. Diseases of the genital organs: vaginal or uterine catarrh (white flow), non-delivery, hydrometra, degeneration of ovaries and testicles, incomplete development, or other testicular anomalies.

2. Weakness of the genital apparatus, as a consequence of premature use of young animals in reproduction, too numerous and close gestations, abuse of stimulants, and onanism.

3. Torpidity of genital life depending on a phlegmatic temperament, a tendency to obesity, or to both causes at the same time. In the cow, a prolonged over-stimulating alimentation and permanent stabling act in the same way,

4. A general weakness due to an insufficient and poor alimentation, excessive work, convalescence, spinal troubles, etc.; also febrile affections of long duration, or constitutional morbid condition.

5. A "psychical disgust" felt by the males against females-the coat of the latter making a disagreeable impression upon them (Haubner-Siedamgrotzky); laziness of the males; the capriciousness of some stallions, which are very fiery for virgin mares and refuse to serve breeding mares.

Treatment. This should vary with the cause; it is, however, always proper to regulate the diet. Weak subjects should be given food which is rich in protein (leguminous, oats, rye, bread, raw eggs, etc.); fat animals should be subjected to a severe diet and should be given as much exercise as possible. The stallion must be mounted or harnessed to heavy loads; the bull can also be harnessed (if accustomed to it from a young age) after having set the nasal ring, or given the freedom of a fenced corral. Added to this, 
the genital activity may be regulated by avoiding too frequent copulations for young males, and in often bringing phlegmatic mares to the stallion, etc.

We resort sometimes to aphrodisiacs, particularly to cantharides powder, the effects of which are especially remarkable at the period of rut, when the heat is slight and imperfectly characterized. To large-sized cows we give every day, for several consecutive days, 5 to 6 grammes of cantharides powder in wine or between two pieces of bread; the dose for the horse is from 1 to 2 grammes. The doses of tincture of cantharides are : for the cow, 20 grammes; for the horse, 10 grammes; for the dog, 0.5 to 1 gramme. In the group of aphrodisiacs are contained all the condiments, the balsams, resins, essential oils, alcohol [pepper, 10 to 15 grammes, for the cow; ginger, cumin, wine (Beaufile), black mustard seed, myrtle berries, aloes (Houdmont and Zündel), flowering tops of sabine, cognac, beer mixture, alcohol, etc.]. All these medicaments produce hyperemia of the abdominal viscera, turgescence of the ovaries and testicles ; they may awaken genesic activity, but when the cause of the anomaly resides in the organic alterations these means are absolutely insufficient.

\section{IMPOTENCE AND STERILITY.}

By the term impotence we designate the lack of aptness of the male to reproduction, and by that of sterility the same anomaly in the female.

I. Impotence of the male. It appears under two very different forms : at times it is characterized by a mechanical impossibility of copulation (true impotence); at other times it is due to the want of spermatic secretion (sterility of the male). For clearness of description we will distinguish these two forms under the names of impotence of copulation and seminal impotence.

a. Impotence of copulation could also bear the name of relative impotence, for it is possible to obtain its cure. It is the result of functional troubles or alterations which prevent coitus. The genesic instinct may be very ardent and the sperm normal.

Its causes consist of very various external anomalies. The principal are :

1. Weakness of the genital apparatus (see Weakening of the Genesic Sense-etiology) produced by chronic cerebral or medullary 
affections. The term "impotence" is specially applied to this weakness. In some cases penile erection is weak, and the intromission of the organ into the vagina is impossible; in others the ejaculation takes place too early, while erection is yet incomplete or before the introduction of the member into the vagina.

2. Certain pathological conditions of the penis: fracture, phimosis, paraphimosis, tumors, anomalies of its size, etc.

3. Locomotion troubles, which render the service impossible or difficult: painful articular affections (spavin, curb) or weakness of the hind quarters (paraplegia).

The treatment of this anomaly must vary with its cause. The principal means to which we may resort are: 1 . Intensive alimentation and the use of electricity. 2. The mechanical relief of the animals at the time of copulating. 3. Surgical intervention (see Weakening of the Genesic Instinct-treatment).

b. Seminal impotence consists either in aspermia, i.e., lack of sperm production, or in azoospermia, or absence of spermatozoa in the ejaculated liquid.

Its most common causes are the complete absence of testicles, their atrophy occurring consecutively to inflammatory processes, degenerative or neoplasmic (carcinoma, aplasia, hyperplasia of the testicles, etc.). We may find seminal impotence in males which have testicles and the other genital organs of perfectly normal appearance, and in which ejaculation takes place perfectly, but the spermatozoa are wanting. This spermatic anomaly is observed in very old or very young stallions. Microscopic examination of the sperm informs us as to the presence or absence of the spermatozoa. In some cases this liquid may contain spermatozoa, but they are dead or immobile-phenomena which are probably due to chronic inflammatory states or degeneration of the seminiferous canaliculi (glandular utricles) or to their congenital aplasia. Seminal impotence is incurable.

II. Sterility : Infecundity of the female. It may be transitory (curable) or persistent (incurable). Its most common causes are: nymphomania, a lessening of the genesic instinct, obstruction of the os uteri by chronic inflammatory processes or by cicatricial contractions-an alteration observed in the cow and the goat (Franck, Harms), contractions of the vagina (sutures of the vulva, changes of relation and direction of the organ), the displacement of the uterus (retroversion, torsion, inversion, prolapsus), uterine 
neoformations, chronic inflammatory processes of the vaginal mucous membrane, of the uterus and Fallopian tubes. The treatment of these accidents is essentially surgical.

Incurable sterility is usually related to the absence of ovaries or to alterations of these organs; aplasia, hyperplasia, atrophy, cystic or carcinomatous degeneration; obstruction of the ovulary fossa by a circumscribed peritonitis (in the mare); also membranous neoformations, which cover up the ovary and prevent the exit of ovules; absence or partial ablation of the uterus, aplasia, and uterine atrophy; hermaphrodism in twin gestations, hybridity, etc.

In the cow Eloire has produced artificial sterility by introducing a foreign body (lead ball, pebble) into the uterus.

\section{ANOMALIES OF LACTATION AND ALTERATIONS OF THE MILK.}

Alterations of the milk are quite frequent in the cow. The veterinarian is more often consulted upon that subject than on the most common diseases of the bovine species. Our present knowledge on this question is particularly due to the researches of Fuchs, Haubner, and Fürstenberg.

Their principal causes are :

1. Irrational alimentation, either excessive or insufficient; a bad quality of food, or tainted. It is often the case with the cow that the alimentary conditions differ considerably from the natural regimen.

2. Gastro-intestinal catarrh, the general morbid conditions, and cachexia.

3. Diseases of the udder.

4. Influences of an infectious kind (micro-organisms), thermic, chemical, electric.

Among these alterations there are some which interest public hygiene and the sanitary police, because they may determine serious accidents in man and the animals of our various domestic species.

According to the standpoint accepted, we may establish a different division. We have adopted the following:

1. Anomalies of secretion : agalorrhea, watery milk, fat milk.

2. Alterations due to external influences, especially to the action of certain micro-organisms : curdled milk, milk not producing any butter, mucous, putrid, blue, red, or yellow milk. 
3. Alterations produced by foreign matters : bitter, rancid, coloring, poisonous, and medicamentous substances, and by pathological products, blood, etc.

\section{Agalorrhea: Drying-up of the Milk Secretion.}

Etiology. Agalorrhea, or agalactia, constitutes one of the first symptoms of the internal diseases, and especially of gastric or intestinal affections. But it may be produced by a food of bad quality, prolonged abstinence, fatiguing work, mastitis, incomplete development or fatty degeneration of the mammæ, and congestion of these organs at the period of heat. Certain phenomena of a psychical kind, such as the taking away of the young, or the change of stable, fear at the time of milking when performed by a stranger, etc., may equally influence the lacteal secretion.

Agalorrhea, quite common in primipara, is more frequent in the mare than in our other domestic females (we must not classify with agalorrhea the vice of "auto-suction," any more than that of suction by other animals. In some cases it occurs without any possibility of detecting its cause. Among the anti-galactopoietic medicaments we must mention: Atropia Belladonna, Hyoscyamus niger, Datura stramonium, Colchicum autumnale, Conium maculatum, and the iodide preparations.

Treatment. If the cause of agalorrhea can be recognized, it should be suppressed first; good food will then have to be given, and preferably liquid food (slops, malt); we may resort finally to galactopoietic medicaments, among which we recognize especially the stibiates (black and golden sulphuret of antimony), sulphur, fennel, juniper berries, the fruit of the cumin, anise, strophanthus, pilocarpine, etc. These agents are given in a separate or associated form.

Galactophor powder, which we employ, is thus composed:

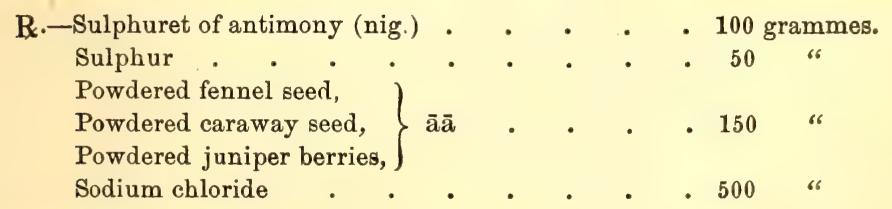

A tablespoonful at each meal for the cow, and a teaspoonful for the goat.

We may observe in the females of our different species an anomaly which is opposed to agalactia, and which consists in the unusual 
appearance of the milk secretion, "a phenomenon which is perhaps due to the imagination of females that they are pregnant and at the period of delivery." Eberhardt ${ }^{1}$ relates the case of a well-bred female retriever dog which was used to drop young ones every year in June; one year when she had not been served she showed symptoms of gestation and gave milk. Friedberger ${ }^{2}$ has observed similar phenomena in a bitch and in a mare. Delafond, ${ }^{3}$ Buffon, ${ }^{4}$ Bassi, ${ }^{5}$ Hering, and others have made similar observations. This anomaly, which is well known in woman, is not rare in the females of our domestic species. In the dog we often have occasion to observe a transient lactation which is independent of gestation and delivery.

"Enzootic agalactia," observed in the ewe and goat by Oreste, Brusasco, and several other authors, an agalactia which was accompanied by lameness, blindness, etc., belongs evidently to the domain of poisonings or infections.

\section{Watery Milk.}

Watery milk is characterized by a relatively too small proportion of fat and casein to the amount of water; its coloration is bluish and its specific gravity higher (the specific gravity of skimmed milk is greater than that of fresh milk). ${ }^{6}$

Etiology. The causes of this anomaly are : a too watery alimentation of poor quality, the exclusive feeding of malt, swill, turnips, and turnip leaves, etc.; stomachal or intestinal diseases, cachectic and hydremic conditions. Watery milk is sometimes a peculiarity of the race.

Treatment. The treatment ought to vary according to the case. Its principal indications are; change of regimen, alimentation with dry food, and the administration of stomachics. The following is a good formula :

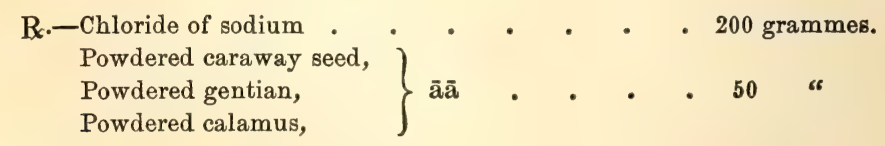

A tablespoonful at each meal.

1 Eberhardt: Magazin, 1859.

2 Friedberger: Münch. Jahresber., 1873-74.

3 Delafond: Recueil Vét, $1857 . \quad$ Buffon: Histoire Naturelle.

5 Bassi : Il Méd. Vét., 1860. 6 Feser: Vortr. f. Thierärzte, Erste Serie. 
If there is any affection of the intestinal canal, or of the blood, etc., it must be combated by an appropriate treatment.

\section{Fat Milk.}

This anomaly is observed in ewes which are subjected to an intensive regimen or an alimentation rich in leguminous plants; it may also appear during the rutting period of the female.

In lambs the ingestion of fat milk produces gastro-intestinal catarrh, diarrhea, etc. We remedy this accident by subjecting the mothers to a cooling diet and exercising them as much as possible. We must especially avoid giving them foods which contain too much nitrogen.

\section{Curdled Milk.}

A too rapid coagulation of the milk, also called caseification, constitutes one of the most frequent alterations of cow's milk; it is also observed in the goat.

Etiology. Its principal causes are: diseases of the digestive apparatus, acid food (malts), affections of the udder and particularly mastitis, hyperemia, and tumefaction of these organs in advanced gestation, nymphomania, overheating of the body by work. Sometimes it is the result of external influences: great heat, very great electric tension of the atmosphere during thunder-storms, damp stables, uncleanliness of the mangers.

According to Fleischmann, it is sometimes determined by the consumption of rusty grain.

Characters. Milk may begin to curdle a few hours after it is drawn, but coagulation happens especially when it is undergoing the boiling process. Milk which is drawn in the evening has more tendency to curdle than that of the morning's milking. Its acidity is very slightly evident (hence the name of sweet curdled milk). The cream forms but a very thin covering, and it is hard to make butter with it. In mastitis, the milk contains flaky clots as it leaves the udder.

Treatment. This is subordinate to the cause. A regulated ventilation, a mild and uniform temperature of the stable, are positively necessary; we should also look to the absolute cleanliness of the dairy utensils. Refrigerant apparatns must be recommended, and some alkaline may be added to the milk (a pinch of bicarbonate of soda or 0.25 gramme of salicylic acid per litre of milk). The in- 
ternal administration of alkalines, especially of soda, is very useful; we could not say the same of acids, which are, however, advised by scme authors. Harms recommends: mixture of fennel and sulphide of antimony, of each 400 grammes, to make six doses, to be given morning and evening. Affections of the digestive apparatus, of the udder, etc., must be the object of special treatment.

The cause of the coagulation of milk was discovered in 1857 by Pasteur; it is the lactic ferment which transforms the sugar into lactic acid, which determines the acidity of the milk and its coagulation. Lister, who has given to this organism the name of Bacterium lactis, has observed that a number of other micro-organisms. produce the acidity of the milk; according to recent researches they are: the micrococcus of osteomyelitis, the Staphylococcus albus, $S$. citreus, S. cereus albus, S. cereus flavus, Streptococcus pyogenes, Bacillus pyogenes foetidus, Streptococus erysipelatosus, Bacterium lactis acidi, Micrococcus et Sphoeroccecus lactis acidi, Bacterium lactis aërogenes, Micrococcus ovalis, and the Streptococcus coli gracilis.

\section{Milk which Does Not Produce any Butter.}

Etiology. Besides the mistakes which may be made in the preparation of the butter, the principal causes of this anomaly are: the diseases of the digestive organs and certain general morbid conditions, nourishment composed of poor or bad quality of food (turnip leaves), udder trouble (in advanced gestation), great heat or cold, and various alterations of the milk (curdled, rancid, putrid milk).

Characters. The milk curdles a short time after being drawn, or only when being boiled, the creamy surface is very thin; the preparation of butter is difficult or impossible. The cream clots and foams in the churn, but the fat does not gather into one mass. When the cream commences to grow old, small yellow spots are formed upon its surface (Harms); then it is unctuous and sticky, the butter it produces is granulous and becomes rapidly rancid.

Treatment. Besides the various galactopoietics (fennel, antimony, cumin, etc.), Haubner and Siedamgrotzky recommends hydrochloric and acetic acids. Alum has also been much praised (in a dose of 8 grammes three times a day), and chalk (in a dose of 50 to 100 grammes three times a day-Harms). When the milk is bitter, we must add to these medicaments a daily dose of 15 grammes of chloride of calcium. 


\section{Putrid Milk.}

Etiology. This alteration, which is quite rare, is occasioned by the introduction of agents of putrid fermentation in the milk. It is generally caused by the uncleanliness of the premises and utensils used in the stable, or vessels in which the milk is deposited. But it may also be dependent upon processes of decomposition which take place in the digestive apparatus after the ingestion of tainted food, or rancid bone-dust, etc. (Haubner and Siedamgrotzky). The principal microbes which determine this alteration are Bacterium termo and B. lineola.

Characters. Three or four days after being drawn gaseous bubbles (sulphuric acid, carburetted hydrogen) appear upon the thin surface of cream formed in the vessels in which the milk has been deposited; they soon open, leaving in their stead small cavities upon whose surface the cream has disappeared (cream which "eats itself away"). Little by little this assumes a dirty-yellow coloration and oil-drops appear in its substance; it takes on a bitter, rancid, putrid flavor (free fat acids, butyric acids, also valerianic, capronic, caprinic, caprylic acids), and does not produce any butter.

Treatment. The prophylaxis consists in disinfection and cleansing of the stables, litter, and vessels destined to receive the milk. When this alteration is due to a gastro-intestinal affection we must administer stomachics and antiseptics internally. We may try alkalines; sea-salt, hyposulphite of soda and hyposulphite of lime.

\section{Mucous, Oily, Viscous, Filamentous Milk.}

Etiology. Filamentous milk is the result of a microbic invasion. In a microscopic examination we find mobile, round, refracting corpuscles, the diameter of which is about $1_{\mu}$, and which appear under the form of isolated micrococci or streptococci (SchmidtMühlheim). The fermentative matter is furnished by the sugar of milk; the albumin or casein solutions are not at all influenced by these micro-organisms, which produce, on the contrary, a very active fermentation when they are deposited in a 1 per cent. solution of sugar of milk. The mucilaginous substance withdrawn from the filamentous milk by the action of alcohol presents exactly the reactions of vegetable mucilage (quince jelly); its production and the disappearance of sugar of milk are correlative phenomena. 
This alteration is frequently observed in the summer-time, when the dairy and stable are dirty and hot; it is sometimes due to an affection of the digestive apparatus, or to the use of decomposed or mouldy alimentary matter; according to Müller, it may be caused by the ingestion of butterwort (Pinguicula), a plant which is used in Sweden in order to produce it artificially.

Filamentous milk contaminates rapidly the whole amount of the draught.

Characters. Two days after being drawn the milk becomes thick, viscous, filamentous; its coagulation is incomplete; the cream is only deposited in a very small layer; it is difficult to obtain butter from it, and this possesses a viscous consistence and a stale and disagreeable taste. The milk often turns into a whitish mass, which is homogeneous, and so thick as not to run out when the vessel in which it was formed is turned upside down. By microscopic examination we find, besides the described microbes, round patches of eleminated casein (sphero-crystals).

Treatment. We should make a complete disinfection of the dairy with sulphuric acid and superheated steam, etc., and see to the cleanliness of the vessels destined to receive the milk. According to Schmidt-Mühlheim, a temperature of $65^{\circ} \mathrm{C}$. destroys the micro-organisms of filamentous milk. In cases of digestive trouble we must resort to stomachic and anti-catarrhal agents. Siedamgrotzky and Haubner recommend hemp leaves (daily dose, 15 grammes).

\section{Blue Milk.}

This alteration, particularly common during summer and spring, and in general during hot or stormy weather, is mostly found in damp dairies, where it may persist for years (Steinhoff has observed it in the same premises for a period of ten years). As a rule it disappears during the cold season-that is to say, in fall and winter, and sometimes also when the air has been purified by thunderstorms or rain. It has been observed that sick animals are predisposed to it.

Etiology. The blue coloration is given to the milk by a particular chromogenic bacterium, the B. syncyanum (Schröter). Fuchs, who described this micro-organism in 1841, has given it the name of Vibrio cyanogenus, and Ehrenberg that of Vibrio syncyanus. Fürstenberg has seen it upon the walls of infected dairies. 
According to Hueppe's recent researches, it appears in the shape of colorless rods, which are very mobile and from 2 to $4 \mu$ long, and which multiply by segmentation or sporulation. In sterilized milk (suppression of the vitality of the lactic ferment) it produces a gray, slaty, or dull-blue coloration; in bouillon, peptonized gelatin, etc., it determines green colorations, which afterward deviate to brownish, and become blue through the action of oxidizing agents. If cultivated on potato it gives a bluish-gray coloration. It decomposes albumin and produces a coloring matter which is similar to aniline-blue. The penetration of this bâcillus into the udder and the alteration of the milk before leaving these organs are yet to be demonstrated. Contamination seems to operate by the intermediary of air and by flies.

Milk which is very albuminous, a condition due to an alimentation rich in protein, or very alkaline, and which has but slight, slow, or want of coagulating properties, is predisposed to this alteration, contrary to what is observed in acid milk. According to Haubner and Siedamgrotzky, it often appears under the influence of a changing of food, through an abrupt change from certain food to another, from a dry to a green diet, especially when the pastures have received a dusting from plaster; it disappears, on the contrary, when the animals are led into spare pastures. Hueppe thinks that certain diets, diseases of the udder, etc., by retarding the acidification of the milk, may have a certain influence upon its production.

Characters. The phenomena which announce this anomaly are peculiar, and have been well described by Fuchs : one or two days after milking, when the milk commences to curdle and to become acid, we see upon the surface of the cream layer small, irregular spots hardly the size of a pin-head and of a clear blue color, which take little by little an indigo or sky-blue shade; they become extended in width and depth, invade the whole creamy surface, even the coagulated milk, the mass of which is sometimes colored blue. Some days later the blue milk is transformed into putrid milk by the development of microbes of putrefaction and of moulds; then it is of a dirty-gray shade. The alterations of albumin and fat give it an abuormal consistence, the casein becomes softened, the butter it produces resembles beef-suet, turns rancid, and soon shows blue spots or bands, often surrounded by a zone of greenish reflex. 
The consumption of blue milk is dangerous for animals and also for man; Mosler has mentioned accidents produced in man by its use; Steinhoff has observed similar occurrences in pigs.

Treatment. This alteration may be avoided by aëration, cleanliness, and disinfection of the stables, dairy, and utensils. The sulphurous acid vapors are perfectly suited for such disinfection. Haubner says the addition of acid whey may replace all other preventive means. In certain cases a change of diet is indicated. According to Zürn, the washing of the udder with an antiseptic solution, thus destroying the micro-organism existing upon the surface (or within it?), would often suffice to prevent this anomaly.

Red milk, which is quite rare, seems to be but a modification of the blue milk; the different chromogenic microbes seem besides to become easily transformed into one another $;^{1}$ in cultures of red milk, Schröter has obtained bluish, and others yellowish, cultures. It is sometimes determined by the Micrococcus prodigiosus (Cohn) or Bacteridium prodigiosum (Schröter), an elliptical, colorless, and mobile bacterium, which produces a coloring matter similar to. fuchsin, and is of mucous consistence, rose at first, and later blood-red; the multiplication of these bacteria goes hand-in-hand with the coagulation and acidification of the milk. Sometimes the red spots on the surface are mixed with blue or yellow spots, which are either isolated or agminated. The production of red milk is announced by the same modifications as those of blue milk.

Yellow milk is also an alteration which is very close to blue milk. It is determined by a colorless and mobile micro-organism, the Bacterium synxanthum (Schröter), which produces a coloring matter varying from a yelk-yellow to lemon-yellow, and which is similar to aniline yellow. This coloration appears quite often at the same time as the green and blue; it is more frequently observed in boiled than in uncooked milk. Within forty-eight hours the yellow spots begin to appear upon the surface of the boiled and coagulated milk; this becomes alkaline at the same time, the quantity of whey increases, and the clots become separated into flakes; within six days it is liquid, watery, and very poor in caseous flakes,

1 The Bulletin of the Central Society of Veterinary Medicine for the year 1877 contains an important communication from Dr. Larcher on red milk (for bibliography, see his paper). This anomaly, generally due to the action of chromogenic microorganisms, may be produced, however, in certain cases by red globules, which become: mixed with the milk. -N. D. T. 
and of a citron-yellow coloration. Lactic acid hinders the development of this bacterium. ${ }^{1}$

\section{Foreign Products in Milk.}

Among the foreign products of milk which present most interest we must mention: the abnormal sapid substances, flavoring or coloring matters, medicines and poisons, the pathological products, pus, blood, and the various micro-organisms of which mention has already been made.

1. Abnormal sapid substances. They are mostly produced by alimentary matters which are undergoing decomposition: rancid cakes, decayed or frozen potatoes or turnips, acid malts ; roots and leaves of turnips, which contain essential oil (Schumacher); fodder of bad quality gathered here and there; tree leaves, chestnuts, Jerusalem artichoke, twigs, dandelion, absinthe, male-fern, garlic, thistles, rape and linseed cake, oat straw and pea grass in too large quantities (Haubner, etc.). The anomalies of sapidity may also be due to unclean utensils : in all these cases the milk takes a disagreeable, rancid and bitter taste. Those which are brought into

1 Dr. Adametz, professor at the Agricultural School of Vienna, has just published an important work in which are explained the most recent acquisitions in the study of milk alterations.

The red coloration may be produced by the Bacillus prodigiosus or Monas prodigiosa (Ehrenberg), and by the Bacterium lactis erythrogenes (Hueppe). The Bacillus prodigiosus causes upon the surface of the milk the formation of a reddish pellicle, the shade of which is variable, but the coloring principle which it generates can only be elaborated in the presence of oxygen; the deep layers preserve their normal color. The Bacterium lactis erythrogenes colors in a uniform red shade the entire mass of the milk. This micro-organism is shown in the shape of very short and immobile rods; cultured in fresh milk it precipitates the casein and colors the serum: within two or three weeks the whole mass becomes a dark blood-red. Light hinders the action of this parasite.

The blue coloration is produced by the Bacillus cyanogenus or Vibrio syncyanus of Ehrenberg, a bacillus with sporulated extremities and animated by very rapid movements. It appears only in milk possessing a certain degree of acidity. We first see the formation of small blue dots upon the cream layer, which become enlarged, and are extended little by little in depth and invade the whole mass.

The yellow coloration is determined by the Bacillus synxanthus, a bacillus of small size, which possesses the power of individual motion.

These coloring principles are altogether different from aniline colors, and do not seem hurtful to health.

Milk constitutes an excellent medium of culture for a large number of microorganisms. The typhic, tuberculous, glanderous, diphtheric, anthrax, and cholera bacilli and the pneumococci multiply very rapidly in it. (Adametz, transl. by Guenot, in Recueil Vét., 1890.)—N. D. T. 
the milk by essential oils of aromatic plants (anise, cumin, fennel, etc.) are much better digested. The treatment consists in a change of regimen of the animals and in keeping the dairy vessels perfectly clean.

2. Flavoring matters. They reach the milk after the ingestion of plants of the Allium genus: yellow garlic (A. ochroleucum or suaveolens, Jacq.), chivet ( $A$. Schoenoprasum, L.), etc., or flavored medicaments: asafoetida, camphor, essence of turpentine, etc. They may also come from the atmosphere (disinfection of the stable or of the dairy with carbolic acid, etc.). Milk placed in a room which has been disinfected by carbolic acid has produced phenomena of phenic poisoning in a dog (Zorn). The experimental researches of Lawson Tait have given similar results.

3. Yellow, red, and blue coloring matters. Yellow and red coloring matters originate, some from vegetables : carrots, rhubarb, madder, saffron, radish, etc.; others, from normal or pathological organic products: bile (icterus), blood, pus, etc. In various affections of the mammæ we observe a colostrum of the color of egg-yelk. Apart from the blue milk of microbic origin, this coloration may also be communicated to the milk by certain plants: Ox tongue (Anchusa officinalis) field shave-grass, horse-tail or scouring rush (Equisetum arvense), yearly mercurial (Mercurialis annua), knotberry (Polygonum aviculare), buckwheat (Sarrasin) (P. fagopyrum), "muscaria à toupet" (Muscari cornosum), flowering rush (Butomus umbellatus), cow-wheat (Melanpyrum arvense), indigo plants, etc. (Hermbstadt).

4. Medicamentous substances. The following may pass into the milk; camphor, ether, essence of turpentine, asafœetida, chloroform, tartar emetic (Günther, Harms), aloes, arsenic (Hertwig, Spinola, Gerlach, etc.); hellebore, belledonna, atropine, henbane, stramonium colchicum (Ratti has observed a cholera-like epidemic after the use of goat's milk containing colchicine), hemlock ; morphine (Scherer, Gorup-Besanez) [we have never succeeded in demonstrating the presence of morphine in the milk, even after the administration of quite large doses of this agent], strychnine, senna, salicylic acid, carbolic acid; various salts (sulphate of soda, of magnesia, borax, etc.); combinations of bismuth; salts of lead (Gerlach), of copper (Grognier, Gerlach), zinc and iron ; compounds of mercurials (Klink) and iodoform (according to Lusansky, the simple administration of iodide compounds to the cow would give 
an iodated milk, which might be used with advantage in therapeutics, as for instance, in syphilis).

5. The milk may serve as a vehicle of pathogenic agents which are susceptible of producing serious disorders in man and animals. The disease assuming most importance in this respect is tuberculosis; there are authentic observations of its transmission to man, and numerous experiments made with milk from tuberculous cows have established that the infecting power of the latter is very great ${ }^{1}$ (see Tuberculosis). Lehmann says that the milk from tuberculous cows is very poor in fat and albumin.

In peri-pneumonia the milk is thick, similar to colostrum; it possesses a particular odor and decomposes very quickly (Fraas); its ingestion may produce vomitings in man (Hankold). In aphthous fever, Herberger has found the milk incompletely coagulated, viscous, mucous, similar to colostrum, of a disagreeable taste and odor, poor in sugar, in casein, and containing sometimes carbonate of ammonia. In bovine pest it is secreted in small quantities and its sugar is almost nil (Monin).

6. Milk containing red globules or hemoglobin. This alteration may depend upon different causes, especially on mastitis, or from contusions of the mammæ, also from brutal handling during milking, and from hard blows given by the calf, etc. (in this latter case usually but one teat gives red milk), congestion of the udder during the rutting period, and the irritation of these organs by turpentine substances (see Enzootic Gastro-enteritis), on hemoglobinemia, on the abrupt change from an insufficient diet to an intensive, nitrogenized feeding (clover pastures-Haubner and Siedamgrotzky), etc. Its characters are the uniform red (hematuria) or striated coloration (mastitis milk, the formation of a red, flaky precipitate, etc.). The treatment should be both local and general. (For further details upon bloody milk, see treatises on obstetrics.)

We ought also to mention the numerous bacteria found in the milk, notwithstanding that they cannot be made apparent as foreign elements. Besides the Bacterium lactis of Lister, the agent producing lactic acid, and which we have already mentioned, Warpmann has found in the milk Bacterium lactis acidi, Sphorococcus lactis acidi, Micrococcus lactis acidi, and Bacterium libatum lactis acidi. The micro-organisms which do not generate acids are,

1 Wesener: Krit. u. exper. Beiträge zur Lehre von der Futterungstuberculose. Freiburg, 1885. 
according to Löffler : the bacillus of the potato (Bacillus mesentericus vulgatus), which is the most frequent, the bacillus of gum (Bacillus liodermos), a butyric acid bacillus, a white bacillus, finally Oidium lactis, Penicillium glaucum, and different species of Aspergillus. ${ }^{1}$

1 Researches upon the subject of bacteria in milk have been made by Miquel, Von Freudenreich, and Knopf. In milk coming from a very cleanly kept farm, Von Freudenreich has found, two hours after milking, 9000 bacteria per cubic centimetre, and at the end of twenty-five hours, at a temperature of $15^{\circ} \mathrm{C}, 5,600,000$. Another sample has given him, within twenty-five hours, and at a temperature of $35^{\circ} \mathrm{C}$. 63,500,000 bacteria. Knopf, of Münich, has obtained still higher figures : 60,000 to 100,000 germs per cubic centimetre, almost immediately after milking, and within five to six hours, that is to say, in the condition in which the milk is delivered to the consumer, 200,090 to 6,000,000. (Annales de Micrographie, 1890, and Centralblatt für Bakter., 1890.)-N. D. T. 


\section{SECTION IV.}

DISEASES OF THE HEART AND LARGE BLOODVESSELS.

\section{GENERAL ANATOMICAL AND PHYSIOLOGICAL CON- SIDERATIONS ON THE DISEASES OF THE HEART. PHYSICAL DIAGNOSIS.}

THE diagnosis of the diseases of the heart requires a perfect knowledge of the anatomy of this organ and of its physiological phenomena in the normal and in pathological conditions. In our domestic animals there is much more difficulty than in man, because the physical examination of the heart is hindered by many circumstances. In quadrupeds, in fact, the heart is more or less completely hidden by the upper attachments of the anterior members; its percussion can only be practised from the left side. In draught horses possessing strong muscles, as well as in the fat pig, it is absolutely impossible. In small animals, its volume varies considerably, according to the race and the individuals; it is difficult to establish its dimensions, even approximately; moreover the number of pulsations do not permit us to define their characters. Before studying the cardiac affections in particular, we shall explain the principles upon which their diagnosis may be established.

I. The relations of the heart vary according to their nature. In the horse alone, the largest part of the left ventricle, that which corresponds to the halfmoon-shaped depression of the lung, is in direct relation with the costal wall-at the surface of the space situated between the third and sixth ribs; consequently, direct percussion of the heart is possible. Cardiac dulness which is perceptible above and behind the seventh rib (eight or nine centimetres behind the posterior edge of the shoulder) is abnormal ; in mediumsized animals its height is extended to about twelve centimetres above the floor of the pectoral cavity; but we must direct attention to the fact that the cardiac dulness begins but three fingerbreadths from the lower edge of the sternum (great pectoral). In the ox, 
where the heart is covered up by a portion of the lungs, a deep percussion reveals, however, a localized dulness between the fourth and sixth ribs. In other animals, the cardiac dulness, when it is perceptible, gives but vague information, on account of the very reduced dimensions of the heart. Sub-dulness may be due to the augmentation of the volume of the lung (emphysema), also to dextro-cardia, pneumothorax, or pneumo-pericardium; the increased dulness is produced by cardiac hypertrophy, dilatation of the heart, exudative pericarditis, hydro-pericarditis, atrophy or retraction of the left pulmonary lobe (tuberculosis, glanders); dextro-cardia may result from a pleuritic exudate, hydrothorax, and pulmonary hepatization; anteversion of the heart is the consequence of tympanites; backward displacement is caused by the adhesions of the pericardium to the diaphragm or to the lung, etc.

II. The weight of the heart, in animals of the same species, varies according to the size, the race, the constitution, age, service, etc. Slight or average increase or lessening of this weight are difficult to recognize. It is proper to take as a base the relation existing between the weight of the body and that of the heart. In the horse this relation is 1 per cent. ( 0.7 to 1.1 per cent., according to Franck); the absolute weight of the heart varies between 2 and 7 kilos; as a general rule, when this organ weighs more than $4 \frac{1}{2}$ kilos (except when the horse is of extraordinary size or of a fine breed), it must be considered as hypertrophied. The relation between the thickness of the right and left cardiac walls is as $1: 2.5$. (In the horse, this thickness is 2 centimetres on the right and $3 \frac{1}{2}$ centimetres on the left.)

In the ox and sheep, the weight of the heart is to that of the body as 1:220 (Colin); this proportion is nearly the same in the pig; in the dog it is as 1:90 (Colin, Negrini), and according to. Raabe, of 1.4 per cent. on an average.

III. In all domestic animals the cardiac or precordial bruit is normally perceptible at the surface of the fifth rib. In healthy carnivorous animals, it is felt on both sides of the chest, because the heart is situated nearer the median line and the diaphragm. The normal cardiac blow is very feeble in the horse, where it is only perceptible on the left; in horses of strongly developed muscles it is imperceptible. The number of pulsations is on an average 28 per minute in the stallion, from 33 to 39 in the gelding, from 34 to 40 in the mare, from 40 to 60 in the ox, from 70 to 80 in the 
sheep, the goat, and large-sized dogs ; from 80 to 120 in small animals. It is lessened in some cerebral affections (subacute encephalitis, immobility, affections of the cervical bulb and vagus nerve centres); it increases in fever, painful diseases, heart diseases, anemia, and hemorrhages. In a normal state, under the influence of exercise, excitement, etc., the cardiac bruit becomes fuller and stronger (this phenomenon is observed in most horses and dogs upon a first examination); it is violent in palpitations, valvular alterations, hypertrophy, in recent endocarditis and pericarditis, in hepatization or pulmonary indurations located upon the anterior part of the lobes, and during the course of serious febrile conditions ; in these latter, its intensity comes in contrast with the weakness of the pulse; the violent bruit is here the expression of the distress of the myocardium. Lessening of the force of the cardiac impulse is due to atony of the heart (insufficient innervation, weakening by very elevated temperatures, fatty degeneration and inflammatory alterations of the cardiac muscle), also to its remoteness from the pectoral walls, due to a pleuritic or pericarditic exudate, by hydrothorax or hydro-pericardium, pneumothorax, or pneumopericardium (rare), pulmonary emphysema, to adhesions with the thickened pericardium, etc. In the horse, the precordial impulse is perceptible upon the right side in dextro-cardia, in right cardiac hypertrophy, in febrile diseases (frequent); often, also, when the chest is narrow, it is felt slightly upon the right side. In pericarditis and endocarditis we observe, beside the cardiac impulse, friction or vibrating noises; these are related to valvular alterations.

IV. The heart sounds, perceived normally by auscultation and so called in contrast to all heart bruits which are pathological, must be distinguished as first and serond; the first is low, long, systolic (it corresponds with the ventricular systole); the second is short, clear, diastolic (it corresponds with the beginning of diastole). The interval between the first and the second sound is short (little pause), between the second and the first it is long (great pause). The two sounds are only positively recognized by this single character. The first is produced by the tension and vibration of the auriculo-ventricular valves; it is perhaps increased by the muscular bruit of the myocardium when it is contracting, by the blood circulation under a strong pressure in the ventricles, and by the concussion of the pectoral walls under cardiac impulsion; the 
second is due exclusively to the rotation of the heart and to closure of the semilunar valves. The precision and elearness of the heart sounds indicate the healthy action of its valvular apparatus.

$\mathrm{V}$. The heart bruits are endocardiac (intra-cardiac) or pericardiac (extra-cardiac).

1. The endocardiac bruits are distinguished as organic (due to an obstacle to the circulation) and anorganic or accidental (produced by irregular undulations of the valves without the existence of a true circulatory obstacle); the importance of these latter is entirely secondary. The organic bruits are produced by valvular alterations occasioning insufficiency or contraction. In contractions the liquid bruit is due to the vibrations of the blood-flow, which is forced through a too narrow opening; in insufficiencies it is produced by the return of the blood in the cardiac compartment which is situated above the diseased valve. The systolic bruits are blowing and buzzing; the diastolic bruits, on the contrary, are quivering and whistling. The first are generally determined by an insufficiency of the auriculo-ventricular valves, mainly of the mitral, sometimes by the contraction of the openings of the aorta and pulmonary vessels. The diastolic bruits indicate a contraction of the auriculo-ventricular openings, especially of the mitral orifice, or an insufficiency of the aortic valves. These alterations are much more rare in the right heart.

2. The pericardiac bruits are friction noises; they are distinguished from the endocardiac bruits by the fact that they are not intimately related to the act of systole or diastole.

In the horse we find quite frequently a doubling of the heart sounds. It is mostly the first which presents this anomaly; it may be observed upon subjects which are otherwise perfectly healthy; it should be counted among the anorganic bruits; it is very probably the consequence of an unequal tension of the mitral or tricuspid valves.

VI. In the horse and the ox we may investigate (count) the pulse at the external maxillary artery (more rarely the brachial, temporal, tibial, and the collateral of the canon); in small animals it is taken at the femoral artery. Outside of their number (see Cardiac Impulse) the pulsations also present peculiar characteristics which cause us to distinguish :

1. Regular pulse (rhythmic) or irregular pulse (arrhythmic), according to the equal or unequal intervals separating the pulsations; 
when a pulsation (every third or fourth, for instance) is wanting, we say that the pulse is intermittent; the intermission may be regular or irregular.

2. Equal or unequal pulse coincide with the regularity or irregularity in volume of the blood-waves. When small waves alternate with the larger ones the pulse is called alternative.

3. Strong or weak pulse (great or small, full or empty pulse) are expressions which indicate the degree of fulness of the blood-wave).

4. Hard or soft pulse (hard and soft artery)-depending upon the degree of distention of the artery. When the pulse is small and hard it is said to be filiform.

5. Accelerated or slow pulse-depending upon the more or less rapid gliding of the blood-wave under the finger.

Among the other anomalies of the pulse we must also mention: the dicrotic, or alternating, in which the curve of the pulse is interrupted in its descent, and the anacrotic pulse, in which the ascending part of the curve is irregular.

The intermittent pulse is often observed in animals which are perfectly healthy (slight troubles of heart innervation); it is also found in diseases of the heart and brain; we have seldom found it in gastric diseases, contrary to what others have advanced; in febrile diseases it is quite frequent, as well as the irregular pulse. The full pulse is observed in cardiac hypertrophy; the hard pulse in painful diseases (colics, tetanus, etc.).

The venous pulse is especially a symptom of cardiac weakness (febrile infectious diseases), valvular lesions, acute heart trouble (traumatic myocarditis), etc.; it results from an abnormal fulness of the right heart. It is ordinarily observed upon the lower part of the jugulars. A slight backward flow of the blood in these veins is observed in the normal condition of a large number of animals; it does not at all indicate the existence of heart disease. In the ox the venous pulse is very common; it has been very erroneously looked upon as a sure symptom of traumatic carditis and pericarditis. There is no true venous pulse except when the compressed blood-wave is very visible and ascends quite high in the jugulars.

\section{Hypertrophy and Dilatation of the Heart.}

If hypertrophy and dilatation of the heart constitute anatomically and clinically two affections, in practice it is very difficult to separ- 
ate them. They not only frequently coexist (in valvular lesions, for instance), especially when cardiopathy is old, but their differential diagnosis is almost impossible, on account of the similarity of the symptoms which they present.

The usual medical terminology indicates the close relationship existing between hypertrophy and dilatation; the first, in fact, is also designated under the name of active dilatation, the second under that of passive dilatation.

Pathological anatomy. Hypertrophy and dilatation are both characterized anatomically by an increase of the size of the heart; but whilst in hypertrophy this alteration is due to the thickening of the walls of the organ, in dilatation it is produced by the enlargement of its cavities and the distention of its walls.

1. We designate under the name of simple cardiac hypertrophy - - a form of augmentation of the volume of the heart which is quite rare-the thickening of the walls of the organ; in the great majority of cases we find at the same time a dilatation of the cavities; this is excentric hypertrophy, or active dilatation of the heart, a form to which we generally make allusion when speaking of cardiac hypertrophy. The existence of a hypertrophy which has been called concentric, and in which the thickening of the walls would correspond with the contraction of the cavities, is problematic. Under the name of false cardiac hypertrophy we designate the increase of the heart's size produced by the development of foreign tissues in the thickness of the myocardium.

True hypertrophies may be entire, partial (involving a single ventricle) or circumscribed (affecting one or several muscular pillars). In hypertrophy of the left heart, the much more frequent form, the organ is elongated, cylindrical ; in right hypertrophy of the left heart it is more or less flattened, and its length is increased; in entire hypertrophy it is round in shape and has often acquired extraordinary dimensions. Stephenson has observed the heart of a horse weighing 16 kilos; Gerlach has found one of $9 \frac{1}{2}$ kilos; Herran ${ }^{1}$ has seen the heart of an ox which measured 35 centimetres in length, weighed 18 kilos, and the walls of which were affected by a calcareous incrustation. The left wall of the heart is frequently thickened to twice or three times its normal size; its structure may be perfectly normal; in most cases, however, the myocardium is harder and of firmer consistence ; sec-

1 Herran: Journ. des Vét. du Midi, 1862. 
tions made into its thickness sometimes show ingrowth of interstitial connective tissue, which are dark-brown or marked with spots or clear streaks (pigment; fatty degeneration of the muscular fibres), according to the duration of the process. On the surface of these spots the muscle is soft and friable. When the right heart is hypertrophied, if we make a traumatic section, its wall remains rigid and its opening gaping.

2. In simple or passive cardiac dilatation the heart is increased in size by the enlargement of its cavities, and its walls are weakened and edged off in proportion. It is mostly found on the right side; when existing in the left heart, if we make a transverse section, the walls drop instead of remaining in a gaping condition. Here, also, the structure of the myocardium may be normal, but, as a rule, the walls are flabby, anemic, soft, friable, of a clear yellow-brown color, affected by fatty degeneration; at times they are very thin, even transparent in certain places, where the two serous membranes are directly back to back; finally, as secondary lesions, we may observe in the myocardium depressions to which have been given the name cardiac aneurisms. Cases of dilatation have been mentioned in which the size of the heart was more than doubled.

Etiology. Excentric hypertrophy of the heart depends upon various causes :

1. Violent exertions performed during work (idiopathic hypertrophy). This pathogenic influence is especially observed in racehorses and hunting-dogs-animals making exertions of short duration, it is true, but which are pushed to their greatest intensity. Reasons of the same kind determine that in a physiological state the heart of horses of a fine breed is more voluminous than that of common horses; for instance, the heart of the thoroughbred English stallion, "Helenus," weighed nearly seven kilos. The mechanism of idiopathic hypertrophy of the heart is that of muscular hypertrophies in general: the contraction of the muscles of the trunk during violent exertions determines a compression of the muscular arteries, the blood can no longer flow therein so abundantly, the arterial pressure increases and produces more violent contractions of the heart, and as a consequence hypertrophy of the myocardium results (Traube).

In very nervous animals, especially in breeds of trained dogs, we observe a special cardiac hypertrophy, the nature of which is 
as yet imperfectly known; we are ignorant as to whether it be a cause or a consequence of the nervousness of these subjects.

2. Vascular alterations which mechanically hinder the circulation, determining a secondary cardiac hypertrophy, which is also called symptomatic hypertrophy.

Among these alterations we must mention : aneurisms of the aorta ; congenital contraction, thrombosis, and the compression of this vessel by neoformations; atheromatous processes and arteriosclerosis.

3. Valvular lesions and troubles of the circulation to which they lead constitute the most frequent cause of cardiac hypertrophy. This hypertrophy well deserves the attribute of "compensatory," for Nature produces it in order to remedy the circulatory troubles.

4. Diseases of the lungs, emphysema, compression of both lobes or of a single one by exudates or transudates, pleural adhesions, glandered or tuberculous interstitial processes, by stoppage of the circulation in a part of the pulmonary vessels, increasing the blood pressure in the right heart, the walls of which become hypertrophied.

5. Pericardiac adhesions, by the constraint which they exercise upon the movements of the heart, produce also a greater activity. of this organ and its hypertrophy.

6. Finally, chronic nephritis becomes almost fatally complicated by cardiac hypertrophy. (Concerning the morbid sympathies existing between these two affections, see Chronic Nephritis.)

Passive dilatation of the heart constitutes generally the final stage of active dilatation. A time comes, in fact, when the hypertrophied heart no longer suffices for the exigencies of circulation; the blood pressure which is felt upon it dilates it mechanically. Fatty degeneration, endocarditis, myocarditis; the alterations of the myocardium in cases of intense fever, infectious diseases, poisonings, anemia, etc., determine cardiac dilatation by the same mechanism. A rapid passive dilatation may be produced in the course of acute pulmonary affections in which serious troubles of the circulation exist, and also a considerable elevation of the blood pressure in the right heart. Chronic pulmonary diseases, on the contrary, produce hypertrophy, except when the animals are extremely debilitated : in this latter case, on account of the want of nutritive matters, a dilatation only is formed.

Symptoms. Common cardiac hypertrophy and compensatory 
hypertrophy are so slowly developed that they may exist for years without becoming apparent by serious general troubles. Hypertrophies which are of some gravity, such as those accompanying chronic nephritis, are marked by a full pulse and a more intense cardiac impulse than in a normal state, with loud and clear sounds, and an increased extent of the precordial dulness. But this characteristic symptomatic picture is rarely observed, for hypertrophy through permanent insufficiency soon makes way for a state of dilatation, relaxation, of the heart, and idiopathic hypertrophy is exceedingly rare.

Most of the cases designated in veterinary publications and in practice under the name of cardiac hypertrophy are certainly only complications of active and passive dilatations, and seen in this. latter affection only. The general symptoms of this double affection are confounded with those of non-compensated valvular insufficiency, and differ essentially from those of common cardiac hypertrophy.

The increased extent of the precordial dulness is a symptom common to both forms of the cardiac dilatation ; in the dog it may be extended to the surface of the last false ribs. The relaxation. of the hypertrophied heart is marked at first by dyspnœa, palpitations, an abnormal pulse, cerebral hyperemia, and anemia, vertiginous attacks $(\mathrm{dog})$, extended circulatory troubles and their consequences. The attention is generally attracted by the strength of the cardiac impulse, which is palpitating, and perceptible on both sides of the body; this is sometimes shaken at each beating, phenomena which are to be recognized at a distance; but in some cases. the impulse is imperceptible; the heart sounds are irregular, rolling, or alternating; the first is strong, metallic, vibrating; the second is very weak or completely obliterated. ${ }^{1}$ The force of the

1 The more or less distinct doubling of the heart bruits is relatively common in the horse. It is most often observed at the first bruit (anapestic bruit-two short and one long), sometimes at the second (galop bruit, dactylic bruit, one long and two short) In most cases it does not seem to have the serious prognostic signification which is. attributed to it in man. It is generally explained by hypertrophy of the left ventricle and the unequal rapidity of function of the tricuspid and mitral valves (nondoubling of the first bruit) or aortic or pulmonary sigmoids (non-doubling of the second bruit). Perhaps, in the horse, it is the expression of a cardiac trouble produced by excessive and repeated exertions to which most draught animals are subject. Whatever the cause may be, it is an established fact that we may observe it upon horses used every day in the hardest kind of service without showing any serious. respiratory or circulatory troubles.-N. D. T. 
pulse is not at all in proportion with the violence of the impulse of the heart; as a rule, it is weak, almost inappreciable; under the influence of moderate exertions it acquires an extraordinary frequency, and becomes irregular and unequal; we observe very often a venous pulse. At rest the respiration is already considerably accelerated; work makes it dyspnœic (cardiac asthma, weak heart); it may become asphyxiating: the animals stagger, fall, and perish from apoplexy. In some cases we observe vertiginous attacks, tremblings, convulsions, and sweating-spells.

The circulatory troubles which occur afterward sometimes affect the encephalon (congestion or cerebral anemia, marked by vertigo and syncopal phenomena), at times the bronchial mucous membrane (bronchitis), the digestive canal (chronic intestinal catarrh), the liver (hepatic stage, cardiac liver), the kidneys (renal infiltration, albuminuria, and oliguria), etc. Finally, we note the appearance of cyanosis and dropsies (ascites, hydro-pericardium, hydrothorax, anasarca). The sternal and lower abdominal regions, and the legs become odematous. Sometimes the animals become emaciated and die of marasmus, at other times they are carried away by a consecutive pulmonary embolism due to the coagulation of the blood in the heart; finally, at times death is the result of cardiac paralysis, of cerebral hemorrhage, or of carbonic intoxication.

The symptoms which have just been explained are most frequently remarked in old dogs, appearing in an alarming dyspnoea or in attacks of vertigo; they are also observed upon brokenwinded horses, but they are rare in subjects of our other domestic species. Herran has recognized cardiac hypertrophy in an ox which wasted away and showed symptoms of dyspnoa; when this animal was obliged to make any exertions he would stop abruptly on account of the tumultuous beatings of the heart.

Differential diagnosis. In the majority of eases hypertrophy and dilatation of the heart are taken for a primary pulmonary disease-pulmonary congestion or pneumonia-because dyspnœa is the predominant manifestation of it, and that which always attracts the attention of the owner. Auscultation and percussion of the lung, the progressive evolution and long duration of the disease, lastly the phenomena observed with reference to the heart, enable us to make the diagnosis.

Cardiac palpitations are distinguished from hpertrophy by the intermittence of their manifestation, by the want of augmentation 
of the precordial dulness, and by the symptoms, which are most alarming. Hydro pericarditis and myocarditis (especially chronic myocarditis) are more difficult to distinguish from hypertrophy; their distinction is often impossible. The differential diagnosis with valvular lesions is relatively simple; these latter are marked by characteristic bruits.

Treatment. The principal indication of the treatment of hypertrophy and dilatation of the heart is to search for the causes of these troubles and to lessen their effects.

Let us first remark that it is always inadvisable to obstruct (by digitalis, for instance) the development of a compensatory hypertrophy. The patients should be protected from the various causes of excitement and fatigue; we must avoid riding or harnessing the horse or letting the dog hunt. Inactivity being necessary if we wish to obtain a cure, we find that the owners decide to sacrifice the animals in most cases. A nitrogenized alimentation is advantageous; it furnishes the cardiac muscle, which is forced into a hypertrophic condition, the necessary materials for the formation of muscular substance.

When the dilatation complicates the hypertrophy we must combat the relaxation and weakness of the heart by cardiacs, stimulants, and tonics. We administer digitalis leaves in small and repeated doses at short intervals (horse, 2 to 5 grammes; dog, 0.1 to 0.3 gramme per day, in an infusion). This agent moderates the activity of the heart, increasing the duration of its resting periods, and permits it to assimilate a greater proportion of nutritive elements; its prolonged use requires some precautions. In certain cases there is advantage in using caffeine (horse and ox, 5 to 10 grammes; small breed of dog, 0.1-0.5 gramme; large dog, 0.5 to 2 grammes), tincture of strophanthus (horse and ox, 10 to 25 grammes ; dog, 10 to 25 drops). In circulatory troubles we must resort to stimulants: (wine, camphor, alcohol, ether, veratrine). In associating these remedies, we may for a certain time counteract the insufficiency of the cardiac muscle, but the complete cure is only possible in cases where hypertrophy is idiopathic and when its causes may be completely suppressed.

[Iodide of potassium is a very valuable drug in this disease, as its action upon the heart is to induce rest of the organ, thus allowing more time for the circulation of the blood in, and the nutrition of its own tissues. In those asthmatic attacks which are induced 
by an overtaxed heart remarkable results are sometimes obtained. In the use of iodide of potassium in diseases of the heart, I have always administered it combined with digitalis, nux vomica, and coca in small doses, using the powdered drug. The dose of iodide of potassium for the horse is from 4 to 8 grammes daily and proportionate doses for other animals. Iodine may also be used in these cases in doses of from 0.50 to 1 gramme daily in the food.-W. L. z.]

\section{PERICARDITIS.}

Inflammation of the pericardium is a frequent disease in the $o x$, whilst it is very rare in the horse and other animals. ${ }^{1}$ Pericarditis of the ox is clearly characterized by its quite special nature, by its causes, which are of a traumatic kind ; finally, by its ordinary complication of myocarditis, which helps to modify its symptomatic expression. In the goat we have also observed a few cases of traumatic pericarditis, while in subjects of our other species this disease is recognized by internal causes. For reasons of an etiological, clinical, anatomical, and therapeutic nature, it is proper to examine in a special article the pericarditis of the ox and that of the goat.

\section{Pericarditis of the $\mathrm{Ox}$ and Goat: Traumatic Pericarditis and Carditis.}

Etiology. Traumatic cardo-pericarditis is by far the most frequent of the cardiac diseases of the ox. We may, indeed, question whether any pericarditis occurs in this animal which is not of a traumatic nature. A priori we must answer in the affirmative, for nothing prevents inflammation of the pericardium developing in the ox (as in the horse) after colds, during muscular or acute articular rheumatism, infectious diseases, puerperal septicemia, pleurisy, etc. Authors have incriminated all these causes at the same time as traumatism. In studying the reported observations, however, we are tempted to believe that most of the cases claimed to be rheumatismal.

1 Pericarditis is frequent in birds. According to its nature and age the alterations: produced by it are very variable. When it is recent the pericardium is greatly congested, unpolished, rough; at a more advanced period its folds are covered by a whitish deposit, of chalky appearance (rheumatismal pericarditis), or joined by inflammatory adhesions for the greater part of the surface; in certain cases we find an abundant liquid collection in it. Sometimes the birds which are affected die quite suddenly, at other times they waste away and die of consumption (see Larcher: Mélanges de Pathologie comparée).-N. D. T. 
pericarditis are in reality of a traumatic nature. It seems to be a habit with practitioners to call "rheumatismal" any pericarditis which is found at the autopsy, in the absence of a vulnerating body within the lesions. As a general rule no account has been taken of the fact, for example, that pointed foreign bodies may return into the stomach after having involved the heart. Anyhow, we must only advance the diagnosis of rheumatismal pericarditis with the greatest reserve. Pericarditis which appears in the course of a septic metritis-more frequent than the traumatic form-is but an isolated phenomenon, the terminal of a long series of organic alterations. (For pericarditis occurring in the course of consumption, see Tuberculosis.)

In the article on traumatic inflammation of the stomach and diaphragm we have mentioned the mode of penetration of foreign bodies into the pericardium; we must, however, remember that this organ is situated at a distance of a few centimetres only from the stomach, from which it is only separated by the diaphragm. This proximity explains the frequency of wounds of the pericardium and the heart by vulnerating bodies which have been accidentally swallowed. Pericarditis consecutive to penetrating traumatism of the chest, to fractures of the ribs, or to blows made by horns (Mathieu), is very rare.

Pathological anatomy. The alterations of the pericardium and of the heart are generally much marked; it is exceptional to find the alterations of inflammatory process close to its commencement. We find a liquid or solid pericardial exudate, adhesions between the heart and the pericardium, its thickening and dilatation, lesions of the myocardium, and secondary alterations in the lungs, diaphragm, etc.

The pericardial exudate is most variable in its composition. It is serous or fibrinous, purulent, hemorrhagic, putrid ; clear or milky, whitish, flaky, yellowish-gray, greenish-yellow, reddish, red-brown, dirty red, etc. ; on microscopic examination, we find elements in it of most varied figures : red and white globules, drops of fat, bacteria, etc. It is often mixed with gas, developed in the pericardium itself or derived from the reticulum; at times this gas has a more or less fetid odor, in other instances it is odorless (pneumo-pericardium). The quantity of exudate is sometimes considerable (ten to fifteen litres); the pericardium is much distended by it, it compresses the lungs, the heart, and especially the auricle and the large 
veins. When the disease is prolonged it ordinarily terminates in chronic dropsy of the pericardium (hydro-pericardium). The pericardial pouch more rarely contains blood which is collected in it after the perforation or rupture of the heart; a quantity amounting to several litres may be found.

Solid exudates are developed at the expense of false fibrinous membranes, in which the cellular elements have been transformed into a connective tissue. They appear in the shape of fibrinous blades or bands which are attached to the epicardium or distended filamentous proliferation between the heart and the parietal fold of the pericardium. The membranous layers which cover up the heart present special characters; they constitute spots of the color of egg-yelk or of an omelet, with a villous, rugous, and papillous surface, which increase the dimensions of the organs; this is mammillated or rugous upon its surface (cor villosum, cor hirsutum). The epicardium itself is thickened, and transformed into a lardaceous mass which may become incrusted by a calcareous substance and form a.globe with a solid shell around the heart. These exudates sometimes undergo fatty degeneration, or become reabsorbed leaving milky spots behind, also callous and cicatricial thickening.

The resistant neoproductions which establish adhesions between the heart and the pericardium represent spots, cords, or some kind of tendons provided with bloodvessels. Sometimes the pericardium is found to be so intimately joined to the heart, that it seems to be completely wanting (cardiac symphysis, ankylosis of the heart).

The pericardium may acquire the thickness of the thumb and over; it is often considerably dilated, its capacity may even reach fifteen litres. The thickenings and adhesions of the pericardium are signs of the long duration of the disease.

The heart is infiltrated with serum; its fibres are softened; we find circumscribed fatty degeneration in it, also centres of partial myocarditis, small intermuscular or cavernous abscesses which are provided with a pyogenic membrane. In certain cases, the cardiac wall is hollowed, the cavity containing the foreign body; in others it shows lesions from ulceration, which are dark red, and surrounded by a yellowish zone (wound of the heart). Sometimes the foreign body is located in the heart, at other times it is only more or less enclosed in the myocardium; finally, in other instances, it swims freely in the pericardium. Camoin has observed a canal which extended from the left ventricle to the reticulum, a canal 
through which the blood runs directly into the stomach. When the duration of the disease has been long, it is exceptional to find the heart hypertrophied or dilated, the compression exerted upon it by the pericardial exudate has almost always made it flaccid, small, and atrophied. ${ }^{1}$

In some cases we observe pleurisy, adhesions of the pleural folds, centres of pneumonia, adhesions between the heart and the diaphragm, as well as the alterations described under the subject of traumatic inflammation of the stomach and diaphragm.

Symptoms. The symptoms of pericarditis by foreign bodies follow those of phlegmasia of the stomach and diaphragm : chronic gastritis appearing without appreciable cause, the evolution of which is insidious and which is accompanied by painful manifestations when breathing, making expulsive exertions, frequent alvine evacuations, diaphragmatic palpitations, etc., enterorrhagias, considerable emaciation-troubles which are intractaule to all medicati $n$.

To these manifestations of chronic catarrh of the stomach are added characteristic cardiac and pulmonary symptoms, as well as general phenomena; in most cases they are developed abruptly after delivery, over-exertion, etc.

1. Cardiac symptoms. At the onset of the affection the heart impulse is strong, palpitating, bounding; later, as the exudate increases, it becomes weak, then disappears completely or is only manifested by an undulatory movement. Percussion, which is very painful for the animals, permits us to determine an increase of precordial dulness, the extent of which is proportioned to the quantity of pericardial exudate; quite frequently we perceive a tympanic resonance extend over the whole region of the heart, and which is due to the driving back of this organ by the liquid exudate and by gases coming from the reticulum or resulting from the decomposition of the exudate; it may be extended to the whole left costal region (pneumothorax, pleurisy, compression of the lung, and atelectasis). In dry pericarditis the palpitation shows a slight friction, which is synchronous with the movements of the heart; when there is a liquid exudate we often observe undulatory bruits.

Auscultation of the heart furnishes precise information; the ear

1 We have observed a case of traumatic pericarditis in which thirteen months elapsed between the accident and death. The foreign body (a nail) was fixed in the cardiac muscie. The heart was atrophied notwithstanding the small amount of exudate (Recueil Vét., 1889).-N. D. T. 
should be placed upon the surface of the left fifth rib, or in front of the anterior extremity, or immediately below the withers ; generally the sounds are absolutely normal, but less clear and strong, especially when masked by other bruits; blowing with the first or second sound of the heart is altogether exceptional, and indieates wounds of the valves or endocarditis. On the other hand, we perceive almost constantly characteristic pericardial bruits, which are variable according to the nature and quanticy of the exudate. When this is fibrinous, at the beginning of the disease, auscultation permits us to recognize a bruit of friction which is pronounced, and is produced by the gliding of the two folds of the pericardium. We may also detect crepitation, shuddering, whizzing, blowing, and rustling. In general, the bruit of friction is rare; it is only produced at the beginning of exudative pericarditis.

The liquid sounds are more frequent; at times there is a sort of splash (similar to the fall of a solid body upon a liquid surface), which at times may be heard at a distance, at other times it is a kind of snapping bruit, a gurgling, rattling fluctuation; in some cases there is a metallic tinkle, similar to that made by small drops; in others, again, especially when the pericardium is extremely distended, it is dull and obscure. While the solidium bruits are closely related to the displacements of the heart, the liquidium, on the contrary, may precede or follow them; quite frequently they disappear momentarily; in the large vessels we sometimes hear a particular blowing; these latter sounds, which are, as a rule, isochronous with the heart movements, are weakened or effaced when the exudate is reabsorbed.

2. Pulmonary symptoms. Compression of the auricles by the exudate soon produces a passive pulmonary hyperemia, which is marked by an acceleration of the respiration upon movement, by slight coughing spells, and by dyspnœa. The pulmonary affection dominates the scene, and may lead to the belief of the existence of pneumonia, of contagious pleuro-pneumonia, or even of tuberculosis. There are cases where the foreign body has produced pleurisy or pneumonia. The persistence of the vesicular murmur, the absence of pulmonary dulness, and the cardiac symptoms, are valuable signs. ${ }^{1}$

3. Symptoms furnished by the jugulars. Four to six

1 In cows affected by traumatie pericarditis, Cadéac and Brissot have observed a pulmonary blowing which is similar to tubular blowing.-N. D. T. 
days after the appearance of pericardial bruits, more rarely only at the end of eight to fourteen days, the jugular veins become enlarged and show a venous pulse; their enlargement is due to pressure exerted by the exudate upon the heart and large venous trunks. At the same time a cold œedema invades the fetlock, the jugular regions, and the larynx when the head is carried low ; this cedema is sometimes extended to the sternal region and even to the abdomen; it has only a true diagnostic value when it is accompanied by particular symptoms furnished by the heart; it may be occasioned by other diseases, and in traumatic pericarditis it is sometimes wanting.

4. General symptoms. The irregularity of the heart's action leads to modifications of the pulse. At the beginning it is much accelerated (120 pulsations or more per minute); later it becomes weak, filiform, sometimes very irregular; its frequency varies with the intensity of the cardiac affection. The temperature may reach $41-42^{\circ}$; it is distributed unequally upon the superficial regions, the extremities are at times cold, at other times hot; we observe chills and trembling. The general condition is greatly affected; the animals are very weak; they are anxious, and their look expresses suffering; the decubitus and the movements of respiration occasion pain and complaints; the extremities are distended and stiff, the elbows are turned from the body. Some patients preserve a standing position and avoid all movement, others stretch themselves upon the soil and refuse to rise, others lie down and rise at every moment. The visible mucous membranes are congested at the start; later they become pale, yellowish, or cyanosed. Emaciation increases ; we observe symptoms of hydremia, at times enterorrhagia; the animals succumb to the progress of marasmus and to the complications of pleurisy (asphyxia) or of pyemia [metastases in the internal organs, metastatic abscess (Schurink), etc.].

Pericarditis generally assumes a chronic course with periodical exacerbations. Death may, however, occur in a few days through perforation of the heart and intra-pericardial hemorrhage. A small number of cured cases have been reported as a result of the retrocession of the foreign body into the stomach. Between the start of traumatic inflammation of the stomach and diaphragm and the appearance of pericarditis, weeks, months, and even years may pass. Everything depends upon the shape of the foreign body, the use made of the animals, the alimentation, the number of gestations 
(progression of the foreign body during working exertions) and other accidental circumstances.

Differential diagnosis. Chronic troubles of digestion, increased activity of the heart, pericardial bruits, venous stagnation, œdema, the chronic course of the affection and its propressive aggravation, are enough for the definition of the diagnosis of traumatic pericarditis. At the beginning the coincidence of the three following symptoms is very significant: first, incurable chronic catarrh of the stomach; secondly, tympanic sound in the region of the heart; thirdly, respiratory troubles (dyspnœa, cough) are shown irrespective of any serious pulmonary alteration (Rothfritz).

In the ox, the frequency of traumatic pericarditis allows us to eliminate the rheumatismal form even when there is a complication of pleurisy; the cardiac symptoms counsel us to decide on the existence of the first. But this is not the case in the horse and the dog. By a careful examination it is always possible to avoid the confusion of traumatic pericarditis of the ox with pulmonary affections. (Concerning the differential diagnosis of pericarditis and endocarditis, see the following article.)

Treatment. We must not prolong the treatment of animals affected by traumatic cardo-pericarditis. On the contrary, we should have them slaughtered as soon as the diagnosis is established. All authors agree on this head. At the outset, when the diagnosis is still doubtful and life is not in danger, it is proper to regulate the heart's action by means of digitalis ( 2 to 5 grammes per day), caffeine ( 5 grammes), tincture of strophanthus (10 to 25 grammes), etc. We have tried to induce the retrogression of the foreign body by exciting the peristaltic movements of the stomach (tartar emetic, salts), but these means are inefficient as a rule. In some cases we resort to surgical treatment, which consists either in the puncture of the distended pericardium (Lydtin, Schmidt), or in the extraction of a foreign body which is perceptible to palpation or visible in the pericardium (Bastian). Meyer has advised this extraction by laparotomy and the opening of the belly. It is doubtful whether such means would give favorable results when pericarditis has already set in.

\section{Pericarditis of the Horse and Other Domestic Animals.}

Etiology. In the large majority of cases pericarditis of the horse, sheep, and dog, is idiopathic. It is very often of a rheuma- 
tismal nature and results from colds, as frequently happens in the horse; it may also be developed in the course of infectious diseases, muscular or acute articular rheumatism, septicemia, contagious pneumonia of the horse, and simple pneumonia. It is sometimes the consequence of the propagation upon the pericardium of an inflammation from a neighboring organ (endocarditis, pleurisy, myocarditis, pneumonia); it sometimes accompanies an empyema developed as a consequence of the opening of a pulmonary abscess in the pleura-here it always starts on the external fold of the pericardium. It may also appear in the course of chronic nephritis. According to Anacker, sheep are subject to an enzootic pericarditis, which is usually fatal within a few days.

In very rare cases it is of a traumatic nature. In a horse, Henniges has found, in the right auricle, a sewing-needle which had reached this place through the osophagus. Finally, it may occur consecutively to fractures of the ribs, to the penetration of shot into the pericardium ( $\mathrm{dog})$, etc.

Pathological anatomy. At the beginning we observe congestion, ecchymosis, and a turbid tumefaction of the serous membrane, which is covered by a gelatinous exudate, spread in a thin layer and easy to detach. According to the nature of the affection, we find in the pericardium a serous or fibrinous exudate (cold), hemorrhagic, purulent, putrid (septic infection), the quantity of which is very variable. In the $\mathrm{dog}$ and the sheep pericarditis generally takes a chronic type; its lesions are those of hydro-pericarditis. At a more advanced period the epicardium becomes covered with rugous lardaceous neoformations (cor villosum), and the heart forms adhesions with the pericardium (cardiac symphysis); it is hypertrophied or atrophied. In the horse, Prakke has found it reduced to the size of a child's fist; in some places its walls were only as thick as a playing-card. The other alterations are similar to those of pericarditis of the ox.

Symptoms. In the beginning the impulse of the heart is palpitating, tumultuous ; later, in proportion to the increase of the quantity of exudate, it gradually weakens; the precordial dulness is extended upward and backward; we observe bruits of friction; the pulse is accelerated, small, irregular, unequal; the respiration is dyspnœic; we observe a venous pulse, the temperature varies from $39^{\circ}$ to $40.5^{\circ}$, etc. In the horse, in one case, Bruckmüller only observed fever, the obliteration of the precordial impulse and diffi- 
culty of the respiration. The course of pericarditis is sometimes acute, at other times chronic; in the acute form, death may happen suddenly through heart rupture. In the dog, the symptoms of hydro-pericardium are the following: intense dyspnoea, cyanosis, swelling of the jugular veins, imperceptible cardiac impulse, which is undulatory or chopping; the pulse is rapid and irregular; we also see dropsies, emaciation, and marasmus.

Differential diagnosis. Pericarditis may be confounded with endocarditis and pleurisy, but it is easy to distinguish it from pulmonary affections. The differential diagnosis of pericarditis and endocarditis must be based upon the very special characters of the bruits localized in the pericardium; these are friction bruits which the observer thinks he hears immediately below the ear; they are sometimes felt, and they are not so closely related to both phases of the cardiac rhythm as the endocarditic bruits; besides, the heartsounds are absolutely normal and without intimate connection with the pericarditic bruits. It is more difficult to distinguish it from pleurisy at the beginning; this latter trouble is also accompanied by a bruit of friction, which, however, is rather isochronous with the respiratory movements than with those of the heart, and besides the circulatory troubles are wanting. Sometimes pericarditis is secondary and is related to the pleural inflammation. In doubtful cases we must conclude the existence of pleurisy (Röll), which is relatively much more frequent than inflammation of the pericardium.

Treatment. In the horse and dog pericarditis is much less serious than in the ox, and its treatment offers many more chances of success. It consists in avoiding excitements, fatigue, and in the use of refrigerants and antiphlogistics: applications of ice or permanent irrigation of cold water; this latter method is the most advantageous for the horse. Internally we give digitalis (for the horse, 10 to 12 grammes per day; for the dog, 0.1 to 0.3 gramme); we may also use tartar emetic and salts. If the trouble shows a chronic course we must resort to the drastics and diuretics. When the quantity of exudate becomes threatening to life, or if it remains stationary, we may puncture the pericardium by means of the trocar. In the horse we practise the puncture in front of the anterior edge of the fifth and sixth ribs, a handbreadth above the floor of the pectoral cavity; it is proper to make a preliminary incision of the shape of a buttonhole into the skin in order to facilitate the introduction of the instrument. In subjects of our small species, 
we must use a capillary needle and Pravaz's syringe. In the dog, we puncture in the centre of the dull zone. The general weakness and atony of the heart must be combated with stimulants: wine, camphor, coffee, alcohol, ether, etc.

We designate under the name of hydro-pericardium or dropsy of the pericardium the collection in it of a variable quantity of transudated liquid. Hydro-pericardium always constitutes a secondary disease which is produced by mechanical obstacles to the circulation of the blood (chronic heart diseases, chronic nephritis, hydremia). From a clinical standpoint it is characterized by the disappearance of the heart impulse, weakness of the sounds of this organ, and the increased extent of the precordial dulness.

\section{PALPITATIONS AND "ABDOMINAL PULSATIONS" OF THE HORSE: CHOREA OF THE DIAPHRAGM.}

Definition. Under the name of "palpitation" in the horse morbid conditions have been described which have nothing in common between themselves, and authors have advanced very different opinions upon the subject. If in certain cases palpitations observed in the horse seem identical with those of man (which are due to a cardiac neurosis), they mostly differ radically from these, and are produced by diaphragmatic spasms. Several reasons, however, have induced us to outline these various complications in a simple article: first, the pathology of the majority of them is of the most obscure kind; again, all are marked by convulsive movements of the chest and abdomen ; finally, all have been confounded under the generic denomination of palpitations.

1. True palpitations are the expression of a cardiac neurosis without any apparent anatomical alterations. Some animals are predisposed to it. Among the causes which determine it we mention: intense excitement produced by excessive exertions, brutal use of the whip (Soumille), catching in the tether during the night (Johne), a too severe pulling upon the reins by the rider ( $\mathrm{Al}$ brecht), etc. The increased force of the cardiac impulse which is observed in the course of hypertrophy of the heart, chronic endocarditis, valvular lesions, must be distinguished from palpitations; the same is the case with the bounding impulse which is observed in anemia, leukemia, and in cases of serious disturbance of general nutrition or heart atrophy. 
The symptoms of nervous palpitations are the violence of the precordial impulse (in some patients it shakes the thorax or the whole body, and is extended some distance); the acceleration of the circulation and of the respiration, the small irregular pulse; restlessness, anxiety, sweating-spells. Auscultation determines the intensity of the sounds, which are sometimes not very distinct, the augmentation in force of the cardiac systole is perceptible upon the abdominal aorta when the ear is placed upon the dorsal region opposite this bloodvessel; we have also observed a venous pulse and visible pulsations upon the carotids (Milanese). We may observe true attacks, during which the animals frequently expel excrements and stretch themselves as if to urinate. In some isolated cases the disease ends with paralysis of the heart or cerebral apoplexy ; in most of these a cure is produced after a variable time, but the attacks may be repeated. The synchronism of the thoracic shaking with the beatings of the heart is a very important diagnostic symptom.

2. Diaphragmatic spasms produce phenomena which are similar to palpitations, with which they have often been confounded by authors. They have also been designated under the name of $a b$ dominal pulsations. They are produced by various causes. Catarrh and inflammation of the stomach or intestine may cause them in a reflex way or through the propagation of the inflammation to the diaphragmatic partition. Bril has seen them appear after vomiting; Berghuis observed them in cases of overloading of the stomach; Oreste, in gastric disturbances, in colics, and after heavy meals; Grosswendt, in enteritis ; Leblanc, Wörz, and others, immediately after the imbibition of cold water; Boiteau, after the ingestion of mouldy hay-that is to say, as the expression of a poisoning; Cartwright, after the administration of aloes pills. It is possible that the cardiac palpitations are accompanied by morbid contractions of the diaphragm - that the diaphragmatic nerve, passing below the base of the heart, is irritated by the violent movements of this organ (Haubner-Siedamgrotzky).

The diaphragmatic spasm, designated very appropriately under the name of chorea of the diaphragm, is accompanied by particular symptoms which are very remarkable. We observe in the left costal region and in the corresponding flank, shocks which at times shake the whole body; they are usually accompanied by a short, jerking expiration (Boiteau, Hering), and by a dull sound which is 
heard at a distance of several paces (Simonin and others); these abrupt and violent shocks, the number of which rarely exceed ten to fifteen, are perceived very distinctly along the diaphragm (Grosswendt); they are gradually attenuated toward the front along the thoracic cavity, or backward in proportion as the point examined is in more or less close proximity to the location of the pelvis; there are cases where they seem localized to a circumscribed region of the diaphragmatic partition; in some patients they produce a hiccough (Wörz) They are mostly not isochronous with the beatings of the heart, and these are not palpitating, but normal, or even weakened. While they do not seem to have any connection with the cardiac systole (Berghuis, Simonin, Boiteau, Bril, Wörz, Hering, Grosswendt, Soumille, etc.), it is generally admitted that they are due to an irritation of the phrenic nerve by the movements of the heart.

Besides these symptoms we often observe a quick and small pulse, an acceleration of the respiration, trembling, anxiety, gaping, frequent extension of a posterior member, manifestations of pain, etc. The duration of the affection varies from a few minutes to two or three days, very rarely longer, though Leblanc has seen it persist for three weeks. The prognosis seems to be less favorable than for palpitations ; of eighteen patients observed by Cartwright six died. ${ }^{1}$ The frequency of the pulsations on the left side (Cartwright found these twelve times on the left in sixteen cases) is undoubtedly due to the situation or distention of the stomach.

Treatment. This consists of the use of antispasmodics, morphine (subcutaneous injections of 0.4 to 0.6 gramme of hydrochlorate of morphine in the horse), bromide of potassium, hydrate of chloral ( 25 to 50 grammes). For true palpitations we may also have recourse to cold irrigations upon the heart region, to irritating cutaneous frictions, and to derivation in the intestinal canal by means of drastics.

Bleeding, which was formerly much favored, is only of doubtful efficiency.

The obscurity which still covers the nature of the morbid con-

1 This gravity of diaphragmatic chorea, as represented by Cartwright's statistics, must be considered as altogether exceptional. Of nine patients which we have observed none have died, recovery being always produced rapidly, whatever the treatment applied. - N. D. T. 
ditions of which we have just spoken is occasioned by the attempt to identify cardiac palpitations and the diaphragmatic spasm.

In comparing the abdominal pulsation to a spasm of the diaphragm we agree with most authors (Apperley and Castley, Beeson, Boiteau, Goubaux, Hering, Cagny). The spasmodic contractions of the diaphragm explain perfectly the intense abdominal shocks. A simple circulatory trouble in the posterior aorta, and still less the aneurism of this bloodvessel, could not produce such serious. effects; the same is the case with the isolated contraction of the abdominal muscles (Delafond), which would make these phenomena similar to expulsive exertions. ${ }^{1}$ Abdominal pulsations have been attributed to an alteration of the hepatic and splenic arteries (Milanese), but this is a purely imaginary theory. Palpitations have also been confounded with the phenomena produced by thrombosis of the crural arteries and of the posterior aorta. From these considerations we must conclude that abdominal pulsations of the horse form an affection which is similar to hiccough in man (neurosis of the diaphragm). They may appear isolated or may coexist with cardiac palpitations.

True palpitations have been observed in the dog and the horse (Fricker, Brusasco); Fricker has seen these in a pet dog which was cured in thirteen days by the administration of small doses of digitalis. In a similar case Brusasco has used with advantage hydrate of chloral, given in doses of 3 grammes.

\section{MYOCARDITIS: INFLAMMATION OF THE CARDIAC MUSCLE.}

Etiology. Myocarditis, which is very rare and difficult to recognize in our animals, is ordinarily of an infectious nature. It may be produced by aphthous fever (Johne), septicemia, pyemia, tuberculosis (Johne), contagious pneumonia of the horse, etc.; in certain cases it is due to colds and to excessive exertions. At times it occurs as a secondary affection through propagation to the myocardium of an inflammation localized at first upon the endocardium

1 The existence of this phase of the disease has been irrefutably established by Delafond (see Bull. Soc. centr. Vét., 1851). In two horses used for surgical experiments we have observed these abdominal shocks, which seemed to originate in the flank, and, like Delafond, we were able, after a laparotomy, to recognize that they were not produced by the diaphragm, but really by the abdominal muscles, and especially by the ilio-abdominalis.-N. D. T. 
or upon the pericardium. Wounds of the heart, which are quite common in the ox, rarer in the horse, commotions and contusions. of this organ (Trélut), may also occasion it.

Pathological anatomy. The alterations of the myocardium are very varied; they depend upon the nature of the trouble, whether there is a parenchymatous, interstitial, indurated, or suppurative myocarditis; but they are always more or less circumscribed, never diffuse.

1. In acute parenchymatous myocarditis, which is extremeiy rare, the inflammation does not exclusively involve the muscular fibres; it is accompanied by an interstitial elementary infiltration. Johne has described, in the ox, two cases of this form of myocarditis; the macroscopic alterations consist of softening centres, which are reddish-gray, yellowish gray, or yellowish-white; on the surface of these lesions the fibrillary aspect of the muscle had disappeared; on section the tissue seemed homogeneous. With the microscope were observed the disappearance of the transverse striæ, granulous. turbidity of the muscular fibres, and a cellular infiltration of the interstitial connective tissue, which was so abundant that the muscular fibres were separated from one another by layers of embryonic. cells which were three to five times thicker than the fibres themselves. We have observed a case entirely similar in a horse which had died from contagious pneumonia. At the autopsy of a mare Sanson also found parenchymatous alterations, ecchymoses, and a yellowish serous infiltration of the myocardium.

2. Indurated chronic myocarditis is marked by the presence, in the myocardium, of milky spots (cardio-sclerosis), true cicatricial centres, with which the muscle is filled and which are especially found toward the apex and in the wall of the left heart. These indurations are always depressed upon the surface of the heart; often we find quite deep excavations upon their area (cardiac aneurism). In some cases we find the muscular fibrils undergoing a process of granulo-fatty degeneration and surrounded by an abundant fibrous connective-tissue neoformation (Friedberger). The process has as an initial lesion a cellular infiltration of the interstitial connective tissue; the induration, the fibrinous transformation, characterize its previous stages; this state leads usually to secondary hypertrophy of the heart. ${ }^{1}$

1 Delamotte has mentioned two cases of cardiac sclerosis in the horse. In one the microscopic examination of the myocardium has been made by Montané. The mus- 
3. Suppurative myocarditis (abscess of the heart) is mainly observed in pyemia, in puerperal metritis of the cow, in omphalo-phlebitis of the foal (Venaert). Sometimes abscesses of the heart are very small, miliary and disseminated throughout the connective tissue, where we see them proliferate until their dimensions are that of a hazel-nut or a chestnut; they are generally caused by embolic infarct (metastatic myocarditis). Abscesses of the heart have been observed in the horse and the ox ; in this latter they may undergo caseous transformation and become incrusted with calcareous salts ("ossification of the heart").

Cardio-sclerosis is not always developed at the expense of the inflammatory process; according to Ziegler, it would frequently be the expression of myomalacia (softening of the myocardium) which is consecutive to necrobiosis by cardiac anemia, and which has often been designated under the name of parenchymatous myocarditis. Cardiac myomalacia occurs generally as a consequence of hemorrhagic infarctus which is produced in the course of an arteriosclerous or embolic process; this infarctus leads sucessively, within a limited territory, to arterial anemia, softening, caseification, resorption of detritus, and finally cicatricial induration. In circumscribed centres of cerebral softening and in renal infarctus, the interstitial connective cicatrices are formed in the same way.

Degenerations of the myocardium observed in the course of infectious diseases represent, so to speak, the first phase of myocarditis, with which they are closely related, but they are much more frequent than myocarditis itself. Among these alterations we may mention: turbid tumefaction, fatty and granulous degeneration, etc. The myocardium, which is flaccid, friable, as if cooked, shows an abnormal coloration, but the cellular infiltration is wanting. The symptoms are similar to those of myocarditis. All these degenerative lesions lead to atony of the heart.

Symptoms. The symptoms consist especially of a functional

cular tissue was overrun by a partial sclerosis located upon the pericardial side, and penetrated in some places the thickness of the ventricle, reaching even as far as the endocardium. The muscular fibres had undergone either colloid degeneration or fatty degeneration, with a return to the embryonic form in both (Revue Vét., 1890). Quite recently we have seen, in a horse affected by chronic valvular endocarditis, a good specimen of interstitial myocarditis, which was characterized by the presence of bands and islands of fibrinous connective tissue disseminated in the thickness of the walls of the left ventricle, and which were accompanied by degenerative alterations and atrophy of the muscular bundles or fibres.-N. D. T. 
trouble of the myocardium, with weakness or insufficiency of the heart; they are nearly the same as in advanced hypertrophy. The precordial impulse, which is strong, irregular, tumultuous, is felt over a greater surface; the eye and ear sometimes perceive it at some distance. The pulse is weak, irregular, often hardly felt; in the horse we may count 80 to 120 pulsations and more per minute. The respiration is accelerated and difficult. We observe also great weakness, trembling, and spells of vertigo. The temperature is more or less high according to the nature and causes of the disease. When this lasts long, phenomena indicating the existence of an obstacle to the return circulation become apparent.

There are cases where inflammation of the myocardium develops very rapidly : the patient may suddenly fall as if struck by a thunderbolt (aphthous fever, tuberculosis). Death happens suddenly through rupture or paralysis of the heart. In acute or suppurating parenchymatous myocarditis we frequently observe this course and termination.

Differential diagnosis. It is very hard to distinguish myocarditis from several other cardiopathies, especially passive dilatation, endocarditis and pericarditis; at times, in reality, the symptoms of these affections have nothing characteristic. As a general rule, we may conclude the existence of myocarditis when, with serious symptoms of cardiac distress, we do not observe any indication of organic alteration of the heart (murmurs, friction bruits, etc.).

Treatment. The treatment of myocarditis must be that for cardiac weakness. Excitements and exertions must be avoided, the myocardium should be sustained by a substantial alimentation, stimulants, digitalis, caffeine, strophanthus, and the administration of antifebriles. The principal indication is to combat the primary affection. Subacute myocarditis does not yield to any treatment.

[The veterinary publications contain a few cases of ossification of the auricles of the heart which have been observed only in old horses. It is mostly located upon the right auricle; at times it is bilateral but irregularly developed, the left auricle being always the least altered. Sometimes it hardly reaches the size of a silver dollar, at other times it is located upon the whole ceiling of the auricle (Barrier and Veret, Nocard, etc.), exceptionally this is entirely involved (Renault, Barthélemy). In a case related by Chuchu (Bull. Soc. centr. Vét., 1884) the right auricle, which was completely ossified, was five times its normal size and weighed 1800 
grammes; at its superior part it was hollowed by a large orifice, like the opening of a large shell, with which the vena cava corresponds. The horse at the autopsy of which this lesion was found was but eight years old; for three years he had done excellent draught service without ever showing any symptoms of uneasiness before the last weeks of his existence. In less than two months he became very thin and weak, to the extent of not being able to rise. In most cases the ossification of the auricle of the heart does not produce any serious trouble of the circulation, because it is limited and isolated, without any concomitant lesions of the orifices and valves. It concerns the anatomo-pathologist much more than the practitioner.]

\section{RUPTURE OF THE HEART.}

Etiology. Outside of the action of traumatisms, this accident is caused by certain alterations of the myocardium which lessen the resistance of the walls of the organ; at times they simply predispose to rupture, in other instances they end in occasioning it.

Among these alterations we must mention : inflammation, softening, abscess formation, weakening of the walls by cardio-sclerosis and aneurism, senile degeneration of the muscular fibres, endocarditis and pericarditis, atheromatous degeneration of the base of the aorta, echinococcus and the nematode worms (Spiroptera) located in the walls of the heart, etc. The principal causes which determine it are commotions of the trunk, violent falls (Hertwig), over-excitement produced by coitus (Hering), the operation of ascites in the $\operatorname{dog}$ (Stockfleth, and acute tympanites in the ox (Anacker, Mayer, Perdan).

Pathological anatomy. Besides the primitive alterations of myocarditis, we find a more or less long tear in the wall of the heart ( 3 to 4 centimetres on the average); it is mostly located upon an auricle, at the base of the aorta, or of the pulmonary ariery (see Rupture of the Large Bloodvessels). The pericardium is distended by a blood collection (hemo-pericardium).

Symptoms. The tearing of the heart is announced by a sudden falling, which is apoplectiform; in sinking, the horse sometimes. utters a piercing cry (Alers); in other cases the respiration becomes. very accelerated; the animal, taken with vertigo, trembling, staggers, drops, and presents symptoms of internal hemorrhage, and 
death happens in convulsions, mostly in less than one hour, sometimes only after several hours.

It is an accident without a remedy. Any treatment is illusory.

\section{ACUTE ENDOCARDITIS.}

Inflammation of the endocardium is much more frequent than that of the myocardium, and it is certainly more common. The horse, ox, and pig are the animals most affected by it; it is relatively rare in animals of our small species; we have observed it but once in the cat. [It is perhaps one of the most common inflammatory diseases in the horse, with the possible exception of pneumonia. Several instances have been brought to my notice in which this condition has been diagnosticated as pleurisy, and there is no doubt that many unrecognized cases are considered and treated as other diseases.-W. L. z.]

Etiology. Acute endocarditis is usually produced by infectious agents which are suspended in the blood. It may happen as an epiphenomenon of a number of infectious diseases: pyemia, septic metritis, aphthous fever, etc. In the horse, in some cases, it complicates simple pneumonia, contagious pneumonia, founder, and violent colics. In the ox it appears often in the course of acute articular rheumatism (Meyer, Ruchte). In the pig, Roth has also seen rheumatismal polyarthritis accompanied by a fatal acute endocarditis. The inflammatory processes of the myocardium and pericardium, of the lungs and pleura, are in some instances propagated to the endocardium. Cold seems to play an important etiological rôle in the horse (idiopathatic endocarditis à frigore-Trasbot). Endocarditis is more rarely of a traumatic nature (Ebinger has observed endocarditis in a cow as a consequence of the fracture of a rib made by a blow from the horn). Finally, predisposing causes seem to exist: advanced age, which is accompanied by certain degenerative processes (arterio-sclerosis, sclerosis of the aortic valves); young age, during which an hereditary influence is sometimes felt (Burke has seen all the puppies of the same litter killed by endocarditis). A first attack of the disease predisposes to a return of the trouble. Among the medicaments the prolonged administration or excessive doses of which produce endocarditis, we must especially mention digitalis.

Wyssokowitsch has made very interesting experimental researches 
in the rabbit on the subject of the etiology of ulcerous endocarditis. By tearing the valves of the heart and injecting into the veins the Streptococcus pyogenes he has produced phenomena similar to those of ulcerous endocarditis (myentic) of man. His experiments have proved that the slightest wound of the valves is sufficient to permit micro-organisms, which are inoffensive in the uninjured circulatory apparatus, to produce a fatal endocarditis. The Staphylococcus pyogenes aureus and cocci of septicemia have given similar results. ${ }^{1}$

Pathological anatomy. The macroscopic alterations vary with the cause and degree of intensity of the disease. In the benign rheumatismal forms the loose edges of the valves, the chordæ tendineæ and the papillary muscles are covered with miliary prominences, which are of a grayish or yellowish-white color, and similar to granulations or small pimples. When these neoformations exist in large numbers the serous membrane is coated with a layer of pustules which give to it a glandulous or warty aspect; they are often covered with a fibrinous, stratified, bloody, or discolored exudate (warty endocarditis). If developed on the loose edge of the valves they may be transformed into enormous polypous vegetations which determine the insufficiency of the valves or the contraction of the orifices. In cases where the granulations are of very small dimensions the endocardium seems slightly rugous; it is tumefied, turbid, red, and covered with an exudate. The subendocardial connective tissue, also the epicardial and interstitial, are overrun with small hemorrhagic centres. In the serous septic forms we observe small mortified spots and ulcerations upon the serous membrane (ulcerous endocarditis). These latter are ordinarily superficial, their dimensions varying from the size of a lentil to that of a twenty-five-cent piece; they are covered with a dirty, spongy, friable necrotic exudate, which may be dragged away by the blood-current into almost any organ. Purulent embolus of

1 In making a culture of the blood obtained from a woman who had died of malignant endocarditis caused by an ulceration of the upper lip, Gilbert and Lion have been able to isolate and cultivate a microbe which seemed to play an important rôle in the development of this cardiopathy. The inoculation of a few drops of the culture of this micro-organism in some rabbits, which were free from any previous valvular traumatism, has produced a vegetating endocarditis in these animals, which was with or without aortitis, and the lesions of which were absolutely identical wlth those which determine this disease in man. The microbe was found in the vegetations of the mitral and tricuspid valves. (Bull. de la Soc. de Biologie, 1888-90.) -N. D. T. 
the lung, kidney, liver, spleen, etc., are thus produced; they may even reach as far as the coronary arteries (abscess of the heart).

In warty endocarditis the microscope shows an exudation and a cellular infiltration of the sub-endocardial connective tissue; in ulcerous endocarditis the tissues are necrosed, and transformed into granulous masses which are rich in micrococci. According to Ziegler, these two forms differ only in the degree of intensity of the process. The first sometimes ends in the resorption of the exudate and recovery, at other times in a connective neoformation of leucocytic origin: the valves become thickened, shrunken, and form abnormal adhesions (insufficiency and contraction of chronic endocarditis); in certain cases the thrombotic exudate, which is carried away by the circulatory flow, is thrown into the kidney, brain, etc., where it produces infarct, which, as a rule, ends in cicatrization. The tissue which is subjacent to the thrombotic exudate becomes necrotic; it is the seat of a true "diphtheric" infiltration.

While in man endocarditis is generally localized in the left heart, in animals it is the right heart which seems to be mostly affected. In nine cases of endocarditis observed by Meyer the inflammation involved the tricuspid valve eight times, the mitral valve four times, and the aortic valves twice only. In the ox right endocarditis is frequent (Bollinger, Ruchte); in man, on the contrary, it is very rare and ordinarily of foetal origin.

Symptoms. Acute endocarditis is marked at first by a tumultuous action of the heart, the beatings of which are strong, violent, are felt over a great surface, and which may also shake the whole body. At times their number exceeds that of the pulsations (Trasbot); in the horse we have found 160 cardiac contractions per 70 pulsations. The circulation is accelerated; in the ox and horse we count 80 to 160 pulsations per minute; the pulse is irregular, intermittent, very weak, often imperceptible.

The heart-sounds are normal at the beginning, but soon they lose their clearness ; the first becomes dull, and is sometimes confounded with the second; at times an auscultation reveals abnormal endocardial bruits, especially a systolic blowing at the first sound. More rarely we perceive a diastolic bruit, which is quivering and vibratory, and is very important from a diagnostic point of view. Hyperthermia is constant. In the septic forms the temperature is always high ; in the horse Trasbot has found it $40^{\circ}$ to $41^{\circ} \mathrm{C}$. 
From the start the respiration is painful and accelerated to such an extent as to induce us to believe in the existence of pneumonia or pleurisy; in some cases it becomes dyspnœic. The general condition is alarming; we observe a great weakness, intermittent colics, then symptoms produced by emboli in the lungs (suffocating spells), in the kidneys (hematuria), in the extremities (lameness), and in the brain (apoplectic phenomena). The septic form is often com- plicated by hemorrhages.

There are cases where the course of the affection is subacute: the patients die within a certain number of hours, or a few days at the most; in other instances it lasts several hours, and again in others it passes into a chronic state. The return of the disease is frequent (Trasbot, Roth). Warty endocarditis is relatively benign, but the septic form is very serious. Its most common termination is death; it is due to septic infection or to the formation of embolic centres in the lungs, the kidneys, the brain, etc. The valvular form especially acquires a serious character through the lesions left behind.

Differential diagnosis. When it assumes the subacute form called foudroyant, endocarditis may be confounded with anthrax, septicemia, encephalitis, petechial fever, etc. We are sometimes embarrassed in making the differential diagnosis between this disease and pneumonia at the beginning, also in cases of pulmonary congestion, œdema of the lung, which is also frequently accompanied by intense dyspnœa. But the difficulties reach their maximum when the observed symptoms are at the same time those of myocarditis and endocarditis. In these cases it is indispensable to make a full and very minute examination of the heart. We must also take this fact into account, that in anemia and leukemia systolic blowings may exist which are similar to those of endocarditis. When this is not accompanied by any abnormal bruit, its intra vitam diagnosis is impossible.

Treatment. The animals must be withdrawn from all excitement and movement; the activity of the heart should be regulated by digitalis; the fever should be combated with quinine, tartar emetic, and other antipyretics: such are the principal indications. In small animals we may use ice upon the precordial region; in others we should make continuous irrigation with cold water. The action of the cutaneous derivatives is of the most uncertain kind. When weakness becomes much marked, it must be combated by the administration of camphor, ether, or alcohol. 


\section{CHRONIC ENDOCARDITIS: VALVULAR ALTERATIONS.}

Etiology. Valvular diseases are extremely common in our domestic animals, especially in the horse and dog. In the majority of cases they are characterized by alterations which are developed in the course of chronic endocarditis, which most often succeeds acute endocarditis. In the ox they are ordinarily preceded by acute articular rheumatism, which is complicated by cardiac accidents (Hering, Joyeux). In the horse they have been observed after influenza (Percival), and in some other acute infectious diseases. Chronic endocarditis recognizes another pathology in which colds, repeated excitements, and violent exertions play the principal rôle. Processes of arterio-sclerosis which develop at the origin of the large bloodvessels (Boisy) also give rise in old animals to valvular alterations. These may also be produced during intra-uterine life; in cases of this kind and in a certain number of others, we must admit a congenital predisposition.

They are exceptionally related to anomalies of development and to neoformations. Johne and Eisenblätter have described congenital alterations of the inter-ventricular wall in the cow. In a dog affected by epileptiform attacks and cyanosis, Franck has found an incomplete obstruction of Botal's canal. Neoplasms of the heart and those which are developed in its neighborhood (carcinoma, sarcoma, myxoma), hypertrophy or tumors of the bronchial ganglions (Johne, Koch, Wolff) may produce contractions of the cardiac orifices. But recorded facts of this kind are very rare; we shall therefore limit ourselves to the study of valvular affections produced by chronic endocarditis.

Pathological anatomy. The alterations of the auriculo-ventricular and sigmoid valves determine the insufficiency or the contraction of the corresponding opening. ${ }^{1}$

Insufficiency of the valves is due to their contraction or shortening

1 Valvular endocarditis as a first effect produces a want of coaptation of the valvular blades-that is to say, an insufficiency. As the process never retrocedes, the valves undergo a double retraction: first, along the vertical axis - that is to say, the free edge toward the investing line; secondly, along the transversal axis or in the direction of the loose edge. This progressive retraction of the valves increases little by little the insufficiency, and ends by leading to the contraction of the corresponding orifice. Valvular insufficiency is thus the first term of a lesion the last stage of which is contraction (Peter). 一N. D. T. 
of their tendons, these membranes having in these cases lost their normal mobility. In the course of chronic endocarditis they sometimes become contracted and undergo an atrophy which may go as far as the almost complete disappearance of their apparatus; at times the contraction of their chordæ tendineæ does not permit of complete unfolding, and at the time of their tension we find between their convergent edges an opening through which the blood flows.

Contraction of the orifices is produced either by the thickening of the valves or by the partial joining of their edges, or, again, by vegetations of the endocardium. We may find the orifices contracted by polypous, warty, cauliflower-shaped neoformations, etc., which are developed on the valvular lumina; sometimes they are almost entirely filled ${ }^{*}$ with neoplasmic masses. The loose edge of the valve is tumefied and spongy, or thickened, or, again, incrusted with calcareous salts ; in other instances, being joined toward their extremities, they form narrow cartilaginous or bony rings, through which the blood is driven with violence.

These alterations are generally added to endocarditis, and they are often found associated (combined valvular affections); cases of simple insufficiency or of idiopathic contraction are very rare. The abnormal bruits they produce will be described further (see symptoms of particular valvular affections, page 475). As to the secondary morbid phenomena produced by circulatory troubles, the principal are: hypertrophy and dilatation of the heart; hyperemia of the liver, brown induration of the lung, obstruction of kidneys and spleen; general dropsy, ascites, hydrothorax, hydro-pericarditis, anasarca, emaciation, hemorrhages, etc.

AfFected animals. Valvular diseases are mostly observed in the dog and horse; they are also quite common in the ox, perhaps on account of the acute articular rheumatism in this species; they are also found in the pig (Daudt), etc., ${ }^{1}$ and in birds. ${ }^{2}$ In man, the

1 Under the name of "chronic pseudo-rouget" (swine plague) Bang has mentioned a case of warty endocarditis observed in an eighteen-months'-old sow. The principal symptoms were the following: bluish-red coloration of the ears, belly, and legs, which spread to the larger part of the body; the rectal temperature was $40.4^{\circ} \mathrm{C}$., the respiration was accelerated and noisy, the heart was depressed, and there was, so to speak, a permanent decubitus. The animal did not touch any liquids and took but a little fresh grass which it kept in the mouth, like a horse when said to be smoking his pipe. At the autopsy, besides abundant dropsies in the great splanchnic cavities, the mitral valve was found uneven, rugous, warty, and so thickened that it obstructed almost entirely the aurico-ventricular orifice.-N. D T.

3 Dr. Larcher: Recueil Vét., 1874. 
left heart is generally affected (in 300 cases of endocarditis, they were found 297 times in the left heart, and in 255 the mitral valve was affected). The 37 observations of valvular affections. contained in our special bibliography ${ }^{1}$ are as follows : 20 cases of alterations of the left heart and 17 of the right; the mitral was found injured 14 times, the tricuspid 15 times, the aortic valve 6 times, that of the pulmonary artery twice. It is especially in the ox that the tricuspid seems to be frequently involved. In the 7 cases observed in this animal, 6 times the lesions were found on the right side, in one case they were bilateral ; they were not seen located upon the left heart in any case. In the horse, on the contrary, the proportion is almost similar to that found in man: in 15 cases which were examined, 7 times the lesions were found to be located upon the left heart, 3 times upon the right, and they were bilateral in 5 cases.

A table of Nocard establishes the relative frequency of the different valvular affections in the horse. Of 42 subjects, 38 showed lesions of the aortic valve; in the other four they were found upon the aortic and mitral valves. The intensity of these alterations was very different: at times the valves were simply thickened and rigid; at others they had undergone cicatricial contraction; in some cases they were covered with fibrinous vegetations; in others, again, they were gnawed, indented, or perforated. The diagnosis had been made during life. In almost all these animals was heard a blowing bruit which covered the whole silent stage. The intensity and sound of these bruits were of the most variable kind. ${ }^{2}$

Symptoms of general valvular affections. Valvular insufficiencies and contractions of the orifices produce a blood stagnation above the lesion-in the left auricle and in the lung, if the mitral is affected, in the right auricle and the general venous system when it is tricuspid ; the diseases of the aortic and pulmonary valve produce a stagnation in the corresponding ventricle. Some circulatory

I See original text.

2 Fourteen observations of chronic endocarditis-in very old horses, as a rulegathered by us, are thus divided as regards the localization of the process: lesions affecting exclusively the sigmoid valves, ten cases; lesions involving the mitral valve exclusively, one case; sigmoidal and mitral lesions, two cases. In none of these cases have we found any alterations of the tricuspid nor of the valves of the pulmonary artery. Thirteen cases of chronic endocarditis which we have observed in the dog are thus divided : four cases of alterations of the mitral and tricuspid; one of mitral and aortic: five of mitral only; two of tricuspid; one of the aortic valves.-N. D. T. 
affections are counterbalanced at the start by an increase of the activity of the heart (compensatory hypertrophy). A compensatory cardiac alteration in this way may not produce any serious symptoms for years; but, after violent exertions or excitements, we observe more or less marked abnormal symptoms. Sooner or later the cardiac muscle becomes tired and is no longer able to respond to the task which has been created by the morbid condition. Circulatory troubles then appear in the lungs and the system of the vena cava. It is often at that period only that the animals are known to be sick and we are called to treat them; in most cases we find an ensemble of symptoms hardly differing from that of advanced cardiac hypertrophy.

[The first symptom of chronic endocarditis which attracts attention is the marked weakness of the animals while at work. They act somewhat like a dummy; they will stop, refusing to advance, and do not respond to the voice or the whip ; this, however, is of but short duration.-W. L. z.]

The principal manifestations of serious valvular lesions are:

1. The acceleration of the pulse and of the heart rhythm, the abnormal excitability of the latter, the irregularity of its contractions and of the pulse; palpitations; in exceptional cases, as in contraction of the aorta in man, a slowing of the pulse is observed.

[The intermissions constitute an entirely special form of rhythmic disorder of the heart, and are not related to any substantial alteration of this organ. Quite common in the horse, they consist in a short suspension of the heart's action, which are generally repeated at every fourth, fifth, or sixth beating. With the exception of the first contraction of each series, which is sometimes rebounding, and the usual very short interval which separates it from the second, the others are normal as to force and rhythm. In some cases these intermissions seem to correspond with a general disturbance of the health, but, like the alterations, they may be seen in animals which are in good condition and are used for hard work without showing any symptom of indisposition.-W. L. z.]

2. The difficulty of breathing, especially during exercise. In the horse we observe true dyspnœic attacks, designated as "cardiac heaves." This dyspnœa, which generally dominates the scene, is explained by the slowing of the pulmonary circulation and the persistent connective tissue alterations (brown induration of the lung, contraction of the pulmonary alveoli after capillary stasis). 
3. Vertiginous paroxysms after exertions (in draught dogs).

4. Cyanosis of the mucous membrane of the skin (in the pig), especially marked upon the ears and the back (Daudt); swelling of the veins (glosso-facial and jugular); venous pulse.

5. Discharges into the splanchnic cavities (ascites, hydrothorax, hydro-pericarditis), and œedema of the belly, sheath, chest, and legs.

6. Albuminuria and lessening of the urinary secretion-phenomena which are due to blood stagnation in the kidneys.

7. Icterus, a consequence of passive hyperemia of the liver.

8. Embolisms of the peripheral organs. They are derived from cardiac thrombus produced by the slowing of the blood-flow. They are observed in the lung, brain, kidney, liver, spleen, in the extremities (here they sometimes produce lameness-Weber), and the articulations (acute secondary articular tumefactions observed in the horse-Percival).

9. Progressive emaciation, weakness, marasmus, easy and frequent perspirations after the slightest efforts-symptoms which coexist with a general apyretic condition.

10. Abnormal bruits which are observed on auscultation.

Symptoms of particular valvular affections. The most important of these diseases are insufficiency of the mitral valves (horse and $\operatorname{dog}$ ), tricuspid (ox), aortics, with contraction of the corresponding orifices; alterations of the orifices and pulmonary valves are much more rare. They do not always remain simple and isolated; on the contrary, they often become combined, and the symptoms by which they are marked may become strangely complicated. Because of the precision of cardiology in human pathology, and the abundance of special works published on vetcrinary matters, also the personal observations that we have gathered, we have decided to describe the particular characters of the different valvular affections; this study may serve as a basis for future works.

I. Mitral insufficiency. It produces at the time of systole a re-flow of blood into the left auricle through the incompletely closed auriculo-ventricular opening.

The vibrations of the refluent blood through the mitral orifice and its meeting with that leaving the pulmonary vein produce an acute systolic bruit (blowing). ${ }^{1}$ The auricle, filled to excess, becomes

1 The seat of the bruit is characteristic of the lesion and its sound from the form of this lesion. In mitral insufficiency the blowing is especially extended to the base of 
dilated and little by little hypertrophied; the discharge of the blood into the pulmonary veins is obstructed, there is blood stagnation in these vessels, in the capillaries and the arteries of the lungs, and finally in the right heart, which in the end becomes atrophied. On account of the increased blood pressure in the pulmonary artery the second sound (pulmonary sound) is strengthened. When the right heart can no longer withstand the circulatory troubles cyanosis occurs, also dyspnœa, swelling of the jugular veins, venous pulse, dropsies, etc. ${ }^{1}$

Characteristic symptoms of mitral insufficiency: Systolic blowing, strong diastolic pulmonary sound, hypertrophy of the right heart, weak pulse, dyspncea, stagnation in the venous system.

Schmidt ${ }^{2}$ has described an interesting case of mitral insufficiency in the horse. Symptoms: pulse accelerated, small, arrhythmic; loud systolic bruit (wheezing) at the apex, increase of the precordial dulness, odema under the chest, ete.

II. Contraction of the mitral orifice. This has as a consequence obstruction to the entrance of blood into the left ventricle at the time of the contraction of the auricle, and produces a diastolic bruit (blowing). The incompletely filled ventricle has a tendency to atrophy; the pulse becomes small and weak. The left auricle becomes dilated and hypertrophied; the pulmonary circulation is interfered with, from which results dilatation and hypertrophy of the right heart; the diastolic pulmonary sound is very loud.

Characteristic symptoms of mitral contraction. Diastolic blowing, strong pulmonary sound, very small pulse, serious circulatory troubles, and especially pulmonary obstruction.

This lesion has been described in the horse and pig by Daudt, Schneidemühl, Appenrodt, and a few others.

III. Tricuspid insufficiency. It permits the return of the blood into the right auricle, and is indicated by a systolic bruit (blowing). The flowing back of the blood into the auricle spreads to the veins (venous pulse), and produces a stagnation in the whole

the heart; it is weaker in the whole height of the posterior half of this organ. It is mild when the insufficiency is simple and coarse when vegetations are present.-L. T.

1 Chronic alterations of the mitral which are evident enough to produce insufficiency, may exceptionally be found at the autopsy of horses not showing any serious respiratory or circulatory trouble during their life. We have obtained a remarkable example of it. - N. D. T.

2 Schmidt: Preuss. Mittheil., 1877-78. 
venous system (cyanosis of the visible mucous membranes, etc.). The right auricle becomes hypertrophied and dilated; later, the ventricle undergoes the same modifications. When the activity of the right heart becomes insufficient, a pulmonary obstruction is produced (dyspnea), also thrombosis of this organ and dropsies consecutive to blood stagnation in the vena cava.

Characteristic symptoms of tricuspid insufficiency: Systolic blowing and venous pulse.

In the ox, Cagny has observed a great distention of the jugular veins, venous pulse, dyspnœea, and dropsies. Schmetz, in a similar case, has only observed a systolic blowing.

IV. Contraction of the tricuspid orifice. It is rare in man, but quite common in the ox. The phenomena produced by it are : a diastolic bruit (blowing), dilatation and hypertrophy of the right auricle, venous pulse, slowing of the blood-flow in the pulmonary artery, partial thrombosis of this vessel and consecutive pulmonary embolism, respiratory troubles, dropsies, finally hypertrophy and dilatation of the right ventricle.

Hering has observed a venous pulse in the jugulars of a horse, also violent beatings of the heart and œdematous infiltrations in the extremities, belly, etc.

At the autopsy of a cow in which grave respiratory troubles had been observed, Eggeling found in almost all the branches of the pulmonary artery, thrombi derived from thickened sigmoid valves; in another he observed pulmonary embolic centres coming from a thrombus of the size of a hen's egg, which was developed upon the lateral wall of the right ventricle and was partially softened; in this case, also, an extremely laborious respiration had been observed during life.

V. Aortic insufficiency. This permits the re-flow of the blood in the aorta into the left ventricle at the time of the diastole and produces a diastolic bruit (blowing), which has sometimes the characters of a shudder. The left ventricle, which is extremely distended, is dilated and hypertrophied. Hyper-functional activity of the heart determines in its turn a very strong pulse, the decreasing curve of which is nevertheless abrupt, because part of the blood which ought to go forward in the arterial vessels comes back in its tracks. The aortic diastolic bruit is sometimes perceptible upon the carotids; in man, auscultation of this vessel by the stethoscope allows us to recognize a systolic bruit that is sharp and short; 
upon the smaller-sized arteries we perceive peculiar vibrations, the crural artery giving the double sound of Traube.

Characteristic symptoms of aortic insufficiency: Diastolic blowing; dilatation and hypertrophy of the left heart, strong arterial pulse (pulsus celer, Corrigan's pulse), ${ }^{1}$ arterial bruits.

Polansky has observed an interesting case of aortic insufficiency in the horse. He has noted an increase of the precordial dulness, violent beatings, a strong diastolic murmur, of metallic resonance, which was also heard in the neighborhood of the dorsal region and upon both carotids, but which became weaker and weaker in proportion to their remoteness from the heart. The pulse, which was broad and full, lifted the finger and was visible in several peripheral arteries, especially in the transversal artery of the face, in the posterior auricular, and anterior and posterior tibials. After a continued moderate exercise for a quarter of an hour, there was perceived in the region of the heart a kind of very distinct diastolic shock, which disappeared as soon as the action of the heart and the respiration had again become calm.

VI. Contraction of the aORTic orifice. It communicates a whirling movement to the blood-flow, which is accompanied by a systolic bruit (blowing). The pulse is very small and slow, the left ventricle becomes hypertrophied; when the contraction is much marked we observe symptoms of cerebral anemia, vertigo, etc.

Characteristic symptoms of aortic contraction: Systolic blowing, a very small pulse, hypertrophy of the left heart.

Bagge has observed two horses in which the first heart-sound was accompanied by a dull humming sound; the hand applied upon the precordial region perceived a shuddering sensation; the pulse was weak. One of these horses was very soft in working; he ate little and had become considerably emaciated. Lustig has described a case of aortic insufficiency and contraction in the horse.

VII. INSUFFICIENCY OF THE VALVES OF THE PULMONARY ORIFICE. It is very rare, and is indicated by a diastolic bruit (blowing) and accompanied by hypertrophy and dilatation of the right ventricle, and marked respiratory and circulatory troubles as soon as the activity of the heart begins to abate.

1 Aortic insufficiency is the only organic lesion of the heart where the pulse is strong. The blood-spurt which is thrown by the ventricular systole into the arterial apparatus, where the tension is we $\mathrm{k}$, lifts the vascular wall abruptly, and produces a full pulsation, which is sometimes rebounding in the horse. - N. D. T. 
Special literature contains but one case of this alteration. Boisy has found, in a cow, the second sound replaced by a rasping bruit; venous pulse was much accentuated, the respiration laborious and subsultory; he observed besides an almnst imperceptible arterial pulse and ascites. At the autopsy this author observed cauliflower vegetations which were developed upon the valves of the pulmonary artery.

VIII. Contraction of the pulmonary orifice. It gives rise to a systolic bruit (a "blowing"), an hypertrophy of the right heart, a stagnation in the venous system, and dyspnœic phenomena.

This alteration is also very rare; Huth has, however, met with it in the horse. During the subject's life he observed the existence of the blowing bruit, which was synchronous with the pulse. After a slight exercise dyspnœa became intense, the heart beat violently, and the breathless animal was compelled to stop.

Diagnosis of valvular affections. A variety of circumstances obscure the diagnosis of valvular diseases. Abnormal systolic and diastolic bruits may exist without the least organic lesion of the valves; they are designated under the name of anorganic bruits; they are observed in anemia, leukemia, etc.; on the other hand, a valvular affection does not always lead to a pathological bruit at the start, its most important diagnostic symptom may be wanting. Two or several valvular alterations are often associated; contraction and insufficiency coexist frequently; the most complex forms are possible. Lastly, in the majority of cases these affections only attract attention and are only recognized in their last stage, when they all become evident by their almost similar functional symptoms (circulatory troubles in the lungs and the venous system). An anatomical diagnosis is, therefore, quite difficult. However, we have to point out that in cases where several valves are in volved, the troubles produced by one of these generally dominate the scene; it is consequently possible to formulate a probable diagnosis. Here are the principal points which permit us to establish a differential diagnosis of the various valvular diseases.

1. The systolic bruit (blowing) exists in mitral or tricuspid insufficiencies, in aortic or pulmonary contractions. The first are quite frequent; the latter is, so to speak, not worth being noticed. Tricuspid insufficiency is especially observed in the ox and is accompanied by a venous pulse. In mitral insufficiency the pulse 
is nearly normal ; in aortic contraction, on the contrary, it is very small and at times slower.

2. A diastolic bruit (blowing) may be derived from a mitral or tricuspid contraction, from an aortic or pulmonary insufficiency. ${ }^{1}$ The latter is extremely rare. Tricuspid contraction, which is frequent in the ox, is accompanied by venous pulse and dyspnœea, phenomena which are consecutive to the formation of thrombosis in the right heart. In mitral contraction the pulse is small and irregular; in aortic insufficiency it is strong and rapid.

Treatment of valvular diseases. As long as valvular affections are compensated, they do not require any treatment. We may be limited to good feeding of the animals, avoiding excitement and violent exertions.

But as soon as we observe symptoms of heart exhaustion, respiratory troubles, venous stagnation, we must resort to certain means which regulate and diminish the circulation, increase the blood pressure in the arterial system, and give to the myocardium the necessary time for its rest and nutrition. In this respect, digitalis constitutes a valuable medicament; it increases arterial pressure, diminishes the pulse, and compensates the circulatory troubles. If used in the form of powder or infusion of 1 to 2 grammes, it is proper to give it at intervals, for several days consecutively, watching attentively the effects produced. Large doses should be avoided: for the dog, we may use an infusion of 1 to 2 grammes of digitalis leaves in 250 grammes of water (one to two tablespoonfuls per day; one to two teaspoonfuls for smaller animals); for large animals, we generally use the powder given upon bread or in the shape of electuary; dose for the horse, 2 to 5 grammes; for the ox, 4 to 8 grammes. Tincture and extract must be abandoned on account of the uncertainty of their containing digitalin. When dropsies exist, caffeine is advisable, its diuretic properties are more evident than those of digitalis and it is less toxic than the latter, especially when we are obliged to prolong its administration ; it is given in the shape of sodo-benzoate of caffeine in a dose of 1 gramme for the dog, 5 to 10 grammes for the horse and ox. The tincture acts in a similar way (horse and ox, 10 to 25 grammes; dog, 10 to 25 drops).

1 In our animals, the diastolic blowing is, in almost all cases, the expression of an aortic insufficiency. The blowing produced by the contraction of one of the auriculovcntricular openings (rare) is only heard toward the end of the great pause, when the corresponding hypertrophied auricle drives the last portion of the blood which it contained through the contracted orifice; it is presystolic.-N. D. T. 
The symptomatic treatment consists in making an outlet for the transudates (ascites, hydrothorax, hydro-pericarditis); also in the administration of diuretics (the cardiacs which have just been mentioned, squill, acetate of potash) ; also diaphoretics and sialagogues (pilocarpine); the drastics may also produce momentary relief. General weakness should be combated with stimulants, and palpitations with narcotics (subcutaneous injections of morphine: for the horse, 0.4 to 0.6 gramme; for the $\mathrm{dog}, 0.01$ to 0.05 gramme; bromide of potassium, urethane, hypnone, etc.).

[One of the best agents to reinforce the action of digitalis is the iodide of potassium in small doses of 4 to 8 grammes for the horse. Its effects are most remarkable when given in combination (see page 366) with other drugs. Very decided improvement can be seen even when used alone.-W. L. z.]

\section{ANEURISM OF THE AORTA.}

Etiology. Aneurism of the aorta-a circumscribed dilatation of this organ-is generally the consequence of a morbid state of its walls, which is itself determined by a chronic endarteritis or a mesarteritis with fatty or calcereous degeneration. Similar vascular alterations are observed on the mesenteric arteries of the horse in verminous aneurism, which is caused by the Strongylus armatus (see Embolic Colics). They are more rarely found to be produced by the Spiroptera sanguinolenta. Traumatic and mechanical influences, such as violent muscular exertions, injuries, falls, may produce aneurism, especially when the arteries are already the seat of a degenerative alteration. At times there seems to exist a true aneurismal diathesis (Raymond).

Pathological anatomy. Aneurisms are sacciform, discoid, or cylindrical. Their volume, which is very variable, may become enormous ; they may exceed a man's head in size. In true aneurism the sac is formed by the vascular membranes; in false aneurism the neighboring organs (intestine, liver, etc.) constitute their walls; some of these become joined to adjacent organs - to the stomach, cæcum, even to the small intestine (a case observed by Schütt). The most frequent are those of the anterior mesenteric; but they are also found at the point of division of the aorta, on the abdominal aorta, thoracic aorta, and the aortic trunk (Konhäuser). Lustig has found a voluminous aneurism in the horse, which occu- 
pied the whole portion of the abdominal aorta situated in front of the point of emergence of the renal arteries, and the diameter of which was $12 \frac{1}{2}$ centimetres. In most cases the three layers of the vascular wall take part in the dilatation, and are affected by atheromatous degeneration; it is thickened in some points and atrophied in others, and contains here and there small calcareous blotches. The contents of the aneurism are formed of fibrinous clots which are ordinarily stratified; these are sometimes organized, at others softened or undergoing purulent destruction. According to Röll, a spontaneous cure may be produced in our animals through cicatricial retraction of those thrombi which are undergoing degeneration. The dilated aorta exerts a compression more or less great upon the organs which are related to it: lungs, stomach, intestine, liver, kidneys, etc.; it may even depress the vertebral column. When the aneurism becomes ruptured the blood flows into the pleura and peritoneum; more rarely into the stomach (Vogel), the intestine, or the rectum (Labat and Cadéac).

Symptoms. Generally aneurism of the aorta is not marked by any serious trouble. At times it produces death abruptly through internal hemorrhage without any symptom indicating this fatal ending, which is almost instantaneous, and is apoplectiform; it occurs as a rule after energetic exertions: the animals are overcome with dyspnoea, stagger, become weak and die. In some cases we have observed alarming phenomena, which are marked by paroxysmal attacks without any immediate fatal termination. In a horse that had made violent exertion Lustig observed staggering of the hind quarters, abrupt falling, intense dyspnoea, and epileptiform attacks, during which the neck and head were held backward, with the legs stiff and extended; within a quarter of an hour the animal had completely recovered. In a horse affected by aneurism of the pulmonary artery the same author has observed vertiginous spells. Barrier has observed considerable emaciation in a dog affected by aneurism of the posterior aorta, notwithstanding that the appetite was preserved; also an interference with the respiration, which was increased under the influence of exercise; paralysis of the hind quarters (symptoms of heart-weakness). ${ }^{1}$

\footnotetext{
1 Aneurisms involving arteries of a small calibre are very rare in our animals. Their rupture may be followed by a hemorrhage which may become rapidly fatal when the blood escapes through a mucous membrane or flows into a splanchnic cavity.-N. D. T.
} 
The symptomatology and diagnosis of aneurism of the aorta are much more complete and precise in man than in our domestic animals. Physical examination upon the latter is nearly always impossible; in the horse, percussion and auscultation of the aorta are impracticable. In man, the characteristic symptoms are the following :

1. Dyspnoea at the time of pulmonary compression.

2. Swelling of the jugulars, vertigo, cyanosis, dropsies, when the aneurismal tumor compresses large veins.

3. Neuralgias of the intercostal and brachial plexus nerves, through the compression and traction exerted by the aneurism.

4. Very small pulse, which is even imperceptible on one side (compression of an arterial trunk through aneurism); keeping back the blood-wave and pulse, as a consequence of the slackening bloodflow at the time it crosses the aneurismal dilatation.

5. Paralysis of the left vocal cord when the aneurism compresses the recurrent nerve (similar to chronic wheezing of the horse).

6. Pulsatile tumor, giving a particular blowing or shuddering bruit; in some cases this tumor is visible; in others it may be recognized through percussion.

The consequences of aneurism of the anterior mesenteric has been described in the article on Thrombo-embolic Colics.

Treatment. In man we generally resort to galvano-puncture, which is even applied upon the pectoral aorta ; injections of ergotin are also used ; internally, we administer sugar of lead or iodide of potassium.

\section{RUPTURE OF THE LARGE VESSELS OF THE PECTORAL AND ABDOMINAL CAVITIES.}

Etiology. Rupture of the large vessels of the pectoral and abdominal cavities must make us presuppose the existence of an alteration of their wall, which is produced, either through chronic endarteritis (arterio-sclerosis or atheromatous degeneration) or by aneurism. The determinating causes are sometimes muscular exertions (violent tractions-Prietsch), at other times a fall (Hering), exceptionally vomiting (Straub), or excitement produced by veratrine used with therapeutic intent (Hering), etc. A partial destruction or perforation of the vascular walls may be the effect of an 
ulcerating process starting from a neighboring organ, or from nematode worms contained in the bloodvessels (armed strongyle in the horse-Durieux; spiroptera in the dog-Morgagni, Mégnin) Animals affected by hypertrophy of the heart are predisposed to vascular lacerations.

Pathological anatomy. These ruptures have been observed in the posterior vena cava (Page, Scruby, Hertwig), the anterior vena cava (Straub), the pulmonary artery (Hering, Hartmann, Prietsch), upon the aortic trunk, in the neighborhood of the semilunar valves; here they are the consequence of the atheromatous alteration of the walls of the aorta (Larcher). When the internal and median membranes are torn, the blood accumulates between the adventitious layer and the middle tunic of the bloodvessel; a dissecting aneurism is formed.

Symptoms. Symptoms of the rupture of a large bloodvessel are those of internal hemorrhage: a sudden appearance of paleness in the mucous membranes; a filiform pulse, which is imperceptible; weakness, staggering, dropping, chilled condition of the extremities. Straub has seen epileptiform attacks preceding the laceration of the anterior vena cava; Hartmann has observed an abundant hemoptysis which was produced by the opening of an aneurism of the pulmonary artery into a large bronchial tube.

It would be of little use to consider the treatment of such accidents, which are always followed by the death of the animal within a short time.

\section{THROMBOSIS OF THE BRANCHES OF THE AORTA.}

\section{THROMBOSIS OF THE ILIAC AND AXILLARY ARTERIES.}

Etiology. Thrombosis of the crural and axillary arteries belongs to the domain of surgery ; lameness of the particular members constitutes the principal symptom of it. The causes of these diseases, however, belong to the jurisdiction of internal pathology; they consist in alterations of the large bloodvessels, and we must therefore consider them here. Its causes are either endarteritis or fibrinous clots deposited upon the vascular wall, which has become rugous, or obstruction of the aortic branches, produced by emboli coming from a diseased heart or from an aneurism. 
Pathological anatomy. Thrombi are relatively frequent in the iliac arteries, they are uncommon on the obturator, still more rare on the axillary and humeral, and quite exceptional on the lumbar arteries. ${ }^{1}$ In cases of thrombosis of the aorta, the end of this and the opening of its divisions are dilated, the walls of its canals are thickened, affected with atheromatous degeneration, the intima looks suspicious, has undergone fatty degeneration, or is calcified, or partially destroyed, ulcerated. Inside of it we find a hard, stratified and organized clot, which is ordinarily of a grayishwhite color, and obstructs the lumen of the bloodvessel, which is often reduced to a narrow canal; this thrombus frequently extends more or less forward into the aorta and backward into its divisions; it is sometimes mounted on the spur of the internal iliac arteries; in some cases one of these is completely obstructed. With this thrombus we may find a sort of connecting lesion, a compensatory cardiac hypertrophy. When the clot is softened, emboli are formed in the arterial divisions of the posterior members.

Symptoms. Arterial thrombi generally do not determine any symptoms during rest ; but when the animals are exercised, mounted, hitched up, or led, within a certain time we observe particular and characteristic symptoms.

1. In thrombus of the femoral artery, which is common in the horse, very rare in the ox (Fordie), we observe from the start a certain constraint in the functional movements of the posterior member, and a weakness which grows more and more marked. After a short exercise the gait becomes embarrassed, the toe of the diseased member is dragged on the ground, the animals tremble, stumble, fall, remain down for a few moments, and manifest intense agitation; the respiratory movements are very accelerated, the beatings of the heart are precipitate and violent, the visible mucous membranes are much injected; the body is covered with perspiration; the temperature of the lame extremity is generally lower than that of the other parts of the body ; the pulsations are very often wanting in the tibial and collateral arteries of the canon. The animals rise within a few minutes and come gradually back to a normal state. If we explore the thrombosed vessels by rectal examination, we find these dilated, thickened, rigid, and filled with a hard elongated body; in most cases we only perceive pulsations at this point. Thrombosis of the peripheric veins sometimes com-

1 Hahn (communicated). 
plicate arterial lesions, and determine gangrene of the member (Cadéac et Malet).

2. Thrombosis of the axillary trunk is marked by a lameness of the corresponding anterior member; the flexion of the bony rays is very limited, the toe is dragged upon the ground; the animals stumble and sometimes fall; we observe trembling in the muscular mass of the shoulder. We have never observed respiratory troubles, or cardiac excitement, or congestion of the mucous membranes. Abnormal phenomena ordinarily disappear in a few minutes.

3. Thrombosis of the arteries of the pelvis lead to paralysis of the rectum, bladder, tail, and croup (Kolb).

Treatment. The treatment of these diseases is quite limited and very uncertain in its results. The therapeutic agents administered internally (iodide of potassium, alkalies) are generally inefficient. Massage of the thrombus through the rectal walls, which is recommended by Colin and Bayer, is more important. It consists of slight and repeated pressures performed upon the obliterated portion of the artery, the clot of which may become loosened and reabsorbed(?); it has the danger, however, of exposing the patient to emboli produced by the débris of the thrombus.

Stimulation of the cardiac muscle by regular exercise has been advised, in order to increase the blood pressure and promote the circulation. However, absolute rest prolonged for several weeks has produced effects which were indeed very satisfactory in some cases (resorption or organization of the clot); in other instances the thrombus increased in size under the influence of immobilization.

Thrombosis of the heart is observed in diseases of the endocardium, particularly in ulcerating valvular endocarditis; here the cardiac weakness and slackening of the blood-flow act as predisposing causes. When they reduce the dimensions of the orifices they are accompanied by general troubles; we then observe symptoms of the contraction of the corresponding orifice and later those of dilatation of the heart. They also determine sometimes emboli in the lungs and peripheral organs. The thrombi adhere firmly to the surface of the endocardium, and must not be confounded with the fibrinous clots found when death is attended with great agony. 


\section{HEMATOZOA.}

The circulatory apparatus of subjects belonging to several animal species, especially the dog, may lodge numerous helminths, for instance: 1. Filaria immitis; 2. Howatozoon subulatum-Leisering, or Strongylus subulatus-Cobbold; 3. Pseudalius inflexus (found in a seal by Hering).

The most important species is the cruel threadworm (Filaria immitis), the discovery of which is erroneously attributed to Leidy, of Philadelphia (1858), for this worm is identical with the Filaria papillosa homatica, described by Delafond and Gruby. According to Krabbe, the adult threadworms reach a length of 15 to 30 centimetres and a thickness of 1 millimetre. We have found specimens measuring 35 centimetres. Oreste has found 75 threadworms in the right heart, where they had determined an obliterating thrombosis like those observed in the pulmonary artery (Serres). In the left heart they may also produce obstruction of the auriculo-ventricular orifice (Silvestri). The embryo of the threadworm has been found in the blood of the dog by hundreds of thousands (Delafond and Gruby); their average length is onefourth of a millimetre and their thickness $5 \mu$.

Symptoms. As a rule, threadworms do not occasion any serious trouble, even when the blood contains them in large quantities. In some cases, though, they produce fatal accidents (apoplectiform death), either in determining thrombosis of the heart and obstruction of its orifices, or in forming miliary emboli in the encephalic capillaries. We have besides observed epileptiform convulsions, dyspnœa, emaciation, great weakness, hemoptysis, enterorrhagia, and even rabiform symptoms.

Treatment. It would be illusory as long as the entrance-door of the parasite remains undiscovered. Were this known it would undoubtedly be possible to make some prophylaxis. Hereditary transmission of threadworms is very doubtful; possibly association with infected animals may cause contamination of young dogs.

According to Manson, the mosquitoes of the tropical regions of the New World ingest the embryo of the threadworm along with human blood; they become, therefore, the intermediary host of the Filaria sanguinis hominis. From the stomach of the mosquito the worm penetrates into the thorax; its migrations then cease, and 
according to Manson it passes through six stages of development. The female mosquito lays its eggs and goes to die in the water; the worms then travel through its tegmentary skeleton and swim freely in the water. Man may thus become infected in two ways: through the sting of a mosquito containing threadworms when it comes to suck blood, or through the ingestion of water holding the embryo of these parasites in suspension. According to Sonsino, the threadworm of human blood is also frequent in Egypt, where it is developed in the body of the common gnat (Culex pipiens). The infection of the dog must occur in the same way. 


\section{SECTION V.}

\section{DISEASES OF THE SKIN.}

\section{GENERAL CONSIDERATIONS.}

Diseases of the external integument are not observed with the same frequency in all our domestic animals. Particularly common in the dog, horse, and sheep, they are rarer in the ox, pig, goat, cat, and birds. In all these species they take the most varied forms. Old authors have arbitrarily applied to them the titles of the skin diseases of man, and such names as tetters (herpes), rash, rot, mange, scab, thrush, grease, etc., have often served to designate cutaneous diseases of a very different nature. ${ }^{1}$ These terms have been perpetuated up to the present time. It would be of advantage to abandon completely this. old nomenclature, but we would not know how to make the change at present without danger of being misunderstood by practitioners; we can, however, suppress part of them without any inconvenience, and refer to certain principal dermatoses those accessory tegumentary lesions described until now as special diseases. Following Hebra's example, we shall extend the pathological outline of eczema; as Gerlach has already proposed, we shall use the term herpes in order to designate diseases determined by vegetable parasites; finally, we shall reserve the expression mange in order to designate diseases produced by the acari.

A rigorously scientific division of diseases of the skin is much more difficult to establish in veterinary than in human medicine. In our animals, in fact, their diagnosis is rendered obscure by the presence of hair and pigment; a diffuse or circumscribed redness is only observed in animals having a white skin; in others it can only be recognized on certain limited regions where pigmentation

1 French authors designate these dermatoses under the generic name of Thrush. The word tetters means a benign affection which is characterized by vesicles which are filled with a clear liquid and are disposed in groups.-N. D. T. 
is wanting. A classification based upon the pathological anatomy could not be accepted, because the same alteration may be found in diseases of a different nature, and because the same dermatosis often shows the most diverse anatomical modifications in the course of its evolution. Thus, the expression papulous rash could not mean a clinical entity: papules may appear in the course of a certain number of skin diseases; on the other hand, several of these (eczema, for instance) evolve with a whole graduating series of anatomical alierations (hyperemia, pimples, vesicles, pustules, scabs, and crusts). We do not see the fitness of elassifying skin diseases in acute and chronic forms; there are some (eczema) which pass rapidly from an acute to a chronic state, or which take both types alternately. The division of dermatoses into idiopathic and symptomatic is not any more warranted; urticaria, for example, which constitutes a cutaneous disease par excellence from a clinical point of view, may be due to external causes (insects), or internal (urticaria $a b$ ingestis), or they may depend on another affection (strangles, etc.). It is the etiology that has offered the most solid basis upon which to establish a classification; such a grouping, however, would be imperfect, for the etiology of a large number of dermatoses is still unknown. The division of these processes into parasitic and non-parasitic must be considered as the most rational, notwithstanding that the field of action of vegetable parasites is not yet distinctly defined. Opinions given at the present time on the pathogenic rôle of some fungi found in the altered epidermis are nothing less than concordant. It is certain, however, that we frequently find the integument invaded by a nonparasitic dermatosis; again, it may be covered by vegetable microorganisms which are absolutely harmless.

In cutaneous diseases produced by parasites we will establish two categories : animal parasitic diseases and vegetable parasitic diseases. Certain eruptions which are due to internal causes (variola, aphthous fever, etc.), and which may be designated under the denomination exanthematous, will be described in the article on Contagious Diseases. We shall not deal with the numerous cutaneous alterations (erysipelas, phlegmon, panaris, skin neoformations, etc.) which belong to the domain of surgery.

[The various expressions used to denominate the primary and secondary morbid phenomena of cutaneous diseases have a special significance and should not be used indifferently. 
By the term efflorescence we designate either a morbid modification located on a circumscribed space of the integument and presenting a determined type in its form, evolution, and anatomical signification (Kaposi), or very slight eruptions, which are of little emportance and without very distinct nosography (Besnier and Doyon).

Spots consist of abnormal colorations of the skin or in depilations which are limited to a circumscribed region.

Papules are small, salient, cutaneous elevations filled solid with the products of an infiltration in the papillary layer of the derma. They may lose their typical character and be crowned by a vesicle or a pustule (papulo-vesiculous and papulo-pustulous alterations).

Vesicles are soft elevations, from the size of a millet-seed to a lentil, the superior part of which is thin and frail and exclusively formed by the epithelial layer of the epidermis, and which contain a serous exudate.

Bulla, bleb, or phlyctcena. These differ only from the vesicles by their larger dimensions.

The very comprehensive term pimple (bouton) refers to the small isolated or discrete papulous cutaneous blotches, which do not end in suppuration.

Pustules are epidermic or dermo-epidermic elevations which are filled with pus. Epidermic pustules (pustulo-vesicle or pustulobulla) leave uninjured the epithelial layer which immediately covers the papilla. Dermic pustules lead to destruction of the whole epidermal and papillary layer.

Excoriations are more or less deep traumatic cutaneous lesions which are determined by the action of a foreign body upon a region which is the seat of pruritus.

The term exulceration must be reserved for superficial lesions of the integument, whether traumatic or not, and characterized by the destruction of the horny coating of the epidermis and the laying bare of the mucous layer.

Ulcerations are losses of substance of variable extent and depth, and affecting the corium, being also accompanied by a purulent secretion, which is more or less abundant and only forming a cicatrix very slowly.

Rhagades (fissures, eracks) are crevices of the epidermis, which sometimes extend to the derma ; the edges of these lesions are ordinarily thick, sharp-cut, the floor being bloody or ulcerated. 
By the expressions phyma or dermatoma we designate more or less salient cutaneous neoformations, which are active and obstinate.

Squamce are epidermic lamellæ which become detached from the cutaneous surface (desquamation) in the shape of small pellicles or thicker scales.

Scabs are masses resulting from desiccation on the surface of the skin-pus, serum, and blood which has been extravasated.

By maculoe we designate the pigmentary spots which persist for more or less time after the different eruptions, or which appear at an advanced stage of their evolution.

Cicatrices are small islands of newly-formed tissue which fill the losses of the dermic substance.

The expression rash is obsolete nowadays, and was applied to cutaneous diseases of chronic course giving origin to crusts and cutaneous exfoliations.]

\section{NON-PARASITIC SKIN DISEASES.}

\section{ERYTHEMA : ERYTHEMATOUS DERIMATITIS.}

Erythema of the skin-hyperemia of the papillary layer and superficial capillaries-is the simplest of tegumentary diseases. Sometimes it is diffuse, at other times more or less circumscribed (roseola). In our large domestic animals it is only found upon the depigmented cutaneous surfaces (upon the white spots of the head and extremities in those animals the skin of which does not contain any pigment (sheep, pig, dog, albino or piebald horses, etc.), it may be found upon the most diverse regions. Its only remarkable symptom is a diffuse or circumscribed redness of the skin, which disappears momentarily through the pressure of the finger, contrary to what takes place when this abnormal coloration is of hemorrhagic nature (petechiæ, ecchymoses, purpura); it is sometimes accompanied by pruritus. When it persists for a certain time it leads to an abundant epithelial degeneration.

Etiology. Erythema sometimes originates quite suddenly and constitutes a real morbid entity, at other times it is but the initial stage of other cutaneous diseases. In the first case it is ordinarily fugacious and does not leave any anatomical alteration behind it.

The principal causes are:

1. Mechanical irritations of the skin [pressures, frictions, shear- 
ing of sheep and dog, contusions during transportation of the animals (pig), etc.]. Here, erythema is called traumatic.

2. Chemical irritations: slight cauterizations, frictions having a carbolic acid or tar base, also cantharides, emetics, sinapisms, etc.; decomposition of urine upon the skin, which is soiled with it in the neighborhood of the vulva or sheath, in paralysis of the posterior quarters; insect stings, and the action of moulds; this form is toxic erythema.

3. Thermic irritations: extreme temperature (first stage of a burn and of congelation), the action of the solar rays falling directly upon the regions which are deprived of pigment: this form is termed caloric or solar erythema.

Treatment. We must suppress its cause or attenuate its effects, cover the skin with compresses of white lotion, and dust it with starch, oxide of zinc, use frictions with a salve of lead or zinc base; finally, when the pruritus is very violent, we use nitrate of silver in a solution of 6 per cent.; such are its principa! indications. In the larger number of cases, cutaneous erythema disappears within a few days without any intervention.

[In mild cases of dermatitis in the horse we may make use of an application composed of ung. zinci oxid. 1 part, hydrarg. oleat. (5 per cent.) 1 part, glycerin 4 parts. This will be found a most efficient preparation, not only curative but as a prophylactic. The action of mercurials in inflammations of t'is skin is too well known to require discussion in this connection.-W. L. z.]

A special form of this disease, which, however, is no longer a simple erythema, is represented by the eruption produced by feeding with buckwheat (fagopyrism). It sometimes develops in the shape of erythema, but oftener it is complicated by an erythematous dermatitis (inflammatory infiltration of the derma and oedema), which may take on the vesicular, bullous, phlegmonous, erysipelatous, or gangrenous forms. Indeed, it is impossible to draw a distinct line between simple hyperemia of the skin and its erythematous inflammation.

The cause of fagopyrism is feeding upon buckwheat (Polygonum fagopyrum) and a few other plants of the same family ( $P$. persicaria, etc.) It may be produced by green buckwheat or by seeds, husks, dry stubbles, etc. But it seems that the influence of sunlight is necessary to its development. It is probable that in the majority of cases its pathogenesis is the following: moulds and other 
fungi existing on the surface of the plant, on reaching the unpigmented skin, already affected by solar erythema, further irritate it and produce a more or less intense dermatitis. Under certain circumstances it is possible that insects may determine what has been called buckwheat eruption (buckwheat fields constitute excellent pastures for bees); it is also possible that an idiosyncrasy existsan individual sensitiveness which favors the development of this affection.

AFFECTED ANimals. The disease is most frequently observed on white, piebald, or spotted sheep, especially on lambs. It is also found in the pig, more rarely in the goat (Hering) or the ox, and in solipeds, the skin of which is partially discolored. Black or spotted black sheep, those living in stables and those going tu pasture only during damp weather or which pasture in the shade, are not subject to it. An eruption may also be produced when the animals are exposed to the sun's rays from eight days to four weeks after having ceased feeding on buckwheat; as soon as they are subjected again to permanent stabling it disappears, to return again if the influence of the sun is felt anew. Hemminger observed a disease similar to fagopyrism in horses working in potato fields at the time when the stems of the latter were in full growth.

Symptoms. They consist of an intense redness (erythema) and and inflammatory tumefaction (erythematous dermatitis) of the ears, eyelids, and face, which extends at times to the guttural region and to the neck and shoulders; these phenomena are accompanied by an intense pruritus, the animals are restless, shake the head, rub themselves, perform uncontrollable movements; we may even observe rabiform symptoms. Sometimes the red and tumefied skin becomes covered with vesicles, from the size of a lentil to that of a pea, containing a yellowish liquid; the small wounds resulting from their rupture become cicatrized and covered with scabs (pimple of the sheep, bullous erysipelas). The intensity of eruption is in proportion to the quantity of buckwheat consumed and the duration of exposure to sunlight. During the winter it is only expressed by itchings and scratching.

In grave cases we see respiratory troubles (erysipelatous inflammation of the respiratory tract), also fever and cerebral symptoms : vertigo, stupefaction, whirling, spasms, intense excitement, etc., which are undoubtedly produced by the propagation of the erysipe- 
latous process of the skin and head to the meninges. These cornplications may lead to death. Vertiginous and epileptiform attacks observed by Rabe ${ }^{1}$ and others on horses and pigs the cutaneous tegument of which was unaffected would induce the supposition that there exists a narcotic poison in buckwheat.

Treatment. From a prophylactic point of view we should avoid driving white sheep into buckwheat fields during hot weather or when the sun is very strong; they must only be taken there toward the end of the day or in cloudy weather. It is always more advisable to have this plant consumed in the sheepfold. When the disease has made its appearance the animals must at once be taken to shady places, or better, into stables. If there are any complications, we should institute local treatment. Erysipelatous inflammation of the skin of the head, accompanied or not by cerebral and general symptoms, must be combated by cooling compresses or lotions with a solution of cresol, and thorough internal administration of antiphlogistics.

\section{ECZEMA.}

Eczema may be defined as a plain dermatitis of multiple form presenting numerous stages of development and very variable in its intensity. Considered in its nature and course, eczema offers the greatest similarity to inflammation of membranous organs, especially the mucous membranes; it has therefore been looked upon as a kind of catarrh of the skin. As with catarrhal inflammation, we may recognize in it serous, mucous, and purulent types, etc., according to the activity of the phlegmasia and the constitution of the affected regions. This polymorphia assumed by eczema in its manifestations has been the cause of the classification of a large number of special diseases under this denomination. These old terms much more easily explain that the same cutaneous affection may present objectively considerable differences; the most simple dermatoses acquire a certain serious character when the animals rub or scratch themselves; the same influence may produce phenomena which are altogether dissimilar, according to the location of the integument upon which it acts, also an individual sensitiveness, the constitution and age of the animals, ete. The numerous influences to which these diseases of the skin are susceptible account readily for the obscurity and confusion which has always existed in this respect.

1 Rabe: Preuss. Mittheil., Bde: xvi. u. xvii. 
For clearness of description it is indispensable to make a judicious selection of them.

Eczema, like any other inflammatory affection, shows different stages of evolution. In our domestic animals it runs through the phases of simple dermatitis in man; thus, according to the nature and aspect of cutaneous affections, we distinguish the following six periods :

1. Erythematous stage. Characterized at the beginning by hyperemia and a superficial exudation, it consequently results in the thickening of the epidermis, which disappears later through a pultaceous desquamation. The chronic condition of erythematous eczema has received the name of squamous eczema; but most frequently this is only the final stage of the eczematous process. Diseases described by the following names are related to this type: pityriasis, psoriasis, squamous herpes, mange, and also in the horse, eczema of the canon and grease.

2. The papular condition consists of small nodes which a microscopic examination shows to be formed of a serous and cellular infiltration and through the swelling of papillary elements. Papulous eczema comprises lichen, rash (strophulus) and heat pimples of the horse and ox.

3. The vesicular condition. At times this is developed directly, at other times it follows one of the preceding stages (when the serous exudation is abundant in the papules and the cells of the Malpighian layer are separated from one another, the liquid exudate reaches immediately the most superficial epidermic layer). It represents in some ways the type of eczema; it is often designated simple eczema. It corresponds with herpes of our domestic animals. Heat-rash of the horse may be related to it, but it has nothing in common with the herpes of man.

4. The moist condition (eczema madidans). It occurs after a spontaneous or artificial opening of the vesicles (frictions); it is the red eczema or moist rash. It is generally observed in the dog in the shape of the rash or mange of obesity.

5. The pustular condition. This is sometimes produced from the outset, at other times it is due to the passage of vesicles into the pustulous stage. After their opening the skin is often transformed into a suppurating surface; to a more or less considerable extent, it is impetiginous eczema. It corresponds to pustulous rash, or to scurvy eczema of the dog or horse. 
6. The scabby stage (scabby rash of our animals). It is the result of the desiccation of the exudate in all the moist forms of the disease. In many cases, and especially in the chronic form, scabby eczema is related to squamous eczema, of which we have just spoken.

These successive stages give an explanation of the multiple forms of eczema. But this disease does not necessarily travel through all these stages; it may pass directly from the first to the last, and there are cases where, having reached a certain phase, it remains stationary; we must point out that, upon the skin which is covered with hair or pigmented, several stages which evolute very rapidly may pass entirely unobserved; such are especially the papular and vesicular stages.

We will now proceed to examine eczema in our different species.

\section{A. Eczema of the Dog.}

Eczema is the most frequent of the cutaneous diseases of the dog. It is ordinarily located upon certain regions; it is especially observed along the dorsal column; it starts at the base of the tail; on the back or neck; from these points it extends and radiates in all directions. The external face of the legs and buttocks are often affected, more rarely the abdominal and lower pectoral regions; the fold of the knee, the neighborhood of the elbow, and the extremity of the calcaneum are also regions which are often affected. It is localized upon the head in some rare cases. Old or very young animals, pet dogs, and delicate subjects with fine skin, also fat animals, are particularly exposed to it. Concerning the races, the rough-coated dogs, bulldogs, and Leonberg dogs seem to furnish the largest contingent to the disease.

Causes. Eczema is generally the result of an irritation of the integument; this irritation may come from shearing or clipping, from repeated pressures and frictions exerted upon certain regions, from dust, dirt, and parasites (fleas, lice, trichodectes), which often abound in the dorsal region, the preferred location of eczema. Frictions or washing with black soap occasion it in a great number of cases. The rôle of fungi in the genesis of eczema is very incompletely known. Zürn was the first to express himself affirmatively concerning the mycotic nature of this disease. In vesicles of the dog, opened with all proper precautions, Müller has found micrococci; he maintains that eczema may occur whenever these parasites in considerable numbers reach a dirty skin, where 
they are developed rapidly. ${ }^{1}$ The adherents of this microbic theory go decidedly too far. However, the bacteria must act here, as in all the various phlegmasias, by increasing the inflammation. The question as yet undecided is, whether common fungi, found upon the surface of the skin, play a pathogenic rôle, or are met with there by accident. Concerning those cases where eczema appears without having been preceded by any cutaneous irritation, internal causes have been incriminated, especially ingestion of a poor quality of food, digestive troubles, plethora, weakness of constitution, etc. But these etiological influences probably act only as predisposing conditions. The cause determining eczema seems to be in all cases a direct irritation of the cutaneous integument.

[From an anatomical point of view we find that the skin of dogs. is naturally predisposed to eczematous diseases, but other conditions must necessarily be present: first, excessive alimentation, an affliction which all house dogs are called upon to endure; secondly, insufficient exercise, which is always associated with the first condition; thirdly, uncleanliness. These are irresistible influences constantly at work in the production of this disease.-W. L. z.]

Symptoms. Symptoms of acute eczema in the dog are of the most complete kind; it is difficult to give a general description of their nature. They vary much, according to the period at which they are observed.

1. In the initial phase, which often passes unnoticed, we find upon the skin which is deprived of pigment light-red spots, of a diameter varying from a pin-head to a pea; their coloration becomes gradually darker, and a small papule is developed in the centre; these spots become enlarged little by little, and also confluent; the skin undergoes tumefaction on their surface; it also becomes thickened, turgescent, and very hot; the hairs are erect in tufts. The slightest touch occasions pruritus, and these compressions, even when slight, produce pain. At a more advanced stage we may observe erythema or erythematous dermatitis. These characters are sometimes greatly modified by frictions and the

1 We may find microphytes as numerous as they are various, and without any pathogenic rôle, upon the surface of the skin and sometimes quite deeply in the excretory canals. When we wish to study vesicular and pustular lesions of the skin from a bacteriological standpoint, it is proper'to press the liquid to be examined or cultured from the deep parts of the derma and not through the epidermic layer; without this precaution the collected product is almost always impure.-N. D. T. 
action of the teeth. Arrived at this stage, the eczema becomes attenuated and disappears in most cases; in others it remains stationary. Impetiginous and scabby forms succeed the papulous stage.

2. When eczema follows a regular course the papules are transformed into vesicles. These last, which contain a clear liquid, are sometimes isolated, at other times in small groups; in the beginning their volume is rarely of larger size than a millet-seed; they often pass unnoticed; it is, besides, quite difficult to discover these upon the hairy integument of the dog. Some dry up in forming small crusts; others burst; they are torn by rubbing, and constitute inflammatory centres, upon the surface of which the hairs stick together and fall out. When the eczema retrocedes the diseased surfaces become covered with thin scabs, the inflammatory processes become attenuated, and the epidermis regenerated. Upon dark-colored skin slightly pigmented spots persist for a long time; they are vestiges of the receded vesicles.

3. Eczema is more frequently irradiating; the vesicles which have become confluent are ruptured, and form red, moist, depilated blotches (red eczema), covered with a serous, sero-plasmic or purulent exudate, which is very sensitive to the touch; they have a remarkable tendency to spread to the neighboring healthy parts (consuming rash or serpiginous eczema); under the influence of friction the disease sometimes ends in purulent or hemorrhagic dermatitis. The cure of red eczema is obtained through drying of the exudate (scabby condition) and regeneration of the subjacent epidermis, which takes place from the periphery to the centre of the blotches.

4. Vesicles are also quite frequently transformed into pustules: they increase in size; their contents are turbid and become purulent (pustulous eczema). If disseminated or agminated, the pustules become torn, and form in some places more or less extended suppurating surfaces. The hair is damp, agglutinated by the exudate, and gathered in packed layers; the hairs fall out on the least pulling, and many are completely detached. The diseased regions, which are covered with a yellowish or greenish-yellow glutinous or creamy pus, are the seat of an intense sensitiveness, and bleed at the least contact; the skin is much thickened (impetiginous eczema). The cure takes place through desiccation of the exudate, which forms crusts, under which suppuration may persist for a certain 
time. In some rare cases, but especially when animals rub or scratch themselver constantly, the process becomes aggravated; a phlegmonous inflammation is produced, which is followed by ulcerative destruction of the derma, with abundant suppuration, extensive depilation, and callous thickening. Similar circumscribed purulent dermatitis accompanies vesiculous or pustulous eruptions which are due to mechanical, chemical or violent thermic irritations.

The different varieties of eczema may pass into a chronic state. In benign papulous or vesiculous forms recurring cases are not rare. Pustulous eczema is remarkable by its tendency to the chronic condition. The persistence of the affection seems to have as a principal cause the rubbing and scratching of the diseased animals.

The manifestations of chronic eczema are permanent hyperemia of the skin, local hyperthermia and hypertrophy, which may treble or quadruple the normal thickness. The surface of these hypertrophies is shiny ; it is the seat of a very active epidermic proliferation and of an abundant desquamation (squamous eczema). Little by little the skin becomes dry, hard, rigid; it is wrinkled, and folds artificially made in it persist for a certain time; the hairs, which are very sparse, are erect, standing in all directions; they are often dried, brittle, or entangled like sheep's wool (compression and atrophy of the hair-bulbs and of their bloodvessels by connective tissue neoformation and retraction of the tissue); the diseased surfaces may be completely denuded. Sometimes the skin presents a granulons aspect, due to thickening, hypertrophy of the papillæ, which are more or less prominent. Alterations produced by serious eczemas predispose to a recurrence of the trouble; an acute outbreak happens in the spring, when the cutaneous functions are especially active.

The general health of animals affected by eczema is ordinarily but little modified. We observe only permanent agitation, restlessness, and intense thirst. When the disease is prolonged and acute outbreaks occur, continual itching, loss of heat and of nutritive fluids, with reflex excitement, produce gradual emaciation and cachexia. Very young or weak animals may succumb.

The duration of acute eczema varies from one to three weeks. Chronic eczema persists often for many months, even for years, showing remissions of variable duration. 
Differential diagnosis. Eczema may be confounded with suppurating dermatitis which is produced by wounds, contusions, cauterizations, burns, cold, tonsurating scurvy, and mange in its various forms.

1. Traumatic dermatitis consists, as a rule, of a circumscribed reactionary phlegmasia, which is developed around a scab produced by cauterization, or a gangrenous blotch, etc.; they coexist, as a rule, with a limited mortification of the skin. Papules, vesicles, or pustules characteristic of eczema are absolutely wanting; but as these lesions may not be perceptible in eczema, a distinction is sometimes quite difficult.

2. Tonsurating thrush is recognized by the rounded form of the eruption, by the absence of pimples, vesicles, pustules, and of pruritus; by its transmissibility ; finally, by the microscopic character of the alterations.

3. Follicular mange is especially olserved upon the head and legs; if contagious it produces but slight itchings; its symptoms are often typical, and in doubtful cases finding of the Demodex assures the diagnosis.

4. Sarcoptic mange affects preferably regions where the hair is not abundant-the lower part of the abdomen and thorax, the internal face of the thighs, the hock, elbow, and the base of the ears. It is contagious and accompanied by very sharp itching. These characters do not always suffice, however, to establish a diagnosis, and sarcoptic mange shows sometimes a great resemblance to eczema ; in such cases the microscope is absolutely necessary in order to establish the difference.

Treatment. The treatment of eczema must vary according to period of the affection and the constitution of the patients. In the majority of cases the eruption would certainly be cured with the help of Nature alone, if we could suppress the irritation produced by the pruritus. But it is extremely difficult to avoid this irritation; in some dogs pruritus is violent and lasting, and the patients are constantly tormented by itching; they overcome all the mechanical devices used in order to prevent them from scratching or rubbing themselves (muzzle, bandages, collars, boots, etc.).

1. In the benign forms or starting stages of eczema (erythematous, papulous, vesiculous stages) we must first try antiphlogistics, emollients, anodynes, and protective dressings. We use, as a rule, 
plain zinc ointment ( 1 part of oxide of zinc to 9 of $\operatorname{lard}^{1}$ ) or lead ointment, and especially Hebra's ointment (a mixture of equal parts of plain litharge and paraffin ointment ${ }^{2}$ ). White precipitate ointment must only be used for dogs with the greatest care, on account of the danger of poisoning through licking. Among the protective dressings we must especially recommend powders which will adhere to the skin of the regions on which they are applied. The following mixture may be used: Zinci oxidi 5 grammes, amidon (starch) 20 grammes.

2. For moist and impetiginous eczema we have used, for a very long time and with success, a solution of caustic (argentum nitras), 6 per cent., which is applied by means of a brush upon the diseased regions, thoroughly cleansed beforehand; a dry scab is formed under which healing progresses very rapidly. Among the ointments we give the preference to nitrate of silver ( 1 per cent. in paraffin ointment) or to tannin (tannic acid 5 grammes, paraffin ointment 50 grammes). Drying powders are very efficient for obstinate moist pı ocesses; we generally prescribe a mixture of fine iodoform powder and oak-bark 1:10, or cresol combined with boric acid ( 2 to 4 per cent.).

3. In chronic eczema our main remedy is tar-in a natural condition, or in an alcoholic solution (beech tar and alcohol, of each 25 grammes), or in a liniment (tar and green soap). We may use the following preparation against chronic impetiginous eczema: beechwood tar and green soap, of each 50 grammes, alcohol q. s. Four to six days after the friction we may detach with care the dry scabs then formed upon the diseased surfaces. In certain cases it is necessary to make a second application. Tar liniment has the advantage of being easily removed by bathing. At the present time we replace tar with cresol; this is not at all toxic, and its action is equal to that of tar; it is proper to use it in the shape of liniment (cresol and green soap, of each 100 grammes, alcohol 50 grammes), creosoted alcohol (1:10-20), or ointment $(1: 10-20$ of paraffin oint-

1 This preparation has also this formula:

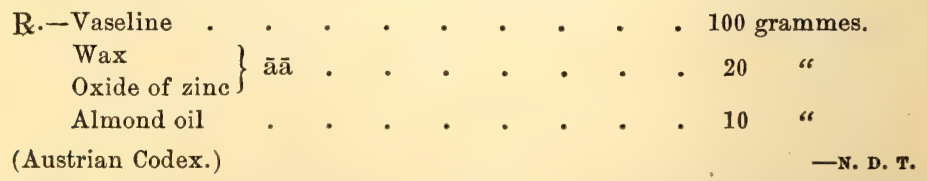

2 Paraffin ointment is composed of four parts of liquid paraffin and one part of solid paraffin. - N. D. T. 
ment). Squamous eczema may also be combated with tar liniment or cresol, or by these agents associated with alcohol $(1: 10-20)$ and by green soap. Among the other agents we have used with advantage chrysarobin (chrysarobin 1-5 grammes, unguentum paraffini 20 grammes) oxynaphthalin $(1: 10)$ as well as natural ichthyol.

The series of medicaments used to combat eczema in the dog is far from being exhausted (naphthol, anthrarobin, resorcin, naphthalin, oxynaphtholic acid, etc.). The enumeration of the many formulæ would be fastidious and useless.

No matter which agent may be used, it is advisable to cut the hair before applying it. The region which has been subjected to friction must be covered with a bandage, in order to prevent the dogs from licking themselves (poisoning).

Internally, it is customary to use arsenous acid for chronic eczematous processes-Fowler's solution, administered for several weeks at a time, in a dose of 5 to 10 drops per day. We have tried it quite frequently, but without much success. But in the moist forms of eczema purgatives will be of excellent service in the beginning of the treatment; desiccation of the blotches is very rapidly produced under their influence. Dietetic means (suppression of meat, etc.) have not, by far, the importance generally attributed to them; they have never given us satisfactory results.

[The first indication in the treatment of eczema is to reduce the food of the patients and to increase their exercise: internally, the administration of magnesii sulphas in sufficiently large dose to produce a laxative effect upon the bowels, giving one dose every alternate day for two or three weeks, will alone cure many cases, especially those that are of but a few weeks' or months' standing. For the last ten years this has been my only treatment for eczematous diseases of a mild type. When the affection is of long standing and more or less extensive, I have invariably associated with this treatment a local application of the iodide of sulphur ointment made according to the following formula :

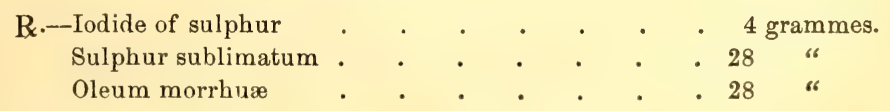

M. et ft. unguentum.

Sig.-One application, repeated if necessary after ten days.

This may be modified by changing the cod-liver for equal parts of cosmoline and lanolin. I have never taken any precaution to 
prevent the patients from licking themselves, as I have thought that while the ingestion of the iodine usually made them sick, it never had any serious consequences, but appeared rather to hasten the cure. Every part of the body should be anointed whether it be diseased or not, and it is a rare condition that will require a second application. I have obtained such good results from this treatment in eczema that $\mathrm{I}$ am almost tempted to look upon it as a specific for this condition.-w. L. Z.]

\section{B. Eczematous Diseases of the Horse.}

Most of the non-parasitic cutaneous diseases of the horse are of a purely eczematous nature. Various dermatoses described as special eruptions must be classified with eczema, and we may without any inconvenience suppress the names applied to some of these. We connect with eczema the following eruptions, which have been considered as so many independent diseases :

1. Lichen and strophulus, summer mange, saddle mange, heat pimples, pimply and tuberculous eruptions-which are but varieties. of papulo-vesicular eczema.

2. Pityriasis and psoriasis - the characteristics of which are those of squamous or chronic eczema.

3. "Mange" of mane and tail, diseases designated under the name of "rat tail" - may be classified with pustular impetiginous and chronic eczemas.

4. Pastern crevices (scratches), salanders and malanders-which represent a local eczema, at first moist and later impetiginous.

\section{Papulo-vesiculous Eczema (Lichen, Strophulus, Heat Pimples, Summer Eruption, Summer Mange, Saddle Mang̀e).}

Etiology. Papulo-vesiculous eczema of the horse is usually limited to certain regions; it may, however, invade the whole surface of the body (during the course of strangles-for instance, where it is developed parallel with urticaria, and during the summer, when it appears in the shape of heat pimples or summer mange). The regions which are mostly affected are those where the sudation is abundant or which are exposed to mechanical irritations : head (halter), the lateral sides of the base of the neck and shoulders, the neighborhood of the withers (collar), the back and costal regions 
(saddle, girth, shafts), hind quarters, breech (breeching of the crupper). ${ }^{1}$

There is no plausible reason to admit the intervention of internal causes adding their action to those of these local irritations: young age, fineness of the integument, and the moulting or shedding season must be considered as causes predisposing to this dermatosis.

Symptoms. Papulo-vesiculous eczema is essentially characterized by the development of numerous papules which are arranged in irregular groups ; at the beginning of the disease they may only be found in passing the hand over the integument; their volume varies between a millet-seed and a pea; their consistence is soft, but later they become hard, and the hair is erect upon their surface. The skin is warm and sometimes slightly tumefied; when it is folded or slightly pressed we observe an intense sensitiveness. At the time of efflorescence there is a moderate pruritus, which disappears when the disease is at its height.

The papules are soon the seat of a sub-epidermic serous exudation (vesiculous condition); small scabs are formed which agglutinate the adjacent hairs into tufts, and carry these along when falling out. Circumscribed depilated spots remain, which are reddish when the skin is deprived of pigment, are covered with a grayish epidermic pellicle when the skin is pigmented, and squamous when exudation has been abundant (exfoliant rash). In cases where the inflammatory process is much extended, whitish blotches persist on the pigmented skin; these are entirely depilated (strophulus).

Treatment. This variety of eczema is benign ; it heals of itself as soon as the different stages of inflammation have ensued; the hair returns and grows slowly. In the majority of cases it is useless to resort to drugs; if, however, the owners prefer to have their animals treated we may advise softening of the scabs and dirt with glycerin, vaseline, or green soap, and friction with alcohol upon the depilated regions, with the object of stimulating the growth of hair. The most favorable preparations are tincture of tar (tar and alcohol) and tincture of creolin (1:5-10 of alcohol). Ichthyol has also been advised (Bass).

1 Acute eczema may be developed by this mechanical action on regions where cutaneous surfaces rub against one another during movements (of the foreleg, groin), especially when the skin is covered with sweat and dust; we give them the name of intertrigo; it is also expressed by the saying that the animal has chafed the foreleg, etc. 
Observations upon the Relations Existing between Prurigo, Pruritus, and Eczema.

1. In man we designate under the name of prurigo a papulous cutaneous eruption, which is preferably located on the surfaces of the lower members. Prurigo is almost exclusively observed in very young children: it is accompanied by intense itching; its course is chronic; it is hereditary. After having persisted for several years it leaves special alterations on the skin (thickening, pigmentation, etc.). It is only curable in a minority of cases. Many patients succumb to the continual and intense itching, the permanent excitement, and to the progressive exhaustion which is produced. It is generally admitted that this eruption, which has very distinct characters, exists in our animals; works on special pathology mention it under the title of pruriginous eruption. It does not seem to us that we are warranted in describing in our veterinary literature a cutaneous affection corresponding with the prurigo of man, for the following reasons :

a. Monographs of prurigo given by veterinarians are contradictory in their essential outline; in going through them we have become convinced that some authors have manufactured a prurigo of animals; several have, moreover, obtained their information from one and the same source.

$b$. The disease claimed to have been observed on the horse, the dog, the ox, and the sheep, does not at all correspond in its symptoms, causes, and course, to the prurigo of man; it is, indeed, impossible to find anything typical at all in the description of pruriginous eruption of animals.

c. The causes given are as diverse as they are flimsy : anomalies of diet, digestive troubles, heating food, changing fróm a poor nutrition to one very nourishing, acidity of the blood, shedding, microbes, etc. It is claimed that micro-organisms have been found, as in eczema.

d. We have never observed true prurigo in the subjects of our different species, and many cases reported under this name were certainly nothing else but papular eczema; the symptomatology of a large number of them answers exactly to that of this latter affection; concerning chronic and incurable cases, they belong to the list of the different kinds of manges. In the interest of accuracy of description, and in order to remain within the domain of facts, 
we do not believe in admitting prurigo among the dermatoses of our animals.

In human medicine the word pruritus serves to designate an itching existing outside of any appreciable alteration of the skin. It is only observed on the extending surfaces of the extremities, on the palms of the hands, soles of the feet, genital organs, and in the neighborhood of the anus. It is evidently an affection of nervous origin. In our animals, the existence of a similar morbid state is not at all demonstrated.

\section{Squamous Chronic Eczema. (Pityriasis, Psoriasis, Mange} of Inanition.)

Etiology. Squamous eczema is described under the name of pityriasis or psoriasis in certain special works; it has been identified with these same affections of the human species. This connection is not well founded. Observation demonstrates that the various forms of eczematous processes may pass into a squamous state after a period of variable duration.

Its causes are those of eczema in general. Among the principal we must mention : uncleanliness, neglected hygiene of the skin, and insufficient alimentation (mange of inanition) or food in a damaged condition. Some fungi have also been incriminated, but without any proof being furnished in support of their pathogenic rôle.

Symptoms. Squamous eczema is marked by the development of small granular or furfuraceous scabs on the surface of the skin, and under which the derma shows chronic alterations, such as thickening, grayish coloration, induration, etc.; itching is rare. It is most frequently seen upon the head (especially on the orbits), on the inner surface of the auricular shell, the neck and shoulders, the base of the mane, elbows, hips, at the base of the tail, behind the knee and in front of the hock (sallenders and mallenders). It has an essentially chronic course; its duration varies from a few months to several years. It may be cured without any intervention.

Treatment. The treatment consists in taking care of the skin, in softening the scabs by means of green soap, and tar or creosote preparations (ointment, liniment, or tincture). Tar and creosote seem to transform the obscure and tenacious chronic inflammatory process into acute phlegmasia which ends rapidly in a cure. Chrysarobin ointment acts in the same way.

The expressions psoriasis and pityriasis, which are borrowed from 
human dermatology, have been applied in veterinary medicine to cutaneous diseases of an eczematous nature.

Psoriasis of man is characterized by an inflammatory infiltration of the papillary body with a horny transformation of the epidermis which covers it. At the outset we observe red pimples ( $P$. punctata) which are transformed later into squamous nacreated blotches ( $P$. guttata); later these blotches may reach a diameter of a silver dollar ( $P$. nummularis); at times they become confluent and cover large surfaces; they often heal from the centre and then form ringor garland-shaped pictures, according to their isolated or confluent condition ( $P$. annularis et gyrata). Its preferred locations are the surfaces of extension of the elbow and carpus, the hairy part of the head, forehead, and ears. We have never observed a similar disease in our domestic animals.

Pityriasis of man is a very rare dermatosis, which by a redness and desquamation sometimes extends over the whole surface of the integument. It determines atrophy, even gangrene of the skin, general emaciation, and finally death. Such a disease does not exist in animals.

3. Chronic Impetiginous Eczema of the Regions which are Covered by the Mane. ("Mange" of Mane and Tail, Plica.) $)^{1}$

The different forms of eczema, but especially the vesiculous and pustular condition, are observed on regions covered with coarse hair, and principally on the upper edge of neck and shoulders (mane mange), and upon the tail (tail mange). As a consequence of the inflammatory action and the nutritive disturbances of the skin, certain pathological products necessarily take part in the process ; at times the mane becomes agglutinated, atrophic changes take place in the skin and connective tissue (plica), sometimes the hair falls out in great quantity, producing the so-called rat tail.

Etiology. The causes of impetiginous eczema located upon the regions covered by the mane and tail are purely local. We must incriminate, first, uncleanliness and neglected hygiene of the skin, which permit of the accumulation upon its surface of hay-dust and a large variety of irritating bodies. Abundant and felted horse-

1 The denomination plica has been adopted in Germany, undoubtedly on account of the similarity of the affection with plica of the human species.-N. D. T. 
hair constitutes a preferred location for numerous parasites (lice, trichodectes, etc.). This disease is, therefore, most commonly found on badly kept farms and in countries where, on account of certain prejudices or superstitious belief, the grooming of the horse is neglected (Tartary, Russia, Poland, etc.). A cause which is entirely the opposite of the preceding, is the abuse of soap on the mane; if the soap is not removed with a free use of water, it becomes the agent of a repeated or permanent irritation of the skin of the neck, and may produce eczema.

Prolonged dampness of the mane (through rain water, repeated washings), leads to maceration of the epidermis and decomposition of the normal secretions of the skin (sebaceous matter, perspiration), like rain rot of the sheep; it thus becomes a direct cause of eczema, which is produced so much easier on account of the fine and tender condition of the skin of the regions protected by the mane, which are thus specially predisposed to this disease. Anterior inflammatory processes which have more or less modified the condition of the skin favor a recurrence of the trouble (squamous eezema).

Symptoms. The first manifestations of eczema on regions covered with horse-hair (the mane) often pass unobserved, either on account of neglect in the eare of the skin, or because the dermal lesions are masked by the cutaneous exfoliation. It is only when the decomposed inflammatory products have aggravated the disease that the owner observes the alterations of the hair and symptoms of pruritus. The animals scratch and rub themselves, they bite the diseased parts. The lesions are then generally the following: the skin is covered with a serous, purulent, bloody, or scabby exudate; vesicles and pustules are often observed in it; the hairs of the mane and tail are agglutinated by perspiration and by a fatty, sticky, dirty, fetid matter; they become firmly packed in a more or less thick layer (Polish plica). Later, when the inflammatory process is extended to the hair follicles, the character of the horse-hair is modified; the hairs become fragile, curl, and through the irregularity of direction the felting which characterizes plica is favored. Upon other regions where the animals rub or bite themselves easily (at the tail, for instance), the hairs become atrophied and fall. The skin of the caudal appendage becomes gradually sclerosed, thickened, and indurated, the cutaneous papillæ become depressed, the horse-hairs do not grow again (rat tail). This is the chronic state of squamous eczema. 
The duration of the process is often very long. It is always difficult to obtain a cure.

Treatment. It consists in cleansing the diseased regions thoroughly and in drying the moist blotches. Before all else it is indispensable to cut the agglutinated horse-hairs at their root. After having rendered the skin accessible to medicament, we apply upon it a liniment of creosol or tar or astringent drying powders (iodoform mixed with powdered oak-bark), or a solution of nitrate of silver, 6 per cent. The latter produces a blackish coloration of the hair on light coats, a circumstance which should be mentioned to the owner. In most cases the trouble necessitates long treatment.

We have considered plica or Polish plica as a phenomenon of wet chronic impetiginous eczema of the upper border of the neck and shoulders, an affection in which the propagation of the inflammation to the hair follicles may determine an alteration of the horse-hair. The descriptions which were formerly applied to plica in the treatises upon veterinary pathology, and the importance which the public has for a long time attributed to it, occasion some astonishment. It was formerly considered as an enzootic disease. It had also been regarded as a favorable premonitory manifestation : its appearance during the course of an internal disease announced its. near cure; its disappearance, on the contrary, predicted a fatal termination.

Some authors, Spinola among them, admit that plica depends upnn a constitutional disease ; Haubner classifies it among nutritive troubles; others maintain that it is determined by fungi. Haselbach has observed it quite recently on one-tenth of the equine population of Poland. He considers it an affection of the mane and tail in which a viscous liquid is excreted from the stem of the hair! Strange opinions formerly advanced as to its nature are rendered still more confusing by the distinction of true and false plica. The latter term has served to designate a simple packing of the hair of the mane and tail by dirt, dust, or other foreign bodies (thistle-heads, etc.).

In the horse, plica is a disease which is entirely due to neglect. This opinion has been admitted for a long time in human medicine, where the disease is considered, besides, as an entanglement of the hair due exclusively to the lack of care and to uncleanliness.

[I have frequently seen this disease under conditions where it was not possible to attribute it to uncleanness or neglect. I have 
seen it in coach horses, in gentlemen's road horses, and in racehorses, and not in any instance could the disease be associated with. neglect or dirt as a cause. When treated early there is a chance of cure, therefore these cases should be cared for as soon as possible. Dress the parts with green soap and allow it to remain for twelve or eighteen hours, then wash thoroughly, dry carefully, and anoint with ammoniated mercury ointment (unguentum hydrargyri ammoniati). The oleate of mercury ointment, 10 per cent., is an excellent preparation for the treatment of this disease, as is also the iodide of sulphur ointment, as advised for eczema in dogs (see page 503), to which should be added two or three grains of corrosive sublimate to the ounce.-W. L. z.]

4. Eczema of the Flexion Surfaces of the Inferior Articulations of the Legs. (Salanders and Malanders: Grease).

The localization of eczema on the flexion surfaces of the extremities has for a long time been designated by special names. The most important is that upon the pasterns, and this, in opposition to the exanthema of horse-pox and dirty grease (Schmutzmauke), is also designated under the name of creviced grease. Eezema of the kneefolds and of the hock (malanders and salanders) is rare in proportion to the preceding; these articulations are more distant from the soil, to the irritating influences of which they are consequently less. exposed. It habitually assumes a squamous form.

Etiology. The flexion surfaces of the inferior articulations of the legs are predisposed to odematous infiltration, because the skin. is the seat of a continual displacement or wrinkling of these surfaces, a circumstance to which is added the irritating action of dust, mud, dampness, and cold. These etiological influences explain why grease is more frequent in winter than in summer, and also more upon the hind legs than on the forelegs.

During the cold season Prietsch has several times observed numerous examples of this disease on street-car horses which had been used on highways where salt had been thrown. Siedamgrotzky, Born, and Jelkmann have not been able to establish the injurious action of this agent by experimentation.

In common horses clipping of the legs sometimes becomes an indirect cause of this eczema by exposing the skin to the action of dampness and cold. 
Traumatic cases of grease, observed by Straub on army horses after manœuvres in stubble fields, have more analogy with infectious dermatitis than simple eczema. Similar lesions to that of grease may be produced by a large number of superficial wounds giving place to a secondary erysipelatous or phlegmonous inflammation. An infectious dermatitis is, too, very often developed in the course of creviced leg grease; it is due to the penetration of septic matters into wounds formed by the tearing of the vesicles, and into the fissures of the skin.

Symptoms. The acute form of grease, malanders and salanders, is indicated by typical symptoms. The skin is at first red, tumefied, painful and hot (erythematous state); it is soon covered with small vesicles (vesiculous state) which burst and discharge a serous, yellowish liquid, which is at first without odor (damp condition). The integument, by the movements of the animals, is formed into thick folds, hetween which crevices are formed (rhagades), the edges of which become inflamed and covered with dry scabs. The hairs are agglutinated, erect, or have fallen out in large quantity. The movements of the corresponding member are stiff and painful.

At this period the salanders and malanders may become healed or pass into a squamous state; in this latter case the skin becomes thickened, the trouble has a tendency toward the chronic condition, and returns of the disease are frequent. Grease produces in the course of time serious alterations in the pastern. The excreted liquid becomes decomposed and macerates the epidermis; a kind of viscous, doughy exudate is formed, which is of bad aspect, of fetid odor, is possessed of irritating properties, and gives rise to ulcerating losses of substance in the skin. The pastern fold becomes also the seat of granulating wounds, more or less extended, also of callous embossed thickening of the skin, which is affected by fissures or deep crevices (a condition formerly designated under the names of "tuberculous disease" and "callous leg grease)." The hairs are erect, standing like bristles. After months or years, cutaneous hypertrophy may be transformed into true elephantiasis, in which case the surface of the skin remains the seat of chronic squamous eczema (squamous grease). ${ }^{1} \quad$ The integument is sometimes tumefied

1 Elephantiasis (elephantiasic fibroma) or pachydermia is essentially characterized by hypertrophy of the skin and subcutaneous connective tissue. It occurs usually after inflammatory obstruction or reiterated odematous infiltrations. In the horse it is quite commonly found on the hind legs, mostly on one only, sometimes upon both. 
as far as the forearm or thigh. Thrush is a frequent phenomenon of chronic grease.

Treatment. The treatment of these eczematous affections varies according to the stage of the disease. At the beginning, attention to cleanliness of the skin, absorbing powders (oxide of zinc and starch), powdered oak-bark, lead or zinc ointment are sufficient. In advanced stages we must resort to more active measures. We seek first of all to prevent the secretions and their decomposition by means of drying powders and astringents. Liniments of creosote or tar are here of some service; drying powders (oak-bark, sulphate of iron, plaster, charcoal, etc.) and astringent baths (solution of alum, decoction of oak-bark, etc.) are also of advantage. When the affection is benign we may obtain the cure by application of continuous iodoform dressing for a certain time. In cases where granulations or callosities exist (elephant's foot) all these means remain without effect-an integral restoration has become impossible. The treatment must then be exclusively surgical-it is that of cutaneous neoformations; we must limit ourselves to extirpation and cauterization of the granulations which are growing too rapidly.

\section{Eczematous Diseases of the Ox.}

Generalized eczema is more rare in the ox than in the horse, but the forms of the eruption are nearly the same in both species. Thus we observe papulo-vesiculous and squamous eczema in the shape of rash, strophulus, scurvy, or inanition mange (Hungerräude); we find also on the extremity of the tail an impetiginous eczema similar to plica. In the ox more than in all other animals a certain alimentary regimen has been incriminated. In old treatises "stalkdisease" " occupies an important place. This disease is nothing

It starts always on the lower parts (pastern, fetlock), but it often extends above the hock. The diseased extremity, which is regularly hypertrophied, is hard and not sensitive to the touch; it may reach enormous proportions, especially on the fetlock; the skin of the coronet sometimes forms a voluminous swelling above the hoof. Lesions of the same nature may be observed on the sheath, the region of the girth, in front of the shoulders, and the dewlap in the ox. A dissection of the diseased tissues shows, in addition to their hardness and fibrous transformation, an enormous dilatation of the lymphatic vessels and sometimes an ossification of the periosteal connective tissue in the metatarsal and phalangeal regions. According to Cadéac, elephantiasis of the ox must be related to anasarca (see Revue Vét., 1884).-N. D. T.

1 Stalk-disease or grass fire is generally described as a vesiculous eruptive disease which is localized on the forelegs, the udder, the lips, and which are accompanied or not by itching. It is not of any gravity; it always ends in a cure. In oxen affected 
else than a papulous eruption at the beginning, which later becomes pustulous, and generally remains localized on the extremities; it would be produced by the ingestion of stalks and grape-vine leaves (see Indications of French Veterinarians in Hering's Pathology, 1858). The influence of alimentation is of chief importance in the variety which is designated "malt eczema" with which stalkdisease has certainly a marked relationship. Compared with maltdisease, all the other forms of eczematous process in the ox lose their importance. Concerning their clinical characters and their treatment, we would refer to what has been said relative to eczema of the horse. We must, however, add that in animals of the bovine species especially, causes which are entirely unlike may determine cutaneous diseases similar in their objective characters.

\section{ECZEMA PRODUCED BY MALT (FOOT RASH OR THRUSH).}

Opinions which have been advanced up to the present time on the nature and essential character of this form of eczema are far from agreeing with each other. Under this name have evidently been confounded dermatoses which are very different in etiology and symptoms. It is sufficient to consult the bibliography of this subject to become convinced that eczema produced by the ingestion of malt (l'eruption de marc; Träberaussshlag-grains or swill of breweries) or the refuse or residues from grapes, has been confounded with mange of the pastern, and especially with grease (eaux aux jambes crevassées). This latter disease, the existence of which has been doubted by many observers, is sometimes very difficult to distinguish clinically from malt exanthema, but the causes of these two morbid states are radically different. While grease is, as in the horse, the result of external influences, and principally from lack of cleanliness, of neglected hygiene of the skin, dampness, cold, etc., the disease which we are about to study is exclusively due to ingestion of potato residues. These two diseases are therefore very distinct eczematous forms.

From this confusion in terminology, opinions which are altogether divergent have originated as to the nature of malt eczema. The various hypotheses will be discussed farther on. We shall

by a dermatosis which showed all the characters of stalk-disease, Railliet and Moreau have found a large number of harvest-ticks (Leptus autumnalis) or Trombidion larva, which they are disposed to consider as the agents of this disease. They propose to give this trouble the name of Trombidian acariasis. 
consider this dermatosis as a toxic exanthema similar to those produced by certain drugs (to mercurial eruption, for instance), and due to the action of a poisonous matter contained in potato residues, perhaps in the tuber and stalks.

Affected animals. Malt or residue eczema has been known for more than half a century. Its appearance is contemporaneous with the introduction of large potato distilleries and the extension of the culture of this plant which took place at that time. Spinola, who observed it for the first time in 1827, gave a good description of it in 1836.

It is found to exist especially in oxen and bulls which are being fattened; it is less frequently observed in heifers ; milch cows generally escape it. While certain animals seem predisposed to it, others possess a true immunity. The gravest cases are observed in the spring and the beginning of summer, at the period of the year when the potato has reached its maximum growth.

It is found to be particularly intense in animals recently introduced into stables where the trouble exists; it is most frequently observed in establishments where the cows are often renewed; here new milch cows may be affected. In the greater number of cases it is localized on the hind legs, where it ascends as far as the hock; in others it affects exclusively the forelegs; the four members are sometimes affected at the same time; exceptionally it is located upon other parts of the body - for instance, on the ribs, back, neck, shoulders, etc. Care, hygiene, and the exposure of the stable have no great influence on its appearance; it may be recognized on model farms which are perfectly kept and where the animals are subject to the greatest care.

Etiology and pathogenesis. This eczema is generally the consequence of alimentation with potato residues. Those from rye and Indian corn are inoffensive; the same must be the case with beer residues, although these have been incriminated by some authors. Its intensity is usually in proportion to the quantity of residue which enters into the ration. Exclusive feeding with malts or a large ingestion of these (80 litres daily per head) will be sure to produce it when at the same time but little dry feed is given. Forty litres of malt per day are sufficient to produce it in some animals, but its course is then ordinarily benign. Residues coming from germinating potatoes or those in advanced fermentation are particularly dangerous. Their harmfulness, which is more or less 
marked according to age, seems also to depend upon a number of conditions: variations in fermentation and its products, diversity of species or growth of the potato, composition of the soil, manuring, etc.; it is possible that, as with lupin, the injurious quality varies with the soil where the tuber has grown. According to Peiffer, malts which have been subjected to a too rapid distillation would be particularly hurtful; their acidity does not seem to play any etiological rôle. According to Baranski the disease made its appearance in Galicia after the introduction of Gleason's potato in that country.

The disease which is here considered is not exclusively produced by malts; raw or cooked potatoes, especially those in course of germination, stalks of the plant, water used for cooking purposes, and acid fermented matters may also occasion it. From this fact we must infer that the potato itself contains a noxious agent, which remains in the residues in a quantity relatively more considerable than in the tuber itself, the carbo-hydrates of which have passed into alcohol. Nothing certain is known concerning the nature of this agent; it is generally admitted that at the time of its elimination by the skin it irritates the latter, inflames it, and thus determines an eczema. If milch cows are rarely affected, it is probably due to the elimination of the toxic principle through the very active lacteal secretion. Among the facts establishing that milk from sick cows is certainly harmful, we may mention that it produces diarrhea in calves (Johne), and occasions an eruption in children. ${ }^{1}$ We have observed that the affection is generally of a benign character in working animals; it is very likely that cutaneous excretion of the pathogenic agent diminishes its force, aided also by a corresponding elimination on the part of the various secretory organs under the influence of exercise. This theory has been objected to on account of the ordinary appearance of eczema on the hind legs. But this may first invade different regions of the body; and, on the other hand, we must take into account the relative delicacy of the skin of the extremities compared with that of the trunk, as well as the irritation exerted upon the posterior extremities by the liquid and even diarrheal excrements dejected by animals fed on malts, in which condition these members are kept in a constant state of dampness and uncleanliness. This weakened resistance of the skin of the extremities to medicamentous exanthema

1 Hering: Jahrbuch für Kinderheilkunde, 1873. 
is well known; we have observed it upon a horse, in which case large doses of bromide of potassium had been administered : a general eruption shows itself, which is mild on the trunk, and very intense, on the contrary, upon the extremities.

Opinions advanced on the subject of the nature of the toxic matter which produces eczema are not based upon any positive facts. The following chemical principles contained in the residues or in the potato itself have been in turn accused :

1. Solanine or solanidin. The affection has been looked upon for a very long time by some authors as a poisoning from solanine. In support of this theory, they claim an increase of solanine in potatoes at the time of germination of the plant and the relative frequency of the disease in animals which are fed with germinating potatoes or with their residues. But in malt or residue eczema we do not observe the symptoms of accidental or experimental poisoning by solanine, such as narcotism, staggering gait, stupefaction, paralysis, etc. (see Poisoning by Solanine); on the other hand, potatoes which are not yet undergoing germination, and which consequently do not contain any solanine, may also cause this disease.

2. Inferior alcohols produced at the same time as spirits of wine. They are found in all inoffensive malts, especially in those of rye; again, the residues of potatoes which are given to animals are almost always free from alcohol ; moreover, these do not exist in potatoes which are in process of germination. Finally, all the symptoms of intoxication by inferior alcohols are altogether different from those of malt disease.

3. The acids contained in potato residues (acetic, lactic, or butyric acids). They are also found in other residues, while they do not exist in potato stalks. The proportion of these acids, too, is extremely variable, and inoffensive malts are often quite loaded with them.

4. Johne has thought himself able to establish the relation of cause and effect between the potash salts contained in the potato and eczema, but nothing justifies this hypothesis : the potash compounds are muscular poisons, and their presence in a large proportion in alimentary matters is not injurious.

Other theORIES UPON THE NATURE OF MALT ECZEMAS. 1. According to Zürn, the fermenting cells (Sprosshefezellen) of beer yeast are transformed into batonets in the intestinal canal, which, 
with other micro-organisms (bacteria and micrococci) of the intestinal contents, would produce a mycotic inflammation of the skin when they are deposited on the latter by the intermediary of excrements. Johne has judiciously opposed the following arguments to this hypothesis: $a$. All malts contain the same yeast cells, all should therefore produce eczema; nevertheless rye and Indian corn malt do not determine it. $b$. The accused yeast and fungi are found in excrements of healthy animals which are fed on malt, and even in those which do not consume any; they must therefore be considered as elements without any etiological importance. $c$. The disease may appear, notwithstanding the most minute care and cleanliness, and it is not found in many unclean and badly kept stables; certain regions of the body's surface may be its seat without having been soiled by excrements. $d$. Contagion by excrements of affected animals has not been established, and attempts at the transmission of the disease through inoculation of malts are always failures; more than that, we have employed for a long time and with success as a method of treatment, lotions of hot malts (quite recently Baranski, on results obtained by himself, has taken up this argument and directed it against Zürn's theory). e. In eczema appearing after the ingestion of raw potatoes, potato stems, etc., there could not arise any question of a pathogenic influence of yeast cells.

2. Rabe considers eczema produced by malts as a simple parasitic (symbiotic) mange (Symbiosis bovis), which could be transmitted by contagion; intermittence in its manifestation would be associated with the biology of the parasites. Johne still further has clearly refuted this view by offering in opposition the following facts: $a$. Acari are only found accessorily in eczema produced by malts, and they are frequently observed in perfectly healthy animals or in subjects which have been recently cured; on the other hand, they are only found in one-third of the patients. $b$. Tail mange often exists in certain stables for years without the appearance of eczema, either in the patients or their neighbors. c. Inoculation of eczerna has always been a failure. $d$. It heals without medical treatment; in order to cause it to disappear it is sufficient to modify the alimentary regimen. e. Mange never spreads so rapidly as eczema. In fine, the very close relations which exist between alimentation with potatoès and the dermatosis of which we are speaking is undeniable. 
Symptoms. The local symptoms generally start two or three weeks after beginning the feeding with malt, sometimes sooner, when this diet is given exclusively or in very large quantity. They consist, first, in a redness or tumefaction of the skin of the pastern; recumbent animals have some trouble in raising themselves to the standing position; the movements of the affected members are constrained, and the walk is stiff. Vesicles which are often confluent and torn appear on the painful hyperemic skin; and moist surfaces are also formed where the derma is exposed. Later these lesions dry up and become scabby; the hairs are erect; the extremity of the legs is congested. The eruption generally invades the whole pastern and extends as far as the knee or the hock; it may even extend upward on the internal side of the thighs as far as the testicles in the male and the udder in the female. In certain cases it spreads on the trunk (abdomen, chest, neck, shoulders, back, etc.), which is at times covered with impetiginous scabs. The tumefied skin becomes wrinkled, cracked, and creviced, and a purulent liquid exudate oozes, which accumulates and forms more or less thick scabs. Besides these local alterations, general troubles exist. At the outset we observe a slight fever, inappetence, a delayed defecation, congestion, a hypersecretion of the conjunctiva, also salivation, etc. To these symptoms are added an obstinate diarrhea, weakness, and emaciation. When the disease is not controlled, the condition becomes aggravated and the animals die from exhaustion, septicemia, or pyemia. In entering stables where eczema exists we may sometimes perceive a peculiar mouldy odor.

The mortality is very variable. In Galicia, during the year 1885, Baranski recorded a loss in the proportion of 20 per cent $(70$ deaths in 380 animals). Serious cases are especially observed in unclean and badly ventilated stables, more rarely in well-kept places; they are mostly found in old animals which have little resistance and in those living in permanent stabling. Some subjects are affected as often as six times in a single year.

In general, eczema produced by malts is a benign disease, cured in two or three weeks; the scabs gradually become detached, the hairs fall, and the epidermis is left bare. When the eruption is neglected, however, it may be complicated with erysipelatous, phlegmonous, or gangrenous dermatitis, which is accompanied by superficial eschars, and sometimes by septic or pyemic infection. 
Differential diagnosis. Eczema produced by malts may be confounded with other affections, among which the most important are :

1. Grease, the objective characters of which are sometimes similar to those of the dermatosis we are now considering. From an etiological standpoint these two diseases are essentially different: the first is produced by influences which are exclusively internal; it is not at all dependent upon the method of feeding. Its alterations do not exceed an erythematous condition; vesicles are exceptionally found in it. In practice we must be specially guided by the indications and the regimen of the animals; all eczemas which are produced by other causes than alimentation with malts must be classified under grease.

2. Foot mange produced by parasites. Mange and eczema are sometimes concomitant; it is possible that the presence of acari favors the development of eczema ; but analyses made from microscopic comparisons show the symptoms and course of these diseases to differ essentially. Parasitic or symbiotic mange is always benign ; its evolution is very slow, and it is rarely located upon the extremities of the legs.

3. Aphthous fever which is localized on the lower part of the legs. It is characterized by a vesiculous exanthema which develops preferably upon the coronet and the integument of the interdigital space; it is extremely contagious. Even in cases where it coexists with malt eczema, the diagnosis is not difficult.

4. Panaris (whitlow). It consists of an infectious inflammation of the integument of the coronet or of the interdigital space, which is more or less extended upon the phalangeal region, and may affect the tendons, articulations, periosteum and the bones. It is a purely surgical trouble of the digits; it is frequent in the ox, but could not be confounded with exanthema produced by malt or residues.

The special symptoms of this affection are often indistinct on account of the agents employed by empyrics, or used by owners, etc. (petroleum and carbolic acid frictions, etc.). The diagnosis may then present some difficulty at the start.

Treatment. The principal indication consists in modifying the alimentary regimen. Malts must be suppressed, or the daily rations reduced to forty or even twenty litres, and we must give a greater proportion of dry fodder or other kinds of food. According to 
Märker ${ }^{1}$ the addition of Indian corn to potato residues, in the proportion of one-third, would suffice to counteract the development of eczema, on the condition of not giving more than seventy or eighty litres of residues per day. A large number of agents have been tried. Rabe recommends a tar and soap liniment (tar 1 part, soap 2 parts, sulphur 1 part, alcohol 2 parts), or carbolated oil in the proportion of 5 per cent.; others use desiccatives or astringents, either in a natural condition or in solution: oak-bark, the powder or a decoction, lead solution, sulphate of copper, sugar of lead, etc. Cresol in the shape of a liniment (cresol and green soap, of each 100 grammes; alcohol 50-500 grammes), or salve (1:10-20), in a water solution ( 2 per cent.), or alcoholic $(1: 10-25)$, may also be used with advantage. Moderate exercise favors the cure.

It is important to keep the places in good order. Uncleanliness. predisposes to the disease and aggravates it when it exists.

\section{Eczematous Diseases of the Sheep.}

Non-parasitic dermatoses of the sheep are very rare, and as yet little known. According to Haubner-Siedamgrotzky, we observe in the sheep, as well as in the horse, a papulous eczema which is marked by a modification of the coat; the wool is in tufts. In the course of distomiasis and other cachectic affections, we may observe a squamous eczema characterized by exfoliation of the superficial epidermic layers. In other cases, this inanition mange is due to the want of care of the skin ; it is then designated "dirty rash."

May has described a pruriginous vesiculous eruption and an impetiginous eczema under the names of prurigo and furfuraceous rash. "Eaux aux jambes sales" (dirty grease) exists also in the sheep, but up to date we have not yet observed authentic facts of eczema produced by malts or residues.

The most frequent eczemas and also the most important of this species is rain rot (Regenfäule), also called moist mange, or fatty mange. In poorly fed animals with sparse wool, and when the superficial layer of the epidermis is macerated by rain or dampness, this disease may be developed. It is accompanied by tumefaction of the skin, formation of vesicles and scabs, depilations, itching, etc. These alterations are usually located upon the back and the lumbar regions; they may extend forward to the withers, neck, shoulders,

1 Märker: Hand̉buch der Spiritusfabrikation, 1877. 
and head, and also backward as far as the base of the tail. In the majority of cases the recovery occurs of itself as soon as the rainy season is over. It is not necessary to resort to therapeutic agents.

This disease is prejudicial chiefly on account of the falling out of wool which it occasions. When it assumes an exceptionally serious character the animals must be kept in the sheepfold.

\section{E. Eczematous Diseases of the Pig.}

In the porcine species we know but "fuliginosis" of young pigs (pitcly mange, scabby rash-Russ). In the beginning this affection is characterized by a vesiculous eczema, which later become pustulous or impetiginous. It affects especially young, weak, or sickly animals [in cases of acute articular rheumatism (Ulrich); in rhachitis (Fünfstück); tuberculosis, hog cholera, etc.]. It is due to various causes (dirty stables, accumulation and decomposition of sebaceous matter, etc.). In most cases it depends upon a constitutional disease and may be compared to scrofulosis in man.

As a general rule it extends over the whole surface of the body; it is indicated by the appearance of vesicles, which rapidly go to the pustulous state and dry up, forming, black, thick, and pitchy crusts, under which the skin excretes a watery serous matter.

As to treatment, we may simply keep the beasts clean, wash them with soap-water, and also feed them well.

Zschokke has described under the name of granulous eruption (Schrottenauschlag) in the pig, a peculiar papulous dermatosis, having a chronic course, which is characterized by circumscribed blotches, of the size of the hand, and located upon the ears, back, croup, and base of the tail. The papules are of a gray-violet coloration, similar to that of lead shot; they are not transformed into pustules and do not produce any itching, which distinguishes them from fuliginosis. According to Zschokke, the morbid process consists in a proliferation of the papillary layer of the derma, and is caused by a specific micrococci. ${ }^{1}$

1 Under the name Scleroderma we designate a cutaneous affection of a hypertrophic nature, which seems peculiar to animals of the porcine species (Gabarret, Lécuyer, Thierry). It is characterized by rigidity and thickening of skin, by induration, sclerosis of the derma, and the disappearance of the adipose tissue (lard routé). It starts as a rule upon the dorsal region; thence it extends to the neck and shoulders, also to the croup ; then it invades the lateral parts of the body, and becoming general, it soon leads to cachexia. The principal alteration is hypertrophic sclerosis of the skin. This membrane may acquire a thickness of five centimetres (two inches) in the dorsal region (see Journ. de Lyon, 1882). - N. D. T. 


\section{URTICARIA.}

Urticaria consists of flattened, regular, distinctly defined pustules, standing more or less high above the surface of the skin; they are circumscribed to a capillary region and are due to a serous transudation which rapidly takes place in the papillary body and the chorion. It may thus be considered as an oedematous affection of the skin or as an acute serous dermatitis in which cellular infiltration has not had time to take place. This last character distinguishes the urticaria blotch from the pimple; it also explains the very rapid disappearance of urticaria by resorption of the serous liquid. The transudation which produces it is the result of a sudden dilatation of the capillaries, which is itself a consequence of a vasomotor irritation or of an embolus. Urticaria can therefore be considered as a vasomotor neurosis, or as an angioneurosis. In certain cases it occurs as a reflex consecutive to the irritation of the skin in others it is of internal origin.

Etiology. 1. Among the external causes, we must mention therapeutic or other agents which interest the skin directly: bites, of insects, hairs of caterpillars, nettles (Urtica dioïca and U. urens). Other irritations may also give rise to it in thin-skinned subjects; for instance, frictions of essence of turpentine used to combat colics in horses.

2. Internal causes produce urticaria only when an individual predisposition exists (young, sanguine, well-fed animals). The nature of this disease is unknown, but it should consist of a disturbance of the nervous vasomotor system. Pug dogs are frequeutly affected with it. An urticaria of internal origin may be produced by substances which, after having entered into the blood, exert a peculiar irritating action on the skin. It occurs in the course of a few infectious diseases (petechial fever, strangles, urticaria of the pig); here, the virulent elements undoubtedly constitute the essential factors. In urticaria generated by troubles of the digestive canal (gastro-intestinal catarrh, icterus), certain products of digestion and abnormal decompositions appear to be the pathogenic agents.

In animals, as in man, there must exist an idiosyncrasy or quite a special receptivity related to certain external influences, or to particular foods, which are liable to produce urticaria. Leguminous 
plants, buckwheat, green potatoes (Lippold), green rye, as also. abrupt changes of regimen, etc., are especially noted for producing this disturbance (in the human species, in predisposed individuals it appears after eating strawberries, raspberries, shrimps, oysters, fish, etc.).

It may occur in the course of other affections of the derma; thus Schindelka has seen it coexisting with English variola (pustulous dermatitis).

3. An abrupt chilling of the skin at the time when the body is much overheated may determine urticaria; the disease is therefore quite frequent in the spring and fall, also during great heats, stormy weather, rain, violent efforts, and rapid movements. It sometimes accompanies rheumatismal hemoglobinemia. In all these cases it seems rather due to the retention of injurious substances in the organism, which has originated under the influence of cold, than to an indirect irritation by this agent. But in a large number of others the cause of the affection cannot be determined.

The result of these considerations shows that urticaria represents mostly a purely symptomatic exanthema; it ought therefore to be considered only as an accessory symptom of the diseases of which we are now speaking. We have nevertheless thought best to describe it separately, because it frequently constitutes the only appreciable manifestation of a morbid complexus, and because in many cases nothing indicates the existence of an internal affection.

Urticaria is frequently observed in the horse, dog, and pig, more rarely in the ox. We shall first consider it as it is manifested in the first three species.

\section{Urticaria of the Horse, Dog, and Ox.}

Symptoms. In the horse it was formerly described under the names of pimple, ecthyma pustules, bubonic fever (Beulenfieber); urticaria is characterized by the sudden appearance of pimples or blotches, which often extend over almost the entire skin within a few hours or in one night. At the beginning we observe tumefactions which are flat, regular, soft, of the size of a pea, on the surface of which the hair stands erect, and which are usually located on both sides of the neck and shoulders, also on the back, the pectoral walls, and the buttocks. As they increase in size others are developed; they often become confluent, and form blotches of the size of a hand or plate, which in certain cases spread to the head, to the 
forward part of the forelegs, to the leg, and the mammæ (Schleg); it modifies greatly the appearance of the animals. Itching rarely exists.

Urticaria blotches are sometimes recognized on the visible mucous membranes, the pituitary, the buccal and vaginal mucous membranes. If they are developed in large numbers on the pituitary they may determine respiratory troubles (Schleg). In a cow Zipperlen found the conjunctiva much tumefied, the vaginal and rectal mucous membranes salient, forming thick swellings outside of the anus and vulva.

In strangles, urticaria of the skin frequently coincides with petechiæ of the pituitary. When the transudated liquid accumulates between Malpighian layer and the epidermis, vesicles may be formed on the surface of the blotches (urticaria vesicles, pomphus). In this case itching is generally intense and the hairs fall out.

Besides these manifestations, we observe also several troubles of the general condition which are subordinate to the nature of the principal disease. We often notice a fever of medium intensity (the distinction between febrile and apyretic urticaria, properly so called, is not of any importance); in addition, there exists weakness during work ; finally, we may sometimes observe the symptoms of a gastro-intestinal catarrh, troubles of appetite, diarrhea, retention of excrements, benign icterus, etc.

The eruption generally disappears very rapidly, often within one or two days, but returns of the disease are not rare; they may give the affection the stamp of chronicity. Some animals are affected several times in the same year.

Treatment. In most cases it is sufficient to subject the animals to a diet and to cover them warmly. When the general condition is at fault, it is advisable to produce a derivation on the intestinal canal. To accomplish this result we may administer any of the purgatives (aloes, calomel, etc.), or the laxative salines. [In the beginning of the disease the best treatment for plethoric horses is bleeding, for others purgation; the effects are most prompt and satisfactory.-W. L. z.]

\section{Urticaria of the Pig.}

Urticaria of the pig has often been confounded with rouget (swine plague) and contagious pneumo-enteritis-diseases which are, however, radically different from it. While it is generally 
sporadic, it must be considered as a specific disease. If we describe it here, it is because, from a practical standpoint, it is preferable to separate it entirely from these two morbid conditions, the infectious and epizootic.

Symptoms. Urticaria is marked by a sudden appearance of blotches on the skin, which disappear rapidly. It is accompanied by gastric troubles and a more or less intense fever.

In animals which have until then been very healthy it suddenly appears, and most frequently during the night, on the upper regions of the trunk, the chest, croup, abdomen, and the upper part of the limbs : flattened pustules of a more or less dark red color, of the size of 1 to 3 centimetres, of 1 to 2 millimetres in thickness, and very sensitive to the touch. By their confluence they form blotches of the size of a saucer; they are swollen (bolster-shaped) on their periphery, and present a variable coloration according to the period at which they are observed and the degree of intensity of the disease; when of recent origin they are of a red tint, while at their period of complete formation they are whitish and surrouuded by a violet zone. These are the only symptoms found in cases of benign course. In forms of some gravity the blotches are hemorrhagic, their color varies from light red to dark red (at the place of the serous transudate there are subcutaneous ecchymotic centres); they are sometimes covered with scabs, which are eliminated within a variable time; in detaching these latter the hemorrhagic blotches appear of rhomboidal shape.

Gastric troubles are marked by a diminution of appetite, constipation, and vomiting. The animals, depressed and weak, fall into their litter; the mucous membranes are much injected, the breathing accelerated, the temperature often rises to $40^{\circ}$. In some instances the gait is stiff, a phenomenon which may lead us to suspect a rheumatismal affection of the legs.

The course of urticaria is ordinarily rapid and its prognosis benign. In less serious cases recovery is produced after one or two days; in others within four to six days. We have never seen a fatal termination. The improvement is announced by expulsion of softened excrementitious matters; exanthema soon retrocedes, the blotches turn pale, become flaccid, and disappear little by little.

Differential diagnosis. Urticaria was formerly considered as a "benign form of rouget" (hog cholera). This erroneous opinion 
still persists, although it is almost half a century since Haubner described urticaria as a pathological entity.

It differs from hog cholera and pneumo-enteritis by the following characters :

1. Urticaria is accompanied by blotches, circumscribed cutaneous prominences, which are generally localized upon the upper regions of the body, while hog cholera and pneumo-enteritis produce a diffuse redness of the skin, which is often limited to the lower parts of the body.

2. It is a disease of little gravity. Hog cholera and pneumoenteritis, on the contrary, are infectious diseases which produce death in a relatively short time (one to three days).

3. It is sporadic, and is marked by non-alarming symptoms, and the febrile reaction produced by it is always light.

Treatment. Benign cases do not require any treatment; it will be sufficient to regulate the diet. In the serious form the treatment ought to be symptomatic : clysters, suppositories, medium doses of calomel (2 to 4 grammes). Haubner recommends nitrate of potash associated with sulphate of soda (nitrate of potash, 5 grammes; sulphate of soda, 50 grammes-in a honey electuary). Cold-water irrigations are sometimes useful in order to reduce the inflammation of the skin.

\section{GANGRENOUS DERMATITIS.}

\section{White Spotted Gangrene: Gangrenous Leg Grease. ${ }^{1}$}

Etiology. Gangrenous dermatitis is most usually observed on piebald animals and on regions of the integument which are deprived of pigment; it exists sometimes in an enzootic state. The sphacela of the extremities which occurs in chronic ergotism has nothing in common with it.

The action of an ardent sun and hot, drying wind (Weber, Bonnaud) should be considered in some cases as a direct cause of gangrenous dermatitis (solar gangrene); this latter is really but an

1 The expressions gangrenous dermatitis and white spotted gangrene (balzanes) well characterize the disease, but practitioners will undoubtedly find that the term gangrenous grease (eaux aux jambes sales) has the disadvantage of establishing a closer relationship between two very dissimilar diseases from a clinical and anatomopathological standpoint.-N. D. T. 
intense degree of erythema. The cutaneous surfaces which are deprived of integument are particularly sensitive to the influence of solar rays as well as to various irritations; they are manifestly predisposed to gangrenous dermatitis. Regions which are covered with white hair must necessarily be most frequently affected by it.

Moulds may determine it in certain cases (trifoliosis, lupinosis). Tainted vetches, green fodder which is covered with blight and rust (Erysibe), and also plant-lice.

"Gangrenous leg grease" is undoubtedly due to infectious processes or to causes of the same nature as grease, but acting in a more energetic way (very intense cold, etc.).

Symptoms. Gangrenous dermatitis starts with symptoms of erythema or of eczema. Tumefaction increases rapidly, the skin cracks, becomes creviced or necrosed upon a more or less extended surface. Sometimes the mortification is limited to the superficial layer of the integument, at other times it affects its whole thickness. In the first case the skin presents on its surface dried, tanned blotches; in the other the sphacelated islands are distinctly defined, then eliminated by suppuration (formation of a disjunctive furrow). The loss of substance is regularly compensated by a granulation of its walls, but sometimes it is complicated with abscesses, detachments, and fistulæ.

Treatment. In the beginning it is proper to cover the diseased region with lead ointment (plumbo-tannic salve) ${ }^{1}$ or carbolated oil, carbolated salve, iodoform or cresol salve, cresol liniment, or cresolated water, etc. Later, when the skin is mortified, we must, by means of a bistoury or scissors, remove the necrosed parts and then apply an antiseptic dressing to the wound.

\section{BULLOUS DERIMATITIS: PEMPHIGUS.}

Bullous dermatitis is characterized by the development of more voluminous vesicles than those of eczema, by bulloe, which may attain the size of a hen's egg.

Etiology. Nothing certain is known concerning the causes of

1 The formula of this preparation is as follows :

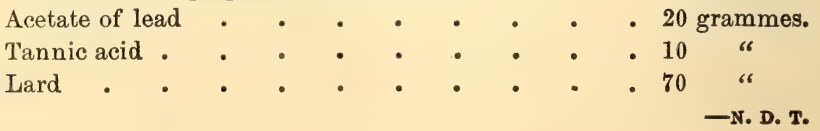


this exanthema, which is extremely rare in our animals. Intense irritations, whose nature is still unknown, undoubtedly act upon the integument in a manner similar to bullous traumatic, thermic (burns) or chemical (cantharides) dermatitis.

Symptoms. In consulting veterinary publications, we do not find any good description of the manifestations of this dermatosis. Loiset has described an enzootic eruption in the ox, characterized by spherical or ovoid bullæ, measuring as much as ten centimetres in circumference, appearing on the croup, the loins, and posterior members; they were filled with a transparent liquid; a short time after their formation they were torn, the moist surfaces thus produced were then covered with scabs, the skin became desquamated, recovery took place rapidly; for some time some shining blotches could be seen to persist at the place where the vesicles had disappeared. Seaman recognized an entirely similar eruption on the ox, which was accompanied by chills and fever. Dieckerhoff observed a bullous dermatitis which was characterized by flat or slightly rounded vesicles, from the dimensions of a walnut to a saucer, which were developed on the skin of the abdomen, head, neck, shoulders, and thorax of five horses; some contained a clear and limpid liquid like water, others a yellowish matter. Most of these would burst within a few days, producing moist secreting surfaces; the patients seemed to experience much itching.

These forms of bullous dermatitis, which seem entirely different from varicella, show a striking analogy with acute pemphigus of man, a disease the etiology and nature of which are yet imperfectly known-some considering it as an acute infectious disease, others as a vasomotor neurosis, others as a dermatomycosis. The cases described in the ox much resemble pemphigus vulyaris of man, a benign disease, which is quite different from pemphigus foliaceus, which often causes death.

Besides the large characteristic vesicle of bullous dermatitis, some authors have found in the horse, upon the lips and nostrils, a specific vesiculous eruption which has been described under the name of "heat pimples" (Haubner) and herpes labialis. This exanthema is characterized by small agminated vesicles, of the size of a lentil, and which contain a transparent liquid. They become rapidly ruptured and cicatrize under a scabby envelope.

We must admit that this specific vesiculous eruption, which is located preferably in the neighborhood of natural openings, shows 
a genuine analogy with facial and genital herpes of man. But the word herpes has been so much used and abused in veterinary medicine that it is impossible to gain any light concerning the nature of a large number of observations described under this appellation. As already proposed by Gerlach, it ought to be reserved to designate herpes tonsurans.

The benign exanthema which has just been mentioned has nothing in common with herpes zoster (zona), which is developed along the cutaneous nerves.

\section{SHEDDING OF HAIR AND WOOL.}

Alopecia.

Shedding of hair or wool, occurring independently of any disease of the integument, may be observed upon the whole body or be limited to circumscribed regions. It is generally considered as the consequence of certain nutritive troubles of the skin (tropho-neuroses), which are accompanied by atrophy of the hair bulbs, but we could not affirm without reserve that it is not of a parasitic nature, although up to the present time no micro-organism could be seriously incriminated.

Etiology. The causes of alopecia are imperfectly known. A general falling out of the hair is always under the influence of a serious alteration of nutrition; it is also designated under the name symptomatic alopecia. It may appear in eachectic conditions during gestation (Koller), ${ }^{1}$ in the course of chronic diseases, of some affection of the blood (petechial fever), under the influence of abundant lactation, prolonged sudations (Hertwig), and circulatory troubles of the skin; it is more rarely due to a spare alimentation, to the ingestion of certain fodders (hay from low, swampy meadows -Fomin). Finally, it is sometimes congenital (Koller). ${ }^{2}$

Areolated alopecia (alopecia areata, area Celsi, area Johnstoni) is considered by some as a tropho-neurosis, that is to say, nutritive trouble, happening in the region of the cutaneous nerve; others classify it among parasitic diseases.

Symptoms. 1. In symptomatic alopecia the whole cutaneous

1 Köller (communication).

${ }^{2}$ Generalized neurotic alopecia has been observed by Joly on an energetic and irritable horse, which had struggled hard for "une prise de longe." In a few days the skiv became absolutely smooth (Presse Vét., 1889).—N. D. T. 
surface becomes bare and the animals may be entirely naked. In the horse alopecia is frequently preceded or accompanied by gastric troubles, œdematous tumefactions of the legs, of the lower abdominal wall, and of the sternal region (Adam, Siedamgrotzky); in other cases no important accessory symptom is observed; the fallen hairs are generally replaced within a few weeks. In the dog, at the same time that alopecia is produced a pigmentation of the skin is developed which seems to be "a protective measure destined to supplement the hair production." On microscopical examination of such modified skin, Siedamgrotzky observed atrophy of the hair and hair bulbs, as well as a black or brown granulous infiltration of the cells of the Malpighian follicles of the sheaths of the root of the hair, of the hair bulbs and sebaceous glands. In the sheep, when the wool falls out in agglomerated tufts, the animals sometimes show large bare blotches.

2. Areolated alopecia is marked in its initial phase by small, bare spots, the periphery of which goes on increasing. It is most frequently observed on the dog, and with the exception of specific fungi, it has absolutely the characters of tonsurating scurvy of the head. Röll found it once in the horse. At the beginning it was marked by small blotches, which were bare of hair; within a year it extended over the greater part of the surface of the body. With atrophy of hair bulbs a very accentuated black pigmentation was observed. Schindelka has described a similar case in the horse. The constant absence of micro-organisms has led to the admission of an atrophic process as a cause of the trouble. The affection is very obstinate; it generally resists all treatment.

Treatment. We may try to stimulate the hair bulbs. Ordinarily we use alcohol, opodeldoc (linimentum saponis comp.), and camphorated alcohol; we may also use diluted tincture of cantharides (in an alcoholic solution of $1: 5$ or in ointment). In areolated alopecia we recommend balsam of Peru, which is a stimulant and an antiseptic at the same time ( 1 part to 10 of alcohol), cresol (1 part to 10-20 of alcohol), and tincture of iodine, to which we add an equal quantity of alcohol, and which is to be applied for several days consecutively by means of a brush.

Trofimo has pointed out a peculiar "pilous" affection, which is identical with the disease of man which Kaposi has designated under the name of Trichorrhexis nodosa. On two-thirds of the horses of an artillery brigade he has seen on the back, lumbar 
region, and croup some spots on the surface of which most of the hairs were broken or swollen at about one centimetre from the root. A microscopic examination showed a very marked fibrillar slitting. Mégnin has mentioned a case almost similar. ${ }^{1}$

\section{CUTANEOUS HEMORRHAGES.}

\section{Hematidrosis: Hematopedesis.}

Etiology. The etiology of numerous cases of hematidrosis reported in our periodical publications is extremely obscure. They have especially been observed in the horse and ox. Several of these cases are very probably only symptomatic hemorrhages, having occurred during the course of serious diseases, such as petechial fever, anthrax, septicemia, and scorbutus. Others would rather belong to the domain of acute exanthema, which are often complicated by capillary ruptures. Excluding these cases, there remains yet a whole category of particular hemorrhages of the cutaneous derma having no relation at all with the secretion of sudorific glands - as the improper denomination of "hematidrosis" might induce us to believe; these are simply hemorrhages by rhexis or by diapedesis. They are yet little known in their etiology and pathology. They are, perhaps, due to a nutritive trouble of the vascular walls. Cutaneous hemorrhages of the ox, especially, belong to this group.

1 Roy has described a similar variety of alopecia existing for five years on the horses of the Ninth Curassiers. It appears toward the end of the fall and disappears in the spring at the time of shedding. At the beginning we observe on the croup and loins lines or sinuous stripes, on the surface of which the hair is dull and erect; they do not harmonize with the coat, because of their darker shade if the horse is a bay, black, or chestnut, and because of their lighter shade when the horse is a gray; they give the coat a zebra-like appearsnce. Later the hairs on these regions all break at the same height, about one millimetre from the root; some bare spots are also formed that one might think had been made with scissors. The diseased surfaces become enlarged, the base of the broken hair falls out; the skin is smooth, white, not inflamed, but covered with an abundant furfur. The disease gradually invades the other regions of the trunk; the legs are almost always preserved from the trouble; itching is never observed. After having persisted for a month or six weeks it disappears without leaving the slightest trace.

We have observed the transmission of this disease by the intermediary of grooming implements and blankets, and it has been communicated experimentally by rubbing healthy horses with pellicles taken from an affected animal. Microscopic study of the hair and the abnormal epidermic productions has not shown any cryptogamic elements in it.-N. D. T. 
In horses of Oriental races, also in Hungarian, Russian, and Tartar horses, etc., we find cutaneous hemorrhages which are, so to speak, physiological, being altogether harmless, and this condition was formerly considered as an auto-therapeusis.

These accidents are explained by the considerable development of the vascular system and the distention of the superficial veins in blooded horses; the increase of the blood pressure by violent muscular efforts would produce the tearing of these dilated vessels. In summer, when the animals are annoyed by itching, slight wounds made by them in scratching themselves may also determine these conditions. ${ }^{1}$

Symptoms. Cutaneous hemorrhages are marked by sweating of blood, the drops pearling on the surface of the skin, especially on the neck and shoulders, on the sides of the chest, and on the extremities. In benign cases the skin only is the seat of these hemorrhages, they are localized, and cease within a few moments; but if a "hemorrhagic diathesis" exists, we may observe epistaxis, enterorrhagia, hematogalactia, and hematuria, a slight fever, gastric symptoms, etc. A serious anemia results which may occasion the death of the animal. In very sanguine horses the hemorrhage appears sometimes as a jet lasting a more or less long time (venous ectasia).

Treatment. It is rare that any interference may become necessary in order to combat localized hemorrhages; we may, however, make cold lotions on the regions which are their seat. In cases of hemophilia, on the contrary, it is proper to institute a new internal treatment, of which styptics constitute the base; we may administer ergot, tannin, sugar of lead, etc.

A large number of morbid conditions of the skin belong to the domain of general pathology: such are exaggerated sudations (hyperidrosis), the pathological secretion of sebaceous glands (seborrhea), scleroderma, affections of the shedding period, and the anom-

1 Drouilly has observed in Hungarian horses cutaneous hemorrhages of parasitic origin, which were produced by a threadworm ( $F$. hemorrhagica, Raill). The female only is known; it is whitish, six to seven centimetres in length, about one-third of a millimetre thick at its centre, and gradually attenuated in its posterior third; its migrations and location are yet unknown. The parasites produce on various regions, but particularly on the ribs, back, and withers, also on the shoulders and the sides of the neck, pustules which are slightly œdematous and which break open a few hours after their appearance, discharging a variable quantity of blood. These hemorrhagic pustules disappear in winter and return in the spring for three or four years successively, and later a final recovery occurs spontaneously.-N. D. T. 
alies of pigmentation; some are of a surgical order : warts, cutaneous horns, elephantiasis, ichthyosis, phlegmons, erysipelas, etc.

Sebaceous pimples (Talgknötchen) constitute a morbid variety, of which we must say a few words. They are the result of the obstruction of the excretory canal and of the sebaceous glands, and of the distention of these latter by secreted matter ; these are phenomena which are accompanied by inflammation of the neighboring cutaneous tissue. They are identical with acne in man; they are firm, and from the size of a lentil to that of a pea. In compressing these a pasty matter is forced out which is similar to boiled gruel. Later the papules are transformed into furuncles. In the horse they are found on the neck, shoulders, and back; in fine breeds of sheep they may be found on the inner surface of the thighs (Negretti) and on the lower abdominal wall (Haubner). Some of the eruptions formerly described under the name of tuberculous eruption seem as if they ought to be classified in this group. In the dog, besides exanthema of typical acne, which is produced by Demodex folliculorum (see Follicular Mange), we find frequently a furunculosis of the median part of the nose produced by prolonged irritation of the muzzle; it is very tenacious and only yields to incisions. ${ }^{1}$

\section{SKIN DISEASES CAUSED BY VEGETABLE PARASITES.}

\section{DERMATOMYCOSES.}

Among the skin diseases of vegeto-parasitic origin, three only are well known in our animals, namely, depilating thrush (herpes tonsurans), favous thrush (favus) and contagious pustulous dermatitis

1 Some rare observations have been related concerning cutaneous tumors of a mycotic nature. In a cow Mollereau has observed on the left side of the upper part of the neck an actinomycotic tumor measuring twenty centimetres in diameter; this was hollowed by a fistulous passage from which flowed a thick white pus, in which the microscope showed numerous actinomycetes. The Botryomycotic neoformations remarked in the horse by Perroncito and Soula affected mainly the subcutaneous tissues. We find in chickens and pigeons a cutaneous trouble which consists of more or less numerous nodules of a round or oblong shape and of yellowish color, their volume varying from a poppy-seed to that of a grain of corn. The largest are found on the base and commissures of the bill, on the nostrils, and under ths nasal mucous membrane, also around the external auditory meatus, upon the border of the eyelids and the face. They form yellowish rugosities upon the comb and small barbel of 
(English, Canadian, or American variola of the horse). The first two are by far the most important. A lengthy discussion has occurred in order to find out if the fungi which determine these belong to two species, or if they constitute but two varieties of the same type. Microscopic examination does not reveal any well-defined difference between the mycelium and the spores of these parasites, and till lately they have been confounded between themselves and also with Ö̈dium albicans. But the recent researches of Grawitz ${ }^{1}$ have established that herpes and favus are produced by two distinct parasitic species. If the spores of both fungi seem morphologically identical, they nevertheless offer remarkable differences in their growth, also in their power to liquefy gelatin, and, again, in the form of the cultures and conditions of reproduction; concerning Oidium lactis, it has nothing in common with them. The nonidentity of these diseases is also indicated by their symptomatology, and the absence of a transition form between the one and the other would itself suffice to make us separate depilating herpes and favus.

Besides these dermatomycoses, the bibliography of the subject contains others in which the nature of the fungi could not be exactly determined. Siedamgrotzky ${ }^{2}$ has described a cutaneous affection which is altogether similar to circinated herpes, but in which it has been impossible to establish the presence of the Trichophyton. In a rooster, Leisering ${ }^{3}$ observed a localized eruption around the cloaca, which was accompanied by falling out of the feathers; it was transmitted to the hens by copulation. By microscopic examination of matter which was obtained from diseased spots, greenish conidia (spores) were found. Leisering ${ }^{4}$ and Engel $^{5}$ have also described a few vegeto-parasitic diseases of the mane and the base of the horse's tail.

\section{DEPILATING THRUSH.}

Etiology. Depilating thrush has been designated by the expressions depilating dermatomycosis, decalvant thrush, tinea scutellata, thrush of the calf's mouth, scurvy of the goat, lambs, etc.; it has

the chickens (Neumann). Rivolta and Silvestrini regard this disease as related to psorospermosis. Cornil and Mégnin look upon it as a form of tuberculo-diphtheria. -N. D. T.

1 Virchow's Archiv, Bd. ciii., 1886.

2 Siedamgrotzky: Sächs. Jahresber., 1872.

3 Leisering: Wochenschr., 1867.

4 Ibid., 1868.

5 Engel: Ibid., 1881. 
also been called lichen. It is due to a vegetable parasite, Trichophyton tonsurans. This fungi, which belongs to the group of Mucedines, was discovered by Gruby in 1842, and studied by Malmsten in 1845. Gerlach has demonstrated that it is the agent of the depilating thrush of our animals; in 1857 he published a work on this disease which is still classical. Contagion operates through grooming utensils, harnesses, blankets, and by direct contact (suckling). It may also occur on pastures where the animals are frequently in immediate contact. Breeding animals of the male sex may communicate the disease to all subjects of their species which cohabit with them.

It exists ordinarily among animals of the bovine species without any distinction as to age; the dog, horse, goat, and cat follow in order; sheep and pigs are rarely affected; it may be transmitted to the rabbit by inoculation. In general, however, it is quite a rare disease, and one which does not exist everywhere; it seems to be stationary on certain premises, and exists permanently in several countries (England, Holland, France, Switzerland), where it is much more common than elsewhere.

Its transmission to man has been mentioned by numerous authors; persons affected by it are generally those working in stables, or who take care of sick animals, or have been engaged in skinning animals affected by thrush, which have been killed for consumption. The dog may communicate it to man by its caresses (Friedberger). In the human species it assumes, at times, an endemic character. In 1840 almost all the inhabitants of a Swiss village (Andelfingen) were contaminated by diseased oxen. In Berlin cases of tranmission of thrush of the dog to man are frequent; in 1887 we observed and treated at the polyclinic of the veterinary school a score of cases of human favus of canine origin. We have observed it several times in all the members of one family.

Morphology And Physiology of Trichophyton tonsuRANS. Microscopic examination of the Tricophyton tonsurans permits us to recognize two kinds of elements in it: filaments and spores.

1. Filaments or mycelia are very fragile elongated tubes, of a thickness of $4 \mu$; they are straight or undulated, simple or articulated, ramified according to the dichotomic type. They form a network around the hair, penetrate into it, and are sometimes so abundant that they constitute a real packing to which the word mycelium is more particularly applied. 
2. Spores or conidia are small spherical or oblong cells, which are refringent, with a very distinct outline, the diameter of which is nearly the same as that of the filaments at the expense of which they are produced by segmentation; they are often shaped like a bead-roll, and exist in much larger number than the filaments, rendering these latter almost imperceptible. As they are very resistant to the various causes of destruction, they preserve their vitality for more than one year (Siedamgrotzky).

The trichophyton vegetates preferably in the packed bulbs and in the hair itself; at first it surrounds the lower parts of the former, with its filaments forming an enveloping cloak or an aliform membrane; later it penetrates into the sheath of the organ, becomes implanted in its root, and produces an inflammation of the follicle, which is soon followed by a loosening and falling out of the hair; lastly it invades even the substance of the latter, which becomes slit, brittle, friable, and sometimes has its deep layers overrun with conidia (spores) to such an extent as to leave no trace of the hair structure. The fungi seem to thrive upon skins that are deeply pigmented.

The dimensions of spores and filaments vary according to the animal species, and they may differ considerably when being examined comparatively with several animals of the same species. Mégnin thought proper to admit two distinct parasitic species: Trichophyton tonsurans in the horse and Trichophyton epilans in the ox. But the dimensions of the parasite are of but secondary importance, depending undoubtedly on more or less favorable conditions of the soil.

According to Grawitz, the fungi of depilating thrush softens gelatin much more rapidly than that of favus. On agar, Trichophyton is developed and produces a kind of grass, while Achorion gives a star-shaped vegetation.

Symptoms. Manifestations of depilating thrush vary according to the animal species, race, localization of the dermatomycosis, its age, and alterations due to rubbings, bites, etc. The characteristic symptom consists of round-shaped spots, mostly located on the head, neck, and legs; these spots are bare or covered with broken hair; at the outset their dimensions are nearly that of a lentil; later they become enlarged, till they reach the size of a silver dollar or over. These spots remain sometimes disseminated, at other times they become confluent and confounded in more or less extended 
blotches; at other times the morbid process becomes weakened, and then disappears from the centre while at the same time it progresses at the periphery (circinated herpes). The disease may invade the greater part of the surface of the body and occasion an almost general dropping of the hair. When examining the fallen or pulled hairs with the microscope, the conidia (spores) and mycelia mentioned above are found in them.

The integument of the affected surfaces is altered in various degrees-at times it is not at all inflamed; upon the thick and resisting skin of the horse we find only an augmentation of the epidermic desquamation, an abundant ashy-gray or slaty, amianthous furfur (herpes tonsurans maculosus); upon the fine skin we observe at the beginning some hyperemia and a slight tumefaction of the diseased spots; later vesicles become developed (herpes tonsurans vesiculosus), which soon burst and dry up, forming thick scabs. In the adult ox these scabs, the color of which is variable, are of the consistence of leather. In the calf, where they appear in a grayish-white color, similar to that of dough, they are deeply slit. Their constitution depends also upon the character of the fleece; they are thick when the hair itself is abundant, while they are very thin upon the regions where the hair is sparse; in the ox they acquire a thickness of one centimetre. After their falling out the suppurating surfaces which they covered often heal by themselves. The following are, briefly expressed, the clinical characters of thrush in our different species:

1. IN THE ox. Gerlach has outlined an excellent description of it. We find upon the head and shoulders, more rarely upon the whole surface of the body, isolated or in groups, spots which are distinctly limited and are salient upon the surface of the skin; at times they are denuded of hair and covered with furfur or whitishgray, amianthous scabs, at other times they are covered with erected hair; they may reach the size of a saucer and become confluent. During its extreme periods-at the start, and when a cure is produced - the disease produces slight itching. Upon the black skin the scabs are thick and are of a grayish tint; upon the discolored integument they are thinner and of a yellowish shade. Upon these we find a viscous purulent liquid, and we observe small suppurative dimples in the derma, which are formed by the dilated, suppurating follicles which are deprived of their hair. The cure is ordinarily produced by a sub-crustaceous cicatrization. When the 
scab falls off we find in its place a blotch, the epidermis of which is slightly desquamated, and where the hairs grow again gradually. The duratinn of the disease varies from six to twelve weeks. In cases where its normal course is interfered with by mechanical influences (scratching, rubbing, etc.), it spreads by a series of inoculations; new blotches are developed in the neighborhood of the healed regions, and it may last for many months, even for years.

In suckling calves it is mainly located in the neighborhood of the mouth (mouth thrush). It has also been observed in the same location upon other sucking animals (lambs, kids, young pigs). Some authors have described an impetiginous eczema which is nonparasitic and limited to the buccal mucous membrane. But the clinical picture and the striking contagious character of this disease indicate that in the majority of cases depilating thrush is indicated, an opinion which has been elsewhere confirmed by the microscopic researches of Hahn, ${ }^{1}$ who found the trichophyton in the buccal lesions. According to this author, the trouble is mainly characterized by the presence upon the surface of the mucous membrane on the edge of the lips, and at times over the whole surface of the body, of spots which become covered with dry, furfuraceous scabs; on those which are developed upon the skin we observe the hair slit and broken by the fungi. It is very obstinate in young animals.

2. IN THE DOG. As a rule, it is located on the head and extremities; at times it extends over the entire surface of the body. It starts with round, isolated, distinctly circumscribed spots ; later we may find upon the whole skin bare island-like spots, which are of circular or elliptic form, and from one to two centimetres in diameter; they are particularly numerous in the neighborhood of the lips, eyes, and extremities. In some cases these islands unite into large blotches, the objective character of which varies according to the location and length of the process; we find quite often the alterations of plain non-inflammatory alopecia in it (area Celsi); frequently also the skin is covered with scabs, sometimes thin, of an amianthous or dirty gray color, at other times this is thicker, agglutinating the hairs at the base; when detached, the integument appears copper-red or red-brown colored and strewn with a large number of small pimples of the size of a millet-seed (compressed tumefied follicles). Sometimes the diseased parts are quite promi-

1 Hähn: München. Jahresber., 1861. 
nent; the adjacent skin is tumefied and swollen. The regions which are first invaded remain pale for a long time; they are smooth or covered with a little epidermic furfur.

3. IN THE HORSE. It is generally observed in the regions upon which the harness is placed (withers, back, croup) and on the flank; it is rarely found on the head. The blotches, the dimensions of which vary from that of a ten-cent piece to a silver dollar, are round, more or less bare, and covered with furfur or scabs. The hairs of the neighboring regions may be pulled out with the slightest traction. Those which grow again in the centre of the blotches are ordinarily of a darker color than the old ones.

4. In sheEP. It is generally located upon the neck, chest, shoulders, and along the back. According to Bräuer, the wool is found to be packed and the skin is squamous or scabby. The patients experience intense itching. At the beginning small tufts appear above the surface of the fleece; later, when they exist in larger numbers, they give to it a slashed appearance.

5. In POULTRy. In birds, depilating thrush is accompanied by dropping of the feathers and a serious hyperemia of the derma in the neighborhood of the papilla.

Diagnosis. The objective symptoms of the disease enable us to recognize it easily. The multiplicity and dissemination of the blotches, the dropping out of the hairs toward their periphery, their amianthous epidermic productions, in the dog especially (lips, cheeks, eyelids); itching being slight, or entirely wanting; lastly, the contagiousness characterize it sufficiently. In doubtful cases microscopic recognition confirms the diagnosis: it is sufficient to pull out a few hairs upon the periphery of the blotches, and make an examination of their roots with an enlargement of 300 to 400 diameters; we may also examine the scabs after having macerated them in a solution of potash of 10 per cent.; this method enables us to isolate the pilous roots which are contained in them. The fungus is also recognized by the microscope, because of the existence of the whitish membrane formed around the base of the hair. In the dog, depilating thrush may be confounded with other dermatoses; it extends at times over the whole surface of the body and offers great similarity to sarcoptic mange. The frequency of the transmissibility of thrush to man and the very intense itching which accompany mange are sufficient differential characters in some cases. But, in a similar occurrence, the practitioner should 
confirm his diagnosis by microscopic examination of the hair or scabs. In the ox we may confound thrush with mercurial eruption.

Prognosis. It is favorable in the sense that the cure is always obtained by an appropriate treatment persistently applied. In adult animals of the bovine species it may occur spontaneously, when the scabs fall and remove the hair at the same time, which are the elements at the expense of which the trichophyton feeds and vegetates. We have also observed cases of spontaneous cure in the dog. In young animals localization of the trouble in the neighborhood of the mouth renders the prehension of food difficult and sometimes causes death by inanition.

Treatment. The prophylactic treatment consists in isolating the sick animals, in cleaning and disinfecting the stables, in destroying the dog-kennels, in warning those persons having the care of the animals of the transmissibility of the disease to man, giving them sufficient instruction to preserve them from it.

The curative treatment comprises two principal indications. We must: 1. Thoroughly clean the diseased regions and remove the scabs with green soap. 2. Apply parasiticide agents upon the parts which have been thus prepared. We have the choice among many remedies: cresol, carbolated creosote, naphthol, camphor or iodoform ointments, with tar $(1: 10)$, with biniodide of mercury, mercurial ointment (we must avoid the use of these latter preparations in the ox); iodine tincture (1:1-5 of alcohol), salicylic acid and alcohol $(1: 10)$, alcoholic solution of sublimate or sublimate ointment ( 1 per cent.), etc. In cases of localized thrush, cresol ointment, tincture of iodine, and an alcoholic solution of salicylic acid have always given us good results.

No matter what remedy may be used, it must be applied several times daily upon the affected surfaces.

\section{Favus: Favous Thrush.}

Etiology. Also called chicken thrush, comb thrush, white comb. This disease is due to a mycelium discovered by Schœnlein in 1839, and designated later by Remak Achorion Schoenleinii. It is rare in the horse, quite frequent in the $\operatorname{dog}$ and cat; it is sometimes found in the rabbit and the mouse. Among the Gallinaceæ, the foreign breeds, the Cochin China and Brahma races, are mostly affected. It is transmissible from animals to man and from man to animals (hereditary favus; Erbgrind). The cat contracts it 
generally by eating mice which are affected by it. Gerlach has not succeeded in communicating it from the chicken to the horse, ox, and dog. In experiments undertaken by Schütz, the inoculation of cultures of favus from the chicken has only given positive results upon poultry; it has remained unsuccessful upon rats, guinea-pigs, pigeons, and mice. The fine and tender skin of young animals is very favorable for the development of Achorion.

Morphological COnsiderations UPON the ACHORIon of Schenlein. This parasite produces, upon the surface of the skin, more or less thick crusts, depressed in the centre, and cup-shapedfavi or scutula ("small shields"), which are formed exclusively of fungi, and represent, so to speak, a pure culture of the achorion. Microscopic examination demonstrates their packed texture and permits us to recognize filaments in it (mycelium, sporophoric tubes) and spores (conidia). Morphologically these elements resemble those of the trichophyton. The filaments, the diameter of which varies from 2 to $5 \mu$, are at times elongated, very fine, not encysted, sometimes shorter and thicker, ramified, articulated, inflated like a club and with granulous contents; they are separated by spherical or ovoid spores; they are quite rare at the beginning of the disease, but they exist in considerable numbers at a more advanced stage; their diameter differs little from that of sporophoric tubes, by which they are generated through segmentation. The achorion penetrates into the pilous follicles; later, it invades the hair itself or the quill of the feather, which becomes atrophied at the root and falls out. Its filaments abound also between the epidermic layers.

Quite recently Schütz has cultivated the achorion of the chicken upon peptonized gelatin at a normal temperature. The inoculation of these cultures upon the chicken has given successful results.

According to Quincke, favous thrush may be produced by three different fungi.

Symptoms. 1. Favus of mammals. The disease is characterized by dry scabs in our animals; they are brownish or yellowish gray or silver white upon their surface, and white or sulphur yellow in their deep layer. These scabs have a circular form, their diameter does not exceed that of a ten-cent piece, their thickness sometimes reaches one-half of a centimetre. They occasion atrophy of the hair and a slight depression of the integument. They are

1 Quincke: Archiv für experiment. Pathol. u. Pharmakol., 1887. 
usually found on the forehead, cheeks, ears, face, abdomen, the external side of the hind legs, in the neighborhood of the claws and on the notch of the auricular concha in the cat. In this animal and the dog St. Cyr has found these scabs split, gray or saffron yellow and of pitchy consistence. At the beginning they are perforated by some stray hairs which soon fall out. Later their central depression becomes more marked and they end in exfoliation. The skin is thinned and sometimes bloody upon their surface. In the horse favi may gather in bands the width of a finger. Itching, which is quite intense in the dog, is wanting or almost unobserved in the cat.

In most cases the course of the affection is rapid, but its prognosis is benign. A cure has been obtained by Siedamgrotzky after eight days of treatment; according to St. Cyr, cure is not delayed longer than from eight to twelve days, the hair begins to grow again within that time.

2. Favus of the chicken: White comb. The parasitic nature of chicken thrush was discovered almost at the same time by Leisering, Gerlach, and Müller. According to Gerlach, the affection is expressed by whitish spots, resembling mould, which appear upon the comb and ears, become enlarged, and soon entirely cover these organs, upon which the vegeto-parasitic coating is transformed into a thick scabby covering. As a rule, it remains localized upon the comb for several months; later it extends to the head, shoulders, back, and lastly over the whole body. The feathers which are at first erected, become loosened and drop out; the skin is covered with crustaceous masses. The animals become emaciated and spread an odor of mouldiness; they may die from exhaustion if the process has been somewhat extended.

Differential diagnosis. Differentiation between favus and tonsurating thrush is mainly based upon the existence of cups, which in the former disease are principally formed of fungi. The scabs of tonsurating thrush have not this particular form, and the elements of trichophyton are not abundant in it; the sporophoric tubes are much more rare in this affection than in favi.

Treatment. The treatment is the same as that of tonsurating thrush. After having detached the crusts the parasiticides indicated in that affection are to be used (cresol, sublimate, carbolic acid, iodine, etc.). 
According to Ercolani, ${ }^{1}$ the achorion might penetrate the horn or the hoof, as in Onychomycosis favosa of man, and occasion troubles in the integumentary membrane, whi-h would lead to a loosening of the podophyllous and keratophyllous lamellæ; "seedy toe" is said to be formed in this way (air-cells of the wall). Ercolani designates this disease onychomycosis and the fungi Achorion keratophagus.

\section{CONTAGIOUS PUSTULOUS DERMATITIS.}

\section{English, Canadian, or American Variola of the Horse:} Contagious Acne.

English or Canadian variola of the horse has nothing in common with the so-called equine variola. It consists of a particular pustulous exanthema, which is essentially contagious and is characterized by circumscribed efflorescences. In its slight form it is marked by vesicles, pustules, and scabs; in its serious form this process is greatly intensified, and is accompanied by suppuration, pimples, ulcers, and tumefaction of the lymphatic ganglions ; but it always develops without producing either general troubles or itchings. Notwithstanding that its duration is quite long, its prognosis is benign.

According to Schindelka, this affection seems to be identical with contagious impetigo of man (Kaposi), a disease which is not serious, which has typical characters, and consists essentially in a pustulous exanthema of the scalp.

HIstory. According to the statement of Axe, who has given to it the name of Canadian contagious pustulous dermatitis, English variola of the horse was introduced into England in 1877 by horses imported from Canada. It spread rapidly in different regions of the Continent, where it was reported in 1879. Its identity with pustulous stomatitis, which was suspected and even affirmed at this epoch, was not well founded. It seems to have been observed at earlier dates. In 1841 and 1842, Goux described upon horses of the French army a similar pustulous eruption remarkable for its extreme contagiousness, its benign course, and its localization (back, shoulders, croup); all the horses of one squadron were affected by it within a couple of weeks; the symp- 
toms were the same as those of English variola. Hering has reported this case under the name of tuberculo-contagious eruption.

Etiology. The disease is communicated to healthy animals through the intermediary of grooming utensils, blankets, girths, and harness; the extent of the affected surface often corresponds exactly to that of blanket, saddle, girth, etc. This mode of development explains why the shoulder and chest are regions but seldom attacked, except in a case mentioned by Giel. The contagion has been recognized by Axe, who also suspected its microbian nature. In the pus and scabs of the invaded regions, Schindelka has constantly found small micrococci which readily absorbed aniline colors; Siedamgrotzky has detected these in the shape of diplococci.

Pustulous dermatitis has been transmitted experimentally upon the horse, goat, and rabbit. In rabbits and guinea-pigs, Siedamgrotzky has seen malignant œedema and septicemia develop after inoculation.

Dieckerhoff and Grawitz, who have given to English variola the name of contagious acne, have been able quite recently to obtain conclusive results from pure cultures of the bacterium (acne bacillus). It is a micro-organism of very small dimensions; its length is about half that of the bacillus of tuberculosis (about $2 \mu$ ); it is divided by segmentation into ovoid or round corpuscles, which gather in twos or fours to form diplococci or small chains; they become very rapidly colored by a watery solution of fuchsine.

In cultures (serum) this bacillus gives characteristic colonies; its most rapid development takes place at $37^{\circ}$; it is killed by a temperature of from $80^{\circ}$ to $90^{\circ}$ lasting for half an hour. If preserved in a dry condition it remains virulent for at least four weeks. It does not produce any putrid decomposition.

Friction with a few drops of water containing this micro-organism, made upon any portion of the horse's skin, is sufficient to determine the manifestation of the specific exanthema. Inoculation is also successful in the ox, sheep, dog, and rabbit. The guinea-pig is killed in forty-eight hours by friction made upon the skin moistened with the culture; we observe the symptoms and lesions of septic intoxication. Subcutaneous injections produce pyemic accidents in the white mouse.

Symptoms. After an incubating period of six to fifteen days (Schindelka), circumscribed efflorescences are formed on the con- 
taminated region, the character of which varies according to the degree of intensity of the affection and the constitution of the skin.

1. In benign cases we observe, in the beginning, spherical or ovoid prominences of the size of a pea, a bean, or a hazel-nut, either isolated or in small groups; they may occupy a surface of the size of a saucer, but they never exist in large numbers ; on these spots the hair is erect and gathered in tufts; the skin is sensitive, hot, slightly tumefied. Upon the surface of these circumscribed inflammatory centres we observe isolated vesicles, which are rarely confluent, of the dimensions of a millet-seed to that of a bean, the contents of which, at first watery and limpid, are soon purulent and turbid (pustules). After one or two days they burst, and their contents dry, forming thick yellow scabs of the size of a ten-cent piece to that of a twenty-five-cent piece. Within a week the scabs and hairs drop out and uncover circular blotches which are entirely depilated and depigmented, but which are already covered with an absolutely normal epidermis. Exanthema only affects the superficial layers of the skin, being arrested at the Malpighian layer; it heals entirely within two weeks without having produced general troubles. Nevertheless, a moderate induration, gradually becoming reabsorbed, is constantly observed on the lymphatic ganglions of the inter-maxillary space and the neighborhood of the pharynx.

2. This slight form, which is common, is subject to numerous more or less serious aberrations, which depend either on the abundance of the infectious elements or on the confluence of the pustules, whether from the want of care of the sick animals, or by frictions of the harness, which irritate and injure the inflamed regions and cause the spread of the bacilli to adjacent parts. No matter what the cause may be in all these cases, the exanthema is radiating, the small inflammatory islands are multiplied-we may count from fifty to sixty, and the number of pustules may increase four or fivefold. In the neighborhood of the first prominences new phlegmasic centres are developed; the scabs are enlarged, and-a circumstance particularly aggravating - the inflammation penetrates dceply into the derma, even into the subcutaneous connective tissue; voluminous furunculous pimples are formed, similar to the small tumors produced by the Estrus larvæ, which are very painful aud rapidly form abscesses; in compressing these, purulent masses are forced out of them and more or less deep ulcers remain in their place; these sometimes become confluent; later they are filled by 
development of the granulations. Lymphangites are derived from these suppurative lesions, which spread in all directions; the neighboring lymphatic ganglions may form abscesses. A cure occurs but slowly; the process often lasts six weeks, two months and over. We may observe several acute outbreaks. But even in the most serious cases the general condition is not much affected, and itching is almost absent.

Differential diagnosis. 1. True variola of the horse is ordinarily localized upon the fold of the pastern ("dirty leg grease") and it is always accompanied by febrile symptoms which are wanting in English variola.

2. Farcy is marked by manifestations showing sometimes a true analogy with those of grave English variola (ulceration, lymphangitis); nevertheless, in the first we observe neither vesicles nor pustules; the pus is liquid, oily, and the cutaneous ulcerations have no tendency to cicatrization.

3. If there is a certain clinical resemblance between pustulous dermatitis and impetiginous eczema, in this case the dimensions of the vesicles and pustules are always illy defined, the pruritus is intense, and contagion is wanting or little marked. Some nonparasitic pustulous eczemas may, however, be transmitted by inoculation. In applying a piece of skin of a young bovine which was affected by mercurial exanthema upon the internal side of the leg of a healthy calf, Friedberger has determined a pustulous eczema.

4. In its initial stage English variola may be confounded with lesions which are produced by compression of the saddle or of the girth, but soon all doubts are removed by the evolution of the pustules.

Treatment. As a prophylactic measure it is proper to isolate the patients and to disinfect the stable utensils, harness, blankets, etc., which have been used for these animals. In benign cases we may confine ourselves to hygienic care. In a form of some gravity the ulcers should be treated by means of antiseptics; Friedberger has obtained good results with carbolic acid; Schindelka and Siedamgrotzky recommend a solution of sublimate at 1 per cent. 


\section{SKIN DISEASES CAUSED BY ANIMAL PARASITES.}

\section{A. MANGE OF DOMESTIC MAMMIFEROUS ANIMALS.}

Mange (scabies) is a contagious cutaneous disease produced by acari. Without acari there is no mange. The word mange was used formerly to designate non-parasitic affections of the skin-an inanition mange, an adipous mange, etc., whilst true psora (itch) was attributed to a dyscrasia of the blood.

The psoroptic acari comprise the three species, Sarcoptes, Dermatokoptes (Psoroptes), and Dermatophagoides (Symbiotes). The Demodex (Acari folliculorum), which produce follicular mange, are not generally considered as forming a part of the group of psoroptic acari.

These parasites give rise to so many separate dermatoses in our domestic animals-each animal species has its acari--that the expression mange must necessarily be incomplete unless the species and variety of the parasite be indicated. Sarcoptic, psoroptic, and symbiotic manges are consequently distinguished.

Mange is frequent in the sheep and dog; it is quite common in the horse, but rare in the ox, cat, and pig. It is considered as very contagious, and is classified among the epizootic diseases. We have preferred to describe it in the section on Diseases of the Skin, for in a work like this the practical side should predominate. Certain manges, indeed, such as those of the ear or feet, are without any importance from a sanitary standpoint, and do not present any clinical interest.

History. Mange in man and in animals has been mentioned from the most remote ages, Moses having already considered mange of animals as a special affection. Acari have also been known for many centuries. According to Gerlach, the Arabian doctor Avenzoar was the first to point them out, in 1174; Fürstenberg says that they are described under the name of suren in Hildegarde's Physica (1200). Wedell found acari in the cat in 1672, Kersting in the horse in 1789, Walz in the sheep in 1809, Gohier in the ox and the dog in 1812, Spinola in the pig in 1846. Numerous works have been published on these parasites. From an entomological and clinical standpoint those of Gerlach and Fürstenbery have 
been excellent guides for investigators. Among recent literature we must particularly mention that of Robin, Mégnin, and Zürn.

Morphology AND BIOLOGY of PSOROPtic ACARI. The specific agents of mange constitute a group of the Arthropodes, of the class Arachnides, the order Acari, and the family Sarcoptes. Their dimensions vary between 0.2 and 0.8 millimetre; their general form is rounded or ovoid; in their immature form they have three pairs of legs, in the adult state they possess four; the latter, with five joints, are fitted with cucurbitula (suction cups), covered with fine hair, and armed with claws (hooks); the head, thorax, and abdomen are not separated; the organs of mastication are represented by mandibles; the cutaneous surface is covered with scales, hair, spikes, or silky hair, etc. The females, which are larger than the males, lay from twenty to twenty-four eggs; at the end of four to seven days the larvæ come out, and after having undergone three to four metamorphoses, arrive at the stage of reproduction from the fourteenth to the seventeenth day. Concerning the psoroptes of the sheep, Gerlach has calculated that one female alone may produce $1,500,000$ individuals in ninety days. The females die in from three to five weeks after laying; the males live about six weeks. If exposed to damp air or placed upon wet manure the acari continue to live from six to eight weeks; in dry air they die within two to three weeks; upon damp ground the eggs remain alive for from two to four weeks; in a dry place they lose their vitality after four to six days. A moderate heat is favorable to their vitality and to the pullulation of the parasites; in warm places, under cover, and during the summer their movements are more active and they multiply more rapidly than under the opposite conditions. According to Gerlach, the medicines which kill these parasites most rapidly are: creosote (fifteen to thirty seconds), caustic potash in a solution of $1: 25$ (two minutes), essence of turpentine (five to ten minutes), petroleum (seven minutes), tar (eight to thirteen minutes), tobacco ( 1 part to 5 of water, ten to twenty minutes; 1 part to ten, two to five hours; 1 part to 50 , four to ten hours), liver of sulphur at 1:10 (in a quarter to half an hour), green soap (half an hour to one hour, etc.). (For further details on the biology of acari, see treatises on general pathology and parasitology.)

KINDS AND SPECIES. We must examine the various kinds successively: 
1. Sarcoptes.

2. Psoroptes (Gervais), Dermatodectes (Gerlach), Dermatokoptes (Fürstenberg).

3. Symbiotes (Gerlach), Chorioptes (Gervais), Dermatophagus (Fürstenberg).

A. The Sarcoptes dig sub-epidermic galleries and live on the plasma cells of the Malpighian layer ; they multiply in these galleries and occasion a very intense dermatitis. They are characterized by their general form, which is that of a shield, and by their small size ( 0.2 to 0.5 millimetre); they can only be seen by means of a microscope. The head, which is horseshoe-shaped, is fitted with two strongly developed conical mandibles; the legs are short, stumpy; the suction cups, which are tulip-shaped, exist in the males on the first, second, and third pair of legs; in the females on the first two pairs only.

Authors disagree on the subject of the varieties in the sarcoptes: Fürstenberg has attempted a division based upon morphological characters; Gerlach has advised a biological grouping; but the inconsistency of these varieties and their exaggerated number has been well pointed out (Zürn, Johne). We have adopted the division established by Siedamgrotzky, who recognizes two principal groups :

a. The large sarcoptes, comprising :

(1) Sarcoptes scabiei of man, horse, and lion.

(2) Sarcoptes squamiferus of the dog, pig, sheep, and goat.

$b$. The small sarcoptes (Sarcoptes minor), which is found in the cat and rabbit.

B. The Psoroptes live upon the surface of the skin; adhere to the fatty matter and the hair; suck the blood and lymph of the integument by means of their buccal organs, producing a more or less intense dermatitis, through the numerous stings which they inflict. They are characterized by their relatively great size $(0.5$ to 0.8 millimetre), which allows them to be seen with the naked eye upon dark surfaces; they are very easily seen with the help of a magnifying glass. The head is elongated and pointed; the mandibles are long, straight, and stinging; the legs are very long; the sucking cups, tulip or trumpet shaped, are carried by articulated pedicles. In the male they are seen upon four pairs of legs; in the female, upon the first, sccond, and fourth pairs only. Zürn divides these as follows: 
a. Psoroptes communis (in the horse, ox, and sheep).

b. Psoroptes cuniculi (acari of the rabbit's ear).

In all sub-species of psoroptes we find interesting morphological variations; Zahn has mentioned some in the horse; we have also seen them several times.

C. The Symbiotes live especially upon the surface of the skin of the extremities and exist on epidermic scabs. They are relatively voluminous ( 0.3 to 0.5 millimetre); their outlines are visible to the naked eye or with the magnifying glass; the head is short and conical, wider than long; the body is ovoid, slightly notched upon the periphery; the legs are long and the sucking cups are shaped like a Roman shield; in the male, they are found upon four pairs of legs; in the female, they are found upon the first, second, and fourth pairs ; the male carries two copulating cups and the young female two copulating tubercles. Zürn has established the following division :

a. Symbiotes communis.

(1) Symbiotes equi et bovis, which occasion mange of the pastern and tail.

(2) Symbiotes ovis, which produces mange of the sheep's leg.

b. Symbiotes felis, canis, and cuniculi (acari of ear mange). ${ }^{1}$

General CHARACTERs of THE DifFerent ACARI; DOMESTIC ANIMALS UPON WHICH THEY ARE OBSERVED. From a clinical standpoint, the three kinds of psoroptic acari produce as many special manges, on account of the different biological conditions of these parasites.

1. Sarcoptic mange starts preferably upon the head and on the regions which are imperfectly protected by hair or wool. In the horse, dog, and pig, it often extends over the whole surface of the body. In the dog its selected place is the base of the ears, the joints, the lower pectoral region, the belly, and the internal side of the extremities; in the sheep it does not invade the parts which are deprived of wool.

2. Psoroptic mange is especially observed upon the protected regions. In the horse it is mostly found upon the inner side of the legs, the neighborhood of the sheath, at the base of the mane and tail; but when the hygiene of the skin is neglected, it may extend over the actual surface of the body. In sheep it affects the parts

1 French authors distinguish only: in sarcoptes, S. scabiei and S. minor; in psoroptes, Ps. communis; in symbiotes, S. communis and S. auricularum.-N. D. T. 
which are covered with wool ; with time it invades the whole surface of the body, except the head.

3. Symbiotic mange affects only certain regions: the extremities of the legs in the horse and sheep (pastern mange), the base of the tail in the ox (tail mange), the auricular concha in the dog, the rabbit, and the cat (ear mange). In the different domestic species we may find the following acari :

A. In the HORSE: Sarcoptes scabiei, Psoroptes communis, Symbiotes equi. Sarcoptic mange is the most important.

B. In the ox: Psoroptes communis, Symbiotes bovis. Psoroptic mange is the most serious.

C. In the SHEEP: Sarcoptes squamiferus, Psoroptes communis, Symbiotes ovis. Psoroptic mange is the principal.

D. In the GoAT : Sarcoptes squamiferus.

E. In the DOG: Sarcoptes squamiferus, Symbiotes canis. Sarcoptic mange is the most important.

F. In the САT: Sarcoptes minor, Symbiotes felis. Sarcoptic mange is the principal.

G. In the PIG: Sarcoptes squamiferus.

H. In the RABbit : Psoroptes and Symbiotes cuniculi.

Concerning the transmissibility of the different manges to animals and man we possess to-day precise facts :

1. All sarcoptes may live for an indefinite period upon man's skin, but the psoroptes and symbiotes die very rapidly ; they occasion but slight irritations.

2. The horse may contract sarcoptic mange of the sheep, pig, dog, and cat.

3. The ox takes the sarcoptes of the horse, sheep, goat, and cat.

4. The sheep contracts sarcoptic mange of the goat.

5. The dog takes the sarcoptes of man, pig, cat, sheep, and goat.

6. The pig contracts sarcoptic mange of the goat.

From this summary we see that sarcoptic mange is especially transmissible from one species to the other.

Symptoms of mange in general. Mange is never developed but by contagion. The incubating period-that is to say, the interval which elapses between the moment when the acari are deposited upon the surface of the body and the appearance of cutaneous alterations-varies according to the number of transmitted parasites. When in small numbers the first manifestations 
of mange are sometimes only apparent at the end of four to six weeks; on the contrary, they may occur at the end of fifteen days. Contamination takes place either by direct contact: immediate, as on pasture or at the stable, etc., or by intermediary agents (litter, blankets, harness, grooming implements, and grooms taking care of the patients). Animals which have not received proper attention to the skin, or are emaciated, weak, and those upon which epidermic exfoliation is abundant, are particularly predisposed to mange.

The first symptoms produced by acari bites are a punctated redness, pimples, and vesicles, augmentation of the epidermic desquamation, the formation of squamæ and scabs. As soon as they appear these lesions are accompanied by an intense pruritus, which becomes unbearable during the night, or under the influence of the solar heat, in hot stables, and after strenuous work; the animals scratch, rub, and bite themselves on the affected regions; these mechanical irritations alter considerably the local symptoms, which are determined by the parasite itself: the hairs fall out or break, the wool is flaky and the skin is bloody, a more intense phlegmasia is formed in the derma, it passes into a chronic state, and leads to persistent alterations.

When the disease reaches its maximum intensity we find the skin moist or bloody, ulcerated, scabby, squamous, thickened and wrinkled, etc.

As a general rule, the course of mange is essentially chronic. It depends upon numerous circumstances, but especially on the parasite species and its habits. While the symbiotes remain stationed in a very limited cutaneous territory, the sarcoptes and psoroptes often invade the whole surface of the body. The constitution of the skin, the care given to it, the stay of the animals in the stable, the season of the year, etc., all influence the course of the trouble. Its manifestations are aggravated by the heat of summer ; it becomes attenuated, on the contrary, during the cold season. In well-cared-for and groomed horses (army horses) it may remain benign and localized for a long time; in the sheep, after shearing, a temporary improvement is regularly noticed because for some time the acari suffer ftom their exposure to the weather. Finally, a large number of agents used in lotions and frictions arrest the extension of most manges, exterminating them by killing the parasites. A spontaneous cure has never been seen; the remissions 
which have been observed during certain seasons in intermittent acariasis must not be looked upon as cures. When mange is generalized the alterations of the skin are usually accompanied by general symptoms: a permanent excitement and a persistent cutaneous exudation delay the growth of young animals, disturb nutrition, and in some cases lead to death through exhaustion and cachexia.

Diagnosis. We possess facts and symptoms which permit us to make a diagnosis of mange, besides the detection of parasites. The enzootic character of the trouble, its contagiousness, its appearance in herds and rapid extension through their distribution are important clinical peculiarities; on the other hand, pruritus is much more intense than in other dermatoses. The knowledge of the selected places of the different manges is valuable from a diagnostic standpoint. In the horse, the localization upon the head (often followed by generalization) indicates sarcoptic mange; the eruption which is exclusively located upon the legs and tail is characteristic of symbiotic mange; the location of this affection upon regions which are protected by mane or tail announces psoroptic mange.

But the safest diagnosis is the microscopic search for acari. We make it by collecting the scabs in a dilute solution of 10 per cent. of potash and examining them by means of a low power. In sarcoptic mange of the dog it is necessary to scrape the skin deeply, in order to find the parasites in the product thus obtained; it may be scraped with a sharp bistoury or we may make thin cuts into it with a razor or scissors. Gerlach has recommended to apply suspected scabs on a man's arm; in the case of sarcoptic mange, pruritus and a slight dermatitis is produced (?) after twelve hours, and in case of psoroptic mange after two hours. For psoroptes and symbiotes it is sometimes sufficient to place the suspected scabs on black paper or in a case having a glass cover, and to expose them to a mild heat or to the sun. Soon we can see, with the magnifying glass or with the naked eye, acari coming out of the scabs and advancing on the surface of the paper or the glass. (For differential diagnosis see the articles devoted to special manges.)

Prognosis. It varies with the species of the parasite and the degree of extension of the disease.

Generalized sarcoptic mange of the horse is, so to speak, incurable; on the other hand, that of the pastern heals in a few days. Generalized psoroptic mange of the sheep is very difficult 
to conquer, while in the horse this same dermatosis, when it is limited, is rapidly brought under control by the action of most anti-psoroptics. If the prognosis is particularly serious in generalized sarcoptic and psoroptic manges, it is much less so when these affections are located upon a limited region, and it is altogether mild for symbiotic mange.

The state of nutrition of the animals has a corresponding relation more or less favorable to the extension of mange and to the therapeutic intervention. Young, weak, and emaciated subjects do not stand energetic treatment well. The temperature, season, condition of the coat, and individual sensitiveness are also circumstances which must be reckoned with. The anti-psoroptic bath of the sheep, for example, could only be used with advantage during the warm season. Abundant epithelial productions (dandruff) often render a cure impossible; all practitioners have observed that sheep mange is extremely hard to cure when care is not taken to shear the animals beforehand. The sensitiveness of certain species to the action of anti-psoroptic medicaments is well known: carbolic acid frequently produces poisoning in the cat; baths are dangerous for this animal, ${ }^{1}$ as well as for the goat. Localization of mange in the neighborhood of sensitive and important organs (upon the eyelids, for instance) may be an obstacle to the cure by rendering the application of certain drugs impossible. Finally, the age of the disease and the possibility of a new infection must be taken into consideration.

Treatment. Mange can only be cured by the use of antipsoroptics. Among these agents, which are very numerous, the least active are the alkalines : potash lye (soda, potash, green soap, ash lye), quicklime, hypochloride of lime, sulphate of potash, ammonia, ammoniacal urine of the ox, etc. All these remedies soften the scabs and squamæ; they are used concurrently with others, which are more efficient, in order to facilitate the penetration of these latter into the thickness of the skin. The various sulphur preparations are also harmless; when alkalines are present they produce sulphate of hydrogen, which is very toxic for the acari. Balsam of Peru and styrax are advisable in certain cases. As more powerful anti-psoroptics we must mention the products of dry distillation of animal or vegetable substances:

1 We must here add that sarcoptic mange in the cat often ends in death-sometimes in a very short time.-L. T. 
cresol, carbolic acid, creosote, tar, petroleum, benzol, fetid animal oil, and essences (essence of turpentine, etc.) The most energetic, but also the most dangerous, are tobacco in infusion or decoction, arsenic, and mercurial preparations.

Concerning the preparation and composition of these agents and of the medicated baths which are used in the different species, we refer the reader to the study of manges in particular. We will only mention here general facts, which may serve as a guide in the treatment of acariases.

1. The prophylaxis includes the isolation and seclusion of mangy animals. During the time of treatment we should prevent any immediate contact of diseased animals with healthy subjects, and avoid mediate contagion-through blankets, grooming implements, litter, and the walls of the stable or kennel. Besides, we must disinfect the stables and utensils used in it.

2. The use of therapeutic agents ought to be preceded by a careful cleaning of the skin; this should be cleared of the scabs and epidermic desquamations.

3. In selecting an anti-psoroptic we should take into consideration the species, the constitution of the subjects, the condition of the skin, the wounds existing upon its surface, etc. In weak animals it is proper to use only harmless drugs; we must avoid mercurial preparations for the ox or carbolic acid for the cat. In the sheep, immediately after shearing, the skin is strewn with a number of small wounds and the cutaneous absorption is rendered very active; at this time irritating agents and toxic baths are absolutely counter-indicated.

4. The treatment must be particularly energetic upon the localities where mange is intense; but in the sheep especially it is essential to treat the parts adjoining the diseased surfaces and even the entire skin, for any region may be the seat of a recent infection.

5. It must be renewed after some time. Very often a first antipsoroptic application only reaches the acari; it does not affect the vitality of the eggs, and in less than a week these latter give birth to new parasites. In quite a number of cases, especially when mange is old and stubborn, it is necessary to make a third application: the eggs being unequally advanced in their development, some of them may have escaped the two former treatments. And in old sarcoptic mange, when the acari have penetrated deeply into 
the skin, a third anti-psoroptic friction is not sufficient (sarcoptic mange of the horse); the final cure can only be obtained by repeating the treatment at short intervals for weeks and even months.

Mange is a very important disease from the standpoint of sanitary police regulation and commercial jurisprudence.

1. It is considered as an epizootic disease by the sanitary police; the German law of June 23, 1880, concerning epizootics includes sarcoptic and psoroptic mange of horse, ass, mule, jennet, and psoroptic mange of the sheep.

2. Sheep mange is regarded as sufficient to set aside a contract of sale in Bavaria, Würtemberg, Baden, Hesse, Hohenzollern, etc., with a delay of fourteen days; in Saxony the delay is extended to fifteen days; in the Duchy of Nassau to twenty-nine days. Mange of the horse sets aside a contract of sale in Prussia, with a delay of fourteen days, and in Saxony with fifteen days' delay. In this latter province mange of the ox is also controlled by law with a delay of fifteen days.

\section{B. MANGE OF DIFFERENT MAMMIFEROUS DOMESTIC ANIMALS IN PARTICULAR.}

\section{a. Manges of the Horse.}

[1. Sarcoptic mange. This affection generally first shows itself upon the head, neck, shoulders, costal walls, but frequently also on the regions where the saddle or girth is placed (contagion by harness). It is indicated by small depilated blotches which gradually become extended; the eruption rarely reaches the whole surface of the body; in most cases it remains located upon the head, neck, and shoulders.

Its other principal symptoms are: a continuous pruritus, which is especially intense during the night and when the skin is overheated; this pruritus forces the animals to scratch and rub themselves and to bite the diseased regions. When touched, rubbed, or slightly scratched on these parts of the body the horse shows, by manifestations not to be mistaken, the pleasant sensations which he feels and the relief he experiences; he stretches his neck and head, bends the spinal column downward and turns up his upper lip like the stallion when smelling at the mare. At the beginning, the alterations of the skin consist of small pimples, on the surface 
of which the hairs fall out or are agglutinated by a viscous liquid; there are sometimes vesicles. Later, the pimples become the seat of an abundant epidermic desquamation and are covered with scabs; these may acquire the thickness of one centimetre; they are mainly formed of epidermic cells which are disposed in stratified layers, in which we may recognize graded galleries. The skin becomes indurated, parchment-like, wrinkled, fissured, creviced, and excretes a serous or purulent exudate; it is rendered bloody here and there by frictions. When the disease is old and more or less generalized the animals become emaciated, fall into a cachectic condition, and may perish from exhaustion.

This mange is transmissible to man, affecting especially the hands and arms of persons having the care of mangy horses (Dupont, Gerlach, Weigel). According to Sick, in 1791 two hundred Hussars of the same regiment were contaminated by their horses.

The diagnosis of sarcoptic mange is difficult at the outset; minute and repeated microscopic examinations are mostly necessary in order to discover the sarcoptes, and there are cases where they are not found, no matter what is done. The only symptom which at this period has a real diagnostic value is a very intense pruritus which is much less evident in non-parasitic pustulous eczema (summer mange, pustulous rash). These intense itchings, which contrast with the slightly marked alterations of the surface of the skin, led to the belief entertained formerly of the existence of prurigo and pruritus in the horse (see Eczema). In cases of old and neglected mange we can easily detect the acari.

The prognosis is more serious than is generally admitted. Concerning curability, sarcoptic mange differs essentially from psoroptic mange, which ordinarily heals quite rapidly; the former, when it has reached an advanced stage, is rebellious to all medication, even the most energetic; almost always, after a treatment which has been prolonged for months, we observe recurrences of the disease which are often taken for successive infections, but which are manifested in cases where the possibility of repeated contagions could not be invoked.

The observations related further on will confirm the decided judgment which we have just given upon the gravity of this mange. The treatment consists of cleansing the diseased surfaces, softening the scabs with carbolated or cresolated glycerin at 5 to 10 per cent., clipping the hair if necessary, and applying green soap, which 
is to be left upon the skin for twenty-four hours. When the integument is thus prepared, we apply the anti-psoric application, which is to be used in the shape of ointment, liniment, or solution.

In benign cases we recommend Vienna tar liniment, which has the following formula: tar and sulphur sublimate, of each 500 grammes; green soap and alcohol, of each 1000 grammes. It is applied in frictions by means of a hard brush, and is left upon the surface of the skin for a period of six to eight days, taking care to make new applications of the preparation whenever it is rubbed off; at the end of eight days we wash the parts that have received treatment with soap and warm water. In repeating this practice two or three times a cure is almost always obtained. ${ }^{1}$

In generalized and persistent mange it is good to change the therapeutic agents. After having tried tar liniment for some time, we may use cresolated liniment (cresol, green soap, of each 500 grammes; alcohol 250 grammes), carbolic acid and creosote (1 part to 10 of oil or alcohol), arsenical vinegar (1 per cent.), styrax, sublimate lotions ( 1 to 3 per cent), decoctions of tobacco ( 5 per cent), etc. Lotions with sublimate solutions are well borne; but in order to obtain a final cure we have had to repeat the use of the solution, 2 to 3 per cent., or add a decoction of tobacco ( 15 per cent).

The following observations show how, in the horse, the treatment of old sarcoptic mange is long and uncertain.

On January 7, 1884, a horse affected by generalized sarcoptic mange was left for treatment at the Veterinary School of Munich. They started to rub it with 1 kilo of green soap. They made after-

1 Trasbot's charge may also be used with advantage:

Benzine

Oil of cade

Coal tar

Mix the coal tar and oil of cade in the mortar and add the benzine.

The Codex gives a somewhat more complex formula :

\begin{tabular}{|c|c|c|c|c|c|c|c|c|c|c|c|}
\hline Benzine & - & - & - & - & • & - & - & • & . & \multicolumn{2}{|c|}{300 grammes. } \\
\hline Oil of cade & - & - & & - & - & $\bullet$ & - & $\bullet$ & - & 100 & "6 \\
\hline Coal tar & - & . & - & - & - & - & - & - & - & 100 & “ \\
\hline Green soap & - & - & - & - & - & - & - & - & - & 100 & 6 \\
\hline Essence of $t$ & pen & & - & . & . & - & - & . & . & 100 & 66 \\
\hline
\end{tabular}

The preferred anti-psoric preparation, at the Veterinary School of Toulouse (Neumann) is cevadilla oil :

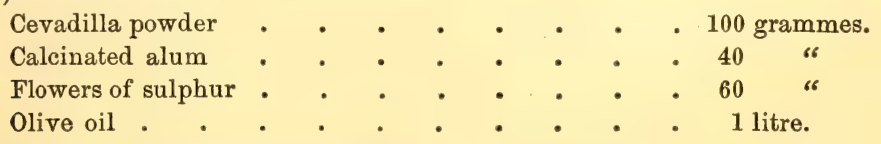

Digest for two hours in a water-bath. 
ward : first, five irrigations with a litre of sublimate solution at $2 \frac{1}{2}$ per cent. at three days' interval ; second, a friction with carbolated soap at 10 per cent.; third, a new irrigation with sublimate. The animal seeming to be cured, was sent home on the first of February. Eight days later he was brought back with the pathognomonic itching. Treatment: washing with carbolated soap; two frictions of carbolated glycerin at 20 per cent.; frictions with carbolated liniment (carbolic acid, 100 grammes ; alcohol, 600 grammes; green soap, 750 grammes). On February 16 th the horse appeared. again to be cured and was delivered to his owner. On March 7th the eruption reappeared. New treatment: Three frictions of Vienna tar liniment; application of carbolated alcohol upon the most affected regions; washing with a sublimate solution. The patient seemed cured for the third time, and left the hospital on April 24th. Six weeks later, mange appeared again and soon radiated over a large surface of the integument; it disappeared only after the use of lotions of sublimate solutions used repeatedly for two months. This time the cure lasted five months. At the end of this period no sarcoptes were found on microscopic examination, but the animal again began to rub and scratch himself. The way he was kept, and the measures which were taken, permit us to exclude absolutely the hypothesis of successive infections.

A second horse, affected by generalized sarcoptic mange of long standing, was treated for five months. Eleven irrigations were made with a solution of sublimate, five frictions of carbolated soap (5-12 per cent.), a friction of Vienna tar liniment, an application of carbolated alcohol by means of a brush, and frequent applications of balsam of Peru. A cure seemed obtained, but the mange returned within four weeks.

A third horse, which had been mangy for years, was cured after a treatment lasting ninety-three days. During the first three weeks, three tar applications were made; as the condition showed little improvement, daily washings with a sublimate solution were resorted to for a period of six weeks (solution of $1-1 \frac{1}{2}$ per cent., three litres daily); during the same period, daily lotions with Hager liquor were made (carbonate of soda, 250 grammes; hydrochlorate of ammonia, 60 grammes; water, 12 litres; carbolic acid and glycerin, of each, 100 grammes), for the purpose of softening the skin, which was dried up and stiff. Four months later this horse was brought back in a mangy condition. The diseased regions 
were treated with an application of carbolated alcohol and frequent washings with sublimate solutions at 2 per cent. In this way a final cure was obtained.

A horse affected by a quite extensive mange was cured, in a relatively short time, by twelve washings of sublimate solution and by frictions with Vienna tar liniment, carbolated alcohol, and balsam of Peru.

These few facts are sufficient to show how tenacious sarcoptic mange is, and how rebellious to all treatment.

2. Psoroptic mange. This form of mange starts with somewhat large spots which are distinctly circumscribed, and are preferably located upon protected regions, especially at the base of the mane, tail, sheath, upon the mammæ and the inner surface of the posterior members; they are more rarely found upon the withers, back, or croup. The first manifestation is the appearance of pimples, of papulous islands produced by the bites of the psoroptes; a lymphoid liquid oozes from these lesions which dries up and forms scabs, which may become as thick as a finger. In addition we observe an abundant epidermic desquamation and falling out of the hair. The skin becomes gradually thicker, acquiring the aspect and consistence of leather; it is also wrinkled. The pruritus is intense ; the integument becomes excoriated, bloody, or suppurating, and is inflamed in its whole thickness by the action of rubbing.

The differential diagnosis of psoroptic and sarcoptic mange is based upon the predilection of the former for protected regions, and on the characters of the parasite. In addition, psoroptic mange progresses in the same way as an oil-spot on a piece of cloth ; when, however, it has invaded a large surface, it may simulate and be mistaken for sarcoptic mange. The intense pruritus which accompanies it, and the distinct definition of the blotches at the beginning of the affection, permit us to distinguish it easily from squamous eczema and from mange of the mane.

A cure is much more rapidly obtained than in sarcoptic mange. We succeed quickly, even with drugs of little energy. Cresolated or carbolated soap, or glycerin associated with these agents (10 parts to 1), used alone or with Vienna tar liniment, are antipsories which suffice in all cases; the latter must not be used when thick crusts are present. In cases of some gravity, the medium duration of the treatment varies from two to three weeks.

3. Symbiotic mange. This is also known under the name of 
pastern mange; it is clearly distinguished from the preceding by its localization upon the extremity of the legs. It is most frequently found in the fold of the pastern; thence it extends to the fetlock, the canon, the knee, or the hock; in exceptional cases it advances as far as the elbow, the shoulder, neck, leg, and thigh. Its development is extremely slow; its generalization takes place only after months.

At the beginning the only important symptom which attracts attention is the habit which the mangy animal has when at rest of abruptly striking the ground with one of the hind legs, doing this at more or less close intervals for hours, but especially during the night. Some horses kick; all scratch and bite themselves in the region of the fetlock. If the legs are examined we there find an abundant epithelial desquamation, also partial depilations, and later scabs, sometimes also crevices. The skin is dry, thick, as if tanned; according to Gerlach, it may be covered after a certain time with horned papillous proliferations.

The diagnosis is easy. This mange is distinctly characterized by its seat, by the abundance of epidermic desquamation and the intensity of the pruritus; this latter symptom distinguishes it from eczematous leg grease (eaux aux jambes).

It is combated by carbolated or cresolated glycerin applications, by carbolated soap or tar ointment (1 part to 10). It disappears. generally in a short time.

Mégnin (according to Delafond) has described it as an intermittent mange; it appears in winter to disappear in the spring. The symbiotes could, however, remain alive during the warm season; they could find sufficient nourishment in the more abundant cutaneous secretions. In winter, on the contrary, they would be forced to attack the skin itself.

Schwartz explains the frequency of wounds of the pastern by a permanent irritation which is felt by the animals affected by symbiotic mange.

\section{b. Sheep Mange.}

Psoroptic mange. Sheep may present three kinds of acariases, like the horse; but the most frequent and the most serious is psoroptic mange. The importance of this trouble, from an agricultural standpoint, is established by numerous documents. Statistics establish that, in 1884, 170,000 sheep had been affected in Germany and 80,000 (61 per cent. of the whole number of all 
flocks) in Alsace-Iurraine. In the last three years, when the German law relati've to epizooty was already in force, the official reports have shown, for Prussia alone, 183,000 mangy sheep; for Bavaria nearly 100,000 , and Würtemberg more than 60,000 . The death-rate amounted to about 2000 head in Prussia. For the district of Greifswald, Fürstenberg estimates the yearly loss of wool at 100,000 marks; this loss amounts to several millions yearly for the whole German Empire.

Transmission of psoroptic mange is generally by immediate contact; a mediate contagion is much less frequent. Contagion will especially tak duced into a healthy flock the animals of which have their coat of wool, or when the temperature is high, during the summer, in hot sheepfolds where the animals are crowded against one another. Such conditions favor the activity of acari and their emigration.

Symptoms. The psoroptes invades the part covered by wool; other parts (belly, lower pectoral region, head, etc.) remain untouched. The croup, base of the tail, back, sides of the thorax, neck, and shoulders are the places chosen by the parasites.

The eruption always commences with small spots, which may be isolated or gathered in groups, according to the mode of infection. By spreading the wool apart at the beginning we observe flattened pimples, of the size of a millet-seed, of a paleyellow or reddish color, which are produced by the bites of the psoroptes. According to the number of the latter, we find the papules disseminated or agminated, forming in this latter case flat pimples, which may reach the size of a silver dollar; the skin of the neighborhood is red, and sensitive to the touch. On the surface of the pimples small vesicles or pustules appear which tear or dry up; an abundant epidermic desquamation is produced at the same time, which forms thick crusts by becoming mixed with the sebaceous matter and with the contents of the pustules; these scabs are sometimes very hard and of a yellow-brownish color; they protect the parasites. The scabs become shrivelled and parchment-like upon the diseased shorn skin. The remaining bits of wool are at first agglutinated by the liquid of the pustules; soon they form scabby masses and detach these from the skin; their roots are loosened; the fleece gradually becomes flaky: more or less large numbers of tufts appear on its surface and fall out or are torn out; the wool has lost its lustre and its softness; it 
is of pale color, as if washed; it may be removed with the greatest ease. The integument is dry, hard, shrivelled, thickened, cracked, creviced; we often see ulcerations upon it which are produced by rubbings.

Besides these local alterations we observe a very intense pruritus. It is generally the first symptom which attracts attention. The animals rub themselves against neighboring objects; they roll, bite themselves, and tear out the wool from the affected regions. If they are rubbed they give evident signs of satisfaction by moving the lips and shaking the head-movements which are altogether peculiar; they open and close their jaws alternately; they move their posterior legs and tail, favoring the action of the hand by the position which they take. The pruritus is especially intense when the animals are exposed to the sun or are kept in a hot sheepfold, after exercise or during the night. Their continually rubbing themselves hastens the falling out of the wool, and produces more or less deep ulcerative lesions of the skin and a diffuse dermatitis.

When mange becomes generalized the permanent excitement, loss of nutritive fluids, and suppression of appetite and rumination lead gradually to emaciation. As a rule, weak animals die (hydremia, cachexia).

Course. The course of this acariasis is slow but continuous. A more or less marked aggravation is observed in the fall and during the winter; some improvement takes place by shearing and washing. The thin skin of animals belonging to improved breeds is much more sensitive to the action of the psoroptes than that of common sheep.

Diagnosis. This is based upon the following: 1 . The existence of a contagious dermatitis in the flock. 2. Persistent itching and particular symptoms observed in certain animals when they are examined. Frictions and scratching, instead of producing relief, very frequently occasion pain ; this fact is often seen when mange is commencing. Let us add that there are shepherds who are clever in hiding pruritus and in misleading the veterinarian, either by tightly squeezing the sheep being examined, or by using certain energetic drugs. 3. The irregularity of the fleece, which is flaky. The condition of the wool, the ease with which it may be torn out, and the existence of depilated surfaces, are so many symptoms which should induce us to suspect mange. 4. The evidence of lesions revealed by a minute examination of the skin. We must 
especially inform ourselves concerning the condition of the integumentary membrane covering these regions: base of the tail, croup, back, and shoulders; it is suffieient to spread the wool apart in order to recognize the alterations of the integument; we may judge of the degree of its sensitiveness by scratching. We must also look for any coloration of the wool resulting from drugs used by the shepherds. 5. A microscopic examination and finding of the psoroptes alone permit us to decide the nature of the trouble with certainty.

Differential diagnosis. The affections which we may confound with psoroptic mange are :

1. Rain rot. This is an eczematous disease, which is produced by prolonged dampness of fleece and skin; it is sometimes observed upon a large number of animals of one flock, exactly like mange. A difference may be made by the pathology of this dermatosis, by its rapid disappearance as soon as the weather becomes favorable or when the animals are kept in the sheepfold; also by the slight intensity of the alterations of the skin and the absence of pruritus.

2. Other cutaneous parasitic eruptions produced by melophages and trichodectes. It is sufficient to examine the wool and skin carefully, in order to be immediately informed.

3. The abnormal production of "yolk" (wool fat) and its accumulation in the fleece. In this affection the bits of wool are agglutinated and some pruritus exists, but the skin is clean and smooth, soft, and free from any lesion.

4. Cauterizations of the skin, made with fraudulent design. They may be recognized by the abnormal coloration which the caustic has left upon the wool and skin, or by the characters of the scab; in some cases, however, this has truly the aspect of mange scab. The course of the disease and the microscopic examination give more precise diagnostic information.

Treatment. We have the choice between two principal methods, frictions and baths. The former constitute a kind of provisory treatment, which is used either as a preparation for baths, or at times when baths cannot be given (cold weather). We apply ointment or liniments on the circumscribed mangy centres (carbolated soap or oil, mercurial ointments, decoction of tobacco, petroleum, etc.). These frictions cannot produce a radical cure, for they only reach the appearing centres, which are more or less old; the others, which are of recent date and disseminated on the 
surface of the body, remain untouched. The toxic property of mercurial ointment has been recognized in all countries; this substance is very dangerous, even for the lambs of the animals which are under treatment; we must abandon its use and replace it by less noxious preparations. The several compositions which we will discuss have been advised for the bath.

1. Walz's lye, the oldest anti-psoric bath, has been extolled by the author, who discovered the psoroptes. It has the following formula: quicklime 4 parts, potash 5 (this may be replaced by 60 parts of wood ashes), fetid animal oil $6, \operatorname{tar} 3$, ox urine 200, water 800 . Walz's lye is the least expensive of all anti-psoric solutions, but the majority of practitioners consider it as insufficient; old and serious cases of mange resist its influence; it has also the disadvantage of coloring the wool brown. It ought only to be used as a preparatory measure for more efficient anti-psoric baths.

2. Gerlach's treatment. This consists of a preparatory bath composed as follows: potash 2 parts, quicklime 1 , water 50 , in a so-called anti-psoric bath. This is a decoction of tobacco at 3 per cent.; it would be more advisable to use a decoction of 5 per cent. (Roloff). It is generally found to be very efficient, especially since the modification added by Roloff. This treatment has the inconvenience of being somewhat complex, and the preparatory bath affects the hands.

3. Ziindel's bath is composed as follows: commercial carbolic acid, $1 \frac{1}{2}$ kilos; quicklime, 1 kilo; soda and green soap, of each 3 kilos; water, 260 litres (per 100 sheep). Its cost price is not high (about 50 cents). Kayser and Ostertag have modified it to advantage by replacing common water with a 2 per cent. decoction of tobacco (tobacco, 5 kilos; water, 250 litres); its price is thus trebled, but its effects are considerably increased; it represents, so to speak, a combination of Gerlach and Zündel's baths. In order to simplify this preparation, Kayser has used nicotina (tobacco lye). ${ }^{1}$

4. In Prussia and Bavaria the Government has recommended a similar composition. We begin by boiling $7 \frac{1}{2}$ kilos of tobacco in 250 litres of water for half an hour; to the warm liquid we add

1 Nicotina contains about 4 per cent. of nicotine; the sheep are dipped for about three minutes in a solution of this substance, then they are rubbed with brushes.N. D. T. 
carbolic acid and potash, of each 1 kilo (cost, 13 to 14 francs). This bath is very energetic, but its preparation requires some time, and it may produce poisoning.

5. The arsenical baths, commended by Tessier and Mathieu, have been variously appreciated. Some have pointed out the dangers to which they expose the animals, whilst others have insisted upon their remarkable efficiency. The numerous poisonings which have been observed have been due to neglect and imprudent manœuvres rather than to cutaneous absorption; they must, however, only be used after the small wounds produced by the process of shearing have healed; shearing should, therefore, be performed eight to fourteen days before the treatment. Tessier's bath has the following formula: arsenous acid, $1 \frac{1}{2}$ kilos; sulphate of iron, 10 kilos; water, 100 litres. $^{1}$ Mathieu replaces sulphate of iron by alum (arsenous acid, 1 kilo; alum, 10 kilos; water, 100 kilos). This last preparation is especially used in Würtemberg and some districts of Bavaria. Kehm recommends a solution prepared with $\frac{1}{2}$ kilo of arsenous acid, 6 kilos of alum, and 100 litres of water. It is necessary to dissolve the arsenous acid beforehand in boiling water, in order to avoid the cauterizations which it may occasion. Putscher $^{2}$ uses also arsenical solutions, for which he prepares the animals by a washing with black soap; in order to obtain the cure he was obliged to give three baths in every instance; he advises a nine days' interval between the baths.

6. Fröhner's cresol bath, adopted by the German Government. Shearing is not absolutely indispensable for the cresol treatment; mangy sheep with their fleece have been subjected to it with success and without any damage to the wool. If the sheep are shorn, the scabs of the most affected regions should be softened; this is done by making daily frictions for three to five days with a cresol liniment (creolin 1 part, alcohol 1, green soap 8). These frictions constitute an important element of the cure.

1 In order to lessen the danger of poisoning, the solution has been rendered bitter through the addition of gentian powder, and more recently with aloes. Trasbot's arsenical bath, which at present replaces Teissier's bath everywhere, has the formula (Codex of 1884):

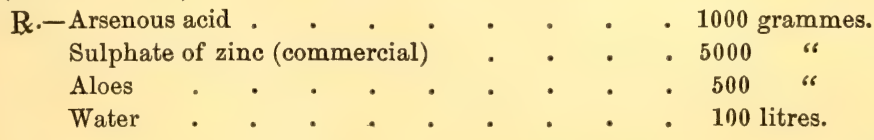

2 Putscher (communication).

-N. D. T. 
The patients thus prepared are bathed twice, at an interval of one week, in an aqueous solution of cresol, $2 \frac{1}{2}$ per cent. ( $6 \frac{1}{2}$ litres of cresol in 250 litres of water-for 100 sheep). The cost of this bath is about 10 francs. It is prepared by adding cresol to warm water $\left(30^{\circ} \mathrm{R}\right.$. $)$ and stirring the solution. Immersion ought to last for three minutes; immediately afterward the sheep is brushed hard upon the whole surface of the body; when this manœuvre is ended, the animal is to be dipped a second time into the liquid for a few moments. The manner of holding the animal in the bath and the various manipulations do not need any particular indication. It is important not to act too precipitately, and to watch the workman well, in order to control the precise execution of the smallest details of the operation; when its rules are neglected a third bath may become necessary. The cresol solution is distinguished from all others by its absolute harmlessness; its preparation is simple, and its price not high; added to these important advantages, the wool is neither colored nor changed, contrary to what is observed with tobacco, arsenic, etc.

No matter which bath is used, the procedure is the same in all cases. The animals must only be subjected to the treatment some time after shearing: eight to ten days for an arsenical bath, three or four days for all others. The solution should be brought to a temperature of about $30^{\circ} \mathrm{R}$. $\left(37.5^{\circ} \mathrm{C}\right.$.) ; it ought to be abundant enough in order to allow for the use of two to three litres of liquid per sheep. The bathing utensil should be placed near the sheepfold, in a cool and shady place; it is of advantage to fix it in the ground at a sufficient depth. The tub used for the brushing should be placed in close proximity. All the animals should be treated successively, beginning with those which seem healthy or which are slightly affected. The whole body, with the exception of the eyes, mouth, and nose, should be dipped into the anti-psoric liquid for about three minutes. In order to immerse the animal, one man must take him by the four legs and another by the head; the latter must take care to apply both thumbs upon the eyes, in order to protect them; the patient is then thrown on its back and plunged in this position. Immediately after the bath it is carried to the brushing tub, where two other men rub it well, particularly upon the dorsal and lumbar regions; it is then plunged again into the anti-psoric solution for a few moments, and is then set free in a clean stable or in a pasture which is exposed to the sun. It 
is important to keep the animals from the rain, which might occasion cold or remove the preparation left upon the surface of the skin. The sheepfolds ought to be disinfected. At the end of a week the second anti-psoric bath is to be given, and within two weeks a third one, if necessary. In all cases it is indispensable to give two at least. During the intervals the regions where the disease persists should be treated. When the cure is ended we must also watch the animals and not neglect the mange blotches which may appear subsequently. Finally, we must avoid, as far as possible, taking the animals into the roads and pastures which they frequented before the treatment.

Sarcoptic Mange. According to the observations of Delafond, Gerlach, our own, and those of a large number of other authors, this mange is exclusively located on the parts of the head which are bare of wool (head mange, black muzzle); it rarely invades the legs. The regions which are usually affected are the lips, the labial commissures, forehead, chin, orbit, cheeks, and the external face of the auricular concha; thick scabs are found in it ( $\frac{1}{2}$ to 1 centimetre), they are grayish, and are strongly adherent to the skin; the pruritus is generally very intense. During the warm season mange extends over nearly the whole head; in winter time it retrocedes. It has no troublesome consequence and disappears rapidly by the application of cresol liniment or a decoction of tobacco. An experimental inoculation upon the laniferous skin of merinos produces only a slight dermatitis.

In long-tailed sheep Roloff ${ }^{1}$ observed a sarcoptic mange similar to goat mange (cuirass-shaped scabs, of stony hardness, which are developed upon the head, and a few other regions).

The most efficient remedies against this psora are creosoted glycerin and carbolated glycerin.

Symbiotic mange. This is also designated under the name of foot mange; it is located upon the hind legs, whence it may extend upward to the testicles or mammæ. It is marked by itching, an abundant epidermic desquamation and the formation of scabs.

\section{c. Mange of the Ox.}

1. Psoroptic mange. This mange, the most frequent in the ox, appears upon the lateral aspect of the neck and shoulders, the

1 Roloff: Berlin. Archiv, 1877. 
base of the horns and the root of the tail ; thence it spreads to the back, costal walls, etc. ; it may invade the entire body.

Its principal manifestations are more or less numerous pimples, exudation, an abundant epidermic desquamation, falling out of the hair, an intense pruritus and the formation of dry, gray-brownish scabs. In the course of time the skin becomes thickened, stiff, acquires the consistence of leather and becomes wrinkled. During extreme seasons Müller has observed alterations of aggravation (winter) and improvement (summer). When the disease spreads over the whole surface of the body, the animals lose flesh and may die. It coexists sometimes with symbiotic mange (Müller). Roll has found it on the buffalo.

The treatment is the same as for other animals; creosote and tobacco decoctions have proved particularly efficient.

2. Symbiotic mance. This is also known under the name of tail mange. It remains generally localized upon the depressions of the posterior part of the pelvis and on the base of the caudal appendage; it may, however, extend over the whole surface of the body (back, neck, internal aspect of the thighs) when the hygiene of the skin is neglected. These cases are rare; those in which mange is propagated to the members (foot mange) are also exceptional. The evidence of symbiotes in malt or residue eczema is without importance (Johne), notwithstanding that Rabe has attempted to assimilate this trouble to foot mange. These morbid conditions may coexist, but they are radically different. Malt eczema may favor the extension of symbiotic mange.

If characterized by abundant desquamation or a formation of scabs, by partial depilations and itching, this mange may persist for years without any discomfort to the animals; its spreading tendency is almost wanting, and its contagiousness hardly noticeable. We are rarely called on to treat it. The mild anti-psorics indicated for the horse (carbolated soap, tar ointment) suffice in every instance.

3. Sarcoptic mange. This acariasis is extremely rare in the ox, which is always contaminated through the horse. It is therefore entirely accidental, and not important; it is sufficient to mention it. Besides, its symptoms are the same as in the horse. 


\section{d. Mange of the Goat. ${ }^{1}$}

SARCOPtIC MANGE of recent origin is always localized upon regions where the hair is sparse, especially on the head, lips, nose, ears; but it is quickly propagated over the whole surface of the body.

It is marked by the presence of furfur and scaly scabs, which are hard, split, and of a grayish blue ; the skin becomes thickened, cracked, wrinkled, parchment-like, and little by little assumes a gray coloration. We notice the appearance of circumscribed depilations on its surface, which gradually become enlarged; in the course of time the animals may become entirely nude. An intense pruritus exists at the same time. A fatal termination is quite frequent in the goat: but of 1000 sick animals observed by Wallraff, 500 died. In a single community Klingau lost 100 head in a year.

It seems to be easily transmitted to man. In one locality Wallraff found almost all the families contaminated. Roloff has by experiment communicated it to sheep with short wool (Somali), and to animals with smooth wool, poor in grease (large-tailed sheep).

For treatment we must resort to ointments with a tar base, sulphur, carbolated glycerin, etc. Baths must be omitted, for they do not agree with the goat.

\section{e. Mange of the Pig.}

Sarcoptic Mange of the pig, which is caused by the Sarcoptes squamiferus, (?) invades the head first, especially the fossa beneath the eye, the eyelids, cheeks, and auricular region; thence it extends upon the neck and shoulders, the back, the inner aspect of the members, and finally over the whole surface of the body. The affected regions are covered with an abundant epidermic desquamation, and also thick scabs, which may reach the thickness of one centimetre, and the color of which varies from a grayish white to a silver-white. Sometimes the animals seem as if dusted with

1 We have found sarcoptic, symbiotic, and posoroptic mange in the goat.

Symbiotic mange, as observed by Delafond, was located upon the upper part of the trunk (neck, fetlock, back, and loins). Mollereau found it localized upon the upper part of the legs (see Bull. Soc. cent. Vét., 1888).

Psoroptic mange, which is localized inside the ear (auricular acariasis), has been seen by Pezas, Mégnin, Nallet, Raillet, and Morot. It may affect both ears (Morot and Nallet) or one only (Pezas). (See Revue Vét., 1889, and Bull. Soc. centr. Vét., 1890.) -N. D. T. 
guano (Müller). The skin is thick, stiff, very hard, and wrinkled. All the bristles become loose and fall out; at times they are agglutinated in tufts. The acari hide themselves under the scabs; it is quite difficult to recognize them upon the living animal.

The treatment consists in softening the scabby masses with green soap or glycerin, and applying tar ointment or sulphur ointment on the diseased regions.

\section{$f$. Manges of the Dog.}

Sarcoptic mange. This is produced by the Sarcoptes squamiferus, and is a disease extremely prevalent in certain countries. In Berlin, among 100 sick dogs, ten have this trouble. During the year 1886-87, out of 8399 animals which were presented at the dog clinic of the Berlin School, 639 were affected by sarcoptic mange. The "dog homes" (boarding kennels) contributed in a. large measure to its dissemination.

Symptoms. The appearance generally on the head, forehead, the base of the ears, the orbits, and sometimes on the abdominal and lower pectoral regions, the root of the tail, the elbows and feet, and exceptionally upon other regions (genital organs). Ordinarily it spreads very rapidly over the whole surface of the body; in one month the entire skin may be invaded. Its manifestations vary according to the degree of resistance of the integument.

At the beginning of the trouble we detect spots especially on surfaces where the skin is bare, which are similar to fleabites, and are located upon the lower abdominal region, as well as on the inner aspect of the legs, where the integument is very thin. The action of friction and rubbing often produce a diffuse redness of the skin. On the surface of the skin where the bites are located, small pimples of the size of a millet-seed or lentil are developed, which may be transformed into vesicles. These latter are frequently observed in considerable numbers, spread upon the whole surface of the body; in the majority of cases they are ruptured, producing large moist surfaces (moist mange); in others, a simple epidermic desquamation is observed (dry mange), less frequently the vesicles pass into a pustulous state. The pustules are ordinarily found upon the thin skin of the abdomen and of the inner aspect of the legs ; they present sometimes a dark central point as if produced with a small needle, and corresponding with the parasite. Vesicles and pustules dry rapidly and form yellowish-gray scabs, 
scales are detached in great abundance, and the hairs fall out, forming extensive bare blotches. The skin itself becomes thickened, wrinkled, and cracked, and more or less deeply creviced. These cutaneous alterations are accompanied by an intense pruritus, which becomes intolerable in hot stables or near the fire, also after various kinds of excitement and exertion. In order to reduce it, the animals scratch themselves constantly; rubbing performed with the fingers upon the diseased regions gives them a relief which they show by moving one of their hind legs, the movements of which are synchronous with those of the hand. When the trouble is old and generalized the dogs lose flesh. Weak subjects may succumb.

Diagnosis. The most important diagnostic symptoms are: intense itching, localization of the disease upon the head, its rapid extension, its transmissibility to other species and to man, wrinkling of the skin and depilations, also the accumulation of squamæ and formation of scabs, the tendency of the eruption to settle on the ears, the lower pectoral region, the belly, elbows, the inner aspect of the thighs, etc. Here again, however, the only characteristic symptom is finding the sarcoptes, by microscopical examination, in the product which is taken from the affected regions. This is quite difficult; as a rule, the parasites are located deeply in the skin, and in order to reach these we must not only remove the scabs, but also the superficial layers of the integument. It has to be scratched by means of a very sharp bistoury till the appearance of blood, or we may make superficial cuts upon it with a pair of scissors. The movements of the acari may also be influenced; they may be attracted upon the surface of the skin through heat; to effect this the animals may be placed near a fire, in addition being covered with a woollen blanket, etc. In some cases, especially when thick scabby blotches have formed, the parasites are easily found. As a last diagnostic method, we may try the application of scabs upon a man's arm. (The sarcoptes are easily found on a dead animal.)

Differential diagnosis. Sarcoptic mange may be confounded with follicular mange. Both are contagious and are marked by an eruption of pimples, falling out of hair, and abundant epidermic exfoliation, etc. The main differences are: the intensity of the pruritus and the rapidity of healing in sarcoptic mange; a particular pustulous character and an extremely easy recognition of Demodex in follicular mange. 
A contagious cutaneous eruption accompanied by an intense pruritus and symptoms which have just been detailed must be considered as sarcoptic mange, even when microscopic research gives a negative result.

Some papulous eczemas are marked by manifestations which are quite similar to those of sarcoptic mange, but the effects of the treatment are sufficient to dispel all doubts. Extended dermatoses which disappear after therapeutic applications are not of a parasitic nature.

In certain doubtful cases the accidental transmission to man permits us to establish a diagnosis. It is not rare in Berlin, where, as previously stated, sarcoptic mange is very common.

Prognosis. Notwithstanding that the prognosis of sarcoptic mange is less serious in the dog than in the horse, the cure is not always effected rapidly; far from it. Old and generalized mange is quite obstinate, and weak and debilitated animals often die during the treatment.

Treatment. It is indispensable to take certain precautions in the case of the dog which may be neglected in other species; he must be prevented from licking himself by tying his mouth, by muzzling or covering him with leather blankets, etc. Long-haired dogs must be clipped, and we must wait for some days before covering the integument with acaridian preparations possessing any toxic property; it is also improper to apply them upon a too large moist surface.

Among the commended remedies against dog mange, the most advisable are :

1. Cresol used in the shape of a liniment (cresol and green soap, of each 1 part; alcohol, $\frac{1}{2}$ part to 10 parts, according to the degree of intensity of the disease and its extension). With this preparation a daily application is to be made upon the diseased surfaces until a cure is effected. In generalized mange we rub every day one-third of the surface of the body. A cure is obtained in from one to three weeks. We may also use cresylic alcohol $(1: 10-20)$, and also cresol soap. Cresol as compared with all other antipsorics has the advantage of being absolutely inoffensive.

2. Tar, which is generally used as a liniment (tar and green soap equal quantities, alcohol q. s.). The whole surface of the body is to be rubbed three times-one-third of it at a time-each day. On the sixth day the animal is washed in a bath, and the treat- 
ment is repeated till cured. Cases of medium gravity require from three to four frictions.

3. Creosote, mixed with olive or rape oil $(1: 10-15)$. It may cure an old generalized mange within a month. It is the most efficient agent, but, unhappily, also the most dangerous. The rules applying to its use are the same as for cresol.

4. Carbolic acid in the shape of carbolated oil at 2-5 per cent., or carbolated glycerin. When these preparations are used it is proper to make the frictions at quite long intervals (carbolic acid. poisoning). During the period of treatment it is prudent to give. every day a small dose of an alkaline sulphate: sulphate of soda, 2 to 5 grammes-producing a formation of sulpho-phenate of soda in the blood, which is harmless.

Among other useful substances we may also recommend balsam. of Peru (especially in the case of delicate animals and in places where mange is localized in the neighborhood of the eyes), styrax, benzol, naphthol, ${ }^{1}$ oxynaphthalin, and ichthyol (Rabe, Friedberger). ${ }^{2}$ Naphthalin has not given more good results at the Berlin clinic than at Dresden, and it has the disadvantage of spreading. a very disagreeable odor. Concerning balsam of Tolu, its action is most uncertain (Siedamgrotzky). Benzine and petroleum has. not given us any success.

Dietetic care is necessary during the whole course of the treatment.

Symbiotes of The Dog. The acarus discovered by Hering in 1836 and designated Sarcoptes Cyanotis by this author, is a symbiote which is found in the product of external otitis of the dog (auricular catarrh). It is not certain that this acarus is the cause

1 The use of naphthol in the therapeutics of cutaneous diseases, parasitic and otherwise, has been studied by A. Josias and Nocard. If associated with lard, or vaseline (4 per cent.), or oil (10 per cent.), this agent has shown itself of much value in the treatment of dermatoses of our animals, especially in sarcoptic mange, in chronic forms of eczema, and in auricular catarrh of the dog. (See Recueil Vét., 1885.) -N. D. T.

2 "Trasbot's charge" (see Sarcoptic Mange of the Horse) is also a very efficient preparation. After having cut the hair and cleansed the skin we use the pure charge, or add an equal quantity of benzine to it. It is applied first on one-half of the body, and forty-eight hours later upon the remaining portion. After a few days a general soaping should be given with lukewarm water. If mange persists in some places, another application of the charge is made. During the whole duration of the treatment we give internally 2 to 5 grammes of bicarbonate of soda, according to the size of the animal. -N. D. T. 
of parasitic otitis. ${ }^{1} \quad$ Nocard has observed epileptiform attacks and deafness as a complication of this disease. Zürn affirms that this acarus is certainly the agent of auricular mange in the cat. ${ }^{2}$

\section{g. Mange of the Cat.}

Sarcoptic Mange. This is caused by the Sarcoptes minor; it is generally localized upon the head; its preferred seat is the bell of the ear. At the beginning it is indicated by small red dots, later by pimples and vesicles; the integument becomes gradually covered with an impetiginous coating; the hairs are erect and fall out partially, the epidermis becomes bare, the exfoliated lamellæ and the dried-up contents of the vesicles form thick yellowish-gray scabs, which may reach a thickness of one centimetre and conceal a large number of acari. The skin becomes stiff, hard, and wrinkled. The eyelids are very frequently tumefied, and a catarrhal or purulent conjunctivitis is developed. The pruritus is very intense; the animals scratch themselves with their paws, shake the head and rub it against hard objects; they are downcast and lose flesh rapidly; some succumb to the trouble.

The eruption may spread to the cervical region, even to the whole surface of the body; the paws, the lumbar region, and the croup are successively invadeed. It is especially in this form of the disease that a fatal termination is encountered.

Treatment. Baths, anti-psoric washings and frictions, upon the whole surface of the body, are means which should be prescribed. Carbolic acid, which is very toxic for the cat, must be entirely excluded.

Helmerich's ointment is an excellent and inoffensive remedy. It has constantly given us good results when the general condition remained satisfactory. If balsam of Peru is more efficient than Helmerich's ointment, it is also more dangerous; we have several times seen a single friction of balsam of Tolu upon the head produce a very intense cerebral excitement, rabiform spells, torpidity, and death. We have also observed these accidents after the application of rancid cod-liver oil, which is, however, recommended by Schwartz. Styrax seems to be less dangerous.

1 Since the publication of Guzzoni's work almost all authors admit that this affection is caused by symbiotes (Symbiotes auricularum, var. canis).-N. D. T.

2 Sulphate of potassium in aqueous solution, 1:5 naphthalin oil and cresolated oil give a rapid cure of auricular acariasis in subjects of our small species.-N. D. T. 
Delafond and Bourguignon, and more recently Johne, ${ }^{1}$ have detected sarcoptic mange upon the wild felines (lion, leopard). It was not produced by Sarcoptes minor but by Sarcoptes scabiei. At the beginning they observed circumscribed psoric centres disseminated on the chest, belly, back, etc. The mange gradually became generalized; the whole body was covered with squamæ, scabs, and depilated spots. The animals fell away rapidly, lost their appetite, and succumbed within a variable time. Aspersion with Zündel's anti-psuric bath has produced fatal poisonings (carbolic acid poisoning). Johne has obtained successful results by proceeding in the following manner: the animals, kept in the narrow cage, were sprinkled with a solution of balsam of Peru (balsam 1 part, alcohol 3 parts), then they were rubbed vigorously by means of brushes fastened to long iron haudles. These manœuvres, which were repeated five times at intervals of eight to ten days, produced an entire cure.

Ear mange of the cat is produced by Symbiotes (S. auricularum, var. cati). Affected animals shake the head, carry it inclined to one side, and show symptoms of otorrhea. We have obtained good results in the treatment of mange by using balsam of Peru mixed with an equal quantity of glycerin. The external auditory meatus must be thoroughly cleansed before injecting the remedy.

\section{h. Mange of the Rabbit.}

1. Sarcoptic mange. This is produced by the Sarcoptes minor and is similar to mange of the cat, it affects the head in preference (nose, lips, forehead), where it produces squamæ and scabs, falling out of the hair, thickening of the skin, itching, etc. The extremities are quite frequently affected at the same time as the head. When it becomes generalized, the subjects grow rapidly emaciated and die. It is advisable to use balsam of Peru or sulphur ointment frictions as in the cat.

2. Ear mange is produced by Symbiotes and Psoroptes cuniculi. It frequently consists of an intense inflammation of the integument of the external auditory meatus and is manifested by the formation of thick crusts of brownish-yellow color, which dry, slit, and render the concha very rigid at times. It may be complicated by alterations of the middle ear ; in some instances the psoroptes invade the whole surface of the body (Zürn).

1 Johne: Berlin. Archiv, 1880. 
The principal indications in treatment are the softening of the scabs, their removal, and injections of glycerin of cresolated or carbolated oil in the external ear ( 2 to 5 per cent.).

Addendum. Mange of the Ferret.

We observe a sarcoptic and symbiotic mange on this animal. ${ }^{1}$

\section{FOLLICULAR MANGE.}

Follicular mange, which is frequent in the dog and cat, less frequent in the pig (Csokor) and ox (Grimm), has sometimes been observed in the sheep (Oschatz), the goat (v. Niederhäusern), the stag (Prietsch), even upon the bat, rat (Hahn), and field mouse (Zschokke). It is produced by the Demodex folliculorum. This acarus exists frequently in the comedones of man's face, (dilated sebaceous glands), without producing any morbid phenomenon.

Natural History. Demodex folliculorum was discovered in 1843 by Henle and Simon in the comedones of man. At the same time Tulk found it upon the dog. It is a parasite of the group of Arthropodes, belonging to the family of the Dermatophiles, the order Acari and class Arachnides. At the present date we distinguish several varieties.

Demodex folliculorum, var. canis, cati, suis (also designated under the name of Demodex phylloides by Csokor), hominis, etc.

In the adult state the Demodex folliculorum is a vermiform parasite, shaped like a laurel leaf and one-third to one-quarter of a milimetre in length; it comprises three parts: the head, thorax, and abdomen, the first two being joined in a single organ. The

1 [Sarcoptic mange, which is determined by Sarcoptes scabiei, var. hydrochori, is at times localized on the head and feet, at other times it is spread over the whole body. It is indicated by a gelatinous pitchy oozing, by depilations, grayish or brownish scabs and an intense pruritus: When the disease is old the parasites are often accumulated upon the ears and feet, on the base of the paws, which become deformed and acquire enormous dimensions.

Symbiotic mange (auricular acariasis) is produced by Symbiotes auricularum, var. furonis. In the generality of cases its spread is so insidious that the troubles produced by it pass unnoticed. We may, however see the patients scratch their ears; we remark also an unusual weakness, depression, and torpidity, but the epileptiform symptoms observed in auricular acariasis of the dog and cat are exceptional in the ferret.

As soon as the parasites exist in large numbers they produce violent inflammatory phenomena, destruction of the tympanic membranes, lesions of the middle ear, caries of the internal ear, or a fatal meningo-encephalitis.] 
rostrum, which is lyre- or horseshoe-shaped, is formed of several chitinoid pieces. In Demodex phylloides it comprises: first, the camerostrum, which is the base of the whole masticating apparatus ; second, a pair of mandibles; third, a pair of maxillæ; fourth, maxillary feelers, poly-articulated and mobile; fifth, a tongue, uneven and stylet-shaped. On each side of the rostrum, and below, we find also two small pimples of clear shade, which Csokor mistook for eyes. The cephalothorax carries upon both of its sides four pairs of short and mobile feet, formed of three parts: hip, leg, and tarsus, the latter being fitted with five chitinoid points similar to claws. The lower aspect of the cephalothorax shows a median stripe and four lateral transverse stripes. The abdomen is long and transversely striated; it is fringed upon its borders and pointed posteriorly. The young parasites are small and narrow, and have but three pairs of legs; their abdomen is destitute of transverse stripes. The eggs are cordiform or fusiform. The larvæ undergo three metamorphoses before reaching the adult state.

Pathological anatomy. On microscopic examination of the skin we observe the demodex in the sebaceous glands (especially in the neighborhood of the excretory canals) and in the upper parts of the hair follicle. They exist in greater or less numbers; thirty to sixty are generally counted in a single gland, sometimes 100 to 200 and over. Almost all have the head directed toward the glandular cul-de-sac. We find eggs and larvæ mixed with the parasites.

When the demodectes are very numerous the sebaceous glands and their excretory canals are greatly dilated and sacciform ; inflammation begins in their neighborhood, an abundant leucocytic migration is produced, the periglandular tissue undergo purulent disintegration, and small abscesses are developed upon the surface of the invaded glands. But the process ends in suppuration only in cases where the acari exist in considerable number. When they are not very numerous they produce simply atrophy and the falling out of the hair.

The most serious alterations are observed in the pig. Several suppurating glands may become confluent and form voluminous purulent islands, which may contain as many as 1000 acari. Deep ulcerations follow these abscesses, the size of which often reaches that of a silver dollar. When the disease lasts for a long time the skin becomes sclerotic; it may become three to four times its normal thickness. 
Symptoms in the dog. Follicular mange is very common in the canine species; it is observed in all races, but retrievers and terriers are predisposed to it. Its intensity and course vary according to the individual : in some it remains localized for three months; in others it spreads rapidly over the entire surface of the body. While it is very contagious, few attempts to transmit it to the healthy dog have been successful. Haubner is the only author who has obtained a positive result. Attempts of experimental transmission to other species have not been more successful. Several cases of accidental transmission to man have, however, been reported. The propagation of the disease to the different regions is by rubbing; the pustules are torn open, and their contents, so to speak, sown upon the whole surface of the body. Extension after a first curative friction takes place in the same way.

The manifestations of follicular mange vary considerably according to the quantity of parasites accumulated in the sebaceous glands.

1. SQuamous Form. In a large number of cases the symptoms are reduced to the falling out of the hair and to an abundant epidermic desquamation; the surface of the skin, which is entirely dry, presents only the symptoms of a slight inflammation (squamous eczema). This form is especially observed upon the eyelids and in the neighborhood of the eye. Within a few days, a peri-ocular depilated blotch is produced, the integument being red and covered with thin epidermic lamellæ. The benign character of these manifestations, especially the absence of pustules, obscures the diagnosis and misleads the practitioner, who regards it as an eczematous disease. Any blepharitis accompanied by depilation should be subjected to microscopic study. This variety of mange quite frequently shows itself by disseminated circumscribed depilations upon the whole surface of the body, without the existence of the slightest pruritus; these symptoms then show a striking resemblance to those of scurvy. The increased pigmentation of the bare regions is a symptom of follicular mange.

2. Pustulous Form. This is more frequent than the preceding; it is characterized by an intense dermatitis with a formation of pimples and pustules, these latter being due to the suppuration of the sebaceous glands, which are overrun by the parasites. At times it is localized upon certain regions; at other times it is extended over the whole surface of the body. In the former case it is located preferably upon the forehead, lips, eyelids, and feet. Its 
chosen places are the head, the guttural and cervical regions, and the inner aspect of the legs.

At the beginning of the process the skin is tumefied, red, and hot; the hair is erect; so-called miliary buttons appear, which are soon transformed into reddish or whitish-blue pustules. The integument may acquire double its normal thickness; it is covered with a greenish-yellow, viscous, purulent, and bloody exudate, and also with dry yellowish or reddish-gray scabs. The hairs which are still upon the diseased regions may be torn out by the slighest traction; their roots are coated with a layer of pus. When the skin is folded or slightly compressed, blood or purulent masses will ooze out of it. The intensity of pruritus varies according to the individuals: at times it is very severe, but in most cases it is almost wanting; as a general rule, it is much less marked than in sarcoptic mange.

When, later, the objective manifestations of the disease are not modified by scratching or rubbing, the skin dries up, becomes wrinkled, and covered with lead-gray epidermic matter or scabs, between which bloody cracks and crevices are formed; it takes the appearance of the bark of a tree or of elephant skin ; it is sometimes strewn with hard pimpled projections. The non-pigmented regions are of a red or coppery shade, or appear as a rosy ground strewn with red-blue pustulous pimples (whence the old denomination, "red mange"). Lastly, we may also observe in some locations small circular depressions and papillary or connective tissue vegetations. The patients are thin and sensitive to cold, their expression is much altered, and the head reminds one of that of the hippopotamus. The general condition, however, is hardly disturbed; the appetite is ordinarily increased, and in some instances we may observe a true "hunger-evil" (the central organic changes being increased as a consequence of the cutaneous irritation).

These symptoms may be greatly modified when the animals scratch or rub themselves. The diseased surfaces are then transformed into suppurating wounds or large ulcerations. The inflammatory cedema of the subcutaneous connective tissue produces enormous tumefactions in some regions-on the head, in the neighborhood of the eyes, upon the neck, etc. The animals in which the disease has reached the degree of marked emaciation spread a particular odor, perceptible at some distance, and have a miserable appearance. They soon die from exhaustion or septicemia. 
Symptoms in the pig. In this animal follicular mange is marked by pimples from the size of a grain of sand to that of a hazelnut, which soon become transformed into inflammatory tumors, the dimensions of which progressively increase, and which, when opened, leave large ulcerations of the skin (Csokor). Varioliform efflorescences may be observed; these have been the cause of confounding follicular mange with variola (Csokor). The disease invades only the "soft regions," and those where the skin is thin : the neighborhood of the groin, neck, sternal region, flanks, abdomen, and the inner aspect of the legs; the upper part of the head, the back, and the external fascia of the legs remain generally untouched. The inside of the pimples contains from 50 to 100 demodectes, that of large abscesses from 500 to 1000 . The parasite is found in it in all degrees of development (the demodex of the pig is about twice as large as that of the $\operatorname{dog}$ ); we may also find "cuirasses" therein, derived from metamorphoses due to the acari. Follicular mange of the pig is much more contagious than that of the dog.

Symptoms in other species. In the cat the eruption is generally localized on the head (nose, eyes, and ears); these symptoms are the same as in the dog. In the ox Grimm observed a case in which the whole surface of the body, with the exception of the head and extremities, was covered with pustules of the size of a pea, on the surface of which the hair had disappeared; on compressing these pustules, a thick and clammy pus, containing a large number of demodectes, would run out of them. The animal was very thin; its development had been delayed. Various modes of treatment were tried, but did not produce any improvement. ${ }^{1}$

Diagnosis. The localization of the trouble upon the head and extremities, and the presence of reddish-blue pustules on these regions, clearly characterize follicular mange, and enable us to recognize it immediately. There are cases (squamous form) where the diagnosis can only be established with certainty by microscopic examination; this is generally very simple, requiring but a few moments. It consists in spreading between two lamellæ the contents of a pustule, and examining the preparation with an enlarge-

\footnotetext{
1 In 1885 Railliet and Nocard observed a case of demodectic mange upon a twoyear-old buck which had been born and raised at the Alfort School. Demodectes existed in large numbers in pustules mainly located upon the costal region and the flank.
} 
ment of fifty diameters. When no pustules exist, we examine the product obtained by scratching the skin or the roots of some hairs pulled out for that purpose.

The affections which may be confounded with follicular mange are:

1. Impetiginous eczema and traumatic or purulent dermatitis.

2. Pustulous exanthema of distemper.

3. Sarcoptic mange.

4. Inflammation of the eyelids (blepharitis).

5. Tonsurating scurvy.

In the dog we frequently observe in the neighborhood of the eyes, cheeks, and lips, rounded, depilated, confluent blotches, which show altogether the objective characters of tonsurating scurvy: redness, accumulation of dry scabs, and absence of pustules. Microscopic examination shows that it is, in fact, the dry form of follicular mange. We must, therefore, never neglect this valuable method of forming the diagnosis-so much the more because we may observe mixed cases in which follicular mange is associated with other dermatoses. It has been seen, namely, to coexist with sarcoptic mange (Siedamgrotzky), with tonsurating scurvy (Friedberger), with exanthema of the disease of young age, and with sarcoptic mange upon the same animal (Fröhner).

Prognosis. Follicular mange must be considered as a very serious disease. If spontaneous, cure is possible (Röll); it is extremely rare. There is no authentic instance of a final cure of generalized mange, even when under treatment for months; but apparent cures, followed by a return of the trouble at a more or less remote time, are not rare; particular instances of this kind are mentioned in our publications.

Disappointments are very common, and we can only be certain of having overcome demodectic mange after having kept a record for months of dogs which are apparently cured. Even in cases where it is localized, the prognosis must be reserved, for the treatment (because of the manipulations which it necessitates) is one cause of the extension of the trouble. At all events, it is proper to advise the owner of the rebellious character of this acariasis. In generalized follicular mange, except where animals of exceptional value are concerned, it is mostly decided to sacrifice the patients.

The prognosis also depends upon the constitution, temperament, and idiosyncrasy of the patients, but we can only judge of its influence by the effects of the treatment. 
Treatment. The number and diversity of medicaments which have been tried against follicular mange testify to the uncertainty and impotence of our armamentarium. It has been recommended to use various preparations containing carbolic acid, cresol, benzol, petroleum, balsam of Peru, styrax, mercurials, cantharides, flowers of sulphur, naphthalin, oil of juniper, sulphur ointments, etc.; also washing of the skin with bran bath, ash lye, and green soap. The medicaments which have given us the best results are balsam of Peru, cantharides ointment, sulphate of potash, and sublimate ointment.

1. Balsam of Peru, advised by Siedamgrotzky, must be used in daily frictions, to be repeated for weeks, taking care to fold the skin beforehand and burst the pustules. This agent cures animals having mange localized in a few regions, and even generalized mange. It has given us successful results when we were able to continue its use for some time; in one case the treatment lasted fifty-eight days. In an animal affected by follicular mange of much gravity we observed at the end of sixteen weeks an evident improvement, which might have been taken for a cure, but the mange returned some time later. In some cases the treatment must be prolonged for several months; it is very expensive, and the disease is generally aggravated during the first weeks of the treatment.

2. The medication used by Brusasco at the Turin School consists in washing the diseased skin with a solution of sulphate of potash (5 grammes; water, 1 litre), and theu rubbing it with cantharides ointment (cantharides ointment, 1 part; lard, 6 parts). It has several times given us a rapid cure in serious cases; but cantharides ointment is extremely dangerous for the eyes-it may produce a purulent panophthalmia which is sometimes fatal ; it is therefore proper in all cases to restrict ourselves to the use of balsam of Peru for the peri-ocular region. Brusasco's treatment is applied in the following manner : on the first day the animal, which has been shorn beforehand, is placed for some time in a bath which is prepared with 200 grammes of sulphate of potash and 70 litres of water (for a large $\operatorname{dog}$ ); on the second day cantharides ointment is applied upon one-third of the body, on the third day upon the second third, and on the fourth day upon the rest. Twenty-four to forty-eight hours afterward the patient is to be washed thoroughly, then he is left quiet for several days, and later the treatment is to be repeated. We do not by any means always 
obtain a cure. Friedberger has treated in this way for sixty-eight days a poodle affected by generalized follicular mange, but without the least success; twelve sulphurated baths were given, nine frictions with cantharides were made upon the whole surface of the body, and nine other partial applications made upon such regions as were particularly affected. Balsam of Peru used in frictions for sixteen weeks has not shown itself to be more efficient.

3. Frictions of sublimate ointment at 1 per cent. and lotions with a solution of this agent concentrated in the same degree, quite often give good results when used long enough. But these agents are dangerous; should the dogs lick themselves a fatal poisoning may be the consequence.

Sulphur baths, which are recommended by Mégnin, ${ }^{1}$ are used by many practitioners. They are continued for two months (a quarter of an hour every day); the sulphuretted hydrogen produced in this way is a very energetic destructive agent for these parasites. In Brusasco's treatment a part of the results obtained must be attributed to it.

We have never obtained any satisfactory results with carbolic acid or benzol. Naphthalin, which has been recommended quite recently, produces but an amelioration, never a cure (Siedamgrotzky's experiences). Cresol is sometimes sufficient for benign cases. The same is the case with ichthyol (Rabe). Bran baths (Martemucci) may act favorably in cleansing the skin and in relieving it from the demodectes deposited upon its surface; but they have not any acaricide action, and cannot by themselves produce a cure.

No matter what treatment used, a long time and great care are necessary in all cases; success is only possible under such conditions. Every day, before applying the treatment, the pustules must be compressed and made to burst. The hair must be cut from time to time. Finally, the patients must be stimulated by an abundant and very nourishing alimentation.

The prophylaxis consists in separating mangy dogs from healthy animals, and in disinfecting the stables or kennels. From a sanitary point of view, it will be proper to watch the patients and kill the incurable animals. Such is the only efficient prophylaxis.

1 Mégnin has also advised bisulphide of carbon ointment:

Bisulphide of carbon

Lard or vaseline

Sig.: One friction morning and evening after having broken the pimples.-N. D. T. 


\section{CUTANEOUS DISEASES PRODUCED BY FILARIA (THREADWORMS).}

1. In the horse we observe a particular disease of the skin which is designated under the names of summer wounds, pruriginous dermatitis (Rivolta) granulous dermatitis (Laulanié). It well deserves the last description, as it is characterized by granulations which are related to the presence of nematode worms. Its first description, which is due to Ercolani, dates back to 1861 (Rivolta). The integument is thickened and covered with vegetations containing small encysted concretions easy of extraction. According to Laulanié, the whole thickness of the skin is overrun with spheric or elliptic granulations, the centre of which, having undergone fatty degeneration, contains a spiral-shaped nematode worm, which is marked by a characteristic transverse striation. In old granulations this parasite is often destroyed, but it may preserve its vitality for years (Laulanié). The process begins with a cellular infiltration of the subcutaneous connective tissue of the derma. The disease retrocedes during the winter, and is aggravated in summer under the more active influence of the circulation. Rivolta thinks that cure can only be obtained by the removal of diseased tissues. Condamine and Drouilly have observed in the horse hemorrhagic pimples produced by threadworms (see page 533, footnote).

It is probable that some of the troubles described under the name of tuberculous eruption by the ancients, especially by Haubner, and sometimes mistaken for farcy, belong to the group of granuloverminous dermatites.

2. In the dog, Siedamgrotzky observed a pustulous eruption which was produced by nematode worms (nemathelminths). It was located principally upon the external aspect of the legs. The pustules, which were small, were surrounded by a red zone and occasioned a very intense pruritus. They contained one, two, or three embryos of nemathelminths, with a tapering or pointed tail, 0.04 to 0.7 millimetre in length, and coming undoubtedly from hair follicles; they have also been found upon the floor of the stable, under the litter near adult subjects, and were about one millimetre in length. Rivolta has also found filaria in several ulcerous lesions of the dog's skin.

Under the name of squamous herpes, Semmer has described, in 
the horse, an affection of the skin which is localized upon certain regions, and due to numerous round worms situated between the epidermis and derma.

\section{SKIN DISEASES CAUSED BY INSECTS.}

Some insects annoy the animals of our different species without producing any true morbid process in the integument. During the warm season they torment and irritate the animals constantly during meals and are the cause of wasting part of the food ; among them we must mention the common fly (Musca domestica) and the corvina fly (Musca corvina), which suck the perspiration. Others -the gadfly of the ox (Tabanus bovinus), the small rain breeze fly (Homatopota; T. pluvialis), the little blinding gadfly ( $T$. coccutiens), etc.- suck the blood, especially in regions where the skin is thin. Others again-the common gnat (Culex pipiens), the stinging stomox (Stomoxys calcitrans), the meteoric anthomyia (Anthomyia or Hydrotoea meteorica, the creeping simulium (Simulium reptens), and the most dangerous of all, Kolumbacz's simulium (S. Columbaczensis) - throw themselves upon the animals and make a great many bites in the skin, which are followed by a more or less intense dermatitis. The larvæ of some live in the integumentary membrane: the calliphora, or blue meat-fly (Musca vomitoria), the carnivorous sarcophagus (Sarcophaga carnaria), the cadaver fly (Musca cadaverina), which deposit their eggs in wounds and ulcerations, finally the hypoderma of the ox (Estrus bovis), the larvæ of which perforate the skin in order to penetrate as far as the subcutaneous connective tissue.

Among these insects those which offer the most interest from a pathological standpoint are :

1. Simulium of Kolumbacz, or spotted simulium, which is of ash-gray color, and about the size of a flea. It was first observed in the neighborhood of Kolumbacz, Hungary (Banat of Temesvar); in 1783 it produced the death of nearly six hundred animals (horses, oxen, pigs, sheep) in this region. It is found in Austria, Bohemia, Prussia, and several German provinces. After freshets immense swarms of these flies are often seen; they come in actual clouds, which fall upon the animals and literally cover them with a blackish mantle, penetrating into the natural orifices (mouth, nose, ears and eyes, vagina and rectum), and covering the 
skin and mucous membranes with bites. Anxious and crazed, their victims run away or throw themselves upon the ground moaning ; many perish on account of the inflammation and obliteration of the bronchi. Those which resist show serious symptoms; the skin becomes covered with a large number of small pimples which are hard and painful, the mucous membranes are inflamed; the inflammatory phenomena persist often for from three to four weeks. On animals which succumb we find a sero-sanguinolent infiltration of the subcutaneous connective tissue, also an inflammation of the exposed mucous membranes; finally, hyperemia and œdema of the lung. When the simulia have penetrated into the pharynx, larynx, and trachea, the mucous membrane of these canals is much tumefied; the respiratory tract is sometimes entirely obstructed. The treatment consists in making cold-water lotions upon the skin, and in practising scarifications upon the accessible regions of the inflamed mucous membranes. As a prophylactic, lotions of tobacco lye have been recommended; we may also use cresol, carbolic acid, tar, asafotida, or a decoction of walnut leaves. in vinegar.

2. Hypoderma of the ox (Estrus or Hypoderma bovis). This fly produces in this animal, sometimes also in the horse, the donkey, and the sheep, nodes which are ordinarily observed upon the shoulders, back, lumbar, croup, and thorax. The eggs of the hypoderma, deposited upon the skin, give birth to larvæ which perforate this membrane and become lodged in the subcutaneous connective tissue, where they remain for about nine months (from July to March). While their development is going on they irritate the neighboring tissues, which become inflamed and infiltrated with pus, and form tumors which may reach the size of a pigeon's egg. When the larva has reached the adult state it leaves its connective tissue covering and escapes; dilating, by means of the posterior part of its body, the cutaneous opening which it has made. These parasites are not generally numerous, but we find at times from fifty to one hundred; then they may occasion serious trouble: the animals become emaciated, now and again they are under the influence of an intense excitement; when at pasture they go through uncontrollable movements, toss the tail, bellow, run toward water, and try to enter the stable. The treatment consists in securing the removal of the ripe larvæ by compressing the tumors, or in incising these by means of a bistoury. As prophylactic means, we 
recommend hygienic care of the skin and frictions upon the upper regions of the trunk with tar liniment, cresol or diluted carbolic acid, solutions of asafoetida, and decoctions of walnut leaves.

Hinrichsen is said to have found the larvæ of the hypoderma in the spinal canal of the ox. He thinks that the animals of the bovine species ingest the eggs of the parasite with the food, and that the young larvæ reach the medullar canal by passing from the stomach or intestine; they live in it for from five to six months; they then emigrate in order to locate themselves under the skin. They have, so far, been found but rarely in the rhachidian duct.

In the dog, Railliet and Lenoir have mentioned lesions similar to those occasioned by the hypoderma of the ox, and which are due to the introduction under the skin of larvæ of Ochromyia anthropophaga. These parasites give rise to the formation of hard, painful tumors, which are of the size of a lentil to that of a nut, and provided with a central cylindrical opening, which becomes softened within six or seven days, allowing the larvæ to escape. They may be found upon various regions, but especially upon the tail, ears, and feet. The extraction of the larvæ is the only efficient treatment.

Disease of the larve of the Lucilia, which exists in lambs, is especially found in Holland. According to Jennes and Van Laer, it is due to the larvæ of the Lucilia sericata. The lucilia deposits its eggs upon the internal aspect of the thighs of lambs, which are affected by diarrhea; the larva seeks those parts of the body where the wool is abundant (the base of the tail, lumbar region, etc.); it forms a sort of nest under the fleece and perforates the skin like a sieve. Adult animals are rarely affected. The parasites must be removed and the skin given a lotion of a carbolated solution or a decoction of tobacco; these are the two principal indications of the treatment.

In tropical countries some meat-flies may occasion the death of young animals of the bovine species. According to Frantzius, ${ }^{1}$ 2000 calves are said to perish every year as a consequence of inflammatory conditions produced by larvæ of these flies upon the umbilical region.

The means directed against winged insects in general are frictions with liquids having a very strong odor : cresol ( 2 per cent), asafoetida (60 grammes dissolved in a glass of vinegar and two

1 Frantzius: Virchow's Archiv, Bd. xliii. 
glasses of water-Martin), benzine, petroleum, fetid animal oil, decoction of walnut leaves or tobacco, bitter decoctions (absinth, gentian, nux vomica), alcoholic extract of Persian insect powder.

\section{LICE (HÆMATOPINUS) AND TRICHODECTES.}

1. Lice are apterous insects, the tubular mouth of which is disposed in the shape of a suction apparatus. They fix their eggs (nits) upon the hair of animals which are affected by them. Most of our domestic species nourish particular species of lice: the horse is affected by the Homatopinus or Pediculus equi; the ox, by the $H$. eurysternus and tenuirostris; the pig suffers from the $H$. suis; the dog from the $H$. pilifer; and the goat from the $H$. stenopsis. The selected regions of these parasites are the neck, back, the base of the tail, and the neighborhood of the horns in the ox, and the inner side of the hind legs in the pig. They are particularly frequent in the dog and ox; in the latter species they may be found upon subjects which are perfectly well kept. In general, they become easily and rapidly developed upon poorly fed, weak and debilitated animais; their presence is almost always a symptom of insufficient care of the skin. They produce itching, partial depilations; besides, we observe nits in more or less large numbers, and an abundant epidermic desquamation; the animals often spread a disagreeable odor, and, in the dog especially, the skin ends by becoming covered with a viscous, impetiginous coating.

2. Trichodectes do not suck the blood; they attack the hair and devour the epidermis. The head is larger than that of lice. They locate in preference upon the head, the neck, and the legs. We find special species of trichodectes in the $\operatorname{dog}(T$. latus), the horse (T. pilosus et parumpilosus), the ox (T. scalaris), the cat ( $T$. subrostratus), the goat (T. climax), and the sheep (T. sphoerocephalus). This latter produces a very intense itching, which may lead to suspicion of the existence of psoroptic mange. The principal therapeutic agents utilized for the destruction of hæmatopinus and trichodectes are: 1. Mercurial ointment. It may be used in small quantity for all domestic species, but it is especially appropriate for the horse, pig, and dog. In the ox, which is very sensitive to mercurial preparations, we have obtained good results by making frictions upon the nape of the neck and the integument at the base of the horns with five grammes of mercurial ointment; we have 
never observed any symptoms of poisoning. In the dog, we may apply a small quantity of ointment under the collar (as much as the size of a pea or acorn); good results are also obtained with lotions of sublimate at 1 per cent., using it upon small surfaces at a time, and carefully drying the skin after each lotion. 2. Persian insect powder spread between the hair. It is generally too weak for large animals. 3. Decoctions of tobacco (4 to 5 per cent.). They are very advisable for the sheep and $\operatorname{dog}_{0}$; they are less adapted for the horse and ox.

Some practitioners use tobacco lye which is known under the name of nicotina (see page 566). We may also try lotions of cresol water ( $2 \frac{1}{2}$ per cent.), a decoction of staphisagria seed in vinegar (1:20), a mixture of powdered cevadilla and staphisagria, white hellebore (of each 1 part) and anise powder ( 2 parts) ; benzol associated with green soap, alcohol or oil (Dinter); carbolic acid in a solution of 5 per cent., essence of anise (mixed with ordinary oil, $1: 10$ ) for pet dogs. Washing with arsenical preparations (arsenous acid, potash, of each 15 grammes, water and vinegar of each $1 \frac{1}{2}$ kilo, recommended by Viborg, later by Schleg, are very efficient, especially for animals of our large species ; but this remedy requires. caution. A decoction of nux vomica, advised by Eck, is very dangerous and must not be used. Concerning fetid animal oil, which is commended by Menkert, it may be replaced with advantage by cresol. Thierry combats trichodectes of the sheep with a mixture of cevadilla seed powder and Persian powder. The eggs of these parasites are rapidly destroyed by vinegar, which dissolves their shell.

\section{VARIOUS OTHER PARASITES OF THE SKIN.}

1. Fleas are seen on the $\operatorname{dog}$ (Pulix sanis) and the cat (Pulex felis) -they suck the blood, cause itching, and produce cutaneous eruptions; their excrements soil the skin, and often accumulate on the base of the hair in thick layers, somewhat similar to coffee-grounds. The most advisable therapeutic agent is Persian insect powder ; it is applied upon the skin after having slightly moistened it. It is proper to remove animals which are thus treated in order to prevent the return upon the integment of the expelled parasites after they have remained in the stables for some time. Baths of cresol water ( 2 per cent.) and washing with this preparation are also very favorable. 
2. Trcks are insects which have the same form as the acarus, but they are larger. They are found in woods, in grass ; they become fixed upon the skin of domestic animals, of which they suck the blood. We recognize the tick of the dog (Ixodes ricinus), which affects the dog, ox, and sheep : the reticulated ixode (Ixodes reticulatus), a parasite of the ox and sheep), and the American ixod( (Ixodes Americanus), which attacks the horse in certain parts of the New World. These insects may be removed by means of common oil, cresol, or carbolated oil ; by essences (turpentine, anise, cumin, fennel, etc.). It is not proper to tear them out; when this is done the head of the parasite remains in the substance of the derma.

3. Hippoвоscж (Pupipares) are observed upon the horse (Hippobosca equina) and the sheep (Melophagus ovinus). The hippoboscæ of the horse are located upon the base of the tail, in the neighborhood of the anus or upon the lower aspect of the abdomen; they irritate the animals more by their rapid movements than by their bites. The melophages are particularly found upon sheep going to pasture; they suck the blood and produce a very intense cutaneous irritation; the animals rub themselves, they bite the fleece and spoil it ; the wool is often colored green from the excrements of these parasites. The agents to be used to combat melophages are decoctions of tobacco, lotions with cresol or carbol solutions, and applications of mercurial ointment ( 3 to 4 grammes per head) along the back and neck. The animals must be shorn beforehand. In experiments instituted to determine the degree of toxicity of cresol against the Melophagus ovinus, Fröhner has obtained the following results: Adult parasites are killed within ten to twelve minutes by a few drops of cresol solution at 3 per cent., and in fifteen to twenty minutes with a solution of 0.51 per cent.; mangy sheep carrying many melophages are entirely freed by a single bath of cresol at 2.5 per cent.

4. Dermanyssus locates in preference upon chickens, pigeons, swallows, and house birds; they lodge also in poultry aviaries and in cages. They are of a blood-red or red-brown color. During the night they overrun any mammals within their reach (oxen, horses, dogs, and cats), and occasion intense itching, even eruptions. They are sometimes found in considerable numbers under blankets (Trasbot). In the horse Steinbach observed a dermanyssic eruption similar to mange; the skin was covered with scabs, which were separated by narrow crevices; the parasites nested in the front 
and back, mane and the tail. In eight cows Möbius observed an affection of the same nature, which was accompanied by rounded depilated spots. They were treated unsuccessfully for several years, when they disappeared spontaneously after the destruction of several swallows' nests which were in the stable. Upon two horses which were tormented by dermanyssi, Prietsch observed a large number of superficial wounds which were located mainly upon the neck and back; the diseased regions were depilated and covered with a furfuraceous matter or thin crusts; itching was very great in the evening and during the night. In animals of the bovine species these parasites, by becoming accumulated in the ear-passage, produce at times an internal otitis (Gassner); they may penetrate into the middle ear and occasion cerebral accidents. In oxen which were killed as being hydrophobic, Stadler and Schuemacher found a considerable quantity of dermanyssi in the external and middle ear. (See Cutaneous Affections of Poultry).

As to treatment, we must remove chicken coops from stables and barns, destroy the swallows' nests these may contain, and use insecticides (cresol, carbolic acid, etc.). The stables must also be disinfeeted.

5. Autumnal lepta (Leptus autumnalis). This is found in dry grass, ripe grain, upon elder and willow trees. It affects the $\operatorname{dog}$ at times (Friedberger), and produces a pustulous eruption in the neighborhood of the muzzle, eyelids, ears, and inner surfaces of the anterior and posterior members, on the belly, genital organs, etc. At the beginning we observe upon the skin small red pimples (pimples or pustules) from the size of a poppy to a hemp-seed, on which the leptus is fixed. They may easily be recognized by their scarlet color. ${ }^{1}$

On pressing these pimples a bloody liquid oozes from them. Later they become larger and may reach the size of a ten-cent silver piece, and may also become confluent; from their surface the hair falls out,.partially or completely. The skin is red and sensitive; generally we find a moderate pruritus. The eruption may last for weeks, but it disappears by using preparations (ointments, glycerole) with a base of carbolic acid or cresol.

1 According to Mégnin this seems like the hexapod larvæ of the silky Trombidion. -N. D. T. 


\section{ADDENDA.}

\section{CUTANEOUS AFFECTIONS OF BIRDS WHICH ARE CAUSED BY ANIMAL PARASITES.}

\section{Mange of Poultry.}

Acari hold the first place among the numerous parasites living upon birds in poultry yards. They produce two principal kinds of mange.

1. Sarcoptic mange, produced by the Dermatoryctes mutans (Ehlers), also designated under the names of Sarcoptes mutans (Robin), Knemidocoptes viviparus (Fürstenberg).

2. Symbiotic mange, which is due to the Symbiotes gallinarum discovered by Caparini. According to Trouessart and Neumann, this parasite is said to be identical with the Epidermoptes bilobatus (Rivolta).

I. Sarcoptic mange. The Sarcoptes mutans was discovered in 1859 by Reynal and Languetin. It resembles closely the sarcoptes of mammals, from which it differs in some of its characters, especially in the disposition of the mandibles. The females are larger than the males ( 0.4 millimetre in length); the body is spheroidal; the males are rather of an ovoid form ( 0.2 millimeter in length). They are of a dirty yellow color. These parasites are provided with four pairs of legs in the adult state, and three pairs when in the larval form; the anus has two long hairs, one on each side. It is a burrowing acarus, digging sub-epidermic galleries.

It is frequent upon the chicken, but we also observe sarcoptic mange upon pigeons and exotic birds; foreign gallinaceous races are predisposed to it. Mégnin found it upon turkeys, pheasants, and small pet birds. In 1885 Railliet described, in the pigeon, a particular mange produced by an acarus related to Sarcoptes mutans, and which he designated Sarcoptes loevis, var. columboe. Quite recently he has found a second variety of the same acarus on the chicken-Sarcoptes laevis, var. gallinoe. The Dermatoryctes described by Friedberger in 1886-87 is very likely identical with the Sarcoptes loevis, Raill. The fact of the great variability of these parasites permits the supposition that Sarcoptes mutans and S. loevis constitute but two varieties of the same species. According to 
Railliet, foot mange determined by S. mutans would be less contagious than that of the body produced by S. loevis.

In the chicken, foot mange (calcareous tarsa, fish-scale disease), is marked by very characteristic symptoms. It is located upon the tarsa, exceptionally on the comb, or on the fleshy lobes of the lower mandibles and the bill (a contagion which is produced by picking and scratching). At the outset we observe upon the anterior aspect of the extremities small grayish spots which gradually eularge and soon form rounded indented scabs; later, the whole surface of the foot is covered with irregular, rugous, grayish-yellow, porous and friable scales, which may reach a thickness of one centimetre, showing an imbricated disposition of the different lamellæ, which are pearly in appearance and aie greasy to the touch. The feet, which are often enlarged and deformed, seem to be coated with lime or clay (calcareous feet). Under the scabs, which are mainly formed of epidermic scales and of dried-up inflammatory exudate, are found the acari ; the skin is red, tumefied, infiltrated with blood and pus; pruritus is quite intense; the animals rub and scratch themselves, and pick the scabby covering. When the trouble is old the movements are painful and the standing position is often tiresome. Emaciation, cachexia, and even death ensue in serious cases.

Its treatment is very simple. The scabs must be removed after having been first softened with tepid water; we then apply one of the following agents upon the diseased surfaces: glycerin, green soap, carbolated or creosotic ointments (1:10 to 20) tar, balsam of Peru, styrax, Helmerich's ointment, etc. We must also disinfect the coops and perches.

II. Symbiotic or rather EPIDERmic mange is generally localized on the cervical region and the thorax ; it may, however, spread very rapidly to the whole surface of the body, even to the comb and to the fleshy lobes of the lower mandibles.

The skin becomes covered with thin, transparent, straw-yellow, stratified epidermic scales, which soon form thick, dirty yellow, hard crusts, similar to dried dough, under which the skin is hyperemic; while it does not produce pruritus, this mange leads quite rapidly to weakness and wasting. Many birds die.

The treatment is the same as that of the preceding trouble. Friedberger observed the coexistence of these two manges upon the same animals.

Under the name of Sarcoptes cysticola (Laminoscoptes gallinarum) 
several authors have described an acarus similar to the sarcoptes, which is found upon the surface of the skin and in the connective tissue. It sometimes occasions a cutaneous eruption which is similar to mange (Unterberger).

\section{Other Cutaneous Diseases of Poultry Caused by Animal} Parasites.

Among other parasites of birds which determine particular diseases we must mention the following:

1. Harpirhynchus of the domestic pigeon. The Harpirhynchus nidulans has its habitat in the bulbs of the feathers, which are swollen into capsules or yellow cysts of the size of a pea to that of an acorn. They are also found in cutaneous tumors of the sternal region and of the lower part of the wings.

The contents of these cysts consist almost exclusively of harpirhynchi; there are found in them by hundreds. When these lesions exist in large number on the surface of the skin the animals lose flesh and die.

2. Vermiform Hypodectes of the pigeon (Hypodectes columbarum). They are located in the subcutaneous cellular tissue, on the surface of the peritoneum, and in the connective tissue of the aorta. This parasite is found to represent the hypopial nympha of an acarina which was before unknown. It occasions slight phlegmasia and moulting trouble, and prevents regeneration of the feathers.

3. Syringophiles of the Chicken ANd pigeon (Syringophilus [Picobia] bipectinatus). They live in the tail quills and wing feathers, producing the deformation and later the falling out of these organs as well as irregularities of the moulting process. The stem of the feathers has lost its transparency; it is generally bent at its extremity, and its puip is replaced by an opaque, purulent matter. These are the only symptoms to which they give rise.

According to Heller, in Schleswig-Holstein 70 to 90 per cent. of the chickens are said to carry Syringophilus bipectinatus. In the peacock we find a distinct species, Syringophilus [Cheyletus] uncinatus.

4. Acarina of pigeon feathers (Zürn). This species is sometimes found in considerable quantity between the feathers; it may occasion emaciation and a fatal exhaustion. According to Zürn, thrush of the anterior regions of the head in the pigeon is 
said to be due to this acarina. (Meyninia asternalis is undoubtedly meant.)

5. Dermoglyph of the Guinea Fowl (Dermoglyphus or Analges minor, var. similis). It was discovered by Nörner within the stem of the feathers of the Guinea fowl. This acarina is entirely harmless, it does not alter the feathers at all, and is of no scientific interest.

6. Dermanyssus of Birds (Dermanyssus avium). This is an acarian sucker, which lives upon the blood of its host; it must be classified among the most dangerous ectoparasites. It is especially found upon chickens, pigeons, sometimes upon house birds. As seen above, the dermanyssi attack also the horse, dog, ox, cat, and man. During the day they stay in the cracks of the floor, walls, or cages, and those on the lower surface of the perches. During the night they spread upon the poultry, covering these with their bites and filling themselves with blood. The birds lose flesh; some, especially young ones, die from exhaustion.

We also find quite frequently the dermanyssi in the nasal cavities, where they may determine a catarrh of the mucous membrane, leading sometimes to the death of young birds (Zürn). They have also been found in the ear passage.

7. The bordered Argas (Argas marginatus s. reflexus) of the pigeon. It is found particularly in France and Italy. Like the dermanyssi, it is a nocturnal blood-sucking parasite.

8. Pennivora. These are the Mallophages or Ricinides, which act upon chickens like the trichodectes on mammals. They live exclusively upon feathers and epidermic débris. They are sometimes found lodged upon the skin by means of their buccal organs (concerning the different families and species, see Zürn's work ${ }^{1}$ ). When they exist in large numbers they cause nutrition troubles. They develop with extraordinary rapidity upon sick birds.

9. The Bird Flea (Pulex avium). It is particularly common upon pigeons.

A great number of drugs may be directed against these parasites. Zürn combats syringophiles and acari, which affect the feathers, with essence of anise and rosemary (diluted in 20 to 50 parts of water or oil), balsam of Peru and styrax ( 1 part to 4 parts of alcohol); he advises keeping the birds perfectly clean and giving them plenty of sand. Sprinkling with a water solution of cresol,

1 Zürn: Die Krankheiten des Hausgeflügels, Weimar, 1852. 
1 per cent., is also very efficient. Against the dermanyssus we generally use Persian insect powder and washings with diluted essence of anise. Every morning the corners and cracks of the woodwork, perches, etc., should be sprinkled with cresol, carbolic acid, or kerosene; the premises should be washed with warm water to which is added a small quantity of anise; the walls ought to be whitewashed ; finally, wash the nests and cages with boiling water. The pennivorous aptera are removed by Persian insect powder, also by any kind of essence, by decoctions of anise, parsley seed, etc. In order to enable the poultry to take a dustbath easily, that they may rid themselves of these parasites, we arrange in a corner of the chicken-yard a layer of sand or a mixture of sand and cinders. It is also advisable to throw a certain quantity of this upon the floor of the coop. 





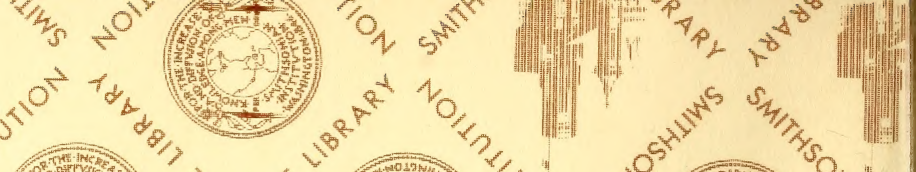

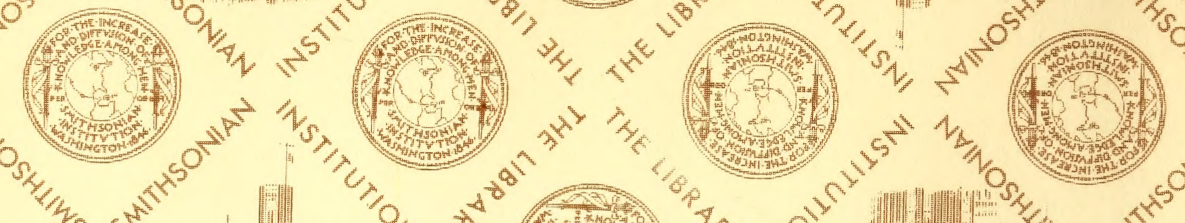

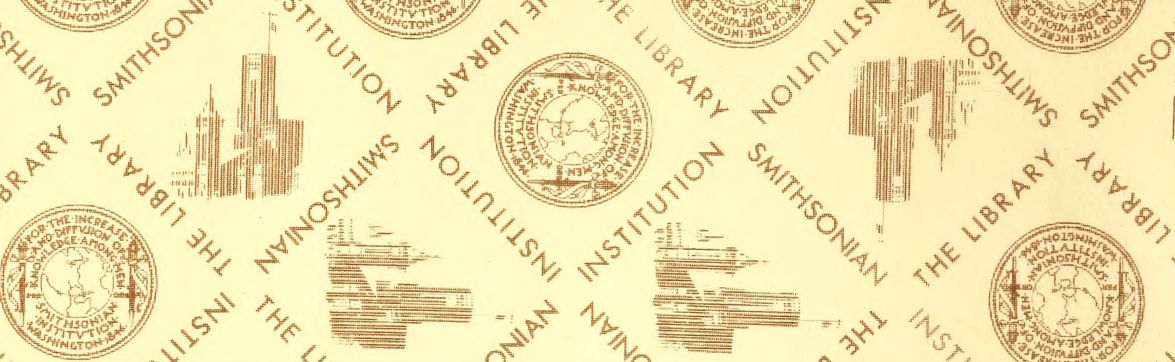

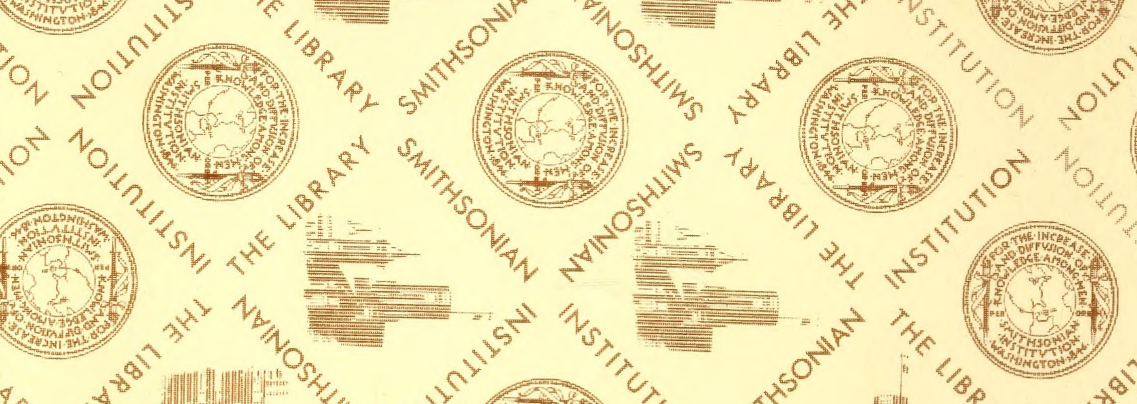

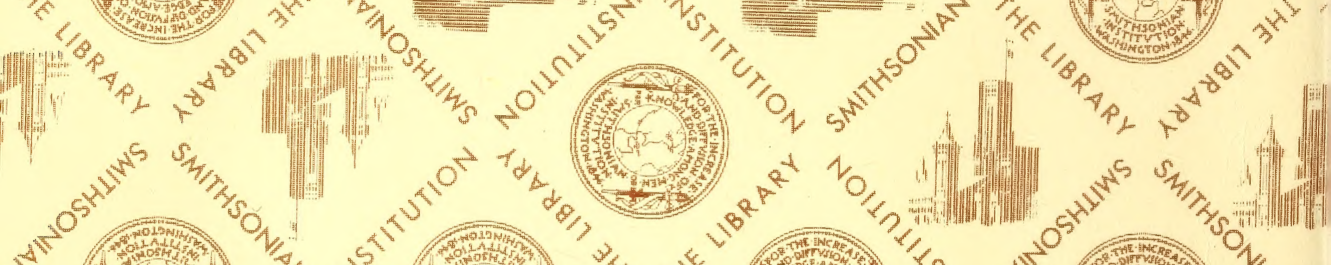

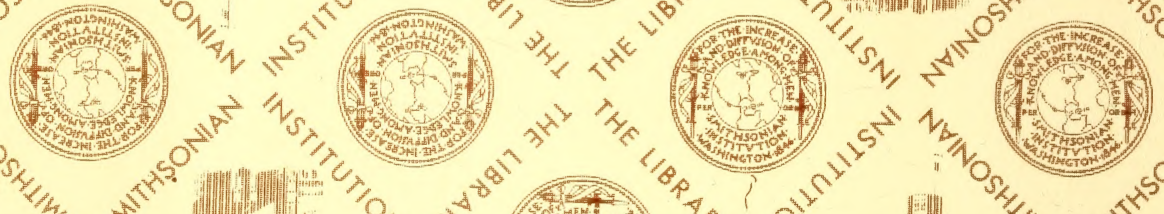

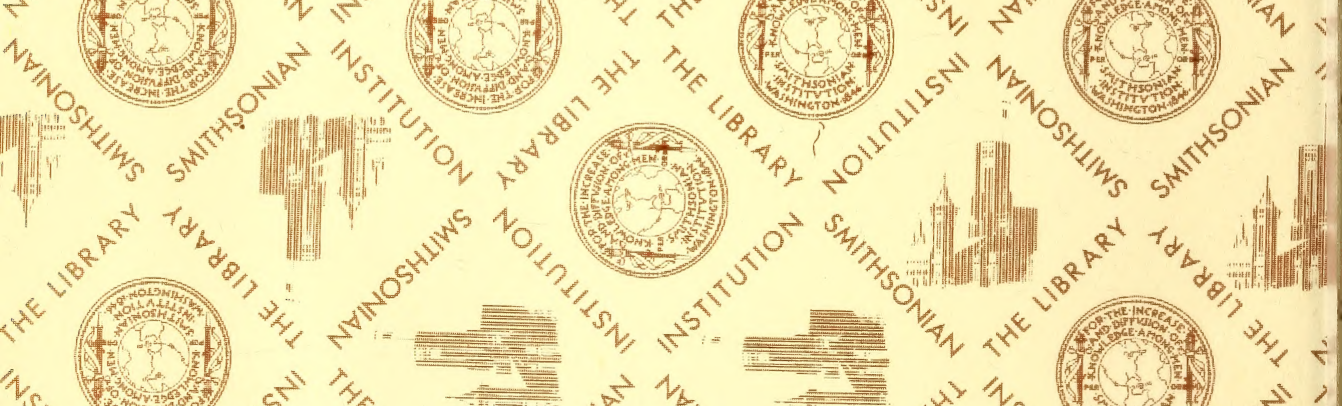
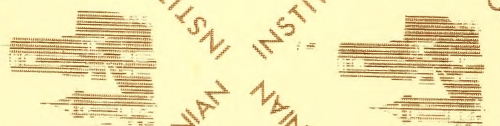
